\title{
Identification of Multivariate Stochastic Functional Models with Applications in Damage Detection of Structures
}

Ph.D. Thesis

John D. Hios

Submitted to the Department of Mechanical Engineering \& Aeronautics University of Patras, Greece

June 2012 



\section{Examination Committee}

- Spilios Fassois, Professor Ph.D. (supervisor)

Department of Mechanical Engineering \& Aeronautics

University of Patras, Greece

- Dimitris Saravanos, Professor Ph.D. (advisor committee member)

Department of Mechanical Engineering \& Aeronautics

University of Patras, Greece

- Anthony Tzes, Professor Ph.D. (advisor committee member)

Department of Electrical \& Computer Engineering

University of Patras, Greece

- George Bitsoris, Professor Ph.D.

Department of Electrical \& Computer Engineering

University of Patras, Greece

- Ioannis Koutrouvelis, Professor Ph.D.

Department of Mathematics

University of Patras, Greece

- Costas Kravaris, Professor Ph.D.

Department of Chemical Engineering

University of Patras, Greece

- Dimitris Skarlatos, Associate Professor Ph.D.

Department of Mechanical Engineering \& Aeronautics

University of Patras, Greece 



\section{Acknowledgments}

This thesis would not have been possible without the guidance, motivation and support from several individuals. I owe my gratitude to all these people who not only made this thesis possible, but because of whom my experience as a Ph.D. student has been one to cherish forever.

I wish to thank my supervisor Prof. Spilios Fassois for giving me the chance, motivation and freedom to complete this thesis. Without his guidance and support this thesis would not have been possible.

Dr. Dimitris Dimogiannopoulos (T.E.I. Piraeus) is greatly acknowledged for his support and inspirational discussions. Also, I would like to thank all the current and past members of the SMSA Laboratory - Lecturer John Sakellariou, Dr. Minas Spyridonakos, Dr. Fotis Kopsaftopoulos, Dr. Dimos Rizos and Dipl-Eng. Paul Michaelides for their support and encouragement.

Last but not least, I would like to thank my family for their support, patience and understanding.

John D. Hios

Patras, June 2012 



\section{Dedication}

To my family,

with love and appreciation 



\section{Contents}

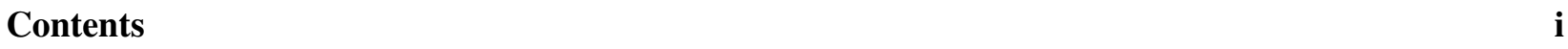

Summary $\quad$ v

$\begin{array}{lll}\text { Nomenclature } & \text { vii }\end{array}$

1 Introduction $\quad 1$

1.1 The general problem and its importance . . . . . . . . . . . . . . . 1

1.2 State-of-the-art . . . . . . . . . . . . . . . . . . . . . . . 2

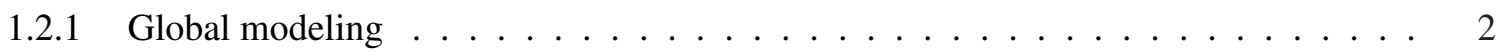

1.2.2 Damage detection under different temperature conditions . . . . . . . . . . . . 4

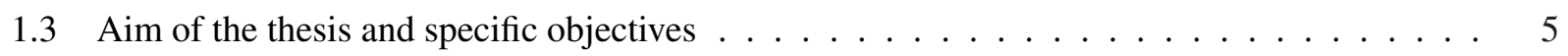

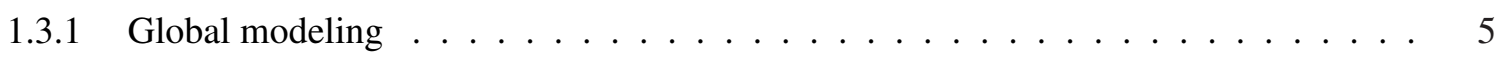

1.3.2 Damage detection under different temperature conditions . . . . . . . . . . . 6

1.4 Novel contributions . . . . . . . . . . . . . . . . . . . . . . . . . . 7

1.5 Overview of the thesis chapters $\ldots \ldots \ldots \ldots \ldots \ldots \ldots$

2 Identification of functionally pooled models: the Functionally Pooled VARX (FP-VARX) parametrization $\quad 13$

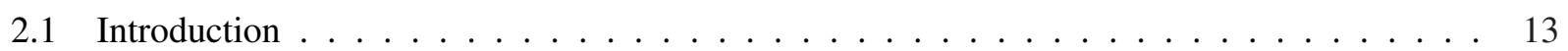

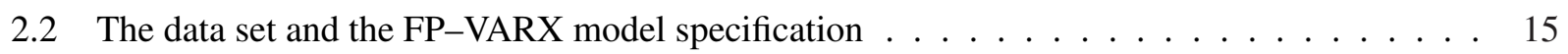

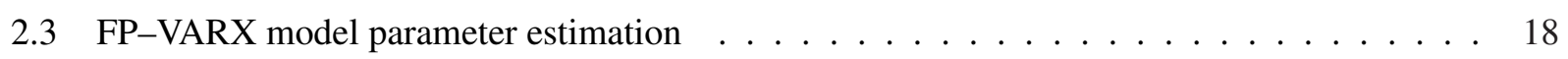

2.3.1 LS-based estimators . . . . . . . . . . . . . . . . . . . . . 18

2.3.2 The conditional ML estimator . . . . . . . . . . . . . . . . . . . 20

2.4 Large sample properties of the LS and ML type estimators . . . . . . . . . . . . . 20

2.4 .1 The LS-based estimators . . . . . . . . . . . . . . . . . . . . . . . 20

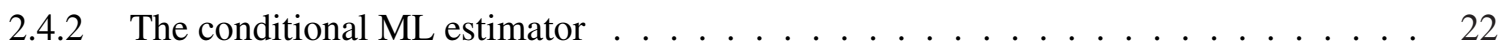

2.4.3 Accuracy of FP-VARX model parameters at the $k$-th operating condition . . . . . 23

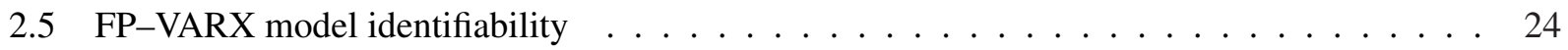


2.6 FP-VARX model structure specification and validation $\ldots \ldots \ldots \ldots \ldots \ldots$

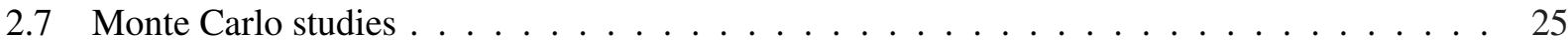

2.7.1 Case study I: An FP-VARX $(2,0)_{[3,3]}$ model $\ldots \ldots \ldots \ldots \ldots \ldots \ldots \ldots$

2.7.2 Case study II: An FP-VARX $(3,1)_{[4,4]}$ model $\ldots \ldots \ldots \ldots \ldots \ldots$

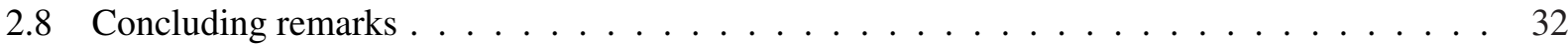

Appendix 2.A: Tutorial example of $\operatorname{FP-VARX}(n a, n b)_{[p a, p b]}$ parameter estimation $\ldots \ldots \ldots$

Appendix 2.B: Additional results on the LS and ML type estimators $\ldots \ldots \ldots \ldots$

Appendix 2.C: Additional results on FP-VARX model identifiability . . . . . . . . . . . . . . . 45

Appendix 2.D: Shifted Chebyshev polynomials of the second kind ～. . . . . . . . . . . . 50

Appendix 2.E: FP-VARX WLS estimation via QR implementation ～. . . . . . . . . . . . . . . 50

Appendix 2.F: Additional results on Monte Carlo studies ～. . . . . . . . . . . . . . . . . . . 54

3 Stochastic identification of temperature effects on the dynamics of a smart composite beam: assessment of multi-model and global model approaches

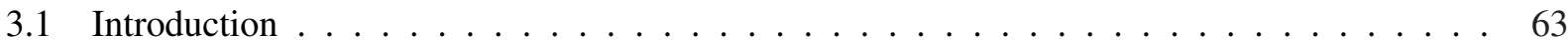

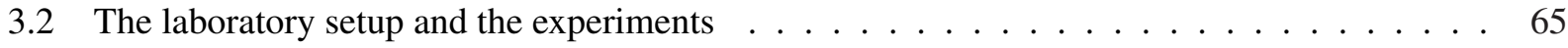

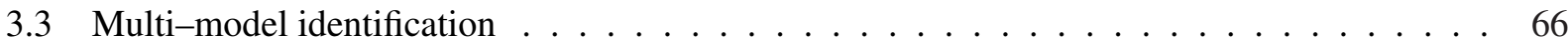

3.3.1 Non-parametric Welch-based multi-model identification . . . . . . . . . . . . 66

3.3.2 Parametric VARX-based multi-model identification . . . . . . . . . . . . 66

3.4 Global model identification . . . . . . . . . . . . . . . . . . . . . . . . . . . . . 69

3.4.1 Classes of stochastic global models . . . . . . . . . . . . . . . . . . 69

3.4.2 Global model identification methodology . . . . . . . . . . . . . . 70

3.4.3 Global model identification results . . . . . . . . . . . . . . . . . . . . 72

3.5 Assessment of the multi-model and global model approaches . . . . . . . . . . . . . . . . 74

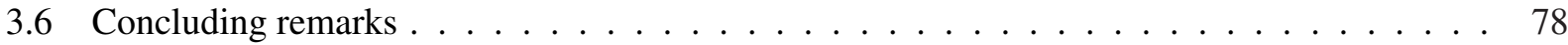

Appendix 3.A: Material properties of the glass-polyester composite and the piezoceramic patches

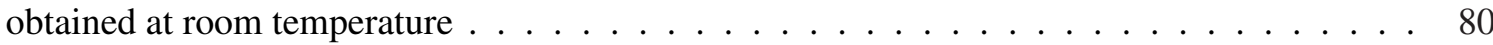

Appendix 3.B: Asymptotic properties of the WLS-based projection coefficient estimator . . . . 80

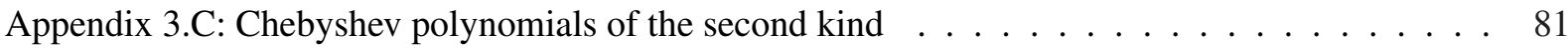

Appendix 3.D: Dependency of natural frequency estimates on temperature . . . . . . . . . . . 81

4 Identification of functionally pooled models: the Functionally Pooled VARMA (FP-VARMA) parametrization

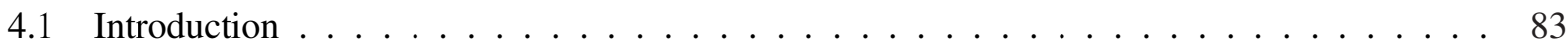

4.2 The data set and the FP-VARMA model specification $\ldots \ldots \ldots \ldots$

$4.3 \quad$ FP-VARMA model parameter estimation . . . . . . . . . . . . . . . . . . . 87

4.3.1 The Two-Stage Least Squares (2SLS) estimator . . . . . . . . . . . . . . . . 87 
4.3.2 The conditional Maximum Likelihood (ML) estimator . . . . . . . . . . . . . . . 90

4.4 Large sample properties of the 2 SLS and ML type estimators _ . . . . . . . . . . . . . 90

4.4.1 The iterated 2 SLS estimator $\ldots \ldots \ldots \ldots \ldots \ldots$

4.4.2 The conditional ML estimator . . . . . . . . . . . . . . . . . . . . 92

4.4.3 Accuracy of FP-VARMA model parameters at the $k$-th operating condition _ . . . 92

4.5 FP-VARMA model identifiability . . . . . . . . . . . . . . . . . . . . 93

4.6 FP-VARMA model structure specification and validation . . . . . . . . . . . . 93

4.6.1 Model order specification . . . . . . . . . . . . . . . . 93

4.6.2 Functional subspace dimensionality specification . . . . . . . . . . . . 98

4.6 .3 Model validation . . . . . . . . . . . . . . . . . . . . . . . . 98

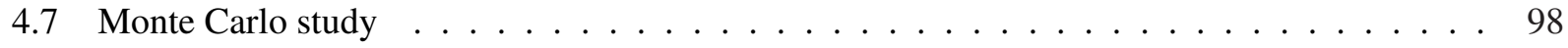

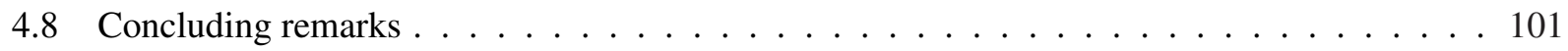

Appendix 4.A: Tutorial example of P-VAR parameter estimation . . . . . . . . . . . . . . . 102

Appendix 4.B: Additional results on the 2 SLS and ML type estimators . . . . . . . . . . . 103

Appendix 4.C: Additional results on FP-VARMA model identifiability . . . . . . . . . . . . . . 111

Appendix 4.D: Tutorial example of FP-VARMA structure specification ～. . . . . . . . . . . 112

Appendix 4.E: Additional Monte Carlo study results . . . . . . . . . . . . . . . . . . . . . . . . . 114

5 Output-only vibration-based statistical damage detection in a composite structure under dif$\begin{array}{ll}\text { ferent temperatures: a global model based approach } & 123\end{array}$

5.1 Introduction . . . . . . . . . . . . . . . . . . . . . . . . . . 123

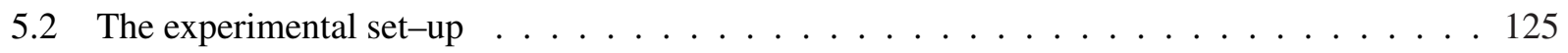

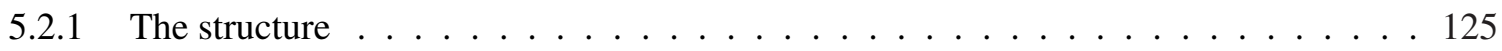

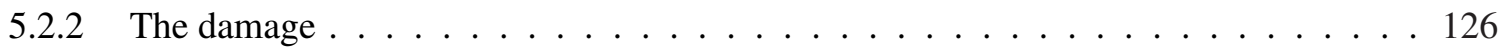

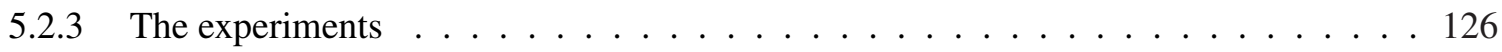

5.3 The stochastic global model based approach to damage detection . . . . . . . . . . . . 126

5.3.1 Baseline phase . . . . . . . . . . . . . . . . . . . . . 127

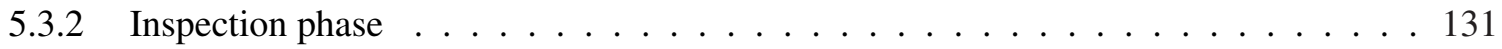

5.4 Experimental damage detection results and assessment $\ldots \ldots \ldots \ldots \ldots$

5.4.1 The stochastic global model based approach . . . . . . . . . . . . . . . . . 135

5.4 .2 Comparisons with alternative methods . . . . . . . . . . . . . . . . . . . 141

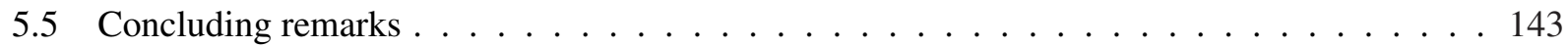

Appendix 5.A: FP-VAR model selection: two-step scheme versus exhaustive search $\ldots \ldots \ldots$ Appendix 5.B: Additional modeling results ． . . . . . . . . . . . . . . . 144

Appendix 5.C: Damage detection based on methods attempting removal of temperature effects $\ldots 148$ 
6 Stochastic identification under multiple operating conditions: assessment of global and conventional VARX modeling approaches

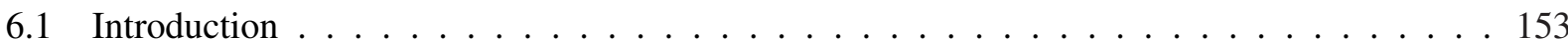

6.2 The P-VARX model class $\ldots \ldots \ldots \ldots \ldots \ldots \ldots$

6.2.1 The data set and the P-VARX model specification $\ldots \ldots \ldots$. . . . . . . 155

6.2.2 P-VARX model parameter estimation . . . . . . . . . . . . . . . . 156

6.2.3 Large sample properties of the LS and ML type estimators . . . . . . . . . . . . . 159

6.2 .4 P-VARX model identifiability . . . . . . . . . . . . . . . . . . . 162

6.2.5 Comparison of P-VARX and FP-VARX model classes. . . . . . . . . . . . . . 163

6.3 The CCP-VARX model class . . . . . . . . . . . . . . . . . . . . . . 167

6.3 .1 The CCP-VARX model specification . . . . . . . . . . . . . . 167

6.3.2 CCP-VARX model parameter estimation . . . . . . . . . . . . . . . . 168

6.3.3 Large sample properties of the LS and ML type estimators . . . . . . . . . . . . . 170

6.3 .4 CCP-VARX model identifiability . . . . . . . . . . . . . . . . . . . . 172

6.3.5 Comparison of CCP-VARX and conventional VARX models . . . . . . . . . . . . 173

6.3.6 Comparison of CCP-VARX and FP-VARX model classes. . . . . . . . . . . . 175

6.4 Monte Carlo studies . . . . . . . . . . . . . . . . . . . . . . . . . . . . . . . 177

6.4.1 Case study I: An FP-VARX $(3,1)_{[4,4]}$ model $\ldots \ldots \ldots \ldots \ldots \ldots \ldots \ldots$

6.4 .2 Case study II: An FP-VAR $(2)_{[4]} \operatorname{model} \ldots \ldots$. . . . . . . . . . . . . . . . . 179

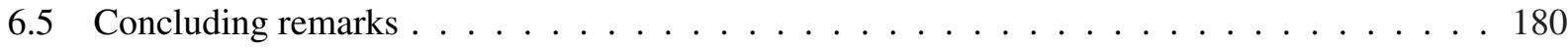

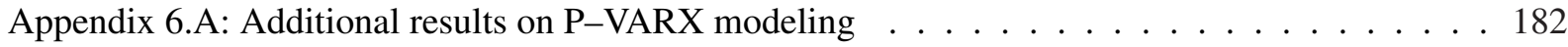

Appendix 6.B: Additional results on CCP-VARX modeling . . . . . . . . . . . . . . . . . . . 194

7 Conclusions and future research 199

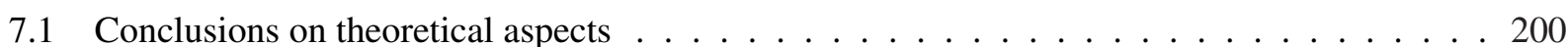

7.2 Conclusions on practical aspects $\ldots \ldots \ldots \ldots \ldots \ldots$

7.3 Future research recommendations $\ldots \ldots \ldots \ldots$. . . . . . . . . . . . . 201

$\begin{array}{ll}\text { Bibliography } & 203\end{array}$

$\begin{array}{ll}\text { List of Figures } & 209\end{array}$

$\begin{array}{ll}\text { List of Tables } & 215\end{array}$

$\begin{array}{ll}\text { Vita } & 217\end{array}$ 


\section{Summary}

This thesis addresses the identification of stochastic systems operating under different conditions, based on data records corresponding to a sample of such operating conditions. This topic is very important, as systems operating under different, though constant, conditions at different occasions (time intervals) are often encountered in practice. Typical examples include mechanical, aerospace or civil structures that operate under different environmental conditions (temperature or humidity, for instance) on different occasions (period of day, and so on). Such different operating conditions may affect the system characteristics, and therefore its dynamics.

Given a set of data records corresponding to distinct operating conditions, it is most desirable to establish a single global model capable of describing the system throughout the entire range of admissible operating conditions. In the present thesis this problem is treated via a novel stochastic Functional Pooling (FP) identification framework which introduces functional dependencies (in terms of the operating condition) in the postulated model structure. The FP framework offers significant advantages over other methods providing global models by interpolating a set of conventional models (one for each operating condition), as it:

(i) treats data records corresponding to different operating conditions simultaneously, and fully takes cross-dependencies into account thus yielding models with optimal statistical accuracy,

(ii) uses a highly parsimonious representation which provides precise information about the system dynamics at any specified operating condition without resorting to customary interpolation schemes,

(iii) allows for the determination of modeling uncertainty at any specified operating condition via formal interval estimates.

To date, all research efforts on the FP framework have concentrated in identifying univariate (single excitation - single response) stochastic models. The present thesis aims at (i) properly formulating and extending the FP framework to the case of multivariate stochastic systems operating under multiple operating conditions, and (ii) introducing an approach based on multivariate FP modeling and statistical hypothesis testing for damage detection under different operating conditions.

The case of multivariate modeling is more challenging compared to its univariate counterpart as the couplings between the corresponding signals lead to more complicated model structures, whereas their nontrivial parametrization raises issues on model identifiability. The main focus of this thesis is on models of the Functionally Pooled Vector AutoRegressive with eXogenous excitation (FP-VARX) form, and Vector AutoRegressive Moving Average (FP-VARMA) form. These models may be thought of as generalizations of their conventional VARX/VARMA counterparts with the important distinction being that the model parameters are explicit functions of the operating condition.

Initially, the identification of FP-VARX models is addressed. Least Squares (LS) and conditional Maximum Likelihood (ML) type estimators are formulated, and their consistency along with their asymptotic normality is established. Conditions ensuring FP-VARX identifiability are postulated, whereas model structure specification is based upon proper forms of information criteria. The performance characteristics of the identification approach are assessed via Monte Carlo studies, which also demonstrate the effectiveness 
of the proposed framework and its advantages over conventional identification approaches based on VARX modeling.

Subsequently, an experimental study aiming at identifying the temperature effects on the dynamics of a smart composite beam via conventional model and novel global model approaches is presented. The conventional model approaches are based on non-parametric and parametric VARX representations, whereas the global model approaches are based on parametric Constant Coefficient Pooled (CCP) and Functionally Pooled (FP) VARX representations. Although the obtained conventional model and global representations are in rough overall agreement, the latter simultaneously use all available data records and offer improved accuracy and compactness. The CCP-VARX representations provide an "averaged" description of the structural dynamics over temperature, whereas their FP-VARX counterparts allow for the explicit, analytical modeling of temperature dependence, and attain improved estimation accuracy.

In addition, the identification of FP-VARMA models is addressed. Two-Stage Least Squares (2SLS) and conditional ML type estimators are formulated, and their consistency and asymptotic normality are established. Furthermore, an effective method for 2SLS model estimation featuring a simplified procedure for obtaining residuals in the first stage is introduced. Conditions ensuring FP-VARMA model identifiability are also postulated. Model structure specification is based upon a novel two-step approach using Canonical Correlation Analysis (CCA) and proper forms of information criteria, thus avoiding the use of exhaustive search procedures. The performance characteristics of the identification approach are assessed via a Monte Carlo study, which also demonstrates the effectiveness of the proposed framework over conventional identification approaches based on VARMA modeling.

An approach based on the novel FP models and statistical hypothesis testing for damage detection under different operating conditions is also proposed. It includes two versions: the first version is based upon the obtained modal parameters, whereas the second version is based upon the discrete-time model parameters. In an effort to streamline damage detection, procedures for compressing the information carried by the modal or the discrete-time model parameters via Principal Component Analysis (PCA) are also employed. The effectiveness of the proposed damage detection approach is assessed on a smart composite beam with hundreds of experiments corresponding to different temperatures. In its present form, the approach relies upon response (output-only) vibration data, although excitation-response data may be also used. FP-VAR modeling is used identify the temperature dependent structural dynamics, whereas a new scheme for model structure selection is introduced which avoids the use of exhaustive search procedures. The experimental results verify the capability of both versions of the approach to infer reliable damage detection under different temperatures. Furthermore, alternative methods attempting removal of the temperature effects from the damage sensitive features are also employed, allowing for a detailed and concise comparison.

Finally, some special topics on global VARX modeling are treated. The focus is on the identification of the Pooled (P) and Constant Coefficient Pooled (CCP) VARX model classes. Although both model classes are of limited scope, they are useful tools for global model identification. In analogy to the FPVARX/VARMA model case, the LS and conditional ML type estimators are studied for both model classes, whereas conditions ensuring model identifiability are also postulated. The relationships interconnecting the P-VARX and CCP-VARX models to the FP-VARX models in terms of compactness and achievable accuracy are studied, whereas their association to the conventional VARX models is also addressed. The effectiveness and performance characteristics of the novel global modeling approaches are finally assessed via Monte Carlo studies.

Keywords - system identification, multivariate stochastic models, functionally pooled models, pooling techniques, stochastic global models, VARX/VARMA modeling, identification algorithms, temperature effects, smart structures, statistical damage detection, vibration-based methods. 


\section{Nomenclature}

\section{Important Conventions and Symbols}

- Boldface upper/lower case symbols designate matrix/column-vector quantities, respectively.

- Matrix/vector transposition is indicated by the superscript ${ }^{T}$.

- Equality by definition is indicated by $\triangleq$.

- A hat designates estimator/ estimate of the indicated quantity; for instance $\widehat{\boldsymbol{\theta}}$ is an estimator / estimate of $\boldsymbol{\theta}$.

- A functional argument in brackets designates function of an integer variable; for instance $\boldsymbol{x}[t]$ is a function of normalized discrete time $(t=1,2, \ldots)$. The conversion from discrete normalized time to analog time is based on $(t-1) T_{\mathrm{S}}$, with $T_{\mathrm{S}}$ standing for the sampling period.

- The subscript " $o$ " designates the true value of the indicated quantity.

- The subscripts "o" and "u" designate quantities associated with the baseline (healthy) and current (healthy or damaged) state of the structure, respectively.

\section{Symbols}

$\boldsymbol{A}_{i, k}, \boldsymbol{B}_{i, k}$

$\boldsymbol{A}_{k}(\mathcal{B}), \boldsymbol{B}_{k}(\mathcal{B})$

$\boldsymbol{A}_{i}, \boldsymbol{B}_{i}$

$\boldsymbol{A}(\mathcal{B}), \boldsymbol{B}(\mathcal{B})$

$\boldsymbol{A}_{i, j}, \boldsymbol{B}_{i, j}, \boldsymbol{C}_{i, j}$

$\boldsymbol{A}_{i}(k), \boldsymbol{B}_{i}(k), \boldsymbol{C}_{i}(k)$

$\boldsymbol{A}[\mathcal{B}, k], \boldsymbol{B}[\mathcal{B}, k], \boldsymbol{C}[\mathcal{B}, k]$

$\mathcal{B}$

$\boldsymbol{D}_{M n y}$

$E\{\cdot\}$

$\boldsymbol{e}_{k}[t, \cdot]$

$f_{\mathrm{n}}$

$f_{\mathrm{s}}$

$G_{j}(k)$
: $\quad \mathrm{AR}, \mathrm{X}$ parameter matrices at operating condition $k$ (P-VARX model)

: $\mathrm{AR}, \mathrm{X}$ matrix polynomials at operating condition $k$ (P-VARX model)

: AR, X parameter matrices (CCP-VARX model)

: $\quad$ AR, $\mathrm{X}$ matrix polynomials (CCP-VARX model)

: $\quad$ AR, $X$ and MA projection matrices (FP-VARX/VARMA models)

: $\mathrm{AR}, \mathrm{X}$ and MA parameter matrices at operating condition $k$ (FP-VARX/VARMA models)

$\mathrm{AR}, \mathrm{X}$ and MA matrix polynomials at operating condition $k$ (FP-VARX/VARMA models)

: $\quad$ backward shift operator $\left(\mathcal{B}^{j} \boldsymbol{x}[t] \triangleq \boldsymbol{x}[t-j]\right)$

: duplication matrix

: statistical expectation

: residual vector at operating condition $k$

: natural frequency $(\mathrm{Hz})$

: sampling frequency $(\mathrm{Hz})$

: basis functions $(j=1,2, \ldots, p)$ 


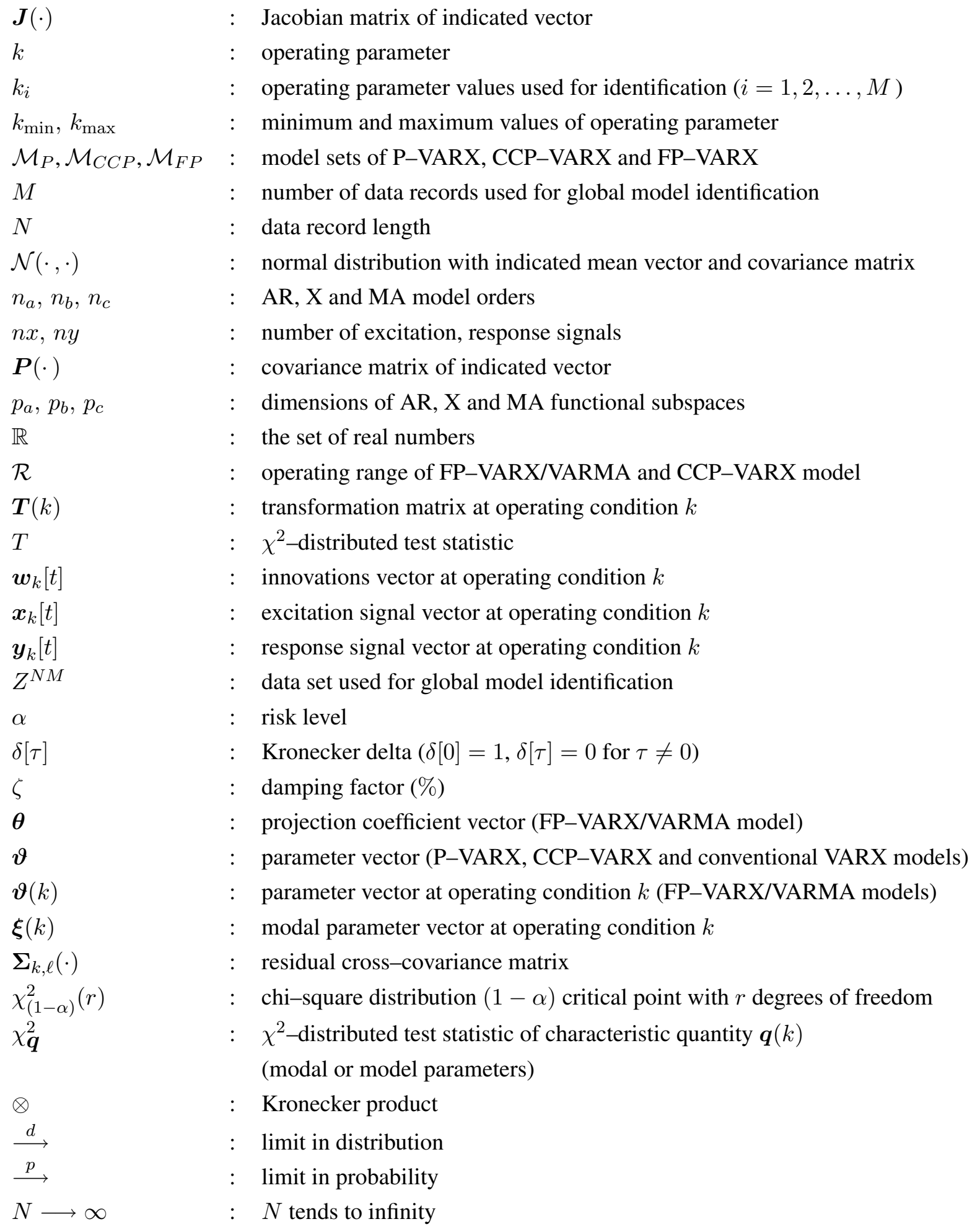

\section{Acronyms}

AIC $:$ Akaike Information Criterion
BIC : Bayesian Information Criterion
CCA $:$ Canonical Correlation Analysis 


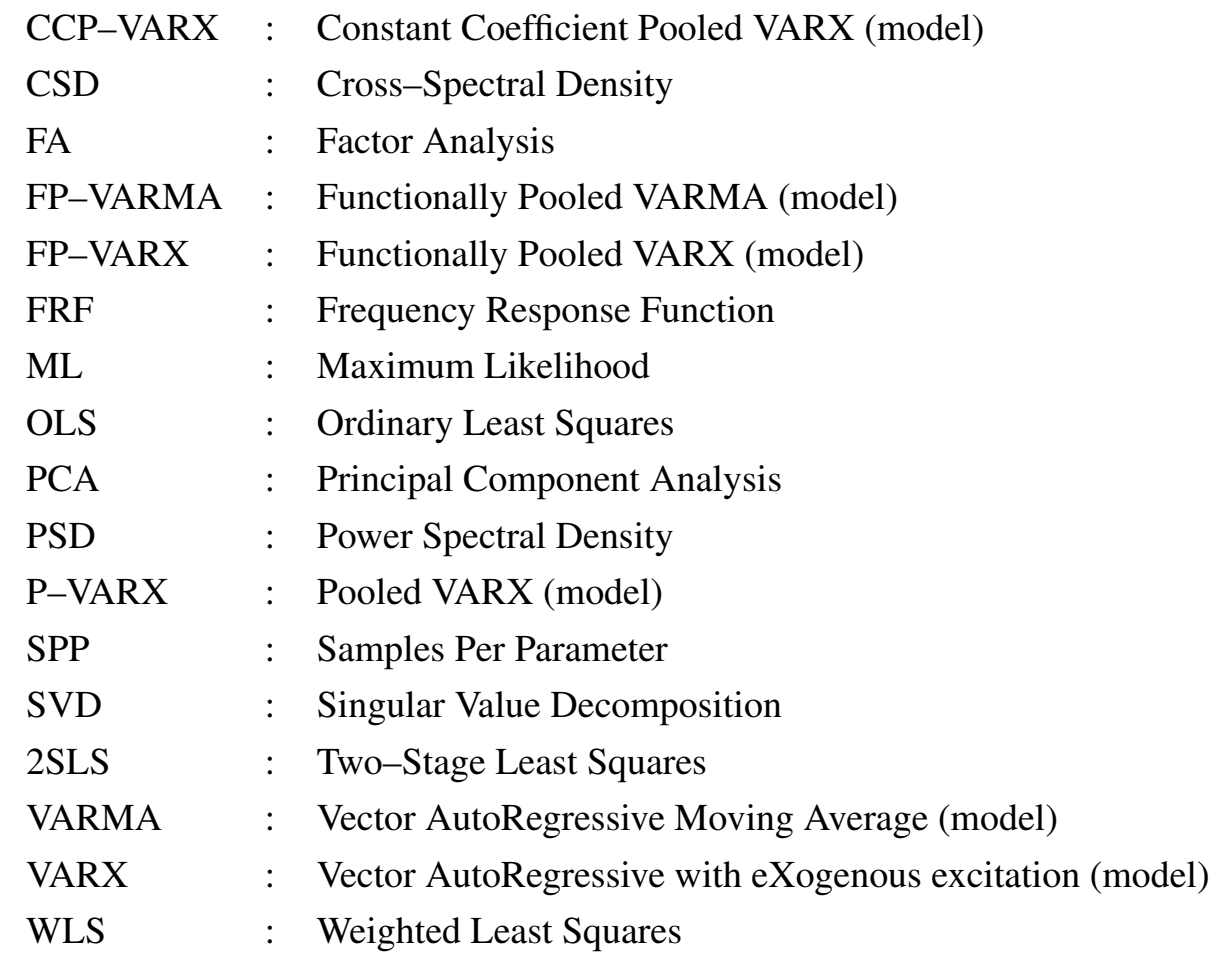




\section{Chapter 1}

\section{Introduction}

\subsection{The general problem and its importance}

System identification is a well-studied interdisciplinary topic which aims at deriving mathematical models for dynamic systems based on measured data [1,2]. Traditionally, the techniques used in system identification yield models capable of representing the system dynamics at a specific operating condition. Nonetheless, systems operating under different, though constant, conditions at different occasions (time intervals) are often encountered in practice. Typically, each operating condition affects the system characteristics and therefore its dynamics. Indicative examples include industrial processes and structures (civil-mechanical-aerospace) under different environmental conditions (such as temperature and/or humidity), vehicles carrying different payloads, or hydraulic systems under different operating pressures on different occasions (period of the day and so on). Oftentimes, data records (comprising of excitation-response, or response-only signals), each one corresponding to a distinct such condition, are obtained. In such cases, it is preferable to use all the available data records in order to identify a single, "global", and compact (parsimonious) model that is capable of accurately describing the system dynamics throughout the entire range of admissible operating conditions. The problem of identifying a global model for a system is presented schematically in Fig. 1.1.

The identification of a single, "global", model is justified from a theoretical and practical viewpoint, as it accounts for the fact that it is the same system that operates under different conditions each time. A global model thereby, allows for a better physical understanding of the system and may contribute in several areas of technical importance including (i) engineering design (for instance, in material characterization or model updating when used in conjunction with analytical models), (ii) automatic control, and (iii) structural health monitoring.

Structural health monitoring has attracted significant attention recently, due to the increased demands posed by modern engineering practice for high performance and safety. A particularly challenging aspect of this application area concerns the influence of environmental factors on the structural dynamics, as it has been widely acknowledged that factors such as wind loads, humidity and temperature can often mask subtle structural changes caused by damage [3]. Given a set of data records from a system operating under different environmental conditions, it would be most desirable to obtain a global model that describes the environment-dependent structural dynamics, and thus being capable of "separating" the effects of the environment from those of damage.

In view of the aforementioned, the motivation of the present thesis may be summarized in (i) the formulation of effective methods for global model identification, and (ii) the postulation of global models to the damage detection of structures under different environmental conditions. 


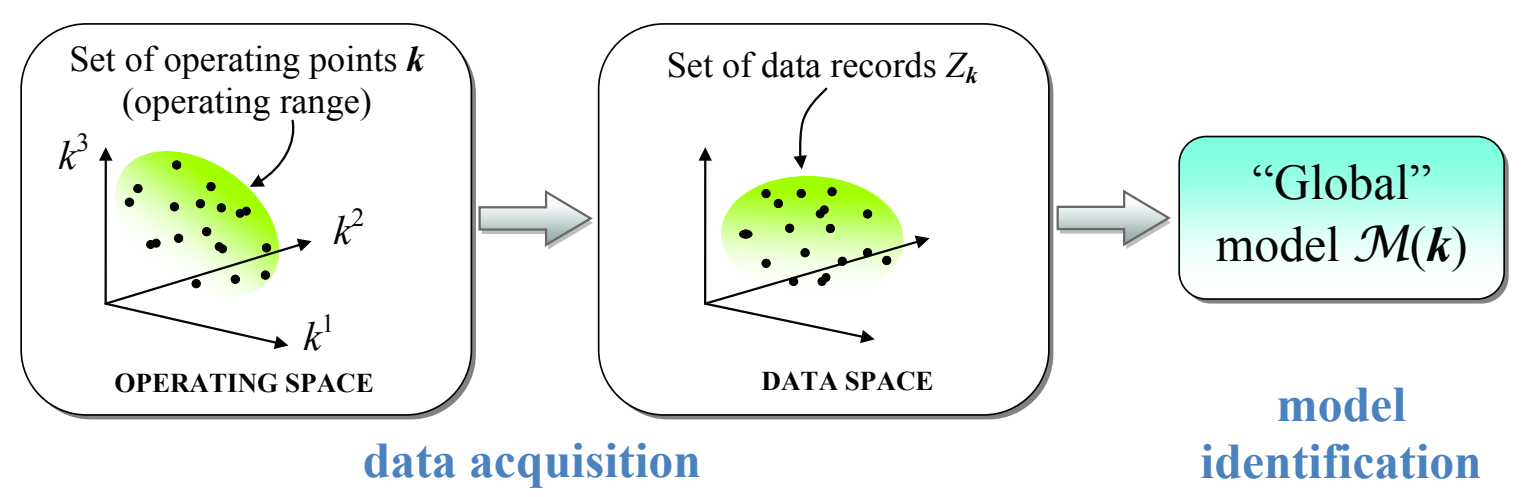

Figure 1.1: Schematic of the global model identification problem. Each operating condition $\boldsymbol{k}$ of the system (not varying with time) is depicted as a point in a generic operating space. The set of all possible operating conditions forms the operating range, whereas for each value of $\boldsymbol{k}$ a data record $Z_{\boldsymbol{k}}$ is obtained which is depicted as a point in a generic data space.

\subsection{State-of-the-art}

\subsubsection{Global modeling}

A "feasible" solution for obtaining a global model could be based upon the "multi-model" approach. According to this, the problem is artificially split into several disjoint subproblems (one per each operating condition), whereas data records from distinct operating conditions are used to obtain a set of models via customary identification techniques. The preceding models may be subsequently "interpolated" in order to yield a single global model valid for the entire operating range (refer to the schematic of Fig. 1.2). This solution (which is closely related to the gain scheduling concept [4, pp. 3-4 and the references therein]) is in fact applied by the "local approach" for Linear Parameter Varying (LPV) model identification [5-9]. Nevertheless, the combined "multi-model" approach with interpolation procedures is both inefficient and statistically suboptimal. Inefficiency has to do with the treatment of multiple identification problems instead of a single one. Statistical suboptimality has to do with (i) the ineffective use of the available information as possible interrelations among different data records are ignored, (ii) the increased estimation error resulting from the potentially large number of estimated parameters (several identified models), and (iii) the separate treatment of the interpolation stage which constitutes an additional source of modeling error. The shortcomings associated with the "multi-model" approach for global model identification are demonstrated in Chapters 2 and 4 by means of simulation studies, whereas in Chapter 3 an experimental study provides a detailed and precise comparison with other global modeling approaches.

A more elaborate solution to this problem consists of simultaneously treating all the available data records (obtained from different operating conditions) in order to identify a single, "global", model. Under the term "global model fitting" in control engineering literature, global descriptions of nonlinear systems having the form of Nonlinear AutoRegressive Moving Average with eXogenous excitation (NARMAX) models with common and constant over the complete operating range parameters are identified [10]. The same principle is also used in statistics and econometrics [11,12], although referred to as "data pooling". Typically, a single linear regression equation, or more complex time-series representations of the Vector AutoRegressive (VAR) form [13], are fitted to all data records obtained from a fixed number of units (operating conditions in the present case) simultaneously. Depending upon the particular set of assumptions with respect to the equation parameters and/or the disturbances, several models have been suggested in literature [11-13]. Nevertheless, these models do not explicitly account for each operating condition. They rather attempt to either "average out" their effects by providing representations characterized by constant parameters, or to provide a measure of model "spread" by treating the parameters as random variables. 


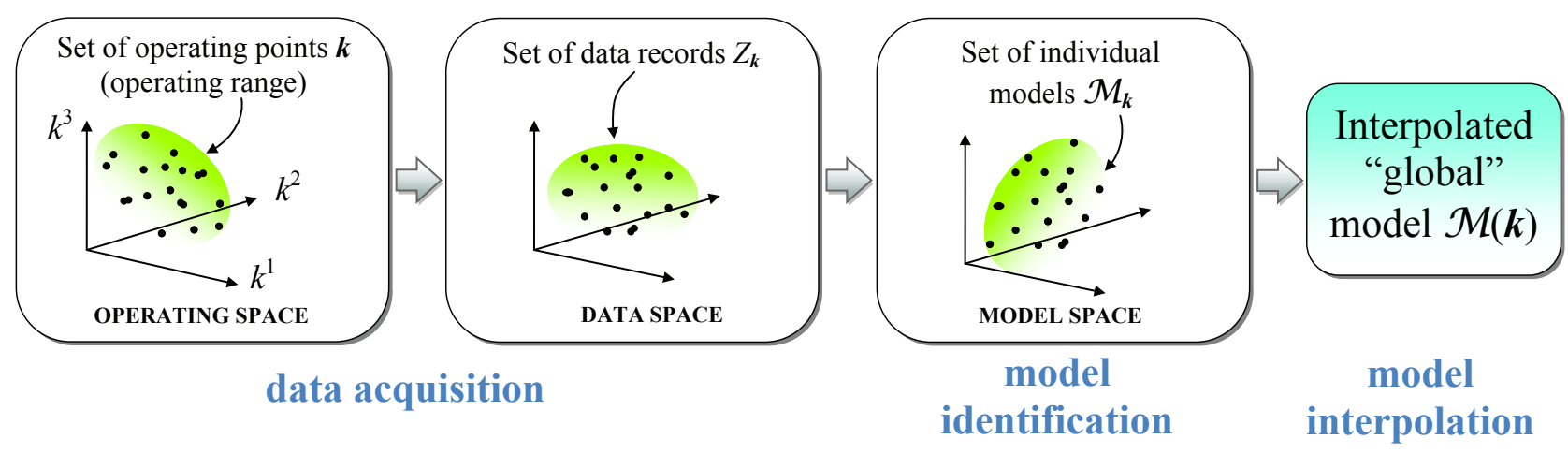

Figure 1.2: Schematic of global model identification based on the "multi-model" approach and interpolation procedures. For every data record $Z_{\boldsymbol{k}}$ belonging to the generic data space an individual model $\mathcal{M}_{\boldsymbol{k}}$ is identified, which corresponds to a point in a generic model space. The set of individual models is subsequently interpolated in order to obtain a "global" model $\mathcal{M}(\boldsymbol{k})$.

In order to effectively overcome the drawbacks of the preceding solutions and enable the identification of compact and accurate global models, a stochastic Functional Pooling identification framework has been recently introduced by Fassois and co-workers [14-17]. This framework is based on:

(1) A stochastic Functionally Pooled (FP) model structure that explicitly allows for system modeling under multiple operating conditions using a single (global) mathematical representation. This global representation is characterized by parameters that explicitly (functionally) depend upon the operating condition. It thus allows for the effective, compact, modeling of the dynamics under all possible conditions, as well as for optimal statistical accuracy due to its limited parametrization and the full accounting of the interrelations among the various data records.

(2) Data pooling techniques, which simultaneously treat as one entity the data obtained from different operating conditions.

(3) Statistical inference techniques for model estimation.

The Functional Pooling framework is distinct in that it allows for the global model to change its parameters in a way that functionally depends upon one or more measurable variables characterizing the operating condition (such as temperature, humidity and so on), and thus referred to as operating parameters. The assignment of each operating condition to a specific value of the operating parameter(s) is an essential practical condition for using the Functional Pooling framework and obtaining global models. The Functional Pooling identification framework is presented schematically in Fig. 1.3.

The class of FP models could be in the most general terms considered to belong to the broader family of LPV models (see [4] and the references therein for details). Despite their superficial resemblance, these two model classes address somewhat different identification problems with quite different perspectives. LPV modeling aims at identifying nonlinear or time-varying systems by means of linear models with parameters depending upon measurable time-varying operating parameters (referred to as scheduling parameters), usually assumed bounded in terms of rate and magnitude [4, pp. 45-48]. For this purpose, LPV model identification is achieved via two major approaches: (i) the local approach and (ii) the global approach. The local approach interpolates a set of linear time-invariant models identified from a multitude of experiments corresponding to different fixed values of the scheduling parameters, thus encompassing the shortcomings of the combined "multi-model" approach and interpolation procedures. On the contrary, the global approach identifies an LPV model from a single identification experiment by exciting the system and the scheduling parameters simultaneously. Unlike the Functional Pooling identification framework, the preceding identification approaches neither treat data obtained from different operating conditions simultaneously nor take 


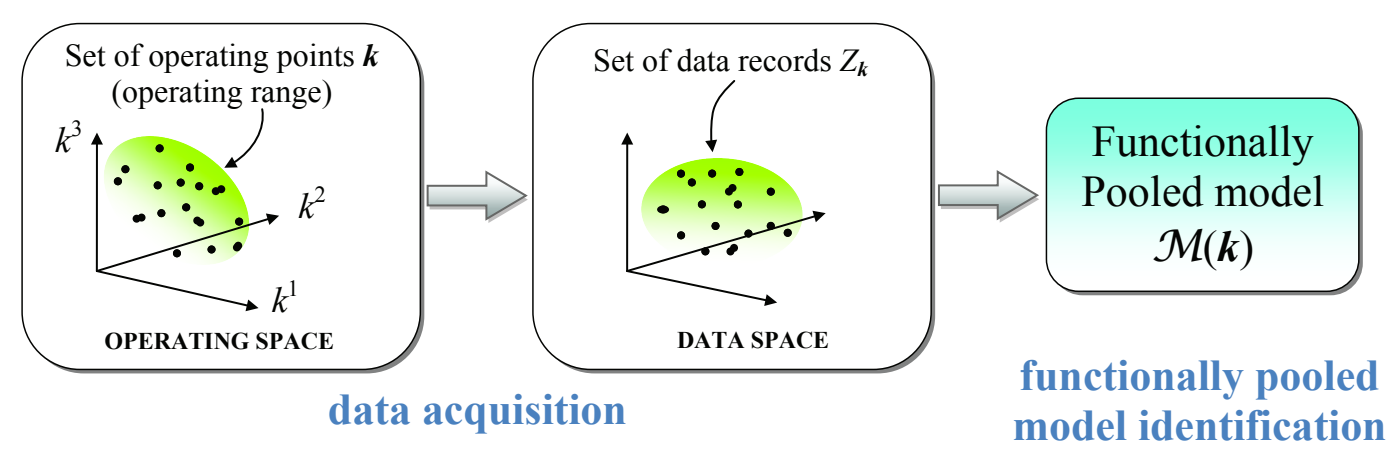

Figure 1.3: Schematic of the Functional Pooling framework for global model identification. The available data records $Z_{\boldsymbol{k}}$ belonging to the generic data space are treated simultaneously using data pooling techniques in order to obtain a "global", functionally pooled, model $\mathcal{M}(\boldsymbol{k})$.

into account the interrelations among the various data records. In view of the above, the FP and LPV models constitute distinct representations with important differences, and therefore should be clearly distinguished.

The research efforts on the Functional Pooling framework have been so far concentrated in identifying univariate (single excitation - single response) stochastic models. The case of FP models with a single (scalar) operating parameter is treated in $[15,16]$, whereas in $[14,17]$ an adaptation to the case of multiple (vector) operating parameters is presented. Some practical damage detection and identification problems have been already addressed by the Functional Pooling framework with very promising results [18-22].

\subsubsection{Damage detection under different temperature conditions}

In recent years, there has been a growing interest in developing methods capable of separating the effects of the environment on the structural dynamics from those of damage, based on vibration data. The motivation lies on the fact that environmental factors - especially temperature - can often mask subtle structural changes caused by damage $[3,23]$. The methods developed to address this problem may be classified into two main classes.

The first class relies upon extracting, from the measured data, characteristic quantities (also referred to as features) that are sensitive to damage but insensitive to temperature changes. Statistical pattern recognition techniques are often used to project the selected features (usually being model or modal parameters, although other quantities may be used also) on lower dimensions using variations of Principal Component Analysis [24-29], and Factor Analysis [30] procedures in order to attenuate the temperature effects. The aforementioned procedures treat temperature as an embedded variable and thus temperature measurements are not required, whereas damage is detected when the corresponding changes in the features are somehow "orthogonal" to the changes caused by temperature. Alternatively, subspace identification techniques may be used to model response vibration data and generate residuals for damage detection [31-33]. The temperature effects in [31] are "averaged out" by merging data from the healthy structure at different (though not necessarily measured) temperatures. A conceptually different approach is followed in [32,33], as a physics-based model of the temperature effects on the structural dynamics is also required. Two versions of the approach for damage detection are formulated. The first version utilizes the physics-based model to obtain a temperature adjusted residual vector for damage detection. The second version treats temperature as a "nuisance" parameter and its effects on the residual vector are eliminated with the aid of the physics-based model. Note that these residual-based approaches detect damages producing changes "orthogonal" to the response spaces spanned by the baseline (healthy) data.

The second class is based upon the direct modeling of temperature effects on the features of interest, 
and may be used when temperature measurements are available. Typically, a conventional structural dynamics model is obtained for each temperature using customary identification techniques. The models are then linked via polynomial regression or interpolation procedures which approximate the dependence of model parameters on temperature [34]. Alternatively, the modal parameters are extracted and are subsequently related to temperature via additional expressions. Static linear regression models associating modal frequency with temperature are employed in [35]. In order to account for the structure's thermal capacity, linear dynamic models describing the modal frequency dynamic dependence on temperature have also been employed $[23,36,37]$. The present class fully exploits the available temperature information, yielding methods with increased sensitivity to the presence of damage. Nonetheless, the methods employed are based upon the "multi-model" approach and interpolation procedures, which embody the limitations stated in the previous subsection.

\subsection{Aim of the thesis and specific objectives}

\subsubsection{Global modeling}

The aim of the present thesis is the proper formulation and extension of the FP framework to the case of multivariate stochastic systems operating under multiple operating conditions. The motivation stems from the fact that oftentimes the joint study of several excitation-response signals is required in order to obtain a better understanding of the system dynamics. The models developed for this purpose obey the following basic assumptions:

A1. They are linear, time-invariant, stochastic dynamic models, which means that the present value of the response depends linearly on the present and past values of the excitation and the disturbance (that is noise due to ambient conditions, measurement errors, and so on), whereas the model parameters do not change with time.

A2. They are parametric models in discrete time, that is finitely parametrized models utilizing sampled versions of the excitation-response signals at discrete, equidistant, time instants (for example, $t=$ $0,1,2, \ldots)$, whereas the time between two consecutive instants is used as time unit.

A3. They are functionally pooled models, which means that multiple data records (each one obtained from a different operating condition) are treated simultaneously to identify a single model. The model parameters depend functionally upon the operating parameter, with the latter quantity being measurable upon operation of the system, bounded, and constant during data acquisition.

The choice of linear parametric models provides important benefits due to their (i) direct relationship with differential equations used in engineering analysis, (ii) increased accuracy and frequency-domain resolution, (iii) parsimonious representation, and (iv) suitability for analysis, prediction, automatic control and structural health monitoring. The price paid for these benefits includes a generally increased identification complexity and the requirement of expert knowledge to derive appropriate models.

Furthermore, the development of multivariate models is more challenging than the univariate case treated in [14-17], as it poses certain technical difficulties which concern:

(i) The increased complexity of model analysis (the model structures involved are more complicated, whereas certain scalar quantities become vectors or matrices and might not commute).

(ii) The model identifiability (their parametrization is nontrivial).

(iii) The increased algorithmic complexity (a large amount of data is processed). 
In this work, the main focus is on models of the Functionally Pooled Vector AutoRegressive with eXogenous excitation (FP-VARX) form, and Vector AutoRegressive Moving Average (FP-VARMA) form characterized by a single (scalar) operating parameter. These models may be thought of as generalizations of their conventional VARX/VARMA counterparts with the important distinction being that the model parameters are explicit functions of the operating parameter.

In order to obtain an FP-VARX/VARMA model, system identification techniques are employed. This is an experimental procedure, requiring the excitation of the system at different, constant, operating conditions (that is, for constant values of the operating parameter). The excitation-response or response-only signals for each distinct operating condition are recorded over a time interval, and a single model is subsequently fitted to all the available data records by assigning suitable numerical values to its parameters. The two main steps in obtaining an FP-VARX/VARMA model consist of (i) determining an appropriate form of the model (the model structure specification problem), and (ii) using statistically based methods to estimate the unknown parameters of the model (the parameter estimation problem).

An effective framework for identifying FP-VARX/VARMA models is presently developed. Estimators for both FP-VARX/VARMA models are formulated by means of the Least Squares (LS), and the conditional Maximum Likelihood (ML) principles. In order to compare the derived LS and ML type estimators in a meaningful way, their large sample properties (consistency, asymptotic distribution and asymptotic efficiency) are studied. Also, conditions ensuring the FP-VARX/VARMA model identifiability are postulated (as opposed to the univariate case [14-17]). The critical problem of FP-VARX/VARMA model structure specification is tackled via effective strategies based on information criteria and statistical decision making tools. The performance characteristics of the derived FP-VARX/VARMA estimators are assessed via Monte Carlo studies, and comparisons which also demonstrate the effectiveness of the proposed framework over its conventional VARX/VARMA based multi-model counterpart are also made.

An experimental study, aiming at identifying the temperature effects on the dynamics of a smart composite beam is also undertaken. Global VARX modeling and multi-model (non-parametric Welch based and parametric VARX) approaches are used to identify the system dynamics under various temperatures. A detailed and precise comparison of the obtained models is thus allowed, as both modeling approaches are assessed in terms of compactness and achievable accuracy, demonstrating the advantages of the FP framework over conventional multi-model approaches.

Finally, two special cases of global models are addressed: the Pooled $(\mathrm{P})$ and the Constant Coefficient Pooled (CCP) VARX models. Although both model classes are of limited scope, they are useful tools for global model identification. In analogy to the FP-VARX/VARMA model case, the LS and conditional ML type estimators are studied for both model classes, whereas conditions ensuring model identifiability are also postulated. The relationships interconnecting the P-VARX and CCP-VARX models to the FP-VARX models in terms of compactness and achievable accuracy are subsequently examined, and their association to the conventional VARX models is also addressed. The effectiveness and performance characteristics of the novel global VARX modeling approaches are finally assessed via Monte Carlo studies.

\subsubsection{Damage detection under different temperature conditions}

This thesis also aims at introducing an approach for effective damage detection under different temperatures based on vibration data. Damages are considered as deviations of the system from its normal behavior that adversely affect its current or future performance, whereas the objective of damage detection is to ascertain if damage is present or not in the system. The fundamental principle upon which vibration-based methods are founded is that small deviations (damage) in a structure cause discrepancies in its vibration response, which in turn may be detected. 
The proposed damage detection approach belongs to the second class of methods (see Section 1.2.2), which is based upon the direct modeling of temperature effects on characteristic quantities obtained from the measured data, and may be used when temperature measurements are available. The strengths and advantages of the approach stem from the statistically efficient stochastic modeling of the dynamics under any temperature via stochastic Functionally Pooled (FP) models, the effective statistical treatment of uncertainties, and statistical decision making to infer damage detection. The global FP models employed are presently characterized by parameters having an explicit functional dependence on temperature. FP modeling offers significant advantages as it (i) treats data records corresponding to various temperatures simultaneously and fully takes cross-dependencies into account, (ii) uses a highly parsimonious representation, (iii) provides precise information about the system dynamics at any specified temperature without resorting to customary interpolation schemes, and (iv) allows for the determination of modeling uncertainty at any specified temperature via formal interval estimates. These characteristics lead to highly accurate and efficient stochastic modeling of the temperature-dependent dynamics, as opposed to the "multi-model" approaches employed by the other methods belonging to the specific class.

The proposed damage detection approach belongs to the statistical time series methods (see the overviews [18,21]), which offer a number of important advantages such as (i) no requirement for physicsbased or finite element models, (ii) use of a partial structural model with a limited number of measurable signals, (iii) inherent accounting for uncertainty, (iv) statistical decision making with specified performance characteristics, and (v) the effective use of natural random vibration data records (no need to interrupt normal operation). On the other hand, the limitations of these methods include the requirement of user expertise, the potentially limited physical insight, and lower sensitivity compared to "local" nondestructive testing-type methods. Although the proposed global model approach shares certain aspects with the Functional Model based damage detection and identification approach of Fassois and co-workers [18-21], it is distinct in its structure and unique in its capability of handling temperature effects.

As with other vibration-based methods [18,21], the proposed approach consists of two phases: (a) The baseline phase, which involves the identification of a global FP model for the healthy structure, and (b) the inspection phase, which includes conventional identification of the current structural state and statistical decision making to test for significant discrepancies between the baseline (healthy) and current characteristic quantities. The approach may be used either with response-only or excitation-response data whenever available. Two versions of the damage detection approach are formulated: the first version uses as a characteristic quantity the obtained modal parameters, whereas the second version uses the discrete-time model parameters. In an effort to streamline damage detection, techniques for compressing the information carried by the characteristic quantities via Principal Component Analysis (PCA) are also investigated.

\subsection{Novel contributions}

The novel contributions of the present thesis are summarized as follows:

- The novel FP-VARX/VARMA model classes are specified and a framework for FP-VARX/VARMA model identification is developed. Effective model structure specification strategies are formulated and conditions ensuring model identifiability are postulated. Assessment of the derived FP-VARX/VARMA estimators' performance characteristics, and demonstration of the proposed framework's effectiveness over its conventional VARX/VARMA based multi-model counterpart is achieved via Monte Carlo studies (Chapters 2 and 4).

- The novel P-VARX and CCP-VARX model classes are specified and their identification is postulated. A comparison of the P-VARX and CCP-VARX model structures with their FP-VARX and conventional VARX counterparts is provided, focusing on the relationships associating the preceding 
structures in terms of model parsimony and achievable accuracy. The effectiveness and performance characteristics of the novel global VARX modeling approaches are assessed via Monte Carlo studies (Chapter 6).

- A detailed and precise comparison of the FP framework with the conventional multi-model approaches is presented, and the obtained models are assessed in terms of compactness and achievable accuracy. The advantages of the FP framework over the conventional multi-model approaches are practically demonstrated, for the first time, by identifying the temperature effect on the dynamics of a smart composite beam using vibration data (Chapter 3 ).

- A novel approach based on (i) global FP models, and (ii) statistical hypothesis testing is developed for damage detection under different operating conditions. The approach is assessed over a large number of vibration experiments performed on a smart composite beam under various temperatures, whereas extensive comparisons with methods attempting removal of the temperature effects from the damagesensitive features are also made (Chapter 5).

\subsection{Overview of the thesis chapters}

The thesis is divided into five main chapters besides the introductory and the conclusive chapters. A short description of the main chapters along with the contributions and innovations of each one is provided below, whereas a schematic overview of the thesis structure along with the relations between the chapters is presented in Fig. 1.4.

\section{Chapter 2. Identification of functionally pooled models: the Functionally Pooled VARX (FP-VARX) parametrization.}

The identification of stochastic systems capable of operating under different conditions is addressed based on data records corresponding to a sample of such operating conditions. The problem is tackled within the novel Functional Pooling framework, which focuses on the identification of Functionally Pooled Vector AutoRegressive with eXogenous excitation (FP-VARX) models. Least Squares (LS) and conditional Maximum Likelihood (ML) type estimators are formulated, and their large sample properties are examined. An additional contribution of this chapter is the postulation of conditions ensuring FP-VARX model identifiability. These conditions are necessary in order to (i) obtain a unique FP-VARX representation (unlike the univariate case $[14,15,17]$ were such a requirement is not needed), and (ii) determine the types of excitation that allow for a properly identified FP-VARX model. Furthermore, the problem of FP-VARX model structure specification is addressed by formulating appropriate information criteria. The performance characteristics of the estimators are finally assessed via Monte Carlo studies, which also demonstrate the effectiveness of the proposed framework over conventional identification approaches.

\section{Chapter contributions:}

(i) Specification of the novel FP-VARX model class.

(ii) Formulation of LS and conditional ML type estimators, and confirmation of their large sample properties (consistency, asymptotic normality).

(iii) Postulation of conditions ensuring FP-VARX model identifiability (uniqueness of representation and informative experiments).

(iv) Formulation of appropriate information criteria to address the problem of FP-VARX model structure specification.

(v) Assessment of the LS and ML type estimators performance characteristics, and demonstration of the 
accuracy of the global FP-VARX modeling approach over its conventional VARX based multi-model counterpart via Monte Carlo experiments.

\section{Chapter 3. Stochastic identification of temperature effects on the dynamics of a smart composite beam: assessment of multi-model and global model approaches.}

The temperature effects on the dynamics of a smart composite beam are experimentally studied via conventional multi-model and novel global model identification approaches. The multi-model approaches are based on non-parametric Welch based and parametric VARX representations, whereas the global model approaches are based on novel Constant Coefficient Pooled (CCP) and Functionally Pooled (FP) VARX parametric representations. The analysis indicates that the obtained multi-model and global model representations are in rough overall agreement. Nevertheless, the latter simultaneously use all available data records offering more compact descriptions of the dynamics, improved numerical robustness and estimation accuracy, which is reflected in significantly reduced modal parameter uncertainties. Although the CCPVARX representations provide only "averaged" descriptions of the structural dynamics over temperature, their FP-VARX counterparts allow for the explicit, analytical modeling of temperature dependence exhibiting a "smooth" deterministic dependency of the dynamics on temperature which is compatible with the physics of the problem. In accordance with previous studies, the obtained natural frequencies decrease with temperature in a weakly nonlinear or approximately linear fashion. The damping factors are less affected, although their dependence on temperature may be of potentially more complex nature.

\section{Chapter contributions:}

(i) Experimental identification of the temperature-dependent dynamics of a smart beam with global models (CCP-VARX and FP-VARX), and multi-model approaches (non-parametric Welch based and parametric VARX representations).

(ii) Assessment of the obtained models in terms of compactness and achievable accuracy.

\section{Chapter 4. Identification of functionally pooled models: the Functionally Pooled VARMA (FP-VARMA) parametrization.}

The identification of stochastic systems capable of operating under different conditions is addressed via models of the Functionally Pooled Vector AutoRegressive Moving Average (FP-VARMA) form. For this purpose, Two-Stage Least Squares (2SLS) and conditional Maximum Likelihood (ML) type estimators are formulated, and their large sample properties are examined. It is worth noting that the 2SLS estimator large sample properties are studied for the first time within the FP framework. Also, an effective method for 2SLS model estimation is introduced, featuring a simplified procedure for obtaining residuals in the first stage (in contrast to the complicated inverse function operators employed in [16]). Furthermore, conditions ensuring FP-VARMA model uniqueness of representation are postulated (as opposed to the univariate case [16]). Model structure specification is based upon a novel two-step approach that avoids the use of exhaustive search procedures. In the first step, model orders are estimated via an effective Canonical Correlation Analysis (CCA) based scheme which avoids specifying the form of the FP-VARMA parameter functional dependency on the operating parameter. This specification is accomplished in the second step by using proper forms of information criteria. The performance characteristics of the identification approach are assessed via a Monte Carlo study, which also demonstrates the effectiveness of the proposed framework over conventional identification approaches.

Chapter contributions:

(i) Specification of the novel FP-VARMA model class.

(ii) Formulation of 2SLS and conditional ML type estimators, and confirmation of their large sample properties (consistency, asymptotic normality). 
(iii) Introduction of a novel method for 2SLS model estimation featuring a simplified procedure for obtaining residuals in the first stage.

(iv) Postulation of conditions ensuring FP-VARMA model identifiability (uniqueness of representation).

(v) Postulation of a novel two-step approach to address the critical problem model structure specification using a canonical correlation analysis (CCA) scheme and information criteria.

(vi) Assessment of the 2SLS and ML type estimators performance characteristics, and demonstration of the accuracy of the global FP-VARMA modeling approach over its conventional VARMA based multimodel counterpart via Monte Carlo experiments.

\section{Chapter 5. Output-only vibration-based statistical damage detection in a composite structure under different temperatures: a global model based approach.}

The statistical damage detection in a composite structure operating under different temperatures based on vibration data is considered. A novel stochastic global model approach is introduced for this purpose using models of the Functionally Pooled (FP) form, which are capable of describing the dynamics under any temperature, and statistical decision making. Two versions of the approach, depending upon the characteristic quantity used in the decision making part are considered. The first version is based upon the obtained modal parameters, whereas the second version is based upon the discrete-time model parameters. In an effort to streamline damage detection, procedures for compressing the information carried by the characteristic quantity via Principal Component Analysis (PCA) are also employed. In its present form, the approach relies upon response (output-only) vibration data, although excitation-response data may be also used. FP-VAR modeling is used identify the temperature dependent structural dynamics, whereas a new scheme for model structure selection is introduced which avoids the use of exhaustive search procedures. The effectiveness of the approach is experimentally confirmed, while comparisons with alternative methods attempting removal of the temperature effects from the damage-sensitive features are also made.

Chapter contributions:

(i) Postulation of an effective statistical approach for structural damage detection under different temperatures.

(ii) Introduction of a new scheme for global (FP) model structure selection which avoids the use of exhaustive search procedures.

(iii) Postulation and testing of two versions of the approach - the first relying on the estimated natural frequencies and the second on the discrete-time model parameters. Techniques for information compression (dimensionality reduction) are also investigated.

(iv) Experimental identification of the temperature-dependent dynamics from output-only data and comparison with those previously identified from input-output data.

(v) Laboratory assessment of the approach with many hundreds of experiments corresponding to different temperatures (proof-of-concept study).

(vi) Extensive comparisons with alternative damage detection methods that attempt potential removal of the temperature effects from the damage-sensitive features.

\section{Chapter 6. Stochastic identification under multiple operating conditions: assessment of global and} conventional VARX modeling approaches.

The identification of multivariate stochastic systems capable of operating under different conditions, based on samples of data records obtained at different operating conditions, is addressed via novel global modeling approaches. The global model classes postulated in this study may be thought as generalizations of 
the conventional Vector AutoRegressive with eXogenous excitation (VARX) model class. The focus is on the Pooled (P) and Constant Coefficient Pooled (CCP) VARX model classes. Although both model classes are of limited scope, they are helpful in FP-VARX/VARMA identification. The P-VARX models are characterized by parameters depending on the operating condition but not expressed as explicit functions of it. These models are valid only at the operating conditions used in the identification procedure, and prove valuable in FP-VARX/VARMA model structure specification as well as in FP-VARMA LS-based estimation. On the other hand, the CCP-VARX models provide simple, "averaged", descriptions of the dynamics over the entire range of operating conditions, due to the assumption of constant model parameters (coefficients). In analogy to the FP-VARX/VARMA model case, LS and conditional ML type estimators are studied for both model classes, whereas conditions ensuring model identifiability are also postulated. Furthermore, their association to the recently introduced Functionally Pooled (FP) VARX and the conventional VARX model classes is also addressed. Least Squares and conditional Maximum Likelihood type estimators for the P-VARX and CCPVARX models are formulated and their large sample properties are studied, whereas conditions ensuring model identifiability are also provided. The effectiveness and performance characteristics of the novel global modeling approaches are finally assessed via Monte Carlo studies.

\section{Chapter contributions:}

(i) Specification of the novel P-VARX and CCP-VARX model classes.

(ii) Formulation of LS and conditional ML type estimators for the P-VARX and CCP-VARX models, and confirmation of their large sample properties (consistency, asymptotic normality).

(iii) The postulation of conditions ensuring P-VARX and CCP-VARX model identifiability (uniqueness of representation and informative experiments).

(iv) The assessment of the global VARX and conventional VARX model structures in terms of model parsimony and achievable accuracy. The effectiveness and performance characteristics of the novel global modeling approaches are further examined via two Monte Carlo studies. 

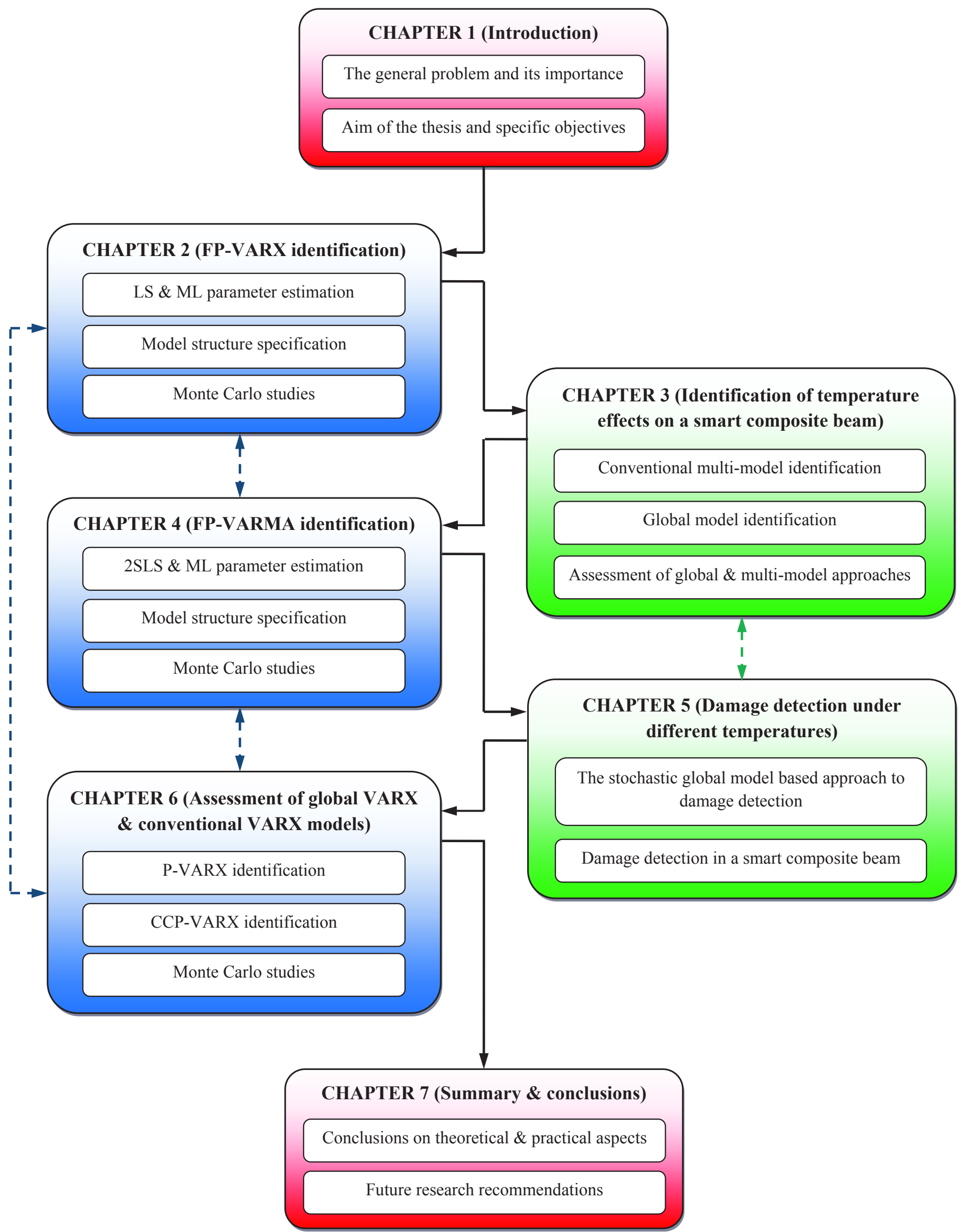

Figure 1.4: Structure of the thesis. 


\section{Chapter 2}

\section{Identification of functionally pooled models: the Functionally Pooled VARX (FP-VARX) parametrization}

\subsection{Introduction}

System identification traditionally addresses the problem of deriving a model capable of representing a dynamic system at a specific operating condition, based on measured data. Nonetheless, systems operating under different, though constant, conditions at different occasions (time intervals) are often encountered in practice. Typical examples include industrial processes, environmental systems, materials and structures (civil-mechanical-aerospace) under different environmental conditions (such as temperature or humidity) on different occasions (period of the day and so on). Oftentimes, data records, each one corresponding to a distinct such condition, are obtained. In such cases, it is preferable to use all the available data records in order to identify a single, "global", and compact (parsimonious) model that is capable of accurately describing the system dynamics throughout the entire range of admissible operating conditions.

A "feasible" solution for obtaining a global model could be based upon the "multi-model" approach. According to this, the problem is artificially split into several disjoint subproblems (one per each operating condition), whereas data records from distinct operating conditions are used to obtain a set of models via customary identification techniques. The preceding models may be subsequently "interpolated" in order to yield a single global model valid for the entire operating range. This solution (which is closely related to the gain scheduling concept [4, pp. 3-4 and the references therein]) is in fact applied by the "local approach" for Linear Parameter Varying (LPV) model identification [5-9]. Nevertheless, the combined "multi-model" approach with interpolation procedures is both inefficient and statistically suboptimal. Inefficiency has to do with the treatment of multiple identification problems instead of a single one. Statistical suboptimality has to do with (i) the ineffective use of the available information as possible interrelations among different data records are ignored, (ii) the increased estimation error resulting from the potentially large number of estimated parameters (several identified models), and (iii) the separate treatment of the parameter interpolation stage which constitutes an additional source of modeling error.

A more elaborate solution to this problem would consist of fitting a single ("global") model on several data records, obtained from different operating conditions, simultaneously. Under the term "global model fitting" in control engineering literature, global descriptions of nonlinear systems having the form of Nonlinear AutoRegressive Moving Average with eXogenous excitation (NARMAX) models with common and constant over the complete operating range parameters are identified [10]. The same principle is also used in 
statistics and econometrics [11,12], although referred to as "data pooling". Usually, a single linear regression equation, or more complex time-series representations of the Vector AutoRegressive (VAR) form [13], are fitted to all data records obtained from a fixed number of units (operating conditions in the present case) simultaneously. Depending upon the particular set of assumptions with respect to the equation parameters and/or the disturbances, several models have been suggested in literature [11-13]. Nevertheless, these models do not explicitly account for each operating condition. They rather attempt to either "average out" their effects by providing representations characterized by constant parameters, or to provide a measure of model "spread" by treating the parameters as random variables.

In order to effectively overcome these restrictions, a "global" modeling approach capable of describing the dynamic behavior of systems operating under different, though constant, operating conditions at different occasions has been introduced by Fassois and co-workers [14-17]. This approach has been formulated within a novel stochastic Functional Pooling framework which is based upon:

(1) A stochastic Functionally Pooled (FP) model structure that explicitly allows for system modeling under multiple operating conditions using a single ("global") mathematical representation. This global representation is characterized by parameters that explicitly (functionally) depend upon the operating condition. It thus allows for the effective, compact, modeling of the dynamics under all possible conditions, as well as for optimal statistical accuracy due to its limited parametrization and the full accounting of the interrelations among the various data records.

(2) Data pooling techniques, which simultaneously treat as one entity the data obtained from different operating conditions.

(3) Statistical inference techniques for model estimation.

The Functional Pooling framework is distinct in that it allows for the global model to change its parameters in a way that functionally depends upon one or more measurable variables characterizing the operating condition (such as temperature, humidity and so on), and thus referred to as operating parameters. The assignment of each operating condition to a specific value of the operating parameter(s) is an essential practical condition for using the Functional Pooling framework and obtaining "global" models.

The class of FP models could be in the most general terms considered to belong to the broader family of LPV models (see [4] and the references therein for details). Despite their superficial resemblance, these two model classes address somewhat different identification problems with quite different perspectives. LPV modeling aims at identifying nonlinear or time-varying systems by means of linear models with parameters depending upon measurable time-varying operating parameters (referred to as scheduling parameters), usually assumed bounded in terms of rate and magnitude [4, pp. 45-48]. For this purpose, LPV model identification is achieved via two major approaches: (i) the local approach and (ii) the global approach. The local approach interpolates a set of linear time-invariant models identified from a multitude of experiments corresponding to different fixed values of the scheduling parameters, thus encompassing the shortcomings of the combined "multi-model" approach and interpolation procedures. On the contrary, the global approach identifies an LPV model from a single identification experiment by exciting the system and the scheduling parameters simultaneously. Unlike the Functional Pooling identification framework, the preceding identification approaches neither treat data obtained from different operating conditions simultaneously nor take into account the interrelations among the various data records. In view of the above, the FP and LPV models constitute distinct representations with important differences, and therefore should be clearly distinguished.

The identification of univariate FP models with a single (scalar) operating parameter is treated in [15, $16]$, whereas an adaptation to the case of multiple (vector) operating parameters is studied in $[14,17]$. Some practical damage detection problems have been already addressed by the Functional Pooling framework with very promising results [18-22,38,39]. An experimental study demonstrating the advantages of the Functional Pooling framework over conventional "multi-model" approaches is provided in [40-42], which 
treats the problem of identifying the dynamics of a composite beam under various temperatures.

This study aims at extending the Functional Pooling framework to the case of multivariate stochastic systems operating under multiple operating conditions. Unlike the univariate case, the richer internal structure of the multivariate models poses certain technical difficulties concerning (i) the increased complexity of model analysis (certain scalar quantities become vectors or matrices, and might not commute), (ii) the model identifiability (their parametrization is nontrivial), and (iii) the increased algorithmic complexity (a large amount of data is processed).

The novel contributions of the present study may be summarized as follows:

(i) The specification of the novel Functionally Pooled Vector AutoRegressive with eXogenous excitation (FP-VARX) model class, which is characterized by a single operating parameter for global model identification.

(ii) The formulation of Least Squares (LS) and conditional Maximum Likelihood (ML) type estimators, and confirmation of their large sample properties (consistency, asymptotic normality).

(iii) The postulation of conditions ensuring FP-VARX model identifiability (uniqueness of representation and informative experiments).

(iv) The formulation of appropriate information criteria to address the problem of FP-VARX model structure specification.

(v) The assessment of the LS and ML type estimators performance characteristics via two Monte Carlo studies, which also aim at demonstrating the accuracy of the FP-VARX modeling approach over its conventional VARX based multi-model counterpart.

The rest of this chapter is organized as follows: The data set and the FP-VARX model specification is presented in Section 2.2. FP-VARX model parameter estimation methods and the estimators' large sample properties are presented in Sections 2.3 and 2.4, respectively. Issues regarding model identifiability are discussed in Section 2.5, whereas model structure specification and validation are addressed in Section 2.6. In Section 2.7 the FP-VARX parameter estimation methods are assessed by means of Monte Carlo studies, whereas the concluding remarks are summarized in Section 2.8.

\subsection{The data set and the FP-VARX model specification}

The identification of a Functionally Pooled model is based upon $M$ sets of excitation-response data records, each one obtained under a different, constant, operating condition. That is, each data record corresponds to a specific, constant, value of a measurable operating parameter $k$. The set of all possible operating conditions belongs to the range $\mathcal{R}=\left[k_{\min }, k_{\max }\right] \subset \mathbb{R}$, and a discretized version $\left\{k_{1}, k_{2}, \ldots, k_{M}\right\}$ of it is used for data acquisition. Assuming $N$-sample-long records, the complete set of data is designated ${ }^{1}$ :

$$
Z^{N M} \triangleq\left\{\boldsymbol{x}_{k}[t], \boldsymbol{y}_{k}[t] \mid k=k_{1}, \ldots, k_{M} ; t=1, \ldots, N\right\}
$$

with $t$ designating the normalized discrete time, $\boldsymbol{x}_{k}[t]$ being the $(n x \times 1)$ excitation vector, whereas $\boldsymbol{y}_{k}[t]$ is the $(n y \times 1)$ response vector corresponding to the $k$-th operating condition.

The FP-VARX representation postulated for treating this problem has the following form:

$$
\boldsymbol{y}_{k}[t]+\sum_{i=1}^{n a} \boldsymbol{A}_{i}(k) \boldsymbol{y}_{k}[t-i]=\sum_{i=0}^{n b} \boldsymbol{B}_{i}(k) \boldsymbol{x}_{k}[t-i]+\boldsymbol{w}_{k}[t]
$$

\footnotetext{
${ }^{1}$ Boldface upper/ lower case symbols designate matrix/ column-vector quantities, respectively.
} 


$$
\begin{gathered}
E\left\{\boldsymbol{w}_{k}[t] \boldsymbol{w}_{\ell}^{T}[t-\tau]\right\}=\boldsymbol{\Sigma}_{k, \ell} \delta[\tau] \quad k, \ell \in \mathcal{R} \\
\boldsymbol{A}_{i}(k)=\sum_{j=1}^{p a} \boldsymbol{A}_{i, j} G_{j}(k), \quad \boldsymbol{B}_{i}(k)=\sum_{j=1}^{p b} \boldsymbol{B}_{i, j} G_{j}(k)
\end{gathered}
$$

with $n a$ and $n b$ designating the corresponding AutoRegressive (AR) and eXogenous (X) model orders. $\boldsymbol{w}_{k}[t]$ is a $(n y \times 1)$ innovations vector which is zero mean, serially uncorrelated, with fully parametrized crosscovariance matrices $\boldsymbol{\Sigma}_{k, \ell} . E\{\cdot\}$ designates statistical expectation, and $\delta[\tau]$ the Kronecker delta. The AR and $\mathrm{X}$ matrices $\boldsymbol{A}_{i}(k)$ and $\boldsymbol{B}_{i}(k)$ are fully parameterized and have dimensions $(n y \times n y)$ and $(n y \times n x)$, respectively. They are expressed as explicit functions of the operating parameter $k$ by belonging to a functional subspace of dimensionality $p=\max (p a, p b)$ spanned by the mutually independent functions $G_{j}(k)$ $(j=1, \ldots, p)$. These functions form a functional basis, with $\boldsymbol{A}_{i, j}$, and $\boldsymbol{B}_{i, j}$ designating the corresponding, fully parametrized, projection matrices. The representation of Eq. (2.2a)-(2.2c) is referred to as an FP-VARX model of orders $(n a, n b)$ and functional subspace dimensionalities $(p a, p b)$, or in short as an $\operatorname{FP}-\operatorname{VARX}(n a, n b)_{[p a, p b]}$ model. It is worth noting that:

(i) All information concerning the interrelations among the data records in $Z^{N M}$ is reflected in the crosscovariance matrices $\boldsymbol{\Sigma}_{k, \ell}$. This knowledge is incorporated into the parameter estimation phase to obtain statistically optimal models (see Section 2.3). An overview of the most common cases of statistical dependencies between the innovations $\boldsymbol{w}_{k}[t]$ belonging to different operating conditions are summarized in Table 2.1.

(ii) The projection of the $\mathrm{AR}$ and $\mathrm{X}$ matrices, $\boldsymbol{A}_{i}(k)$ and $\boldsymbol{B}_{i}(k)$, on the functional basis $G_{j}(k)(j=$ $1, \ldots, p)$ allows for models capable of representing the system dynamics everywhere within $\mathcal{R}=$ $\left[k_{\min }, k_{\max }\right]$, and not only on the distinct (sample) values $\left\{k_{1}, k_{2}, \ldots, k_{M}\right\}$ involved in $Z^{N M}$.

(iii) The form of functional dependence is an important issue. Physical insight may be used for this, although experience has shown that orthogonal polynomials (for instance, Chebyshev or Legendre), or trigonometric functions are sufficient, as any given function may be approximated by any orthogonal set (refer to [38-42] for practical applications, where Chebyshev polynomials of the second kind are used). Nevertheless, the specific selection might affect model compactness (see Section 2.6). Of course, a practical limitation posed is that the selected functional subspace dimensionality must be smaller than the number of available data records $M$, that is $p<M$. form:

The FP-VARX $(n a, n b)_{[p a, p b]}$ model in Eq. (2.2a)-(2.2c) may be rewritten in the following polynomial

$$
\begin{gathered}
\boldsymbol{A}[\mathcal{B}, k] \boldsymbol{y}_{k}[t]=\boldsymbol{B}[\mathcal{B}, k] \boldsymbol{x}_{k}[t]+\boldsymbol{w}_{k}[t], \quad k \in \mathcal{R} \\
\boldsymbol{A}[\mathcal{B}, k] \triangleq \boldsymbol{I}_{n y}+\boldsymbol{A}_{1}(k) \mathcal{B}+\ldots+\boldsymbol{A}_{n a}(k) \mathcal{B}^{n a} \\
\boldsymbol{B}[\mathcal{B}, k] \triangleq \boldsymbol{B}_{0}(k)+\boldsymbol{B}_{1}(k) \mathcal{B}+\ldots+\boldsymbol{B}_{n b}(k) \mathcal{B}^{n b}
\end{gathered}
$$

with the matrix polynomials $\boldsymbol{A}[\mathcal{B}, k], \boldsymbol{B}[\mathcal{B}, k]$ having degrees $n a, n b$ respectively, whereas $\mathcal{B}$ designates the backward shift operator. The preceding model satisfies the following assumptions.

\section{Assumptions on the model structure}

A1. All zeros of $\operatorname{det}(\boldsymbol{A}[\mathcal{B}, k])$ lie outside the unit circle for the distinct (sample) values $\left\{k_{1}, k_{2}, \ldots, k_{M}\right\}$ ("stability condition").

A2. The polynomial matrices $\boldsymbol{A}[\mathcal{B}, k], \boldsymbol{B}[\mathcal{B}, k]$ are left coprime for the distinct (sample) values $\left\{k_{1}, k_{2}\right.$, $\left.\ldots, k_{M}\right\}$ ("irreducibility condition"). 
Table 2.1: Overview of innovation dependency types.

\begin{tabular}{|c|c|c|c|c|c|c|}
\hline $\begin{array}{l}\text { Innovations } \\
\text { dependency type }\end{array}$ & $\begin{array}{l}\text { Interrelations among } \\
\text { different operating conditions } \\
k, \ell \in \mathcal{R}=\left[k_{\text {min }}, k_{\text {max }}\right] \subset \mathbb{R}\end{array}$ & $\begin{array}{l}\text { Innovi } \\
E\{\boldsymbol{w}[ \\
\boldsymbol{w}[t]=\end{array}$ & $\begin{array}{l}\text { ions cova } \\
\left.\boldsymbol{w}^{T}[t]\right\} \\
{\left[\boldsymbol{w}_{k_{1}}^{T}[t]\right.}\end{array}$ & $\begin{array}{l}\text { ariance mi } \\
=\boldsymbol{\Sigma}, \text { with } \\
\boldsymbol{w}_{k_{2}}^{T}[t]\end{array}$ & $\begin{array}{l}\text { atrix } \\
\text { : } \\
\end{array}$ & $\left.\boldsymbol{w}_{k_{M}}^{T}[t]\right]^{T}$ \\
\hline Contemporaneously correlated & $E\left\{\boldsymbol{w}_{k}[t] \boldsymbol{w}_{\ell}^{T}[t]\right\}=\boldsymbol{\Sigma}_{k, \ell}, \forall k, \ell \in \mathcal{R}$ & $\Sigma=$ & $\begin{array}{c}\boldsymbol{\Sigma}_{k_{1}, k_{1}} \\
\boldsymbol{\Sigma}_{k_{2}, k_{1}} \\
\vdots \\
\boldsymbol{\Sigma}_{k_{M}, k_{1}}\end{array}$ & $\begin{array}{c}\boldsymbol{\Sigma}_{k_{1}, k_{2}} \\
\boldsymbol{\Sigma}_{k_{2}, k_{2}} \\
\vdots \\
\boldsymbol{\Sigma}_{k_{M}, k_{2}}\end{array}$ & $\begin{array}{l}\ldots \\
\ldots \\
\ddots \\
\cdots\end{array}$ & $\left.\begin{array}{c}\boldsymbol{\Sigma}_{k_{1}, k_{M}} \\
\boldsymbol{\Sigma}_{k_{2}, k_{M}} \\
\vdots \\
\boldsymbol{\Sigma}_{k_{M}, k_{M}}\end{array}\right]$ \\
\hline Groupwise heteroscedastic & $E\left\{\boldsymbol{w}_{k}[t] \boldsymbol{w}_{\ell}^{T}[t]\right\}=\boldsymbol{\Sigma}_{k, \ell} \delta[k-\ell], \forall k, \ell \in \mathcal{R}$ & $\boldsymbol{\Sigma}=$ & $\begin{array}{c}\boldsymbol{\Sigma}_{k_{1}, k_{1}} \\
\mathbf{0} \\
\vdots \\
\mathbf{0}\end{array}$ & $\begin{array}{c}\mathbf{0} \\
\boldsymbol{\Sigma}_{k_{2}, k_{2}} \\
\vdots \\
\mathbf{0}\end{array}$ & $\begin{array}{l}\cdots \\
\cdots \\
\ddots \\
\cdots\end{array}$ & $\left.\begin{array}{c}\mathbf{0} \\
\mathbf{0} \\
\vdots \\
\boldsymbol{\Sigma}_{k_{M}, k_{M}}\end{array}\right]$ \\
\hline Groupwise homoscedastic & $E\left\{\boldsymbol{w}_{k}[t] \boldsymbol{w}_{\ell}^{T}[t]\right\}=\overline{\boldsymbol{\Sigma}} \delta[k-\ell], \forall k, \ell \in \mathcal{R}$ & $\Sigma=$ & $\begin{array}{cc}\bar{\Sigma} & \mathbf{0} \\
\mathbf{0} & \overline{\boldsymbol{\Sigma}} \\
\vdots & \vdots \\
\mathbf{0} & \mathbf{0}\end{array}$ & $\left.\begin{array}{cc}\ldots & 0 \\
\ldots & 0 \\
\ddots & \vdots \\
\ldots & \bar{\Sigma}\end{array}\right]$ & & \\
\hline
\end{tabular}

A3. $\operatorname{rank}\left[\boldsymbol{A}_{n a}(k) \vdots \boldsymbol{B}_{n b}(k)\right]=n y$ for the distinct (sample) values $\left\{k_{1}, k_{2}, \ldots, k_{M}\right\}$ ("identifiability condition").

A4. The basis functions $G_{j}(k)$ and subspace dimensionalities $(p a, p b)$ are known.

\section{Assumptions on $x_{k}[t], \boldsymbol{w}_{k}[t]$ and $k$}

A5. The sequence $\boldsymbol{x}_{k}[t]$ is ergodic covariance stationary and is persistently exciting of order $(n b+1)$ for the distinct (sample) values $\left\{k_{1}, k_{2}, \ldots, k_{M}\right\}$.

A6. $\boldsymbol{w}_{k}[t]$ is a sequence of independent and identically distributed (i.i.d.) random vectors with zero mean and positive-definite covariance $\boldsymbol{\Sigma}_{k, k}$ (white noise). Furthermore, $\boldsymbol{w}_{k}[t]$ is uncorrelated with $\boldsymbol{x}_{\ell}[t-$ $j], \forall j \geq 0$ and $k, \ell \in \mathcal{R}$.

A7. Each value of the operating parameter $k$ corresponds to a specific operating condition. The operating parameter $k$ is measurable upon operation of the system, bounded (that is, $k \in \mathcal{R}=\left[k_{\text {min }}, k_{\text {max }}\right] \subset$ $\mathbb{R})$, and remains constant during data acquisition.

Remark 1. The assumptions A1 and A5 ensure the covariance stationarity of the response vectors $\boldsymbol{y}_{k}[t]$ for the distinct (sample) values $\left\{k_{1}, k_{2}, \ldots, k_{M}\right\}$. This means that the responses are completely described by their mean and covariance, whereas to derive the large sample properties of the LS and ML estimators (see Section 2.4) the sums of $\boldsymbol{y}_{k}[t]$ over time may be replaced with their corresponding expectations.

Remark 2. The assumption A2 ensures that there are no common left factors in the AR and X polynomials of the model except for unimodular matrices (that is, matrices with constant determinant), whereas assumption A3 ensures that the common left factors are constant matrices (refer to [2, pp. 182-183], for the corresponding conditions on conventional VARX models). When assumptions A2-A4 hold, uniqueness of representation for the $\operatorname{FP}-\operatorname{VARX}(n a, n b)_{[p a, p b]}$ model in Eq. (2.2a)-(2.2c) is warranted (see Section 2.5).

Remark 3. The assumption A7 poses some practical conditions in order to obtain an FP model. It ensures that (i) the system dynamics at two different operating conditions are not described by the same VARX representation, and (ii) data records suitable for FP-VARX identification are obtained (see Section 2.3). 


\subsection{FP-VARX model parameter estimation}

The FP-VARX model estimation problem may be stated as: "Given the data record $Z^{N M}$, identify the model $\mathcal{M}(\boldsymbol{\theta})$ from the following set $\mathcal{M}$ of all candidate FP-VARX models that "best" fits the observed responses":

$$
\begin{array}{r}
\mathcal{M} \triangleq\left\{\mathcal{M}(\boldsymbol{\theta}): \boldsymbol{A}[\mathcal{B}, k, \boldsymbol{\theta}] \boldsymbol{y}_{k}[t]=\boldsymbol{B}[\mathcal{B}, k, \boldsymbol{\theta}] \boldsymbol{x}_{k}[t]+\boldsymbol{e}_{k}[t, \boldsymbol{\theta}] \mid \boldsymbol{A}[\mathcal{B}, k, \boldsymbol{\theta}], \boldsymbol{B}[\mathcal{B}, k, \boldsymbol{\theta}]\right. \\
\text { obey A1 - A4 } \left., E\left\{\boldsymbol{e}_{k}[t, \boldsymbol{\theta}] \boldsymbol{e}_{\ell}^{T}[t-\tau, \boldsymbol{\theta}]\right\}=\boldsymbol{\Sigma}_{k, \ell}(\boldsymbol{\theta}) \delta[\tau] \quad k, \ell \in \mathcal{R}\right\}
\end{array}
$$

with $\boldsymbol{e}_{k}[t, \boldsymbol{\theta}]$ being the $(n y \times 1)$ residual (one-step-ahead prediction error) vector corresponding to the $k$-th operating condition. The residuals, for an accurate model, should be zero mean, serially uncorrelated, with positive-definite covariance matrices $\boldsymbol{\Sigma}_{k, k}(\boldsymbol{\theta})$ (white noise, see assumption A6 in Section 2.2). The FP-VARX models are parametrized in terms of the projection coefficient vector $\boldsymbol{\theta}$ defined as:

$$
\boldsymbol{\theta} \triangleq \operatorname{vec}\left(\left[\boldsymbol{A}_{1,1} \boldsymbol{A}_{1,2} \ldots \boldsymbol{A}_{n a, p a} \vdots \boldsymbol{B}_{0,1} \boldsymbol{B}_{0,2} \ldots \boldsymbol{B}_{n b, p b}\right]^{T}\right)
$$

with $\operatorname{vec}(\cdot)$ designating the operator that transforms the indicated matrix into a vector by stacking its columns.

Parameter estimation is presently based on properly formulated Least Squares (LS) and conditional Maximum Likelihood (ML) estimators.

\subsubsection{LS-based estimators}

The FP-VARX $(n a, n b)_{[p a, p b]}$ model of Eq. (2.4) may be equivalently expressed in a linear regression form as:

$$
\boldsymbol{y}_{k}[t]=\boldsymbol{\Phi}_{k}^{T}[t] \boldsymbol{\theta}+\boldsymbol{e}_{k}[t, \boldsymbol{\theta}]
$$

where:

$$
\begin{gathered}
\boldsymbol{\Phi}_{k}^{T}[t]=\boldsymbol{I}_{n y} \otimes \boldsymbol{\phi}_{k}^{T}[t] \\
\boldsymbol{\phi}_{k}[t]=\left[\begin{array}{c}
-\boldsymbol{y}_{k}[t-1] \\
\vdots \\
-\boldsymbol{y}_{k}[t-n a]
\end{array}\right] \otimes\left[\begin{array}{c}
G_{1}(k) \\
\vdots \\
\boldsymbol{x}_{p a}[t] \\
\vdots \\
\\
{\left[\begin{array}{c}
\boldsymbol{x}_{k}[t-n b]
\end{array}\right] \otimes\left[\begin{array}{c}
G_{1}(k) \\
\vdots \\
G_{p b}(k)
\end{array}\right]}
\end{array}\right.
\end{gathered}
$$

with $\otimes$ designating the Kronecker product ${ }^{2}$.

Model parameter estimation requires stacking the equations of the form (2.6a) for each distinct operating condition, $\left\{k_{1}, k_{2}, \ldots, k_{M}\right\}$ one below the other. This procedure is referred to as pooling:

$$
\begin{gathered}
\boldsymbol{y}[t]=\boldsymbol{\Phi}^{T}[t] \boldsymbol{\theta}+\boldsymbol{e}[t, \boldsymbol{\theta}] \\
\boldsymbol{y}[t]=\left[\begin{array}{c}
\boldsymbol{y}_{k_{1}}[t] \\
\boldsymbol{y}_{k_{2}}[t] \\
\vdots \\
\boldsymbol{y}_{k_{M}}[t]
\end{array}\right], \quad \boldsymbol{\Phi}^{T}[t]=\left[\begin{array}{c}
\boldsymbol{\Phi}_{k_{1}}^{T}[t] \\
\boldsymbol{\Phi}_{k_{2}}^{T}[t] \\
\vdots \\
\boldsymbol{\Phi}_{k_{M}}^{T}[t]
\end{array}\right], \quad \boldsymbol{e}[t, \boldsymbol{\theta}]=\left[\begin{array}{c}
\boldsymbol{e}_{k_{1}}[t, \boldsymbol{\theta}] \\
\boldsymbol{e}_{k_{2}}[t, \boldsymbol{\theta}] \\
\vdots \\
\boldsymbol{e}_{k_{M}}[t, \boldsymbol{\theta}]
\end{array}\right]
\end{gathered}
$$

\footnotetext{
${ }^{2}$ The Kronecker product of an $(m \times n)$ matrix $\boldsymbol{A}=\left[a_{i, j}\right]$ and a $(p \times q)$ matrix $\boldsymbol{B}=\left[b_{i, j}\right]$ is $\boldsymbol{A} \otimes \boldsymbol{B}=\left[a_{i, j} \boldsymbol{B}\right][43$, p. 3].
} 
Notice that the term functional pooling signifies the functional dependence of each equation on the operating parameter $k$. Based upon the above linear regression framework, the projection vector $\boldsymbol{\theta}$ may be estimated using a criterion that minimizes a scalar positive function of the residual sequence $\boldsymbol{e}[t, \boldsymbol{\theta}]$ with respect to $\boldsymbol{\theta}$, that is ${ }^{3}$ :

$$
\widehat{\boldsymbol{\theta}}_{L S}=\arg \min _{\boldsymbol{\theta}}\left(\frac{1}{N M} \sum_{t=1}^{N} \boldsymbol{e}^{T}[t, \boldsymbol{\theta}] \boldsymbol{H} \boldsymbol{e}[t, \boldsymbol{\theta}]\right)
$$

where "arg min" designates the minimizing argument, and $\boldsymbol{H}$ is a $(M n y \times M n y)$ symmetric, positivedefinite weighting matrix.

A simplified example of FP-VARX model parameter estimation via LS is provided in Appendix 2.A.

\subsubsection{The Ordinary Least Squares (OLS) estimator}

The OLS estimator is based upon minimizing the criterion in Eq. (2.8) for $\boldsymbol{H}=\boldsymbol{I}_{M n y}$, which leads to the following estimators for the projection coefficient vector $\boldsymbol{\theta}$ and the covariance matrix $\boldsymbol{\Sigma}$ of $\boldsymbol{w}[t]$ (defined similarly to $\boldsymbol{e}[t, \boldsymbol{\theta}]$ in Eq. (2.7b); see also Table 2.1):

$$
\begin{gathered}
\widehat{\boldsymbol{\theta}}_{O L S}=\left[\frac{1}{N M} \sum_{t=1}^{N} \boldsymbol{\Phi}[t] \boldsymbol{\Phi}^{T}[t]\right]^{-1}\left[\frac{1}{N M} \sum_{t=1}^{N} \boldsymbol{\Phi}[t] \boldsymbol{y}[t]\right] \\
\widehat{\boldsymbol{\Sigma}}_{O L S}=\frac{1}{N} \sum_{t=1}^{N} \boldsymbol{e}\left[t, \widehat{\boldsymbol{\theta}}_{O L S}\right] \boldsymbol{e}^{T}\left[t, \widehat{\boldsymbol{\theta}}_{O L S}\right]
\end{gathered}
$$

\subsubsection{The Weighted Least Squares (WLS) estimator}

The WLS estimator is based upon minimizing the criterion in Eq. (2.8) for $\boldsymbol{H}=\boldsymbol{\Sigma}^{-1}$, which leads to the following estimators for $\boldsymbol{\theta}$ and $\boldsymbol{\Sigma}$ :

$$
\begin{gathered}
\widehat{\boldsymbol{\theta}}_{W L S}=\left[\frac{1}{N M} \sum_{t=1}^{N} \boldsymbol{\Phi}[t] \boldsymbol{\Sigma}^{-1} \boldsymbol{\Phi}^{T}[t]\right]^{-1}\left[\frac{1}{N M} \sum_{t=1}^{N} \boldsymbol{\Phi}[t] \boldsymbol{\Sigma}^{-1} \boldsymbol{y}[t]\right] \\
\widehat{\boldsymbol{\Sigma}}_{W L S}=\frac{1}{N} \sum_{t=1}^{N} \boldsymbol{e}\left[t, \widehat{\boldsymbol{\theta}}_{W L S}\right] \boldsymbol{e}^{T}\left[t, \widehat{\boldsymbol{\theta}}_{W L S}\right]
\end{gathered}
$$

Remark 4. Note that $\Sigma$ in Eq. (2.10a) is usually unavailable. Thus, to obtain a practically applicable estimator, an OLS-based estimate of the covariance matrix is inserted into Eq. (2.10a) in order to compute $\widehat{\boldsymbol{\theta}}_{W L S}$, while the final residual covariance is obtained as in Eq. $(2.10 \mathrm{~b})$. The procedure may be continued by inserting the resulting $\widehat{\boldsymbol{\Sigma}}_{W L S}$ into Eq. (2.10a) in order to obtain a new WLS estimate, and repeated until $\widehat{\boldsymbol{\theta}}_{W L S}$ converges (iterated WLS estimation).

Remark 5. Note that the LS-based parameter estimation of conventional VARX models, with fully parametrized AR and X polynomial matrices, yields identical OLS and WLS estimators [2, pp. 233-235]. This does not hold true for the FP-VARX models, as these estimators are different (compare Eq. (2.9a) and Eq. (2.10a)), except for special cases.

In view of the preceding remark, the following proposition holds for the WLS and OLS estimators.

\footnotetext{
${ }^{3}$ Symbols in hat designate estimators/estimates.
} 
Proposition 2.3.1. Under the assumption of groupwise homoscedastic innovations (see Table 2.1), the WLS projection coefficient estimator is identical to its OLS counterpart.

Proof. See Appendix 2.B.1.

\subsubsection{The conditional ML estimator}

Let $\boldsymbol{w}[t]$ be a Gaussian white noise process, that is $\boldsymbol{w}[t] \sim \mathcal{N}(\mathbf{0}, \boldsymbol{\Sigma})$, with $\mathcal{N}(\cdot, \cdot)$ designating the normal distribution with indicated mean and covariance. The ML estimators of $\boldsymbol{\theta}$ and $\boldsymbol{\Sigma}$ are:

$$
\begin{aligned}
& \widehat{\boldsymbol{\theta}}_{M L}=\arg \min _{\boldsymbol{\theta}} \ln \left(\operatorname{det}\left(\frac{1}{N} \sum_{t=1}^{N} \boldsymbol{e}[t, \boldsymbol{\theta}] \boldsymbol{e}^{T}[t, \boldsymbol{\theta}]\right)\right) \\
& \widehat{\boldsymbol{\Sigma}}_{M L}=\frac{1}{N} \sum_{t=1}^{N} \boldsymbol{e}\left[t, \widehat{\boldsymbol{\theta}}_{M L}\right] \boldsymbol{e}^{T}\left[t, \widehat{\boldsymbol{\theta}}_{M L}\right]
\end{aligned}
$$

with $\ln (\cdot)$ designating the natural logarithm and $\operatorname{det}(\cdot)$ the determinant. Notice that obtaining $\widehat{\boldsymbol{\theta}}_{M L}$ requires the use of iterative optimization techniques as discussed in [2, pp. 212-214].

\subsection{Large sample properties of the LS and ML type estimators}

The consistency and the asymptotic distribution of the LS and the conditional ML type estimators are studied under the assumption of exactly known model structure (model orders $(n a, n b)$, basis functions $G_{j}(k)$, and subspace dimensionalities $(p a, p b)$; see assumption A4 in Section 2.2).

\subsubsection{The LS-based estimators}

Theorem 2.4.1. (The LS-based estimator consistency and asymptotic distribution): Let $\boldsymbol{\theta}_{o}$ be the system's true projection coefficient vector and $\boldsymbol{\Sigma}_{o}$ the true (positive-definite) covariance matrix of $\boldsymbol{w}[t]$, which is additionally assumed to have bounded fourth-order moments. Consider the vector $\boldsymbol{\sigma}_{o}$, comprising of the non-identical elements on and below the main diagonal of $\boldsymbol{\Sigma}_{o}$, that is $\operatorname{vec}\left(\boldsymbol{\Sigma}_{o}\right)=\boldsymbol{D}_{M n y} \boldsymbol{\sigma}_{o}$, with $\boldsymbol{D}_{M n y}$ designating a duplication matrix of proper dimensions. Assuming that $E\left\{\boldsymbol{\Phi}[t] \boldsymbol{\Phi}^{T}[t]\right\}$ is a finite positive definite matrix, then the estimators $\widehat{\boldsymbol{\theta}}_{L S}$ and $\widehat{\boldsymbol{\Sigma}}_{L S}$ are consistent, that is:

$$
\begin{aligned}
& \widehat{\boldsymbol{\theta}}_{L S} \stackrel{p}{\longrightarrow} \boldsymbol{\theta}_{O} \quad(N \longrightarrow \infty) \\
& \widehat{\boldsymbol{\Sigma}}_{L S} \stackrel{p}{\longrightarrow} \boldsymbol{\Sigma}_{O} \quad(N \longrightarrow \infty)
\end{aligned}
$$

with “ $\stackrel{p}{\longrightarrow}$ ” designating the limit in probability 4 .

The limiting distribution of $\widehat{\boldsymbol{\theta}}_{L S}$ is:

$$
\sqrt{N M}\left(\widehat{\boldsymbol{\theta}}_{L S}-\boldsymbol{\theta}_{o}\right) \stackrel{d}{\longrightarrow} \mathcal{N}\left(\mathbf{0}, \boldsymbol{P}_{L S}\right) \quad(N \longrightarrow \infty)
$$

with $\boldsymbol{P}_{L S}=M E\left\{\boldsymbol{\Phi}[t] \boldsymbol{H} \boldsymbol{\Phi}^{T}[t]\right\}^{-1} E\left\{\boldsymbol{\Phi}[t] \boldsymbol{H} \boldsymbol{\Sigma}_{o} \boldsymbol{H} \boldsymbol{\Phi}^{T}[t]\right\} E\left\{\boldsymbol{\Phi}[t] \boldsymbol{H} \boldsymbol{\Phi}^{T}[t]\right\}^{-1}$ designating the asymptotic normalized covariance matrix and “ $\stackrel{d}{\longrightarrow}$ ” the limit in distribution ${ }^{5}$.

\footnotetext{
${ }^{4}$ Let $\left\{\boldsymbol{x}_{N}\right\} N=1,2, \ldots$ be an indexed sequence of $(n \times 1)$ stochastic vector variables, and $\boldsymbol{x}$ a $(n \times 1)$ stochastic vector variable. Then, $\boldsymbol{x}_{N} \stackrel{p}{\longrightarrow} \boldsymbol{x}$ if $\forall \epsilon>0, \operatorname{Pr}\left(\left|x_{i N}-x_{i}\right|>\epsilon\right) \longrightarrow \mathbf{0}$ as $N \longrightarrow \infty$ for $i=1, \ldots, n$ [44, pp. 681-682].

${ }^{5}$ Let the joint distribution functions of $\boldsymbol{x}_{N}$ and $\boldsymbol{x}$ be $F_{N}$ and $F$, respectively. Then, $\boldsymbol{x}_{N} \stackrel{d}{\longrightarrow} \boldsymbol{x}$ if for all real vectors $\boldsymbol{c}$ for which $F$ is continuous, $F_{N}(c) \longrightarrow F(c)$ as $N \longrightarrow \infty[44$, p. 682].
} 
The limiting distribution of $\widehat{\boldsymbol{\sigma}}_{L S}$ is:

$$
\sqrt{N M}\left(\widehat{\boldsymbol{\sigma}}_{L S}-\boldsymbol{\sigma}_{o}\right) \stackrel{d}{\longrightarrow} \mathcal{N}\left(\mathbf{0}, 2 M \boldsymbol{D}_{M n y}^{+}\left(\boldsymbol{\Sigma}_{o} \otimes \boldsymbol{\Sigma}_{o}\right)\left(\boldsymbol{D}_{M n y}^{+}\right)^{T}\right) \quad(N \longrightarrow \infty)
$$

with the superscript "+" designating the Moore-Penrose generalized inverse matrix 6 .

Proof. See Appendix 2.B.2.

Remark 6. The definition of $\boldsymbol{\sigma}$ for the FP-VARX models is in analogy to the corresponding conventional $\operatorname{VAR}(\mathrm{X})$ model case $[44$, p. 90]. Note however that in conventional VARX models, all non-identical elements of the corresponding residual covariance matrix are on and below (or above) the main diagonal, due to its symmetric structure. This is not the case for the FP-VARX models, as the covariance $\boldsymbol{\Sigma}$ has a richer structure with identical elements at certain locations below the main diagonal (refer to Table 2.1, and the example of Appendix 2.A).

Remark 7. The duplication matrix $\boldsymbol{D}_{M n y}$ contains zeros and ones at proper locations, and it is defined in analogy to the conventional $\operatorname{VAR}(\mathrm{X})$ model case [44, p. 92]. Care has to be taken when considering different types of dependencies between the innovations, as the structure of the covariance matrix $\boldsymbol{\Sigma}$ changes, thus affecting the dimensions and structure of the duplication matrix. Indeed, the dimensions of $\boldsymbol{D}_{M n y}$ are $\left((M n y)^{2} \times \frac{1}{4} M n y(M+1)(n y+1)\right)$ for contemporaneously correlated innovations, $\left((M n y)^{2} \times\right.$ $\left.\frac{1}{2} M n y(n y+1)\right)$ for groupwise heteroscedastic innovations, and $\left((M n y)^{2} \times \frac{1}{2} n y(n y+1)\right)$ for groupwise homoscedastic innovations (refer to Table 2.1, and the example of Appendix 2.A).

The following propositions and corollaries hold in view of Theorem 2.4.1.

Proposition 2.4.1. (OLS estimator large sample properties): Under the assumptions of Theorem 2.4.1, the estimators $\widehat{\boldsymbol{\theta}}_{O L S}$ and $\widehat{\boldsymbol{\Sigma}}_{O L S}$ are consistent, whereas $\widehat{\boldsymbol{\theta}}_{O L S}$ and $\widehat{\boldsymbol{\sigma}}_{O L S}$ are asymptotically normally distributed, that is:

$$
\sqrt{N M}\left(\widehat{\boldsymbol{\theta}}_{O L S}-\boldsymbol{\theta}_{o}\right) \stackrel{d}{\longrightarrow} \mathcal{N}\left(\mathbf{0}, \boldsymbol{P}_{O L S}\right) \quad(N \longrightarrow \infty)
$$

with $\boldsymbol{P}_{O L S}=M E\left\{\boldsymbol{\Phi}[t] \boldsymbol{\Phi}^{T}[t]\right\}^{-1} E\left\{\boldsymbol{\Phi}[t] \boldsymbol{\Sigma}_{o} \boldsymbol{\Phi}^{T}[t]\right\} E\left\{\boldsymbol{\Phi}[t] \boldsymbol{\Phi}^{T}[t]\right\}^{-1}$, and:

$$
\sqrt{N M}\left(\widehat{\boldsymbol{\sigma}}_{O L S}-\boldsymbol{\sigma}_{o}\right) \stackrel{d}{\longrightarrow} \mathcal{N}\left(\mathbf{0}, 2 M \boldsymbol{D}_{M n y}^{+}\left(\boldsymbol{\Sigma}_{o} \otimes \boldsymbol{\Sigma}_{o}\right)\left(\boldsymbol{D}_{M n y}^{+}\right)^{T}\right) \quad(N \longrightarrow \infty)
$$

Proof. The proof follows directly from Theorem 2.4.1 by setting $\boldsymbol{H}=\boldsymbol{I}_{M n y}$.

Estimates of the covariances for $\widehat{\boldsymbol{\theta}}_{O L S}$ and $\widehat{\boldsymbol{\sigma}}_{O L S}$ are thus given by:

$$
\begin{aligned}
\widehat{\boldsymbol{P}}\left(\widehat{\boldsymbol{\theta}}_{O L S}\right)= & {\left[\sum_{t=1}^{N} \boldsymbol{\Phi}[t] \boldsymbol{\Phi}^{T}[t]\right]^{-1}\left[\sum_{t=1}^{N} \boldsymbol{\Phi}[t] \widehat{\boldsymbol{\Sigma}}_{O L S} \boldsymbol{\Phi}^{T}[t]\right]\left[\sum_{t=1}^{N} \boldsymbol{\Phi}[t] \boldsymbol{\Phi}^{T}[t]\right]^{-1} } \\
& \widehat{\boldsymbol{P}}\left(\widehat{\boldsymbol{\sigma}}_{O L S}\right)=\frac{2}{N} \boldsymbol{D}_{M n y}^{+}\left(\widehat{\boldsymbol{\Sigma}}_{O L S} \otimes \widehat{\boldsymbol{\Sigma}}_{O L S}\right)\left(\boldsymbol{D}_{M n y}^{+}\right)^{T}
\end{aligned}
$$

with $\boldsymbol{P}(\cdot)$ designating the covariance matrix of the indicated vector.

\footnotetext{
${ }^{6}$ The Moore-Penrose generalized inverse of an $(m \times n)$ matrix $\boldsymbol{A}$ is, $\boldsymbol{A}^{+}=\left(\boldsymbol{A}^{T} \boldsymbol{A}\right)^{-1} \boldsymbol{A}^{T}$ [43, p. 35].
} 
Corollary 2.4.1. (OLS estimator statistical accuracy): Under the assumptions of Theorem 2.4.1, $\widehat{\boldsymbol{\theta}}_{O L S}$ is asymptotically inefficient, as $\boldsymbol{P}_{O L S}$ does not reach the Cramér-Rao lower bound, except for the case of groupwise homoscedastic innovations, whereas $\widehat{\boldsymbol{\sigma}}_{O L S}$ is always asymptotically efficient.

Proof. See Appendix 2.B.4.1.

Proposition 2.4.2. (WLS estimator large sample properties): Under the assumptions of Theorem 2.4.1, the estimators $\widehat{\boldsymbol{\theta}}_{W L S}$ and $\widehat{\boldsymbol{\Sigma}}_{W L S}$ are consistent, whereas $\widehat{\boldsymbol{\theta}}_{W L S}$ and $\widehat{\boldsymbol{\sigma}}_{W L S}$ are asymptotically normally distributed, that is:

$$
\sqrt{N M}\left(\widehat{\boldsymbol{\theta}}_{W L S}-\boldsymbol{\theta}_{o}\right) \stackrel{d}{\longrightarrow} \mathcal{N}\left(\mathbf{0}, \boldsymbol{P}_{W L S}\right) \quad(N \longrightarrow \infty)
$$

with $\boldsymbol{P}_{W L S}=M E\left\{\boldsymbol{\Phi}[t] \boldsymbol{\Sigma}_{o}^{-1} \boldsymbol{\Phi}^{T}[t]\right\}^{-1}$, and:

$$
\sqrt{N M}\left(\widehat{\boldsymbol{\sigma}}_{W L S}-\boldsymbol{\sigma}_{o}\right) \stackrel{d}{\longrightarrow} \mathcal{N}\left(\mathbf{0}, 2 M \boldsymbol{D}_{M n y}^{+}\left(\boldsymbol{\Sigma}_{o} \otimes \boldsymbol{\Sigma}_{o}\right)\left(\boldsymbol{D}_{M n y}^{+}\right)^{T}\right) \quad(N \longrightarrow \infty)
$$

Proof. The proof follows directly from Theorem 2.4.1 by setting $\boldsymbol{H}=\boldsymbol{\Sigma}_{o}^{-1}$.

Estimates of the covariances for $\widehat{\boldsymbol{\theta}}_{W L S}$ and $\widehat{\boldsymbol{\sigma}}_{W L S}$ are thus given by:

$$
\begin{gathered}
\widehat{\boldsymbol{P}}\left(\widehat{\boldsymbol{\theta}}_{W L S}\right)=\left[\sum_{t=1}^{N} \boldsymbol{\Phi}[t] \widehat{\boldsymbol{\Sigma}}_{W L S}^{-1} \boldsymbol{\Phi}^{T}[t]\right]^{-1} \\
\widehat{\boldsymbol{P}}\left(\widehat{\boldsymbol{\sigma}}_{W L S}\right)=\frac{2}{N} \boldsymbol{D}_{M n y}^{+}\left(\widehat{\boldsymbol{\Sigma}}_{W L S} \otimes \widehat{\boldsymbol{\Sigma}}_{W L S}\right)\left(\boldsymbol{D}_{M n y}^{+}\right)^{T}
\end{gathered}
$$

Corollary 2.4.2. (WLS estimator statistical accuracy): Under the assumptions of Theorem 2.4.1, $\widehat{\boldsymbol{\theta}}_{W L S}$ and $\widehat{\boldsymbol{\sigma}}_{W L S}$ are asymptotically statistically efficient, as their corresponding covariance matrices reach the Cramér-Rao lower bound, thereby providing optimal accuracy.

Proof. See Appendix 2.B.4.2.

\subsubsection{The conditional ML estimator}

Theorem 2.4.2. (The ML-based estimator consistency and asymptotic distribution): Let $\overline{\boldsymbol{\theta}}_{o}=\left[\boldsymbol{\theta}_{o}^{T} \vdots \boldsymbol{\sigma}_{o}^{T}\right]^{T}$ be the system's true parameter vector. Assuming that $\boldsymbol{\Sigma}_{o}$ and $E\left\{\boldsymbol{\Phi}[t] \boldsymbol{\Phi}^{T}[t]\right\}$ are positive-definite matrices, then the estimator $\widehat{\overline{\boldsymbol{\theta}}}_{M L}$ is consistent, that is:

$$
\widehat{\overline{\boldsymbol{\theta}}}_{M L} \stackrel{p}{\longrightarrow} \overline{\boldsymbol{\theta}}_{O} \quad(N \longrightarrow \infty)
$$

The limiting distribution of the ML estimator $\widehat{\overline{\boldsymbol{\theta}}}_{M L}$ is given by:

$$
\sqrt{N M}\left(\widehat{\overline{\boldsymbol{\theta}}}_{M L}-\overline{\boldsymbol{\theta}}_{o}\right) \stackrel{d}{\longrightarrow} \mathcal{N}\left(\mathbf{0}, \boldsymbol{P}_{M L}\right) \quad(N \longrightarrow \infty)
$$

with, $\boldsymbol{P}_{M L}=\left[\begin{array}{cc}M E\left\{\boldsymbol{\Phi}[t] \boldsymbol{\Sigma}_{o}^{-1} \boldsymbol{\Phi}^{T}[t]\right\}^{-1} & \mathbf{0} \\ \mathbf{0} & 2 M \boldsymbol{D}_{M n y}^{+}\left(\boldsymbol{\Sigma}_{o} \otimes \boldsymbol{\Sigma}_{o}\right)\left(\boldsymbol{D}_{M n y}^{+}\right)^{T}\end{array}\right]$

Proof. See Appendix 2.B.3. 
Table 2.2: Overview of asymptotic properties for the LS and ML type FP-VARX parameter estimators.

\begin{tabular}{lccc}
\hline $\begin{array}{l}\text { Innovation } \\
\text { dependency type }\end{array}$ & $\begin{array}{c}\text { Ordinary Least Squares } \\
(\text { OLS })\end{array}$ & $\begin{array}{c}\text { Weighted Least Squares } \\
\text { (WLS) }\end{array}$ & $\begin{array}{c}\text { Maximum Likelihood } \\
\text { (ML) }\end{array}$ \\
\hline $\begin{array}{l}\text { Contemporaneously } \\
\text { correlated }\end{array}$ & $\begin{array}{c}\text { consistent } \\
\text { normally distributed } \\
\text { statistically inefficient }\end{array}$ & $\begin{array}{c}\text { consistent } \\
\text { normally distributed } \\
\text { statistically efficient }\end{array}$ & $\begin{array}{c}\text { consistent } \\
\text { normally distributed } \\
\text { statistically efficient }\end{array}$ \\
$\begin{array}{l}\text { Groupwise } \\
\text { heteroscedastic }\end{array}$ & consistent & consistent & consistent \\
& normally distributed & normally distributed & normally distributed \\
statistically inefficient & statistically efficient & statistically efficient \\
homoscedastic & consistent & consistent & consistent \\
& normally distributed & normally distributed & normally distributed \\
& statistically efficient & statistically efficient & statistically efficient \\
\hline
\end{tabular}

Remark 8. Note that the ML estimator properties are in analogy to those of conventional VAR(X) models [44, p. 93]. Also, the upper left block matrix of $\boldsymbol{P}_{M L}$ corresponds to the asymptotic covariance of $\widehat{\boldsymbol{\theta}}_{M L}$, and is identical to its WLS counterpart (see Proposition 2.4.2) suggesting that $\widehat{\boldsymbol{\theta}}_{M L}$ and $\widehat{\boldsymbol{\theta}}_{W L S}$ are asymptotically equivalent.

As in the LS-based case, estimates of the covariances for $\widehat{\boldsymbol{\theta}}_{M L}$ and $\widehat{\boldsymbol{\sigma}}_{M L}$ are given by:

$$
\begin{gathered}
\widehat{\boldsymbol{P}}\left(\widehat{\boldsymbol{\theta}}_{M L}\right)=\left[\sum_{t=1}^{N} \boldsymbol{\Phi}[t] \widehat{\boldsymbol{\Sigma}}_{M L}^{-1} \boldsymbol{\Phi}^{T}[t]\right]^{-1} \\
\widehat{\boldsymbol{P}}\left(\widehat{\boldsymbol{\sigma}}_{M L}\right)=\frac{2}{N} \boldsymbol{D}_{M n y}^{+}\left(\widehat{\boldsymbol{\Sigma}}_{M L} \otimes \widehat{\boldsymbol{\Sigma}}_{M L}\right)\left(\boldsymbol{D}_{M n y}^{+}\right)^{T}
\end{gathered}
$$

An immediate result of Theorem 2.4.2 is the following corollary.

Corollary 2.4.3. (ML estimator statistical accuracy): Under the assumptions of Theorem 2.4.2, $\widehat{\overline{\boldsymbol{\theta}}}_{M L}$ is asymptotically statistically efficient, as $\boldsymbol{P}_{M L}$ reaches the Cramér-Rao lower bound, thereby providing optimal accuracy.

Proof. See Appendix 2.B.4.3.

An overview of the asymptotic properties of the LS and ML type parameter estimators for the different types of innovation dependency is provided in Table 2.2.

\subsubsection{Accuracy of FP-VARX model parameters at the $k$-th operating condition}

Once an FP-VARX model has been obtained, information on the AR and X parameter matrices for any operating condition $k \in \mathcal{R}$ is readily available. The following relationships associate the (LS or ML based) projection coefficient vector estimate $\widehat{\boldsymbol{\theta}}$ to the parameter vector $\widehat{\boldsymbol{\vartheta}}(k)$ comprising all elements of the AR and $\mathrm{X}$ matrices corresponding to the $k$-th operating condition (see also Eq. (2.2c) and Eq. (2.5)):

$$
\begin{gathered}
\widehat{\boldsymbol{\vartheta}}(k)=\boldsymbol{T}(k) \widehat{\boldsymbol{\theta}} \quad k \in \mathcal{R} \\
\boldsymbol{P}(\widehat{\boldsymbol{\vartheta}}(k))=\boldsymbol{T}(k) \boldsymbol{P}(\widehat{\boldsymbol{\theta}}) \boldsymbol{T}^{T}(k) \\
\boldsymbol{\vartheta}(k)=\operatorname{vec}\left(\left[\boldsymbol{A}_{1}(k) \boldsymbol{A}_{2}(k) \ldots \boldsymbol{A}_{n a}(k) \vdots \boldsymbol{B}_{0}(k) \boldsymbol{B}_{1}(k) \ldots \boldsymbol{B}_{n b}(k)\right]^{T}\right)
\end{gathered}
$$


with $\boldsymbol{T}(k)$ designating a transformation matrix whose entries are zeros and basis functions $G_{j}(k)$ at proper locations (refer to the example of Appendix 2.A). Notice that in Eq. (2.19b) an estimate $\widehat{\boldsymbol{P}}(\widehat{\boldsymbol{\theta}})$ of the projection coefficient covariance $\boldsymbol{P}(\widehat{\boldsymbol{\theta}})$ is used in practice.

\subsection{FP-VARX model identifiability}

An important issue in FP-VARX modeling is to ensure that the identification procedure (i) yields a unique projection coefficient vector $\boldsymbol{\theta}$ and (ii) the identified model converges to the exact model structure (when this belongs to $\mathcal{M}(\boldsymbol{\theta})$ ). In this section the conditions under which the projection coefficients of an FP$\operatorname{VARX}(n a, n b)_{[p a, p b]}$ model can be recovered by means of the complete data set $Z^{N M}$ are studied.

The conditions for uniqueness of the $\operatorname{FP}-\operatorname{VARX}(n a, n b)_{[p a, p b]}$ projection coefficients are provided by the following theorem.

Theorem 2.5.1. (FP-VARX uniqueness of representation): Consider the $F P-V A R X(n a, n b)_{[p a, p b]}$ model of Eq. (2.3a)-(2.3c). The model's transfer function matrices:

$$
\boldsymbol{G}[\mathcal{B}, k] \triangleq \boldsymbol{A}^{-1}[\mathcal{B}, k] \boldsymbol{B}[\mathcal{B}, k], \quad k=k_{1}, k_{2}, \ldots, k_{M}
$$

are unique in the class of matrix fraction descriptions of degrees $(n a, n b)$ with known basis functions $G_{j}(k)$ and subspace dimensionalities $(p a, p b)$ if and only if:

$$
\begin{gathered}
\boldsymbol{A}[\mathcal{B}, k], \boldsymbol{B}[\mathcal{B}, k] \quad \text { i left coprime for } k=k_{1}, k_{2}, \ldots, k_{M} \\
\operatorname{rank}\left[\boldsymbol{A}_{n a}(k) \vdots \boldsymbol{B}_{n b}(k)\right]=n y \text { for } k=k_{1}, k_{2}, \ldots, k_{M}
\end{gathered}
$$

Proof. See Appendix 2.C.1.

The following lemma delineates the types of excitation that allow for distinguishing between different $\operatorname{FP}-\operatorname{VARX}(n a, n b)_{[p a, p b]}$ models belonging to $\mathcal{M}$.

Lemma 2.5.1. (Informative experiments): Consider the $F P-V A R X(n a, n b)_{[p a, p b]}$ models belonging to the set $\mathcal{M}$ in Eq. (2.4). Then the experiments performed at the distinct (sample) operating conditions $\left\{k_{1}, k_{2}, \ldots, k_{M}\right\}$ using persistently exciting inputs $\boldsymbol{x}_{k}[t]$ of order $(n b+1)$ are sufficiently informative with respect to $\mathcal{M}$, regardless of the AR polynomial degree, provided that the corresponding residuals $\boldsymbol{e}_{k}[t, \boldsymbol{\theta}]$ are persistently exciting.

Proof. See Appendix 2.C.2.

Note that the concepts of "informative" experiments and "persistently exciting" inputs are in analogy to the conventional VARX model case [1, pp. 411-415].

The following theorem relates the persistent excitation property with the LS and ML estimator consistency.

Theorem 2.5.2. The consistency of the FP-VARX $(n a, n b)_{[p a, p b]} L S$ and ML based estimators, provided that the residuals $\boldsymbol{e}_{k}[t, \boldsymbol{\theta}]$ are persistently exciting, is satisfied if and only if:

$$
\begin{aligned}
& \boldsymbol{x}_{k}[t]\left(k=k_{1}, k_{2}, \ldots, k_{M}\right) \text { is persistently exciting of order }(n b+1) . \\
& \sum_{k=k_{1}}^{k_{M}} \boldsymbol{g}_{j}(k) \boldsymbol{g}_{j}^{T}(k)>\mathbf{0}, \text { with } \boldsymbol{g}_{j}(k)=\left[G_{1}(k) \ldots G_{p j}(k)\right]^{T} \text { for } j=a, b .
\end{aligned}
$$

Proof. See Appendix 2.C.3. 


\subsection{FP-VARX model structure specification and validation}

In FP-VARX identification, model structure specification refers to the problem of determining the AR, $\mathrm{X}$ model orders $(n a, n b)$, as well as the dimensionalities of the functional subspaces $(p a, p b)$. A sensible approach to tackle this problem is via an exhaustive search procedure, where for each specific $n$ ( $n=$ $2,3, \ldots), \operatorname{FP}-\operatorname{VARX}(n, n)_{[p, p]}$ models are fitted to the data for $p=2,3, \ldots(p<M)$, until an adequate model, say FP-VARX $\left(n^{*}, n^{*}\right)_{\left[p^{*}, p^{*}\right]}$, is obtained. To avoid potential over-specification in the AR/X part functional subspaces, lower dimensionalities are also considered, until an adequate model is obtained.

Model adequacy may be quantified by criteria such as the determinant or trace of the estimated innovations (residual) covariance matrix $\widehat{\boldsymbol{\Sigma}}$, or statistical decision tools, such as minimization of the Akaike Information Criterion (AIC) and the Bayessian Information Criterion (BIC) (see [1, pp. 505-507] and [44, pp. 147-150]). The adaptation of these criteria to the FP models are natural extensions of their conventional model counterparts as seen below [15]:

$$
\begin{gathered}
\mathrm{AIC}=\ln (\operatorname{det}(\widehat{\boldsymbol{\Sigma}}))+\frac{2 d_{\mathcal{M}}}{N} \\
\mathrm{BIC}=\ln (\operatorname{det}(\widehat{\boldsymbol{\Sigma}}))+d_{\mathcal{M}} \frac{\ln (N)}{N}
\end{gathered}
$$

with $d_{\mathcal{M}}$ designating the number of estimated parameters.

Notice that in order to determine the most compact FP-VARX representation, different basis function families should be tested via the AIC and/or BIC criteria.

The selected FP-VARX model is finally accepted as an accurate representation of the system once properly validated. Model validation is generally based upon examining (i) the residual whiteness as well as the uncorrelatedness between residuals and excitations by means of the sample Cross-Correlation Function (CCF) [1, pp. 511-514], and (ii) the predictive and/or simulation ability of the model within a properly selected validation data set (not previously used in the estimation procedure) [1, p. 510].

\subsection{Monte Carlo studies}

The performance characteristics of the Ordinary Least Squares (OLS), Weighted Least Squares (WLS) and conditional Maximum Likelihood (ML) based estimators are assessed by means of two Monte Carlo studies.

\subsubsection{Case study I: An FP-VARX $(2,0)_{[3,3]}$ model}

An FP-VARX $(2,0)_{[3,3]}$ model with three excitations $(n x=3)$, two responses $(n y=2)$, and zero excitation delay (that is, $\boldsymbol{B}_{0}(k) \neq \mathbf{0}$ ) is used to generate data. The functional subspaces consist of the first three shifted Chebyshev polynomials of the second kind (see [45, p. 782], and also Appendix 2.D), that is $p=3$. The modeling procedure relies upon $M=21$ operating conditions with $k \in[0,20]$, obtained at unit increments. All excitation sequences are Gaussian white, mutually independent, with zero mean and unit variance. The innovations for each operating condition $k$ are (i) zero mean Gaussian white, and (ii) uncorrelated with those of any other operating condition (that is, $\boldsymbol{\Sigma}_{k, \ell}=\mathbf{0}$ ), which implies that no interrelations exist among the obtained data records (groupwise heteroscedastic innovations; refer to Table 2.1). Moreover, for each response, the innovations' standard deviation over the model's noise-free response standard deviation is set to $10 \%$ (that is, $\sigma_{w_{i k}} / \sigma_{y_{i k}}=10 \%, i=1,2$; see also Fig. 2.1). The resulting colored noise standard deviation over the model's response standard deviation, and the noise-free response standard deviation over the model's response standard deviation for each operating condition $k$ (that is, $\sigma_{n_{i k}} / \sigma_{y_{i k}}$ and $\sigma_{y_{i k}^{*}} / \sigma_{y_{i k}}$, $i=1,2$ respectively; see also Fig. 2.1) are shown in Fig. 2.2. 


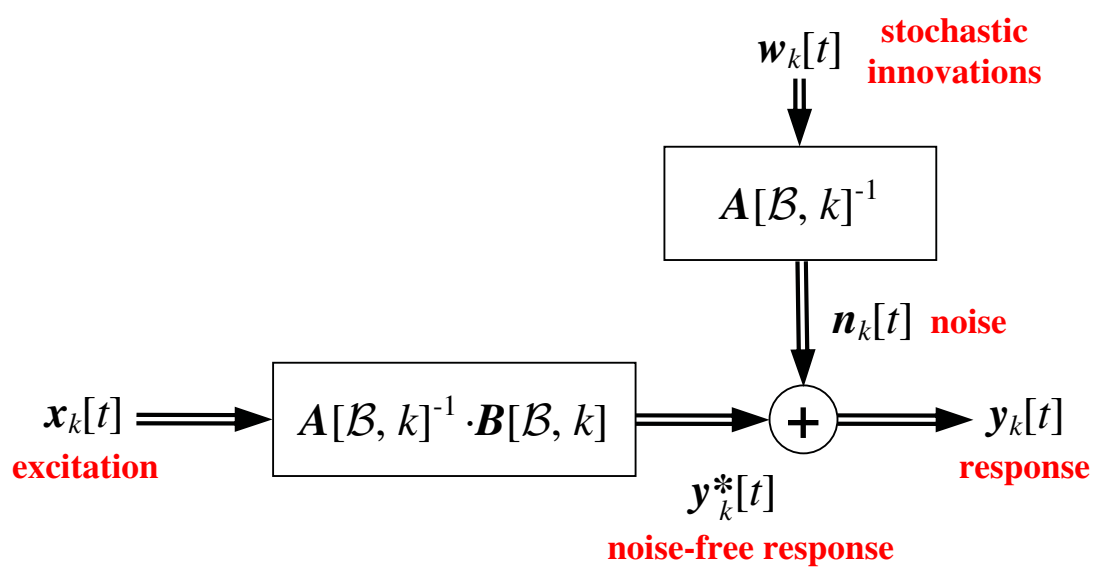

Figure 2.1: Schematic of the FP-VARX model structure.
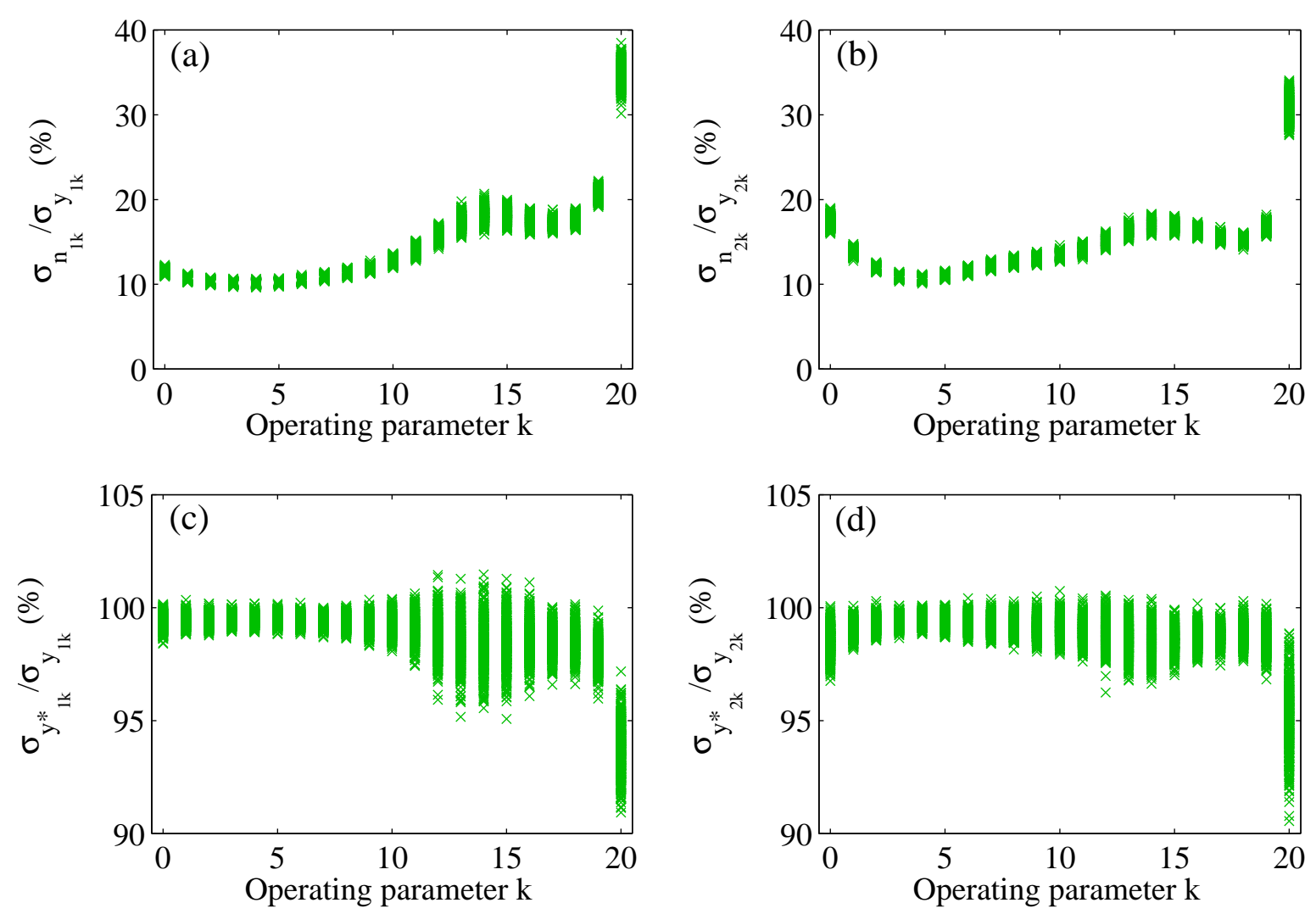

Figure 2.2: Presence of noise on the data generated by the $\operatorname{FP}-\operatorname{VARX}(2,0)_{[3,3]}$ model for each operating condition $k$; (a),(b) ratio of colored noise standard deviation over the model's response standard deviation; (c),(d) ratio of noise-free response standard deviation over the model's response standard deviation (" $\times$ " marks designate the value of a single experiment; 500 Monte Carlo runs).

The study encompasses 500 Monte Carlo runs, and parameter estimation is based upon $N=2,000$ sample-long data records. The OLS and WLS estimation are based upon a QR implementation (see Appendix 2.E). The WLS estimation is non-iterative, whereas the ML estimation is based upon a quasi-Newton optimization algorithm [46, MATLAB ${ }^{\circledR}$ function "fminunc.m"], and uses the WLS parameter vector mean estimate as initial conditions (details in Table 2.3). 
Table 2.3: Details on the Monte Carlo study for FP-VARX $(2,0)_{[3,3]}$ model identification.

\begin{tabular}{|c|c|c|}
\hline $\begin{array}{l}\text { No. of excitations: } n x=3 \\
\text { No. of responses: } n y=2 \\
\text { Innovations dependency type: }\end{array}$ & $\begin{array}{l}\text { Operating points: } M=21(k \in[0,20], \Delta k=1) \\
\text { No. of Monte Carlo runs: } 500 \\
\text { groupwise heteroscedastic }\end{array}$ & $\begin{array}{l}\text { Func. subspace: spanned by Chebyshev II polynomials } \\
\text { Data length: } N=2,000 \text { samples } \\
\text { Innovation sequence type: Gaussian white noise } \\
\text { Excitation sequence type: Gaussian white noise }\end{array}$ \\
\hline \multirow[t]{2}{*}{$\begin{array}{l}\text { FP-VARX } \\
\text { identification } \\
\text { details }\end{array}$} & \multicolumn{2}{|l|}{$\begin{array}{l}\text { WLS method: non-iterative algorithm via } \\
\qquad \text { QR implementation }\end{array}$} \\
\hline & \multicolumn{2}{|l|}{$\begin{array}{l}\text { Structure specification: information criteria (AIC, BIC) } \\
\text { Number of estimated parameters: } 42\end{array}$} \\
\hline $\begin{array}{l}\text { conventional VARX } \\
\text { identification details }\end{array}$ & \multicolumn{2}{|c|}{$\begin{array}{l}\text { OLS method: QR implementation } \\
\text { Number of estimated parameters: } 294 \text { ( } 14 \text { parameters per operating point) }\end{array}$} \\
\hline
\end{tabular}
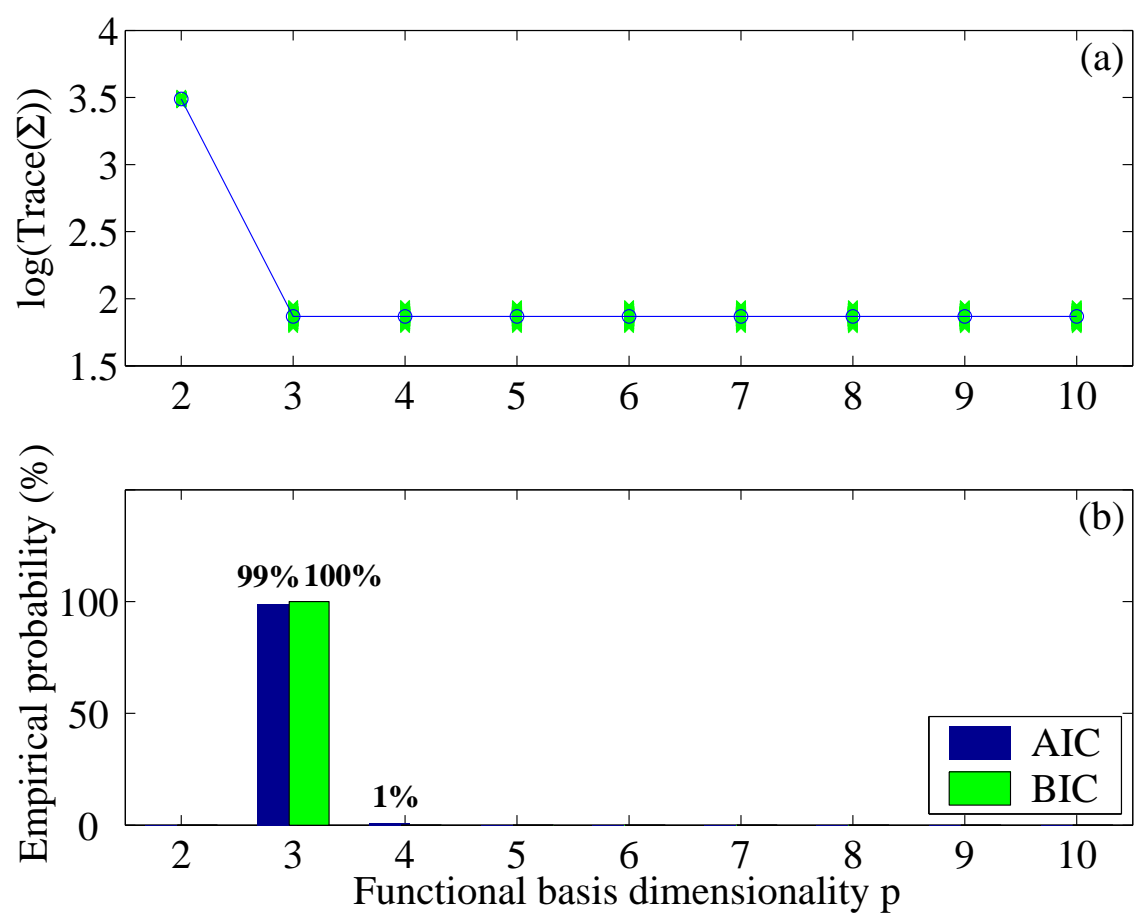

Figure 2.3: FP-VARX $(2,0)_{[p, p]}$ model structure specification results for the WLS-based estimator: (a) residual covariance matrix trace (" $\times$ " marks designate the value of a single experiment and " $\circ$ " their corresponding sample mean), (b) functional subspace dimensionality specification via the AIC and BIC (500 Monte Carlo runs per model).

Indicative structure specification results from the successive fitting of $\mathrm{FP}-\operatorname{VARX}(2,0)_{[p, p]}$ models with increasing basis dimensionality $p=2,3, \ldots, 10$ via WLS are presented in Fig. 2.3 (similar results are obtained for OLS and ML). The trace of the resulting residual covariance matrix, shown in Fig. 2.3(a), decreases monotonically, although once the correct functional basis dimensionality $(p=3)$ is exceeded, the reduction is insignificant. Fig. 2.3(b) depicts the probability of attaining minimal AIC and BIC values for increasing basis dimensionality. The success rates (\%) for both criteria are excellent, as minimum values are attained at $p=3$ in the vast majority of the runs.

Indicative interval parameter estimates (depicting sample mean \pm sample standard deviation) for the OLS, WLS and ML estimators along with the corresponding actual values are presented in Fig. 2.4. Excellent agreement is observed among the actual values and their estimated counterparts. The results indicate that all three estimators yield essentially unbiased estimates with the WLS and ML, expectedly, providing 

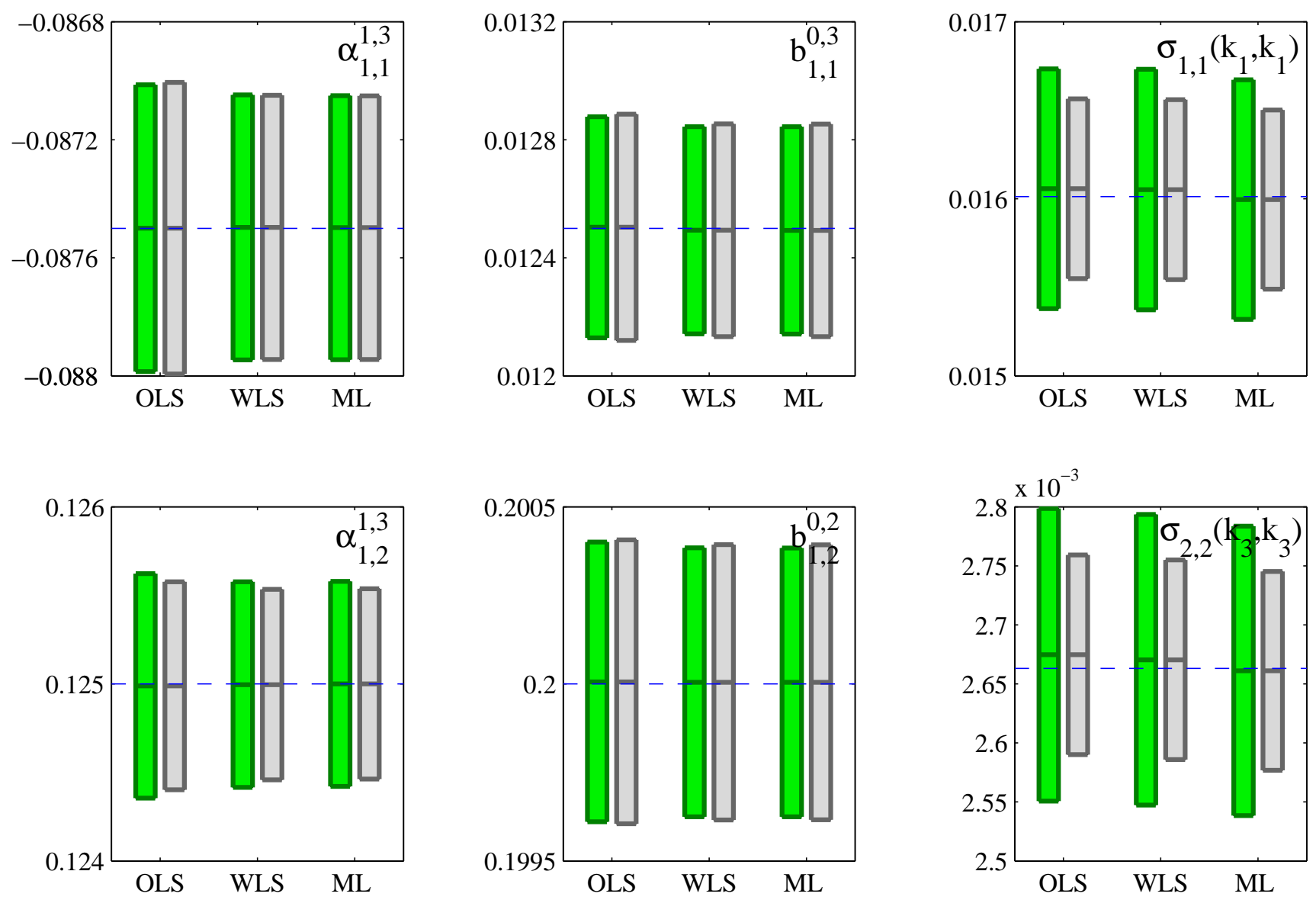

Figure 2.4: $\operatorname{FP}-\operatorname{VARX}(2,0)_{[3,3]}$ actual values (--) and Monte Carlo estimates based upon the OLS, WLS, and ML methods for selected projection coefficients and innovation variances (500 Monte Carlo runs per method; $a_{m, n}^{i, j}$ and $b_{m, n}^{i, j}$ designate the $(m, n)$-th elements of $\boldsymbol{A}_{i, j}$ and $\boldsymbol{B}_{i, j}$ respectively; $\sigma_{m, n}(k, \ell)$ designates the $(m, n)$-th element of $\boldsymbol{\Sigma}_{k, \ell}$; the dark green boxes indicate the sample mean \pm sample standard deviation; the light grey boxes indicate the sample mean \pm mean standard deviation computed through the asymptotic distribution).

better accuracy for the coefficients of projection. Furthermore, the very good agreement between the sample standard deviations and the corresponding values from the asymptotic analysis confirms the validity of the derived normal distribution for the projection coefficient estimators. The corresponding results for the innovation variance estimates are somewhat inferior, yet the validity of the asymptotic analysis is also confirmed. Additional results are presented in Appendix 2.F.1.

Partial results comparing the accuracy of the identified $\operatorname{FP}-\operatorname{VARX}(2,0)_{[3,3]}$ model via WLS with its multi-model counterpart are shown in Fig. 2.5. A conventional $\operatorname{VARX}(2,0)$ model is identified for each operating condition, and parameter estimation is based upon OLS with QR implementation (refer to [1, pp. 318-320], and also [47, MATLAB ${ }^{\circledR}$ function “arx.m”]). The interval parameter estimates (depicting sample mean $\pm 2 \times$ sample standard deviation) indicate very good agreement among theoretical and estimated values for all operating conditions. However, the $\operatorname{FP}-\operatorname{VARX}(2,0)_{[3,3]}$ based estimates, as expected, provide better accuracy than their conventional $\operatorname{VARX}(2,0)$ based counterparts. Since the assumption of groupwise heteroscedastic innovations implies that no interrelations exist among the obtained data records, the only source of inaccuracy for the multi-model approach is the large number of estimated parameters for the same amount of data (a total of 294 parameters for the $21 \operatorname{VARX}(2,0)$ models versus 42 parameters of the $\operatorname{FP}-\operatorname{VARX}(2,0)_{[3,3]}$ model). 

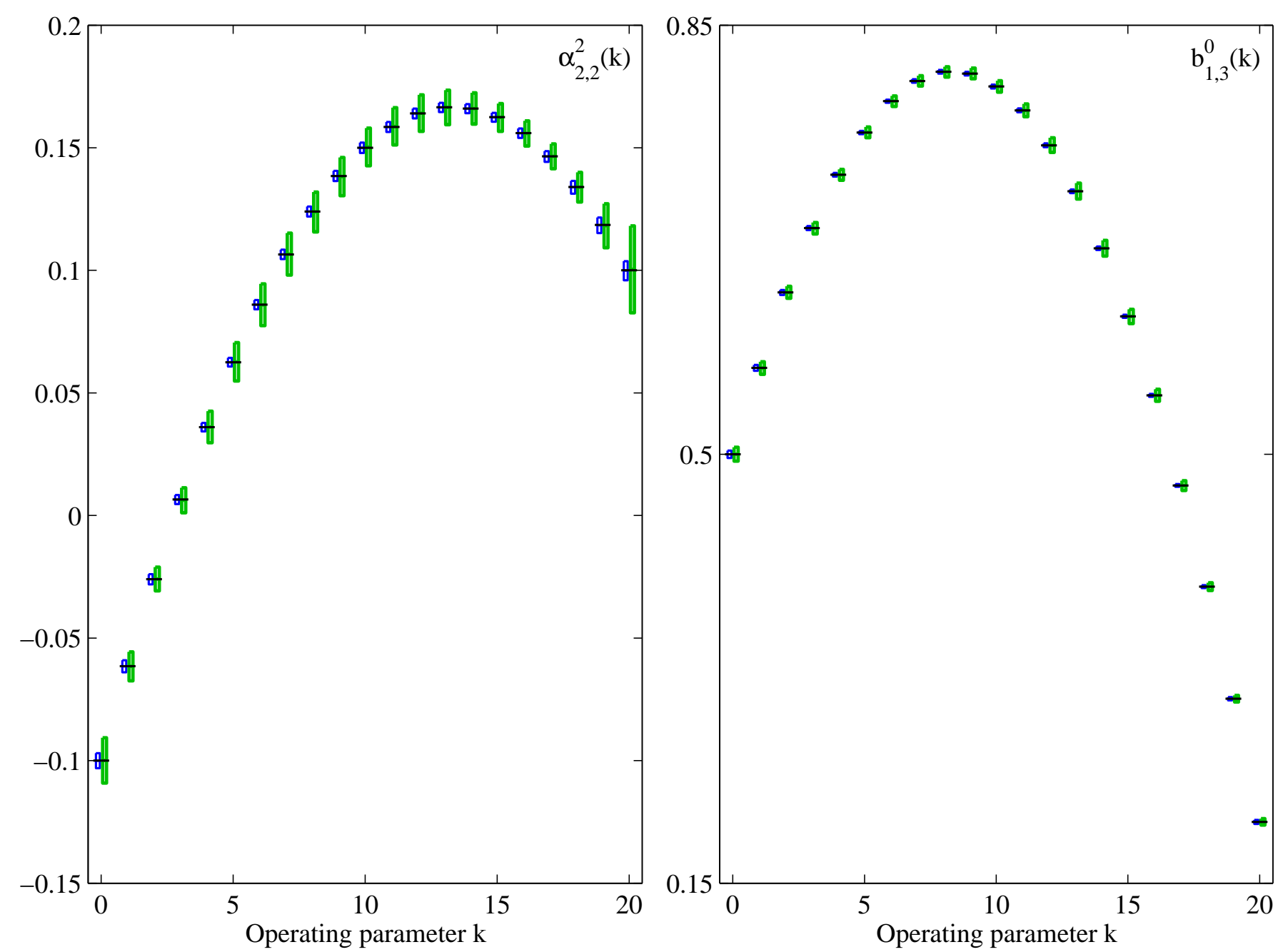

Figure 2.5: Dependence of certain AutoRegressive and eXogenous parameters on the operating parameter $k$ (boxes indicate sample mean estimates $\pm 2 \times$ mean sample standard deviation computed through the asymptotic distribution): (一) theoretical values; (blue boxes) FP-VARX $(2,0)_{[p, p]}$ model; (green boxes) conventional VARX $(2,0)$ models (500 Monte Carlo runs per model; $a_{m, n}^{i}(k)$ and $b_{m, n}^{i}(k)$ designate the $(m, n)$-th elements of $\boldsymbol{A}_{i}(k)$ and $\boldsymbol{B}_{i}(k)$, respectively).

\subsubsection{Case study II: An FP-VARX $(3,1)_{[4,4]}$ model}

An FP-VARX $(3,1)_{[4,4]}$ model with two excitations $(n x=2)$, two responses $(n y=2)$, and zero excitation delay (that is, $\boldsymbol{B}_{0}(k) \neq \mathbf{0}$ ) is considered in the second study. The functional subspaces consist of the first four shifted Chebyshev polynomials of the second kind (see Appendix 2.D), that is $p=4$. The modeling procedure relies upon $M=11$ operating conditions with $k \in[0,10]$, obtained at unit increments. All excitation sequences are Gaussian white, mutually independent, with zero mean and unit variance. The innovations for each operating condition $k$ are (i) zero mean Gaussian white, and (ii) uncorrelated with those of any other operating condition (that is, $\boldsymbol{\Sigma}_{k, \ell}=\mathbf{0}$ ), which implies that no interrelations exist among the obtained data records (groupwise heteroscedastic innovations; refer to Table 2.1). Moreover, for each response, the innovations' standard deviation over the model's noise-free response standard deviation is set to $10 \%$ (that is, $\sigma_{w_{i k}} / \sigma_{y_{i k}}=10 \%, i=1,2$; see Fig. 2.1). The resulting colored noise standard deviation over the model's response standard deviation, and the noise-free response standard deviation over the model's response standard deviation for each operating condition $k$ (that is, $\sigma_{n_{i k}} / \sigma_{y_{i k}}$ and $\sigma_{y_{i k}^{*}} / \sigma_{y_{i k}}, i=1,2$ respectively; see 

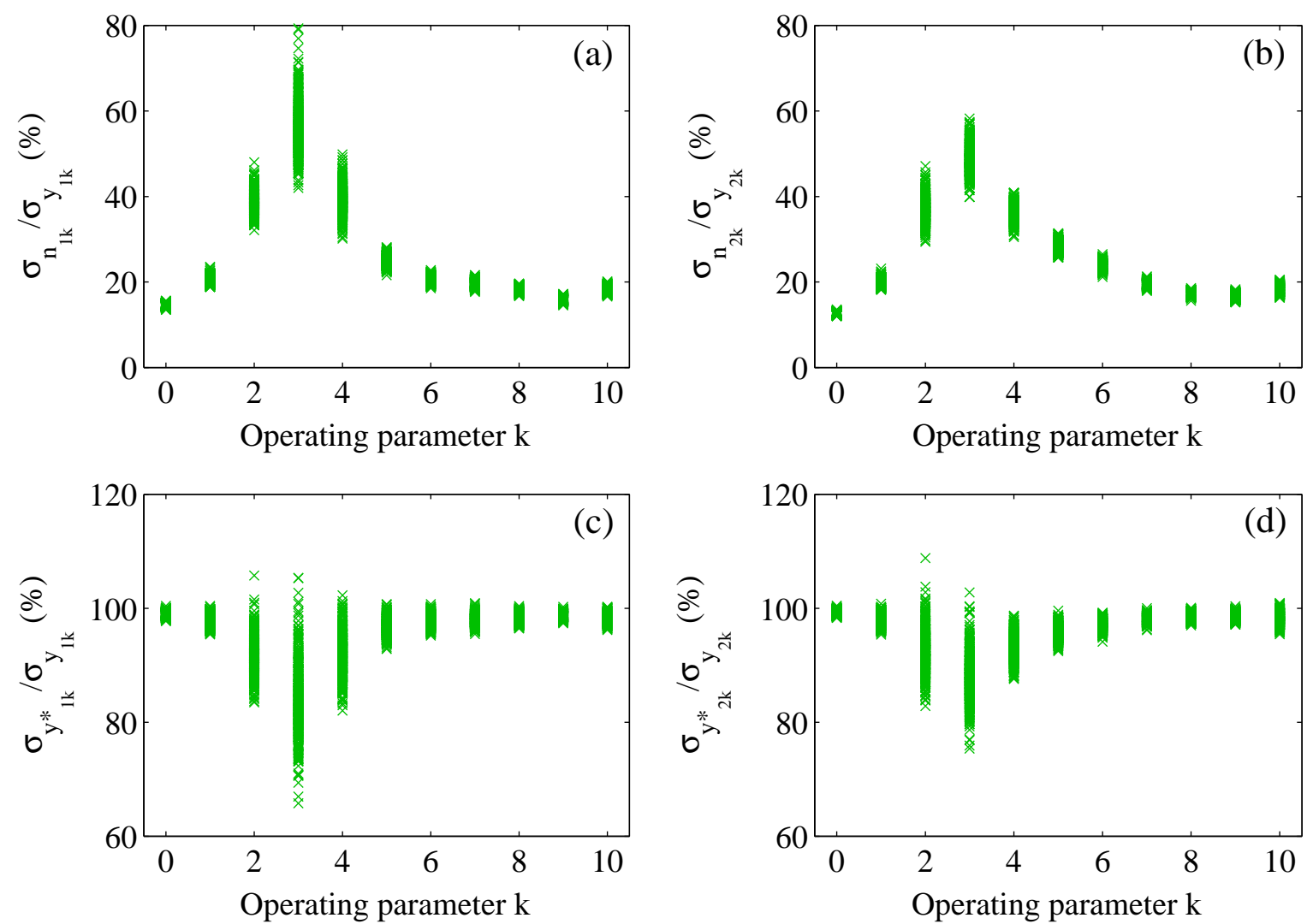

Figure 2.6: Presence of noise on the data generated by the $\operatorname{FP}-\operatorname{VARX}(3,1)_{[4,4]}$ model for each operating condition $k$; (a),(b) ratio of colored noise standard deviation over the model's response standard deviation; (c),(d) ratio of noise-free response standard deviation over the model's response standard deviation (" $\times$ " marks designate the value of a single experiment; 500 Monte Carlo runs).

Table 2.4: Details on the Monte Carlo study for FP-VARX $(3,1)_{[4,4]}$ model identification.

\begin{tabular}{|c|c|c|}
\hline $\begin{array}{l}\text { No. of excitations: } n x=2 \\
\text { No. of responses: } n y=2 \\
\text { Innovations dependency type: }\end{array}$ & $\begin{array}{l}\text { Operating points: } M=11(k \in[0,10], \Delta k=1) \\
\text { No. of Monte Carlo runs: } 500 \\
\text { groupwise heteroscedastic }\end{array}$ & $\begin{array}{l}\text { Func. subspace: spanned by Chebyshev II polynomials } \\
\text { Data length: } N=2,000 \text { samples } \\
\text { Innovation sequence type: Gaussian white noise } \\
\text { Excitation sequence type: Gaussian white noise }\end{array}$ \\
\hline \multirow[t]{2}{*}{$\begin{array}{l}\text { FP-VARX } \\
\text { identification } \\
\quad \text { details }\end{array}$} & $\begin{array}{l}\text { WLS method: non-iterative algorithm via } \\
\text { QR implementation }\end{array}$ & $\begin{array}{l}\text { ML method: quasi-Newton optimization algorithm } \\
\text { init. values from WLS mean estimate } \\
\text { max. number of iterations }=500 \\
\text { estimated pars termination tol. }=10^{-6} \\
\text { objective function termination tol. }=10^{-2}\end{array}$ \\
\hline & $\begin{array}{l}\text { Structure specification: information criteria (AIC, } \\
\text { Number of estimated parameters: } 80\end{array}$ & \\
\hline $\begin{array}{l}\text { conventional VARX } \\
\text { identification details }\end{array}$ & $\begin{array}{l}\text { OLS method: QR implementation } \\
\text { Number of estimated parameters: } 220 \text { (20 paramet }\end{array}$ & rs per operating point) \\
\hline
\end{tabular}

also Fig. 2.1) are shown in Fig. 2.6.

The study encompasses 500 Monte Carlo runs, and parameter estimation is based upon $N=2,000$ sample-long data records. The OLS and WLS estimation are based upon a QR implementation (see Appendix 2.E). The WLS estimation is non-iterative, whereas the ML estimation is based upon a quasi-Newton optimization algorithm [46, MATLAB ${ }^{\circledR}$ function "fminunc.m"], and uses the WLS parameter vector mean estimate as initial conditions (details in Table 2.4). 

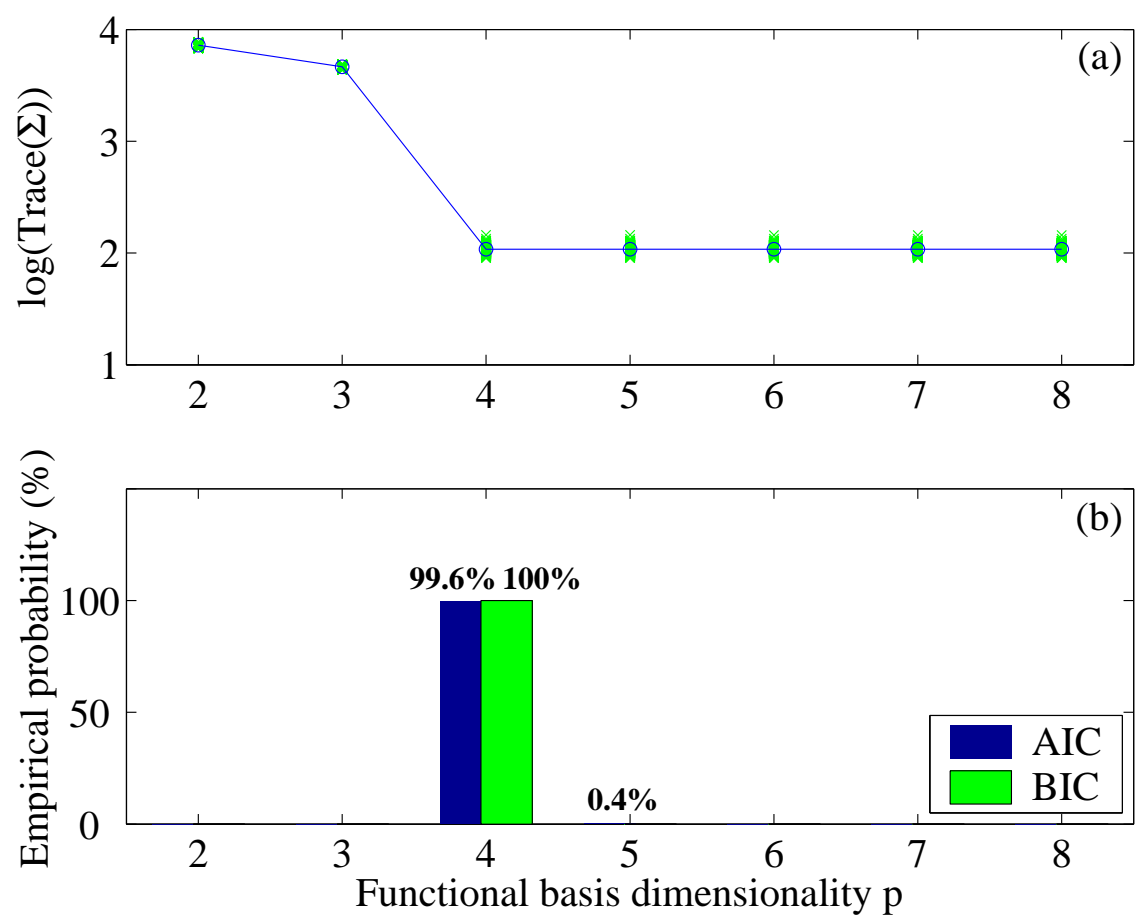

Figure 2.7: FP-VARX $(3,1)_{[p, p]}$ model structure specification results for the WLS-based estimator: (a) residual covariance matrix trace (" $\times$ " marks designate the value of a single experiment and "O" their corresponding sample mean), (b) functional subspace dimensionality specification via the AIC and BIC (500 Monte Carlo runs per model).

Indicative structure specification results from the successive fitting of FP-VARX $(3,1)_{[p, p]}$ models with increasing basis dimensionality $p=2,3, \ldots, 8$ via WLS are presented in Fig. 2.7 (similar results are obtained for OLS and ML). The trace of the resulting residual covariance matrix, shown in Fig. 2.7(a), decreases monotonically, although once the correct functional basis dimensionality $(p=4)$ is exceeded, the reduction is insignificant. Fig. 2.7(b) depicts the probability of attaining minimal AIC and BIC values for increasing basis dimensionality. The success rates (\%) for both criteria are excellent, as minimum values are attained at $p=4$ in the vast majority of the runs.

Indicative interval parameter estimates (depicting sample mean \pm sample standard deviation) for the OLS, WLS and ML estimators along with the corresponding actual values are presented in Fig. 2.8. Excellent agreement is observed among the actual values and their estimated counterparts. The results indicate that all three estimators yield essentially unbiased estimates with the WLS and ML, expectedly, providing better accuracy for the coefficients of projection. Furthermore, the very good agreement between the sample standard deviations and the corresponding values from the asymptotic analysis confirms the validity of the derived normal distribution for the projection coefficient estimators. The corresponding results for the innovation variance estimates are somewhat inferior, yet the validity of the asymptotic analysis is also confirmed. Additional results are presented in Appendix 2.F.2.

Partial results comparing the accuracy of the identified FP-VARX $(3,1)_{[4,4]}$ model via WLS with its multi-model counterpart are shown in Fig. 2.9. A conventional $\operatorname{VARX}(3,1)$ model is identified for each operating condition, and parameter estimation is based upon OLS with QR implementation (refer to [1, pp. 318-320], and also [47, MATLAB ${ }^{\circledR}$ function "arx.m"]). The interval parameter estimates (depicting sample mean $\pm 2 \times$ sample standard deviation) indicate very good agreement among theoretical and estimated values for all operating conditions. However, the FP-VARX $(3,1)_{[4,4]}$ based estimates, as expected, provide better accuracy than their conventional $\operatorname{VARX}(3,1)$ based counterparts. Since the assumption of group- 

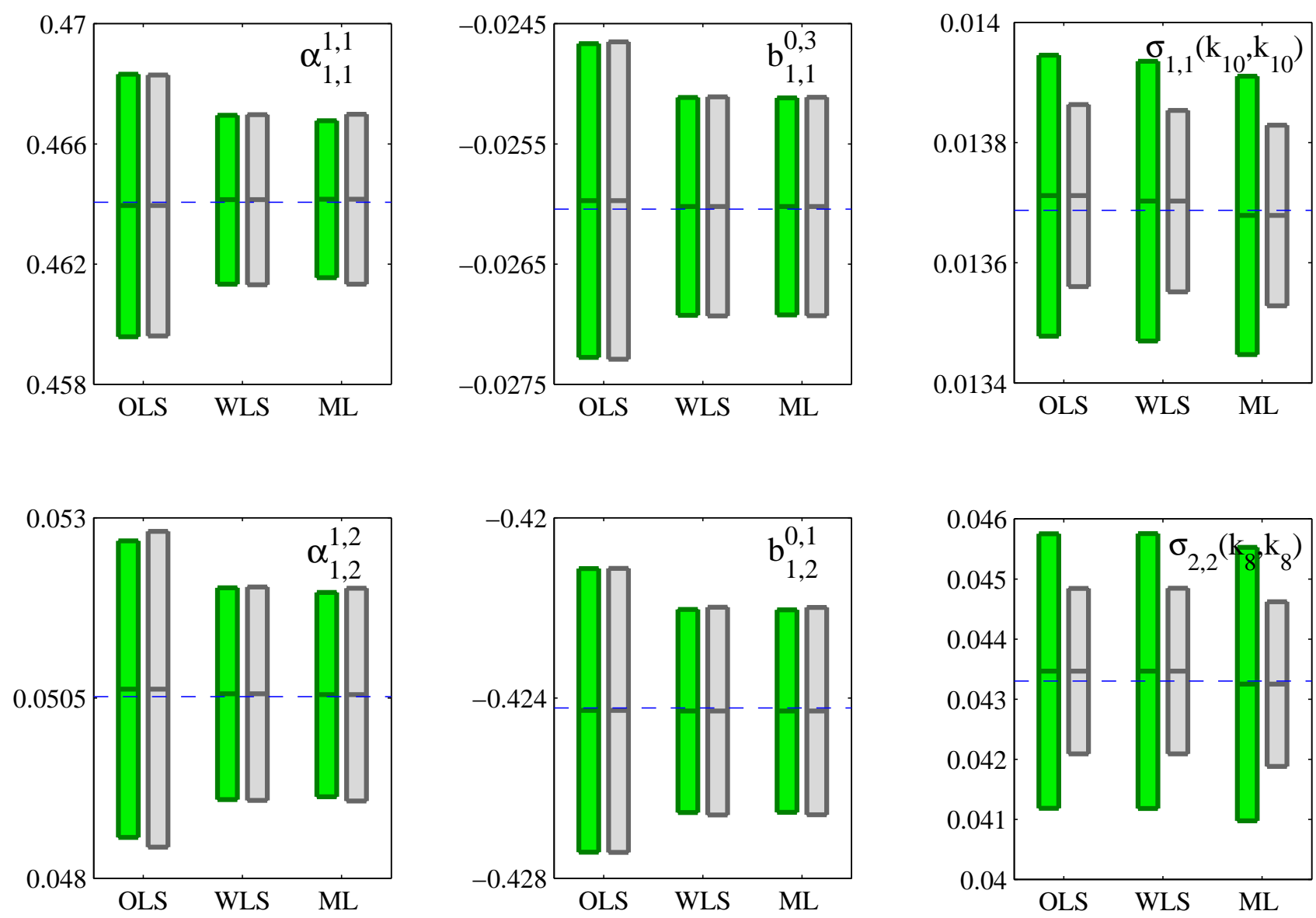

Figure 2.8: FP-VARX $(3,1)_{[4,4]}$ actual values (--) and Monte Carlo estimates based upon the OLS, WLS, and ML methods for selected projection coefficients and innovation variances (500 Monte Carlo runs per method; $a_{m, n}^{i, j}$ and $b_{m, n}^{i, j}$ designate the $(m, n)$-th elements of $\boldsymbol{A}_{i, j}$ and $\boldsymbol{B}_{i, j}$ respectively; $\sigma_{m, n}(k, \ell)$ designates the $(m, n)$-th element of $\boldsymbol{\Sigma}_{k, \ell}$; the dark green boxes indicate the sample mean \pm sample standard deviation; the light grey boxes indicate the sample mean \pm mean standard deviation computed through the asymptotic distribution).

wise heteroscedastic innovations implies that no interrelations exist among the obtained data records, the only source of inaccuracy for the multi-model approach is the large number of estimated parameters for the same amount of data (a total of 220 parameters for the $11 \operatorname{VARX}(3,1)$ models versus 80 parameters of the $\operatorname{FP}-\operatorname{VARX}(3,1)_{[4,4]}$ model $)$.

\subsection{Concluding remarks}

The identification of multivariate stochastic systems capable of operating under different conditions was addressed, based on data records corresponding to a sample of such operating conditions. The focus was on the identification of FP-VARX models within a novel Functional Pooling framework. LS and conditional ML type estimators were formulated, and their consistency along with their asymptotic normality was established. Conditions ensuring FP-VARX identifiability were also postulated. Model structure specification was based upon proper forms of the information criteria AIC and BIC. The performance characteristics of the LS and conditional ML type estimators were assessed by means of Monte Carlo studies, and the results suggested that the ML and WLS estimators attain better accuracy over their OLS counterpart. Finally, 

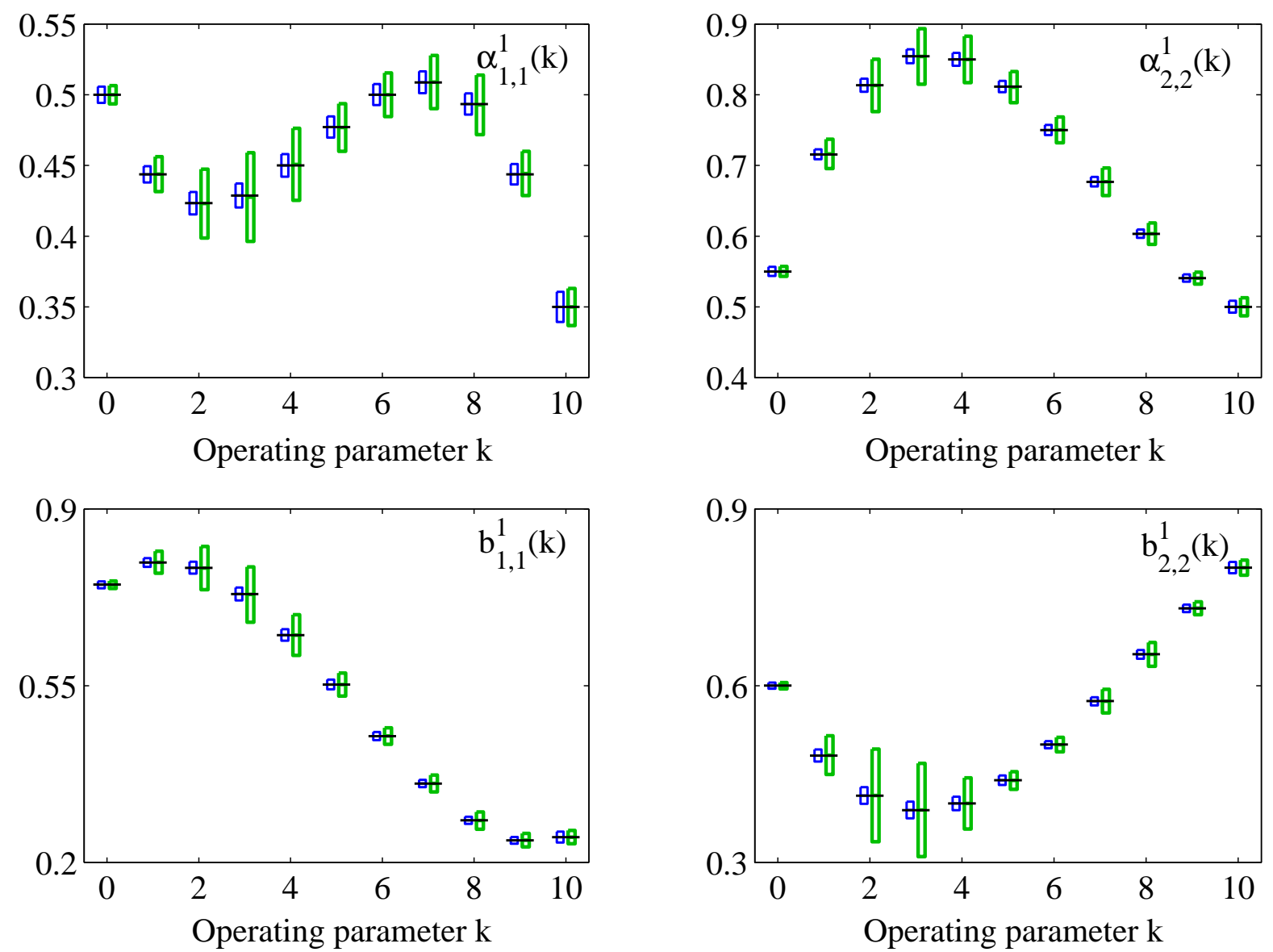

Figure 2.9: Dependence of certain AutoRegressive and eXogenous parameters on the operating parameter $k$ (boxes indicate sample mean estimates $\pm 2 \times$ mean sample standard deviation computed through the asymptotic distribution): (一) actual values; (blue boxes) FP-VARX $(3,1)_{[p, p]}$ model; (green boxes) conventional VARX $(3,1)$ models (500 Monte Carlo runs per model; $a_{m, n}^{i}(k)$ and $b_{m, n}^{i}(k)$ designate the $(m, n)$-th elements of $\boldsymbol{A}_{i}(k)$ and $\boldsymbol{B}_{i}(k)$, respectively).

comparing the FP-VARX modeling approach with its conventional VARX based multi-model counterpart demonstrated the effectiveness of the novel framework as a useful tool for global system modeling.

\section{Appendix 2.A: Tutorial example of FP-VARX $(n a, n b)_{[p a, p b]}$ parameter estima- tion}

The identification of an FP-VARX $(1,0)_{[2,2]}$ model with two excitations, two responses (that is, $n x=n y=$ 2 ), and zero excitation delay (that is, $\boldsymbol{B}_{0}(k) \neq \mathbf{0}$ ), is considered. The AR and X matrices are fully parameterized and belong to functional subspaces of dimensionality $p a=p b=2$, spanned by the mutually independent functions $G_{1}(k)$ and $G_{2}(k)$. The FP-VARX parameter estimation is based upon $M=2$ sets of excitation-response data of $N$ samples each (that is, $k=k_{1}, k_{2}$ and $t=1,2, \ldots, N$ ). The model is of the following form:

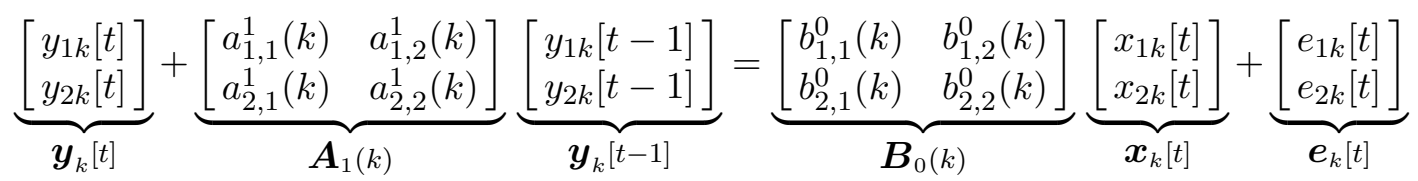


with:

$$
\begin{aligned}
& \boldsymbol{A}_{1}(k)=\underbrace{\left[\begin{array}{ll}
a_{1,1}^{1,1} & a_{1,2}^{1,1} \\
a_{2,1}^{1,1} & a_{2,1}^{1,1}
\end{array}\right]}_{\boldsymbol{A}_{1,1}} G_{1}(k)+\underbrace{\left[\begin{array}{rr}
a_{1,1}^{1,2} & a_{1,2}^{1,2} \\
a_{2,1}^{1,2} & a_{2,2}^{1,2}
\end{array}\right]}_{\boldsymbol{A}_{1,2}} G_{2}(k) \\
& \boldsymbol{B}_{0}(k)=\underbrace{\left[\begin{array}{ll}
b_{1,1}^{0,1} & b_{1,2}^{0,1} \\
b_{2,1}^{0,1} & b_{2,2}^{0,1}
\end{array}\right]}_{\boldsymbol{B}_{0,1}} G_{1}(k)+\underbrace{\left[\begin{array}{rr}
b_{1,1}^{0,2} & b_{1,2}^{0,2} \\
b_{2,2}^{0,2} & b_{2,2}^{0,2}
\end{array}\right]}_{\boldsymbol{B}_{0,2}} G_{2}(k)
\end{aligned}
$$

Expanding AR and $\mathrm{X}$ matrices on the 2-dimensional functional subspaces, yields:

$$
\begin{aligned}
{\left[\begin{array}{l}
y_{1 k}[t] \\
y_{2 k}[t]
\end{array}\right]=} & -\left[\begin{array}{ll}
a_{1,1}^{1,1} G_{1}(k)+a_{1,1}^{1,2} G_{2}(k) & a_{1,2}^{1,1} G_{1}(k)+a_{1,2}^{1,2} G_{2}(k) \\
a_{2,1}^{1,1} G_{1}(k)+a_{2,1}^{1,2} G_{2}(k) & a_{2,2}^{1,1} G_{1}(k)+a_{2,2}^{1,2} G_{2}(k)
\end{array}\right]\left[\begin{array}{l}
y_{1 k}[t-1] \\
y_{2 k}[t-1]
\end{array}\right] \\
& +\left[\begin{array}{ll}
b_{1,1}^{0,1} G_{1}(k)+b_{1,1}^{02} G_{2}(k) & b_{1,2}^{0,1} G_{1}(k)+b_{1,2}^{0,2} G_{2}(k) \\
b_{2,1}^{0,1} G_{1}(k)+b_{2,1}^{02} G_{2}(k) & b_{2,2}^{0,1} G_{1}(k)+b_{2,2}^{0,2} G_{2}(k)
\end{array}\right]\left[\begin{array}{l}
x_{1 k}[t] \\
x_{2 k}[t]
\end{array}\right]+\left[\begin{array}{l}
e_{1 k}[t] \\
e_{2 k}[t]
\end{array}\right]
\end{aligned}
$$

Standard matrix calculus leads to:

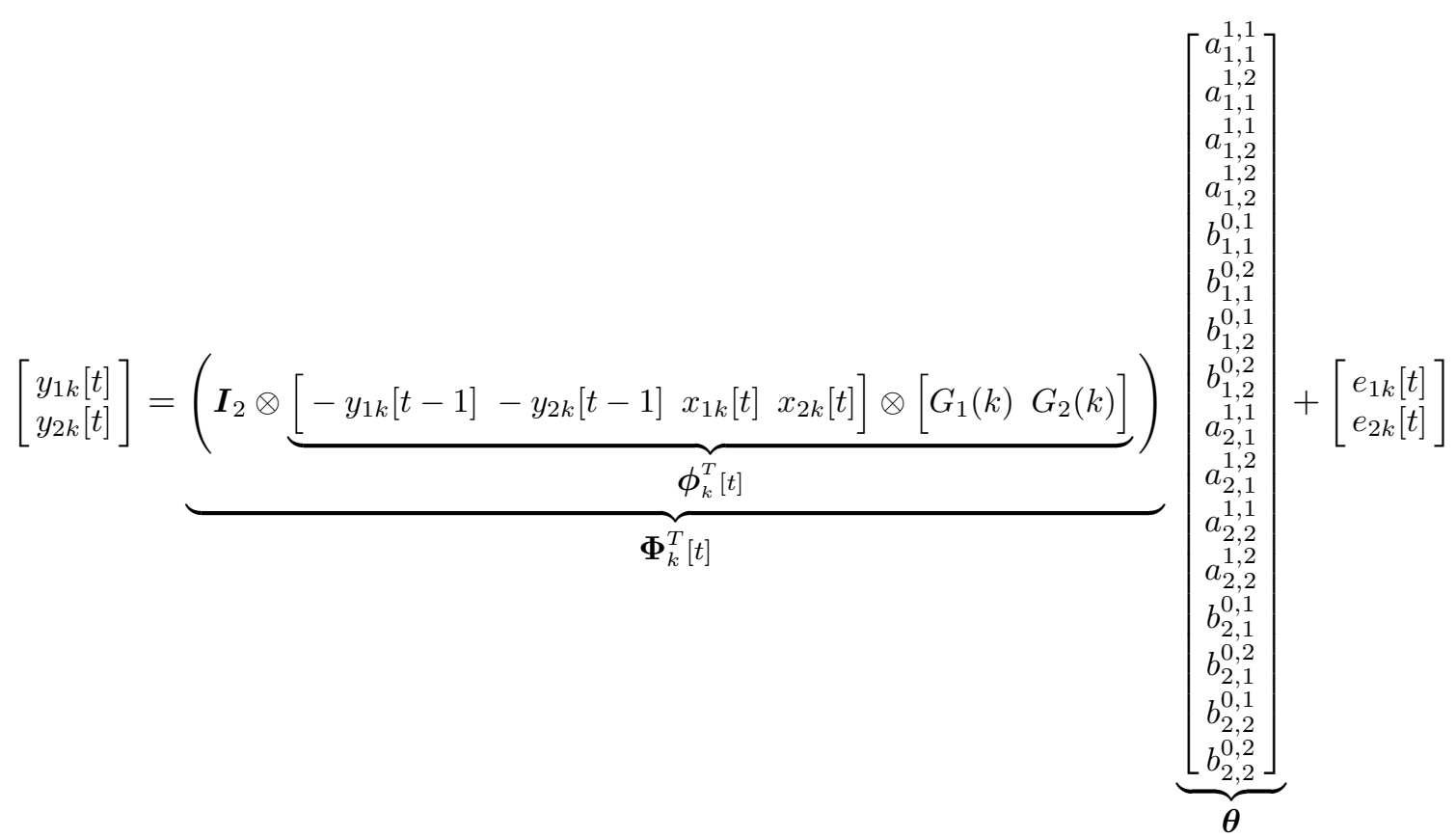

Pooling together the preceding equations which belong to the operating conditions $k=k_{1}, k_{2}$ yields:

$$
\underbrace{\left[\begin{array}{c}
\boldsymbol{y}_{k_{1}}[t] \\
\ldots \ldots \\
\boldsymbol{y}_{k_{2}}[t]
\end{array}\right]}_{\boldsymbol{y}[t]}=\underbrace{\left[\begin{array}{c}
\boldsymbol{\Phi}_{k_{1}}^{T}[t] \\
\ldots \ldots \\
\boldsymbol{\Phi}_{k_{2}}^{T}[t]
\end{array}\right]}_{\boldsymbol{\Phi}^{T}[t]} \boldsymbol{\theta}+\underbrace{\left[\begin{array}{c}
\boldsymbol{e}_{k_{1}}[t] \\
\ldots \ldots \\
\boldsymbol{e}_{k_{2}}[t]
\end{array}\right]}_{\boldsymbol{e}[t]}
$$

Under the assumption of contemporaneously correlated innovations, the corresponding covariance matrix $\mathbf{\Sigma}$ 
is given by:

$$
\boldsymbol{\Sigma}=\left[\begin{array}{c|c}
\boldsymbol{\Sigma}_{k_{1}, k_{1}} & \boldsymbol{\Sigma}_{k_{1}, k_{2}} \\
\hline \boldsymbol{\Sigma}_{k_{2}, k_{1}} & \boldsymbol{\Sigma}_{k_{2}, k_{2}}
\end{array}\right]=\left[\begin{array}{ll|ll}
\sigma_{1,1}\left(k_{1}, k_{1}\right) & \sigma_{1,2}\left(k_{1}, k_{1}\right) & \sigma_{1,1}\left(k_{1}, k_{2}\right) & \sigma_{1,2}\left(k_{1}, k_{2}\right) \\
\sigma_{2,1}\left(k_{1}, k_{1}\right) & \sigma_{2,2}\left(k_{1}, k_{1}\right) & \sigma_{2,1}\left(k_{1}, k_{2}\right) & \sigma_{2,2}\left(k_{1}, k_{2}\right) \\
\hline \sigma_{1,1}\left(k_{2}, k_{1}\right) & \sigma_{1,2}\left(k_{2}, k_{1}\right) & \sigma_{1,1}\left(k_{2}, k_{2}\right) & \sigma_{1,2}\left(k_{2}, k_{2}\right) \\
\sigma_{2,1}\left(k_{2}, k_{1}\right) & \sigma_{2,2}\left(k_{2}, k_{1}\right) & \sigma_{2,1}\left(k_{2}, k_{2}\right) & \sigma_{2,2}\left(k_{2}, k_{2}\right)
\end{array}\right]
$$

Note that due to the symmetry of the covariance matrix $\Sigma$ above, the following relationships are satisfied:

$$
\begin{aligned}
& \sigma_{1,2}\left(k_{1}, k_{1}\right)=\sigma_{2,1}\left(k_{1}, k_{1}\right) \quad \sigma_{1,2}\left(k_{2}, k_{2}\right)=\sigma_{2,1}\left(k_{2}, k_{2}\right) \\
& \sigma_{1,1}\left(k_{1}, k_{2}\right)=\sigma_{1,1}\left(k_{2}, k_{1}\right) \quad \sigma_{2,2}\left(k_{1}, k_{2}\right)=\sigma_{2,2}\left(k_{2}, k_{1}\right) \\
& \sigma_{1,2}\left(k_{1}, k_{2}\right)=\sigma_{2,1}\left(k_{1}, k_{2}\right)=\sigma_{1,2}\left(k_{1}, k_{2}\right)=\sigma_{2,1}\left(k_{2}, k_{1}\right)
\end{aligned}
$$

The vector $\boldsymbol{\sigma}$, comprising all non-identical elements on and below the main diagonal of $\boldsymbol{\Sigma}$, and the duplication matrix $\boldsymbol{D}_{M n y}$ satisfy the following relationship:

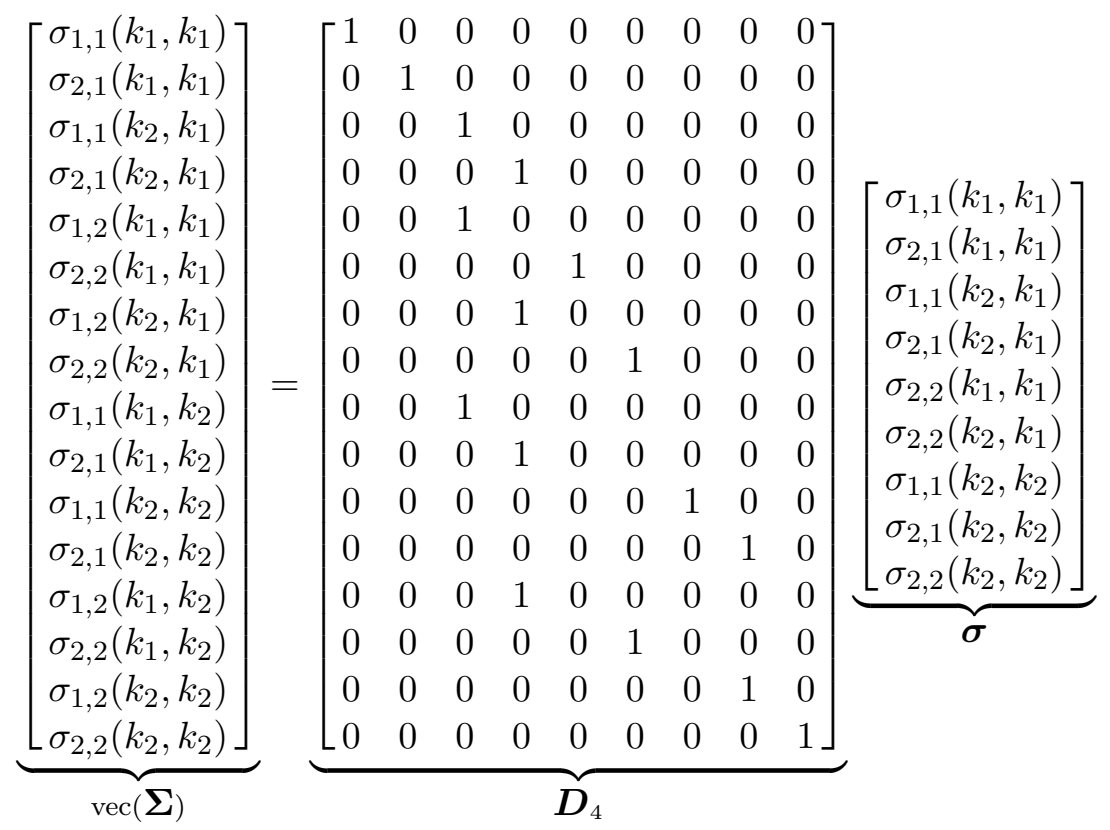

The WLS estimators are given by:

$$
\begin{gathered}
\widehat{\boldsymbol{\theta}}_{W L S}=\left[\frac{1}{2 N} \sum_{t=1}^{N} \boldsymbol{\Phi}[t] \widehat{\boldsymbol{\Sigma}}^{-1} \boldsymbol{\Phi}^{T}[t]\right]^{-1}\left[\frac{1}{2 N} \sum_{t=1}^{N} \boldsymbol{\Phi}[t] \widehat{\boldsymbol{\Sigma}}^{-1} \boldsymbol{y}[t]\right] \\
\widehat{\boldsymbol{\Sigma}}_{W L S}=\frac{1}{N} \sum_{t=1}^{N} \boldsymbol{e}\left[t, \widehat{\boldsymbol{\theta}}_{W L S}\right] \boldsymbol{e}^{T}\left[t, \widehat{\boldsymbol{\theta}}_{W L S}\right]
\end{gathered}
$$

whereas the corresponding covariance estimates are given by:

$$
\begin{gathered}
\widehat{\boldsymbol{P}}\left(\widehat{\boldsymbol{\theta}}_{W L S}\right)=\left[\sum_{t=1}^{N} \boldsymbol{\Phi}[t] \widehat{\boldsymbol{\Sigma}}^{-1} \boldsymbol{\Phi}^{T}[t]\right]^{-1} \\
\widehat{\boldsymbol{P}}\left(\widehat{\boldsymbol{\sigma}}_{W L S}\right)=\frac{2}{N} \boldsymbol{D}_{4}^{+}\left(\widehat{\boldsymbol{\Sigma}}_{W L S} \otimes \widehat{\boldsymbol{\Sigma}}_{W L S}\right)\left(\boldsymbol{D}_{4}^{+}\right)^{T}
\end{gathered}
$$


Using Eq. (2.A.7a) and Eq. (2.A.7c), estimates of the FP-VARX $(1,0)_{[2,2]}$ model parameters and their covariance for any arbitrary operating condition $k$ are given by:

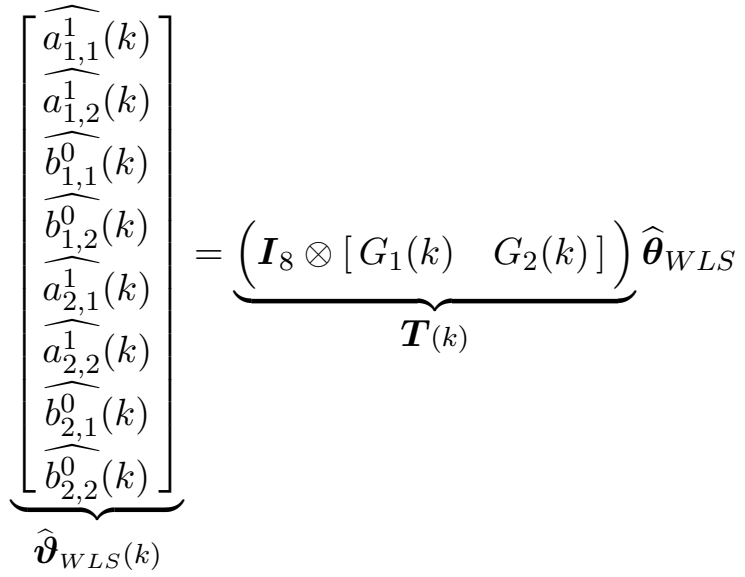

$$
\begin{aligned}
& \widehat{\boldsymbol{P}}\left(\widehat{\boldsymbol{\vartheta}}_{W L S}(k)\right)=\boldsymbol{T}(k) \widehat{\boldsymbol{P}}\left(\widehat{\boldsymbol{\theta}}_{W L S}\right) \boldsymbol{T}^{T}(k)
\end{aligned}
$$

\section{Appendix 2.B: Additional results on the LS and ML type estimators}

\section{B.1 Proof of Proposition 2.3.1}

Under the assumption of groupwise homoscedastic innovations (see Table 2.1), the WLS estimator yields:

$$
\begin{aligned}
\widehat{\boldsymbol{\theta}}_{W L S} & =\left[\frac{1}{N M} \sum_{t=1}^{N} \boldsymbol{\Phi}[t] \boldsymbol{\Sigma}^{-1} \boldsymbol{\Phi}^{T}[t]\right]^{-1}\left[\frac{1}{N M} \sum_{t=1}^{N} \boldsymbol{\Phi}[t] \boldsymbol{\Sigma}^{-1} \boldsymbol{y}[t]\right] \\
& =\left[\frac{1}{N M} \sum_{k=k_{1}}^{k_{M}} \sum_{t=1}^{N} \boldsymbol{\Phi}_{k}[t] \overline{\boldsymbol{\Sigma}}^{-1} \boldsymbol{\Phi}_{k}^{T}[t]\right]^{-1}\left[\frac{1}{N M} \sum_{k=k_{1}}^{k_{M}} \sum_{t=1}^{N} \boldsymbol{\Phi}_{k}[t] \overline{\boldsymbol{\Sigma}}^{-1} \boldsymbol{y}_{k}[t]\right]
\end{aligned}
$$

The double sum in the first bracket of Eq. (2.B.1) may be rewritten as ${ }^{7,8,9}$ :

$$
\begin{aligned}
{\left[\frac{1}{N M} \sum_{k=k_{1}}^{k_{M}} \sum_{t=1}^{N} \boldsymbol{\Phi}_{k}[t] \overline{\boldsymbol{\Sigma}}^{-1} \boldsymbol{\Phi}_{k}^{T}[t]\right]^{-1} } & =\left[\frac{1}{N M} \sum_{k=k_{1}}^{k_{M}} \sum_{t=1}^{N}\left(\boldsymbol{I}_{n y} \otimes \boldsymbol{\phi}_{k}[t]\right)\left(\overline{\boldsymbol{\Sigma}}^{-1} \otimes 1\right)\left(\boldsymbol{I}_{n y} \otimes \boldsymbol{\phi}_{k}^{T}[t]\right)\right]^{-1} \\
& =\left[\frac{1}{N M} \sum_{k=k_{1}}^{k_{M}} \sum_{t=1}^{N}\left(\overline{\boldsymbol{\Sigma}}^{-1} \otimes \boldsymbol{\phi}_{k}[t] \boldsymbol{\phi}_{k}^{T}[t]\right)\right]^{-1} \\
& =[\frac{1}{N M} \overline{\boldsymbol{\Sigma}}^{-1} \otimes \underbrace{\left.\left(\sum_{k=k_{1}}^{k_{M}} \sum_{t=1}^{N} \boldsymbol{\phi}_{k}[t] \boldsymbol{\phi}_{k}^{T}[t]\right)\right]^{-1}}_{\mathbf{\Phi}} \\
& =N M\left(\overline{\boldsymbol{\Sigma}} \otimes \boldsymbol{\Phi}^{-1}\right)
\end{aligned}
$$

${ }^{7}$ Let $\boldsymbol{A}, \boldsymbol{B}, \boldsymbol{C}$ and $\boldsymbol{D}$ be matrices of compatible dimensions, then $(\boldsymbol{A} \otimes \boldsymbol{B})(\boldsymbol{C} \otimes \boldsymbol{D})=\boldsymbol{A} \boldsymbol{C} \otimes \boldsymbol{B} \boldsymbol{D}$ [43, p. 19].

${ }^{8}$ Let $\boldsymbol{A}, \boldsymbol{B}, \boldsymbol{C}$ and $\boldsymbol{D}$ be matrices of compatible dimensions, $\boldsymbol{A} \otimes(\boldsymbol{B} \pm \boldsymbol{C})=\boldsymbol{A} \otimes \boldsymbol{B} \pm \boldsymbol{A} \otimes \boldsymbol{C}$ [43, p. 19].

${ }^{9}$ Let the $(m \times m)$ matrix $\boldsymbol{A}$ and the $(n \times n)$ matrix $\boldsymbol{B}$ be non-singular, then $(\boldsymbol{A} \otimes \boldsymbol{B})^{-1}=\boldsymbol{A}^{-1} \otimes \boldsymbol{B}^{-1}$ [43, p. 19]. 
The double sum in the second bracket of Eq. (2.B.1) may be rewritten as ${ }^{7,8}$ :

$$
\begin{aligned}
\frac{1}{N M} \sum_{k=k_{1}}^{k_{M}} \sum_{t=1}^{N} \boldsymbol{\Phi}_{k}[t] \overline{\boldsymbol{\Sigma}}^{-1} \boldsymbol{y}_{k}[t] & =\frac{1}{N M} \sum_{k=k_{1}}^{k_{M}} \sum_{t=1}^{N}\left(\boldsymbol{I}_{n y} \otimes \boldsymbol{\phi}_{k}[t]\right)\left(\overline{\boldsymbol{\Sigma}}^{-1} \otimes 1\right) \boldsymbol{y}_{k}[t] \\
& =\frac{1}{N M} \sum_{k=k_{1}}^{k_{M}} \sum_{t=1}^{N}\left(\overline{\boldsymbol{\Sigma}}^{-1} \otimes \boldsymbol{\phi}_{k}[t]\right) \boldsymbol{y}_{k}[t]
\end{aligned}
$$

Combining Eq. (2.B.2) and Eq. (2.B.3) into Eq. (2.B.1) yields for the WLS estimator ${ }^{7,8}$ :

$$
\widehat{\boldsymbol{\theta}}_{W L S}=\sum_{k=k_{1}}^{k_{M}} \sum_{t=1}^{N}\left(\boldsymbol{I}_{n y} \otimes \boldsymbol{\Phi}^{-1} \boldsymbol{\phi}_{k}[t]\right) \boldsymbol{y}_{k}[t]
$$

The OLS estimator may be manipulated in a similar manner as seen below:

$$
\begin{aligned}
\widehat{\boldsymbol{\theta}}_{O L S} & =\left[\frac{1}{N M} \sum_{t=1}^{N} \boldsymbol{\Phi}[t] \boldsymbol{\Phi}^{T}[t]\right]^{-1}\left[\frac{1}{N M} \sum_{t=1}^{N} \boldsymbol{\Phi}[t] \boldsymbol{y}[t]\right] \\
& =\left[\frac{1}{N M} \sum_{k=k_{1}}^{k_{M}} \sum_{t=1}^{N} \boldsymbol{\Phi}_{k}[t] \boldsymbol{\Phi}_{k}^{T}[t]\right]^{-1}\left[\frac{1}{N M} \sum_{k=k_{1}}^{k_{M}} \sum_{t=1}^{N} \boldsymbol{\Phi}_{k}[t] \boldsymbol{y}_{k}[t]\right]
\end{aligned}
$$

The double sum in the first bracket of Eq. (2.B.5) may be rewritten as ${ }^{7-9}$ :

$$
\begin{aligned}
{\left[\frac{1}{N M} \sum_{k=k_{1}}^{k_{M}} \sum_{t=1}^{N} \boldsymbol{\Phi}_{k}[t] \boldsymbol{\Phi}_{k}^{T}[t]\right]^{-1} } & =\left[\frac{1}{N M} \sum_{k=k_{1}}^{k_{M}} \sum_{t=1}^{N}\left(\boldsymbol{I}_{n y} \otimes \boldsymbol{\phi}_{k}[t]\right)\left(\boldsymbol{I}_{n y} \otimes \boldsymbol{\phi}_{k}^{T}[t]\right)\right]^{-1} \\
& =\left[\frac{1}{N M} \sum_{k=k_{1}}^{k_{M}} \sum_{t=1}^{N}\left(\boldsymbol{I}_{n y} \otimes \boldsymbol{\phi}_{k}[t] \boldsymbol{\phi}_{k}^{T}[t]\right)\right]^{-1} \\
& =[\frac{1}{N M} \boldsymbol{I}_{n y} \otimes \underbrace{\left(\sum_{k=k_{1}}^{k_{M}} \sum_{t=1}^{N} \boldsymbol{\phi}_{k}[t] \boldsymbol{\phi}_{k}^{T}[t]\right)}_{\mathbf{\Phi}}]^{-1} \\
& =N M\left(\boldsymbol{I}_{n y} \otimes \boldsymbol{\Phi}^{-1}\right)
\end{aligned}
$$

The double sum in the second bracket of Eq. (2.B.5) may be rewritten as ${ }^{7,8}$ :

$$
\frac{1}{N M} \sum_{k=k_{1}}^{k_{M}} \sum_{t=1}^{N} \boldsymbol{\Phi}_{k}[t] \boldsymbol{y}_{k}[t]=\frac{1}{N M} \sum_{k=k_{1}}^{k_{M}} \sum_{t=1}^{N}\left(\boldsymbol{I}_{n y} \otimes \boldsymbol{\phi}_{k}[t]\right) \boldsymbol{y}_{k}[t]
$$

Combining Eq. (2.B.6) and Eq. (2.B.7) into Eq. (2.B.5) yields for the WLS estimator ${ }^{7,8}$ :

$$
\widehat{\boldsymbol{\theta}}_{O L S}=\sum_{k=k_{1}}^{k_{M}} \sum_{t=1}^{N}\left(\boldsymbol{I}_{n y} \otimes \boldsymbol{\Phi}^{-1} \boldsymbol{\phi}_{k}[t]\right) \boldsymbol{y}_{k}[t]
$$

By virtue of Eq. (2.B.4) and Eq. (2.B.8) it holds that $\widehat{\boldsymbol{\theta}}_{W L S}=\widehat{\boldsymbol{\theta}}_{O L S}$, which completes the proof. 


\section{B.2 Proof of Theorem 2.4.1}

The consistency and asymptotic distribution of the LS-based estimators is derived in the following paragraphs.

Consistency of projection coefficient estimator To establish consistency, the LS-based estimator is written as:

$$
\widehat{\boldsymbol{\theta}}_{L S}=\boldsymbol{\theta}_{o}+\left[\frac{1}{N M} \sum_{t=1}^{N} \boldsymbol{\Phi}[t] \boldsymbol{H} \boldsymbol{\Phi}^{T}[t]\right]^{-1}\left[\frac{1}{N M} \sum_{t=1}^{N} \boldsymbol{\Phi}[t] \boldsymbol{H} \boldsymbol{w}[t]\right]
$$

The inverted matrix in the expression above is assumed to be a finite positive definite matrix (refer to Appendix 2.C.3 for details). Furthermore, under fairly general conditions on $\boldsymbol{y}_{k}[t]$ and $\boldsymbol{x}_{k}[t]$ (that is zero-mean, ergodic and stationary processes [2, pp. 547-550]), it holds that ${ }^{10}$ :

$$
\begin{gathered}
\frac{1}{N M} \sum_{t=1}^{N} \boldsymbol{\Phi}[t] \boldsymbol{H} \boldsymbol{\Phi}^{T}[t] \stackrel{p}{\longrightarrow} \frac{1}{M} E\left\{\boldsymbol{\Phi}[t] \boldsymbol{H} \boldsymbol{\Phi}^{T}[t]\right\} \quad(N \longrightarrow \infty) \\
\frac{1}{N M} \sum_{t=1}^{N} \boldsymbol{\Phi}[t] \boldsymbol{H} \boldsymbol{w}[t] \stackrel{p}{\longrightarrow} \mathbf{0} \quad(N \longrightarrow \infty)
\end{gathered}
$$

which implies that ${ }^{11}$ :

$$
\widehat{\boldsymbol{\theta}}_{L S} \stackrel{p}{\longrightarrow} \boldsymbol{\theta}_{O} \quad(N \longrightarrow \infty)
$$

Remark 9. Note, that in the case of conventional VARX models with fully parametrized AR and X polynomial matrices, the LS-based estimator is independent of the weighting matrix $\boldsymbol{H}$ [2, pp. 233-235], and thus not involved in the proof of consistency. This is not the case for the FP-VARX models, however the consistency result is not affected, as it is in analogy to the conventional VARX models [44, p. 196 and p. 397].

Asymptotic distribution of projection coefficient estimator To establish the LS-based estimator asymptotic distribution, Eq. (2.B.9) may be rewritten as follows:

$$
\sqrt{N M}\left(\widehat{\boldsymbol{\theta}}_{L S}-\boldsymbol{\theta}_{o}\right)=\left[\frac{1}{N M} \sum_{t=1}^{N} \boldsymbol{\Phi}[t] \boldsymbol{H} \boldsymbol{\Phi}^{T}[t]\right]^{-1}\left[\frac{1}{\sqrt{N M}} \sum_{t=1}^{N} \boldsymbol{\Phi}[t] \boldsymbol{H} \boldsymbol{w}[t]\right]
$$

Under the assumption that $\boldsymbol{y}_{k}[t]$ and $\boldsymbol{x}_{k}[t]$ are zero-mean, ergodic and stationary processes, the limiting distribution of the rightmost quantity in Eq. (2.B.11) is found by means of a central limit theorem ${ }^{12}$ [2, p.

\footnotetext{
${ }^{10}$ Let the stationary stochastic processes $z_{1}[t]$ and $z_{2}[t]$ be given by $z_{1}[t]=G(B) w_{1}[t]$ and $z_{2}[t]=H(B) w_{2}[t]$, with $G(B)$ and $H(B)$ stable filters, and $\boldsymbol{w}[t]=\left[w_{1}[t] w_{2}[t]\right]^{T}$ a zero-mean white noise with positive-definite covariance matrix and finite fourth order moments. Then, $1 / N \sum_{t=1}^{N} z_{1}[t] z_{2}[t] \stackrel{p}{\longrightarrow} E\left\{z_{1}[t] z_{2}[t]\right\} \quad(N \longrightarrow \infty)$ [2, pp. 547-550].

${ }^{11}$ If $\boldsymbol{x}_{N} \stackrel{p}{\longrightarrow} \boldsymbol{x}$ and $\boldsymbol{A}_{N} \stackrel{p}{\longrightarrow} \boldsymbol{A}$, then $\boldsymbol{A}_{N} \boldsymbol{x}_{N} \stackrel{p}{\longrightarrow} \boldsymbol{A} \boldsymbol{x} \quad(N \longrightarrow \infty)$ [44, p. 683].

${ }^{12}$ Consider $\boldsymbol{x}_{N}=(1 / \sqrt{N}) \sum_{t=1}^{N} \boldsymbol{z}[t]$, where $\boldsymbol{z}[t]$ is a zero-mean vector stationary process given by $\boldsymbol{z}[t]=\boldsymbol{\Phi}[t] \boldsymbol{v}[t]$. The entries of $\boldsymbol{\Phi}[t]$ and $\boldsymbol{v}[t]$ are stationary, possibly correlated, ARMA processes with zero means and underlying white noise sequences with finite fourth-order moments. The elements of $\boldsymbol{\Phi}[t]$ may also contain a bounded deterministic term. Assume that the limit $\boldsymbol{P}=\lim _{N \longrightarrow \infty} E\left\{\boldsymbol{x}_{N} \boldsymbol{x}_{N}^{T}\right\}$ exists and is nonsingular. Then $\boldsymbol{x}_{N}$ is asymptotically Gaussian distributed, $\boldsymbol{x}_{N} \stackrel{d}{\longrightarrow} \mathcal{N}(\mathbf{0}, \boldsymbol{P})$ $(N \longrightarrow \infty)[2$, p. 550].
} 
550], and is given by:

$$
\frac{1}{\sqrt{N M}} \sum_{t=1}^{N} \boldsymbol{\Phi}[t] \boldsymbol{H} \boldsymbol{w}[t] \stackrel{d}{\longrightarrow} \mathcal{N}\left(\mathbf{0}, \frac{1}{M} E\left\{\boldsymbol{\Phi}[t] \boldsymbol{H} \boldsymbol{\Sigma}_{o} \boldsymbol{H} \boldsymbol{\Phi}^{T}[t]\right\}\right) \quad(N \longrightarrow \infty)
$$

By virtue of Eq. (2.B.10a) and Eq. (2.B.12), the asymptotic distribution of the LS estimator $\widehat{\boldsymbol{\theta}}_{L S}$ is ${ }^{13}$ :

$$
\sqrt{N M}\left(\widehat{\boldsymbol{\theta}}_{L S}-\boldsymbol{\theta}_{o}\right) \stackrel{d}{\longrightarrow} \mathcal{N}\left(\mathbf{0}, \boldsymbol{P}_{L S}\right) \quad(N \longrightarrow \infty)
$$

with $\boldsymbol{P}_{L S}=M E\left\{\boldsymbol{\Phi}[t] \boldsymbol{H} \boldsymbol{\Phi}^{T}[t]\right\}^{-1} E\left\{\boldsymbol{\Phi}[t] \boldsymbol{H} \boldsymbol{\Sigma}_{o} \boldsymbol{H} \boldsymbol{\Phi}^{T}[t]\right\} E\left\{\boldsymbol{\Phi}[t] \boldsymbol{H} \boldsymbol{\Phi}^{T}[t]\right\}^{-1}$.

Remark 10. Note, that unlike the case of conventional VARX models [44, p. 196 and p. 397], the asymptotic covariance matrix $\boldsymbol{P}_{L S}$ is affected from the choice of $\boldsymbol{H}$. The cases where optimal statistical accuracy is obtained by the LS estimator is discussed in Appendix 2.B.4.

Consistency of the residual covariance matrix estimator The consistency of $\widehat{\boldsymbol{\Sigma}}_{L S}$ follows directly from the lemma below.

Lemma 2.B.1. Let $\boldsymbol{\theta}_{o}$ be the FP-VARX model true projection coefficient vector and $\boldsymbol{\Sigma}_{o}$ the true covariance matrix of $\boldsymbol{w}[t]$, which is additionally assumed to have bounded fourth-order moments. Then, for any consistent projection coefficient estimator $\widehat{\boldsymbol{\theta}}$, the following covariance matrix estimator is consistent:

$$
\widehat{\boldsymbol{\Sigma}}=\frac{1}{N} \sum_{t=1}^{N} \boldsymbol{e}[t, \widehat{\boldsymbol{\theta}}] \boldsymbol{e}^{T}[t, \widehat{\boldsymbol{\theta}}]=\frac{1}{N} \sum_{t=1}^{N}\left(\boldsymbol{y}[t]-\boldsymbol{\Phi}^{T}[t] \widehat{\boldsymbol{\theta}}\right)\left(\boldsymbol{y}[t]-\boldsymbol{\Phi}^{T}[t] \widehat{\boldsymbol{\theta}}\right)^{T} \stackrel{p}{\longrightarrow} \boldsymbol{\Sigma}_{o}(N \longrightarrow \infty)
$$

Proof. The proof is in analogy to the conventional VAR(X) model case [44, pp. 75-76].

Asymptotic distribution of the residual covariance matrix estimator To establish the asymptotic distribution of $\widehat{\Sigma}_{L S}$ the following lemma is useful.

Lemma 2.B.2. Let $\widehat{\boldsymbol{\theta}}$ be a consistent estimator of the FP-VARX projection coefficients $\boldsymbol{\theta}_{o}$ so that $\sqrt{N}\left(\widehat{\boldsymbol{\theta}}-\widehat{\boldsymbol{\theta}}_{o}\right)$ converges in distribution. Consider the covariance matrix estimator:

$$
\widehat{\boldsymbol{\Sigma}}=\frac{1}{N} \sum_{t=1}^{N} \boldsymbol{e}[t, \widehat{\boldsymbol{\theta}}] \boldsymbol{e}^{T}[t, \widehat{\boldsymbol{\theta}}]
$$

then, the following limit in probability holds:

$$
\sqrt{N}\left(\widehat{\boldsymbol{\Sigma}}-\frac{1}{N} \sum_{t=1}^{N} \boldsymbol{w}[t] \boldsymbol{w}^{T}[t]\right) \stackrel{p}{\longrightarrow} \mathbf{0} \quad(N \longrightarrow \infty)
$$

Proof. The FP-VARX linear regression of Eq. (2.6a) is rewritten as:

$$
\boldsymbol{y}_{k}[t]=\boldsymbol{\Theta}^{T} \boldsymbol{\phi}_{k}[t]+\boldsymbol{e}_{k}[t]
$$

with $\boldsymbol{\Theta}^{T}=\left[\boldsymbol{A}_{1,1} \boldsymbol{A}_{1,2} \ldots \boldsymbol{A}_{n a, p a} \vdots \boldsymbol{B}_{0,1} \boldsymbol{B}_{0,2} \ldots \boldsymbol{B}_{n b, p b}\right]$.

\footnotetext{
${ }^{13}$ Let $\boldsymbol{x}_{N} \stackrel{d}{\longrightarrow} \mathcal{N}(\mathbf{0}, \boldsymbol{P})$ and $\boldsymbol{y}_{N}=\boldsymbol{A}_{N} \boldsymbol{x}_{N}+\boldsymbol{b}_{N}$, with $\boldsymbol{A}_{N} \stackrel{p}{\longrightarrow} \boldsymbol{A}$ and $\boldsymbol{b}_{N} \stackrel{p}{\longrightarrow} \boldsymbol{b}(N \longrightarrow \infty)$. Then, $\boldsymbol{y}_{N} \stackrel{d}{\longrightarrow} \mathcal{N}\left(\boldsymbol{b}, \boldsymbol{A} \boldsymbol{P} \boldsymbol{A}^{T}\right)$ $(N \longrightarrow \infty)$ [2, p. 551].
} 
Stacking the available observations for time samples $t=1,2, \ldots N$ one besides the other yields:

$$
\boldsymbol{Y}_{k}=\boldsymbol{\Theta}^{T} \widetilde{\boldsymbol{\Phi}}_{k}+\boldsymbol{E}_{k}
$$

where:

$$
\begin{aligned}
\boldsymbol{Y}_{k} & =\left[\begin{array}{llll}
\boldsymbol{y}_{k}[1] & \boldsymbol{y}_{k}[2] & \ldots & \boldsymbol{y}_{k}[N]
\end{array}\right] \\
\widetilde{\boldsymbol{\Phi}}_{k} & =\left[\begin{array}{llll}
\boldsymbol{\phi}_{k}[1] & \boldsymbol{\phi}_{k}[2] & \ldots & \phi_{k}[N]
\end{array}\right] \\
\boldsymbol{E}_{k} & =\left[\begin{array}{llll}
\boldsymbol{e}_{k}[1] & \boldsymbol{e}_{k}[2] \ldots \boldsymbol{e}_{k}[N]
\end{array}\right]
\end{aligned}
$$

The following cross-covariance estimator:

$$
\widehat{\boldsymbol{\Sigma}}_{k, \ell}=\frac{1}{N} \sum_{t=1}^{N} \boldsymbol{e}_{k}[t, \widehat{\boldsymbol{\theta}}] \boldsymbol{e}_{\ell}^{T}[t, \widehat{\boldsymbol{\theta}}]=\frac{1}{N}\left(\boldsymbol{Y}_{k}-\widehat{\boldsymbol{\Theta}}^{T} \widetilde{\boldsymbol{\Phi}}_{k}\right)\left(\boldsymbol{Y}_{\ell}-\widehat{\boldsymbol{\Theta}}^{T} \widetilde{\boldsymbol{\Phi}}_{\ell}\right)^{T} \quad k, \ell \in\left\{k_{1}, k_{2}, \ldots, k_{M}\right\}
$$

may be rewritten as:

$$
\begin{aligned}
\frac{1}{N}\left(\boldsymbol{Y}_{k}-\widehat{\boldsymbol{\Theta}}^{T} \widetilde{\boldsymbol{\Phi}}_{k}\right)\left(\boldsymbol{Y}_{\ell}-\widehat{\boldsymbol{\Theta}}^{T} \widetilde{\boldsymbol{\Phi}}_{\ell}\right)^{T}= & \left(\boldsymbol{\Theta}_{o}-\widehat{\boldsymbol{\Theta}}\right)^{T}\left[\frac{\widetilde{\boldsymbol{\Phi}}_{k} \widetilde{\boldsymbol{\Phi}}_{\ell}^{T}}{N}\right]\left(\boldsymbol{\Theta}_{o}-\widehat{\boldsymbol{\Theta}}\right)+\frac{\boldsymbol{W}_{k} \boldsymbol{W}_{\ell}^{T}}{N} \\
& +\left(\boldsymbol{\Theta}_{o}-\widehat{\boldsymbol{\Theta}}\right)^{T} \frac{\widetilde{\boldsymbol{\Phi}}_{k} \boldsymbol{W}_{\ell}^{T}}{N}+\frac{\boldsymbol{W}_{k} \widetilde{\boldsymbol{\Phi}}_{\ell}^{T}}{N}\left(\boldsymbol{\Theta}_{o}-\widehat{\boldsymbol{\Theta}}\right)
\end{aligned}
$$

with $\boldsymbol{W}_{k}$ designating the innovations matrix, defined similar to $\boldsymbol{E}_{k}$ in Eq. (2.B.17d), whereas $\boldsymbol{\Theta}_{o}$ designates the true projection coefficient matrix.

Under the assumption that $\boldsymbol{y}_{k}[t]$ and $\boldsymbol{x}_{k}[t]$ are zero-mean, ergodic and stationary processes, the following limits in probability are zero ${ }^{12,14}$ :

$$
\begin{gathered}
\left(\boldsymbol{\Theta}_{o}-\widehat{\boldsymbol{\Theta}}\right)^{T}\left[\frac{\widetilde{\boldsymbol{\Phi}}_{k} \widetilde{\boldsymbol{\Phi}}_{\ell}^{T}}{N}\right] \sqrt{N}\left(\boldsymbol{\Theta}_{o}-\widehat{\boldsymbol{\Theta}}\right) \stackrel{p}{\longrightarrow} \mathbf{0} \quad(N \longrightarrow \infty) \\
\left(\boldsymbol{\Theta}_{o}-\widehat{\boldsymbol{\Theta}}\right)^{T} \frac{\widetilde{\boldsymbol{\Phi}}_{k} \boldsymbol{W}_{\ell}^{T}}{\sqrt{N}} \stackrel{p}{\longrightarrow} \mathbf{0} \quad(N \longrightarrow \infty) \\
\frac{\boldsymbol{W}_{k} \widetilde{\boldsymbol{\Phi}}_{\ell}^{T}}{\sqrt{N}}\left(\boldsymbol{\Theta}_{o}-\widehat{\boldsymbol{\Theta}}\right) \stackrel{p}{\longrightarrow} \mathbf{0} \quad(N \longrightarrow \infty)
\end{gathered}
$$

Thereby, Eq. (2.B.19) yields ${ }^{15}$ :

$$
\sqrt{N}\left(\widehat{\boldsymbol{\Sigma}}_{k, \ell}-\frac{\boldsymbol{W}_{k} \boldsymbol{W}_{\ell}^{T}}{N}\right)=\sqrt{N}\left(\widehat{\boldsymbol{\Sigma}}_{k, \ell}-\frac{1}{N} \sum_{t=1}^{N} \boldsymbol{w}_{k}[t] \boldsymbol{w}_{\ell}^{T}[t]\right) \stackrel{p}{\longrightarrow} \mathbf{0} \quad(N \longrightarrow \infty)
$$

The preceding result implies that, $\sqrt{N}\left(\widehat{\boldsymbol{\Sigma}}-\frac{1}{N} \sum_{t=1}^{N} \boldsymbol{w}[t] \boldsymbol{w}^{T}[t]\right) \stackrel{p}{\longrightarrow} \mathbf{0} \quad(N \longrightarrow \infty)$, which completes the proof of Lemma 2.B.2.

\footnotetext{
${ }^{14}$ Let $\boldsymbol{x}_{N} \stackrel{d}{\longrightarrow} \boldsymbol{x}$ and $\boldsymbol{A}_{N} \stackrel{p}{\longrightarrow} \mathbf{0}$, then $\boldsymbol{A}_{N} \boldsymbol{x}_{N} \stackrel{p}{\longrightarrow} \mathbf{0}(N \longrightarrow \infty)$ [44, p. 684].

${ }^{15}$ Let $\boldsymbol{x}_{N} \stackrel{p}{\longrightarrow} \boldsymbol{x}$ and $\boldsymbol{y}_{N} \stackrel{p}{\longrightarrow} \boldsymbol{y}$, then $\left(\boldsymbol{x}_{N} \pm \boldsymbol{y}_{N}\right) \stackrel{p}{\longrightarrow}(\boldsymbol{x} \pm \boldsymbol{y})(N \longrightarrow \infty)$ [44, p. 683].
} 
By virtue of Lemma 2.B.2, $\sqrt{N}\left(\widehat{\boldsymbol{\sigma}}_{L S}-\widehat{\boldsymbol{\sigma}}_{M L}\right) \stackrel{p}{\longrightarrow} \mathbf{0}(N \longrightarrow \infty)$, implying that $\sqrt{N}\left(\widehat{\boldsymbol{\sigma}}_{L S}-\boldsymbol{\sigma}_{o}\right)$ and $\sqrt{N}\left(\widehat{\boldsymbol{\sigma}}_{M L}-\boldsymbol{\sigma}_{o}\right)$ have the same asymptotic distribution ${ }^{16}$, thus (see also Appendix 2.B.3):

$$
\sqrt{N M}\left(\widehat{\boldsymbol{\sigma}}_{L S}-\boldsymbol{\sigma}_{o}\right) \stackrel{d}{\longrightarrow} \mathcal{N}\left(\mathbf{0}, 2 M \boldsymbol{D}_{M n y}^{+}\left(\boldsymbol{\Sigma}_{o} \otimes \boldsymbol{\Sigma}_{o}\right)\left(\boldsymbol{D}_{M n y}^{+}\right)^{T}\right) \quad(N \longrightarrow \infty)
$$

which completes the proof of Theorem 2.4.1.

\section{B.3 Proof of Theorem 2.4.2}

The joint $\log$-likelihood function for an FP-VARX process $\boldsymbol{y}[t]$ with zero mean, independent and normally (Gaussian) distributed, innovations having a covariance matrix $\boldsymbol{\Sigma}$ is given by:

$$
L(\boldsymbol{\theta}, \boldsymbol{\Sigma})=-\frac{N M n y}{2} \ln (2 \pi)-\frac{N}{2} \ln (\operatorname{det}(\boldsymbol{\Sigma}))-\frac{1}{2} \sum_{t=1}^{N} \boldsymbol{e}^{T}[t, \boldsymbol{\theta}] \boldsymbol{\Sigma}^{-1} \boldsymbol{e}[t, \boldsymbol{\theta}]
$$

which is in analogy to the conventional VARX case [2, p. 210], and the following assumptions hold:

(i) The projection coefficient vector $\boldsymbol{\theta}$ and the covariance matrix $\boldsymbol{\Sigma}$ are independently parametrized.

(ii) All elements of $\boldsymbol{\Sigma}$ are unknown.

The following composite parameter vector $\overline{\boldsymbol{\theta}}=\left[\boldsymbol{\theta}^{T} \vdots \boldsymbol{\sigma}^{T}\right]^{T}$ is considered, with $\boldsymbol{\sigma}$ being a vector comprising of the non-identical elements on and below the main diagonal of $\boldsymbol{\Sigma}$. Care has to be taken not to select all elements on and below the main diagonal of $\boldsymbol{\Sigma}$ as in the conventional $\operatorname{VAR}(\mathrm{X})$ model case, because the covariance $\Sigma$ has a richer structure with identical elements at certain locations below the main diagonal (refer to Appendix 2.A).

Consistency of parameter estimator The first order Taylor series expansion ${ }^{17}$ of $\partial L(\overline{\boldsymbol{\theta}}) / \partial \overline{\boldsymbol{\theta}}$ around the system's true parameter vector $\overline{\boldsymbol{\theta}}_{o}$ is given by:

$$
\widehat{\overline{\boldsymbol{\theta}}}_{M L} \approx \overline{\boldsymbol{\theta}}_{o}-\left.\left[\left.\frac{1}{N} \frac{\partial^{2} L(\overline{\boldsymbol{\theta}})}{\partial \overline{\boldsymbol{\theta}} \partial \overline{\boldsymbol{\theta}}^{T}}\right|_{\overline{\boldsymbol{\theta}}_{=} \overline{\boldsymbol{\theta}}_{o}}\right]^{-1} \frac{1}{N} \frac{\partial L(\overline{\boldsymbol{\theta}})}{\partial \overline{\boldsymbol{\theta}}}\right|_{\overline{\boldsymbol{\theta}}=\overline{\boldsymbol{\theta}}_{o}}
$$

Obviously, due to the partitioning of $\overline{\boldsymbol{\theta}}$ the first and second order partial derivatives ${ }^{18}$ are rewritten as:

$$
\frac{\partial L(\overline{\boldsymbol{\theta}})}{\partial \overline{\boldsymbol{\theta}}}=\left[\begin{array}{c}
\frac{\partial L(\overline{\boldsymbol{\theta}})}{\partial \boldsymbol{\theta}} \\
\cdots \cdots \cdots \\
\frac{\partial L(\overline{\boldsymbol{\theta}})}{\partial \boldsymbol{\sigma}}
\end{array}\right], \quad \frac{\partial^{2} L(\overline{\boldsymbol{\theta}})}{\partial \overline{\boldsymbol{\theta}} \partial \overline{\boldsymbol{\theta}}^{T}}=\left[\begin{array}{cc}
\frac{\partial^{2} L(\overline{\boldsymbol{\theta}})}{\partial \boldsymbol{\theta} \partial \boldsymbol{\theta}^{T}} & \frac{\partial^{2} L(\overline{\boldsymbol{\theta}})}{\partial \boldsymbol{\theta} \partial \boldsymbol{\sigma}^{T}} \\
\frac{\partial^{2} L(\overline{\boldsymbol{\theta}})}{\partial \boldsymbol{\sigma} \partial \boldsymbol{\theta}^{T}} & \frac{\partial^{2} L(\overline{\boldsymbol{\theta}})}{\partial \boldsymbol{\sigma} \partial \boldsymbol{\sigma}^{T}}
\end{array}\right]
$$

\footnotetext{
${ }^{16}$ Let $\boldsymbol{x}_{N} \stackrel{d}{\longrightarrow} \boldsymbol{x}$ and $\left(\boldsymbol{x}_{N}-\boldsymbol{y}_{N}\right) \stackrel{p}{\longrightarrow} \mathbf{0}$, then $\boldsymbol{y}_{N} \stackrel{d}{\longrightarrow} \boldsymbol{x}(N \longrightarrow \infty)$ [44, p. 683].

${ }^{17}$ Let $\boldsymbol{\theta}_{N}=\boldsymbol{\theta}_{o}+O_{p}\left(r_{N}\right)$ be a $(n \times 1)$ random vector sequence, where $r_{N}=o(1)$, and let $g: \mathbb{R}^{n} \longrightarrow \mathbb{R}$ be a function with continuous partial derivatives of order two at $\boldsymbol{\theta}_{o}$. Then, $g\left(\boldsymbol{\theta}_{N}\right)=g\left(\boldsymbol{\theta}_{o}\right)+\frac{\partial g\left(\boldsymbol{\theta}_{o}\right)}{\partial \boldsymbol{\theta}^{T}}\left(\boldsymbol{\theta}_{N}-\boldsymbol{\theta}_{o}\right)+O_{p}\left(r_{N}^{2}\right)$.

(Let $\left\{a_{N}\right\}$ be a sequence of real numbers and $\left\{b_{N}\right\}$ a sequence of positive real numbers. Then $\left\{a_{N}\right\}$ is said to be of smaller order than $\left\{b_{N}\right\}\left(a_{N}=o\left(b_{N}\right)\right)$ if $a_{N} / b_{N} \longrightarrow 0(N \longrightarrow \infty)$. If $\left\{\boldsymbol{A}_{N}=\left[a_{i j, N}\right]\right\}$ is a sequence of random matrices, then $\boldsymbol{A}_{N}$ is said to be at most of order in probability $\left(\boldsymbol{A}_{N}=O_{p}\left(b_{N}\right)\right)$ if $\forall \epsilon>0, \exists c_{\epsilon}: \forall N \operatorname{Pr}\left(\left|a_{i j, N}\right|>c_{\epsilon} b_{N}\right) \leq \epsilon$ for $i=1,2, \ldots, m$, $j=1,2, \ldots, n)[44$, p. 685].

${ }^{18}$ Let $f(\boldsymbol{\theta})$ be a scalar function that depends on the $(n \times 1)$ vector $\boldsymbol{\theta}$. Then, $\frac{\partial f}{\partial \boldsymbol{\theta}^{T}}=\left[\frac{\partial f}{\partial \boldsymbol{\theta}_{1}} \ldots \frac{\partial f}{\partial \boldsymbol{\theta}_{n}}\right]$ and $\frac{\partial^{2} f}{\partial \boldsymbol{\theta} \partial \boldsymbol{\theta}^{T}}=$ $\left[\begin{array}{ccc}\frac{\partial^{2} f}{\partial \theta_{1} \partial \theta_{1}} & \cdots & \frac{\partial^{2} f}{\theta_{1} \partial \theta_{n}} \\ \vdots & \ddots & \vdots \\ \frac{\partial^{2} f}{\theta_{n} \partial \theta_{1}} & \cdots & \frac{\partial^{2} f}{\theta_{n} \partial \theta_{n}}\end{array}\right]$ 
and their block elements are computed by the following relationships $19,20,21,22,23,24,25,26,27$ :

$$
\begin{gathered}
\frac{\partial L(\overline{\boldsymbol{\theta}})}{\partial \boldsymbol{\theta}}=\sum_{t=1}^{N} \boldsymbol{\Phi}[t] \boldsymbol{\Sigma}^{-1} \boldsymbol{e}^{T}[t, \boldsymbol{\theta}] \\
\frac{\partial L(\overline{\boldsymbol{\theta}})}{\partial \boldsymbol{\theta} \partial \boldsymbol{\theta}^{T}}=-\sum_{t=1}^{N} \boldsymbol{\Phi}[t] \boldsymbol{\Sigma}^{-1} \boldsymbol{\Phi}^{T}[t] \\
\frac{\partial L(\overline{\boldsymbol{\theta}})}{\partial \operatorname{vec}(\boldsymbol{\Sigma})}=-\frac{N}{2} \operatorname{vec}\left(\boldsymbol{\Sigma}^{-1}\right)+\frac{N}{2} \operatorname{vec}\left[\boldsymbol{\Sigma}^{-1}\left(\frac{1}{N} \sum_{t=1}^{N} \boldsymbol{e}[t, \boldsymbol{\theta}] \boldsymbol{e}^{T}[t, \boldsymbol{\theta}]\right) \boldsymbol{\Sigma}^{-1}\right] \\
\frac{\partial^{2} L(\overline{\boldsymbol{\theta}})}{\partial \operatorname{vec}(\boldsymbol{\Sigma}) \partial \operatorname{vec}(\boldsymbol{\Sigma})^{T}}=\frac{N}{2}\left[\left(\boldsymbol{\Sigma}^{-1} \otimes \boldsymbol{\Sigma}^{-1}\right)-\left(\boldsymbol{\Sigma}^{-1} \otimes \boldsymbol{\Sigma}^{-1}\left(\frac{1}{N} \sum_{t=1}^{N} \boldsymbol{e}[t, \boldsymbol{\theta}] \boldsymbol{e}^{T}[t, \boldsymbol{\theta}]\right) \boldsymbol{\Sigma}^{-1}\right)\right. \\
\left.-\left(\boldsymbol{\Sigma}^{-1}\left(\frac{1}{N} \sum_{t=1}^{N} \boldsymbol{e}[t, \boldsymbol{\theta}] \boldsymbol{e}^{T}[t, \boldsymbol{\theta}]\right) \boldsymbol{\Sigma} \otimes \boldsymbol{\Sigma}^{-1}\right)\right] \\
\frac{\partial^{2} L(\overline{\boldsymbol{\theta}})}{\partial \operatorname{vec}(\boldsymbol{\Sigma}) \partial \boldsymbol{\theta}^{T}}=-\frac{1}{2}\left(\boldsymbol{\Sigma}^{-1} \otimes \boldsymbol{\Sigma}^{-1}\right) \sum_{t=1}^{N}\left[\left(\boldsymbol{I}_{M n y} \otimes \boldsymbol{e}[t, \boldsymbol{\theta}]\right)+\left(\boldsymbol{e}[t, \boldsymbol{\theta}] \otimes \boldsymbol{I}_{M n y}\right)\right] \boldsymbol{\Phi}^{T}[t]
\end{gathered}
$$

Note that $\boldsymbol{\sigma}$ and $\boldsymbol{\Sigma}$ are related via the expression $\operatorname{vec}(\boldsymbol{\Sigma})=\boldsymbol{D}_{M n y} \boldsymbol{\sigma}$, with $\boldsymbol{D}_{M n y}$ being a duplication matrix of proper dimensions, defined in analogy to the conventional VARX model case [44, p. 92], thereby ${ }^{28}$ :

$$
\begin{gathered}
\frac{\partial L(\overline{\boldsymbol{\theta}})}{\partial \boldsymbol{\sigma}}=\boldsymbol{D}_{M n y}^{T} \frac{\partial L(\overline{\boldsymbol{\theta}})}{\partial \operatorname{vec}(\boldsymbol{\Sigma})} \\
\frac{\partial^{2} L(\overline{\boldsymbol{\theta}})}{\partial \boldsymbol{\sigma} \partial \boldsymbol{\sigma}^{T}}=\boldsymbol{D}_{M n y}^{T} \frac{\partial^{2} L(\overline{\boldsymbol{\theta}})}{\partial \operatorname{vec}(\boldsymbol{\Sigma}) \partial \operatorname{vec}(\boldsymbol{\Sigma})^{T}} \boldsymbol{D}_{M n y} \\
\frac{\partial^{2} L(\overline{\boldsymbol{\theta}})}{\partial \boldsymbol{\sigma} \partial \boldsymbol{\theta}^{T}}=\boldsymbol{D}_{M n y}^{T} \frac{\partial^{2} L(\overline{\boldsymbol{\theta}})}{\partial \operatorname{vec}(\boldsymbol{\Sigma}) \partial \boldsymbol{\theta}^{T}}
\end{gathered}
$$

${ }^{19}$ Let $\boldsymbol{\Omega}$ be a symmetric $(n \times n)$ matrix and $\boldsymbol{c}(\boldsymbol{\theta})$ an $(n \times 1)$ vector that depends on the $(m \times 1)$ vector $\boldsymbol{\theta}$. Then, $\frac{\partial \boldsymbol{c}^{T}(\boldsymbol{\theta}) \boldsymbol{\Omega} \boldsymbol{c}(\boldsymbol{\theta})}{\partial \boldsymbol{\theta}^{T}}=$ $2 \boldsymbol{c}^{T}(\boldsymbol{\theta}) \boldsymbol{\Omega} \frac{\partial \boldsymbol{c}(\boldsymbol{\theta})}{\partial \boldsymbol{\theta}^{T}}$, and $\frac{\partial^{2} \boldsymbol{c}^{T}(\boldsymbol{\theta}) \boldsymbol{\Omega} \boldsymbol{c}(\boldsymbol{\theta})}{\partial \boldsymbol{\theta} \partial \boldsymbol{\theta}^{T}}=2\left[\frac{\partial \boldsymbol{c}^{T}(\boldsymbol{\theta})}{\partial \boldsymbol{\theta}} \boldsymbol{\Omega} \frac{\partial \boldsymbol{c}(\boldsymbol{\theta})}{\partial \boldsymbol{\theta}^{T}}+\left[\boldsymbol{c}^{T}(\boldsymbol{\theta}) \boldsymbol{\Omega} \otimes \boldsymbol{I}_{m}\right] \frac{\partial \operatorname{vec}\left(\partial \boldsymbol{c}^{T}(\boldsymbol{\theta}) / \partial \boldsymbol{\theta}\right)}{\partial \boldsymbol{\theta}^{T}}\right]$ [43, p. 175].

${ }^{20}$ Let $\boldsymbol{A}$ be an $(m \times n)$ matrix and $\boldsymbol{\theta}$ be an $(n \times 1)$ vector, then $\frac{\partial \boldsymbol{A} \boldsymbol{\theta}}{\partial \boldsymbol{\theta}^{T}}=\boldsymbol{A}$ and $\frac{\partial \boldsymbol{\theta}^{T} \boldsymbol{A}^{T}}{\partial \boldsymbol{\theta}}=\boldsymbol{A}^{T}$ [43, p. 174].

${ }^{21}$ If $\boldsymbol{A}$ is a non-singular $(m \times m)$ matrix with $\operatorname{det}(\boldsymbol{A})>0$, then $\frac{\partial \ln \operatorname{det}(\boldsymbol{A})}{\partial \boldsymbol{A}}=\left(\boldsymbol{A}^{T}\right)^{-1}$ [43, p. 182].

${ }^{22}$ Let $\boldsymbol{A}, \boldsymbol{B}$ and $\boldsymbol{C}$ be $(m \times m)$ matrices with $\boldsymbol{A}$ non-singular, then $\frac{\partial \operatorname{tr}\left(\boldsymbol{B} \boldsymbol{A}^{-1} \boldsymbol{C}\right)}{\partial \boldsymbol{A}}=-\left(\boldsymbol{A}^{-1} \boldsymbol{C} \boldsymbol{B} \boldsymbol{A}^{-1}\right)^{T}$ [43, p. 179].

${ }^{23}$ Suppose $\boldsymbol{\theta}$ is $(m \times 1), \boldsymbol{B}(\boldsymbol{\theta})$ is $(n \times p), \boldsymbol{A}$ is $(k \times n)$, and $\boldsymbol{C}$ is $(p \times q)$ and the latter two matrices do not depend on $\boldsymbol{\theta}$. Then, $\frac{\partial \operatorname{vec}(\boldsymbol{A} \boldsymbol{B} \boldsymbol{C})}{\partial \boldsymbol{\theta}^{T}}=\left(\boldsymbol{C}^{T} \otimes \boldsymbol{A}\right) \frac{\partial \operatorname{vec}(\boldsymbol{B})}{\partial \boldsymbol{\theta}^{T}}[43$, p. 188].

${ }^{24}$ If $\boldsymbol{\theta}$ is $(m \times 1), \boldsymbol{A}(\boldsymbol{\theta})$ is $(n \times p), \boldsymbol{D}(\boldsymbol{\theta})$ is $(q \times r)$, and $\boldsymbol{C}$ is $(p \times q)$ and does not depend on $\boldsymbol{\theta}$. Then, $\frac{\partial \operatorname{vec}(\boldsymbol{A C} \boldsymbol{D})}{\partial \boldsymbol{\theta}^{T}}=$ $\left(\boldsymbol{I}_{r} \otimes \boldsymbol{A} \boldsymbol{C}\right) \frac{\partial \mathrm{vec}(\boldsymbol{D})}{\partial \boldsymbol{\theta}^{T}}+\left(\boldsymbol{D}^{T} \boldsymbol{C}^{T} \otimes \boldsymbol{I}_{n}\right) \frac{\partial \mathrm{vec}(\boldsymbol{A})}{\partial \boldsymbol{\theta}^{T}}[43$, p. 196].

${ }^{25}$ If $\boldsymbol{A}$ is a non-singular $(m \times m)$ matrix, then $\frac{\partial \operatorname{vec}(\boldsymbol{A})^{-1}}{\partial \operatorname{vec}(\boldsymbol{A})^{T}}=-\left(\boldsymbol{A}^{-1}\right)^{T} \otimes \boldsymbol{A}^{-1}$ [43, p. 198].

${ }^{26}$ If $\boldsymbol{\theta}$ is $(m \times 1), \boldsymbol{A}(\boldsymbol{\theta})$ is $(n \times p)$ and $\boldsymbol{B}$ is $(p \times q)$, then, $\frac{\partial \operatorname{vec}(\boldsymbol{A} \boldsymbol{B})}{\partial \boldsymbol{\theta}^{T}}=\left(\boldsymbol{I}_{q} \otimes \boldsymbol{A}\right) \frac{\partial \mathrm{vec}(\boldsymbol{B})}{\partial \boldsymbol{\theta}^{T}}+\left(\boldsymbol{B}^{T} \otimes \boldsymbol{I}_{n}\right) \frac{\partial \mathrm{vec}(\boldsymbol{A})}{\partial \boldsymbol{\theta}^{T}}[43$, p. 196]. ${ }^{27}$ Let, $\boldsymbol{A}, \boldsymbol{B}$ and $\boldsymbol{C}$ be matrices of appropriate dimensions, then $\operatorname{vec}(\boldsymbol{A} \boldsymbol{B} \boldsymbol{C})=\left(\boldsymbol{C}^{T} \otimes \boldsymbol{A}\right) \operatorname{vec}(\boldsymbol{B})=(\boldsymbol{I} \otimes \boldsymbol{A} \boldsymbol{B}) \operatorname{vec}(\boldsymbol{C})=$ $\left(\boldsymbol{C}^{T} \boldsymbol{B}^{T} \otimes \boldsymbol{I}\right) \operatorname{vec}(\boldsymbol{A})[43$, p. 97].

${ }^{28}$ Let $\boldsymbol{a}$ and $\boldsymbol{\theta}$ be $(m \times 1)$ and $(n \times 1)$ vectors, respectively, and suppose $\boldsymbol{h}(\boldsymbol{a})$ is $(p \times 1)$ and $\boldsymbol{g}(\boldsymbol{\theta})$ is $(m \times 1)$. Then, with $\boldsymbol{a}=\boldsymbol{g}(\boldsymbol{\theta}), \frac{\partial \boldsymbol{h}(\boldsymbol{g}(\boldsymbol{\theta}))}{\partial \boldsymbol{\theta}^{T}}=\frac{\partial \boldsymbol{h}(\boldsymbol{a})}{\partial \boldsymbol{a}^{T}} \frac{\partial \boldsymbol{g}(\boldsymbol{\theta})}{\partial \boldsymbol{\theta}^{T}}(p \times n)[43$, p. 174]. 
At $\overline{\boldsymbol{\theta}}=\overline{\boldsymbol{\theta}}_{o}$, the following limits in probability hold ${ }^{10}$ (under the assumption that $\boldsymbol{y}_{k}[t]$ and $\boldsymbol{x}_{k}[t]$ are zero-mean, ergodic and stationary processes):

$$
\begin{gathered}
\left.\frac{1}{N} \frac{\partial L(\overline{\boldsymbol{\theta}})}{\partial \overline{\boldsymbol{\theta}}}\right|_{\overline{\boldsymbol{\theta}}=\overline{\boldsymbol{\theta}}_{o}} \stackrel{p}{\longrightarrow} \mathbf{0} \quad(N \longrightarrow \infty) \\
\left.\frac{1}{N} \frac{\partial^{2} L(\overline{\boldsymbol{\theta}})}{\partial \overline{\boldsymbol{\theta}} \partial \overline{\boldsymbol{\theta}}^{T}}\right|_{\overline{\boldsymbol{\theta}}_{\overline{\boldsymbol{\theta}}} \overline{\boldsymbol{\theta}}_{o}} \stackrel{p}{\longrightarrow}\left[\begin{array}{cc}
E\left\{\boldsymbol{\Phi}[t] \boldsymbol{\Sigma}_{o}^{-1} \boldsymbol{\Phi}^{T}[t]\right\} \\
\mathbf{0} & \mathbf{0} \\
\frac{1}{2} \boldsymbol{D}_{M n y}^{T}\left(\boldsymbol{\Sigma}_{o}^{-1} \otimes \boldsymbol{\Sigma}_{o}^{-1}\right) \boldsymbol{D}_{M n y}
\end{array}\right](N \longrightarrow \infty)
\end{gathered}
$$

Thus, the probability limit in Eq. (2.B.23) yields ${ }^{11}$ :

$$
\widehat{\overline{\boldsymbol{\theta}}}_{M L} \stackrel{p}{\longrightarrow} \overline{\boldsymbol{\theta}}_{O} \quad(N \longrightarrow \infty)
$$

which is in analogy to the conventional VARX model case.

Asymptotic distribution of parameter estimator As in the conventional VARX model case, to derive the asymptotic distribution of $\widehat{\overline{\boldsymbol{\theta}}}_{M L}$, Eq. (2.B.23) is rewritten as follows:

$$
\sqrt{N M}\left(\widehat{\overline{\boldsymbol{\theta}}}_{M L}-\overline{\boldsymbol{\theta}}_{o}\right) \approx-\left[\left.\frac{1}{N M} \frac{\partial^{2} L(\overline{\boldsymbol{\theta}})}{\partial \overline{\boldsymbol{\theta}} \partial \overline{\boldsymbol{\theta}}^{T}}\right|_{\overline{\boldsymbol{\theta}}=\overline{\boldsymbol{\theta}}_{o}}\right]^{-1}\left[\left.\frac{1}{\sqrt{N M}} \frac{\partial L(\overline{\boldsymbol{\theta}})}{\partial \overline{\boldsymbol{\theta}}}\right|_{\overline{\boldsymbol{\theta}}_{=} \overline{\boldsymbol{\theta}}_{o}}\right]
$$

The limiting distribution of the rightmost bracketed quantity is found by means of a central limit theorem $^{12}$ [2, p. 550] yielding:

$$
\left.\frac{1}{\sqrt{N M}} \frac{\partial L(\overline{\boldsymbol{\theta}})}{\partial \overline{\boldsymbol{\theta}}}\right|_{\overline{\boldsymbol{\theta}}_{=} \overline{\boldsymbol{\theta}}_{o}} \stackrel{d}{\longrightarrow} \mathcal{N}\left(\mathbf{0},\left[\begin{array}{cc}
\frac{1}{M} E\left\{\boldsymbol{\Phi}[t] \boldsymbol{\Sigma}_{o}^{-1} \boldsymbol{\Phi}^{T}[t]\right\} & \mathbf{0} \\
\mathbf{0} & \frac{1}{2 M} \boldsymbol{D}_{M n y}^{T}\left(\boldsymbol{\Sigma}_{o} \otimes \boldsymbol{\Sigma}_{o}\right)^{-1} \boldsymbol{D}_{M n y}
\end{array}\right]\right)
$$

and the asymptotic distribution of the ML estimator ${ }^{13}$ is:

$$
\sqrt{N M}\left(\widehat{\overline{\boldsymbol{\theta}}}_{M L}-\overline{\boldsymbol{\theta}}_{o}\right) \stackrel{d}{\longrightarrow} \mathcal{N}\left(\mathbf{0},\left[\begin{array}{cc}
M E\left\{\boldsymbol{\Phi}[t] \boldsymbol{\Sigma}_{o}^{-1} \boldsymbol{\Phi}^{T}[t]\right\}^{-1} & \mathbf{0} \\
\mathbf{0} & 2 M \boldsymbol{D}_{M n y}^{+}\left(\boldsymbol{\Sigma}_{o} \otimes \boldsymbol{\Sigma}_{o}\right)\left(\boldsymbol{D}_{M n y}^{+}\right)^{T}
\end{array}\right]\right)(N \longrightarrow \infty)
$$

which completes the proof of Theorem 2.4.2.

\section{B.4 Statistical accuracy of LS and ML estimators}

In order to study the statistical accuracy of the LS and ML estimators, the following proposition is useful.

Proposition 2.B.1. (Cramér-Rao lower bound for FP-VARX models): The asymptotic Cramér-Rao lower bound for a consistent and asymptotically normal estimator $\widehat{\overline{\boldsymbol{\theta}}}=\left[\widehat{\boldsymbol{\theta}}^{T}: \widehat{\boldsymbol{\sigma}}^{T}\right]^{T}$ of the FP-VARX model is:

$$
E\left\{\left(\widehat{\overline{\boldsymbol{\theta}}}-\overline{\boldsymbol{\theta}}_{o}\right)\left(\widehat{\overline{\boldsymbol{\theta}}}-\overline{\boldsymbol{\theta}}_{o}\right)^{T}\right\}=\left[\begin{array}{cc}
\frac{1}{N} E\left\{\boldsymbol{\Phi}[t] \boldsymbol{\Sigma}_{o}^{-1} \boldsymbol{\Phi}^{T}[t]\right\}^{-1} & \mathbf{0} \\
\mathbf{0} & \frac{2}{N} \boldsymbol{D}_{M n y}^{+}\left(\boldsymbol{\Sigma}_{o} \otimes \boldsymbol{\Sigma}_{o}\right)\left(\boldsymbol{D}_{M n y}^{+}\right)^{T}
\end{array}\right]
$$


Proof. By definition, the Cramér-Rao lower bound equals the inverse of the Fisher information matrix, which is given by the following relationship [2, pp. 560-563]:

$$
\mathcal{F}=-\left.E\left\{\frac{\partial^{2} L(\overline{\boldsymbol{\theta}})}{\partial \overline{\boldsymbol{\theta}} \partial \overline{\boldsymbol{\theta}}^{T}}\right\}\right|_{\overline{\boldsymbol{\theta}}=\overline{\boldsymbol{\theta}}_{o}}
$$

By virtue of Eq. (2.B.24a), the Fisher information matrix $\mathcal{F}$ is rewritten as:

$$
\mathcal{F}=-\left.E\left\{\left[\begin{array}{cc}
\frac{\partial^{2} L(\overline{\boldsymbol{\theta}})}{\partial \boldsymbol{\theta} \partial \boldsymbol{\theta}^{T}} & \frac{\partial^{2} L(\overline{\boldsymbol{\theta}})}{\partial \boldsymbol{\theta} \partial \boldsymbol{\sigma}^{T}} \\
\frac{\partial^{2} L(\overline{\boldsymbol{\theta}})}{\partial \boldsymbol{\sigma} \partial \boldsymbol{\theta}^{T}} & \frac{\partial^{2} L(\overline{\boldsymbol{\theta}})}{\partial \boldsymbol{\sigma} \partial \boldsymbol{\sigma}^{T}}
\end{array}\right]\right\}\right|_{\boldsymbol{\theta}=\boldsymbol{\theta}_{o}, \boldsymbol{\sigma}_{=} \boldsymbol{\sigma}_{o}}
$$

It follows from Eq. (2.B.24c), Eq. (2.B.24h) and Eq. (2.B.24i) that $\mathcal{F}$ is block diagonal, since:

$$
\begin{gathered}
-\left.E\left\{\frac{\partial^{2} L(\overline{\boldsymbol{\theta}})}{\partial \boldsymbol{\theta} \partial \boldsymbol{\theta}^{T}}\right\}\right|_{\boldsymbol{\theta}=\boldsymbol{\theta}_{o}}=N E\left\{\boldsymbol{\Phi}[t] \boldsymbol{\Sigma}_{o}^{-1} \boldsymbol{\Phi}^{T}[t]\right\} \\
-\left.E\left\{\frac{\partial^{2} L(\overline{\boldsymbol{\theta}})}{\partial \boldsymbol{\sigma} \partial \boldsymbol{\sigma}^{T}}\right\}\right|_{\boldsymbol{\sigma}_{=} \boldsymbol{\sigma}_{o}}=\frac{N}{2} \boldsymbol{D}_{M n y}^{T}\left(\boldsymbol{\Sigma}_{o} \otimes \boldsymbol{\Sigma}_{o}\right)^{-1} \boldsymbol{D}_{M n y} \\
-\left.E\left\{\frac{\partial^{2} L(\overline{\boldsymbol{\theta}})}{\partial \boldsymbol{\theta} \partial \boldsymbol{\sigma}^{T}}\right\}\right|_{\boldsymbol{\theta}=\boldsymbol{\theta}_{o}, \boldsymbol{\sigma}_{=} \boldsymbol{\sigma}_{o}=\mathbf{0}}
\end{gathered}
$$

whereas subsequent inversion ${ }^{29}$ of $\mathcal{F}$ leads to Eq. (2.B.28), which completes the proof.

It follows from Proposition 2.B.1 that the Cramér-Rao lower bounds of the optimal FP-VARX estimators for $\boldsymbol{\theta}$ and $\boldsymbol{\sigma}$ are given by:

$$
\begin{array}{r}
E\left\{\sqrt{N M}\left(\widehat{\boldsymbol{\theta}}-\boldsymbol{\theta}_{o}\right)\left(\widehat{\boldsymbol{\theta}}-\boldsymbol{\theta}_{o}\right)^{T} \sqrt{N M}\right\}=M E\left\{\boldsymbol{\Phi}[t] \boldsymbol{\Sigma}_{o}^{-1} \boldsymbol{\Phi}^{T}[t]\right\}^{-1} \\
E\left\{\sqrt{N M}\left(\widehat{\boldsymbol{\sigma}}-\boldsymbol{\sigma}_{o}\right)\left(\widehat{\boldsymbol{\sigma}}-\boldsymbol{\sigma}_{o}\right)^{T} \sqrt{N M}\right\}=2 M \boldsymbol{D}_{M n y}^{+}\left(\boldsymbol{\Sigma}_{o} \otimes \boldsymbol{\Sigma}_{o}\right)\left(\boldsymbol{D}_{M n y}^{+}\right)^{T}
\end{array}
$$

\section{B.4.1 Proof of Corollary 2.4.1}

The following matrix is nonnegative-definite by construction:

$$
\frac{1}{M} E\left\{\left[\begin{array}{cc}
\boldsymbol{\Phi}[t] \boldsymbol{\Sigma}_{o}^{-1} \boldsymbol{\Phi}^{T}[t] & \boldsymbol{\Phi}[t] \boldsymbol{\Phi}^{T}[t] \\
\boldsymbol{\Phi}[t] \boldsymbol{\Phi}^{T}[t] & \boldsymbol{\Phi}[t] \boldsymbol{\Sigma}_{o} \boldsymbol{\Phi}^{T}[t]
\end{array}\right]\right\} \geq \mathbf{0}
$$

Thereby $^{30}$ :

$$
\frac{1}{M} E\left\{\boldsymbol{\Phi}[t] \boldsymbol{\Sigma}_{o}^{-1} \boldsymbol{\Phi}^{T}[t]\right\}-\frac{1}{M} E\left\{\boldsymbol{\Phi}[t] \boldsymbol{\Phi}^{T}[t]\right\} E\left\{\boldsymbol{\Phi}[t] \boldsymbol{\Sigma}_{o} \boldsymbol{\Phi}[t]\right\}^{-1} E\left\{\boldsymbol{\Phi}[t] \boldsymbol{\Phi}^{T}[t]\right\} \geq \mathbf{0}
$$

$$
\begin{aligned}
& { }^{29} \text { Let } \boldsymbol{A}_{i} \text { be }\left(m_{i} \times m_{i}\right) \text { non-singular matrices }(i=1, \ldots, r) \text {, then }\left[\begin{array}{lll}
\boldsymbol{A}_{1} & & \mathbf{0} \\
& \ddots & \\
\mathbf{0} & & \boldsymbol{A}_{r}
\end{array}\right]^{-1}=\left[\begin{array}{ccc}
\boldsymbol{A}_{1}^{-1} & & \mathbf{0} \\
& \ddots & \\
& & \boldsymbol{A}_{r}^{-1}
\end{array}\right] \text { [43, p. 147]. } \\
& { }^{30} \text { Consider the symmetric matrix } \boldsymbol{S}=\left[\begin{array}{cc}
\boldsymbol{A} & \boldsymbol{B} \\
\boldsymbol{B}^{T} & \boldsymbol{C}
\end{array}\right] \text {, then } \boldsymbol{S} \geq \mathbf{0} \Longleftrightarrow \boldsymbol{A}-\boldsymbol{B} \boldsymbol{C}^{-1} \boldsymbol{B}^{T} \geq \mathbf{0}[48, \text { p. 462]. }
\end{aligned}
$$


The above inequality is easily rewritten as ${ }^{31}$ :

$$
M E\left\{\boldsymbol{\Phi}[t] \boldsymbol{\Sigma}_{o}^{-1} \boldsymbol{\Phi}^{T}[t]\right\}^{-1} \leq M E\left\{\boldsymbol{\Phi}[t] \boldsymbol{\Phi}^{T}[t]\right\}^{-1} E\left\{\boldsymbol{\Phi}[t] \boldsymbol{\Sigma}_{o} \boldsymbol{\Phi}[t]\right\} E\left\{\boldsymbol{\Phi}[t] \boldsymbol{\Phi}^{T}[t]\right\}^{-1}=\boldsymbol{P}_{O L S}
$$

which implies that $\widehat{\boldsymbol{\theta}}_{O L S}$ is in general statistically inefficient (compare Eq. (2.B.32a) and Eq. (2.B.35)).

The equality in Eq. (2.B.35) holds under the assumption of groupwise homoscedastic innovations (see also Table 2.1), as straightforward calculations on the OLS asymptotic normalized covariance matrix, similar to the proof of Proposition 2.3.1 (see Appendix 2.B.1), yield:

$$
\boldsymbol{P}_{O L S}=M E\left\{\boldsymbol{\Phi}[t] \boldsymbol{\Phi}^{T}[t]\right\}^{-1} E\left\{\boldsymbol{\Phi}[t] \boldsymbol{\Sigma}_{o} \boldsymbol{\Phi}^{T}[t]\right\} E\left\{\boldsymbol{\Phi}[t] \boldsymbol{\Phi}^{T}[t]\right\}^{-1}=M E\left\{\sum_{k=k_{1}}^{k_{M}} \boldsymbol{\Phi}_{k}[t] \overline{\boldsymbol{\Sigma}}_{o}^{-1} \boldsymbol{\Phi}_{k}^{T}[t]\right\}^{-1}
$$

The Cramér-Rao lower bound in Eq. (2.B.32a) becomes:

$$
E\left\{\sqrt{N M}\left(\widehat{\boldsymbol{\theta}}-\boldsymbol{\theta}_{o}\right)\left(\widehat{\boldsymbol{\theta}}-\boldsymbol{\theta}_{o}\right)^{T} \sqrt{N M}\right\}=M E\left\{\boldsymbol{\Phi}[t] \boldsymbol{\Sigma}_{o}^{-1} \boldsymbol{\Phi}^{T}[t]\right\}^{-1}=M E\left\{\sum_{k=k_{1}}^{k_{M}} \boldsymbol{\Phi}_{k}[t] \overline{\boldsymbol{\Sigma}}_{o}^{-1} \boldsymbol{\Phi}_{k}^{T}[t]\right\}^{-1}
$$

In view of Eq. (2.B.36)-(2.B.37) the statistical optimality of $\widehat{\boldsymbol{\theta}}_{O L S}$ for the case of groupwise homoscedastic innovations is confirmed.

Furthermore, the asymptotic efficiency of $\widehat{\boldsymbol{\sigma}}_{O L S}$ follows directly from Eq. (2.B.32b).

\section{B.4.2 Proof of Corollary 2.4.2}

The asymptotic efficiency of $\widehat{\boldsymbol{\theta}}_{W L S}$, and $\widehat{\boldsymbol{\sigma}}_{W L S}$ follows directly from Eq. (2.B.32a)-(2.B.32b).

\section{B.4.3 Proof of Corollary 2.4.3}

The asymptotic efficiency of $\widehat{\boldsymbol{\theta}}_{M L}$, and $\widehat{\boldsymbol{\sigma}}_{M L}$ follows directly from Eq. (2.B.32a)-(2.B.32b).

\section{Appendix 2.C: Additional results on FP-VARX model identifiability}

\section{C.1 Proof of Theorem 2.5.1}

Stacking the FP-VARX $(n a, n b)_{[p a, p b]}$ representations of Eq. (2.3a)-(2.3c), for each distinct (sample) operating condition $\left\{k_{1}, k_{2}, \ldots, k_{M}\right\}$ one below the other, yields the following expression:

$$
\boldsymbol{A}(\mathcal{B}) \boldsymbol{y}[t]=\boldsymbol{B}(\mathcal{B}) \boldsymbol{x}[t]+\boldsymbol{w}[t]
$$

where:

$$
\boldsymbol{A}(\mathcal{B}) \triangleq\left[\begin{array}{cccc}
\boldsymbol{A}\left[\mathcal{B}, k_{1}\right] & \mathbf{0} & \ldots & \mathbf{0} \\
\mathbf{0} & \boldsymbol{A}\left[\mathcal{B}, k_{2}\right] & \ldots & \mathbf{0} \\
\vdots & \vdots & \ddots & \vdots \\
\mathbf{0} & \mathbf{0} & \ldots & \boldsymbol{A}\left[\mathcal{B}, k_{M}\right]
\end{array}\right], \boldsymbol{B}(\mathcal{B}) \triangleq\left[\begin{array}{cccc}
\boldsymbol{B}\left[\mathcal{B}, k_{1}\right] & \mathbf{0} & \ldots & \mathbf{0} \\
\mathbf{0} & \boldsymbol{B}\left[\mathcal{B}, k_{2}\right] & \ldots & \mathbf{0} \\
\vdots & \vdots & \ddots & \vdots \\
\mathbf{0} & \mathbf{0} & \ldots & \boldsymbol{B}\left[\mathcal{B}, k_{M}\right]
\end{array}\right]
$$

\footnotetext{
${ }^{31}$ Let $\boldsymbol{A}$ and $\boldsymbol{B}$ be $(m \times m)$ symmetric matrices, and assume that either $\boldsymbol{A}$ and $\boldsymbol{B}$ are positive-definite or $\boldsymbol{A}$ and $\boldsymbol{B}$ are negativedefinite. If $\boldsymbol{A} \leq \boldsymbol{B}$, then $\boldsymbol{B}^{-1} \leq \boldsymbol{A}^{-1}$. If, in addition $\boldsymbol{A}<\boldsymbol{B}$, then $\boldsymbol{B}^{-1}<\boldsymbol{A}^{-1}$ [48, p. 476].
} 
The preceding matrix polynomials may be written as (see also Eq. (2.3b) and Eq. (2.3c)):

$$
\boldsymbol{A}(\mathcal{B}) \triangleq \boldsymbol{I}_{M n y}+\boldsymbol{A}_{1} \mathcal{B}+\ldots+\boldsymbol{A}_{n a} \mathcal{B}^{n a}, \quad \boldsymbol{B}(\mathcal{B}) \triangleq \boldsymbol{B}_{0}+\boldsymbol{B}_{1} \mathcal{B}+\ldots+\boldsymbol{B}_{n b} \mathcal{B}^{n b}
$$

with (see also Eq. (2.2c)):

$$
\boldsymbol{A}_{i} \triangleq\left[\begin{array}{cccc}
\boldsymbol{A}_{i}\left(k_{1}\right) & \mathbf{0} & \ldots & \mathbf{0} \\
\mathbf{0} & \boldsymbol{A}_{i}\left(k_{2}\right) \ldots & \mathbf{0} \\
\vdots & \vdots & \ddots & \vdots \\
\mathbf{0} & \mathbf{0} & \ldots & \boldsymbol{A}_{i}\left(k_{M}\right)
\end{array}\right], \boldsymbol{B}_{i} \triangleq\left[\begin{array}{cccc}
\boldsymbol{B}_{j}\left(k_{1}\right) & \mathbf{0} & \ldots & \mathbf{0} \\
\mathbf{0} & \boldsymbol{B}_{j}\left(k_{2}\right) \ldots & \mathbf{0} \\
\vdots & \vdots & \ddots & \vdots \\
\mathbf{0} & \mathbf{0} & \ldots & \boldsymbol{B}_{j}\left(k_{M}\right)
\end{array}\right], \begin{gathered}
i=1,2, \ldots, n a \\
j=0,1, \ldots, n b
\end{gathered}
$$

The similarity of the FP-VARX representation in Eq. (2.C.1a)-(2.C.1d) with the conventional VARX models is obvious, thereby following the same reasoning of conventional VARX modeling, the above parametrization is unique in the class of matrix fraction descriptions (MFD) of degrees $(n a, n b)$ if and only if [2, pp. 182-183]:

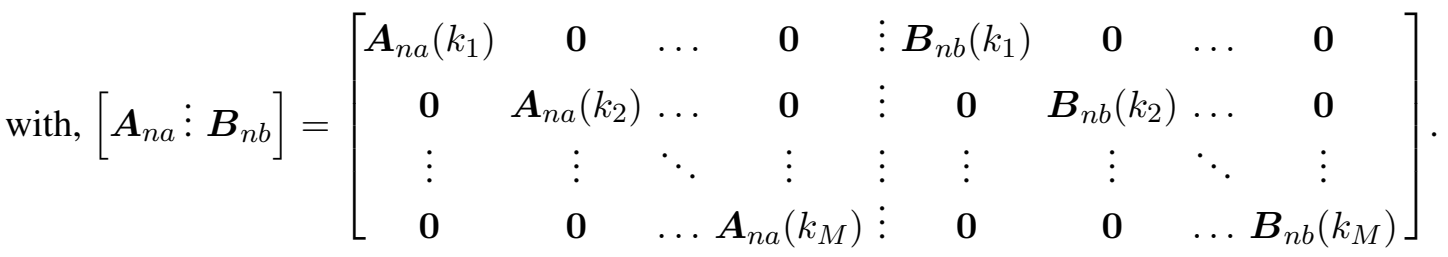

Notice that unlike the conventional VARX case, the matrices $\boldsymbol{A}(\mathcal{B}), \boldsymbol{B}(\mathcal{B})$ are block diagonal. Hence, there exists a block diagonal unimodular matrix implying that their corresponding block elements $\boldsymbol{A}[\mathcal{B}, k]$, $\boldsymbol{B}[\mathcal{B}, k]\left(k=k_{1}, k_{2}, \ldots, k_{M}\right)$ are left coprime.

Rearranging the columns of the composite matrix $\left[\boldsymbol{A}_{n a} \vdots \boldsymbol{B}_{n b}\right]$ yields:

$$
\operatorname{rank}\left[\boldsymbol{A}_{n a} \vdots \boldsymbol{B}_{n b}\right]=\operatorname{rank}\left[\begin{array}{cccc}
{\left[\boldsymbol{A}_{n a}\left(k_{1}\right) \vdots \boldsymbol{B}_{n b}\left(k_{1}\right)\right]} & \mathbf{0} & \ldots & \mathbf{0} \\
\mathbf{0} & {\left[\boldsymbol{A}_{n a}\left(k_{2}\right) \vdots \boldsymbol{B}_{n b}\left(k_{2}\right)\right]} & \ldots & \mathbf{0} \\
\vdots & \vdots & \ddots & \vdots \\
\mathbf{0} & \mathbf{0} & \ldots\left[\boldsymbol{A}_{n a}\left(k_{M}\right) \vdots \boldsymbol{B}_{n b}\left(k_{M}\right)\right]
\end{array}\right]
$$

implying that ${ }^{32}$ :

$$
\sum_{k=k_{1}}^{k_{M}} \operatorname{rank}\left[\boldsymbol{A}_{n a}(k) \vdots \boldsymbol{B}_{n b}(k)\right]=M n y
$$

which indicates that, $\operatorname{rank}\left[\boldsymbol{A}_{n a}(k) \vdots \boldsymbol{B}_{n b}(k)\right]=n y$ for $k=k_{1}, k_{2}, \ldots, k_{M}{ }^{33}$.

The uniqueness of the $\mathrm{AR}$ and $\mathrm{X}$ matrices $\boldsymbol{A}_{i}(k)$ and $\boldsymbol{B}_{i}(k)\left(k=k_{1}, k_{2}, \ldots, k_{M}\right)$, respectively, implies that a unique projection coefficient vector will be obtained from their projection on the functional basis $G_{j}(k)$.

\footnotetext{
${ }^{32}$ Consider the $(m \times n)$ matrix $\boldsymbol{A}$ and the $(\ell \times q)$ matrix $\boldsymbol{B}$, then $\operatorname{rank} \boldsymbol{A}+\operatorname{rank} \boldsymbol{B}=\operatorname{rank}\left[\begin{array}{cc}\boldsymbol{A} & \mathbf{0} \\ \mathbf{0} & \boldsymbol{B}\end{array}\right][43, \mathrm{p} .145]$.

${ }^{33}$ Consider the $(m \times n)$ matrix $\boldsymbol{A}$, then $\operatorname{rank} \boldsymbol{A} \leq \min (m, n)$ [43, p. 61].
} 


\section{C.2 Proof of Lemma 2.5.1}

Consider the FP-VARX representation in Eq. (2.C.1a)-(2.C.1d) with the following transfer function and noise shaping filter matrix polynomials $\boldsymbol{G}(\mathcal{B}, \boldsymbol{\theta})$ and $\boldsymbol{H}(\mathcal{B}, \boldsymbol{\theta})$, respectively:

$$
\boldsymbol{G}(\mathcal{B}, \boldsymbol{\theta}) \triangleq \boldsymbol{A}^{-1}(\mathcal{B}, \boldsymbol{\theta}) \boldsymbol{B}(\mathcal{B}, \boldsymbol{\theta}), \quad \boldsymbol{H}(\mathcal{B}, \boldsymbol{\theta}) \triangleq \boldsymbol{A}^{-1}(\mathcal{B}, \boldsymbol{\theta})
$$

The following projection coefficient vectors $\boldsymbol{\theta}_{1}$ and $\boldsymbol{\theta}_{2}$ are assumed to corresponding in two different models in $\mathcal{M}(\boldsymbol{\theta})$, and the following quantities are introduced: $\Delta \boldsymbol{G}(\mathcal{B})=\boldsymbol{G}\left(\mathcal{B}, \boldsymbol{\theta}_{2}\right)-\boldsymbol{G}\left(\mathcal{B}, \boldsymbol{\theta}_{1}\right), \Delta \boldsymbol{H}(\mathcal{B})=$ $\boldsymbol{H}\left(\mathcal{B}, \boldsymbol{\theta}_{2}\right)-\boldsymbol{H}\left(\mathcal{B}, \boldsymbol{\theta}_{1}\right)$ and $\Delta \boldsymbol{e}[t]=\boldsymbol{e}\left[t, \boldsymbol{\theta}_{2}\right]-\boldsymbol{e}\left[t, \boldsymbol{\theta}_{1}\right]$.

Then, in analogy to the conventional VARX model case, the experiments at the distinct operating conditions $\left\{k_{1}, k_{2}, \ldots, k_{M}\right\}$ are sufficiently informative with respect to $\mathcal{M}(\boldsymbol{\theta})$, if $E\left\{\Delta \boldsymbol{e}[t] \Delta \boldsymbol{e}^{T}[t]\right\}=\mathbf{0}$ implies that the following conditions hold in the frequency domain [1, p. 412]:

$$
\begin{gathered}
\Delta \boldsymbol{H}\left(e^{-j \omega}\right) \triangleq \mathbf{0} \\
\Delta \boldsymbol{G}\left(e^{-j \omega}\right) \boldsymbol{\Phi}_{\boldsymbol{x}}(\omega) \Delta \boldsymbol{G}^{T}\left(e^{j \omega}\right) \triangleq \mathbf{0}
\end{gathered}
$$

with $e$ designating Euler's number (that is the base of natural logarithms), $j$ is the imaginary unit (satisfying $\left.j^{2}=-1\right), \omega \in[-\pi, \pi]$ is the angular frequency, whereas $\boldsymbol{\Phi}_{\boldsymbol{x}}(\omega)$ is the spectral density ${ }^{34}$ of $\boldsymbol{x}[t]$ [2, p. 55].

The preceding identities for the $\operatorname{FP}-\operatorname{VARX}(n a, n b)_{[p a, p b]}$ model yield:

$$
\begin{gathered}
\boldsymbol{A}\left(e^{-j \omega}, \boldsymbol{\theta}_{1}\right) \triangleq \boldsymbol{A}\left(e^{-j \omega}, \boldsymbol{\theta}_{2}\right) \\
\left(\boldsymbol{B}\left(e^{-j \omega}, \boldsymbol{\theta}_{1}\right)-\boldsymbol{B}\left(e^{-j \omega}, \boldsymbol{\theta}_{2}\right)\right) \boldsymbol{\Phi}_{\boldsymbol{x}}(\omega)\left(\boldsymbol{B}\left(e^{j \omega}, \boldsymbol{\theta}_{1}\right)-\boldsymbol{B}\left(e^{j \omega}, \boldsymbol{\theta}_{2}\right)\right)^{T} \triangleq \mathbf{0}
\end{gathered}
$$

The matrix polynomial in Eq. (2.C.4b) is of degree at most $n b$, nonetheless from [2, p. 122], it follows that it is identically zero. Hence the covariance matrix below is positive-definite, which means that $\boldsymbol{x}[t]$ is persistently exciting of order $(n b+1)$ :

$$
\boldsymbol{\Gamma}_{\boldsymbol{x} \boldsymbol{x}}(n b+1)=\left[\begin{array}{cccc}
\boldsymbol{R}_{\boldsymbol{x} \boldsymbol{x}}(0) & \boldsymbol{R}_{\boldsymbol{x} \boldsymbol{x}(1)} & \ldots & \boldsymbol{R}_{\boldsymbol{x} \boldsymbol{x}}(n b) \\
\boldsymbol{R}_{\boldsymbol{x} \boldsymbol{x}(-1)} & \boldsymbol{R}_{\boldsymbol{x} \boldsymbol{x}(0)} & \ldots & \boldsymbol{R}_{\boldsymbol{x} \boldsymbol{x}(n b-1)} \\
\vdots & \vdots & \ddots & \vdots \\
\boldsymbol{R}_{\boldsymbol{x} \boldsymbol{x}(-n b)} & \boldsymbol{R}_{\boldsymbol{x} \boldsymbol{x}(-n b+1)} & \ldots & \boldsymbol{R}_{\boldsymbol{x} \boldsymbol{x}(0)}
\end{array}\right]>\mathbf{0}
$$

The covariance $\boldsymbol{\Gamma}_{\boldsymbol{x} \boldsymbol{x}}(n b+1)$ is by construction symmetric, hence every principal submatrix is positivedefinite $^{35}$. This implies that the following group of covariance matrices corresponding to $\boldsymbol{x}_{k}[t]$ at the distinct operating conditions $\left\{k_{1}, k_{2}, \ldots, k_{M}\right\}$ are also positive definite:

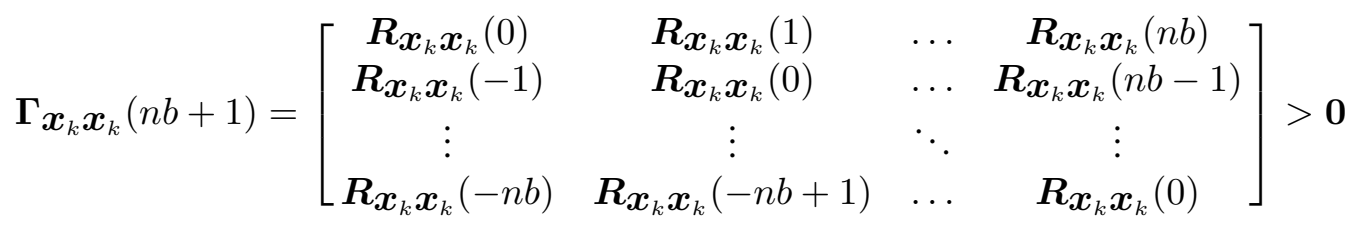

\footnotetext{
${ }^{34}$ The spectral density of $\boldsymbol{x}[t]$ is: $\boldsymbol{\Phi} \boldsymbol{x}(\omega) \triangleq \frac{1}{2 \pi} \sum_{\tau=-\infty}^{+\infty} \boldsymbol{R} \boldsymbol{x} \boldsymbol{x}[\tau] e^{-j \tau \omega}$, with $\boldsymbol{R} \boldsymbol{x} \boldsymbol{x}[\tau]=E\left\{\boldsymbol{x}[t] \boldsymbol{x}^{T}[t-\tau]\right\}$ designating the covariance matrix function of $\boldsymbol{x}[t]$ and $\tau$ the discrete time lag [2, p. 55].

${ }^{35}$ Consider the $(m \times m)$ matrix $\boldsymbol{A}$, if like-numbered rows and columns of $\boldsymbol{A}$ are deleted, then the resulting square submatrix of $\boldsymbol{A}$ is a principal submatrix of $\boldsymbol{A}$. Also, if $\boldsymbol{A}>\mathbf{0} \Longleftrightarrow$ every principal submatrix of $\boldsymbol{A}$ is positive-definite [48, pp. 87-88 and p. 463].
} 
In view of Eq. (2.C.6), the experiments at the distinct operating conditions $\left\{k_{1}, k_{2}, \ldots, k_{M}\right\}$ are informative with respect to $\mathcal{M}(\boldsymbol{\theta})$ when the corresponding excitations $\boldsymbol{x}_{k}[t]$ are persistently exciting of order $(n b+1)$.

Remark 11. The persistent excitation condition implies that the spectral density of $\boldsymbol{x}_{k}[t]$ is non-zero on at least $(n b+1)$ distinct frequencies in the interval $(-\pi, \pi]$. In practice, the excitation $\boldsymbol{x}_{k}[t]$ is usually a broad-band signal, for instance white noise, which satisfies the preceding requirement.

\section{C.3 Proof of Theorem 2.5 .2}

To establish the consistency of the WLS (the results apply directly on the OLS case) or the ML estimator, the second order derivatives of the corresponding minimization criteria have to be asymptotically positivedefinite. It is easy to observe that the following inequality holds ${ }^{36}$ :

$$
E\left\{\boldsymbol{\Phi}[t] \boldsymbol{\Sigma}^{-1} \boldsymbol{\Phi}^{T}[t]\right\} \geq \lambda_{\min }\left(\boldsymbol{\Sigma}^{-1}\right) E\left\{\boldsymbol{\Phi}[t] \boldsymbol{\Phi}^{T}[t]\right\} \geq 0
$$

with $\lambda_{\min }(\cdot)$ denoting the minimum eigenvalue of the indicated matrix. The last inequality implies that the second order derivative is positive-definite when the right hand expectation is also positive-definite (note that $\lambda_{\min }\left(\boldsymbol{\Sigma}^{-1}\right)>0$, by virtue of assumption A6). Therefore, the problem is simplified into finding the conditions for which the right hand expectation in Eq. (2.C.7) is positive-definite.

Using Eq. (2.6b)-(2.6c), simple algebraic calculations lead to ${ }^{7,8}$ :

$$
\begin{aligned}
& E\left\{\boldsymbol{\Phi}[t] \boldsymbol{\Phi}^{T}[t]\right\}=\boldsymbol{I}_{n y} \otimes \sum_{k=k_{1}}^{k_{M}} E\left\{\boldsymbol{\phi}_{k}[t] \boldsymbol{\phi}_{k}^{T}[t]\right\} \\
& \quad=\boldsymbol{I}_{n y} \otimes \sum_{k=k_{1}}^{k_{M}} E\left\{\left[\begin{array}{ll}
\left(\boldsymbol{\phi}_{y k}[t] \boldsymbol{\phi}_{y k}^{T}[t] \otimes \boldsymbol{g}_{a}(k) \boldsymbol{g}_{a}^{T}(k)\right) & \left.\left(\boldsymbol{\phi}_{y k}[t] \boldsymbol{\phi}_{x k}^{T}[t] \otimes \boldsymbol{g}_{a}(k) \boldsymbol{g}_{b}^{T}(k)\right)\right] \\
\left(\boldsymbol{\phi}_{x k}[t] \boldsymbol{\phi}_{y k}^{T}[t] \otimes \boldsymbol{g}_{b}(k) \boldsymbol{g}_{a}^{T}(k)\right) & \left.\left.\left(\boldsymbol{\phi}_{x k}[t] \boldsymbol{\phi}_{x k}^{T}[t] \otimes \boldsymbol{g}_{b}(k) \boldsymbol{g}_{b}^{T}(k)\right)\right]\right\} \mathbf{0}
\end{array}\right.\right.
\end{aligned}
$$

with, $\phi_{y k}[t]=\left[-\boldsymbol{y}_{k}^{T}[t-1] \ldots-\boldsymbol{y}_{k}^{T}[t-n a]\right]^{T}, \phi_{x k}[t]=\left[\boldsymbol{x}_{k}^{T}[t] \ldots \boldsymbol{x}_{k}^{T}[t-n b]\right]^{T}$, whereas $\boldsymbol{g}_{j}(k)=\left[G_{1}(k) \ldots G_{p j}(k)\right]^{T}$ for $j=a, b$.

Thus, what remains is to find the conditions under which the sum of expectations above is positivedefinite.

For this purpose, the following regressor covariance matrix is introduced (the excitation and response signals $\boldsymbol{y}_{k}[t]$ and $\boldsymbol{x}_{k}[t]$ are assumed zero-mean with no loss of generality):

$$
\boldsymbol{\Gamma}_{k}=E\left\{\left[\begin{array}{c}
\phi_{y k}[t] \\
\phi_{x k}[t]
\end{array}\right]\left[\begin{array}{ll}
\phi_{y k}^{T}[t] & \left.\phi_{x k}^{T}[t]\right]
\end{array}\right\}\right.
$$

and designating as $\boldsymbol{R}_{\boldsymbol{u}_{k}}^{k} \boldsymbol{v}_{k}(\tau)=E\left\{\boldsymbol{u}_{k}[t] \boldsymbol{v}_{k}^{T}[t-\tau]\right\}$ the cross-correlation between $\boldsymbol{u}_{k}[t]$ and $\boldsymbol{v}_{k}[t]$ at lag $\tau$, the covariance $\Gamma_{k}$ may be alternatively written as:

$$
\boldsymbol{\Gamma}_{k}=\left[\begin{array}{ccc|ccc}
\boldsymbol{R}_{\boldsymbol{y}_{k}} \boldsymbol{y}_{k}(0) & \ldots & \boldsymbol{R}_{\boldsymbol{y}_{k}} \boldsymbol{y}_{k}(n a-1) & -\boldsymbol{R}_{\boldsymbol{y}_{k} \boldsymbol{x}_{k}(-1)} & \ldots & -\boldsymbol{R}_{\boldsymbol{y}_{k}} \boldsymbol{x}_{k}(n b-1) \\
\vdots & \ddots & \vdots & \vdots & \ddots & \vdots \\
\boldsymbol{R}_{\boldsymbol{y}_{k} \boldsymbol{y}_{k}(-n a+1)} \ldots & \boldsymbol{R}_{\boldsymbol{y}_{k} \boldsymbol{y}_{k}(0)} & -\boldsymbol{R}_{\boldsymbol{y}_{k} \boldsymbol{x}_{k}(-n a)} \ldots & -\boldsymbol{R}_{\boldsymbol{y}_{k} \boldsymbol{x}_{k}(-n a+n b)} \\
\hline-\boldsymbol{R}_{\boldsymbol{x}_{k} \boldsymbol{y}_{k}(1)} & \ldots & -\boldsymbol{R}_{\boldsymbol{x}_{k} \boldsymbol{y}_{k}(n a)} & \boldsymbol{R}_{\boldsymbol{x}_{k} \boldsymbol{x}_{k}(0)} & \ldots & \boldsymbol{R}_{\boldsymbol{x}_{k} \boldsymbol{x}_{k}(n b)} \\
\vdots & \ddots & \vdots & \vdots & \ddots & \vdots \\
-\boldsymbol{R}_{\boldsymbol{x}_{k} \boldsymbol{y}_{k}(-n b+1)} & \ldots & -\boldsymbol{R}_{\boldsymbol{x}_{k} \boldsymbol{y}_{k}(-n b+n a)} & \boldsymbol{R}_{\boldsymbol{x}_{k} \boldsymbol{x}_{k}(-n b)} & \ldots & \boldsymbol{R}_{\boldsymbol{x}_{k} \boldsymbol{x}_{k}(0)}
\end{array}\right]
$$

\footnotetext{
${ }^{36}$ Consider the $(m \times m)$ symmetric matrix $\boldsymbol{A}$, then $\lambda_{\min }(\boldsymbol{A}) \boldsymbol{I}_{m} \leq \boldsymbol{A} \leq \lambda_{\max }(\boldsymbol{A}) \boldsymbol{I}_{m}$ [48, p. 467].
} 


$$
=\left[\begin{array}{c|c}
\boldsymbol{\Gamma}_{\boldsymbol{y}_{k}} \boldsymbol{y}_{k}(n a) & -\boldsymbol{\Gamma} \boldsymbol{y}_{k} \boldsymbol{x}_{k}(n a, n b+1) \\
\hline-\left(\boldsymbol{\Gamma} \boldsymbol{y}_{k} \boldsymbol{x}_{k}(n a, n b+1)\right)^{T} & \boldsymbol{\Gamma} \boldsymbol{x}_{k} \boldsymbol{x}_{k}(n b+1)
\end{array}\right]
$$

Therefore, the asymptotic positive-definiteness of the second derivative implies the following equivalence:

$$
\begin{aligned}
& E\left\{\boldsymbol{\Phi}[t] \boldsymbol{\Sigma}^{-1} \boldsymbol{\Phi}^{T}[t]\right\}>\mathbf{0} \Longleftrightarrow \\
& \sum_{k=k_{1}}^{k_{M}}\left[\begin{array}{cc}
\boldsymbol{\Gamma} \boldsymbol{y}_{k} \boldsymbol{y}_{k}(n a) \otimes \boldsymbol{g}_{a}(k) \boldsymbol{g}_{a}^{T}(k) & -\boldsymbol{\Gamma} \boldsymbol{y}_{k} \boldsymbol{x}_{k}(n a, n b+1) \otimes \boldsymbol{g}_{a}(k) \boldsymbol{g}_{b}^{T}(k) \\
-\left(\boldsymbol{\Gamma} \boldsymbol{y}_{k} \boldsymbol{x}_{k}(n a, n b+1)\right)^{T} \otimes \boldsymbol{g}_{b}(k) \boldsymbol{g}_{a}^{T}(k) & \boldsymbol{\Gamma} \boldsymbol{x}_{k} \boldsymbol{x}_{k}(n b+1) \otimes \boldsymbol{g}_{b}(k) \boldsymbol{g}_{b}^{T}(k)
\end{array}\right]>\mathbf{0}
\end{aligned}
$$

In order to continue the analysis, the $\operatorname{FP}-\operatorname{VARX}(n a, n b)_{[p a, p b]}$ model may be written as follows (refer to Fig. 2.1):

$$
\boldsymbol{y}_{k}[t]=\underbrace{\boldsymbol{A}^{-1}[\mathcal{B}, k] \boldsymbol{B}[\mathcal{B}, k] \boldsymbol{x}_{k}[t]}_{\boldsymbol{y}_{k}^{*}[t]}+\underbrace{\boldsymbol{A}^{-1}[\mathcal{B}, k] \boldsymbol{e}_{k}[t]}_{\boldsymbol{n}_{k}[t]}
$$

with $\boldsymbol{y}_{k}^{*}[t]$ and $\boldsymbol{n}_{k}[t]$ designating the part of the response due to the excitation and the additional noise respectively.

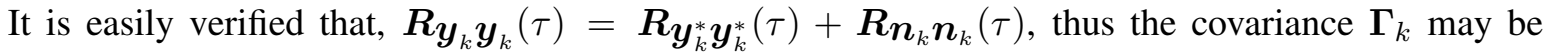
alternatively written as:

$$
\begin{aligned}
& \boldsymbol{\Gamma}_{k}=\left[\begin{array}{ccc|ccc}
\boldsymbol{R}_{\boldsymbol{y}_{k}^{*} \boldsymbol{y}_{k}^{*}(0)} & \ldots & \boldsymbol{R}_{\boldsymbol{y}_{k}^{*} \boldsymbol{y}_{k}^{*}(n a-1)} & -\boldsymbol{R}_{\boldsymbol{n}_{k} \boldsymbol{x}_{k}(-1)} \ldots & -\boldsymbol{R}_{\boldsymbol{n}_{k} \boldsymbol{x}_{k}(n b-1)} \\
\vdots & \ddots & \vdots & \vdots & \ddots & \vdots \\
\boldsymbol{R}_{\boldsymbol{y}_{k}^{*} \boldsymbol{y}_{k}^{*}(-n a+1)} \ldots & \boldsymbol{R}_{\boldsymbol{y}_{k}^{*} \boldsymbol{y}_{k}^{*}(0)} & -\boldsymbol{R}_{\boldsymbol{n}_{k} \boldsymbol{x}_{k}(-n a)} & \ldots & -\boldsymbol{R}_{\boldsymbol{n}_{k} \boldsymbol{x}_{k}}(-n a+n b) \\
\hline-\boldsymbol{R}_{\boldsymbol{x}_{k}} \boldsymbol{n}_{k}(1) & \ldots & -\boldsymbol{R}_{\boldsymbol{x}_{k}} \boldsymbol{n}_{k}(n a) & \boldsymbol{R}_{\boldsymbol{x}_{k} \boldsymbol{x}_{k}(0)}(0) & \ldots & \boldsymbol{R}_{\boldsymbol{x}_{k} \boldsymbol{x}_{k}(n b)} \\
\vdots & \ddots & \vdots & \vdots & \ddots & \vdots \\
-\boldsymbol{R}_{\boldsymbol{x}_{k} \boldsymbol{n}_{k}(-n b+1)} \ldots & -\boldsymbol{R}_{\boldsymbol{x}_{k} \boldsymbol{n}_{k}(-n b+n a)} & \boldsymbol{R}_{\boldsymbol{x}_{k} \boldsymbol{x}_{k}(-n b)} \ldots & \boldsymbol{R}_{\boldsymbol{x}_{k} \boldsymbol{x}_{k}(0)}
\end{array}\right] \\
& +\left[\begin{array}{ccc|ccc}
\boldsymbol{R}_{\boldsymbol{n}_{k} \boldsymbol{n}_{k}(0)} & \ldots & \boldsymbol{R}_{\boldsymbol{n}_{k} \boldsymbol{n}_{k}(n a-1)} & \mathbf{0} & \ldots & \mathbf{0} \\
\vdots & \ddots & \vdots & \vdots & \ddots & \vdots \\
\boldsymbol{R}_{\boldsymbol{n}_{k} \boldsymbol{n}_{k}(-n a+1)} \ldots & \boldsymbol{R}_{\boldsymbol{n}_{k} \boldsymbol{n}_{k}(0)} & \mathbf{0} & \ldots & \mathbf{0} \\
\hline \mathbf{0} & \ldots & \mathbf{0} & \mathbf{0} & \ldots & \mathbf{0} \\
\vdots & \ddots & \vdots & \vdots & \ddots & \vdots \\
\mathbf{0} & \ldots & \mathbf{0} & \mathbf{0} & \ldots & \mathbf{0}
\end{array}\right] \\
& =\left[\begin{array}{c|c}
\boldsymbol{\Gamma} \boldsymbol{y}_{k}^{*} \boldsymbol{y}_{k}^{*}(n a) & -\boldsymbol{\Gamma} \boldsymbol{n}_{k} \boldsymbol{x}_{k}(n a, n b+1) \\
\hline-\left(\boldsymbol{\Gamma}_{\boldsymbol{n}_{k}} \boldsymbol{x}_{k}(n a, n b+1)\right)^{T} & \boldsymbol{\Gamma}_{\boldsymbol{x}_{k}} \boldsymbol{x}_{k}(n b+1)
\end{array}\right]+\left[\begin{array}{c|c}
\boldsymbol{\Gamma} \boldsymbol{n}_{k} \boldsymbol{n}_{k}(n a) & \mathbf{0} \\
\hline \mathbf{0} & \mathbf{0}
\end{array}\right]
\end{aligned}
$$

It then follows that the asymptotic positive-definiteness of the second order derivative implies that ${ }^{37}$ :

$$
E\left\{\boldsymbol{\Phi}[t] \boldsymbol{\Sigma}^{-1} \boldsymbol{\Phi}^{T}[t]\right\}>\mathbf{0} \Longleftrightarrow \mid \begin{aligned}
& \boldsymbol{C}=\sum_{k=k_{1}}^{k_{M}} \boldsymbol{\Gamma} \boldsymbol{x}_{k} \boldsymbol{x}_{k}(n b+1) \otimes \boldsymbol{g}_{b}(k) \boldsymbol{g}_{b}^{T}(k)>\mathbf{0} \\
& \boldsymbol{A}=\sum_{k=k_{1}}^{k_{M}}\left(\boldsymbol{\Gamma} \boldsymbol{y}_{k}^{*} \boldsymbol{y}_{k}^{*}(n a)+\boldsymbol{\Gamma} \boldsymbol{n}_{k} \boldsymbol{n}_{k}(n a)\right) \otimes \boldsymbol{g}_{a}(k) \boldsymbol{g}_{a}^{T}(k)>\mathbf{0}
\end{aligned}
$$

\footnotetext{
${ }^{37}$ Consider the symmetric matrix $\boldsymbol{S}=\left[\begin{array}{cc}\boldsymbol{A} & \boldsymbol{B} \\ \boldsymbol{B}^{T} & \boldsymbol{C}\end{array}\right]$, then $\boldsymbol{S}>\mathbf{0} \Longleftrightarrow \boldsymbol{A}>\mathbf{0}, \boldsymbol{C}>\mathbf{0}$ [48, p. 462].
} 
In view of Lemma 2.5.1, it holds that $\boldsymbol{\Gamma}_{\boldsymbol{x}_{k}} \boldsymbol{x}_{k}(n b+1)>\mathbf{0}$, thus condition (I) yields ${ }^{36,38}$ :

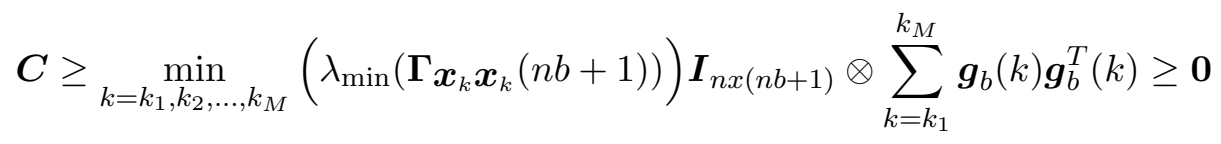

with "min" denoting the minimum with respect to the distinct (sample) values $\left\{k_{1}, k_{2}, \ldots, k_{M}\right\}$. The above inequality implies that condition (I) is satisfied when:

(a) $\boldsymbol{x}_{k}[t]\left(k=k_{1}, k_{2}, \ldots, k_{M}\right)$ is persistently exciting of order $(n b+1)$.

(b) $\sum_{k=k_{1}}^{k_{M}} \boldsymbol{g}_{b}(k) \boldsymbol{g}_{b}^{T}(k)>\mathbf{0}$

Condition (II) requires that $\left(\sum_{k=k_{1}}^{k_{M}} \boldsymbol{\Gamma}_{\boldsymbol{n}_{k}} \boldsymbol{n}_{k}(n a) \otimes \boldsymbol{g}_{a}(k) \boldsymbol{g}_{a}^{T}(k)\right)$ is positive-definite ${ }^{39}$, which is satisfied when:

(c) $\boldsymbol{e}_{k}[t]\left(k=k_{1}, k_{2}, \ldots, k_{M}\right)$ is persistently exciting.

(d) $\quad \sum_{k=k_{1}}^{k_{M}} \boldsymbol{g}_{a}(k) \boldsymbol{g}_{a}^{T}(k)>\mathbf{0}$

Thereby, under weak conditions on the residuals (that is, $\boldsymbol{e}_{k}[t]$ being persistently exciting), the consistency of the LS and ML based estimators implies the following equivalence:

$$
E\left\{\boldsymbol{\Phi}[t] \boldsymbol{\Sigma}^{-1} \boldsymbol{\Phi}^{T}[t]\right\}>\mathbf{0} \Longleftrightarrow \mid \begin{aligned}
& \boldsymbol{x}_{k}[t]\left(k=k_{1}, k_{2}, \ldots, k_{M}\right) \text { is persistently exciting of order }(n b+1) . \\
& \sum_{k=k_{1}}^{k_{M}} \boldsymbol{g}_{j}(k) \boldsymbol{g}_{j}^{T}(k)>\mathbf{0}, j=a, b
\end{aligned}
$$

\section{Appendix 2.D: Shifted Chebyshev polynomials of the second kind}

The shifted Chebyshev polynomials of the second kind are a set of orthogonal polynomials over the range $x \in[0,+1] \subset \mathbb{R}$. They are denoted $G_{n}(x)$ and obey the following recurrence relation [45, p. 782]:

$$
G_{n+1}(x)=(-2+4 x) G_{n}(x)-G_{n-1}(x), \text { with } G_{1}(x)=1, G_{2}(x)=4 x-2 .
$$

An illustration of the polynomials for $n=1,2,3,4$ is provided in Fig. 2.10. In the present framework it is assumed for convenience that $x=k /(M-1)$, since $k \in[0, M-1]$ and is discretized at unit increments, that is $\Delta k=1$ ( $M$ experiments).

\section{Appendix 2.E: FP-VARX WLS estimation via QR implementation}

\section{E.1 The basic QR method}

In order to obtain a FP-VARX WLS estimate $\widehat{\boldsymbol{\theta}}_{W L S}$, the linear regression equations of the form Eq. (2.6a) are stacked one below the other, for each time instant $t=1,2, \ldots, N$, as follows:

$$
\boldsymbol{y}_{k}=\boldsymbol{\Phi}_{k}^{T} \boldsymbol{\theta}+\boldsymbol{e}_{k}
$$

\footnotetext{
${ }^{38}$ Consider the $(m \times m)$ matrices $\boldsymbol{A}_{1}, \boldsymbol{A}_{2}, \boldsymbol{B}_{1}, \boldsymbol{B}_{2} \geq \mathbf{0}$, and assume that $\mathbf{0} \leq \boldsymbol{A}_{1} \leq \boldsymbol{B}_{1}$ and $\mathbf{0} \leq \boldsymbol{A}_{2} \leq \boldsymbol{B}_{2}$, then $\mathbf{0} \leq \boldsymbol{A}_{1} \otimes \boldsymbol{A}_{2} \leq \boldsymbol{B}_{1} \otimes \boldsymbol{B}_{2}$ [48, p. 589].

${ }^{39}$ Consider the $(m \times m)$ symmetric matrices $\boldsymbol{A}$ and $\boldsymbol{B}$, if $\boldsymbol{A} \geq \mathbf{0}$ and $\boldsymbol{B}>\mathbf{0}$, then $\boldsymbol{A}+\boldsymbol{B}>\mathbf{0}$ [48, p. 460].
} 


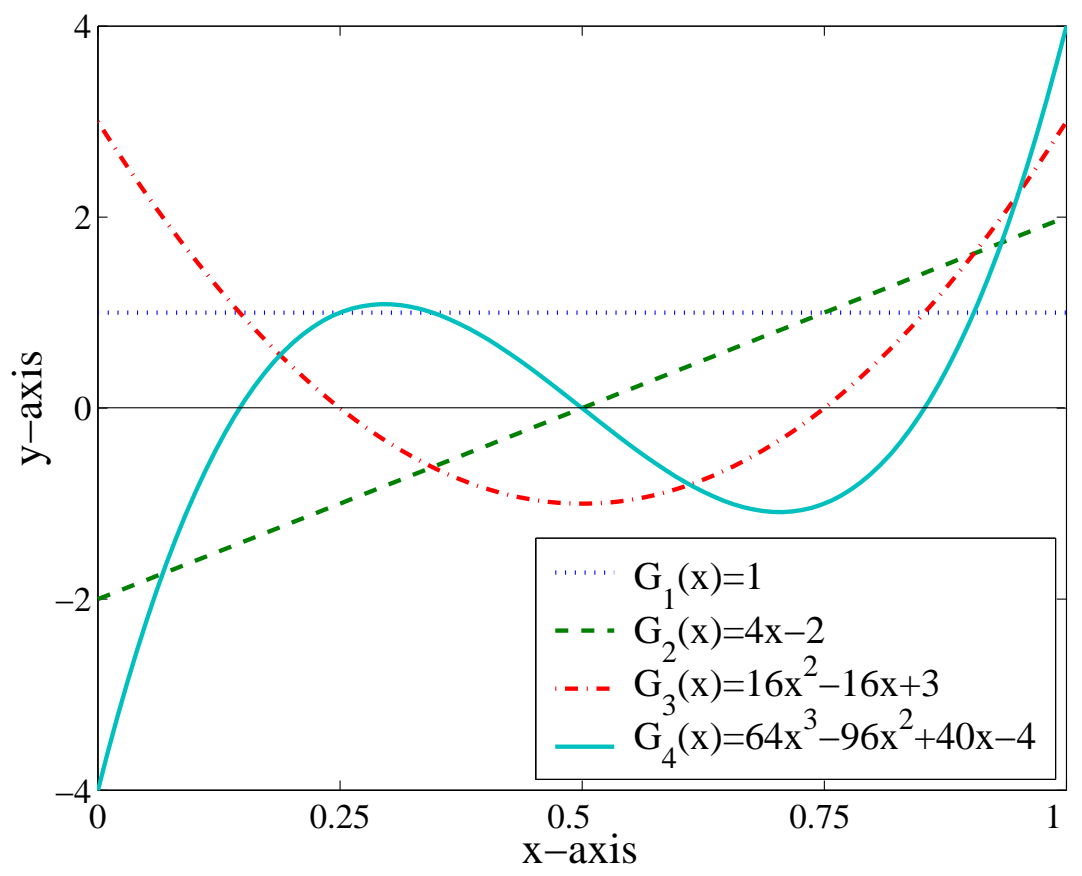

Figure 2.10: Indicative shifted Chebyshev polynomials of the second kind.

with:

$$
\boldsymbol{y}_{k}=\left[\begin{array}{c}
\boldsymbol{y}_{k}[1] \\
\boldsymbol{y}_{k}[2] \\
\vdots \\
\boldsymbol{y}_{k}[N]
\end{array}\right], \quad \boldsymbol{\Phi}_{k}^{T}=\left[\begin{array}{c}
\boldsymbol{\Phi}_{k}^{T}[1] \\
\boldsymbol{\Phi}_{k}^{T}[2] \\
\vdots \\
\boldsymbol{\Phi}_{k}^{T}[N]
\end{array}\right], \quad \boldsymbol{e}_{k}=\left[\begin{array}{c}
\boldsymbol{e}_{k}[1] \\
\boldsymbol{e}_{k}[2] \\
\vdots \\
\boldsymbol{e}_{k}[N]
\end{array}\right]
$$

Pooling the equations of the form Eq. (2.E.1a) for the operating conditions $\left\{k_{1}, k_{2}, \ldots, k_{M}\right\}$ yields:

$$
\boldsymbol{y}=\boldsymbol{\Phi}^{T} \boldsymbol{\theta}+\boldsymbol{e}
$$

with:

$$
\boldsymbol{y}=\left[\begin{array}{c}
\boldsymbol{y}_{k_{1}} \\
\boldsymbol{y}_{k_{2}} \\
\vdots \\
\boldsymbol{y}_{k_{M}}
\end{array}\right], \quad \boldsymbol{\Phi}^{T}=\left[\begin{array}{c}
\boldsymbol{\Phi}_{k_{1}}^{T} \\
\boldsymbol{\Phi}_{k_{2}}^{T} \\
\vdots \\
\boldsymbol{\Phi}_{k_{M}}^{T}
\end{array}\right], \quad \boldsymbol{e}=\left[\begin{array}{c}
\boldsymbol{e}_{k_{1}} \\
\boldsymbol{e}_{k_{2}} \\
\vdots \\
\boldsymbol{e}_{k_{M}}
\end{array}\right]
$$

The corresponding WLS criterion for the general case of contemporaneously correlated innovations (see Table 2.1) is equivalently expressed as (refer to Eq. (2.8)):

$$
\begin{aligned}
J\left(\boldsymbol{\theta}, Z^{N M}\right) & =\frac{1}{N M} \sum_{t=1}^{N} \boldsymbol{e}^{T}[t] \boldsymbol{\Sigma}^{-1} \boldsymbol{e}[t]=\frac{1}{N M}\left(\boldsymbol{y}-\boldsymbol{\Phi}^{T} \boldsymbol{\theta}\right)^{T} \breve{\boldsymbol{\Sigma}}^{-1}\left(\boldsymbol{y}-\boldsymbol{\Phi}^{T} \boldsymbol{\theta}\right) \\
& =\frac{1}{N M}\left\|\boldsymbol{y}-\boldsymbol{\Phi}^{T} \boldsymbol{\theta}\right\|_{\breve{\boldsymbol{\Sigma}}^{-1}}^{2}
\end{aligned}
$$

with:

$$
\breve{\boldsymbol{\Sigma}}=\left[\begin{array}{cccc}
\left(\boldsymbol{I}_{N} \otimes \boldsymbol{\Sigma}_{k_{1}, k_{1}}\right) & \left(\boldsymbol{I}_{N} \otimes \boldsymbol{\Sigma}_{k_{1}, k_{2}}\right) & \ldots & \left(\boldsymbol{I}_{N} \otimes \boldsymbol{\Sigma}_{k_{1}, k_{M}}\right) \\
\left(\boldsymbol{I}_{N} \otimes \boldsymbol{\Sigma}_{k_{2}, k_{1}}\right) & \left(\boldsymbol{I}_{N} \otimes \boldsymbol{\Sigma}_{k_{2}, k_{2}}\right) & \ldots & \left(\boldsymbol{I}_{N} \otimes \boldsymbol{\Sigma}_{k_{2}, k_{M}}\right) \\
\vdots & \vdots & \ddots & \vdots \\
\left(\boldsymbol{I}_{N} \otimes \boldsymbol{\Sigma}_{k_{M}, k_{1}}\right) & \left(\boldsymbol{I}_{N} \otimes \boldsymbol{\Sigma}_{k_{M}, k_{2}}\right) & \ldots & \left(\boldsymbol{I}_{N} \otimes \boldsymbol{\Sigma}_{k_{M}, k_{M}}\right)
\end{array}\right]
$$


and $\|\cdot\|_{\breve{\Sigma}^{-1}}$ designating the weighted norm of the indicated vector with respect to $\breve{\Sigma}^{-1}$.

The WLS criterion in Eq. (2.E.3a) may then be rewritten as follows:

$$
J\left(\boldsymbol{\theta}, Z^{N M}\right)=\frac{1}{N M}\left\|\overline{\boldsymbol{y}}-\overline{\boldsymbol{\Phi}}^{T} \boldsymbol{\theta}\right\|^{2}
$$

with $\overline{\boldsymbol{y}}=\boldsymbol{F}^{-1} \boldsymbol{y}, \overline{\boldsymbol{\Phi}}^{T}=\boldsymbol{F}^{-1} \boldsymbol{\Phi}^{T}$ and $\boldsymbol{F}$ being a lower triangular matrix obtained by the Cholesky factorization $^{40}$ of $\breve{\Sigma}[49$, p. 185]. tions:

The $Q R$ method aims at indirectly "solving" the following (overdetermined) system of linear equa-

$$
\overline{\boldsymbol{\Phi}}^{T} \boldsymbol{\theta}=\overline{\boldsymbol{y}}
$$

by left multiplication with a $(M N n y \times M N n y)$ orthonormal matrix ${ }^{41} \boldsymbol{Q}$, so that the following system of linear equations is obtained:

$$
\boldsymbol{Q} \overline{\boldsymbol{\Phi}}^{T} \boldsymbol{\theta}=\boldsymbol{Q} \overline{\boldsymbol{y}}
$$

The preceding transformation does not affect the WLS criterion in Eq. (2.E.4), since it holds that $\left\|\boldsymbol{Q}\left(\overline{\boldsymbol{y}}-\overline{\boldsymbol{\Phi}}^{T} \boldsymbol{\theta}\right)\right\|^{2}=\left\|\overline{\boldsymbol{y}}-\overline{\boldsymbol{\Phi}}^{T} \boldsymbol{\theta}\right\|^{2}$.

Thus, choosing $Q$ so that $Q \bar{\Phi}^{T}$ is upper triangular, yields the following factorization for the augmented matrix $\left[\overline{\boldsymbol{\Phi}}^{T} \overline{\boldsymbol{y}}\right]$ (see [49, pp. 207-212]):

$$
\boldsymbol{Q}\left[\begin{array}{ll}
\overline{\boldsymbol{\Phi}}^{T} & \overline{\boldsymbol{y}}
\end{array}\right]=\left[\begin{array}{cc}
\boldsymbol{R} & \boldsymbol{d} \\
\mathbf{0} & \boldsymbol{\epsilon}
\end{array}\right]
$$

where $\boldsymbol{d}$ is a $(n \theta \times 1)$ vector, $\boldsymbol{\epsilon}$ is a $((M N n y-n \theta) \times 1)$ vector, $\boldsymbol{R}$ is a $(n \theta \times n \theta)$ square upper triangular matrix, and $n \theta$ is the dimension of the FP-VARX projection coefficient vector $\boldsymbol{\theta}$. The preceding matrix transformation is also known as "QR factorization" and the corresponding WLS criterion becomes:

$$
J\left(\boldsymbol{\theta}, Z^{N M}\right)=\frac{1}{N M}\left\|\boldsymbol{Q}\left(\overline{\boldsymbol{y}}-\overline{\mathbf{\Phi}}^{T} \boldsymbol{\theta}\right)\right\|^{2}=\frac{1}{N M}\left(\|\boldsymbol{d}-\boldsymbol{R} \boldsymbol{\theta}\|^{2}+\|\boldsymbol{\epsilon}\|^{2}\right)
$$

Assuming that $\boldsymbol{R}$ is nonsingular (which is equivalent to $\overline{\boldsymbol{\Phi}}^{T}$ being of full rank, and also to $\overline{\boldsymbol{\Phi}} \overline{\boldsymbol{\Phi}}^{T}$ being non-singular), $J\left(\boldsymbol{\theta}, Z^{N M}\right)$ is minimized when $\boldsymbol{\theta}$ satisfies the following relationship:

$$
\boldsymbol{R} \widehat{\boldsymbol{\theta}}_{W L S}=\boldsymbol{d}
$$

The solution of the linear system of equations in Eq. (2.E.9) is trivial, as $\boldsymbol{R}$ is triangular. The main advantage of the $Q R$ method is its robustness to rounding errors as opposed to a "direct" solution of Eq. (2.E.5).

Remark 12. The covariance estimate of $\widehat{\boldsymbol{\theta}}_{W L S}$, is readily obtained by $\widehat{\boldsymbol{P}}\left(\widehat{\boldsymbol{\theta}}_{W L S}\right)=\boldsymbol{R}^{-1}\left(\boldsymbol{R}^{-1}\right)^{T}$, since:

$$
\boldsymbol{R}^{-1}\left(\boldsymbol{R}^{-1}\right)^{T}=\left(\overline{\mathbf{\Phi}} \overline{\boldsymbol{\Phi}}^{T}\right)^{-1}=\left(\boldsymbol{\Phi} \breve{\Sigma}^{-1} \boldsymbol{\Phi}^{T}\right)^{-1}
$$

Remark 13. When OLS is considered, $\boldsymbol{F}^{-1}=\boldsymbol{I}_{M n y}$ is set in Eq. (2.E.5)-(2.E.9). Note however that the covariance estimate of $\widehat{\boldsymbol{\theta}}_{O L S}$ will not equal $\boldsymbol{R}^{-1}\left(\boldsymbol{R}^{-1}\right)^{T}$ in this case.

\footnotetext{
${ }^{40}$ Consider the $(m \times m)$ real, positive-definite, matrix $\boldsymbol{A}$, then $\exists$ a unique real lower triangular $(m \times m)$ matrix $\boldsymbol{B}$ with positive diagonal elements, such that $\boldsymbol{A}=\boldsymbol{B} \boldsymbol{B}^{T}$ [43, p. 86].

${ }^{41}$ Consider the $(m \times m)$ orthonormal matrix $\boldsymbol{Q}$, then $\boldsymbol{Q} \boldsymbol{Q}^{T}=\boldsymbol{I}_{m}[48$, p. 179].
} 


\section{E.2 Adaptation of the $Q R$ method to the sequential processing of data}

The transformation of the augmented matrix $\left[\overline{\boldsymbol{\Phi}}^{T} \quad \overline{\boldsymbol{y}}\right]$ in Eq. (2.E.7) into upper triangular requires a significant amount of computer storage, due to its potential high dimensionality. To address this problem, an

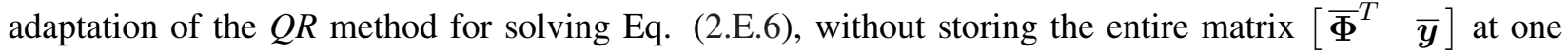
time [49, pp. 207-212], is presented.

This adaptation proves very useful in FP-VARX model parameter estimation, as it provides a means of conserving computational resources while preserving excellent numerical stability properties. For the sake of brevity, the analysis followed hereafter is confined to the general case of contemporaneously correlated innovations (see Table 2.1).

The augmented matrix $\left[\begin{array}{ll}\overline{\boldsymbol{\Phi}}^{T} & \overline{\boldsymbol{y}}\end{array}\right]$ may be rewritten in the following partitioned form:

$$
\left[\begin{array}{ll}
\overline{\boldsymbol{\Phi}}^{T} & \overline{\boldsymbol{y}}
\end{array}\right]=\boldsymbol{F}^{-1}\left[\begin{array}{ll}
\boldsymbol{\Phi}^{T} & \boldsymbol{y}
\end{array}\right]=\left[\begin{array}{c}
{\left[\begin{array}{cc}
\overline{\boldsymbol{\Phi}}_{k_{1}}^{T} & \overline{\boldsymbol{y}}_{k_{1}} \\
{\left[\begin{array}{cc}
\overline{\boldsymbol{\Phi}}_{k_{2}}^{T} & \overline{\boldsymbol{y}}_{k_{2}}
\end{array}\right]} \\
\vdots \\
\overline{\boldsymbol{\Phi}}_{k_{M}}^{T} & \overline{\boldsymbol{y}}_{k_{M}}
\end{array}\right]}
\end{array}\right]
$$

with $\boldsymbol{F}^{-1}$ being lower triangular ${ }^{41}$ :

$$
\boldsymbol{F}^{-1}=\left[\begin{array}{cccc}
\boldsymbol{F}_{k_{1}, k_{1}} & \mathbf{0} & \ldots & \mathbf{0} \\
\boldsymbol{F}_{k_{2}, k_{1}} & \boldsymbol{F}_{k_{2}, k_{2}} & \ldots & \mathbf{0} \\
\vdots & \vdots & \ddots & \vdots \\
\boldsymbol{F}_{k_{M}, k_{1}} & \boldsymbol{F}_{k_{M}, k_{2}} & \ldots & \boldsymbol{F}_{k_{M}, k_{M}}
\end{array}\right]
$$

and also:

$$
\left[\begin{array}{ll}
\overline{\boldsymbol{\Phi}}_{k}^{T} & \overline{\boldsymbol{y}}_{k}
\end{array}\right]=\sum_{j=k_{1}}^{k} \boldsymbol{F}_{k, j}\left[\begin{array}{ll}
\boldsymbol{\Phi}_{j}^{T} & \boldsymbol{y}_{j}
\end{array}\right], k, j=k_{1}, \ldots, k_{M}
$$

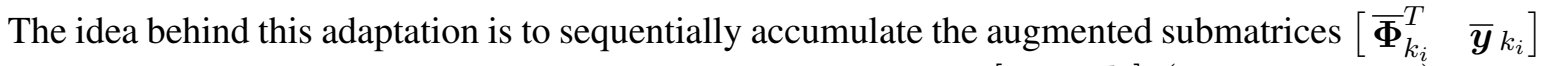
contained in Eq. (2.E.10a), in order to construct a sequence of matrices $\left[\begin{array}{ll}\boldsymbol{R}_{i} & \boldsymbol{d}_{i}\end{array}\right],(i=1,2, \ldots, M)$ so that the solution of the following LS problem:

$$
\boldsymbol{R}_{i} \boldsymbol{\theta}=\boldsymbol{d}_{i}
$$

equals the solution of the following system of equations:

$$
\left[\begin{array}{c}
\overline{\boldsymbol{\Phi}}_{k_{1}}^{T} \\
\overline{\boldsymbol{\Phi}}_{k_{2}}^{T} \\
\vdots \\
\mathbf{\boldsymbol { \Phi }}_{k_{i}}^{T}
\end{array}\right] \boldsymbol{\theta}=\left[\begin{array}{c}
\overline{\boldsymbol{y}}_{k_{1}} \\
\overline{\boldsymbol{y}}_{k_{2}} \\
\vdots \\
\overline{\boldsymbol{y}}_{k_{i}}
\end{array}\right]
$$

The proposed sequential accumulation algorithm is described as follows:

At the initialization stage, a null matrix is formed, designated as $\left[\begin{array}{ll}\boldsymbol{R}_{0} & \boldsymbol{d}_{0}\end{array}\right]$. During the $i$-th stage of the algorithm $(i=1,2, \ldots, M)$, the matrix $\left[\begin{array}{ll}\boldsymbol{R}_{i-1} & \boldsymbol{d}_{i-1}\end{array}\right]$ from the $(i-1)$-th stage and the new data matrix $\left[\begin{array}{cc}\overline{\boldsymbol{\Phi}}_{k_{i}}^{T} & \overline{\boldsymbol{y}}_{k_{i}}\end{array}\right]$ are merged to form the following augmented matrix:

$$
\left[\begin{array}{cc}
\widetilde{\boldsymbol{\Phi}}_{i}^{T} & \widetilde{\boldsymbol{y}}_{i}
\end{array}\right]=\left[\begin{array}{cc}
\boldsymbol{R}_{i-1} & \boldsymbol{d}_{i-1} \\
\overline{\mathbf{\Phi}}_{k_{i}}^{T} & \overline{\boldsymbol{y}}_{k_{i}}
\end{array}\right]
$$

\footnotetext{
$164]$.

${ }^{41}$ Consider the $(m \times m)$ upper (lower) triangular matrix $\boldsymbol{A}$, then $\boldsymbol{A}^{-1}$ is upper (lower) triangular if $\boldsymbol{A}$ is non-singular [43, p.
} 
which is subsequently reduced to a triangular form via $Q R$ factorization (see previous section), as seen below:

$$
\boldsymbol{Q}_{i}\left[\begin{array}{cc}
\widetilde{\boldsymbol{\Phi}}_{i}^{T} & \widetilde{\boldsymbol{y}}_{i}
\end{array}\right]=\left[\begin{array}{cc}
\boldsymbol{R}_{i} & \boldsymbol{d}_{i} \\
\mathbf{0} & \boldsymbol{\epsilon}_{i}
\end{array}\right]
$$

The procedure is repeated $M$ times, and at the $M$-th (final) stage of the algorithm, the resulting triangular matrix is:

$$
\boldsymbol{Q}_{M}\left[\widetilde{\boldsymbol{\Phi}}_{M}^{T} \quad \widetilde{\boldsymbol{y}}_{M}\right]=\left[\begin{array}{cc}
\boldsymbol{R}_{M} & \boldsymbol{d}_{M} \\
\mathbf{0} & \boldsymbol{\epsilon}_{M}
\end{array}\right] \triangleq\left[\begin{array}{cc}
\boldsymbol{R} & \boldsymbol{d} \\
\mathbf{0} & \boldsymbol{\epsilon}
\end{array}\right]
$$

whereas the corresponding FP-VARX WLS estimate is given by:

$$
\widehat{\boldsymbol{\theta}}_{W L S}=\boldsymbol{R}^{-1} \boldsymbol{d}
$$

The solution obtained from Eq. (2.E.15a) and Eq. (2.E.15b) is equivalent to solving the system of linear equations in Eq. (2.E.5) with the basic QR method presented in the previous section, as it can be verified [49, p. 209] that there exists an orthogonal matrix $\boldsymbol{Q}$ so that (compare to Eq. (2.E.7)):

$$
\boldsymbol{Q}\left[\begin{array}{ll}
\overline{\boldsymbol{\Phi}}^{T} & \overline{\boldsymbol{y}}
\end{array}\right]=\left[\begin{array}{cc}
\boldsymbol{R} & \boldsymbol{d} \\
\mathbf{0} & \boldsymbol{\epsilon}
\end{array}\right]
$$

\section{Appendix 2.F: Additional results on Monte Carlo studies}

\section{F.1 Case study I: FP-VARX $(2,0)_{[3,3]}$ model}

Additional FP-VARX $(2,0)_{[3,3]}$ identification results from the Monte Carlo study are presented in this section. The data tabulated in Tables 2.5-2.6 correspond to the interval parameter estimates of the AR, X projection coefficients and cross-covariance matrices as estimated by the LS-based and ML methods along with their actual values. The results confirm the excellent agreement observed among the actual values and their estimated counterparts. Furthermore, it is verified that all three estimators yield essentially unbiased estimates with the WLS and ML, expectedly, providing better accuracy for the coefficients of projection.

In Fig. 2.11-2.12, the magnitude and phase of the frequency response function between the $1^{\text {st }}$ output and $1^{\text {st }}$ input (denoted by $G_{11}$ ) versus frequency and the operating parameter $k$ for the actual system is compared to its mean estimated counterparts via the LS and ML estimators. The agreement between each estimate and the actual frequency response function is excellent, and confirms the effectiveness of the formulated estimators.

The projection of the function minimized in the ML method (equal to the negative value of the concentrated log-likelihood; see Eq. (2.11a)) on selected FP-VARX $(2,0)_{[3,3]}$ model parameters is shown in Fig. 2.13. The obtained surfaces in Fig. 2.13(a),(b) indicate that the minimization criterion is a smooth function of the model parameters with a single global minimum value. The minimum corresponds to the actual FP-VARX $(2,0)_{[3,3]}$ projection coefficient values (see contour plots in Fig. 2.13(c),(d)), thus ensuring the convergence of the iterative optimization algorithm used to obtain the ML estimates. 
Table 2.5: Monte Carlo estimation results for the AutoRegressive and eXogenous projection coefficients of the FP-VARX $(2,0)_{[3,3]}$ model (500 runs per method; sample mean estimate \pm sample standard deviation).

\begin{tabular}{|c|c|c|c|c|}
\hline Coefficient & Actual Value & OLS & WLS & ML \\
\hline$\alpha_{1,1}^{1,1}$ & .41250 & $0.41249 \pm 8.4 \times 10^{-4}$ & $0.41250 \pm 7.6 \times 10^{-4}$ & $0.41250 \pm 7.6 \times 10^{-4}$ \\
\hline$\alpha_{1,1}^{1,2}$ & 0.32500 & $0.32496 \pm 5.4 \times 10^{-4}$ & $0.32497 \pm 4.8 \times 10^{-4}$ & $0.32497 \pm 4.8 \times 10^{-4}$ \\
\hline$\alpha_{1,1}^{1,1}$ & -0.08750 & $-0.08750 \pm 4.9 \times 10^{-4}$ & $-0.08750 \pm 4.5 \times 10^{-4}$ & $-0.08750 \pm 4.5 \times 10^{-4}$ \\
\hline$\alpha_{1,2}^{1,1}$ & -0.12500 & $-0.12508 \pm 1.2 \times 10^{-3}$ & $-0.12506 \pm 1.1 \times 10^{-3}$ & $-0.12507 \pm 1.1 \times 10^{-3}$ \\
\hline$\alpha_{1,2}^{1,2}$ & 0.17500 & $0.17497 \pm 6.3 \times 10^{-4}$ & $0.17497 \pm 5.9 \times 10^{-4}$ & $0.17497 \pm 5.9 \times 10^{-4}$ \\
\hline$\alpha_{1,2}^{1,3}$ & 0.12500 & $0.12499 \pm 6.3 \times 10^{-4}$ & $0.12500 \pm 5.8 \times 10^{-4}$ & $0.12500 \pm 5.8 \times 10^{-4}$ \\
\hline$\alpha_{1,1}^{2,1}$ & -0.01250 & $-0.01257 \pm 8.9 \times 10^{-4}$ & $-0.01255 \pm 8.3 \times 10^{-4}$ & $-0.01254 \pm 8.3 \times 10^{-4}$ \\
\hline$\alpha_{1,1}^{2,2}$ & 0.12500 & $0.12496 \pm 6.7 \times 10^{-4}$ & $0.12497 \pm 5.9 \times 10^{-4}$ & $0.12497 \pm 5.9 \times 10^{-4}$ \\
\hline$\alpha_{1,1}^{2,3}$ & 0.13750 & $0.13751 \pm 5.4 \times 10^{-4}$ & $0.13751 \pm 4.9 \times 10^{-4}$ & $0.13751 \pm 4.9 \times 10^{-4}$ \\
\hline$\alpha_{1,2}^{2,1}$ & -0.27500 & $-0.27498 \pm 1.0 \times 10^{-3}$ & $-0.27497 \pm 9.2 \times 10^{-4}$ & $-0.27498 \pm 4.9 \times 10^{-4}$ \\
\hline$\alpha_{1,2}^{2,2}$ & -0.20000 & $-0.20000 \pm 6.2 \times 10^{-4}$ & $-0.20001 \pm 5.8 \times 10^{-4}$ & $-0.20001 \pm 5.7 \times 10^{-4}$ \\
\hline$\alpha_{1,2}^{2,3}$ & 0.02500 & $0.02505 \pm 5.3 \times 10^{-4}$ & $0.02504 \pm 4.9 \times 10^{-4}$ & $0.02504 \pm 4.9 \times 10^{-4}$ \\
\hline$\alpha_{2,1}^{1,1}$ & 0.38750 & $0.38747 \pm 6.9 \times 10^{-4}$ & $0.38747 \pm 6.1 \times 10^{-4}$ & $0.38747 \pm 6.1 \times 10^{-4}$ \\
\hline$\alpha_{2,1}^{1,2}$ & 0.32500 & $0.32494 \pm 6.3 \times 10^{-4}$ & $0.32496 \pm 4.8 \times 10^{-4}$ & $0.32490 \pm 4.8 \times 10^{-4}$ \\
\hline$\alpha_{2,1}^{1,3}$ & -0.01250 & $-0.01250 \pm 5.7 \times 10^{-4}$ & $-0.01250 \pm 4.6 \times 10^{-4}$ & $-0.01250 \pm 4.7 \times 10^{-4}$ \\
\hline$\alpha_{2,2}^{1,1}$ & 0.28750 & $0.28755 \pm 9.3 \times 10^{-4}$ & $0.28753 \pm 8.3 \times 10^{-4}$ & $0.28754 \pm 8.4 \times 10^{-4}$ \\
\hline$\alpha_{2,2}^{1,2}$ & -0.10000 & $-0.09997 \pm 6.4 \times 10^{-4}$ & $-0.09997 \pm 5.2 \times 10^{-4}$ & $-0.09996 \pm 5.2 \times 10^{-4}$ \\
\hline$\alpha_{2,2}^{1,3}$ & -0.36250 & $-0.36250 \pm 6.2 \times 10^{-4}$ & $-0.36249 \pm 5.0 \times 10^{-4}$ & $-0.36249 \pm 5.0 \times 10^{-4}$ \\
\hline$\alpha_{2,1}^{2,1}$ & 0.11875 & $0.11877 \pm 7.0 \times 10^{-4}$ & $0.11876 \pm 6.1 \times 10^{-4}$ & $0.11876 \pm 6.1 \times 10^{-4}$ \\
\hline$\alpha_{2,1}^{2,2}$ & 0.21250 & $0.21252 \pm 7.4 \times 10^{-4}$ & $0.21252 \pm 5.6 \times 10^{-4}$ & $0.21253 \pm 5.6 \times 10^{-4}$ \\
\hline$\alpha_{2,1}^{2,3}$ & 0.01875 & $0.01875 \pm 6.3 \times 10^{-4}$ & $0.01875 \pm 4.9 \times 10^{-4}$ & $0.01875 \pm 4.9 \times 10^{-4}$ \\
\hline$\alpha_{2,2}^{2,1}$ & 0.11250 & $0.11250 \pm 8.6 \times 10^{-4}$ & $0.11248 \pm 7.6 \times 10^{-4}$ & $0.11248 \pm 7.6 \times 10^{-4}$ \\
\hline$\alpha_{2,2}^{2,2}$ & 0.05000 & $0.04998 \pm 6.1 \times 10^{-4}$ & $0.04997 \pm 5.1 \times 10^{-4}$ & $0.04997 \pm 5.1 \times 10^{-4}$ \\
\hline$\alpha_{2,2}^{2,3}$ & -0.03750 & $-0.03751 \pm 5.8 \times 10^{-4}$ & $-0.03752 \pm 4.9 \times 10^{-4}$ & $-0.03752 \pm 4.9 \times 10^{-4}$ \\
\hline$b_{1,1}^{0,1}$ & 0.21250 & $0.21246 \pm 5.8 \times 10^{-4}$ & $0.21247 \pm 5.4 \times 10^{-4}$ & $0.21247 \pm 5.4 \times 10^{-4}$ \\
\hline$b_{1,1}^{0,2}$ & -0.27500 & $-0.27502 \pm 4.0 \times 10^{-4}$ & $-0.27502 \pm 3.9 \times 10^{-4}$ & $-0.27502 \pm 3.9 \times 10^{-4}$ \\
\hline$b_{1,1}^{1,1}$ & 0.01250 & $0.01250 \pm 3.7 \times 10^{-4}$ & $0.01249 \pm 3.5 \times 10^{-4}$ & $0.01249 \pm 3.5 \times 10^{-4}$ \\
\hline$b_{1,2}^{0,1}$ & -0.16250 & $-0.16251 \pm 5.6 \times 10^{-4}$ & $-0.16251 \pm 5.3 \times 10^{-4}$ & $-0.16251 \pm 5.3 \times 10^{-4}$ \\
\hline $\begin{array}{l}1,2 \\
b_{1,2}^{0,2}\end{array}$ & 0.20000 & $0.20001 \pm 3.9 \times 10^{-4}$ & $0.20000 \pm 3.8 \times 10^{-4}$ & $0.20000 \pm 3.8 \times 10^{-4}$ \\
\hline $\begin{array}{l}1,2 \\
b_{1,2}^{0,3}\end{array}$ & -0.01250 & $-0.01249 \pm 4.0 \times 10^{-4}$ & $-0.01249 \pm 3.8 \times 10^{-4}$ & $-0.01249 \pm 3.8 \times 10^{-4}$ \\
\hline$b_{1,3}^{0,1}$ & 0.68750 & $0.68750 \pm 5.6 \times 10^{-4}$ & $0.68750 \pm 5.4 \times 10^{-4}$ & $0.68750 \pm 5.4 \times 10^{-4}$ \\
\hline$b_{1,3}^{0,2}$ & -0.07500 & $-0.07499 \pm 4.2 \times 10^{-4}$ & $-0.07498 \pm 4.0 \times 10^{-4}$ & $-0.07498 \pm 4.0 \times 10^{-4}$ \\
\hline $\begin{array}{l}1,0 \\
b_{1,3}^{0,3}\end{array}$ & -0.11250 & $-0.11252 \pm 4.2 \times 10^{-4}$ & $-0.11251 \pm 3.5 \times 10^{-4}$ & $-0.11251 \pm 3.5 \times 10^{-4}$ \\
\hline$b_{2,1}^{0,1}$ & 0.36250 & $0.36253 \pm 5.1 \times 10^{-4}$ & $0.36251 \pm 4.6 \times 10^{-4}$ & $0.36251 \pm 4.6 \times 10^{-4}$ \\
\hline$b_{2,1}^{0,1}$ & 0.10000 & $0.10001 \pm 4.2 \times 10^{-4}$ & $0.10001 \pm 3.3 \times 10^{-4}$ & $0.10001 \pm 3.3 \times 10^{-4}$ \\
\hline$b_{2,1}^{0,3}$ & -0.13750 & $-0.13747 \pm 4.5 \times 10^{-4}$ & $-0.13747 \pm 3.7 \times 10^{-4}$ & $-0.13747 \pm 3.7 \times 10^{-4}$ \\
\hline$b_{2,2}^{0,1}$ & -0.10000 & $-0.10000 \pm 4.8 \times 10^{-4}$ & $-0.09999 \pm 4.3 \times 10^{-4}$ & $-0.09999 \pm 4.3 \times 10^{-4}$ \\
\hline$b_{2,2}^{0,2}$ & -0.17500 & $-0.17500 \pm 4.0 \times 10^{-4}$ & $-0.17499 \pm 3.2 \times 10^{-4}$ & $-0.17499 \pm 3.2 \times 10^{-4}$ \\
\hline $\begin{array}{l}2,2 \\
b_{2,2}^{0,3}\end{array}$ & 0.05000 & $0.05002 \pm 4.4 \times 10^{-4}$ & $0.05002 \pm 3.5 \times 10^{-4}$ & $0.05002 \pm 3.2 \times 10^{-4}$ \\
\hline$b_{2,3}^{0,1}$ & 0.31250 & $0.31247 \pm 5.0 \times 10^{-4}$ & $0.31247 \pm 4.3 \times 10^{-4}$ & $0.31247 \pm 4.3 \times 10^{-4}$ \\
\hline$b_{2,3}^{0,2}$ & -0.07500 & $-0.07504 \pm 4.2 \times 10^{-4}$ & $-0.07502 \pm 3.2 \times 10^{-4}$ & $-0.07502 \pm 3.2 \times 10^{-4}$ \\
\hline$b_{2,3}^{0,3}$ & -0.08750 & $-0.08748 \pm 4.4 \times 10^{-4}$ & $-0.08747 \pm 3.6 \times 10^{-4}$ & $-0.08747 \pm 3.6 \times 10^{-4}$ \\
\hline
\end{tabular}


Table 2.6: Monte Carlo estimation results for innovations variance of the FP-VARX $(2,0)_{[3,3]}$ model (500 runs per method; sample mean estimate \pm sample standard deviation).

\begin{tabular}{|c|c|c|c|c|}
\hline Coefficient & ctual Value & OLS & WLS & ML \\
\hline$\sigma_{1,1}\left(k_{1}, k_{1}\right)$ & 0.01601 & $01606 \pm 6.8 \times 10^{-4}$ & $01605 \pm 6.8 \times 10^{-4}$ & $0.01600 \pm 6.8 \times 10^{-4}$ \\
\hline$\sigma_{2,2}\left(k_{1}, k_{1}\right)$ & 0.01188 & $0.01190 \pm 5.9 \times 10^{-4}$ & $0.01191 \pm 5.9 \times 10^{-4}$ & $0.01187 \pm 5.9 \times 10^{-4}$ \\
\hline$\sigma_{1,1}\left(k_{2}, k_{2}\right)$ & 0.01335 & $0.01339 \pm 5.6 \times 10^{-4}$ & $0.01338 \pm 5.6 \times 10^{-4}$ & $0.01334 \pm 5.5 \times 10^{-4}$ \\
\hline$\sigma_{2,2}\left(k_{2}, k_{2}\right)$ & 0.00503 & $0.00505 \pm 2.3 \times 10^{-4}$ & $0.00504 \pm 2.3 \times 10^{-4}$ & $0.00503 \pm 2.3 \times 10^{-4}$ \\
\hline$\sigma_{1,1}\left(k_{3}, k_{3}\right)$ & 0.01193 & $.01197 \pm 5.2 \times 10^{-4}$ & $0.01196 \pm 5.1 \times 10^{-4}$ & $0.01192 \pm 5.1 \times 10^{-4}$ \\
\hline$\sigma_{2,2}\left(k_{3}, k_{3}\right)$ & 0.00266 & $0.00267 \pm 1.2 \times 10^{-4}$ & $0.00267 \pm 1.2 \times 10^{-4}$ & $0.00266 \pm 1.2 \times 10^{-4}$ \\
\hline$\sigma_{1,1}\left(k_{4}, k_{4}\right)$ & 0.01099 & $0.01103 \pm 4.8 \times 10^{-4}$ & $0.01103 \pm 4.8 \times 10^{-4}$ & $0.01099 \pm 4.8 \times 10^{-4}$ \\
\hline$\sigma_{2,2}\left(k_{4}, k_{4}\right)$ & 0.00193 & $0.00194 \pm 8.3 \times 10^{-5}$ & $0.00194 \pm 8.3 \times 10^{-5}$ & $0.00193 \pm 8.3 \times 10^{-5}$ \\
\hline$\sigma_{1,1}\left(k_{5}, k_{5}\right)$ & 0.01024 & $0.01027 \pm 4.5 \times 10^{-4}$ & $0.01027 \pm 4.5 \times 10^{-4}$ & $0.01023 \pm 4.5 \times 10^{-4}$ \\
\hline$\sigma_{2,2}\left(k_{5}, k_{5}\right)$ & 0.00211 & $0.00212 \pm 9.3 \times 10^{-5}$ & $0.00212 \pm 9.2 \times 10^{-5}$ & $0.00211 \pm 9.2 \times 10^{-5}$ \\
\hline$\sigma_{1,1}\left(k_{6}, k_{6}\right)$ & 0.00966 & $.00970 \pm 3.9 \times 10^{-4}$ & $0.00970 \pm 3.9 \times 10^{-4}$ & $0.00966 \pm 3.9 \times 10^{-4}$ \\
\hline$\sigma_{2,2}\left(k_{6}, k_{6}\right)$ & 0.00305 & $0.00307 \pm 1.4 \times 10^{-4}$ & $0.00306 \pm 1.4 \times 10^{-4}$ & $0.00305 \pm 1.4 \times 10^{-4}$ \\
\hline$\sigma_{1,1}\left(k_{7}, k_{7}\right)$ & 0.00920 & $0.00924 \pm 4.0 \times 10^{-4}$ & $0.00923 \pm 3.9 \times 10^{-4}$ & $0.00920 \pm 3 . \times 10^{-4}$ \\
\hline$\sigma_{2,2}\left(k_{7}, k_{7}\right)$ & 0.00466 & $0.00468 \pm 2.3 \times 10^{-4}$ & $0.00467 \pm 2.3 \times 10^{-4}$ & $0.00466 \pm 2.3 \times 10^{-4}$ \\
\hline$\sigma_{1,1}\left(k_{8}, k_{8}\right)$ & 00892 & $6 \pm 4.0 \times 10^{-4}$ & $00895 \pm 3.9 \times 10^{-4}$ & $2 \pm 3.9 \times 10^{-4}$ \\
\hline$\sigma_{2,2}\left(k_{8}, k_{8}\right)$ & 0.00674 & $0.00676 \pm 3.4 \times 10^{-4}$ & $0.00676 \pm 3.4 \times 10^{-4}$ & $0.00673 \pm 3.3 \times 10^{-4}$ \\
\hline$\sigma_{1,1}\left(k_{9}, k_{9}\right)$ & 00894 & $0.00898 \pm 3.9 \times 10^{-4}$ & $0.00897 \pm 3.9 \times 10^{-4}$ & $0.00894 \pm 3.8 \times 10^{-4}$ \\
\hline$\sigma_{2,2}\left(k_{9}, k_{9}\right)$ & 0.00903 & $0.00907 \pm 4.5 \times 10^{-4}$ & $0.00906 \pm 4.5 \times 10^{-4}$ & $0.00903 \pm 4.5 \times 10^{-4}$ \\
\hline$\sigma_{1,1}\left(k_{10}, k_{10}\right)$ & 0.00947 & $0.00950 \pm 4.7 \times 10^{-4}$ & $0.00950 \pm 4.7 \times 10^{-4}$ & $0.00946 \pm 4.7 \times 10^{-4}$ \\
\hline$\sigma_{2,2}\left(k_{10}, k_{10}\right)$ & 106 & $.01110 \pm 6.0 \times 10^{-4}$ & $0.01110 \pm 6.0 \times 10^{-4}$ & $0.01106 \pm 6.0 \times 10^{-4}$ \\
\hline$\sigma_{1,1}\left(k_{11}, k_{11}\right)$ & 1093 & $0.01097 \pm 6.1 \times 10^{-4}$ & $0.01096 \pm 6.0 \times 10^{-4}$ & $0.01092 \pm 6.0 \times 10^{-4}$ \\
\hline$\overline{\sigma_{2,2}\left(k_{11}, k_{11}\right)}$ & 0.01262 & $0.01266 \pm 6.8 \times 10^{-4}$ & $0.01266 \pm 6.8 \times 10^{-4}$ & $0.01262 \pm 6.8 \times 10^{-4}$ \\
\hline$\sigma_{1,1}\left(k_{12}, k_{12}\right)$ & 0.01370 & $0.01374 \pm 8.8 \times 10^{-4}$ & $0.01374 \pm 8.8 \times 10^{-4}$ & $0.01369 \pm 8.7 \times 10^{-4}$ \\
\hline$\sigma_{2,2}\left(k_{12}, k_{12}\right)$ & 0.01344 & $0.01349 \pm 7.9 \times 10^{-4}$ & $0.01349 \pm 7.9 \times 10^{-4}$ & $0.01344 \pm 7.9 \times 10^{-4}$ \\
\hline$\sigma_{1,1}\left(k_{13}, k_{13}\right)$ & 0.01741 & $0.01748 \pm 1.3 \times 10^{-3}$ & $0.01747 \pm 1.3 \times 10^{-3}$ & $0.01741 \pm 1.3 \times 10^{-3}$ \\
\hline$\sigma_{2,2}\left(k_{13}, k_{13}\right)$ & 0.01336 & $0.01341 \pm 8.2 \times 10^{-4}$ & $0.01340 \pm 8.2 \times 10^{-4}$ & $0.01335 \pm 8.1 \times 10^{-4}$ \\
\hline$\overline{\sigma_{1,1}\left(k_{14}, k_{14}\right)}$ & 0.02071 & $0.02079 \pm 1.6 \times 10^{-3}$ & $0.02078 \pm 1.6 \times 10^{-3}$ & $0.02071 \pm 1.6 \times 10^{-4}$ \\
\hline$\overline{\sigma_{2,2}\left(k_{14}, k_{14}\right)}$ & 0.01222 & $0.01227 \pm 7.6 \times 10^{-4}$ & $0.01227 \pm 7.6 \times 10^{-4}$ & $0.01222 \pm 7.6 \times 10^{-4}$ \\
\hline$\overline{\sigma_{1,1}}\left(k_{15}, k_{15}\right)$ & 0.02055 & $0.02061 \pm 1.7 \times 10^{-3}$ & $0.02062 \pm 1.7 \times 10^{-3}$ & $0.02054 \pm 1.7 \times 10^{-4}$ \\
\hline$\sigma_{2,2}\left(k_{15}, k_{15}\right)$ & 0.01003 & $0.01007 \pm 6.0 \times 10^{-4}$ & $0.01006 \pm 6.0 \times 10^{-4}$ & $0.01003 \pm 6.0 \times 10^{-4}$ \\
\hline$\sigma_{1,1}\left(k_{16}, k_{16}\right)$ & 0.01682 & $0.01687 \pm 1.2 \times 10^{-3}$ & $0.01687 \pm 1.2 \times 10^{-3}$ & $0.01681 \pm 1.2 \times 10^{-3}$ \\
\hline$\sigma_{2,2}\left(k_{16}, k_{16}\right)$ & 0.00766 & $0.00769 \pm 3.8 \times 10^{-4}$ & $0.00769 \pm 3.8 \times 10^{-4}$ & $0.00766 \pm 3.8 \times 10^{-4}$ \\
\hline$\sigma_{1,1}\left(k_{17}, k_{17}\right)$ & 0.01194 & $0.01198 \pm 8.0 \times 10^{-4}$ & $0.01198 \pm 8.0 \times 10^{-4}$ & $0.01194 \pm 8.0 \times 10^{-4}$ \\
\hline$\sigma_{2,2}\left(k_{17}, k_{17}\right)$ & 0.00602 & $0.00605 \pm 2.7 \times 10^{-4}$ & $0.00604 \pm 2.7 \times 10^{-4}$ & $0.00602 \pm 2.7 \times 10^{-4}$ \\
\hline$\sigma_{1,1}\left(k_{18}, k_{18}\right)$ & 0.00783 & $0.00786 \pm 4.3 \times 10^{-4}$ & $0.00785 \pm 4.3 \times 10^{-4}$ & $0.00783 \pm 4.3 \times 10^{-4}$ \\
\hline$\sigma_{2,2}\left(k_{18}, k_{18}\right)$ & 0.00539 & $0.00541 \pm 2.5 \times 10^{-4}$ & $0.00540 \pm 2.5 \times 10^{-4}$ & $0.00538 \pm 2.5 \times 10^{-4}$ \\
\hline$\sigma_{1,1}\left(k_{19}, k_{19}\right)$ & 0.00501 & $0.00503 \pm 2.6 \times 10^{-4}$ & $0.00503 \pm 2.6 \times 10^{-4}$ & $0.00501 \pm 2.6 \times 10^{-4}$ \\
\hline$\sigma_{2,2}\left(k_{19}, k_{19}\right)$ & 0.00598 & $0.00601 \pm 3.0 \times 10^{-4}$ & $0.00600 \pm 3.0 \times 10^{-4}$ & $0.00598 \pm 3.0 \times 10^{-4}$ \\
\hline$\sigma_{1,1}\left(k_{20}, k_{20}\right)$ & 0.00334 & $0.00335 \pm 1.7 \times 10^{-4}$ & $0.00335 \pm 1.7 \times 10^{-4}$ & $0.00334 \pm 1.7 \times 10^{-4}$ \\
\hline$\sigma_{2,2}\left(k_{20}, k_{20}\right)$ & 0.00876 & $0.00880 \pm 4.7 \times 10^{-4}$ & $0.00878 \pm 4.7 \times 10^{-4}$ & $0.00875 \pm 4.7 \times 10^{-4}$ \\
\hline$\sigma_{1,1}\left(k_{21}, k_{21}\right)$ & 0.00289 & $0.00290 \pm 1.5 \times 10^{-4}$ & $0.00289 \pm 1.5 \times 10^{-4}$ & $0.00288 \pm 1.4 \times 10^{-4}$ \\
\hline$\sigma_{2,2}\left(k_{21}, k_{21}\right)$ & 0.03598 & $0.03606 \pm 2.7 \times 10^{-3}$ & $0.03609 \pm 2.7 \times 10^{-3}$ & $0.03600 \pm 2.7 \times 10^{-3}$ \\
\hline
\end{tabular}



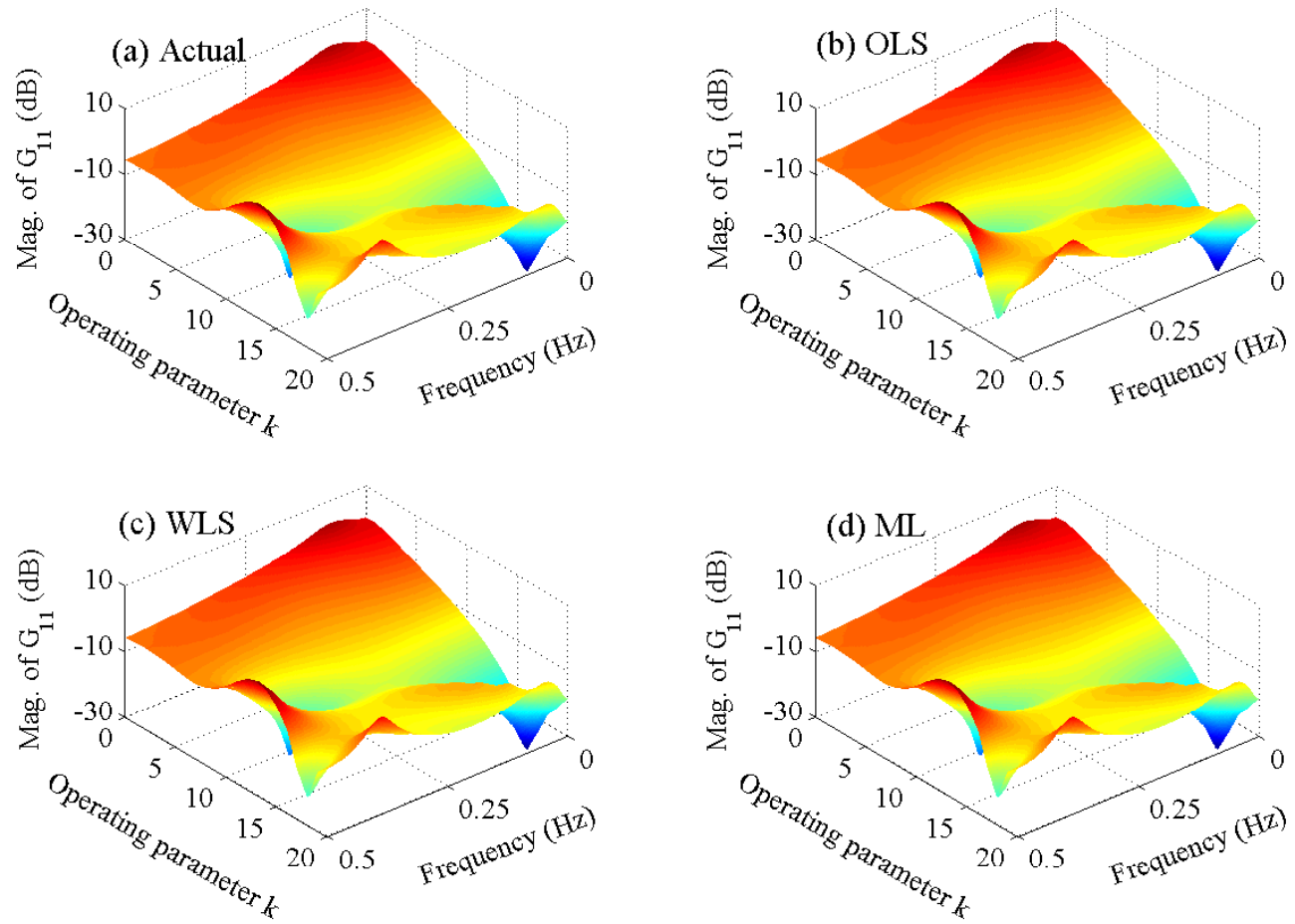

Figure 2.11: FP-VARX $(2,0)_{[3,3]}$ model based frequency response magnitude for $G_{11}$ with respect to frequency and operating parameter $k$ : (a) actual system, (b) OLS mean estimate, (c) WLS mean estimate and, (d) ML mean estimate.
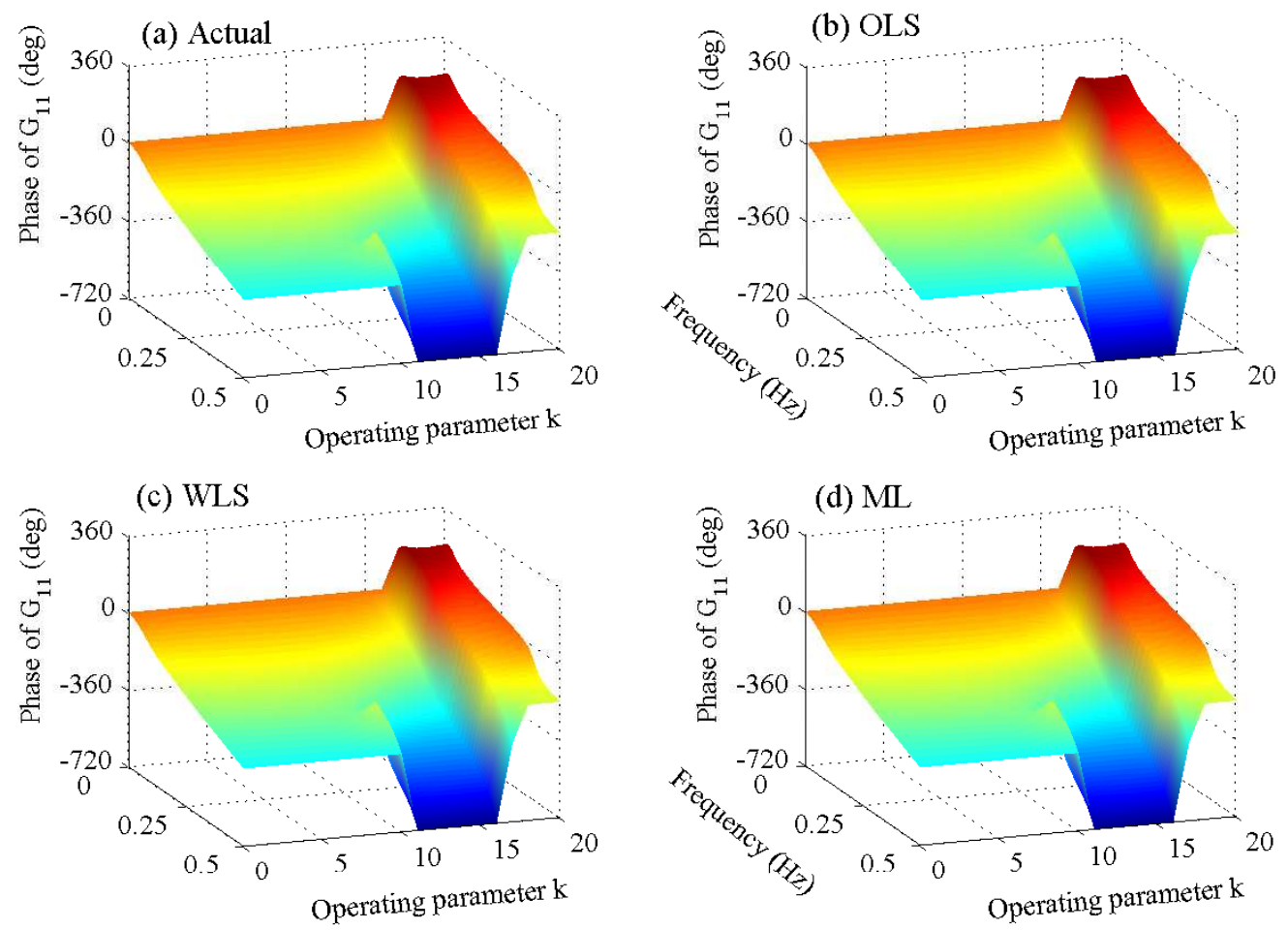

Figure 2.12: $\operatorname{FP}-\operatorname{VARX}(2,0)_{[3,3]}$ model based frequency response phase for $G_{11}$ with respect to frequency and operating parameter $k$ : (a) actual system, (b) OLS mean estimate, (c) WLS mean estimate and, (d) ML mean estimate. 

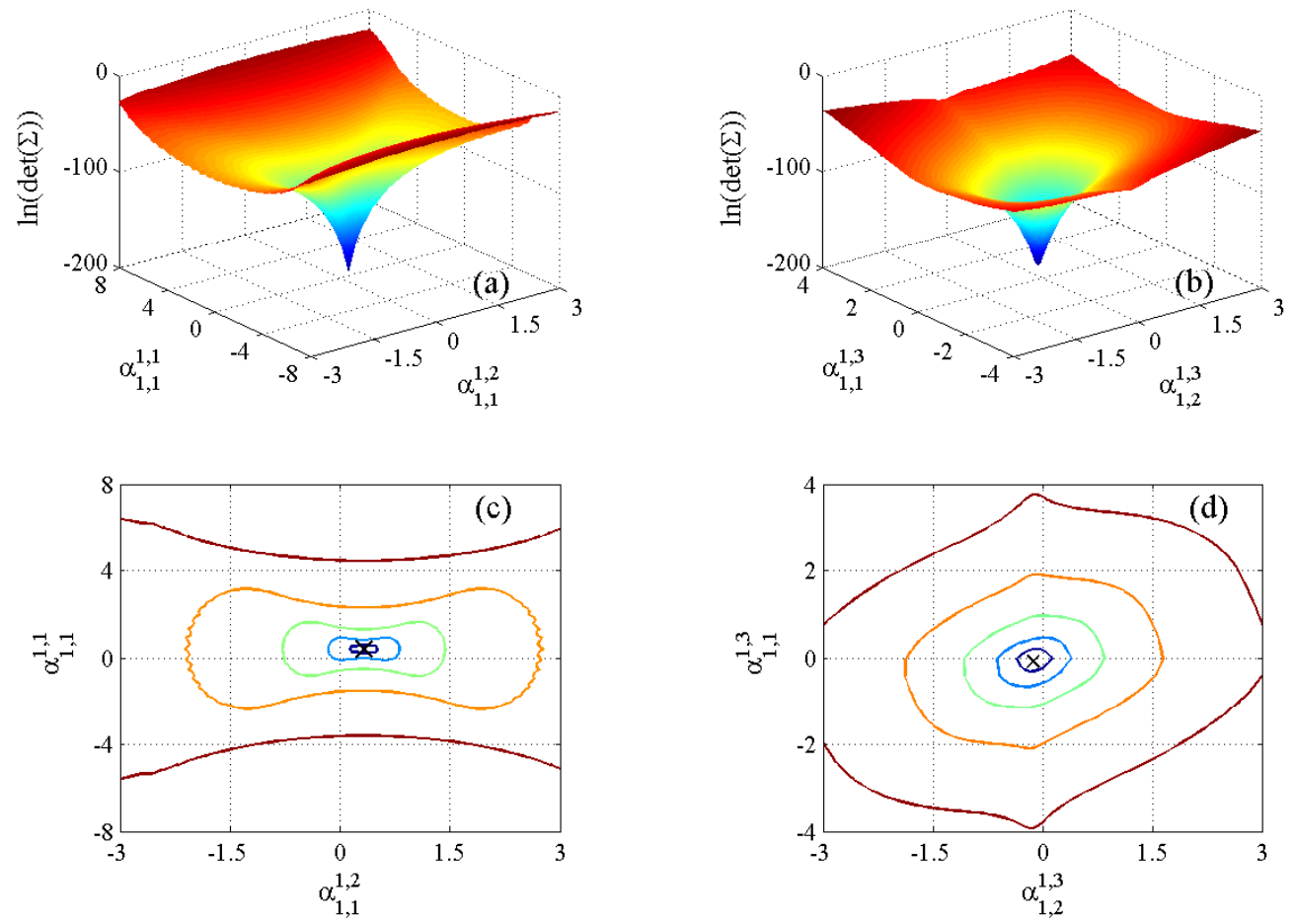

Figure 2.13: Projection of the function minimized in the ML method on selected FP-VARX $(2,0)_{[3,3]}$ model parameters: (a),(c) surface and contour plots by projecting on $\alpha_{1,1}^{1,1}$ and $\alpha_{1,1}^{1,2}$; (b),(d) surface and contour plots by projecting on $\alpha_{1,1}^{1,3}$ and $\alpha_{1,2}^{1,3}$ (the “ $\times$ ” marks designate the actual FP-VARX $(2,0)_{[3,3]}$ model parameters).

\section{F.2 Case study II: FP-VARX $(3,1)_{[4,4]}$ model}

Additional FP-VARX $(3,1)_{[4,4]}$ identification results from the Monte Carlo study are presented in this section. The data tabulated in Tables 2.7-2.9 correspond to the interval parameter estimates of the AR, X projection coefficients and cross-covariance matrices as estimated by the LS and ML methods along with their actual values. The results confirm the excellent agreement observed among the actual values and their estimated counterparts. Furthermore, it is verified that all three estimators yield essentially unbiased estimates with the WLS and ML, expectedly, providing better accuracy for the coefficients of projection.

In Fig. 2.14-2.15, the magnitude and phase of the frequency response function between the $2^{\text {nd }}$ output and $2^{\text {nd }}$ input (denoted by $G_{22}$ ) versus frequency and the operating parameter $k$ for the actual system is compared to its mean estimated counterparts via the LS and ML estimators. The agreement between each estimate and the actual frequency response function is excellent, and confirms the effectiveness of the formulated estimators.

The projection of the function minimized in the ML method (equal to the negative value of the concentrated log-likelihood; see Eq. (2.11a)) on selected FP-VARX $(3,1)_{[4,4]}$ model parameters is shown in Fig. 2.16. The obtained surfaces in Fig. 2.16(a),(b) indicate that the minimization criterion is a smooth function of the model parameters with a single global minimum value. The minimum corresponds to the actual FP-VARX $(2,0)_{[3,3]}$ projection coefficient values (see contour plots in Fig. 2.16(c),(d)), thus ensuring the convergence of the iterative optimization algorithm used to obtain the ML estimates. 
Table 2.7: Monte Carlo estimation results for the AutoRegressive projection coefficients of the FP$\operatorname{VARX}(3,1)_{[4,4]}$ model (500 runs per method; sample mean estimate \pm sample standard deviation).

\begin{tabular}{|c|c|c|c|c|}
\hline Coefficient & Actual Value & OLS & WLS & ML \\
\hline$\alpha_{1,1}^{1,1}$ & 0.46406 & $0.46395 \pm 4.4 \times 10^{-3}$ & $0.46414 \pm 2.8 \times 10^{-3}$ & $0.46416 \pm 2.6 \times 10^{-3}$ \\
\hline$\alpha_{1,1}^{1,2}$ & 0.01458 & $0.01459 \pm 2.7 \times 10^{-3}$ & $0.01462 \pm 1.9 \times 10^{-3}$ & $0.01462 \pm 2.0 \times 10^{-3}$ \\
\hline$\alpha_{1,1}^{1,3}$ & -0.01302 & $-0.01301 \pm 1.8 \times 10^{-3}$ & $-0.01304 \pm 1.2 \times 10^{-3}$ & $-0.01304 \pm 1.2 \times 10^{-3}$ \\
\hline$\alpha_{1,1}^{1,4}$ & -0.02604 & $-0.02603 \pm 1.8 \times 10^{-3}$ & $-0.02605 \pm 1.1 \times 10^{-3}$ & $-0.02604 \pm 1.2 \times 10^{-3}$ \\
\hline$\alpha_{1,2}^{1,1}$ & -0.34219 & $-0.34226 \pm 5.1 \times 10^{-3}$ & $-0.34229 \pm 2.7 \times 10^{-3}$ & $-0.34223 \pm 2.5 \times 10^{-3}$ \\
\hline$\alpha_{1,2}^{1,2}$ & 0.05052 & $0.05062 \pm 2.1 \times 10^{-3}$ & $0.05056 \pm 1.5 \times 10^{-3}$ & $0.05055 \pm 1.4 \times 10^{-3}$ \\
\hline$\alpha_{1,2}^{1,3}$ & 0.03906 & $0.03909 \pm 1.8 \times 10^{-3}$ & $0.03912 \pm 1.0 \times 10^{-3}$ & $0.03910 \pm 9.8 \times 10^{-4}$ \\
\hline$\alpha_{1,2}^{1,4}$ & -0.00651 & $-0.00670 \pm 1.4 \times 10^{-3}$ & $-0.00664 \pm 8.8 \times 10^{-4}$ & $-0.00664 \pm 8.6 \times 10^{-4}$ \\
\hline$\alpha_{1,1}^{2,1}$ & 0.65469 & $0.65469 \pm 3.0 \times 10^{-3}$ & $0.65472 \pm 1.7 \times 10^{-3}$ & $0.65471 \pm 1.5 \times 10^{-3}$ \\
\hline$\alpha_{1,1}^{2,2}$ & -0.09167 & $-0.09164 \pm 2.2 \times 10^{-3}$ & $-0.09166 \pm 1.3 \times 10^{-3}$ & $-0.09165 \pm 1.2 \times 10^{-3}$ \\
\hline$\alpha_{1,1}^{2,3}$ & -0.14323 & $-0.14324 \pm 1.1 \times 10^{-3}$ & $-0.14323 \pm 6.7 \times 10^{-4}$ & $-0.14323 \pm 6.4 \times 10^{-4}$ \\
\hline$\alpha_{1,1}^{2,4}$ & 0.05208 & $0.05209 \pm 1.4 \times 10^{-3}$ & $0.05211 \pm 7.8 \times 10^{-4}$ & $0.05211 \pm 7.4 \times 10^{-4}$ \\
\hline$\alpha_{1,2}^{2,1}$ & -0.05684 & $-0.05691 \pm 6.4 \times 10^{-3}$ & $-0.05700 \pm 3.3 \times 10^{-3}$ & $-0.05696 \pm 2.7 \times 10^{-3}$ \\
\hline$\alpha_{1,2}^{2,2}$ & -0.10840 & $-0.10825 \pm 2.5 \times 10^{-3}$ & $-0.10830 \pm 1.7 \times 10^{-3}$ & $-0.10830 \pm 1.6 \times 10^{-3}$ \\
\hline$\alpha_{1,2}^{2,3}$ & 0.04395 & $0.04403 \pm 2.4 \times 10^{-3}$ & $0.04402 \pm 1.2 \times 10^{-3}$ & $0.04401 \pm 1.1 \times 10^{-3}$ \\
\hline$\alpha_{1,2}^{1,4}$ & 0.08545 & $0.08532 \pm 1.5 \times 10^{-3}$ & $0.08535 \pm 9.3 \times 10^{-4}$ & $0.08535 \pm 9.0 \times 10^{-4}$ \\
\hline$\alpha_{1,1}^{3,1}$ & 0.12344 & $0.12333 \pm 3.2 \times 10^{-3}$ & $0.12346 \pm 2.4 \times 10^{-3}$ & $0.12349 \pm 2.4 \times 10^{-3}$ \\
\hline $\begin{array}{l}1,1 \\
\alpha_{1,1}^{3,2}\end{array}$ & 0.07708 & $0.07718 \pm 2.5 \times 10^{-3}$ & $0.07717 \pm 1.6 \times 10^{-3}$ & $0.07716 \pm 1.6 \times 10^{-3}$ \\
\hline$\alpha_{1,1}^{3,1}$ & -0.09115 & $-0.09112 \pm 1.5 \times 10^{-3}$ & $-0.09116 \pm 9.7 \times 10^{-4}$ & $-0.09116 \pm 9.6 \times 10^{-3}$ \\
\hline $\begin{array}{l}1,1 \\
\alpha_{1,1}^{3,4}\end{array}$ & -0.02604 & $-0.02611 \pm 1.5 \times 10^{-3}$ & $-0.02608 \pm 9.4 \times 10^{-4}$ & $-0.02608 \pm 9.2 \times 10^{-4}$ \\
\hline $\begin{array}{l}1,1 \\
\alpha_{1,2}^{3,1}\end{array}$ & 0.21328 & $0.21321 \pm 5.2 \times 10^{-3}$ & $0.21317 \pm 2.8 \times 10^{-3}$ & $0.21321 \pm 2.5 \times 10^{-3}$ \\
\hline$\alpha_{1,2}^{3,2}$ & -0.03255 & $-0.03237 \pm 2.0 \times 10^{-3}$ & $-0.03244 \pm 1.5 \times 10^{-3}$ & $-0.03245 \pm 1.4 \times 10^{-3}$ \\
\hline $\begin{array}{l}1,2 \\
\alpha_{1,2}^{3,3}\end{array}$ & 0.04557 & $0.04560 \pm 1.8 \times 10^{-3}$ & $0.04560 \pm 1.0 \times 10^{-3}$ & $0.04559 \pm 9.4 \times 10^{-4}$ \\
\hline $\begin{array}{l}1,2 \\
\alpha_{1,2}^{3,4}\end{array}$ & 0.01628 & $0.01608 \pm 1.4 \times 10^{-3}$ & $0.01616 \pm 9.0 \times 10^{-4}$ & $0.01617 \pm 8.9 \times 10^{-4}$ \\
\hline$\alpha_{2,1}^{1,2}$ & -0.50781 & $-0.50795 \pm 4.8 \times 10^{-3}$ & $-0.50784 \pm 3.1 \times 10^{-3}$ & $-0.50785 \pm 2.8 \times 10^{-3}$ \\
\hline$\alpha_{2,1}^{1,2}$ & 0.11302 & $0.11308 \pm 3.3 \times 10^{-3}$ & $0.11305 \pm 2.2 \times 10^{-3}$ & $0.11304 \pm 2.3 \times 10^{-3}$ \\
\hline$\alpha_{2,1}^{1,1}$ & 0.16927 & $0.16930 \pm 2.1 \times 10^{-3}$ & $0.16926 \pm 1.4 \times 10^{-3}$ & $0.16925 \pm 1.3 \times 10^{-3}$ \\
\hline$\alpha_{2,1}^{1,4}$ & -0.00651 & $-0.00656 \pm 2.1 \times 10^{-3}$ & $-0.006536 \pm 1.3 \times 10^{-3}$ & $-0.006544 \pm 1.4 \times 10^{-3}$ \\
\hline$\alpha_{22}^{11}$ & 0.73984 & $0.73991 \pm 5.6 \times 10^{-3}$ & $0.73997 \pm 3.1 \times 10^{-3}$ & $0.74001 \pm 2.6 \times 10^{-3}$ \\
\hline$\alpha_{2,2}^{1,2}$ & -0.07109 & $-0.07104 \pm 2.6 \times 10^{-3}$ & $-0.07107 \pm 1.6 \times 10^{-3}$ & $-0.07107 \pm 1.6 \times 10^{-3}$ \\
\hline$\alpha_{2,2}^{1,3}$ & -0.07162 & $-0.07154 \pm 1.9 \times 10^{-3}$ & $-0.07158 \pm 1.2 \times 10^{-3}$ & $-0.07159 \pm 1.1 \times 10^{-3}$ \\
\hline$\alpha_{2,2}^{1,4}$ & 0.02930 & $0.02930 \pm 1.7 \times 10^{-3}$ & $0.02930 \pm 1.1 \times 10^{-3}$ & $0.02931 \pm 1.1 \times 10^{-3}$ \\
\hline$\alpha_{2,1}^{2,1}$ & 153 & $0.24435 \pm 3.1 \times 10^{-3}$ & $0.24439 \pm 1.8 \times 10^{-3}$ & $0.24437 \pm 1.5 \times 10^{-3}$ \\
\hline$\alpha_{2,1}^{2,2}$ & 0.09505 & $0.09511 \pm 2.7 \times 10^{-3}$ & $0.09511 \pm 1.5 \times 10^{-3}$ & $0.09512 \pm 1.4 \times 10^{-3}$ \\
\hline$\alpha_{2,1}^{2,3}$ & -0.00651 & $-0.006527 \pm 1.2 \times 10^{-3}$ & $-0.00652 \pm 8.0 \times 10^{-4}$ & $-0.00652 \pm 7.5 \times 10^{-4}$ \\
\hline$\alpha_{2,1}^{2,1}$ & -0.01628 & $-0.01632 \pm 1.6 \times 10^{-3}$ & $-0.01632 \pm 9.3 \times 10^{-4}$ & $-0.01633 \pm 8.6 \times 10^{-4}$ \\
\hline$\alpha_{2,2}^{2,1}$ & 0.83437 & $0.83451 \pm 6.9 \times 10^{-3}$ & $0.83455 \pm 3.8 \times 10^{-3}$ & $0.83459 \pm 2.9 \times 10^{-3}$ \\
\hline$\alpha_{2,2}^{2,2}$ & -0.04115 & $-0.04101 \pm 3.0 \times 10^{-3}$ & $-0.04103 \pm 1.9 \times 10^{-3}$ & $-0.04103 \pm 1.9 \times 10^{-3}$ \\
\hline$\alpha_{2,2}^{2,3}$ & -0.07813 & $-0.07813 \pm 2.7 \times 10^{-3}$ & $-0.07815 \pm 1.4 \times 10^{-3}$ & $-0.07816 \pm 1.1 \times 10^{-3}$ \\
\hline$\alpha_{2,2}^{2,4}$ & 0.04557 & $0.04555 \pm 1.8 \times 10^{-3}$ & $0.04556 \pm 1.1 \times 10^{-3}$ & $0.04556 \pm 1.1 \times 10^{-3}$ \\
\hline$\alpha_{2,1}^{3,1}$ & -0.26016 & $-0.26026 \pm 3.6 \times 10^{-3}$ & $-0.26016 \pm 2.5 \times 10^{-3}$ & $-0.26014 \pm 2.5 \times 10^{-3}$ \\
\hline$\alpha_{2,1}^{3,2}$ & -0.16276 & $-0.16277 \pm 3.1 \times 10^{-3}$ & $-0.16273 \pm 2.0 \times 10^{-3}$ & $-0.16273 \pm 1.9 \times 10^{-3}$ \\
\hline$\alpha_{2,1}^{3,3}$ & 0.13672 & $0.13685 \pm 1.7 \times 10^{-3}$ & $0.13673 \pm 1.1 \times 10^{-3}$ & $0.13671 \pm 1.1 \times 10^{-3}$ \\
\hline$\alpha_{2,1}^{3,4}$ & 0.08138 & $0.08136 \pm 1.9 \times 10^{-3}$ & $0.08139 \pm 1.2 \times 10^{-3}$ & $0.081385 \pm 1.2 \times 10^{-3}$ \\
\hline$\alpha_{2,2}^{3,1}$ & -0.00391 & $-0.00375 \pm 5.6 \times 10^{-3}$ & $-0.00371 \pm 3.1 \times 10^{-3}$ & $-0.00367 \pm 2.5 \times 10^{-3}$ \\
\hline$\alpha_{2,2}^{3,2}$ & 0.04453 & $0.04463 \pm 2.6 \times 10^{-3}$ & $0.04461 \pm 1.6 \times 10^{-3}$ & $0.04460 \pm 1.7 \times 10^{-3}$ \\
\hline$\alpha_{2,2}^{3,3}$ & 0.08464 & $0.08463 \pm 1.9 \times 10^{-3}$ & $0.08461 \pm 1.2 \times 10^{-3}$ & $0.08460 \pm 1.0 \times 10^{-3}$ \\
\hline$\alpha_{2,2}^{3,4}$ & -0.00977 & $-0.00977 \pm 1.8 \times 10^{-3}$ & $-0.00977 \pm 1.1 \times 10^{-3}$ & $-0.00977 \pm 1.1 \times 10^{-3}$ \\
\hline
\end{tabular}


Table 2.8: Monte Carlo estimation results for the eXogenous projection coefficients of the FP$\operatorname{VARX}(3,1)_{[4,4]}$ model (500 runs per method; mean estimate \pm standard deviation).

\begin{tabular}{|c|c|c|c|c|}
\hline Coefficient & Actual Value & OLS & WLS & ML \\
\hline$b_{1,1}^{0,1}$ & 0.82813 & $0.82784 \pm 3.1 \times 10^{-3}$ & $0.82801 \pm 2.3 \times 10^{-3}$ & $0.82801 \pm 2.3 \times 10^{-3}$ \\
\hline$b_{1,1}^{1,1}$ & -0.04116 & $-0.04090 \pm 2.9 \times 10^{-3}$ & $-0.04105 \pm 1.8 \times 10^{-3}$ & $-0.04106 \pm 1.8 \times 10^{-3}$ \\
\hline$b_{1,1}^{0,3}$ & -0.02604 & $-0.02597 \pm 1.3 \times 10^{-3}$ & $-0.02602 \pm 9.1 \times 10^{-4}$ & $-0.02602 \pm 9.0 \times 10^{-4}$ \\
\hline$b_{1,1}^{0,4}$ & 0.04557 & $0.04540 \pm 1.8 \times 10^{-3}$ & $0.04551 \pm 1.1 \times 10^{-3}$ & $0.04551 \pm 1.0 \times 10^{-3}$ \\
\hline$b_{1,2}^{0,1}$ & -0.42422 & $-0.42427 \pm 3.1 \times 10^{-3}$ & $-0.42429 \pm 2.3 \times 10^{-3}$ & $-0.42429 \pm 2.2 \times 10^{-3}$ \\
\hline$b_{1,2}^{0,2}$ & 0.39140 & $0.39150 \pm 2.8 \times 10^{-3}$ & $0.39155 \pm 1.8 \times 10^{-3}$ & $0.39155 \pm 1.7 \times 10^{-3}$ \\
\hline$b_{1,2}^{0,3}$ & 0.14974 & $0.14973 \pm 1.3 \times 10^{-3}$ & $0.14973 \pm 8.6 \times 10^{-4}$ & $0.14973 \pm 8.6 \times 10^{-4}$ \\
\hline$b_{1,2}^{0,4}$ & -0.12695 & $-0.12699 \pm 1.8 \times 10^{-3}$ & $-0.12701 \pm 1.1 \times 10^{-3}$ & $-0.12700 \pm 1.1 \times 10^{-3}$ \\
\hline$b_{1,1}^{1,1}$ & 0.53906 & $0.53897 \pm 5.7 \times 10^{-3}$ & $0.53925 \pm 3.5 \times 10^{-3}$ & $0.53924 \pm 3.1 \times 10^{-3}$ \\
\hline$b_{1,1}^{1,2}$ & -0.19010 & $-0.19006 \pm 3.8 \times 10^{-3}$ & $-0.19009 \pm 2.4 \times 10^{-3}$ & $-0.19008 \pm 2.4 \times 10^{-3}$ \\
\hline$b_{1,1}^{1,3}$ & -0.01302 & $-0.01299 \pm 2.1 \times 10^{-3}$ & $-0.01309 \pm 1.4 \times 10^{-3}$ & $-0.01308 \pm 1.3 \times 10^{-3}$ \\
\hline$b_{11}^{1,4}$ & 0.03255 & $0.03258 \pm 2.4 \times 10^{-3}$ & $0.03262 \pm 1.4 \times 10^{-3}$ & $0.03262 \pm 1.4 \times 10^{-3}$ \\
\hline$b_{1,2}^{1,1}$ & 0.26016 & $0.25989 \pm 4.8 \times 10^{-3}$ & $0.25986 \pm 2.9 \times 10^{-3}$ & $0.25990 \pm 2.7 \times 10^{-3}$ \\
\hline$b_{1,2}^{1,2}$ & -0.15078 & $-0.15073 \pm 5.5 \times 10^{-3}$ & $-0.15062 \pm 2.5 \times 10^{-3}$ & $-0.15065 \pm 2.2 \times 10^{-3}$ \\
\hline$b_{1,2}^{1,3}$ & -0.13672 & $-0.13668 \pm 1.5 \times 10^{-3}$ & $-0.13664 \pm 1.0 \times 10^{-3}$ & $-0.13664 \pm 1.0 \times 10^{-3}$ \\
\hline$b_{1,2}^{1,4}$ & 0.08789 & $0.08780 \pm 3.1 \times 10^{-3}$ & $0.08776 \pm 1.5 \times 10^{-3}$ & $0.08777 \pm 1.3 \times 10^{-3}$ \\
\hline$b_{2,1}^{0,1}$ & -0.36406 & $-0.36396 \pm 3.6 \times 10^{-3}$ & $-0.36399 \pm 2.4 \times 10^{-3}$ & $-0.36399 \pm 2.4 \times 10^{-3}$ \\
\hline$b_{2,1}^{0,2}$ & 0.18073 & $0.18071 \pm 3.4 \times 10^{-3}$ & $0.18077 \pm 2.1 \times 10^{-3}$ & $0.18077 \pm 2.1 \times 10^{-3}$ \\
\hline$b_{2,1}^{0,3}$ & 0.01302 & $0.01309 \pm 1.4 \times 10^{-3}$ & $0.01307 \pm 9.8 \times 10^{-4}$ & $0.01307 \pm 9.9 \times 10^{-4}$ \\
\hline$b_{2,1}^{0,4}$ & -0.07162 & $-0.07162 \pm 2.0 \times 10^{-3}$ & $-0.07165 \pm 1.2 \times 10^{-3}$ & $-0.07165 \pm 1.2 \times 10^{-3}$ \\
\hline$b_{2,2}^{0,1}$ & 0.19063 & $0.19097 \pm 3.6 \times 10^{-3}$ & $0.19071 \pm 2.4 \times 10^{-3}$ & $0.19070 \pm 2.4 \times 10^{-3}$ \\
\hline$b_{2,2}^{0,2}$ & -0.77187 & $-0.77213 \pm 3.5 \times 10^{-3}$ & $-0.77188 \pm 2.0 \times 10^{-3}$ & $-0.77187 \pm 2.0 \times 10^{-3}$ \\
\hline$b_{2,2}^{0,3}$ & 0.07813 & $0.07802 \pm 1.4 \times 10^{-3}$ & $0.07810 \pm 9.2 \times 10^{-4}$ & $0.07811 \pm 9.3 \times 10^{-4}$ \\
\hline$b_{2,2}^{0,4}$ & 0.42969 & $0.42984 \pm 2.1 \times 10^{-3}$ & $0.42967 \pm 1.2 \times 10^{-3}$ & $0.42967 \pm 1.3 \times 10^{-3}$ \\
\hline$b_{2,1}^{1,1}$ & 0.09844 & $0.09836 \pm 6.4 \times 10^{-3}$ & $0.09823 \pm 4.1 \times 10^{-3}$ & $0.09822 \pm 3.4 \times 10^{-3}$ \\
\hline$b_{2,1}^{1,2}$ & -0.22448 & $-0.22446 \pm 4.4 \times 10^{-3}$ & $-0.22436 \pm 2.8 \times 10^{-3}$ & $-0.22435 \pm 2.8 \times 10^{-3}$ \\
\hline$b_{2,1}^{1,3}$ & 0.11719 & $0.11710 \pm 2.4 \times 10^{-3}$ & $0.11719 \pm 1.7 \times 10^{-3}$ & $0.11717 \pm 1.6 \times 10^{-3}$ \\
\hline$b_{2,1}^{1,4}$ & 0.14974 & $0.14976 \pm 2.7 \times 10^{-3}$ & $0.14968 \pm 1.6 \times 10^{-3}$ & $0.14967 \pm 1.6 \times 10^{-3}$ \\
\hline$b_{2,2}^{1,1}$ & 0.50469 & $0.50478 \pm 5.5 \times 10^{-3}$ & $0.50468 \pm 3.1 \times 10^{-3}$ & $0.50468 \pm 2.9 \times 10^{-3}$ \\
\hline$b_{2,2}^{1,2}$ & 0.08906 & $0.08881 \pm 6.4 \times 10^{-3}$ & $0.08891 \pm 3.1 \times 10^{-3}$ & $0.08889 \pm 2.6 \times 10^{-3}$ \\
\hline$b_{2,2}^{1,3}$ & 0.06510 & $0.06514 \pm 1.7 \times 10^{-3}$ & $0.06514 \pm 1.1 \times 10^{-3}$ & $0.06514 \pm 1.1 \times 10^{-3}$ \\
\hline$b_{2,2}^{1,4}$ & -0.01953 & $-0.01937 \pm 3.6 \times 10^{-3}$ & $-0.01942 \pm 1.8 \times 10^{-3}$ & $-0.01941 \pm 1.5 \times 10^{-3}$ \\
\hline
\end{tabular}


Table 2.9: Monte Carlo estimation results for innovations variance of the FP-VARX $(3,1)_{[4,4]}$ model $(500$ runs per method; sample mean estimate \pm sample standard deviation).

\begin{tabular}{|c|c|c|c|c|}
\hline Coefficient & Actual Value & OLS & WLS & ML \\
\hline$\sigma_{1,1}\left(k_{1}, k_{1}\right)$ & 0.01177 & $0.01190 \pm 5.5 \times 10^{-4}$ & $0.01177 \pm 5.4 \times 10^{-4}$ & $0.01171 \pm 5.4 \times 10^{-4}$ \\
\hline$\sigma_{2,2}\left(k_{1}, k_{1}\right)$ & 0.01112 & $0.01129 \pm 5.3 \times 10^{-4}$ & $0.01112 \pm 5.2 \times 10^{-4}$ & $0.01106 \pm 5.2 \times 10^{-4}$ \\
\hline$\sigma_{1,1}\left(k_{2}, k_{2}\right)$ & 0.07650 & $0.07685 \pm 5.6 \times 10^{-3}$ & $0.07678 \pm 5.6 \times 10^{-3}$ & $0.07639 \pm 5.5 \times 10^{-3}$ \\
\hline$\sigma_{2,2}\left(k_{2}, k_{2}\right)$ & 0.09187 & $0.09234 \pm 6.8 \times 10^{-3}$ & $0.09221 \pm 6.8 \times 10^{-3}$ & $0.09175 \pm 6.8 \times 10^{-3}$ \\
\hline$\sigma_{1,1}\left(k_{3}, k_{3}\right)$ & 38265 & $0.38374 \pm 5.2 \times 10^{-2}$ & $0.38425 \pm 5.3 \times 10^{-2}$ & $0.38233 \pm 5.2 \times 10^{-2}$ \\
\hline$\sigma_{2,2}\left(k_{3}, k_{3}\right)$ & 68132 & $0.68240 \pm 1.0 \times 10^{-1}$ & $0.68419 \pm 1.0 \times 10^{-1}$ & $0.68077 \pm 1.0 \times 10^{-1}$ \\
\hline$\sigma_{1,1}\left(k_{4}, k_{4}\right)$ & 0.69120 & $0.69213 \pm 1.1 \times 10^{-1}$ & $0.69393 \pm 1.1 \times 10^{-1}$ & $0.69047 \pm 1.1 \times 10^{-1}$ \\
\hline$\sigma_{2,2}\left(k_{4}, k_{4}\right)$ & 39050 & $0.89215 \pm 1.2 \times 10^{-1}$ & $0.89417 \pm 1.2 \times 10^{-1}$ & $0.88970 \pm 1.2 \times 10^{-1}$ \\
\hline$\sigma_{1,1}\left(k_{5}, k_{5}\right)$ & 0.23563 & $0.23646 \pm 3.3 \times 10^{-2}$ & $0.23661 \pm 3.3 \times 10^{-2}$ & $0.23542 \pm 3.3 \times 10^{-2}$ \\
\hline$\sigma_{2,2}\left(k_{5}, k_{5}\right)$ & 0.22485 & $0.22564 \pm 1.9 \times 10^{-2}$ & $0.22572 \pm 1.9 \times 10^{-2}$ & $0.22459 \pm 1.9 \times 10^{-2}$ \\
\hline$\sigma_{1,1}\left(k_{6}, k_{6}\right)$ & 0.06641 & $0.06670 \pm 5.8 \times 10^{-3}$ & $0.06666 \pm 5.8 \times 10^{-3}$ & $0.06633 \pm 5.8 \times 10^{-3}$ \\
\hline$\sigma_{2,2}\left(k_{6}, k_{6}\right)$ & 0.05754 & $0.05788 \pm 3.6 \times 10^{-3}$ & $0.05774 \pm 3.6 \times 10^{-3}$ & $0.05746 \pm 3.6 \times 10^{-3}$ \\
\hline$\sigma_{1,1}\left(k_{7}, k_{7}\right)$ & 0.03328 & $0.03343 \pm 2.5 \times 10^{-3}$ & $0.03340 \pm 2.5 \times 10^{-3}$ & $0.03323 \pm 2.5 \times 10^{-3}$ \\
\hline$\sigma_{2,2}\left(k_{7}, k_{7}\right)$ & 0.03446 & $0.03465 \pm 2.1 \times 10^{-3}$ & $0.03458 \pm 2.1 \times 10^{-3}$ & $0.03441 \pm 2.1 \times 10^{-3}$ \\
\hline$\sigma_{1,1}\left(k_{8}, k_{8}\right)$ & 0.02704 & $0.02716 \pm 1.8 \times 10^{-3}$ & $0.02714 \pm 1.8 \times 10^{-3}$ & $0.02700 \pm 1.8 \times 10^{-3}$ \\
\hline$\sigma_{2,2}\left(k_{8}, k_{8}\right)$ & 0.04330 & $0.04347 \pm 2.3 \times 10^{-3}$ & $0.04347 \pm 2.3 \times 10^{-3}$ & $0.04325 \pm 2.3 \times 10^{-3}$ \\
\hline$\sigma_{1,1}\left(k_{9}, k_{9}\right)$ & 0.02275 & $0.02286 \pm 1.4 \times 10^{-3}$ & $0.02283 \pm 1.4 \times 10^{-3}$ & $0.02271 \pm 1.4 \times 10^{-3}$ \\
\hline$\sigma_{2,2}\left(k_{9}, k_{9}\right)$ & 0.04310 & $0.04329 \pm 2.3 \times 10^{-3}$ & $0.04326 \pm 2.3 \times 10^{-3}$ & $0.04305 \pm 2.3 \times 10^{-3}$ \\
\hline$\sigma_{1,1}\left(k_{10}, k_{10}\right)$ & 0.01426 & $0.01434 \pm 7.0 \times 10^{-4}$ & $0.01431 \pm 7.0 \times 10^{-4}$ & $0.01424 \pm 7.0 \times 10^{-4}$ \\
\hline$\sigma_{2,2}\left(k_{10}, k_{10}\right)$ & 0.02317 & $0.02327 \pm 1.2 \times 10^{-3}$ & $0.02324 \pm 1.2 \times 10^{-3}$ & $0.02312 \pm 1.2 \times 10^{-3}$ \\
\hline$\sigma_{1,1}\left(k_{11}, k_{11}\right)$ & 0.02034 & $0.02038 \pm 1.2 \times 10^{-3}$ & $0.02036 \pm 1.2 \times 10^{-3}$ & $0.02026 \pm 1.2 \times 10^{-3}$ \\
\hline$\sigma_{2,2}\left(k_{11}, k_{11}\right)$ & 0.03673 & $0.03679 \pm 3.0 \times 10^{-3}$ & $0.03678 \pm 3.0 \times 10^{-3}$ & $0.03650 \pm 3.0 \times 10^{-3}$ \\
\hline
\end{tabular}
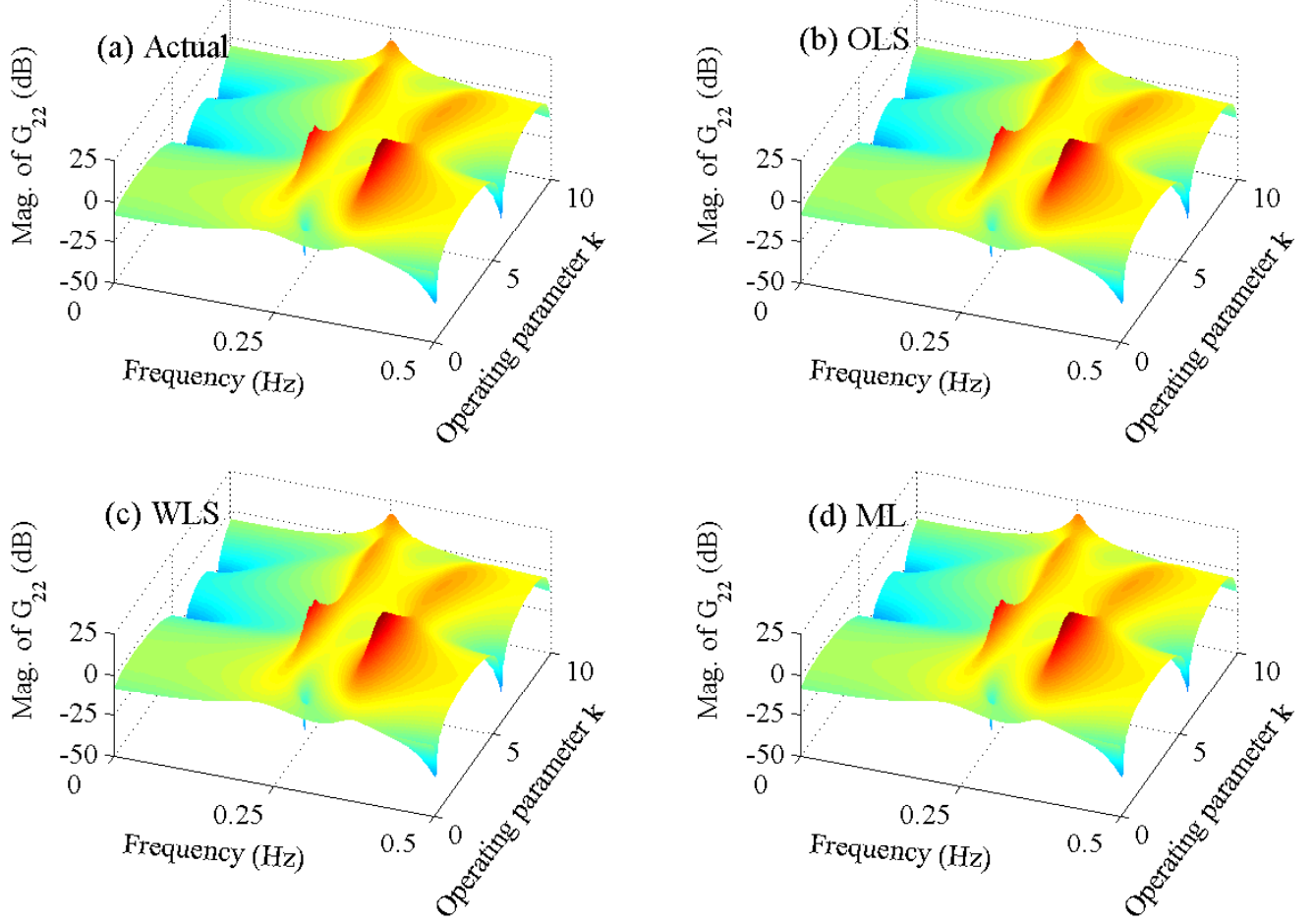

Figure 2.14: FP-VARX $(3,1)_{[4,4]}$ model based frequency response magnitude for $G_{22}$ with respect to frequency and operating parameter $k$ : (a) actual system, (b) OLS mean estimate, (c) WLS mean estimate and, (d) ML mean estimate. 

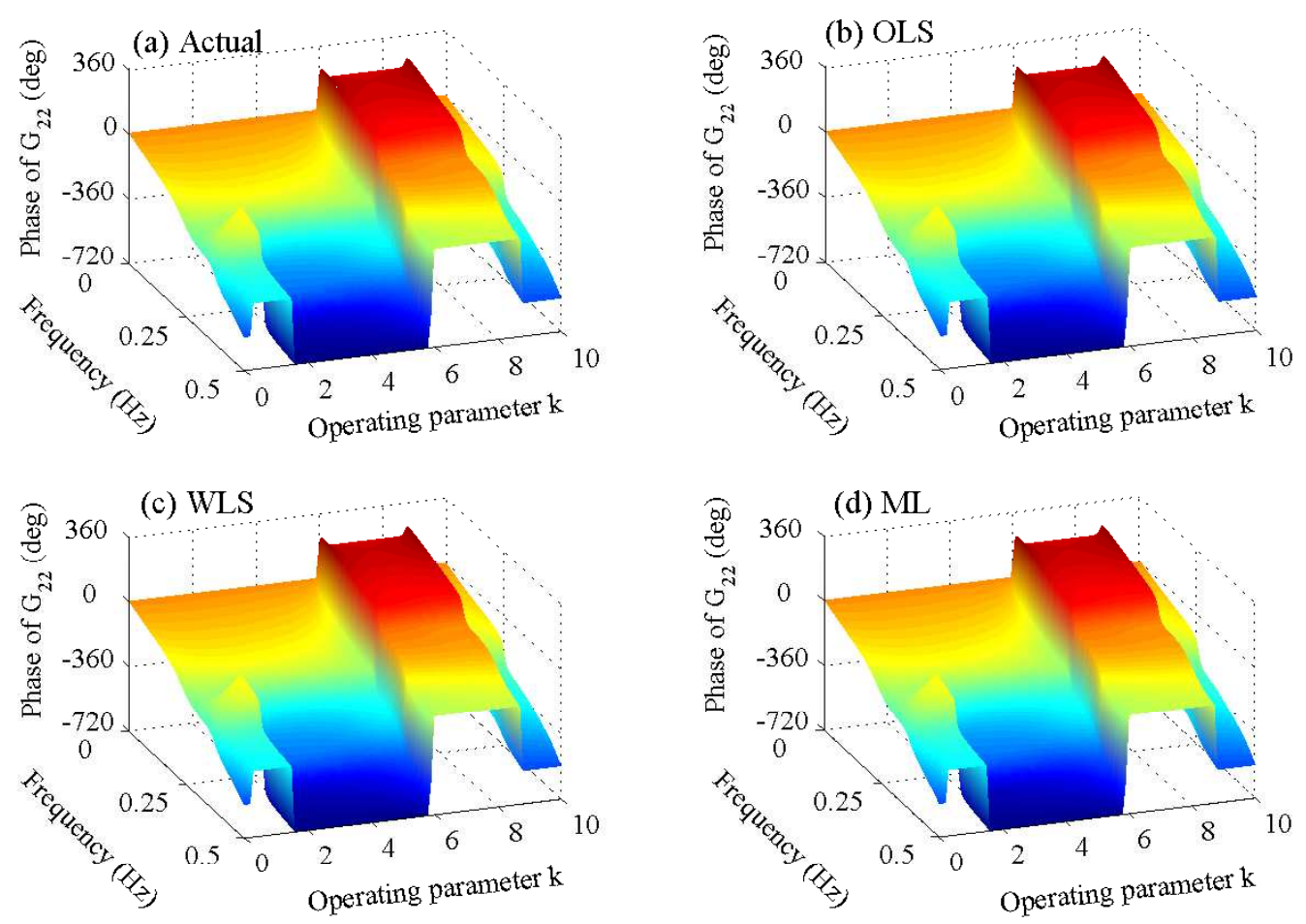

Figure 2.15: $\operatorname{FP}-\operatorname{VARX}(3,1)_{[4,4]}$ model based frequency response phase for $G_{22}$ with respect to frequency and operating parameter $k$ : (a) actual system, (b) OLS mean estimate, (c) WLS mean estimate and, (d) ML mean estimate.
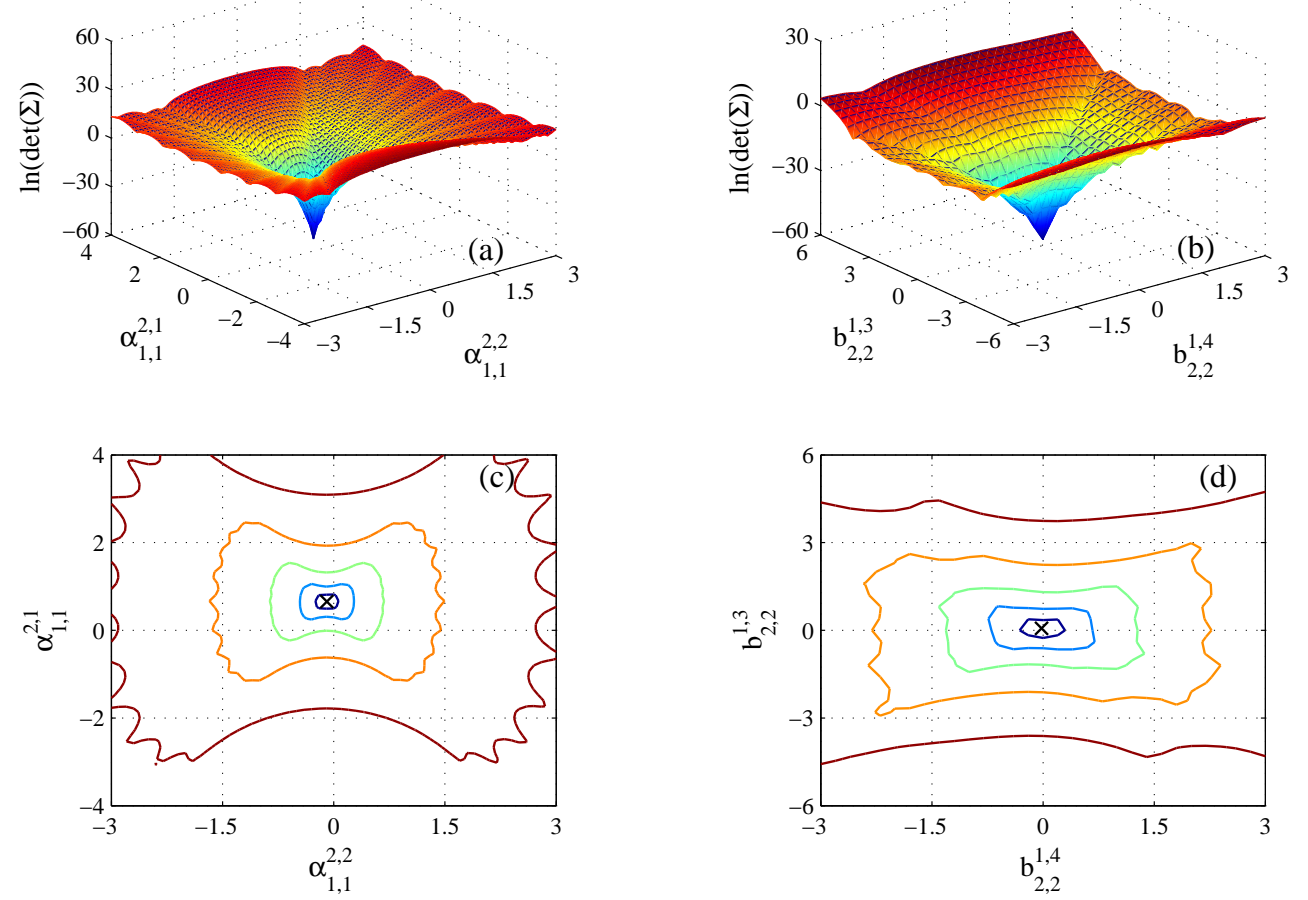

Figure 2.16: Projection of the function minimized in the ML method on selected FP-VARX $(3,1)_{[4,4]}$ model parameters: (a),(c) surface and contour plots by projecting on $\alpha_{1,1}^{2,1}$ and $\alpha_{1,1}^{2,2}$; (b),(d) surface and contour plots by projecting on $b_{2,2}^{1,3}$ and $b_{2,2}^{1,4}$ (the " $\times$ " marks designate the actual FP-VARX $(3,1)_{[4,4]}$ model parameters). 


\section{Chapter 3}

\section{Stochastic identification of temperature effects on the dynamics of a smart composite beam: assessment of multi-model and global model approaches}

\subsection{Introduction}

The demands posed by modern engineering applications for high performance and safety have underscored the necessity to understand the way key environmental factors, such as temperature or humidity, affect the dynamics of a structure. This knowledge is valuable, as it may be used in engineering design, structural health monitoring, and so on.

In recent years, there has been a growing interest in the analysis of temperature effects on the modal parameters (natural frequencies, damping factors) of simple structural elements, such as beams and plates. Analytical finite element [50,51] or physics-based [52,53] models are often used for this purpose. In all studies the modal frequencies decrease as temperature increases and vice versa, since the temperature increase contributes to the reduction of the overall structural stiffness. Yet, the exact nature of the modal frequency dependence on temperature may differ due to design and material selection. Damping factors exhibit a rather more complex dependence on temperature, with the selected materials and the corresponding temperature range playing important roles.

Experimental studies, aiming at extracting the stiffness and/or damping properties of (mainly) fiberreinforced composites under various environmental conditions, have also attracted significant attention [5458]. Vibration testing procedures are used in $[54,55]$ to obtain the modal parameters of carbon fiberreinforced panels exposed to different temperatures. The results in [55] are also found to be in good agreement with finite element based analysis. In [56] the influence of several factors - including temperature on the damping properties of composite materials is studied. Fatigue testing experiments are carried out on specimens of different fiber materials and their loss factors (equal to twice the value of the corresponding damping factors) are obtained. In [57,58], the temperature effect on the damping properties of glass fiberreinforced beams is studied using vibration testing. As in analytical studies, modal frequencies decrease with increasing temperature and vice versa, whereas loss factors generally exhibit a more complex dependence on temperature, which is influenced by the selected materials and temperature range. Recent studies by the present authors based on a smart composite beam and novel stochastic global models have revealed similar behavior [40-42]. 
Other experimental studies examine the dependence of modal parameters on temperature for civil structures, such as bridges [23,35,36, 59-62]. These studies are damage detection oriented and focus on separating temperature from damage effects on the modal parameters. For this purpose, the modal parameters are obtained using customary system identification techniques at each temperature. The problem is split into several disjoint subproblems (one per considered temperature) and a model is identified based upon a single data record at a time. This procedure is hereby referred to as the multi-model approach. The identified modal parameters are subsequently related to temperature via additional expressions. A variety of such expressions have been suggested depending upon the linear or nonlinear nature of the dependence. Static linear regression models associating modal frequency (or damping) with temperature in one or more locations on the structure are very common $[35,59,62]$. In order to account for the structure's thermal capacity, linear dynamic models describing the modal frequency dependence on temperature have been also employed $[23,36]$. Static nonlinear mappings between modal frequency and temperature using neural networks [60] or regression models [61] have been reported as well.

The aim of the present study is the identification of the temperature effects on the dynamics of a smart composite beam via conventional multi-model and novel global model approaches. The multi-model approaches are based on non-parametric (Welch type [63, pp. 76-77]) and parametric Vector AutoRegressive with eXogenous excitation (VARX [64]) representations. The global approaches are based on novel Constant Coefficient Pooled (CCP) and Functionally Pooled (FP) VARX representations (the terms model and representation are interchangeably used). Early results on the topic have been presented in Hios and Fassois [40-42].

The global model approaches aim at circumventing the shortcomings associated with their conventional multi-model counterparts. These may be summarized as follows:

1. The artificial splitting of the identification problem into disjoint subproblems, which results into a large number of separate models. This is practically and theoretically not the best solution, since it is awkward to handle, and does not account for the fact that it is a single structure that operates under different conditions each time.

2. The number of parameters needed to be estimated in the multi-model approaches is proportional to the number of data records. Thus, increasing the number of data records (each one corresponding to a distinct temperature) does not lead to higher statistical accuracy, causing the estimates to be statistically suboptimal.

3. The various data records are not treated as a single entity. As a result, not all available information is extracted, since the potential interrelations among the records are ignored. This constitutes an additional statistical suboptimality source.

The global approaches are based on a recently introduced novel statistical pooling framework, which allows for (a) the postulation of a single, stochastic, global representation of the specified type, and (b) the simultaneous and statistically optimal treatment of all available data records [14-16]. The two types of stochastic global representations that are presently postulated are:

1. Constant Coefficient Pooled VARX (CCP-VARX) representations $[41,42,65]$. These are nevertheless limited in scope since they are characterized by constant coefficients, that is coefficients not depending on temperature. As a consequence, they may only be employed for providing a simple, "overall", description of the dynamics, since their characteristics are "averaged" over temperature.

2. Functionally Pooled VARX (FP-VARX) representations (refer to Chapter 2, Section 2.2, and also [40-42]). These are more elaborate, allowing for the explicit, analytical, modeling of temperature dependence, and constituting the essential representation of choice for the problem. 

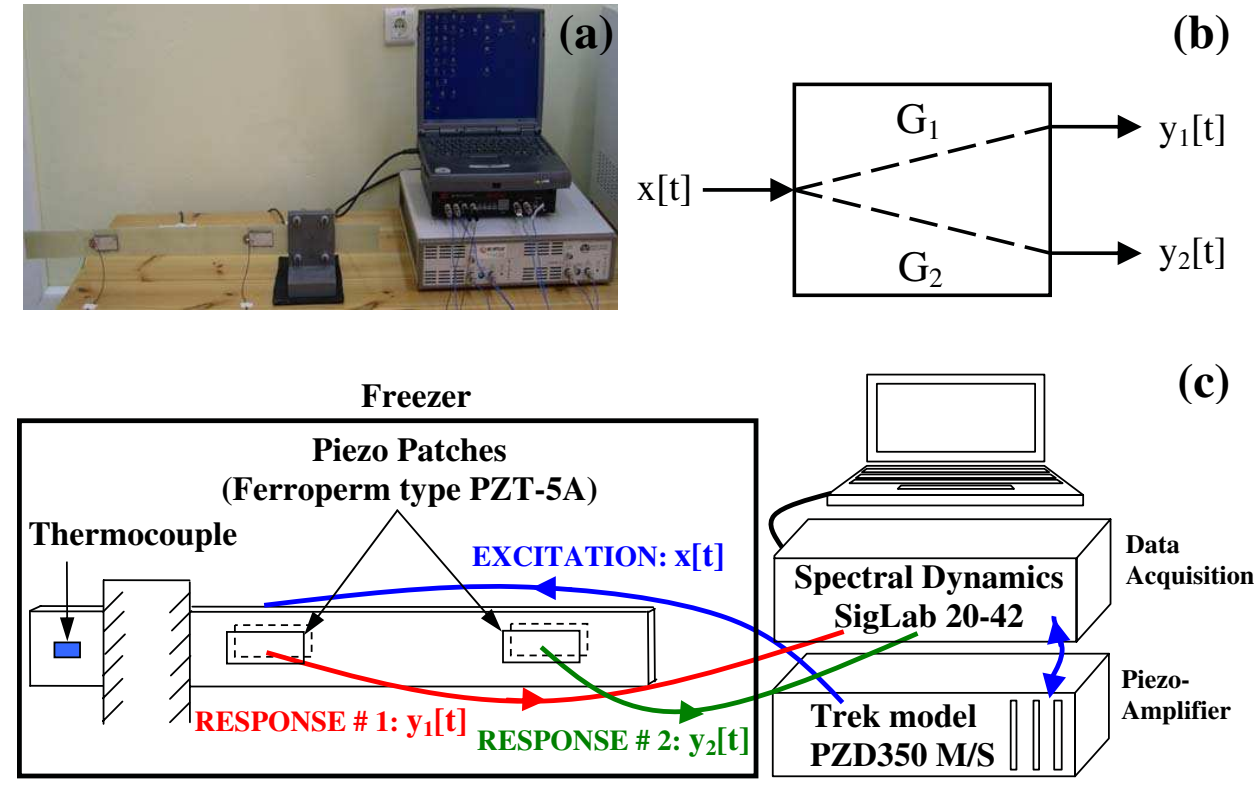

Figure 3.1: (a) Photo of the smart beam; (b) schematic representation of the considered transfer functions $G_{1}, G_{2} ;$ (c) schematic diagram of the laboratory setup.

The rest of this chapter is organized as follows: The laboratory setup and the experiments are described in Section 3.2. Multi-model (non-parametric and parametric VARX type) identification is treated in Section 3.3, whereas global model identification is treated in Section 3.4. The obtained results are assessed in Section 3.5, and the conclusions are summarized in Section 3.6 .

\subsection{The laboratory setup and the experiments}

The structure under test is a cantilever beam with integrated piezoceramic transducers (Fig. 5.1(a)). Such structures are referred to as "smart" due to their potential of performing advanced functions, such as vibration control and health monitoring. The $800 \times 52.5 \times 3 \mathrm{~mm}$ (effective length equal to $600 \mathrm{~mm}$ ) beam is made of a 3-ply glass-polyester composite with fibers oriented along the longitudinal direction, while four $50 \times 25 \times$ $1 \mathrm{~mm}$ piezoceramic patches are bonded to both sides of it. One of the patches near the clamped end excites the structure, whereas the response is measured by the other patches which act as sensors (Fig. 5.1(c)).

The smart beam is placed in a freezer, and the experiments are carried out under quasi-static thermal conditions. The smart beam temperature is obtained from a digital thermometer with a K-type bead thermocouple attached behind the clamp to avoid interference with its vibratory motion (Fig. 5.1(c)). The study focuses on the $[-20,+20]^{\circ} \mathrm{C}$ temperature range, and a total of 21 experiments are performed at $2^{\circ} \mathrm{C}$ increments. The actuator is driven by a properly amplified stochastic, zero-mean, Gaussian white voltage with bandwidth $[0,5,000] \mathrm{Hz}$ and a discretization frequency of $12,800 \mathrm{~Hz}$. All excitation-response signals are collected by a data acquisition device at a sampling frequency of $f_{\mathrm{s}}=2,560 \mathrm{~Hz}$.

The analysis is confined within the $[100,950] \mathrm{Hz}$ range, due to the beam's "rich" modal content therein. The signals are bandpass filtered by means of a digital Chebyshev II filter of order $n=33$. The response signals are designated as $y_{1}[t]$ and $y_{2}[t]$, whereas the excitation as $x[t]$ (Fig. 5.1(b),(c)). Following sample mean subtraction, each signal is scaled to unit variance in order to enhance numerical accuracy. The experimental and data acquisition details are summarized in Table 3.1, while in Appendix 3.A the material properties of the composite beam and the piezoceramic patches are provided. 
Table 3.1: Experimental and data acquisition details.

\begin{aligned} & \hline Beam: $800 \times 52.5 \times 3 \mathrm{~mm}$ 3-ply glass-polyester composite with isopthalic polyester \\ & resin matrix and fibers oriented in the longitudinal direction (that is $\left.[0]_{3}\right) \\ &$ Actuators/Sensors: $50 \times 25 \times 1 \mathrm{~mm}$ Ferroperm type PZT-5A piezoceramic patches \\ & Data acquisition device: Spectral Dynamics SigLab $20-42 \\ &$ Actuator amplifier: Trek PZD350 M/S \\ & Temperature measurements: K-type bead thermocouple \\ & Temperature range: ${[-20,+20]^{\circ} \mathrm{C}$, with $2^{\circ} \mathrm{C}$ increments $} \\ &$ Number of experiments: $M=21 \\ &$ Signal bandwidth: ${[100,950] \mathrm{Hz}$ (bandpass filter: Chebyshev II of order $n=33) } \\ &$ Sampling frequency: $f_{\mathrm{s}}=2,560 \mathrm{~Hz} \\ &$ Excitation: Gaussian white noise \\ & Measured signal length: $N=76,800$ samples $(\approx 30 \mathrm{~s}) \\ &$\hline\end{aligned}

\subsection{Multi-model identification}

\subsubsection{Non-parametric Welch-based multi-model identification}

The Welch spectral method [63, pp. 76-77] is used to estimate the Frequency Response Functions (FRFs) corresponding to the transfer functions $G_{1}$, relating $x[t]$ to $y_{1}[t]$, and $G_{2}$ relating $x[t]$ to $y_{2}[t]$ (Fig. 5.1(b)), for each considered temperature. Once an FRF estimate is available, the structure's modal parameters are obtained by the peak-picking method [66, pp. 163-164].

Identification results: The analysis is based on $N=70,000$ sample long $(\approx 27 \mathrm{~s})$ segments of the excitation-response signals for each temperature. An $L=25,600$ sample long Hanning time data window is used yielding FRF estimates with a frequency resolution equal to $\Delta f=0.1 \mathrm{~Hz}$, whereas overlapping is set to $80 \%$ (Table 3.2). The estimated FRFs [67, MATLAB ${ }^{\circledR}$ function "tfe.m"] reveal the presence of six well-separated modes with low damping $(\zeta<1 \%)$ within the $[100,950] \mathrm{Hz}$ frequency range (see Section 3.5, Fig. 3.5(a)). Furthermore, the coherence function estimate [63, p. 454], [67, MATLAB ${ }^{\circledR}$ function "cohere.m"] appears uniformly flat and near unity for the majority of frequencies in the range of interest (see Section 3.5, Fig. 3.5(b)).

\subsubsection{Parametric VARX-based multi-model identification}

Parametric multi-model identification is based on Vector AutoRegressive with eXogenous excitation (VARX) models [64] of the following form ${ }^{1}$ :

$$
\begin{gathered}
\boldsymbol{y}[t]+\sum_{i=1}^{n a} \boldsymbol{A}_{i} \boldsymbol{y}[t-i]=\sum_{i=0}^{n b} \boldsymbol{B}_{i} \boldsymbol{x}[t-i]+\boldsymbol{e}[t, \boldsymbol{\vartheta}] \\
E\left\{\boldsymbol{e}[t, \boldsymbol{\vartheta}] \boldsymbol{e}^{T}[t-\tau, \boldsymbol{\vartheta}]\right\}=\boldsymbol{\Sigma}(\boldsymbol{\vartheta}) \delta[\tau] \\
\boldsymbol{\vartheta}=\operatorname{vec}\left(\left[\boldsymbol{A}_{1} \boldsymbol{A}_{2} \ldots \boldsymbol{A}_{n a} \vdots \boldsymbol{B}_{0} \boldsymbol{B}_{1} \ldots \boldsymbol{B}_{n b}\right]^{T}\right)
\end{gathered}
$$

where $t$ is the normalized discrete time, while the AutoRegressive (AR) and the eXogenous (X) model orders are designated as $n a$ and $n b$, respectively. $\boldsymbol{y}[t]$ is the $(n y \times 1)$ response vector, $\boldsymbol{x}[t]$ the $(n x \times 1)$ excitation

\footnotetext{
${ }^{1}$ Boldface upper/ lower case symbols designate matrix/ column-vector quantities, respectively
} 
Table 3.2: Conventional multi-model and global model identification approaches and estimation details.

\begin{tabular}{|c|c|c|c|}
\hline \multirow{5}{*}{$\begin{array}{l}\text { Conventional } \\
\text { multi-model } \\
\text { identification }\end{array}$} & $\begin{array}{l}\text { Non-parametric } \\
\text { approach (Welch) }\end{array}$ & $\begin{array}{l}\text { Data length: } N=70,000 \text { samples } \\
\text { Data segment overlap: } 80 \%\end{array}$ & $\begin{array}{l}\text { Hanning window: } L=25,600 \text { samples } \\
\text { Frequency resolution: } \Delta f=0.1 \mathrm{~Hz}\end{array}$ \\
\hline & Parametric & Data length: $N=2,000$ samples & Parameter estimation: OLS (QR) \\
\hline & VARX & Selected model: VARX $(23,23)$ & \\
\hline & & Number of estimated parameters: 2,940 & Samples per parameter: $\mathrm{SPP}=42.9$ \\
\hline & & Condition numbers : OLS inverted matrix & $\sim 10^{6}$; par. estimator covariance $\sim 10^{15}$ \\
\hline \multirow{8}{*}{$\begin{array}{l}\text { Stochastic } \\
\text { global model } \\
\text { identification }\end{array}$} & \multirow{4}{*}{$\begin{array}{l}\text { CCP-VARX } \\
\text { approach }\end{array}$} & Data length: $N=2,000$ samples & Par. estimation: non-iterative WLS (QR) \\
\hline & & odel: CCP-VARX $(23,23)$ & \\
\hline & & Number of estimated parameters: 140 & Samples per parameter: $\mathrm{SPP}=900$ \\
\hline & & Condition number: WLS inverted matrix & $10^{5} ;$ par. estimator covariance $\sim 10^{11}$ \\
\hline & \multirow{4}{*}{$\begin{array}{l}\text { FP-VARX } \\
\text { approach }\end{array}$} & Data length: $N=2,000$ samples & Par. estimation: non-iterative WLS (QR) \\
\hline & & $\begin{array}{l}\text { Selected model: FP-VARX }(23,23)_{[4,3]} \\
\text { with Chebyshev II functional subspaces }\end{array}$ & \\
\hline & & Number of estimated parameters: 512 & Sampl \\
\hline & & Condition numbers: WLS inverted matri & $\sim 10^{6}$; par. \\
\hline $\begin{array}{l}\text { Modal uncertainty } \\
\text { estimation }\end{array}$ & \multicolumn{3}{|c|}{$\begin{array}{l}\text { Numerical differentiation via the central difference approximation [70] } \\
\text { Perturbations within } 10^{-5}-10^{-6} \text { times the standard deviation of the AR parameters }\end{array}$} \\
\hline
\end{tabular}

vector, and $\boldsymbol{e}[t, \boldsymbol{\vartheta}]$ is a $(n y \times 1)$ residual (one-step-ahead prediction error) vector that should be a zero mean uncorrelated sequence with a fully parametrized, positive-definite, covariance matrix $\boldsymbol{\Sigma}(\boldsymbol{\vartheta}) . E\{\cdot\}$ designates statistical expectation, and $\delta[\tau]$ the Kronecker delta. The AR and X parameter matrices, designated as $\boldsymbol{A}_{i}$ and $\boldsymbol{B}_{i}$, are fully parametrized and have dimensions $(n y \times n y)$ and $(n y \times n x)$, respectively. The model is parametrized in terms of the parameter vector $\vartheta$, comprising all elements of the AR and X matrices, whereas the $\operatorname{vec}(\cdot)$ operator transforms the indicated matrix into a vector by stacking its columns. The representation of Eq. (3.1a)-(3.1c) is referred to as a VARX model of orders $(n a, n b)$, or in short as a VARX $(n a, n b)$ model.

Model parameter estimation is accomplished by minimizing an Ordinary Least Squares (OLS) criterion [1, p. 206]. Model order specification may be based upon the determinant (or trace) of the residual covariance matrix, or the Bayessian Information Criterion (BIC) (see [1, pp. 505-507] and [64, 68]). In addition, frequency stabilization diagrams $[64,68]$, which depict the estimated frequencies for increasing model order, may be used. Once a proper model has been selected, modal information in the form of natural frequencies, damping factors and mode shapes is readily obtained $[68,69]$, and a quantitative measure of the modal parameter accuracy may be derived in the form of statistical uncertainty.

The approach for estimating uncertainty is based upon relating the model parameter uncertainties with those of the corresponding modal parameters. Let $\boldsymbol{\xi}=\boldsymbol{f}\left(\boldsymbol{\vartheta}_{A R}\right)$ represent the nonlinear functional relationship between the $(r \times 1)$ modal parameter vector $\boldsymbol{\xi}$ and the $\left(n a n y^{2} \times 1\right)$ parameter vector $\boldsymbol{\vartheta}_{A R}$ comprising all elements from the AR matrices. Linearizing this relationship using a first order Taylor series expansion yields for the covariance matrices $\boldsymbol{P}(\widehat{\boldsymbol{\xi}})$ and $\boldsymbol{P}\left(\widehat{\boldsymbol{\vartheta}}_{A R}\right)$ of the estimators $\widehat{\boldsymbol{\xi}}$ and $\widehat{\boldsymbol{\vartheta}}_{A R}$, respectively $[70]^{2}:$

$$
\boldsymbol{P}(\widehat{\boldsymbol{\xi}}) \approx \boldsymbol{J}\left(\widehat{\boldsymbol{\vartheta}}_{A R}\right) \boldsymbol{P}\left(\widehat{\boldsymbol{\vartheta}}_{A R}\right) \boldsymbol{J}^{T}\left(\widehat{\boldsymbol{\vartheta}}_{A R}\right)
$$

where $\boldsymbol{J}\left(\widehat{\boldsymbol{\vartheta}}_{A R}\right)$ is the $\left(r \times n a n y^{2}\right)$ Jacobian matrix $\frac{\partial \boldsymbol{f}\left(\widehat{\boldsymbol{\vartheta}}_{A R}\right)}{\partial \boldsymbol{\vartheta}_{A R}^{T}}$. To compute $\boldsymbol{P}(\widehat{\boldsymbol{\xi}})$, the covariance matrix of $\widehat{\boldsymbol{\vartheta}}_{A R}$ is replaced by its estimate $\widehat{\boldsymbol{P}}\left(\widehat{\boldsymbol{\vartheta}}_{A R}\right)$ (which is a portion of the full parameter vector estimator covariance matrix). A simple way to obtain the Jacobian matrix is via numerical differentiation using the central dif-

\footnotetext{
${ }^{2}$ Symbols in hat designate estimators/estimates.
} 

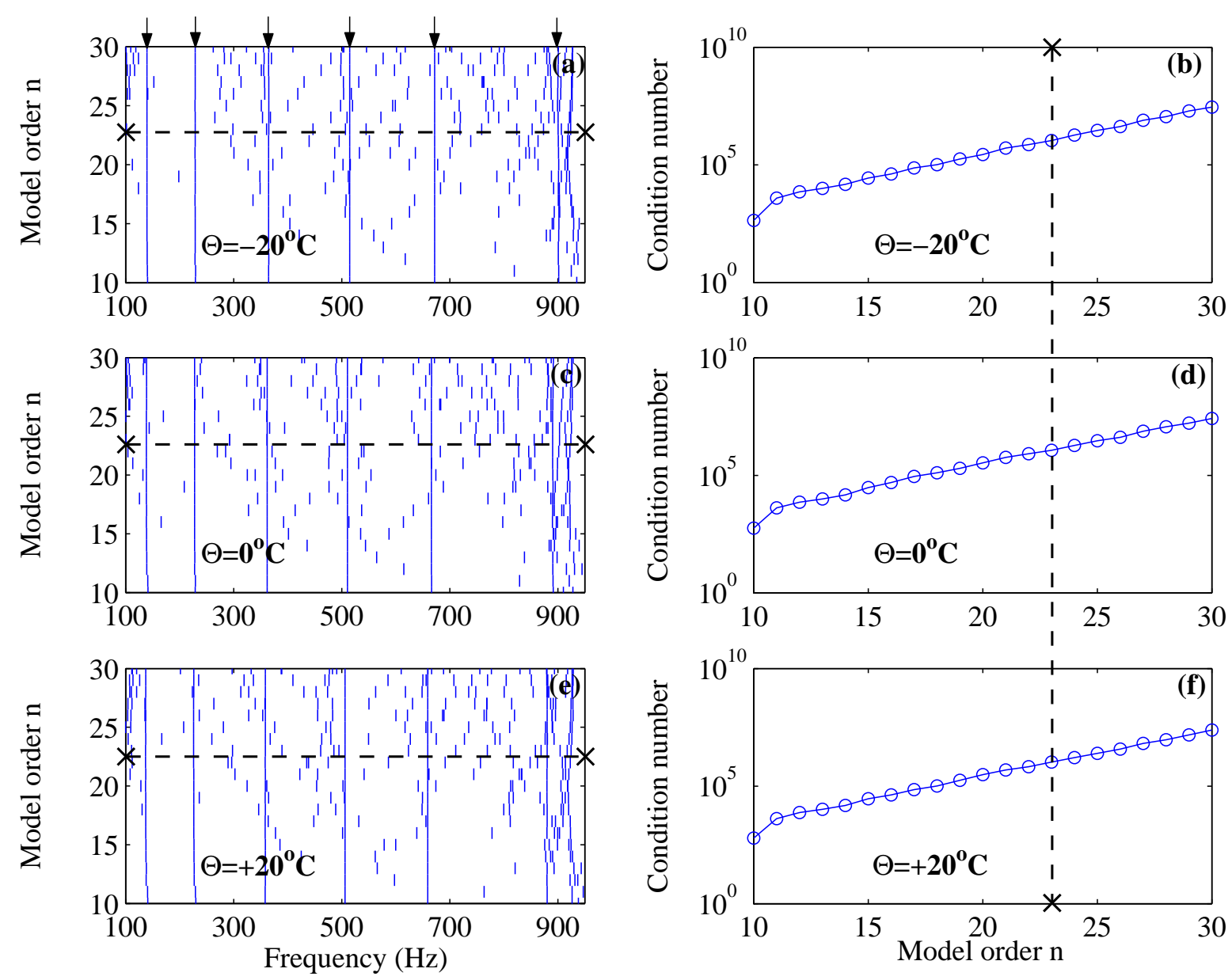

Figure 3.2: Parametric VARX-based multi-model approach: $\operatorname{VARX}(n, n)$ model order specification for three distinct temperatures; (a),(c),(e) frequency stabilization diagrams (the arrows at the top indicate identified modes); (b),(d),(f) condition numbers for the OLS estimator inverted matrix versus model order. (In all cases the vertical/horizontal dashed lines indicate the selected model order).

ference approximation [70]. In this case, the $i$-th column of $\boldsymbol{J}(\widehat{\boldsymbol{\vartheta}})$, designated as $\boldsymbol{j}_{i}\left(\widehat{\boldsymbol{\vartheta}}_{A R}\right)$, is approximated as:

$$
\boldsymbol{j}_{i}\left(\widehat{\boldsymbol{\vartheta}}_{A R}\right) \approx \frac{\boldsymbol{f}\left(\widehat{\boldsymbol{\vartheta}}_{A R}+\boldsymbol{c}\right)-\boldsymbol{f}\left(\widehat{\boldsymbol{\vartheta}}_{A R}-\boldsymbol{c}\right)}{2 c_{i}}
$$

where $c$ is an $\left(n a n y^{2} \times 1\right)$ null vector save for the $i$-th element, designated as $c_{i}$, which holds a properly chosen value causing a small perturbation in the $i$-th element of $\widehat{\vartheta}_{A R}$.

Identification results: The analysis uses $N=2,000$ sample long $(\approx 0.78 \mathrm{~s})$ data records from each temperature. Parameter estimation is based upon OLS with QR implementation (refer to [1, pp. 318-320], and also [47, MATLAB ${ }^{\circledR}$ function "arx.m”]). As the main interest lies in extracting the structure's modal parameters, model overdetermination with subsequent elimination of the "extraneous" numerical modes by means of frequency stabilization diagrams is employed. A succession of $\operatorname{VARX}(n, n)$ models with $n=10,11, \ldots, 30$ are fitted to the data for each temperature and the frequency stabilization diagrams (Fig. 3.2(a),(c),(e)) indicate that higher frequency modes "stabilize" for $n \geq 20$. A $\operatorname{VARX}(23,23)$ model is thus selected, providing a compromise between model compactness and estimation accuracy for each considered temperature 


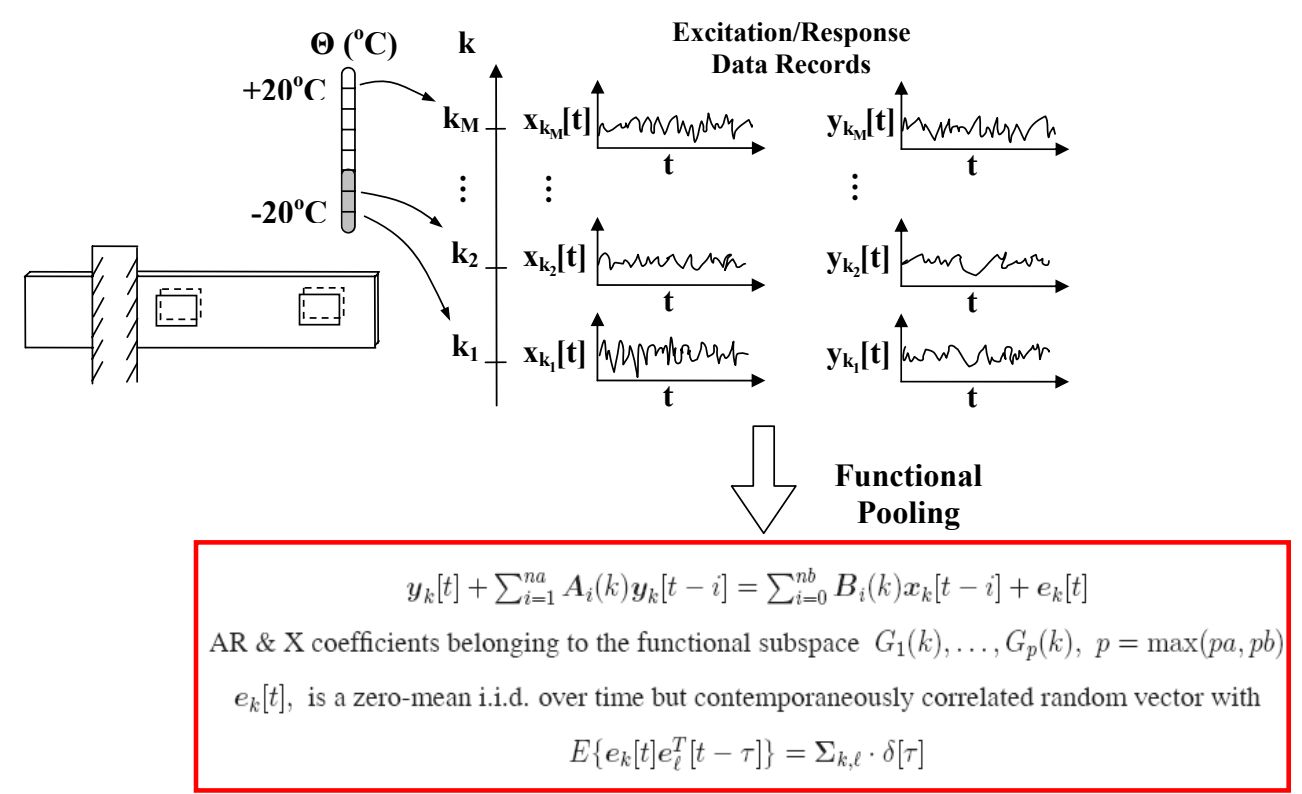

Figure 3.3: Schematic representation of the global model identification problem, showing the operating parameter $k$ (temperature), the excitation/response data records corresponding to different temperatures, and the FP-VARX model class.

(140 estimated parameters and an OLS estimator inverted matrix with condition number $\sim 10^{6}$; see Fig. 3.2(b),(d),(f)). The resulting VARX-based multi-model representation contains 2, 940 parameters, that is 140 parameters (of a single $\operatorname{VARX}(23,23)$ model) for each one of the $M=21$ experiments (temperatures), yielding an SPP (Samples Per Parameter, that is the ratio of all excitation-response signal samples over the number of estimated parameters) value of 42.9. A summary of the estimation details is provided in Table 3.2 .

\subsection{Global model identification}

\subsubsection{Classes of stochastic global models}

The identification of global models is based upon $M$ sets of excitation-response data records, each one obtained under a distinct temperature. That is, each data record corresponds to a specific value of the temperature $k$ (more generally referred to as the operating parameter). The set of all possible temperatures belongs to the range $\mathcal{R} \triangleq\left[k_{\min }, k_{\max }\right] \subset \mathbb{R}$, whereas a discretized version $\left\{k_{1}, k_{2}, \ldots, k_{M}\right\}$ of it is used for data acquisition. Assuming $N$ sample-long data records, the complete data set is designated:

$$
Z^{N M} \triangleq\left\{\boldsymbol{x}_{k}[t], \boldsymbol{y}_{k}[t] \mid k=k_{1}, \ldots, k_{M} ; t=1, \ldots, N\right\}
$$

with $t$ indicating the normalized discrete time, $\boldsymbol{x}_{k}[t]$ the $(n x \times 1)$ excitation vector, and $\boldsymbol{y}_{k}[t]$ the $(n y \times 1)$ response vector corresponding to the $k$-th temperature condition (presently $n x=1, n y=2$ ). A schematic representation of global model identification adapted to the current problem is depicted in Fig. 3.3.

\subsubsection{The CCP-VARX model class.}

The Constant Coefficient Pooled VARX (CCP-VARX) representations may be thought of as generalizations of their conventional VARX counterparts (Eq. (3.1a)-(3.1b)) in that they simultaneously consider the 
complete data set $Z^{N M}$. Their main characteristic is the use of (fully parametrized) common and constant over the complete temperature range $\mathrm{AR}$ and $\mathrm{X}$ parameter matrices. The representations are of the form $[40-42,65]$ :

$$
\begin{gathered}
\boldsymbol{y}_{k}[t]+\sum_{i=1}^{n a} \boldsymbol{A}_{i} \boldsymbol{y}_{k}[t-i]=\sum_{i=0}^{n b} \boldsymbol{B}_{i} \boldsymbol{x}_{k}[t-i]+\boldsymbol{e}_{k}[t, \boldsymbol{\vartheta}] \\
E\left\{\boldsymbol{e}_{k}[t, \boldsymbol{\vartheta}] \boldsymbol{e}_{\ell}^{T}[t-\tau, \boldsymbol{\vartheta}]\right\}=\boldsymbol{\Sigma}_{k, \ell}(\boldsymbol{\vartheta}) \delta[\tau] \quad k, \ell \in \mathcal{R} \\
\boldsymbol{\vartheta}=\operatorname{vec}\left(\left[\boldsymbol{A}_{1} \boldsymbol{A}_{2} \ldots \boldsymbol{A}_{n a} \vdots \boldsymbol{B}_{0} \boldsymbol{B}_{1} \ldots \boldsymbol{B}_{n b}\right]^{T}\right)
\end{gathered}
$$

with $\boldsymbol{e}_{k}[t, \boldsymbol{\vartheta}]$ designating the $(n y \times 1)$ residual (one-step-ahead prediction error) vector corresponding to the $k$-th operating condition. The residuals should be zero mean and uncorrelated with fully parametrized cross-covariance matrices $\boldsymbol{\Sigma}_{k, \ell}(\boldsymbol{\vartheta})$. The representation of Eq. (3.5a)-(3.5c) is parametrized in terms of the parameter vector $\vartheta$, comprising all elements from the AR and X matrices, and is referred to as a CCP-VARX representation of orders $(n a, n b)$, or in short as a CCP-VARX $(n a, n b)$ representation.

\subsubsection{The FP-VARX model class.}

The Functionally Pooled VARX (FP-VARX) representations may be thought of as generalizations of their CCP-VARX counterparts, with the important distinction being that the AR and X parameter matrices are explicit functions of the operating condition. Thus the FP-VARX representation is of the form (refer to Chapter 2, Section 2.2, and also [40-42]):

$$
\begin{gathered}
\boldsymbol{y}_{k}[t]+\sum_{i=1}^{n a} \boldsymbol{A}_{i}(k) \boldsymbol{y}_{k}[t-i]=\sum_{i=0}^{n b} \boldsymbol{B}_{i}(k) \boldsymbol{x}_{k}[t-i]+\boldsymbol{e}_{k}[t, \boldsymbol{\theta}] \\
E\left\{\boldsymbol{e}_{k}[t, \boldsymbol{\theta}] \boldsymbol{e}_{\ell}^{T}[t-\tau, \boldsymbol{\theta}]\right\}=\boldsymbol{\Sigma}_{k, \ell}(\boldsymbol{\theta}) \delta[\tau] \quad k, \ell \in \mathcal{R} \\
\boldsymbol{A}_{i}(k)=\sum_{j=1}^{p a} \boldsymbol{A}_{i, j} G_{j}(k), \quad \boldsymbol{B}_{i}(k)=\sum_{j=1}^{p b} \boldsymbol{B}_{i, j} G_{j}(k) \\
\boldsymbol{\theta}=\operatorname{vec}\left(\left[\boldsymbol{A}_{1,1} \boldsymbol{A}_{1,2} \ldots \boldsymbol{A}_{n a, p a}: \boldsymbol{B}_{0,1} \boldsymbol{B}_{0,2} \ldots \boldsymbol{B}_{n b, p b}\right]^{T}\right)
\end{gathered}
$$

where the AR and X parameter matrices, designated as $\boldsymbol{A}_{i}(k)$ and $\boldsymbol{B}_{i}(k)$, are fully parametrized and have dimensions $(n y \times n y)$ and $(n y \times n x)$, respectively. These matrices are modelled as explicit functions of the operating parameter (temperature) $k$, by belonging to a functional subspace of dimensionality $p=\max (p a, p b)$ spanned by the mutually independent functions $G_{j}(k)(j=1, \ldots, p)$. The preceding functions form a functional basis, while $\boldsymbol{A}_{i, j}, \boldsymbol{B}_{i, j}$ represent the corresponding, fully parametrized, projection matrices. The representation is parametrized in terms of the projection coefficient vector $\boldsymbol{\theta}$, comprising all elements of the AR and X projection matrices. The representation of Eq. (3.6a)-(3.6d) is referred to as an FP-VARX representation of orders $(n a, n b)$ and functional subspace dimensionalities $(p a, p b)$, or in short as an FP$\operatorname{VARX}(n a, n b)_{[p a, p b]}$ representation.

\subsubsection{Global model identification methodology}

For the sake of generality, the FP-VARX representation case is treated. In order to estimate the FP$\operatorname{VARX}(n a, n b)_{[p a, p b]}$ parameters, Eq. (3.6a) is cast into a linear regression form:

$$
\boldsymbol{y}_{k}[t]=\boldsymbol{\Phi}_{k}^{T}[t] \boldsymbol{\theta}+\boldsymbol{e}_{k}[t, \boldsymbol{\theta}]
$$


with:

$$
\begin{gathered}
\boldsymbol{\Phi}_{k}^{T}[t]=\boldsymbol{I} \otimes \boldsymbol{\phi}_{k}^{T}[t] \\
\boldsymbol{\phi}_{k}[t]=\left[\begin{array}{c}
-\boldsymbol{y}_{k}[t-1] \\
\vdots \\
-\boldsymbol{y}_{k}[t-n a]
\end{array}\right] \otimes\left[\begin{array}{c}
G_{1}(k) \\
\vdots \\
\boldsymbol{x}_{k}[t] \\
\vdots \\
G_{p a}(k)
\end{array}\right] \\
{\left[\begin{array}{c}
G_{1}(k) \\
\vdots \\
\boldsymbol{x}_{k b}(t-n b]
\end{array}\right]}
\end{gathered}
$$

and $\otimes$ designating the Kronecker product ${ }^{3}$.

Model parameter estimation requires stacking the equations of the form (3.7a), for each distinct operating condition, say $\left\{k_{1}, k_{2}, \ldots, k_{M}\right\}$, one below the other. This procedure is referred to as pooling, while the term functional pooling signifies the functional dependence of each equation on the operating parameter (temperature) $k$ :

$$
\boldsymbol{y}[t]=\boldsymbol{\Phi}^{T}[t] \boldsymbol{\theta}+\boldsymbol{e}[t, \boldsymbol{\theta}]
$$

with:

$$
\boldsymbol{y}[t]=\left[\begin{array}{c}
\boldsymbol{y}_{k_{1}}[t] \\
\boldsymbol{y}_{k_{2}}[t] \\
\vdots \\
\boldsymbol{y}_{k_{M}}[t]
\end{array}\right], \quad \boldsymbol{\Phi}^{T}[t]=\left[\begin{array}{c}
\boldsymbol{\Phi}_{k_{1}}^{T}[t] \\
\boldsymbol{\Phi}_{k_{2}}^{T}[t] \\
\vdots \\
\boldsymbol{\Phi}_{k_{M}}^{T}[t]
\end{array}\right], \quad \boldsymbol{e}[t, \boldsymbol{\theta}]=\left[\begin{array}{c}
\boldsymbol{e}_{k_{1}}[t, \boldsymbol{\theta}] \\
\boldsymbol{e}_{k_{2}}[t, \boldsymbol{\theta}] \\
\vdots \\
\boldsymbol{e}_{k_{M}}[t, \boldsymbol{\theta}]
\end{array}\right]
$$

The following Weighted Least Squares (WLS) criterion may be then used for estimating $\boldsymbol{\theta}$ (refer to Chapter 2, Section 2.3):

$$
J\left(\boldsymbol{\theta}, Z^{N M}\right)=\frac{1}{N M} \sum_{t=1}^{N} \boldsymbol{e}^{T}[t, \boldsymbol{\theta}] \boldsymbol{\Sigma}^{-1} \boldsymbol{e}[t, \boldsymbol{\theta}]
$$

with $\boldsymbol{\Sigma}$ designating the $(M n y \times M n y)$ innovations covariance matrix. Assuming that the innovations of each operating condition $k$ have different covariance matrices $\boldsymbol{\Sigma}_{k, k}$ and are uncorrelated with those of any other operating condition (groupwise heteroscedasticity; refer to Chapter 2, Table 2.1), the covariance matrix has the block diagonal form:

$$
\boldsymbol{\Sigma}=\left[\begin{array}{cccc}
\boldsymbol{\Sigma}_{k_{1}, k_{1}} & \mathbf{0} & \ldots & \mathbf{0} \\
\mathbf{0} & \boldsymbol{\Sigma}_{k_{2}, k_{2}} & \ldots & \mathbf{0} \\
\vdots & \vdots & \ddots & \vdots \\
\mathbf{0} & \mathbf{0} & \ldots & \boldsymbol{\Sigma}_{k_{M}, k_{M}}
\end{array}\right]
$$

Minimization of $J\left(\boldsymbol{\theta}, Z^{N M}\right)$ with respect to $\boldsymbol{\theta}$ then yields the WLS estimator:

$$
\widehat{\boldsymbol{\theta}}_{W L S}=\left[\frac{1}{N M} \sum_{k=k_{1}}^{k_{M}} \sum_{t=1}^{N} \boldsymbol{\Phi}_{k}[t] \boldsymbol{\Sigma}_{k, k}^{-1} \boldsymbol{\Phi}_{k}^{T}[t]\right]^{-1}\left[\frac{1}{N M} \sum_{k=k_{1}}^{k_{M}} \sum_{t=1}^{N} \boldsymbol{\Phi}_{k}[t] \boldsymbol{\Sigma}_{k, k}^{-1} \boldsymbol{y}_{k}[t]\right]
$$

Note that the matrices $\boldsymbol{\Sigma}_{k, k}\left(k=k_{1}, \ldots, k_{M}\right)$ are normally unavailable. Thus, to obtain a practically applicable estimator, the criterion in Eq. (3.9) is minimized by replacing $\boldsymbol{\Sigma}^{-1}$ with the identity matrix (Ordinary Least Squares (OLS) estimation). The OLS estimates $\widehat{\Sigma}_{k, k}$ are subsequently inserted into Eq. (3.11) to

\footnotetext{
${ }^{3}$ The Kronecker product of an $(m \times n)$ matrix $\boldsymbol{A}=\left[a_{i, j}\right]$ and a $(p \times q)$ matrix $\boldsymbol{B}=\left[b_{i, j}\right]$ is $\boldsymbol{A} \otimes \boldsymbol{B}=\left[a_{i, j} \boldsymbol{B}\right]$ [43, p. 3].
} 
compute $\widehat{\boldsymbol{\theta}}_{W L S}$, while the residual covariance matrices and the parameter vector estimator covariance are obtained as (see also Appendix 3.B):

$$
\begin{gathered}
\widehat{\boldsymbol{\Sigma}}_{k, k}=\frac{1}{N} \sum_{t=1}^{N} \boldsymbol{e}_{k}\left[t, \widehat{\boldsymbol{\theta}}_{W L S}\right] \boldsymbol{e}_{k}^{T}\left[t, \widehat{\boldsymbol{\theta}}_{W L S}\right], \quad k=k_{1}, \ldots, k_{M} \\
\widehat{\boldsymbol{P}}\left(\widehat{\boldsymbol{\theta}}_{W L S}\right)=\left[\sum_{k=k_{1}}^{k_{M}} \sum_{t=1}^{N} \boldsymbol{\Phi}_{k}[t] \widehat{\boldsymbol{\Sigma}}_{k, k}^{-1} \boldsymbol{\Phi}_{k}^{T}[t]\right]^{-1}
\end{gathered}
$$

Alternatively, the procedure may be continued by inserting the estimates $\widehat{\boldsymbol{\Sigma}}_{k, k}$ into Eq. (3.11) in order to obtain a new WLS estimate, and repeated until $\widehat{\boldsymbol{\theta}}_{W L S}$ converges (iterated WLS estimation).

The determination of the AR, $\mathrm{X}$ model orders $(n a, n b)$, as well as the dimensionalities of the functional subspaces $(p a, p b)$, is important in FP-VARX identification. A sensible approach consists of fitting FP$\operatorname{VARX}(n, n)_{[p, p]}$ models with increasing order $n$ and increasing basis dimensionality $p$ on the data set $Z^{N M}$, until an adequate model, say FP-VARX $\left(n^{*}, n^{*}\right)_{\left[p^{*}, p^{*}\right]}$, is obtained. To avoid potential over-specification in the AR and X part functional subspaces, lower dimensionalities are also considered, until an adequate model is obtained.

Model adequacy may be judged like before, using criteria such as the determinant (or trace) of the estimated innovations covariance matrix $\widehat{\boldsymbol{\Sigma}}$ or the BIC (see Chapter 2, Section 2.6). In addition, frequency stabilization diagrams depicting the estimated frequencies as functions of the operating parameter $k$ for increasing basis dimensionality $p$, may also be used. Adequacy is in this case is considered to be attained as soon as the estimated frequencies of each operating condition converge.

Once an FP-VARX model has been obtained, information on the AR and X parameter matrices for any intermediate temperature is readily available. The following relationships associate the projection coefficient vector estimate $\widehat{\boldsymbol{\theta}}$ to the parameter vector $\widehat{\boldsymbol{\vartheta}}(k)$ comprising all elements of the AR and X matrices corresponding to the $k$-th temperature condition (see also Eq. (3.6c)-(3.6d)):

$$
\begin{gathered}
\widehat{\boldsymbol{\vartheta}}(k)=\boldsymbol{T}(k) \widehat{\boldsymbol{\theta}} \quad k \in \mathcal{R} \\
\boldsymbol{P}(\widehat{\boldsymbol{\vartheta}}(k))=\boldsymbol{T}(k) \boldsymbol{P}(\widehat{\boldsymbol{\theta}}) \boldsymbol{T}^{T}(k) \\
\boldsymbol{\vartheta}(k)=\operatorname{vec}\left(\left[\boldsymbol{A}_{1}(k) \boldsymbol{A}_{2}(k) \ldots \boldsymbol{A}_{n a}(k) \vdots \boldsymbol{B}_{0}(k) \boldsymbol{B}_{1}(k) \ldots \boldsymbol{B}_{n b}(k)\right]^{T}\right)
\end{gathered}
$$

with $\boldsymbol{T}(k)$ designating a transformation matrix whose entries are zeros and basis functions $G_{j}(k)$ at proper locations (see also Chapter 2, Appendix 2.A). Presently, the projection coefficient covariance $\boldsymbol{P}(\widehat{\boldsymbol{\theta}})$ in Eq. (3.13b) is replaced by its WLS-based estimate from Eq. (3.12b). The determination of modal parameter uncertainty follows the procedure discussed in Subsection 3.3.2.

\subsubsection{Global model identification results}

\subsubsection{The CCP-VARX approach.}

The analysis uses $N=2,000$ sample-long $(\approx 0.78 \mathrm{~s})$ data records for each temperature. The CCP-VARX orders are set to $n a=n b=23$ for direct comparison with the parametric VARX-based multi-model representation, and parameter estimation is based upon non-iterative WLS estimation (QR implementation; see Chapter 2, Appendix 2.E). The identified CCP-VARX $(23,23)$ representation has 140 parameters (SPP=900) and condition number $\sim 10^{5}$ for the WLS estimator inverted matrix (see Table 3.2 for details). 

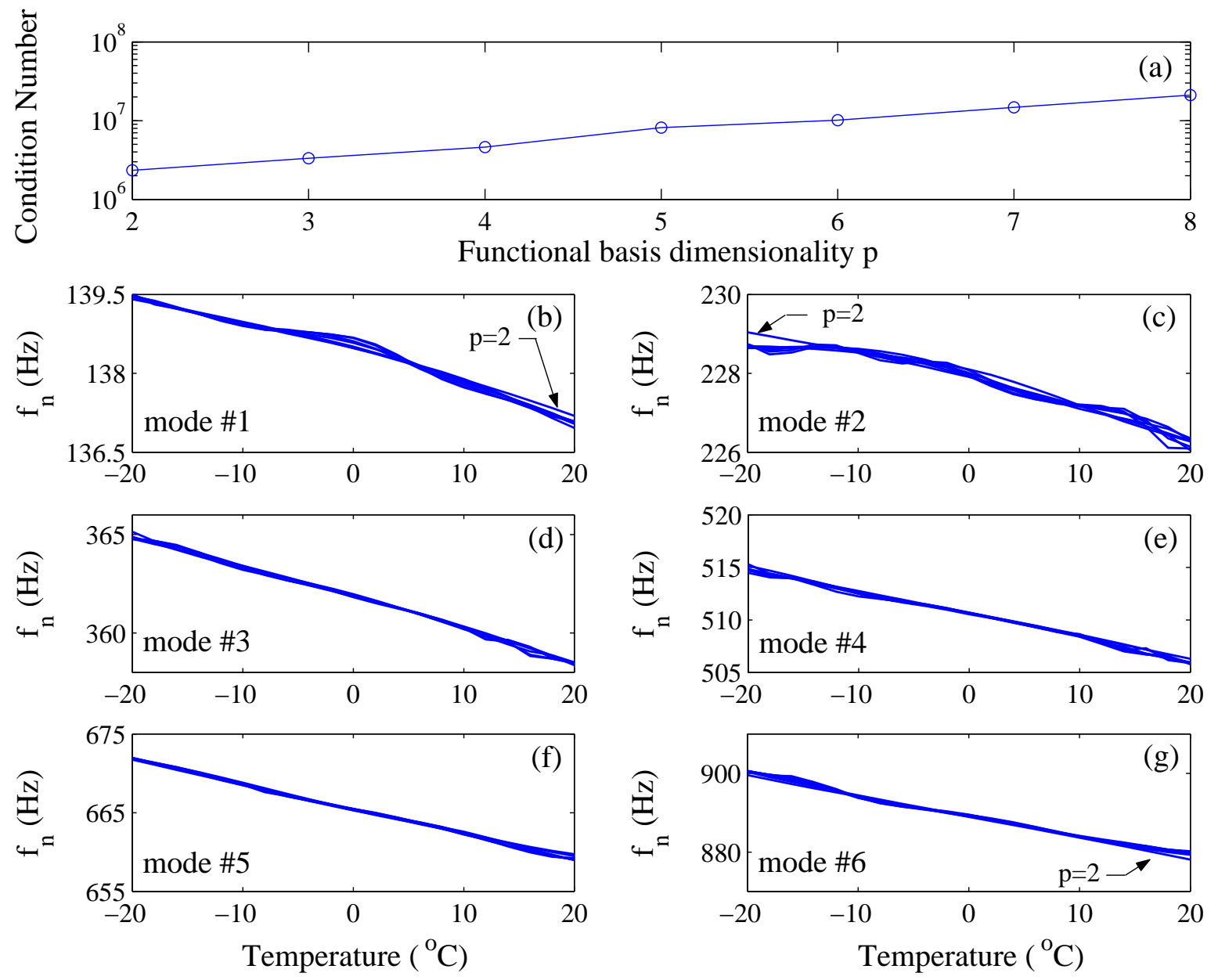

Figure 3.4: $\operatorname{FP}-\operatorname{VARX}(23,23)_{[p, p]}$ structure specification: (a) condition number of the WLS estimator inverted matrix versus functional basis dimensionality $p ;(\mathrm{b})-(\mathrm{g})$ convergence of the natural frequency $\left(f_{\mathrm{n}}\right)$ versus temperature curves for increasing functional subspace dimensionality $p=2,3, \ldots, 8$ (the arrows indicate curves for $p=2$; convergence occurs for $p \geq 3$ ).

\subsubsection{The FP-VARX approach.}

As in the previous case, $N=2,000$ sample-long $(\approx 0.78 \mathrm{~s})$ data records from each temperature are utilized. The AR and X parameters are projected into functional subspaces spanned by Chebyshev polynomials of the second kind (Appendix 3.C). In order to obtain results directly comparable to those of the CCP-VARX and the parametric VARX-based multi-model approaches, the FP-VARX orders are set to $n a=n b=23$, confining the search to specifying the appropriate functional subspace dimensionalities. A succession of $\operatorname{FP}-\operatorname{VARX}(23,23)_{[p, p]}$ representations with increasing dimensionality $p=2,3, \ldots, 8$ are fitted to the data based upon non-iterative WLS estimation (QR implementation; see Chapter 2, Appendix 2.E).

The diagrams in Fig. 3.4(b)-(g) depict the convergence of the natural frequency versus temperature curves for $\operatorname{FP}-\operatorname{VARX}(23,23)_{[p, p]}$ representations with increasing subspace dimensionality $p=2,3, \ldots, 8$. The convergence is very good and rapid (for $p \geq 3$ ). The $\operatorname{FP}-\operatorname{VARX}(23,23)_{[4,3]}$ representation with functional subspaces spanned by the first four Chebyshev polynomials for the AR part and the first three for the $\mathrm{X}$ part is a balanced compromise between model accuracy and parameter parsimony. It is characterized by 512 parameters $\left(\mathrm{SPP}=246.1\right.$ ) and condition number $\sim 10^{6}$ for the WLS estimator inverted matrix (see Table 3.2 for details). 

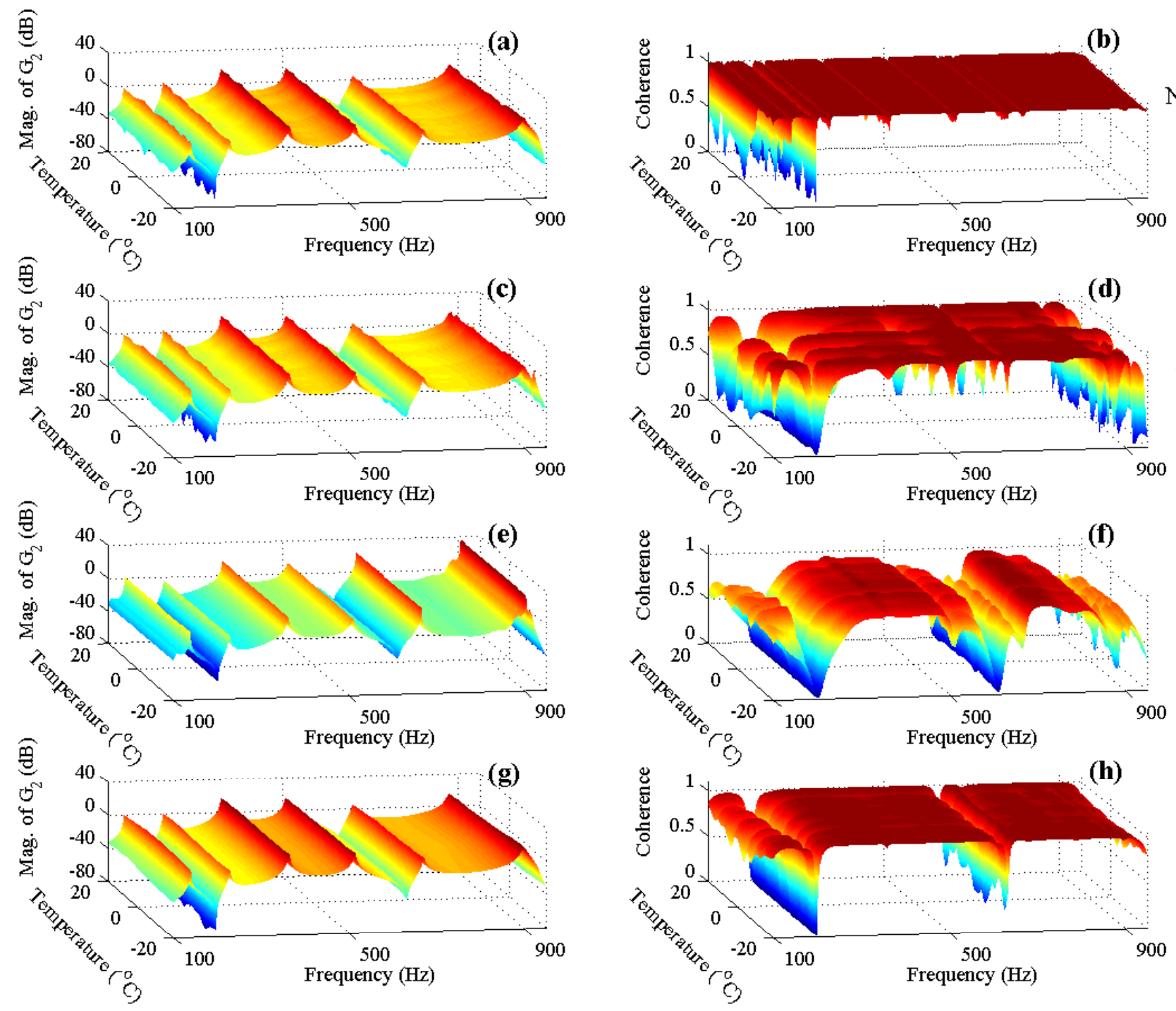

Non-parametric

Welch-based

multi-model approach

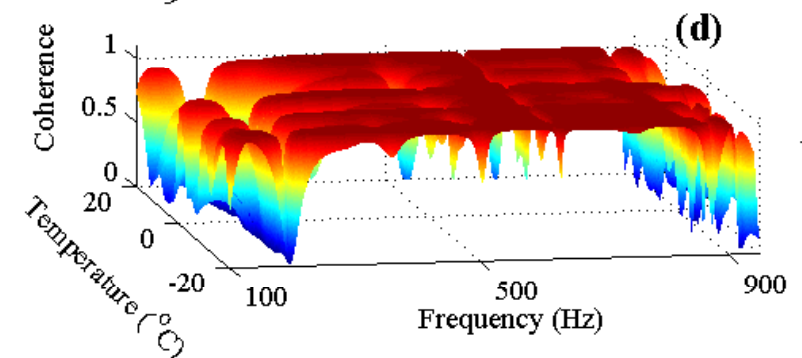

Parametric

VARX-based multi-model approach

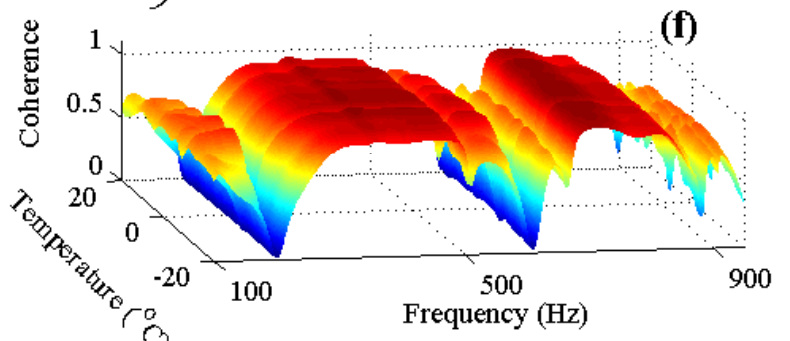

Global CCP-VARX approach

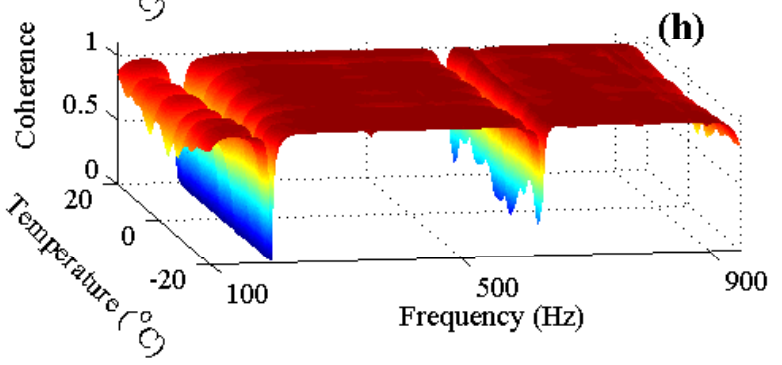

Global FP-VARX approach

Figure 3.5: FRF magnitude and coherence versus frequency and temperature $\left(G_{2}\right.$ transfer function): (a),(b) non-parametric Welch-based multi-model estimates; (c),(d) parametric VARX $(23,23)$-based multi-model estimates; (e),(f) CCP-VARX $(23,23)$ estimates; (g),(h) FP-VARX $(23,23)_{[4,3]}$ estimates.

\subsection{Assessment of the multi-model and global model approaches}

Indicative identification results $\left(G_{2}\right.$ transfer function) are presented in Fig. 3.5. The agreement among the FP-VARX and multi-model FRFs is good. Furthermore note that, unlike the multi-model based FRFs, the FP-VARX $(23,23)_{[4,3]}$ based ones are intrinsic functions of both frequency and temperature, and thus capable of providing a "smooth" and "natural" model dependence on temperature (Fig. 3.5(g)). The corresponding coherence appears uniform, with values near unity for all considered temperatures. The close resemblance of the $\operatorname{FP}-\operatorname{VARX}(23,23)_{[4,3]}$ and the non-parametric Welch-based estimates builds further confidence on the FP-VARX approach, as it provides an accurate and valid, under all considered temperatures, representation of the dynamics. The CCP-VARX $(23,23)$ representation identifies the first four modes fairly well, but exhibits noteworthy discrepancies for frequencies greater than $520 \mathrm{~Hz}$ (Fig. 3.5(e)). This is due to the assumption of constant AR and X parameter matrices, and it is also reflected in the poor coherence estimate (Fig. 3.5(f)). 

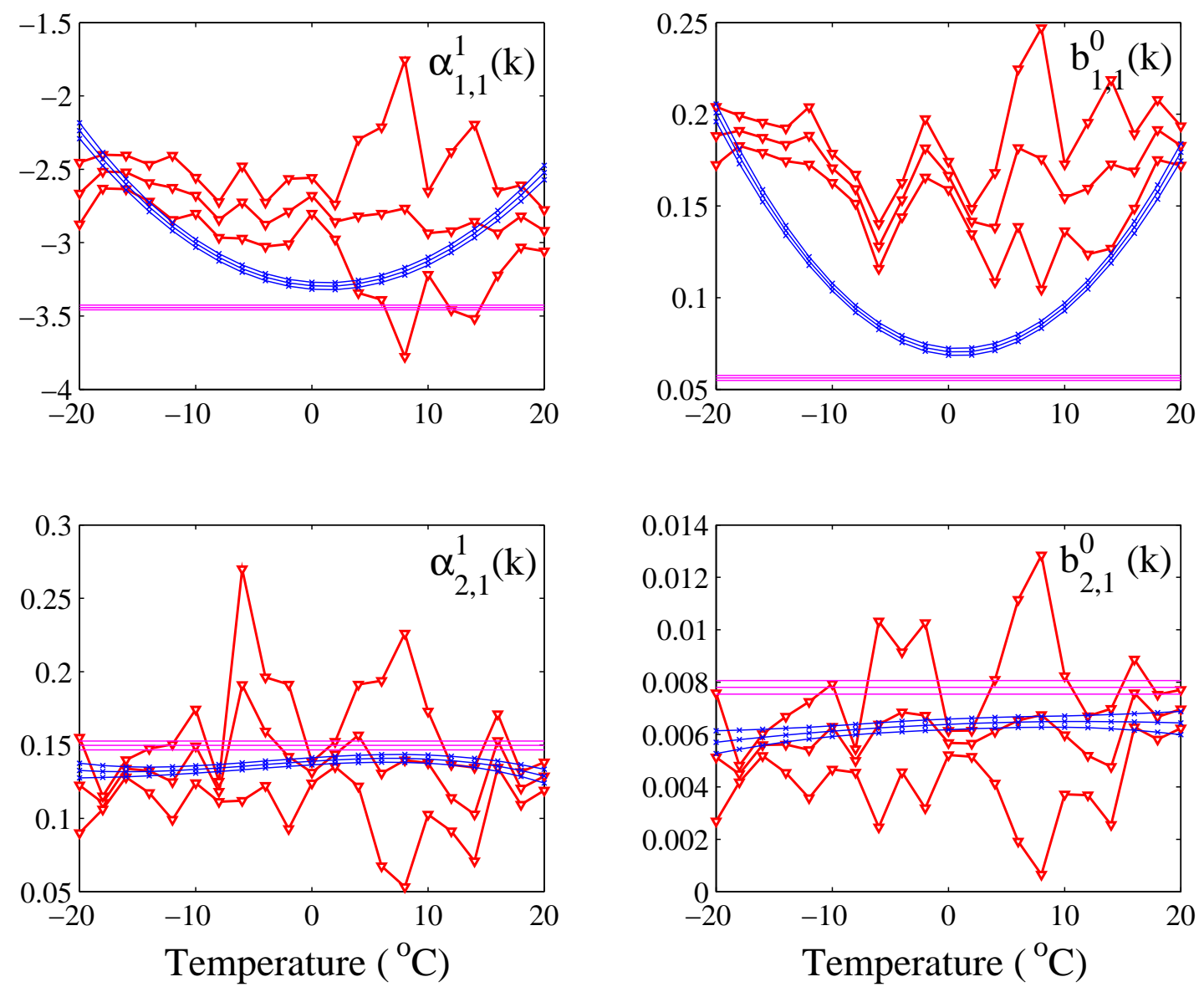

Figure 3.6: Dependence of indicative AutoRegressive and eXogenous parameters on temperature (point estimate $\pm 1.96 \times$ standard deviation; $a_{m, n}^{i}(k)$ and $b_{m, n}^{i}(k)$ designate the $(m, n)$-th elements of $\boldsymbol{A}_{i}(k)$ and $\boldsymbol{B}_{i}(k)$, respectively): parametric $\operatorname{VARX}(23,23)$-based multi-model representation $(-\nabla)$, CCP$\operatorname{VARX}(23,23)$ representation $(-)$, and $\operatorname{FP}-\operatorname{VARX}(23,23)_{[4,3]}$ representation $(*)$.

The temperature dependence of indicative parameters from the identified VARX-based multi-model and global representations is presented in Fig. 3.6. The uncertainty associated with each parameter is given by its $95 \%$ confidence interval (point estimate $\pm 1.96 \times$ standard deviation). The "smooth" dependence of the $\operatorname{FP}-\operatorname{VARX}(23,23)_{[4,3]}$ parameters on temperature contrasts the statistically "biased" parameters of the CCP$\operatorname{VARX}(23,23)$ model and the "noisy" $\operatorname{VARX}(23,23)$-based multi-model parameters. The comparably lower uncertainty of the global representations over their VARX-based multi-model counterpart may be attributed to the smaller condition numbers of the global model parameter vector estimator covariance matrices (see Table 3.2). The model parameter uncertainty levels also reflect on the modal parameters via Eq. (3.2), implying that the modal uncertainties of the global representations are expected to be smaller compared to their VARX-based multi-model counterparts.

The dependence of the identified (by all approaches) natural frequencies on temperature is presented in Fig. 3.7. Excellent agreement is observed among the multi-model and FP-VARX approaches. The FP$\operatorname{VARX}(23,23)_{[4,3]}$ representation provides smooth dependency on temperature for all six modes, which is compatible with the physics of the problem. On the other hand, the CCP-VARX $(23,23)$ representation "averages out" the effects of temperature, which is expected as a direct consequence of the constant AR and X parameter matrices. This "averaging" effect is evident for modes 1-5; for mode 6 three distinct frequencies are identified (Fig. 3.7(f)). This may be attributed to the comparably heavier dependence on temperature (higher slope) for that mode. The natural frequencies exhibit a clear decreasing trend with increasing tem- 

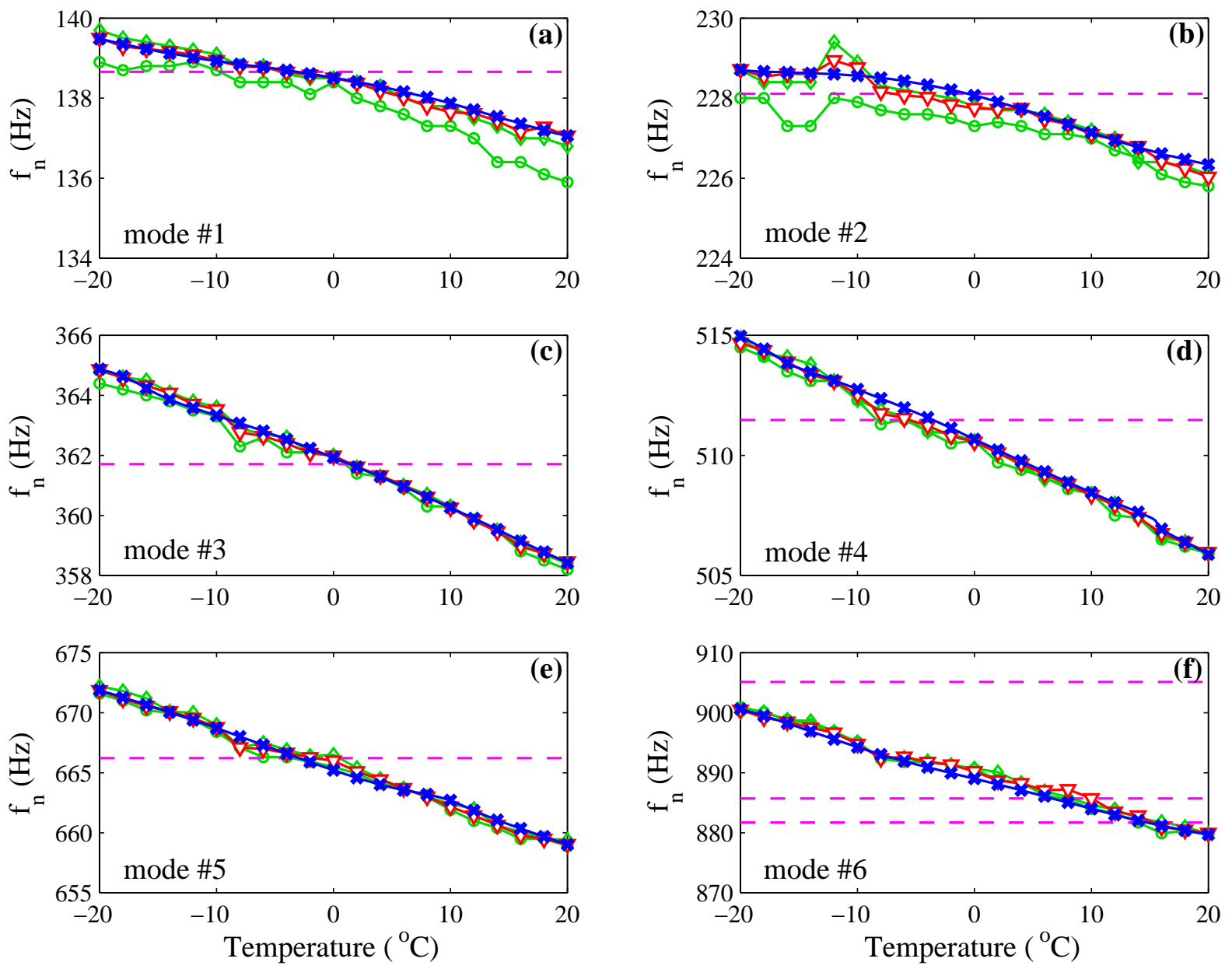

Figure 3.7: Dependence of the estimated natural frequencies $\left(f_{\mathrm{n}}\right)$ on temperature: non-parametric Welchbased multi-model representation $\left(-\circ-G_{1} ; \multimap-G_{2}\right)$, VARX $(23,23)$ multi-model representation $\left(-\nabla^{-}\right)$, CCP-VARX $(23,23)$ representation (--), and FP-VARX $(23,23)_{[4,3]}$ representation $(*)$.

perature, whereas the dependence on temperature is more pronounced for increasing mode number (slopes ranging from $0.06 \mathrm{~Hz} /{ }^{\circ} \mathrm{C}$ for mode 1 to $0.52 \mathrm{~Hz} /{ }^{\circ} \mathrm{C}$ for mode 6 according to the $\mathrm{FP}-\operatorname{VARX}(23,23)_{[4,3]}$ estimates). Furthermore, it appears that the first two natural frequencies exhibit a weakly nonlinear dependence on temperature, whereas the rest exhibit a predominantly linear dependence.

Similarly good agreement among the multi-model and the FP-VARX based damping factor estimates is observed for all six modes (Fig. 3.8). The smooth $\operatorname{FP}-\operatorname{VARX}(23,23)_{[4,3]}$ damping factor dependency with temperature is even more pronounced when compared to its multi-model based counterparts. It is noteworthy that the discordance of the non-parametric Welch-based damping factor estimates for $G_{1}$ in modes 1-2 is due to the peak-picking method, which provides "noisy" estimates when the FRF peaks are not sufficiently clear. The CCP-VARX $(23,23)$ representation "averages out" the temperature effects for the damping factors of modes 1-4, yet underestimates the damping factors of modes 5-6 (Fig. 3.8(e),(f)). The overall trend for the damping factor estimates over temperature appears to be sensibly constant (indicating a rather weak but potentially more complex dependence), which complies with that reported in [54] over a similar temperature range (although different composite material and boundary conditions). Furthermore, the range of the obtained damping factors is in agreement with those in [57] (same composite material but different boundary conditions).

The uncertainties associated with the obtained modal parameter estimates for the VARX-based multi- 

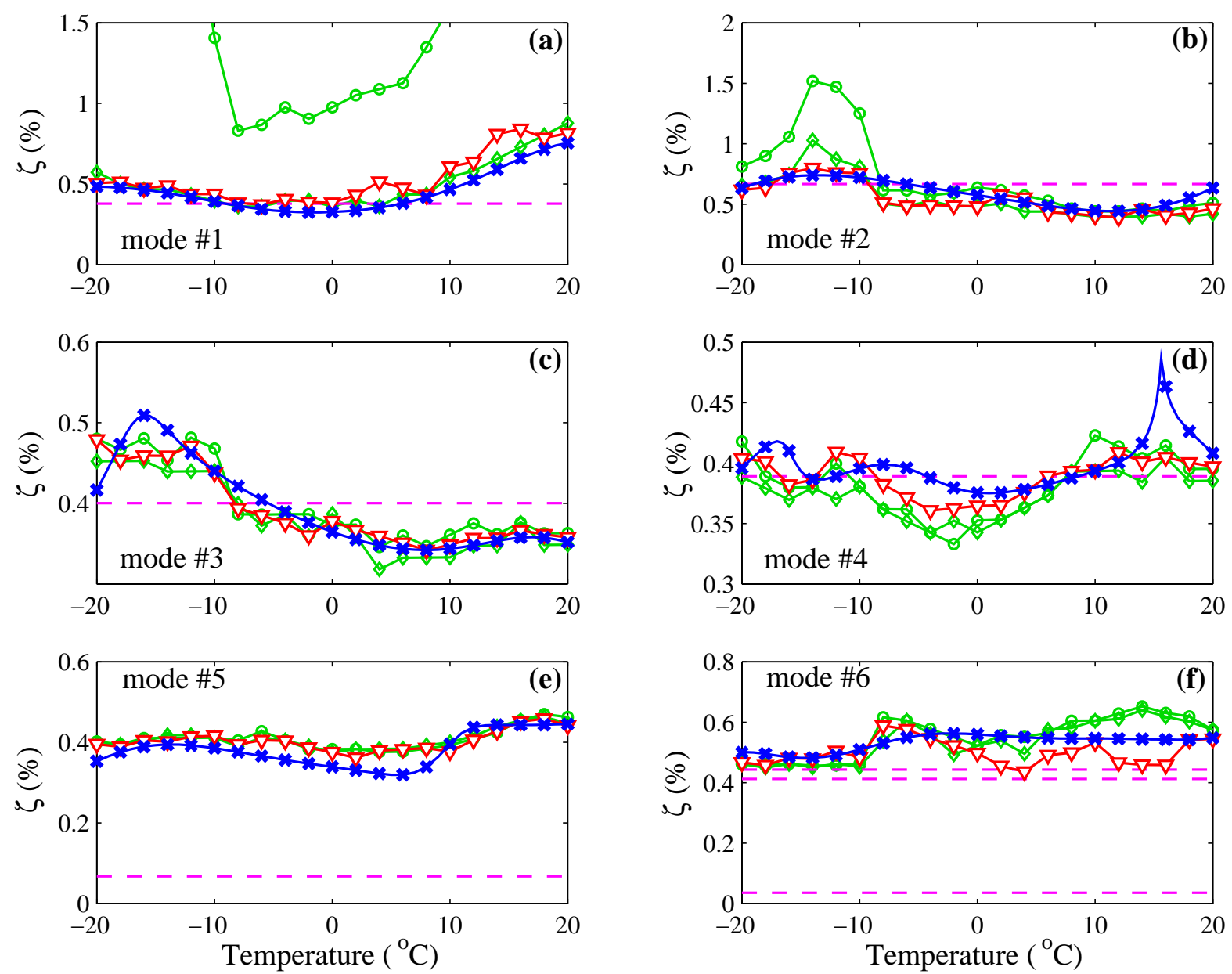

Figure 3.8: Dependence of the estimated damping factors $(\zeta)$ on temperature: non-parametric Welch-based multi-model representation $\left(-\circ-G_{1} ; \multimap-G_{2}\right)$, VARX $(23,23)$ multi-model representation $(-\nabla-)$, CCP$\operatorname{VARX}(23,23)$ representation $(--)$, and $\operatorname{FP}-\operatorname{VARX}(23,23)_{[4,3]}$ representation $(*)$.

model and global representations are presented next. Fig. 3.9 -3.10 present the corresponding natural frequency and damping factor point estimates, and their uncertainties as \pm one standard deviation zones.

The $\operatorname{VARX}(23,23)$ multi-model based natural frequency uncertainties are quite larger compared to their FP-VARX $(23,23)_{[4,3]}$ counterparts (Fig. 3.9(a),(b), see also Appendix 3.D). Notice in the former case and for a certain temperature, the mode 6 uncertainty becomes excessively large. This may be attributed to the numerical differentiation used in the uncertainty computation. Nevertheless, the alternative use of analytical differentiation [70] is not recommended as it would require the inversion of the parameter vector estimator covariance matrix which is presently characterized by large condition numbers $\left(\sim 10^{11}\right.$ and $\sim$ $10^{15}$; see Table 3.2). Furthermore, the $\operatorname{FP}-\operatorname{VARX}(23,23)_{[4,3]}$ uncertainty appears more "evenly" distributed over temperature. The CCP-VARX $(23,23)$ based uncertainties appear quantitatively similar to their FP$\operatorname{VARX}(23,23)_{[4,3]}$ based counterparts; a fact attributed to the increased statistical accuracy attained via data pooling.

Similar observations hold for uncertainties of the damping factors, as those corresponding to the $\operatorname{VARX}(23,23)$ multi-model representation are quite larger than those of their FP-VARX $(23,23)_{[4,3]}$ counterparts (Fig. 3.10(a),(b)). Notice that in the former case, the mode 6 damping factor uncertainty becomes excessively large for certain temperatures. Finally, the weak dependence of damping factors on temperature is observed on this diagram as well. 

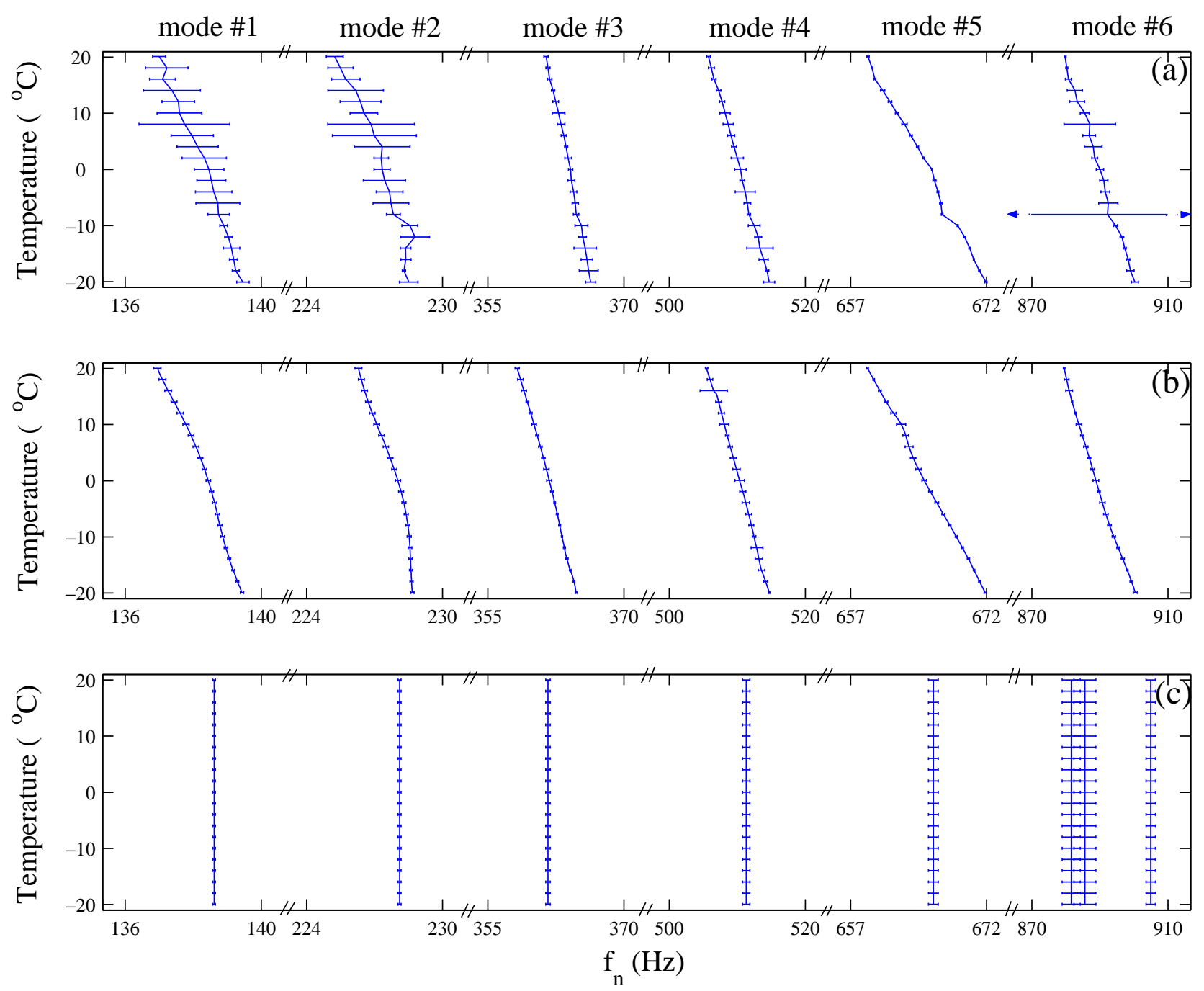

Figure 3.9: Natural frequency $\left(f_{\mathrm{n}}\right)$ estimates and their uncertainties ( \pm one standard deviation): (a) $\operatorname{VARX}(23,23)$ multi-model representation, (b) $\operatorname{FP}-\operatorname{VARX}(23,23)_{[4,3]}$ representation, and (c) CCP$\operatorname{VARX}(23,23)$ representation (the horizontal arrows indicate large uncertainty estimates).

\subsection{Concluding remarks}

The identification of temperature effects on the dynamics of a smart composite beam was addressed via conventional multi-model and novel global model approaches. The multi-model approaches are based on non-parametric and parametric VARX representations, whereas the global approaches are based on parametric CCP-VARX and FP-VARX representations. The main conclusions drawn are:

(i) The multi-model and global representations identified are in rough overall agreement. Yet, the global models are advantageous in that they simultaneously use all available data records, thus offering improved numerical robustness and much more compact representations of the dynamics.

(ii) Among the global representations, the CCP-VARX ones are limited in scope, as they are characterized by AR and X parameters that do not depend on temperature and provide only "averaged" descriptions of the dynamics over temperature. On the other hand, the FP-VARX constitute the essential representations of choice as they allow for the explicit, analytical, modeling of temperature dependence. Along with their compactness and improved numerical robustness, they achieve improved estimation accu- 

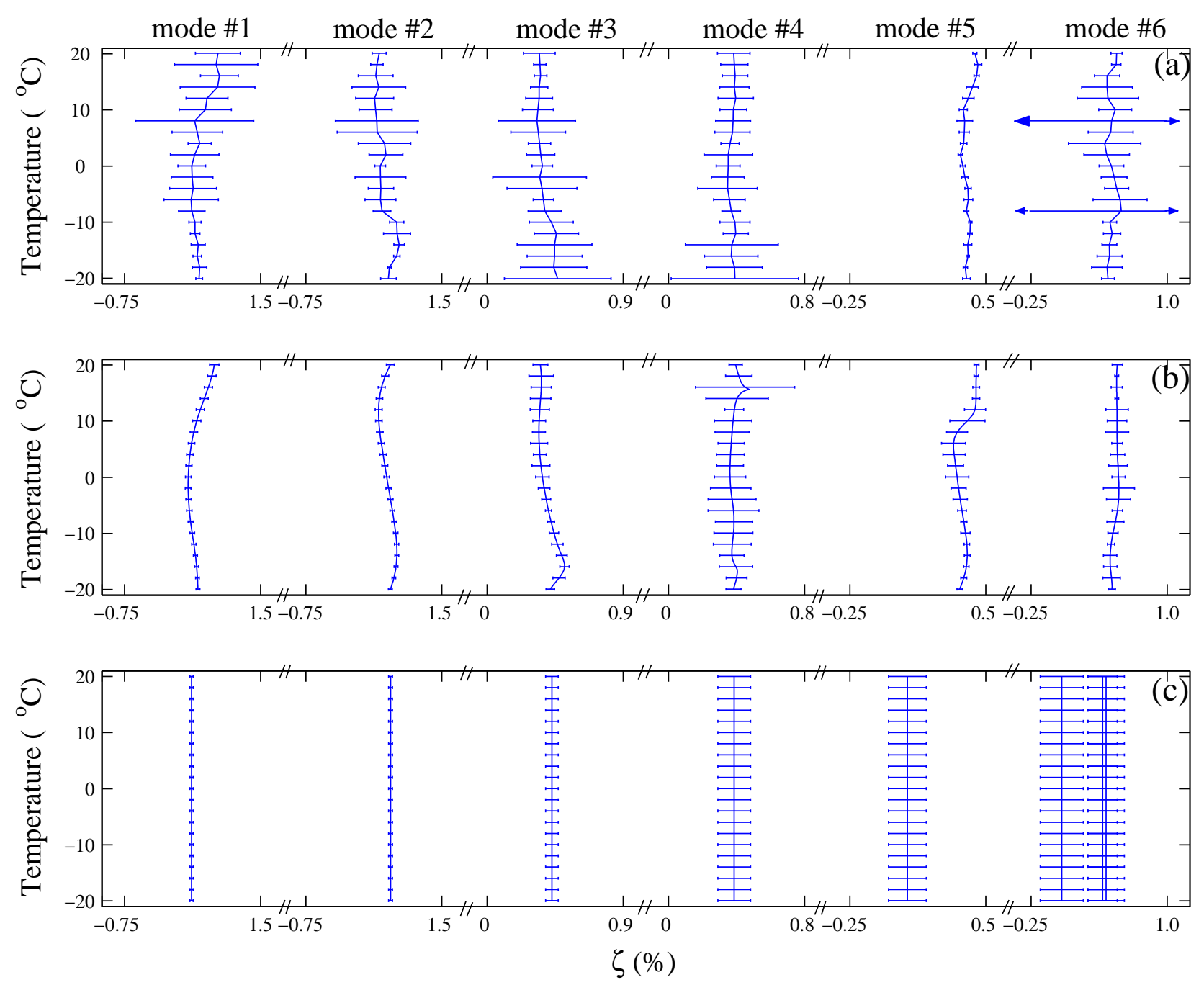

Figure 3.10: Damping factor $(\zeta)$ estimates and their uncertainties ( \pm one standard deviation): (a) $\operatorname{VARX}(23,23)$ multi-model representation, (b) $\operatorname{FP}-\operatorname{VARX}(23,23)_{[4,3]}$ representation, and (c) CCP$\operatorname{VARX}(23,23)$ representation (the horizontal arrows indicate large uncertainty estimates).

racy over their multi-model counterparts, which is reflected in significantly smaller modal parameter estimate uncertainties.

(iii) In accordance with previous studies, the natural frequency estimates decrease with temperature in a weakly nonlinear or approximately linear fashion. This dependence becomes more evident for higher frequency modes. Nevertheless, the damping factor estimates remain sensibly constant, indicating a dependence on temperature that is weaker, but of potentially more complex nature.

Overall, the global modeling approach - and specifically the one based on FP-VARX representations provides a convenient means of identifying the structural dynamics dependence on temperature and performing uncertainty analysis, and is recommended for achieving the highest accuracy and maximum information extraction from experimental data. Indeed, its advantages over the conventional multi-model approach in terms of achievable accuracy, numerical robustness, physical insight, temperature interpolation capability, and, last but not least, compactness of representation are important, and underscore its usefulness as a tool for precise mathematical modeling, improved physical understanding, structural health monitoring, and automatic control. 
Appendix 3.A: Material properties of the glass-polyester composite and the piezoceramic patches obtained at room temperature [71]

\begin{tabular}{|c|c|c|}
\hline & glass-polyester & PZT-5A \\
\hline \multicolumn{3}{|c|}{ Elastic Properties } \\
\hline$E_{11}$ & 25.8 & 58.8 \\
\hline$E_{22}$ & 8.7 & 58.8 \\
\hline$E_{33}$ & 8.7 & 43.1 \\
\hline$G_{23}$ & 2.4 & 23 \\
\hline$G_{13}$ & 3.5 & 23 \\
\hline$G_{12}$ & 3.5 & 21.2 \\
\hline$v_{12}$ & 0.34 & 0.37 \\
\hline$v_{13}$ & 0.34 & 0.39 \\
\hline$v_{23}$ & 0.47 & 0.39 \\
\hline \multicolumn{3}{|c|}{ Damping Properties (\%) } \\
\hline$n_{11}$ & 0.8 & 0 \\
\hline$n_{12}$ & 1.7 & 0 \\
\hline$n_{14}$ & 1.9 & 0 \\
\hline$n_{16}$ & 2.05 & 0 \\
\hline \multicolumn{3}{|c|}{ Piezoelectric Coefficients $\left(10^{-12} \mathrm{~m} / \mathrm{V}\right)$} \\
\hline$d_{31}$ & 0 & -170 \\
\hline$d_{32}$ & 0 & -170 \\
\hline$d_{24}$ & 0 & 56 \\
\hline$d_{15}$ & 0 & 506 \\
\hline \multicolumn{3}{|c|}{ Electric Permittivity } \\
\hline$\varepsilon_{11} / \varepsilon_{0}$ & 3.5 & 1,800 \\
\hline$\varepsilon_{22} / \varepsilon_{0}$ & 3 & 180 \\
\hline$\varepsilon_{33} / \varepsilon_{0}$ & 3 & 1,800 \\
\hline \multicolumn{3}{|c|}{ Mass Density $\left(\mathrm{kg} / \mathrm{m}^{3}\right)$} \\
\hline$\rho$ & 1,672 & 7,700 \\
\hline
\end{tabular}

\section{Appendix 3.B: Asymptotic properties of the WLS-based projection coefficient estimator}

Let $\boldsymbol{\theta}_{o}$ be the system's true projection coefficient vector and $\boldsymbol{w}[t]$ a sequence of identically and independently distributed (i.i.d.), zero mean, $(M n y \times 1)$ random vectors with positive definite covariance matrix $\boldsymbol{\Sigma}_{o}$ and bounded fourth-order moments. Assuming that $E\left\{\boldsymbol{\Phi}[t] \boldsymbol{\Phi}^{T}[t]\right\}$ is a finite positive definite matrix, then the following properties hold asymptotically (that is , as $N \longrightarrow \infty$ ) for the WLS estimator $\widehat{\boldsymbol{\theta}}_{W L S}$ :

- consistency: $\quad \widehat{\boldsymbol{\theta}}_{W L S} \stackrel{p}{\longrightarrow} \boldsymbol{\theta}_{O}$

- asymptotic normality: $\quad \widehat{\boldsymbol{\theta}}_{W L S} \sim \mathcal{N}\left(\boldsymbol{\theta}_{o}, \frac{1}{N} E\left\{\boldsymbol{\Phi}[t] \boldsymbol{\Sigma}_{o}^{-1} \boldsymbol{\Phi}^{T}[t]\right\}^{-1}\right)$

- asymptotic efficiency: $\underbrace{\boldsymbol{P}\left(\widehat{\boldsymbol{\theta}}_{W L S}\right)=\frac{1}{N} E\left\{\boldsymbol{\Phi}[t] \boldsymbol{\Sigma}_{o}^{-1} \boldsymbol{\Phi}^{T}[t]\right\}^{-1}}_{\text {Cramér-Rao lower bound }}$

Notice that $\mathcal{N}(\cdot, \cdot)$, above, designates Gaussian distribution with the indicated mean and variance. 


\section{Appendix 3.C: Chebyshev polynomials of the second kind}

The Chebyshev polynomials of the second kind are a set of orthogonal polynomials over the range $x \in$ $[-1,+1] \subset \mathbb{R}$. They are presently designated as $G_{n}(x)$ and obey the following recurrence relation [45, p. 782]:

$$
G_{n+1}(x)=2 x \cdot G_{n}(x)-G_{n-1}(x), \text { with } G_{1}(x)=1, G_{2}(x)=2 x .
$$

In the present framework $x=k /(M-1)$, since the operating parameter $k \in[-20,+20]^{\circ} C$ is discretized at increments $\Delta k=2^{\circ} C(M=21$ experiments $)$, thus yielding $k_{1}=-20^{\circ} C, k_{2}=-18^{\circ} C, \ldots, k_{21}=+20^{\circ} C$.

\section{Appendix 3.D: Dependency of natural frequency estimates on temperature}

The dependency of natural frequency estimates on temperature, for the FP-VARX and VARX-based multimodel approaches, is presented in Table 3.3. The data tabulated correspond to certain of the results (a temperature increment of $4^{\circ} \mathrm{C}$ is used) in Fig. 3.9(a),(b), and show the natural frequency point estimates along with their uncertainties ( \pm one standard deviation). The standard deviation obtained by the VARXbased multi-model approach at $-8^{\circ} \mathrm{C}$ for mode 6 is excessively large due to the numerical differentiation used.

Table 3.3: Natural frequency $\left(f_{\mathrm{n}}\right)$ estimates and their uncertainties ( \pm one standard deviation) for the VARXbased multi-model and FP-VARX approaches.

\begin{tabular}{|c|c|c|c|c|c|c|c|}
\hline \multirow{2}{*}{$\begin{array}{c}\text { Temperature } \\
\left({ }^{\circ} \mathrm{C}\right)\end{array}$} & \multicolumn{6}{|c|}{ Natural frequency point estimate \pm one standard deviation $(\mathrm{Hz})$} & \\
\hline & mode \#1 & mode \#2 & mode \#3 & mode \#4 & mode \#5 & mode \#6 & \\
\hline \multirow{2}{*}{-20} & $139.48 \pm 0.05$ & $228.71 \pm 0.07$ & $364.87 \pm 0.09$ & $514.96 \pm 0.10$ & $671.87 \pm 0.11$ & $900.60 \pm 0.58$ & FP-VARX \\
\hline & $139.50 \pm 0.18$ & $228.72 \pm 0.34$ & $364.86 \pm 0.73$ & $514.67 \pm 0.81$ & $671.85 \pm 0.12$ & $900.48 \pm 1.02$ & VARX \\
\hline \multirow{2}{*}{-16} & $139.23 \pm 0.04$ & $228.64 \pm 0.07$ & $364.24 \pm 0.09$ & $513.84 \pm 0.47$ & $670.66 \pm 0.09$ & $898.23 \pm 0.22$ & FP-VARX \\
\hline & $139.23 \pm 0.12$ & $228.61 \pm 0.19$ & $364.32 \pm 0.84$ & $513.89 \pm 0.66$ & $670.54 \pm 0.06$ & $898.48 \pm 0.50$ & VARX \\
\hline \multirow{2}{*}{-12} & $139.02 \pm 0.05$ & $228.60 \pm 0.08$ & $363.58 \pm 0.11$ & $513.12 \pm 0.86$ & $669.39 \pm 0.11$ & $895.56 \pm 0.41$ & FP-VARX \\
\hline & $139.08 \pm 0.11$ & $228.94 \pm 0.53$ & $363.71 \pm 0.54$ & $513.10 \pm 0.49$ & $669.55 \pm 0.09$ & $896.65 \pm 0.52$ & VARX \\
\hline \multirow{2}{*}{-8} & $138.85 \pm 0.06$ & $228.51 \pm 0.09$ & $363.07 \pm 0.10$ & $512.37 \pm 0.36$ & $668.01 \pm 0.11$ & $893.03 \pm 0.53$ & FP-VARX \\
\hline & $138.79 \pm 0.32$ & $228.16 \pm 0.25$ & $362.77 \pm 0.38$ & $511.75 \pm 0.24$ & $667.09 \pm 0.09$ & $892.40 \pm$ large & VARX \\
\hline \multirow{2}{*}{-4} & $138.70 \pm 0.06$ & $228.33 \pm 0.09$ & $362.53 \pm 0.10$ & $511.57 \pm 0.53$ & $666.59 \pm 0.21$ & $890.90 \pm 0.78$ & FP-VARX \\
\hline & $138.65 \pm 0.53$ & $228.02 \pm 0.48$ & $362.40 \pm 0.48$ & $511.24 \pm 1.44$ & $666.64 \pm 0.11$ & $891.79 \pm 1.31$ & VARX \\
\hline \multirow{2}{*}{0} & $138.51 \pm 0.07$ & $228.07 \pm 0.11$ & $361.93 \pm 0.27$ & $510.67 \pm 0.69$ & $665.21 \pm 0.24$ & $889.01 \pm 0.86$ & FP-VARX \\
\hline & $138.51 \pm 0.44$ & $227.75 \pm 0.29$ & $361.98 \pm 0.31$ & $510.56 \pm 0.83$ & $665.99 \pm 0.04$ & $890.22 \pm 1.06$ & VARX \\
\hline \multirow{2}{*}{+4} & $138.29 \pm 0.07$ & $227.73 \pm 0.12$ & $361.29 \pm 0.19$ & $509.77 \pm 0.45$ & $664.01 \pm 0.30$ & $887.08 \pm 0.44$ & FP-VARX \\
\hline & $138.18 \pm 0.60$ & $227.75 \pm 1.01$ & $361.33 \pm 0.22$ & $509.65 \pm 0.36$ & $664.43 \pm 0.12$ & $888.21 \pm 2.58$ & VARX \\
\hline \multirow{2}{*}{+8} & $138.02 \pm 0.08$ & $227.35 \pm 0.12$ & $360.61 \pm 0.15$ & $508.88 \pm 0.25$ & $663.19 \pm 0.31$ & $885.04 \pm 0.48$ & FP-VARX \\
\hline & $137.80 \pm 1.33$ & $227.35 \pm 1.57$ & $360.61 \pm 0.54$ & $508.80 \pm 0.76$ & $663.00 \pm 0.29$ & $887.23 \pm 7.58$ & VARX \\
\hline \multirow{2}{*}{+12} & $137.71 \pm 0.09$ & $226.95 \pm 0.12$ & $359.90 \pm 0.15$ & $508.03 \pm 0.43$ & $661.86 \pm 0.30$ & $883.00 \pm 0.28$ & FP-VARX \\
\hline & $137.62 \pm 0.48$ & $226.97 \pm 0.74$ & $359.84 \pm 0.41$ & $507.92 \pm 0.37$ & $661.43 \pm 0.20$ & $883.51 \pm 2.19$ & VARX \\
\hline \multirow{2}{*}{+16} & $137.36 \pm 0.10$ & $226.61 \pm 0.13$ & $359.15 \pm 0.28$ & $506.93 \pm 1.98$ & $660.35 \pm 0.16$ & $881.17 \pm 0.99$ & FP-VARX \\
\hline & $137.16 \pm 0.38$ & $226.43 \pm 0.52$ & $358.97 \pm 0.31$ & $506.75 \pm 0.28$ & $659.78 \pm 0.10$ & $880.94 \pm 0.88$ & VARX \\
\hline \multirow{2}{*}{+20} & $137.06 \pm 0.11$ & $226.34 \pm 0.15$ & $358.42 \pm 0.23$ & $505.88 \pm 0.18$ & $659.01 \pm 0.09$ & $879.69 \pm 0.23$ & FP-VARX \\
\hline & $137.06 \pm 0.19$ & $226.03 \pm 0.31$ & $358.46 \pm 0.31$ & $505.97 \pm 0.28$ & $659.04 \pm 0.13$ & $879.95 \pm 0.26$ & VARX \\
\hline
\end{tabular}




\section{Chapter 4}

\section{Identification of functionally pooled models: the Functionally Pooled VARMA (FP-VARMA) parametrization}

\subsection{Introduction}

System identification traditionally aims at deriving a model capable of representing a dynamic system at a specific operating condition, based on measured data. Yet, in many cases, a system may operate under different, though constant, conditions at different occasions (time intervals). As an example consider a mechanical, aerospace or civil structure that operates under different environmental conditions (such as temperature or humidity) on different occasions (period of the day and so on). Oftentimes, data records, each one corresponding to a distinct such condition, are obtained. In such cases, it is most desirable to simultaneously treat all the available data records in order to identify a single, "global", and compact (parsimonious) model that is capable of accurately describing the dynamics under any operating condition within a specified operating range.

A "feasible" approach to handle this identification problem consists of splitting it into several disjoint subproblems (one per each operating condition) and use customary identification techniques in order to obtain a set of models, each one based on a data record corresponding to a distinct operating condition. This procedure is hereby referred to as the "multi-model" approach. The preceding models may be subsequently "interpolated" in order to yield a single global model valid for the entire operating range. This solution (which is closely related to the gain scheduling concept [4, pp. 3-4 and the references therein]) is in fact applied by the "local approach" for Linear Parameter Varying (LPV) model identification [5-9]. Nevertheless, the combined "multi-model" approach with interpolation procedures is both inefficient and statistically suboptimal. Inefficiency has to do with the treatment of several identification problems instead of a single one. Statistical suboptimality has to do with (i) the ineffective use of the available information as possible interrelations among different data records are ignored, (ii) the increased estimation error resulting from the potentially large number of estimated parameters (several identified models), and (iii) the separate treatment of the parameter interpolation stage which constitutes an additional source of modeling error.

In order to effectively overcome these drawbacks and enable the identification of compact and accurate "global" models, a stochastic Functional Pooling identification framework has been recently introduced by Fassois and co-workers [14-17]. This framework is based on:

(1) A stochastic Functionally Pooled (FP) model structure that explicitly allows for system modeling under multiple operating conditions using a single ("global") mathematical representation. This global 
representation is characterized by parameters that explicitly (functionally) depend upon the operating condition. It thus allows for the effective, compact, modeling of the dynamics under all possible conditions, as well as for optimal statistical accuracy due to its limited parametrization and the full accounting of the interrelations among the various data records.

(2) Data pooling techniques, which simultaneously treat as one entity the data obtained from different operating conditions.

(3) Statistical inference techniques for model estimation.

The pooling idea has been motivated by modeling techniques used in statistics and econometrics [1113]. Nevertheless, the models treated in that context do not explicitly account for each operating condition, but rather attempt to either "average out" their effects by providing representations characterized by constant parameters, or to provide a measure of model "spread" by treating the model parameters as random variables. On the other hand, the present Functional Pooling framework, is distinct in that it allows for the global model to change its parameters in a way that functionally depends upon one or more measurable variables characterizing the operating condition (such as temperature, humidity and so on), and thus referred to as operating parameters.

The class of FP models could be in the most general terms considered to belong to the broader family of LPV models (see [4] and the references therein for details). Despite their superficial resemblance, these two model classes address somewhat different identification problems with quite different perspectives. LPV modeling aims at identifying nonlinear or time-varying systems by means of linear models with parameters depending upon measurable time-varying operating parameters (referred to as scheduling parameters), usually assumed bounded in terms of rate and magnitude [4, pp. 45-48]. For this purpose, LPV model identification is achieved via two major approaches: (i) the local approach and (ii) the global approach. The local approach interpolates a set of linear time-invariant models identified from a multitude of experiments corresponding to different fixed values of the scheduling parameters, thus encompassing the shortcomings of the combined "multi-model" approach and interpolation procedures. On the contrary, the global approach identifies an LPV model from a single identification experiment by exciting the system and the scheduling parameters simultaneously. Unlike the Functional Pooling identification framework, the preceding identification approaches neither treat data obtained from different operating conditions simultaneously nor take into account the interrelations among the various data records. In view of the above, the FP and LPV models constitute distinct representations with important differences, and therefore should be clearly distinguished.

The identification of univariate FP models with a single (scalar) operating parameter is treated in $[15,16]$, whereas whereas in $[14,17]$ the case of multiple (vector) operating parameters is studied. The Functional Pooling framework has been already applied on damage detection problems with very promising results [18-22, 38,39]. Furthermore, a practical demonstration of this framework and its advantages over conventional "multi-model" approaches is provided in [40-42], where the problem of identifying the dynamics of a composite beam under various temperatures is treated.

This study aims at extending the Functional Pooling framework to the case of multivariate stochastic systems operating under multiple operating conditions. Unlike the univariate case, the richer internal structure of the multivariate models poses certain technical difficulties concerning (i) the increased complexity of model analysis (certain scalar quantities become vectors or matrices, and might not commute), (ii) the model identifiability (their parametrization is nontrivial), and (iii) the increased algorithmic complexity (a large amount of data is processed).

The novel contributions of the present study may be summarized as follows:

(i) The specification of the novel Functionally Pooled Vector AutoRegressive Moving Average (FPVARMA) model class characterized by a single operating parameter for global model identification. 
(ii) The formulation of Two-Stage Least Squares (2SLS) and conditional Maximum Likelihood (ML) type estimators, and confirmation of their large sample properties (consistency, asymptotic normality). Note that the generalization of the 2SLS and ML estimators to treat the case where measurable exogenous excitation is present (that is FP-VARMAX models, with X standing for exogenous) is trivial.

(iii) The introduction of an effective method for 2SLS model estimation featuring a simplified procedure for obtaining residuals in the first stage, which avoids the use of complicated inverse function operators employed in [16].

(iv) The postulation of conditions ensuring FP-VARMA model identifiability.

(v) The postulation of a novel two-step approach to address the critical problem model structure specification. In the first step, model orders are estimated via an effective Canonical Correlation Analysis (CCA) based scheme which avoids specifying the form of the FP-VARMA parameter functional dependency on the operating parameter. This specification is accomplished in the second step by using proper forms of information criteria.

(vi) The assessment of the 2SLS and ML type estimators performance characteristics via a Monte Carlo study, which also aims at demonstrating the accuracy of the FP-VARMA modeling approach over its conventional VARMA based multi-model counterpart.

It is worth noting that earlier results on the topic have been presented in Hios and Fassois [72].

The rest of this chapter is organized as follows: The data set and the FP-VARMA model specification is presented in Section 4.2. The model parameter estimation methods and the estimators' large sample properties are presented in Sections 4.3 and 4.4, respectively. FP-VARMA model identifiability is discussed in Section 4.5, whereas the problems of model structure specification and validation are addressed in Section 4.6. In Section 4.7, the effectiveness of the FP-VARMA identification approach is assessed via a Monte Carlo study, and concluding remarks are summarized in Section 4.8 .

\subsection{The data set and the FP-VARMA model specification}

FP-VARMA model identification is based upon $M$ sets of $N$ sample-long data records, each one obtained under a different, constant, operating condition. That is, each data record corresponds to a specific, constant, value of a measurable operating parameter $k$. The set of all possible operating conditions belongs to the range $\mathcal{R}=\left[k_{\min }, k_{\max }\right] \subset \mathbb{R}$ while a discretized version $\left\{k_{1}, k_{2}, \ldots, k_{M}\right\}$ of it is used for data acquisition. The complete data set is designated as ${ }^{1}$ :

$$
Z^{N M} \triangleq\left\{\boldsymbol{y}_{k}[t] \mid k=k_{1}, k_{2}, \ldots, k_{M} ; t=1,2, \ldots, N\right\}
$$

with $t$ indicating the normalized discrete time and $\boldsymbol{y}_{k}[t]$ the $(n y \times 1)$ measurable response vector corresponding to the $k$-th operating condition.

The FP-VARMA representation postulated is of the form:

$$
\begin{gathered}
\boldsymbol{y}_{k}[t]+\sum_{i=1}^{n a} \boldsymbol{A}_{i}(k) \boldsymbol{y}_{k}[t-i]=\boldsymbol{w}_{k}[t]+\sum_{i=1}^{n c} \boldsymbol{C}_{i}(k) \boldsymbol{w}_{k}[t-i] \\
E\left\{\boldsymbol{w}_{k}[t] \boldsymbol{w}_{\ell}^{T}[t-\tau]\right\}=\boldsymbol{\Sigma}_{k, \ell} \delta[\tau] \quad k, \ell \in \mathcal{R} \\
\boldsymbol{A}_{i}(k)=\sum_{j=1}^{p a} \boldsymbol{A}_{i, j} G_{j}(k), \boldsymbol{C}_{i}(k)=\sum_{j=1}^{p c} \boldsymbol{C}_{i, j} G_{j}(k)
\end{gathered}
$$

\footnotetext{
${ }^{1}$ Boldface upper/ lower case symbols designate matrix/ column-vector quantities, respectively.
} 
with $n a$ and $n c$ indicating the corresponding AutoRegressive (AR) and Moving Average (MA) model orders. $\boldsymbol{w}_{k}[t]$ is a $(n y \times 1)$ innovations vector which is zero mean, serially uncorrelated, with fully parametrized cross-covariance matrices $\boldsymbol{\Sigma}_{k, \ell} . E\{\cdot\}$ designates statistical expectation, and $\delta[\tau]$ the Kronecker delta. The $\mathrm{AR}$ and MA matrices $\boldsymbol{A}_{i}(k)$ and $\boldsymbol{C}_{i}(k)$ are fully parameterized and have dimensions $(n y \times n y)$. They are expressed as explicit functions of the operating parameter $k$ by belonging to a functional subspace of dimension $p=\max (p a, p c)$ spanned by the mutually independent functions $G_{j}(k)(j=1, \ldots, p)$. These functions form a functional basis, with $\boldsymbol{A}_{i, j}$ and $\boldsymbol{C}_{i, j}$ designating the corresponding, fully parametrized, projection matrices. The representation of Eq. (4.2a)-(4.2c) is referred to as an FP-VARMA model of orders $(n a, n c)$ and subspace dimensionalities $(p a, p c)$, or in short as an FP-VARMA $(n a, n c)_{[p a, p c]}$ model. It is worth noting that:

(i) All information concerning the interrelations among the data records in $Z^{N M}$ is reflected in the crosscovariance matrices $\boldsymbol{\Sigma}_{k, \ell}$. This knowledge is incorporated into the parameter estimation phase to obtain statistically optimal models (see Section 4.3). An overview of the most common cases of statistical dependencies between the innovations $\boldsymbol{w}_{k}[t]$ belonging to different operating conditions are summarized in Table 2.1 (Chapter 2).

(ii) The projection of $\boldsymbol{A}_{i}(k)$ and $\boldsymbol{C}_{i}(k)$ on the functional basis $G_{j}(k)(j=1, \ldots, p)$ allows for models capable of representing the system dynamics everywhere within $\mathcal{R}=\left[k_{\min }, k_{\max }\right]$, and not only at the distinct (sample) values $\left\{k_{1}, k_{2}, \ldots, k_{M}\right\}$ involved in $Z^{N M}$.

(iii) The form of functional dependence is an important issue. Physical insight may be used for this, although experience has shown that orthogonal polynomials (for instance, Chebyshev or Legendre), or trigonometric functions are sufficient, as any given function may be approximated by any orthogonal set (refer to [38-42] for practical applications, where Chebyshev polynomials of the second kind are used). Nevertheless, the specific selection might affect model compactness (see Subsection 4.6.2). Of course, a practical limitation posed is that the selected functional subspace dimensionality must be smaller than the number of available data records $M$, that is $p<M$. mial form:

The FP-VARMA $(n a, n c)_{[p a, p c]}$ model in Eq. (4.2a)-(4.2c) may be rewritten in the following polyno-

$$
\begin{gathered}
\boldsymbol{A}[\mathcal{B}, k] \boldsymbol{y}_{k}[t]=\boldsymbol{C}[\mathcal{B}, k] \boldsymbol{w}_{k}[t], \quad k \in \mathcal{R} \\
\boldsymbol{A}[\mathcal{B}, k] \triangleq \boldsymbol{I}_{n y}+\boldsymbol{A}_{1}(k) \mathcal{B}+\ldots+\boldsymbol{A}_{n a}(k) \mathcal{B}^{n a} \\
\boldsymbol{C}[\mathcal{B}, k] \triangleq \boldsymbol{I}_{n y}+\boldsymbol{C}_{1}(k) \mathcal{B}+\ldots+\boldsymbol{C}_{n c}(k) \mathcal{B}^{n c}
\end{gathered}
$$

with $\mathcal{B}$ designating the backward shift operator. The following assumptions are made for the model:

A1. All zeros of $\operatorname{det}(\boldsymbol{A}[\mathcal{B}, k])$ lie outside the unit circle for the distinct (sample) values $\left\{k_{1}, k_{2}, \ldots, k_{M}\right\}$ ("stability condition").

A2. All zeros of $\operatorname{det}(\boldsymbol{C}[\mathcal{B}, k])$ lie outside the unit circle for the distinct (sample) values $\left\{k_{1}, k_{2}, \ldots, k_{M}\right\}$ ("invertibility condition").

A3. The polynomials $\boldsymbol{A}[\mathcal{B}, k]$ and $\boldsymbol{C}[\mathcal{B}, k]$ are left coprime for the distinct (sample) values $\left\{k_{1}, k_{2}, \ldots, k_{M}\right\}$ ("irreducibility condition").

A4. $\operatorname{rank}\left[\boldsymbol{A}_{n a}(k) \vdots \boldsymbol{C}_{n c}(k)\right]=n y$ for the distinct (sample) values $\left\{k_{1}, k_{2}, \ldots, k_{M}\right\}$ ("identifiability condition").

A5. The basis functions $G_{j}(k)$ and subspace dimensionalities $(p a, p c)$ are known.

A6. $\boldsymbol{w}_{k}[t]$ is a sequence of independent and identically distributed (i.i.d.) random vectors with zero mean and positive-definite covariance $\boldsymbol{\Sigma}_{k, k}$ (white noise). 
A7. Each value of the operating parameter $k$ corresponds to a specific operating condition. The operating parameter $k$ is measurable upon operation of the system, bounded (that is, $k \in \mathcal{R}=\left[k_{\text {min }}, k_{\text {max }}\right] \subset$ $\mathbb{R})$, and remains constant during data acquisition.

Remark 1. The assumption A1 ensures the covariance stationarity of the response vectors $\boldsymbol{y}_{k}[t]$ for the distinct (sample) values $\left\{k_{1}, k_{2}, \ldots, k_{M}\right\}$. This means that the responses are completely described by their mean and covariance, whereas to derive the large sample properties of the 2SLS and ML estimators (see Section 4.4) the sums of $\boldsymbol{y}_{k}[t]$ over time may be replaced with their corresponding expectations.

Remark 2. The assumption A2 ensures the stability of the prediction error sequence for the distinct (sample) values $\left\{k_{1}, k_{2}, \ldots, k_{M}\right\}$. This assumption implies stationarity of the prediction error sequence, meaning that asymptotically summations over time may be replaced with the corresponding expectations (see Section 4.4).

Remark 3. The assumption A3 ensures that there are no common left factors in the AR and MA polynomials of the model except for unimodular matrices (that is, matrices with constant determinant), whereas assumption A4 ensures that the common left factors are constant matrices (refer to [2, pp. 182-183], for the corresponding conditions on conventional VARX models). When assumptions A3-A5 hold, uniqueness of representation for the FP-VARMA $(n a, n c)_{[p a, p c]}$ model in Eq. (4.2a)-(4.2c) is warranted (see Section 4.5).

Remark 4. The assumption A7 poses some practical conditions in order to obtain a Functionally Pooled model. It ensures that (i) the system dynamics at two different operating conditions are not described by the same VARMA representation, and (ii) data records suitable for FP-VARMA identification are obtained (see Section 4.3).

\subsection{FP-VARMA model parameter estimation}

The FP-VARMA model estimation problem may be stated as: "Given the data record $Z^{N M}$, identify the model $\mathcal{M}(\boldsymbol{\theta})$ from the following set $\mathcal{M}$ of all candidate FP-VARMA models that "best" fits the observed responses":

$$
\begin{array}{r}
\mathcal{M} \triangleq\left\{\mathcal{M}(\boldsymbol{\theta}): \boldsymbol{A}[\mathcal{B}, k, \boldsymbol{\theta}] \boldsymbol{y}_{k}[t]=\boldsymbol{C}[\mathcal{B}, k, \boldsymbol{\theta}] \boldsymbol{e}_{k}[t, \boldsymbol{\theta}] \mid \boldsymbol{A}[\mathcal{B}, k, \boldsymbol{\theta}], \boldsymbol{C}[\mathcal{B}, k, \boldsymbol{\theta}]\right. \text { obey A1 - A4, } \\
\left.E\left\{\boldsymbol{e}_{k}[t, \boldsymbol{\theta}] \boldsymbol{e}_{\ell}^{T}[t-\tau, \boldsymbol{\theta}]\right\}=\boldsymbol{\Sigma}_{k, \ell}(\boldsymbol{\theta}) \delta[\tau] \quad k, \ell \in \mathcal{R}\right\}
\end{array}
$$

with $\boldsymbol{e}_{k}[t, \boldsymbol{\theta}]$ being the $(n y \times 1)$ residual (one-step-ahead prediction error) vector corresponding to the $k$-th operating condition. The residuals, for an accurate model, should be zero mean, serially uncorrelated, with positive-definite covariance matrices $\boldsymbol{\Sigma}_{k, k}(\boldsymbol{\theta})$ (white noise; see assumption A6 in Section 4.2). The FPVARMA models are parametrized in terms of the projection coefficient vector $\boldsymbol{\theta}$ defined as:

$$
\boldsymbol{\theta} \triangleq \operatorname{vec}\left(\left[\boldsymbol{A}_{1,1} \boldsymbol{A}_{1,2} \ldots \boldsymbol{A}_{n a, p a} \vdots \boldsymbol{C}_{1,1} \boldsymbol{C}_{1,2} \ldots \boldsymbol{C}_{n c, p c}\right]^{T}\right)
$$

with $\operatorname{vec}(\cdot)$ designating the operator that transforms the indicated matrix into a vector by stacking its columns.

Parameter estimation is presently based on properly formulated Two-Stage Least Squares (2SLS) and conditional Maximum Likelihood (ML) estimators.

\subsubsection{The Two-Stage Least Squares (2SLS) estimator}

The 2SLS based estimation is characterized by simplicity and computational efficiency, as it resorts exclusively on linear operations which are described below. 
Stage 1 Estimates $\widetilde{\boldsymbol{e}}_{k}[t]$ of the residuals $\boldsymbol{e}_{k}[t, \boldsymbol{\theta}]$ are obtained by first fitting an auxiliary Pooled VAR (PVAR) model of sufficiently high order $m a$ :

$$
\boldsymbol{y}_{k}[t]+\sum_{i=1}^{m a} \widetilde{\boldsymbol{A}}_{i, k} \boldsymbol{y}_{k}[t-i]=\widetilde{\boldsymbol{e}}_{k}[t], k \in\left\{k_{1}, k_{2}, \ldots, k_{M}\right\}
$$

Notice that this is not a functionally pooled model, because the AR matrices $\widetilde{\boldsymbol{A}}_{i, k}$ are not explicit functions of the operating parameter $k$. The P-VAR models are valid for the operating conditions $k \in$ $\left\{k_{1}, k_{2}, \ldots, k_{M}\right\}-$ they do not account for any operating condition within $\mathcal{R}$. Their main advantage is their simple and easy to identify structure, at the cost of reduced accuracy due to the increased number of estimated model parameters.

To estimate the P-VAR $(m a)$ model parameters (that is, the elements of the AR matrices), Eq. (4.6) is expressed in a linear regression form as:

$$
\boldsymbol{y}_{k}[t]=\widetilde{\boldsymbol{\Phi}}_{k}^{T}[t] \boldsymbol{\vartheta}_{k}+\widetilde{\boldsymbol{e}}_{k}[t]
$$

where:

$$
\begin{gathered}
\widetilde{\boldsymbol{\Phi}}_{k}^{T}[t]=\boldsymbol{I}_{n y} \otimes \widetilde{\boldsymbol{\phi}}_{k}^{T}[t] \\
\widetilde{\boldsymbol{\phi}}_{k}[t]=\left[\begin{array}{llll}
-\boldsymbol{y}_{k}^{T}[t-1]-\boldsymbol{y}_{k}^{T}[t-2] \ldots-\boldsymbol{y}_{k}^{T}[t-m a
\end{array}\right]^{T} \\
\boldsymbol{\vartheta}_{k}=\operatorname{vec}\left(\left[\begin{array}{llll}
\tilde{\boldsymbol{A}}_{1, k} & \tilde{\boldsymbol{A}}_{2, k} & \ldots & \tilde{\boldsymbol{A}}_{m a, k}
\end{array}\right]^{T}\right)
\end{gathered}
$$

with $\otimes$ designating Kronecker product ${ }^{2}$.

Model parameter estimation requires stacking the equations of the form (4.7a) for each of the distinct operating conditions $\left\{k_{1}, k_{2}, \ldots, k_{M}\right\}$ one below the other. This procedure is referred to as pooling:

$$
\begin{gathered}
\boldsymbol{y}[t]=\widetilde{\boldsymbol{\Phi}}^{T}[t] \boldsymbol{\vartheta}+\widetilde{\boldsymbol{e}}[t] \\
\boldsymbol{y}[t]=\left[\begin{array}{c}
\boldsymbol{y}_{k_{1}}[t] \\
\boldsymbol{y}_{k_{2}}[t] \\
\vdots \\
\boldsymbol{y}_{k_{M}}[t]
\end{array}\right], \widetilde{\boldsymbol{\Phi}}^{T}[t]=\left[\begin{array}{cccc}
\widetilde{\boldsymbol{\Phi}}_{k_{1}}^{T}[t] & \mathbf{0} & \ldots & \mathbf{0} \\
\mathbf{0} & \widetilde{\boldsymbol{\Phi}}_{k_{2}}^{T}[t] & \ldots & \mathbf{0} \\
\vdots & \vdots & \ddots & \vdots \\
\mathbf{0} & \mathbf{0} & \ldots & \widetilde{\boldsymbol{\Phi}}_{k_{M}}^{T}[t]
\end{array}\right], \boldsymbol{\vartheta}=\left[\begin{array}{c}
\boldsymbol{\vartheta}_{k_{1}} \\
\boldsymbol{\vartheta}_{k_{2}} \\
\vdots \\
\boldsymbol{\vartheta}_{k_{M}}
\end{array}\right], \widetilde{\boldsymbol{e}}[t]=\left[\begin{array}{c}
\widetilde{\boldsymbol{e}}_{k_{1}}[t] \\
\widetilde{\boldsymbol{e}}_{k_{2}}[t] \\
\vdots \\
\widetilde{\boldsymbol{e}}_{k_{M}}[t]
\end{array}\right]
\end{gathered}
$$

The parameter vector $\vartheta$ may then be estimated by minimizing a Weighted Least Squares (WLS) criterion as follows ${ }^{3}$ :

$$
\widehat{\boldsymbol{\vartheta}}_{W L S}=\arg \min _{\boldsymbol{\vartheta}}\left(\frac{1}{N M} \sum_{t=1}^{N} \widetilde{\boldsymbol{e}}^{T}[t] \boldsymbol{\Sigma}^{-1} \widetilde{\boldsymbol{e}}[t]\right)
$$

with "arg min" designating the minimizing argument, and $\boldsymbol{\Sigma}$ being the covariance matrix of $\boldsymbol{w}[t]$ (refer to Chapter 2, Table 2.1). The obtained WLS estimators have the form:

$$
\widehat{\boldsymbol{\vartheta}}_{W L S}=\left[\sum_{t=1}^{N} \widetilde{\boldsymbol{\Phi}}[t] \boldsymbol{\Sigma}^{-1} \widetilde{\boldsymbol{\Phi}}^{T}[t]\right]^{-1}\left[\sum_{t=1}^{N} \widetilde{\boldsymbol{\Phi}}[t] \boldsymbol{\Sigma}^{-1} \boldsymbol{y}[t]\right]
$$

\footnotetext{
${ }^{2}$ The Kronecker product of an $(m \times n)$ matrix $\boldsymbol{A}=\left[a_{i, j}\right]$ and a $(p \times q)$ matrix $\boldsymbol{B}=\left[b_{i, j}\right]$ is $\boldsymbol{A} \otimes \boldsymbol{B}=\left[a_{i, j} \boldsymbol{B}\right][43$, p. 3].

${ }^{3}$ The hat designates estimators/estimates.
} 


$$
\widehat{\boldsymbol{\Sigma}}_{W L S}=\frac{1}{N} \sum_{t=1}^{N} \widetilde{\boldsymbol{e}}\left[t, \widehat{\boldsymbol{\vartheta}}_{W L S}\right] \widetilde{\boldsymbol{e}}^{T}\left[t, \widehat{\boldsymbol{\vartheta}}_{W L S}\right]
$$

A simplified example of P-VAR model parameter estimation via WLS is provided in Appendix 4.A.

Notice, that an alternative way to estimate the residuals $\boldsymbol{e}_{k}[t, \boldsymbol{\theta}]$ is by approximating the FP-VARMA model with an FP-VAR model of sufficiently high order [16]. Such an approach however is not appealing, as it requires the approximation of the matrix polynomial filter $\boldsymbol{C}^{-1}[\mathcal{B}, k] \boldsymbol{A}[\mathcal{B}, k]$ (refer to Eq. (4.3a)-(4.3c)). The procedure involves complicated polynomial algebra operations and results in a matrix polynomial with increased functional subspace dimensionality. This is not desired in practical applications, especially when a limited amount of identification experiments $M$ is available.

Stage 2 The FP-VARMA model is now approximated as:

$$
\boldsymbol{y}_{k}[t]+\sum_{i=1}^{n a} \boldsymbol{A}_{i}(k) \boldsymbol{y}_{k}[t-i]=\boldsymbol{e}_{k}[t, \boldsymbol{\theta}]+\sum_{i=1}^{n c} \boldsymbol{C}_{i}(k) \widehat{\widetilde{\boldsymbol{e}}}_{k}[t-i, \boldsymbol{\theta}]
$$

which is expressed in the following linear regression form:

$$
\boldsymbol{y}_{k}[t]=\boldsymbol{\Phi}_{k}^{T}[t, \boldsymbol{\theta}] \boldsymbol{\theta}+\boldsymbol{e}_{k}[t, \boldsymbol{\theta}]
$$

where $\boldsymbol{\theta}$ is the projection coefficient vector and:

$$
\begin{gathered}
\boldsymbol{\Phi}_{k}^{T}[t, \boldsymbol{\theta}]=\boldsymbol{I}_{n y} \otimes \boldsymbol{\phi}_{k}^{T}[t, \boldsymbol{\theta}] \\
\boldsymbol{\phi}_{k}[t, \boldsymbol{\theta}]=\left[\begin{array}{c}
{\left[\begin{array}{c}
-\boldsymbol{y}_{k}[t-1] \\
\vdots \\
-\boldsymbol{y}_{k}[t-n a]
\end{array}\right] \otimes\left[\begin{array}{c}
G_{1}(k) \\
\vdots \\
G_{p a}(k)
\end{array}\right]} \\
{\left[\begin{array}{c}
\widehat{\boldsymbol{e}}_{k}[t-1, \boldsymbol{\theta}] \\
\vdots \\
\widehat{\widetilde{\boldsymbol{e}}}_{k}[t-n c, \boldsymbol{\theta}]
\end{array}\right] \otimes\left[\begin{array}{c}
G_{1}(k) \\
\vdots \\
G_{p c}(k)
\end{array}\right]}
\end{array}\right.
\end{gathered}
$$

Pooling together the equations of the form (4.12a) from all available operating conditions $\left\{k_{1}, k_{2}\right.$, $\left.\ldots, k_{M}\right\}$, yields:

$$
\begin{gathered}
\boldsymbol{y}[t]=\boldsymbol{\Phi}^{T}[t, \boldsymbol{\theta}] \boldsymbol{\theta}+\boldsymbol{e}[t, \boldsymbol{\theta}] \\
\boldsymbol{y}[t]=\left[\begin{array}{c}
\boldsymbol{y}_{k_{1}}[t] \\
\boldsymbol{y}_{k_{2}}[t] \\
\vdots \\
\boldsymbol{y}_{k_{M}}[t]
\end{array}\right], \quad \boldsymbol{\Phi}^{T}[t, \boldsymbol{\theta}]=\left[\begin{array}{c}
\boldsymbol{\Phi}_{k_{1}}^{T}[t, \boldsymbol{\theta}] \\
\boldsymbol{\Phi}_{k_{2}}^{T}[t, \boldsymbol{\theta}] \\
\vdots \\
\boldsymbol{\Phi}_{k_{M}}^{T}[t, \boldsymbol{\theta}]
\end{array}\right], \quad \boldsymbol{e}[t, \boldsymbol{\theta}]=\left[\begin{array}{c}
\boldsymbol{e}_{k_{1}}[t, \boldsymbol{\theta}] \\
\boldsymbol{e}_{k_{2}}[t, \boldsymbol{\theta}] \\
\vdots \\
\boldsymbol{e}_{k_{M}}[t, \boldsymbol{\theta}]
\end{array}\right]
\end{gathered}
$$

Notice that the term functional pooling signifies the functional dependence of the pooled expression in Eq. (4.13a)- (4.13b) on the operating parameter $k$.

The projection coefficient vector $\boldsymbol{\theta}$ may be estimated by minimizing a WLS criterion as follows:

$$
\widehat{\boldsymbol{\theta}}_{2 S L S}=\arg \min _{\boldsymbol{\theta}}\left(\frac{1}{N M} \sum_{t=1}^{N} \boldsymbol{e}^{T}[t, \boldsymbol{\theta}] \boldsymbol{\Sigma}^{-1} \boldsymbol{e}[t, \boldsymbol{\theta}]\right)
$$


with $\boldsymbol{\Sigma}$ designating the covariance matrix of $\boldsymbol{w}[t]$ (defined similarly to $\boldsymbol{e}[t, \boldsymbol{\theta}]$ in Eq. (4.13b); see also assumption A5 in Section 4.2, and Table 2.1 in Chapter 2). The obtained 2SLS estimators have the form:

$$
\begin{gathered}
\widehat{\boldsymbol{\theta}}_{2 S L S}=\left[\sum_{t=1}^{N} \boldsymbol{\Phi}[t, \boldsymbol{\theta}] \boldsymbol{\Sigma}^{-1} \boldsymbol{\Phi}^{T}[t, \boldsymbol{\theta}]\right]^{-1}\left[\sum_{t=1}^{N} \boldsymbol{\Phi}[t, \boldsymbol{\theta}] \boldsymbol{\Sigma}^{-1} \boldsymbol{y}[t]\right] \\
\widehat{\boldsymbol{\Sigma}}_{2 S L S}=\frac{1}{N} \sum_{t=1}^{N} \boldsymbol{e}\left[t, \widehat{\boldsymbol{\theta}}_{2 S L S}\right] \boldsymbol{e}^{T}\left[t, \widehat{\boldsymbol{\theta}}_{2 S L S}\right]
\end{gathered}
$$

Since $\boldsymbol{\Sigma}$ in Eq. (4.15a) is usually unavailable, $\widehat{\boldsymbol{\theta}}_{2 S L S}$ is obtained in practice by inserting an estimate from Stage 1 (refer to Eq. (4.10b)) into Eq. (4.15a), while the final residual covariance is obtained as in Eq. (4.15b). The procedure may be continued by updating the matrix $\boldsymbol{\Phi}[t, \boldsymbol{\theta}]$ (see Eq. (4.12b)-(4.12c)) and inserting the resulting $\widehat{\boldsymbol{\Sigma}}_{2 S L S}$ into Eq. (4.15a) in order to obtain a new estimate, and repeated until $\widehat{\boldsymbol{\theta}}_{2 S L S}$ converges (iterated 2SLS).

\subsubsection{The conditional Maximum Likelihood (ML) estimator}

Let $\boldsymbol{w}[t]$ be a Gaussian white noise process, that is $\boldsymbol{w}[t] \sim \mathcal{N}(\mathbf{0}, \boldsymbol{\Sigma})$, with $\mathcal{N}(\cdot, \cdot)$ designating the normal distribution with indicated mean and covariance. The ML estimators of $\boldsymbol{\theta}$ and $\boldsymbol{\Sigma}$ are:

$$
\begin{gathered}
\widehat{\boldsymbol{\theta}}_{M L}=\arg \min _{\boldsymbol{\theta}} \ln \left(\operatorname{det}\left(\frac{1}{N} \sum_{t=1}^{N} \boldsymbol{e}[t, \boldsymbol{\theta}] \boldsymbol{e}^{T}[t, \boldsymbol{\theta}]\right)\right) \\
\widehat{\boldsymbol{\Sigma}}_{M L}=\frac{1}{N} \sum_{t=1}^{N} \boldsymbol{e}\left[t, \widehat{\boldsymbol{\theta}}_{M L}\right] \boldsymbol{e}^{T}\left[t, \widehat{\boldsymbol{\theta}}_{M L}\right]
\end{gathered}
$$

with $\ln (\cdot)$ designating the natural logarithm, and $\operatorname{det}(\cdot)$ the determinant. In analogy to conventional VARMA models, $\boldsymbol{e}[t, \boldsymbol{\theta}]$ is a nonlinear function of $\boldsymbol{\theta}$, hence the estimation of $\boldsymbol{\theta}$ requires an iterative search procedure [44, pp. 470-472].

\subsection{Large sample properties of the 2SLS and ML type estimators}

The consistency and the asymptotic distribution of the iterated 2SLS and conditional ML type estimators are studied in this section. Notice that the exact structure (model orders $(n a, n c)$, basis functions $G_{j}(k)$, and subspace dimensionalities $(p a, p c)$ ) are considered known (see assumption A5 in Section 4.2).

\subsubsection{The iterated 2SLS estimator}

Theorem 4.4.1. (The iterated 2SLS estimator consistency and asymptotic distribution): Let $\boldsymbol{\theta}_{o}$ be the system's true projection coefficient vector and $\boldsymbol{\Sigma}_{o}$ the true (positive-definite) covariance matrix of $\boldsymbol{w}[t]$, which is additionally assumed to have bounded fourth-order moments. Consider the vector $\boldsymbol{\sigma}_{o}$, comprising of the non-identical elements on and below the main diagonal of $\boldsymbol{\Sigma}_{o}$, that is $\operatorname{vec}\left(\boldsymbol{\Sigma}_{o}\right)=\boldsymbol{D}_{M n y} \boldsymbol{\sigma}_{o}$, with $\boldsymbol{D}_{M n y}$ designating a duplication matrix of proper dimensions. If the matrix product $E\left\{\boldsymbol{\Phi}\left[t, \boldsymbol{\theta}_{o}\right] \boldsymbol{\Sigma}_{o}^{-1} \boldsymbol{\Phi}^{T}\left[t, \boldsymbol{\theta}_{o}\right]\right\}^{-1}$ $E\left\{\boldsymbol{\Phi}\left[t, \boldsymbol{\theta}_{o}\right] \boldsymbol{\Sigma}_{o}^{-1} \boldsymbol{\Psi}^{T}\left[t, \boldsymbol{\theta}_{o}\right]\right\}$, with $\boldsymbol{\Psi}[t, \boldsymbol{\theta}]=-\frac{\partial \boldsymbol{e}^{T}[t, \boldsymbol{\theta}]}{\partial \boldsymbol{\theta}}$, has eigenvalues inside the unit circle centered at $(1,0)$ in the complex plane, then the iterated $2 S L S$ estimator $\widehat{\boldsymbol{\theta}}_{2 S L S}$ is locally convergent to $\boldsymbol{\theta}_{o}$, and the estimators $\widehat{\boldsymbol{\theta}}_{2 S L S}$ and $\widehat{\boldsymbol{\Sigma}}_{2 S L S}$ are consistent, that is:

$$
\widehat{\boldsymbol{\theta}}_{2 S L S} \stackrel{p}{\longrightarrow} \boldsymbol{\theta}_{O} \quad(N \longrightarrow \infty)
$$




$$
\widehat{\boldsymbol{\Sigma}}_{2 S L S} \stackrel{p}{\longrightarrow} \boldsymbol{\Sigma}_{o}(N \longrightarrow \infty)
$$

with “ $\stackrel{p}{\longrightarrow}$ " designating the limit in probability ${ }^{4}$.

The limiting distribution of $\widehat{\boldsymbol{\theta}}_{2 S L S}$ is:

$$
\sqrt{N M}\left(\widehat{\boldsymbol{\theta}}_{2 S L S}-\boldsymbol{\theta}_{o}\right) \stackrel{d}{\longrightarrow} \mathcal{N}\left(\mathbf{0}, \boldsymbol{P}_{2 S L S}\right) \quad(N \longrightarrow \infty)
$$

with $\boldsymbol{P}_{2 S L S}=M E\left\{\boldsymbol{\Phi}\left[t, \boldsymbol{\theta}_{o}\right] \boldsymbol{\Sigma}^{-1} \boldsymbol{\Psi}^{T}\left[t, \boldsymbol{\theta}_{o}\right]\right\}^{-1} E\left\{\boldsymbol{\Phi}\left[t, \boldsymbol{\theta}_{o}\right] \boldsymbol{\Sigma}^{-1} \boldsymbol{\Phi}^{T}\left[t, \boldsymbol{\theta}_{o}\right]\right\} E\left\{\boldsymbol{\Phi}\left[t, \boldsymbol{\theta}_{o}\right] \boldsymbol{\Sigma}^{-1} \boldsymbol{\Psi}^{T}\left[t, \boldsymbol{\theta}_{o}\right]\right\}^{-T}$, and $\stackrel{d}{\longrightarrow}$ ” designating the limit in distribution ${ }^{5}$.

The limiting distribution of $\widehat{\sigma}_{2 S L S}$ is:

$$
\sqrt{N M}\left(\widehat{\boldsymbol{\sigma}}_{2 S L S}-\boldsymbol{\sigma}_{o}\right) \stackrel{d}{\longrightarrow} \mathcal{N}\left(\mathbf{0}, 2 M \boldsymbol{D}_{M n y}^{+}\left(\boldsymbol{\Sigma}_{o} \otimes \boldsymbol{\Sigma}_{o}\right)\left(\boldsymbol{D}_{M n y}^{+}\right)^{T}\right) \quad(N \longrightarrow \infty)
$$

with the superscript “+” designating the Moore-Penrose generalized inverse matrix 6 .

Proof. See Appendix 4.B.1.

Remark 5. The definition of $\boldsymbol{\sigma}$ for the FP-VARMA models is in analogy to the corresponding conventional VARMA model case [44, p. 480]. Note however that in conventional VARMA models, all non-identical elements of the corresponding residual covariance matrix are on and below (or above) the main diagonal, due to its symmetric structure. This is not the case for the FP-VARMA models, as the covariance $\boldsymbol{\Sigma}$ has a richer structure with identical elements at certain locations below (or above) the main diagonal (refer to Chapter 2, Table 2.1).

Remark 6. The duplication matrix $\boldsymbol{D}_{M n y}$ contains zeros and ones at proper locations, and it is defined in analogy to the conventional VARMA model case [44, p. 480]. Care has to be taken when considering different types of dependencies between the innovations, as the structure of the covariance matrix $\boldsymbol{\Sigma}$ changes, thus affecting the dimensions and structure of the duplication matrix. Indeed, the dimensions of $\boldsymbol{D}_{M n y}$ are $\left((M n y)^{2} \times \frac{1}{4} M n y(M+1)(n y+1)\right)$ for contemporaneously correlated innovations, $\left((M n y)^{2} \times\right.$ $\left.\frac{1}{2} M n y(n y+1)\right)$ for groupwise heteroscedastic innovations, and $\left((M n y)^{2} \times \frac{1}{2} n y(n y+1)\right)$ for groupwise homoscedastic innovations (refer to Chapter 2, Table 2.1).

Estimates of the covariances for $\widehat{\boldsymbol{\theta}}_{2 S L S}$ and $\widehat{\boldsymbol{\sigma}}_{2 S L S}$ are thus given by:

$$
\begin{gathered}
\widehat{\boldsymbol{P}}\left(\widehat{\boldsymbol{\theta}}_{2 S L S}\right)=\left[\sum_{t=1}^{N} \boldsymbol{\Phi}\left[t, \widehat{\boldsymbol{\theta}}_{2 S L S}\right] \widehat{\boldsymbol{\Sigma}}_{2 S L S}^{-1} \boldsymbol{\Psi}^{T}\left[t, \widehat{\boldsymbol{\theta}}_{2 S L S}\right]\right]^{-1}\left[\sum_{t=1}^{N} \boldsymbol{\Phi}\left[t, \widehat{\boldsymbol{\theta}}_{2 S L S}\right] \widehat{\boldsymbol{\Sigma}}_{2 S L S}^{-1} \boldsymbol{\Phi}^{T}\left[t, \widehat{\boldsymbol{\theta}}_{2 S L S}\right]\right] \\
{\left[\sum_{t=1}^{N} \boldsymbol{\Phi}\left[t, \widehat{\boldsymbol{\theta}}_{2 S L S}\right] \widehat{\boldsymbol{\Sigma}}_{2 S L S}^{-1} \boldsymbol{\Psi}^{T}\left[t, \widehat{\boldsymbol{\theta}}_{2 S L S}\right]\right]^{-T}} \\
\widehat{\boldsymbol{P}}\left(\widehat{\boldsymbol{\sigma}}_{2 S L S}\right)=\frac{2}{N} \boldsymbol{D}_{M n y}^{+}\left(\widehat{\boldsymbol{\Sigma}}_{2 S L S} \otimes \widehat{\boldsymbol{\Sigma}}_{2 S L S}\right)\left(\boldsymbol{D}_{M n y}^{+}\right)^{T}
\end{gathered}
$$

The following corollary holds in view of Theorem 4.4.1.

\footnotetext{
${ }^{4}$ Let $\left\{\boldsymbol{x}_{N}\right\} N=1,2, \ldots$ be an indexed sequence of $(n \times 1)$ stochastic vector variables, and $\boldsymbol{x}$ a $(n \times 1)$ stochastic vector variable. Then, $\boldsymbol{x}_{N} \stackrel{p}{\longrightarrow} \boldsymbol{x}$ if $\forall \epsilon>0, \operatorname{Pr}\left(\left|x_{i N}-x_{i}\right|>\epsilon\right) \longrightarrow \mathbf{0}$ as $N \longrightarrow \infty$ for $i=1, \ldots, n$ [44, pp. 681-682].

${ }^{5}$ Let the joint distribution functions of $\boldsymbol{x}_{N}$ and $\boldsymbol{x}$ be $F_{N}$ and $F$, respectively. Then, $\boldsymbol{x}_{N} \stackrel{d}{\longrightarrow} \boldsymbol{x}$ if for all real vectors $\boldsymbol{c}$ for which $F$ is continuous, $F_{N}(\boldsymbol{c}) \longrightarrow F(\boldsymbol{c})$ as $N \longrightarrow \infty$ [44, p. 682].

${ }^{6}$ The Moore-Penrose generalized inverse of an $(m \times n)$ matrix $\boldsymbol{A}$ is, $\boldsymbol{A}^{+}=\left(\boldsymbol{A}^{T} \boldsymbol{A}\right)^{-1} \boldsymbol{A}^{T}$ [43, p. 35].
} 
Corollary 4.4.1. (The iterated 2SLS estimator statistical accuracy): Under the assumptions of Theorem 4.4.1, $\widehat{\boldsymbol{\theta}}_{2 S L S}$ is in general asymptotically inefficient, as $\boldsymbol{P}_{2 S L S}$ does not reach the Cramér-Rao lower bound, except for the case of pure linear regressions (for instance, FP-VAR $(X)$ models), whereas $\widehat{\sigma}_{2 S L S}$ is always asymptotically efficient.

Proof. See Appendix 4.B.4.1.

\subsubsection{The conditional ML estimator}

Theorem 4.4.2. (The ML-based estimator consistency and asymptotic distribution): Let $\overline{\boldsymbol{\theta}}_{o}=\left[\boldsymbol{\theta}_{o}^{T} \vdots \boldsymbol{\sigma}_{o}^{T}\right]^{T}$ be the system's true parameter vector. Assuming that $\boldsymbol{\Sigma}_{o}$ is positive-definite, then the estimator $\widehat{\overline{\boldsymbol{\theta}}}_{M L}$ is consistent, that is:

$$
\widehat{\overline{\boldsymbol{\theta}}}_{M L} \stackrel{p}{\longrightarrow} \overline{\boldsymbol{\theta}}_{O} \quad(N \longrightarrow \infty)
$$

The limiting distribution of the ML estimator $\widehat{\overline{\boldsymbol{\theta}}}_{M L}$ is given by:

$$
\sqrt{N M}\left(\widehat{\overline{\boldsymbol{\theta}}}_{M L}-\overline{\boldsymbol{\theta}}_{o}\right) \stackrel{d}{\longrightarrow} \mathcal{N}\left(\mathbf{0}, \boldsymbol{P}_{M L}\right) \quad(N \longrightarrow \infty)
$$

with, $\boldsymbol{P}_{M L}=\left[\begin{array}{cc}M E\left\{\boldsymbol{\Psi}\left[t, \boldsymbol{\theta}_{o}\right] \boldsymbol{\Sigma}^{-1} \boldsymbol{\Psi}^{T}\left[t, \boldsymbol{\theta}_{o}\right]\right\}^{-1} & \mathbf{0} \\ \mathbf{0} & 2 M \boldsymbol{D}_{M n y}^{+}\left(\boldsymbol{\Sigma}_{o} \otimes \boldsymbol{\Sigma}_{o}\right)\left(\boldsymbol{D}_{M n y}^{+}\right)^{T}\end{array}\right]$.

Proof. See Appendix 4.B.3.

Remark 7. Note that the ML estimator properties are in analogy to those of conventional VARMA models [44, p. 480]. Also, note that the upper left block matrix of $\boldsymbol{P}_{M L}$, corresponding to the asymptotic covariance of $\widehat{\boldsymbol{\theta}}_{M L}$, differs from its 2SLS-based counterpart suggesting that $\widehat{\boldsymbol{\theta}}_{M L}$ and $\widehat{\boldsymbol{\theta}}_{2 S L S}$ are generally not equivalent.

As in the iterated 2SLS case, estimates of the covariances for $\widehat{\boldsymbol{\theta}}_{M L}$ and $\widehat{\boldsymbol{\sigma}}_{M L}$ are given by:

$$
\begin{aligned}
& \widehat{\boldsymbol{P}}\left(\widehat{\boldsymbol{\theta}}_{M L}\right)=\left[\sum_{t=1}^{N} \boldsymbol{\Psi}\left[t, \widehat{\boldsymbol{\theta}}_{M L}\right] \widehat{\boldsymbol{\Sigma}}_{M L}^{-1} \boldsymbol{\Psi}^{T}\left[t, \widehat{\boldsymbol{\theta}}_{M L}\right]\right]^{-1} \\
& \widehat{\boldsymbol{P}}\left(\widehat{\boldsymbol{\sigma}}_{M L}\right)=\frac{2}{N} \boldsymbol{D}_{M n y}^{+}\left(\widehat{\boldsymbol{\Sigma}}_{M L} \otimes \widehat{\boldsymbol{\Sigma}}_{M L}\right)\left(\boldsymbol{D}_{M n y}^{+}\right)^{T}
\end{aligned}
$$

An immediate result of Theorem 4.4.2 is the following corollary.

Corollary 4.4.2. (ML estimator statistical accuracy): Under the assumptions of Theorem 4.4.2, $\widehat{\overline{\boldsymbol{\theta}}}_{M L}$ is asymptotically statistically efficient, as $\boldsymbol{P}_{M L}$ reaches the Cramér-Rao lower bound, thereby providing optimal accuracy.

Proof. See Appendix 4.B.4.2.

\subsubsection{Accuracy of FP-VARMA model parameters at the $k$-th operating condition}

Once an FP-VARMA model has been obtained, information on the AR and MA parameter matrices for any operating condition $k \in \mathcal{R}$ is readily available. The following relationships associate the (2SLS or ML 
based) projection coefficient vector estimate $\widehat{\boldsymbol{\theta}}$ to the parameter vector $\widehat{\boldsymbol{\vartheta}}(k)$ which comprises all elements of the AR and MA matrices corresponding to the $k$-th operating condition (see also Eq. (4.2c) and Eq. (4.5)):

$$
\begin{gathered}
\widehat{\boldsymbol{\vartheta}}(k)=\boldsymbol{T}(k) \widehat{\boldsymbol{\theta}} \quad k \in \mathcal{R} \\
\boldsymbol{P}(\widehat{\boldsymbol{\vartheta}}(k))=\boldsymbol{T}(k) \boldsymbol{P}(\widehat{\boldsymbol{\theta}}) \boldsymbol{T}^{T}(k) \\
\boldsymbol{\vartheta}(k)=\operatorname{vec}\left(\left[\boldsymbol{A}_{1}(k) \boldsymbol{A}_{2}(k) \ldots \boldsymbol{A}_{n a}(k) \vdots \boldsymbol{C}_{1}(k) \boldsymbol{C}_{2}(k) \ldots \boldsymbol{C}_{n c}(k)\right]^{T}\right)
\end{gathered}
$$

with $\boldsymbol{T}(k)$ designating a transformation matrix whose entries are zeros and basis functions $G_{j}(k)$ at proper locations (see also Chapter 2, Appendix 2.A). Notice that in Eq. (4.21b) an estimate $\widehat{\boldsymbol{P}}(\widehat{\boldsymbol{\theta}})$ of the projection coefficient covariance $\boldsymbol{P}(\widehat{\boldsymbol{\theta}})$ is used in practice.

\subsection{FP-VARMA model identifiability}

An important issue in FP-VARMA modeling is to ensure that the identification procedure yields a unique projection coefficient vector $\boldsymbol{\theta}$. In this section the conditions under which the projection coefficients of an FP-VARMA $(n a, n c)_{[p a, p c]}$ model can be recovered by means of the complete data set $Z^{N M}$ are studied.

The conditions for uniqueness of the $\operatorname{FP}-\operatorname{VARMA}(n a, n c)_{[p a, p c]}$ projection coefficients are provided by the following theorem.

Theorem 4.5.1. (FP-VARMA uniqueness of representation): Consider the FP-VARMA $(n a, n c)_{[p a, p c]}$ model of Eq. (4.3a)-(4.3c). The model's noise shaping filter matrix polynomials:

$$
\boldsymbol{H}[\mathcal{B}, k] \triangleq \boldsymbol{A}^{-1}[\mathcal{B}, k] \boldsymbol{C}[\mathcal{B}, k], \quad k=k_{1}, k_{2}, \ldots, k_{M}
$$

are unique in the class of matrix fraction descriptions of degrees $(n a, n c)$ with known basis functions $G_{j}(k)$ and subspace dimensionalities $(p a, p c)$ if and only if:

$$
\begin{gathered}
\boldsymbol{A}[\mathcal{B}, k], \boldsymbol{C}[\mathcal{B}, k] \quad: \quad \text { left coprime for } k=k_{1}, k_{2}, \ldots, k_{M} \\
\operatorname{rank}\left[\boldsymbol{A}_{n a}(k) \vdots \boldsymbol{C}_{n c}(k)\right]=n y \text { for } k=k_{1}, k_{2}, \ldots, k_{M}
\end{gathered}
$$

Proof. See Appendix 4.C.1.

\subsection{FP-VARMA model structure specification and validation}

In FP-VARMA identification, model structure specification refers to the problem of determining the AR and MA model orders $(n a, n c)$, as well as the corresponding functional subspace dimensionalities ( $p a, p c)$. A two-step approach that aims at splitting the combined problem of model order and functional subspace specification into two separate, easier to handle, subproblems, is presently postulated. The approach relies upon a Canonical Correlation Analysis (CCA) scheme and proper forms of information criteria.

\subsubsection{Model order specification}

In the first step, the FP-VARMA model orders are specified by means of a CCA scheme which is based on the work of Tsay [73] and exploits the correlation structure of ARMA processes. The advantages of the scheme are twofold: First, it is quite general as it operates directly on the data set $Z^{N M}$, without any requirements on the functional dependency form of the FP-VARMA parameters over the operating parameter $k$. Second, it avoids the direct estimation of various FP-VARMA models, thus being computationally effective. 
Basic scheme: Specification of overall FP-VARMA model orders The CCA scheme for FP-VARMA model order specification consists of two important entities:

(1) The echelon FP-VARMA representation. This representation considers $\operatorname{FP}-\operatorname{VARMA}(n, n)_{[p a, p c]} \bmod -$ els as in Eq. (4.2a), rewritten in the following echelon form:

$$
\boldsymbol{A}_{0}^{\#}(k) \boldsymbol{y}_{k}[t]+\sum_{i=1}^{n} \boldsymbol{A}_{i}^{\#}(k) \boldsymbol{y}_{k}[t-i]=\boldsymbol{C}_{0}^{\#}(k) \boldsymbol{w}_{k}[t]+\sum_{i=1}^{n} \boldsymbol{C}_{i}^{\#}(k) \boldsymbol{w}_{k}[t-i] \quad k \in \mathcal{R}
$$

with $\boldsymbol{A}_{0}^{\#}(k)=\boldsymbol{C}_{0}^{\#}(k)$ being lower triangular matrices having ones on the diagonal, in analogy to the conventional VARMA model case [74, p. 54].

Stacking the representations in Eq. (4.24) for each distinct (sample) operating condition $\left\{k_{1}, k_{2}\right.$, $\left.\ldots, k_{M}\right\}$ one below the other yields the following echelon FP-VARMA representation:

$$
\boldsymbol{A}_{0}^{\#} \boldsymbol{y}[t]+\sum_{i=1}^{n} \boldsymbol{A}_{i}^{\#} \boldsymbol{y}[t-i]=\boldsymbol{C}_{0}^{\#} \boldsymbol{w}[t]+\sum_{i=1}^{n} \boldsymbol{C}_{i}^{\#} \boldsymbol{w}[t-i]
$$

where:

$$
\boldsymbol{A}_{i}^{\#} \triangleq\left[\begin{array}{cccc}
\boldsymbol{A}_{i}^{\#}\left(k_{1}\right) & \mathbf{0} & \ldots & \mathbf{0} \\
\mathbf{0} & \boldsymbol{A}_{i}^{\#}\left(k_{2}\right) & \ldots & \mathbf{0} \\
\vdots & \vdots & \ddots & \vdots \\
\mathbf{0} & \mathbf{0} & \ldots & \boldsymbol{A}_{i}^{\#}\left(k_{M}\right)
\end{array}\right], \boldsymbol{C}_{i}^{\#} \triangleq\left[\begin{array}{cccc}
\boldsymbol{C}_{i}^{\#}\left(k_{1}\right) & \mathbf{0} & \ldots & \mathbf{0} \\
\mathbf{0} & \boldsymbol{C}_{i}^{\#}\left(k_{2}\right) & \ldots & \mathbf{0} \\
\vdots & \vdots & \ddots & \vdots \\
\mathbf{0} & \mathbf{0} & \ldots & \boldsymbol{C}_{i}^{\#}\left(k_{M}\right)
\end{array}\right]
$$

with $i=0,1, \ldots, n$ and $\boldsymbol{A}_{0}^{\#}=\boldsymbol{C}_{0}^{\#}$ being lower triangular matrices having ones on the diagonal, since $\boldsymbol{A}_{0}^{\#}(k)=\boldsymbol{C}_{0}^{\#}(k)$ for $k=k_{1}, k_{2}, \ldots, k_{M}$.

(2) The canonical correlations between the following vectors:

$$
\begin{aligned}
& \boldsymbol{p}[t]=\left[\boldsymbol{y}^{T}[t-1] \boldsymbol{y}^{T}[t-2] \ldots \boldsymbol{y}^{T}[t-s]\right]^{T} \quad \text { ("past" vector) } \\
& \boldsymbol{f}[t]=\left[\boldsymbol{y}^{T}[t] \boldsymbol{y}^{T}[t+1] \ldots\right]^{T} \quad \text { ("future" vector) }
\end{aligned}
$$

Note that $s$ in Eq. (4.26a) is selected so that $\boldsymbol{p}[t]$ contains all information needed in predicting future observations of $\boldsymbol{y}[t]$. In practice, $s$ is selected equal to the P-VAR model order that minimizes proper forms of information criteria (refer to Subsection 4.6.2) when fitted to $Z^{N M}$, in analogy to the conventional VARMA model [73].

CCA is the statistical interpretation of the notion of principal angles and principal directions in linear subspaces $^{7}$ [75, p. 603]. The aim is to find two sets of basis vectors, one for each random variable (presently the "past" and "future" vectors $\boldsymbol{p}[t], \boldsymbol{f}[t]$ ), such that the projections of the random variables onto these basis vectors are (i) mutually maximized, and (ii) internally uncorrelated. The resulting basis vectors (principal directions) are referred to as the canonical vectors, the projections of the random variables onto their associated canonical vectors yield the canonical variates, and the correlation coefficients (cosines of principal angles) between the canonical variates are the canonical correlations.

\footnotetext{
${ }^{7}$ The principal angles $0 \leq \theta_{1} \leq \theta_{2} \leq \ldots \leq \theta_{\min \left(d_{1}, d_{2}\right)} \leq \pi / 2$ between the subspaces $S_{1}$ and $S_{1}$ belonging in $\mathbb{R}^{m}$ with dimensions $d_{1}$ and $d_{2}$, respectively, and the corresponding principal directions $\boldsymbol{u}_{i} \in S_{1}$ and $\boldsymbol{v}_{i} \in S_{2}$ are defined recursively by:$$
\cos \left(\theta_{i}\right)=\max _{\boldsymbol{u} \in S_{1}} \max _{\boldsymbol{v} \in S_{2}} \boldsymbol{u}^{T} \boldsymbol{v}=\boldsymbol{u}_{i}^{T} \boldsymbol{v}_{i}
$$

subject to: $\|\boldsymbol{u}\|=\|\boldsymbol{v}\|=1$, with $\boldsymbol{u}^{T} \boldsymbol{u}_{j}=0$ and $\boldsymbol{v}^{T} \boldsymbol{v}_{j}=0$ for $j=1, \ldots, i-1$ [75, p. 603].
} 
A canonical correlation $\rho \geq 0$ between $\boldsymbol{p}[t]$ and $\boldsymbol{f}[t]$ is obtained from the eigensystem:

$$
\begin{aligned}
& \left(\boldsymbol{\Gamma}_{\boldsymbol{p} \boldsymbol{p}}^{-1} \boldsymbol{\Gamma}_{\boldsymbol{p} \boldsymbol{f}} \boldsymbol{\Gamma}_{\boldsymbol{f} \boldsymbol{f}}^{-1} \boldsymbol{\Gamma}_{\boldsymbol{f} \boldsymbol{p}}\right) \boldsymbol{v}_{p}=\rho^{2} \boldsymbol{v}_{p} \\
& \left(\boldsymbol{\Gamma}_{\boldsymbol{f} \boldsymbol{f}}^{-1} \boldsymbol{\Gamma}_{\boldsymbol{f} \boldsymbol{p}} \boldsymbol{\Gamma}_{\boldsymbol{p} \boldsymbol{p}}^{-1} \boldsymbol{\Gamma}_{\boldsymbol{p} \boldsymbol{f}}\right) \boldsymbol{v}_{f}=\rho^{2} \boldsymbol{v}_{f}
\end{aligned}
$$

with $\boldsymbol{\Gamma}_{\boldsymbol{p} \boldsymbol{f}}$ being the covariance between $\boldsymbol{p}[t]$ and $\boldsymbol{f}[t]$, and so on. The eigenvectors $\boldsymbol{v}_{p}$ and $\boldsymbol{v}_{f}$ correspond to the canonical vectors associated to $\rho$, whereas $x[t]=\boldsymbol{v}_{f}^{T} \boldsymbol{f}[t]$ and $z[t]=\boldsymbol{v}_{p}^{T} \boldsymbol{p}[t]$ designate the corresponding canonical variates.

Note that if $\rho=0$, then $x[t]=\boldsymbol{v}_{f}^{T} \boldsymbol{f}[t]$ is uncorrelated with all linear combinations of $\boldsymbol{p}[t]$ and conversely. More generally, it holds that if there exist $q$ (where $q \leq \operatorname{dim}(\boldsymbol{f}[t]) \leq \operatorname{dim}(\boldsymbol{p}[t])$, and $\operatorname{dim}(\cdot)$ designating the dimensionality of the indicated vector) linearly independent linear combinations of $\boldsymbol{f}[t]$ which are completely uncorrelated with $\boldsymbol{p}[t]$, then there are $q$ zero canonical correlations between $\boldsymbol{f}[t]$ and $\boldsymbol{p}[t]$ [74, pp. 58-61]. This last property is the cornerstone of the CCA scheme.

Rewriting the echelon FP-VARMA representation of Eq. (4.25a)-(4.25b) as:

$$
\begin{aligned}
\boldsymbol{A}_{0}^{\#} \boldsymbol{y}[t+n]+\sum_{i=1}^{n} \boldsymbol{A}_{i}^{\#} \boldsymbol{y}[t+n-i] & =\left[\boldsymbol{A}_{n}^{\#} \boldsymbol{A}_{n-1}^{\#} \ldots \boldsymbol{A}_{0}^{\#}: \mathbf{0} \ldots\right] \boldsymbol{f}[t] \\
& =\boldsymbol{C}_{0}^{\#} \boldsymbol{w}[t+n]+\sum_{i=1}^{n} \boldsymbol{C}_{i}^{\#} \boldsymbol{w}[t+n-i]
\end{aligned}
$$

indicates that (Mny) linear combinations of $\boldsymbol{f}[t]$ are uncorrelated to $\boldsymbol{p}[t]$, which implies the presence of (Mny) zero canonical correlations between $\boldsymbol{f}[t]$ and $\boldsymbol{p}[t]$. The zero canonical correlations and their associated canonical variates are readily obtained from Eq. (4.27a)-(4.27b), thus providing information on the overall FP-VARMA model orders via Eq. (4.28).

In fact, the examination of the zero canonical correlations between $\boldsymbol{f}[t]$ and $\boldsymbol{p}[t]$ may be used to specify the overall AR/MA polynomial degrees corresponding to each row of the echelon FP-VARMA representation (Kronecker indices). For this purpose, let $\boldsymbol{a}_{i}^{T}(n y(m-1)+j)$ designate the $(n y(m-1)+j)-$ th row of $\boldsymbol{A}_{i}^{\#}(j=1, \ldots, n y$ and $m=1, \ldots, M)$, which corresponds to the $j$-th time-series of the operating condition $k_{m}$. Then, the cross-covariance function of the response $\boldsymbol{R}_{\boldsymbol{y} \boldsymbol{y}}(\tau)=E\left\{\boldsymbol{y}[t] \boldsymbol{y}^{T}[t-\tau]\right\}$, obeys the following relationship (similar to the Yule-Walker equations; refer to [74, p. 28]):

$$
\boldsymbol{a}_{0}^{T}(n y(m-1)+j) \boldsymbol{R}_{\boldsymbol{y} \boldsymbol{y}}^{T}(h+1)+\sum_{i=1}^{h_{j, m}} \boldsymbol{a}_{i}^{T}(n y(m-1)+j) \boldsymbol{R}_{\boldsymbol{y} \boldsymbol{y}}^{T}(h+1-i)=\mathbf{0} \forall h \geq h_{j, m}
$$

with $h_{j, m}$ designating the corresponding Kronecker index, and $n=\max \left(h_{1,1}, h_{2,1}, \ldots, h_{n y, M}\right)$ in analogy to the conventional VARMA models [74, pp. 52-56]. The preceding relationship implies that $\boldsymbol{p}[t]$ is uncorrelated to the linear combination, $\boldsymbol{a}_{0}^{T}(n y(m-1)+j) \boldsymbol{y}\left[t+h_{j, m}\right]+\sum_{i=1}^{h_{j, m}} \boldsymbol{a}_{i}^{T}(n y(m-1)+j) \boldsymbol{y}\left[t+h_{j, m}-i\right]$.

Thereby, a systematic procedure where CCA is performed sequentially, by adding elements to $f[t]$, one at a time (starting with $y_{1, k_{1}}[t]$ - the first element of the response vector $\boldsymbol{y}_{k_{1}}[t]$ ), may be used to determine the (Mny) zero canonical correlations of the echelon FP-VARMA representation.

Let $y_{j, k_{m}}\left[t+h_{j, m}\right](j=1, \ldots, n y$ and $m=1, \ldots, M)$ be the most recent element added in $\boldsymbol{f}[t]$, then the following hypothesis testing problem is considered for the smallest canonical correlation $\rho$ :

$$
\begin{array}{ll}
H_{0}: & \rho=0 \\
H_{1}: & \rho>0
\end{array}
$$

with $H_{0}$ and $H_{1}$ designating the null and alternative hypothesis, respectively. 


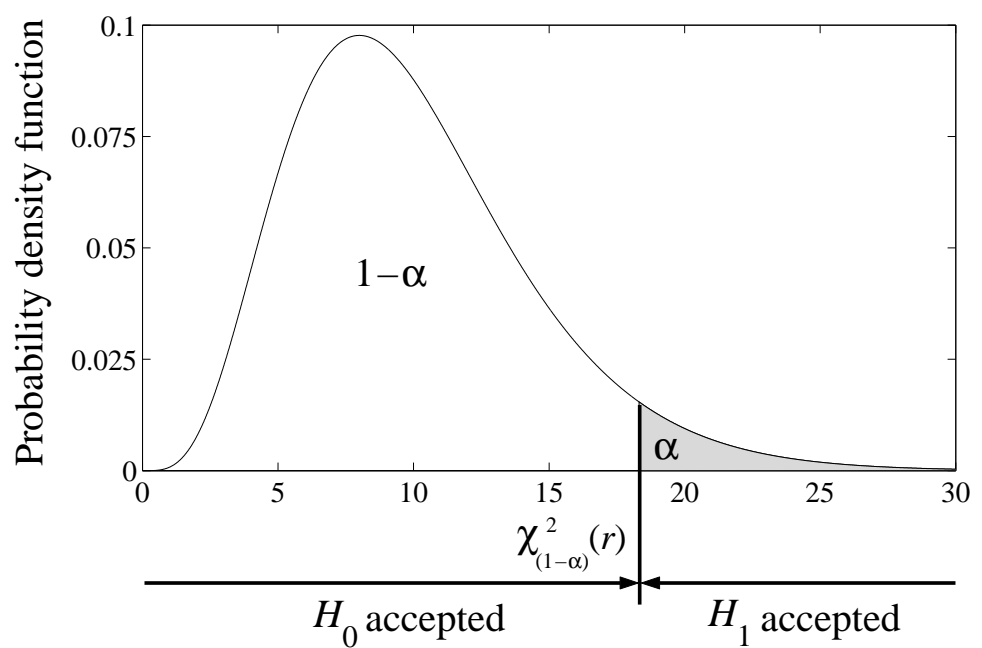

Figure 4.1: Statistical hypothesis testing based upon a $\chi^{2}$-distributed statistic (one-tailed test; $\alpha=0.05$, $r=10)$.

In practice however, estimates of the canonical correlations are obtained by replacing the covariances in Eq. (4.27a)-(4.27b) with their corresponding sample values. Under $H_{0}$ the test statistic below asymptotically follows a $\chi^{2}$ (chi-square) distribution with $(M n y s-\operatorname{dim}(\boldsymbol{f}[t])+1)$ degrees of freedom (in analogy to [73] for the conventional VARMA model case $\left.^{8}\right)$ :

$$
T=-(N-s) \ln \left(1-\frac{\widehat{\rho}^{2}}{\widehat{d}}\right) \sim \chi^{2}(\text { Mnys }-\operatorname{dim}(\boldsymbol{f}[t])+1)
$$

where $\widehat{d}=1+2 \sum_{v=1}^{h_{m, j}} \widehat{\rho}_{x x}(v) \widehat{\rho}_{z z}(v)$ (for $h_{m, j}=0$, then $\widehat{d}=1$ ), with $\widehat{\rho}_{x x}(v)$ and $\widehat{\rho}_{z z}(v)$ designating the lag- $v$ sample autocorrelation coefficients corresponding to the estimates of $x[t]$ and $z[t]$.

This leads to the following test at the $\alpha$ risk level (that is, probability of accepting $H_{1}$ while $H_{0}$ is true equals $\alpha$ ):

$$
\begin{array}{cc}
T<\chi_{(1-\alpha)}^{2}(M n y s-\operatorname{dim}(\boldsymbol{f}[t])+1) & \Longrightarrow H_{0} \text { is accepted } \\
\text { Else } & \Longrightarrow H_{1} \text { is accepted }
\end{array}
$$

with $\chi_{(1-\alpha)}^{2}(M n y s-\operatorname{dim}(\boldsymbol{f}[t])+1)$ designating the distribution's $(1-\alpha)$ critical point (see Fig. 4.1).

If $H_{0}$ is accepted, $y_{j, k_{m}}\left[t+h_{j, m}\right]$ is removed from $\boldsymbol{f}[t]$ and all terms $y_{j, k_{m}}[t+i]\left(i>h_{j, m}\right)$ are excluded from consideration, since they obey Eq. (4.29). The procedure is completed when all (Mny) zero canonical correlations are found, and the identified model is an $\operatorname{FP}-\operatorname{VARMA}(n, n)$ with $n=\max \left(h_{j, m}\right)$.

\footnotetext{
${ }^{8}$ Suppose that $\boldsymbol{y}[t]$ is a $(n y \times 1)$ stationary Gaussian conventional VARMA process. Let $\boldsymbol{p}[t]=\left[\boldsymbol{y}^{T}[t-1] \boldsymbol{y}^{T}[t-2] \ldots \boldsymbol{y}^{T}[t-\right.$ $s]]^{T}$ be a past vector with a pre-specified $s>0$ that contains all the information needed in predicting the future observations of $\boldsymbol{y}[t]$, and $\boldsymbol{f}[t]=\left[\ldots y_{j}\left[t+h_{j}\right]\right]^{T}$ be a future sub-vector of $\boldsymbol{y}[t]$ by adding elements to it sequentially, one at a time, starting with the first element of the response vector $\boldsymbol{y}[t]$. Let $y_{j}\left[t+h_{j}\right](j=1, \ldots, n y)$ be the most recent element added in $\boldsymbol{f}[t]$, with $\rho$ designating the corresponding smallest canonical correlation between $\boldsymbol{f}[t]$ and $\boldsymbol{p}[t]$, whereas $x[t]=\boldsymbol{v}_{f}^{T} \boldsymbol{f}[t]$ and $z[t]=\boldsymbol{v}_{p}^{T} \boldsymbol{p}[t]$ designate the corresponding canonical variates. Also, let $\widehat{\rho}$ be the corresponding smallest sample canonical correlation. Under the null hypothesis $\left(H_{0}: \rho=0\right)$ that the smallest canonical correlation $\rho$ between $\boldsymbol{f}[t]$ and $\boldsymbol{p}[t]$ is zero, but all the other canonical correlations are non-zero, the test statistic below asymptotically follows a $\chi^{2}$ (chi-square) distribution with $(n y s-\operatorname{dim}(\boldsymbol{f}[t])+1)$ degrees of freedom:$$
T=-(N-s) \ln \left(1-\frac{\widehat{\rho}^{2}}{\widehat{d}}\right) \sim \chi^{2}(n y s-\operatorname{dim}(\boldsymbol{f}[t])+1)
$$

where $\widehat{d}=1+2 \sum_{v=1}^{h_{j}} \widehat{\rho}_{x x}(v) \widehat{\rho}_{z z}(v)$ (for $h_{j}=0$, then $\widehat{d}=1$ ), with $\widehat{\rho}_{x x}(v)$ and $\widehat{\rho}_{z z}(v)$ designating the lag- $v$ sample autocorrelation coefficients corresponding to the estimates of $x[t]$ and $z[t][73]$.
} 
Refined scheme: Reduction of MA order A refinement on the basic scheme is employed so that the limitation of specifying an FP-VARMA $(n, n)$ model is removed, and further information about the model orders may be obtained. The refined scheme utilizes the lag $-j$ shifted "past" vector:

$$
\boldsymbol{p}_{j}[t]=\left[\boldsymbol{y}^{T}[t-j] \boldsymbol{y}^{T}[t-j-1] \ldots \boldsymbol{y}^{T}[t-j-s]\right]^{T}
$$

where $j$ satisfies $j \geq 1-n$, and $n=\max \left(h_{1,1}, h_{2,1}, \ldots, h_{n y, M}\right)=\max (n a, n c)$ for a given FP$\operatorname{VARMA}(n a, n c)$ model. Only the case where $n a=n$ and $n c<n$ is considered as this occurs more often in practice.

The idea is based on the fact that when a zero canonical correlation exists between $\boldsymbol{p}[t]$ and $\boldsymbol{f}[t]=$ $\left[\ldots y_{j, k_{m}}\left[t+h_{j, m}\right]\right]^{T}$, while it has been verified from the basic scheme that $h_{j, m}=n$, then the corresponding row of the echelon FP-VARMA model for $n a=n$ and $n c<n$ is an $\mathrm{MA}(u)$ process with $u \leq n-1$, that is:

$$
\begin{aligned}
x[t]=\boldsymbol{v}_{f}^{T}[t] \boldsymbol{f}[t] & =\underbrace{\boldsymbol{a}_{0}^{T}(n y(m-1)+j) \boldsymbol{y}[t+n]+\sum_{i=1}^{n} \boldsymbol{a}_{i}^{T}(n y(m-1)+j) \boldsymbol{y}[t+n-i]}_{\operatorname{MA}(u), u \leq n-1} \\
& =\underbrace{\boldsymbol{c}_{0}^{T}(n y(m-1)+j) \boldsymbol{w}[t+n]+\sum_{i}^{n c} \boldsymbol{c}_{i}^{T}(n y(m-1)+j) \boldsymbol{w}[t+n-i]}_{i=1}
\end{aligned}
$$

Thus, $x[t]$ is a linear combination of $\{\boldsymbol{w}[t+n], \ldots, \boldsymbol{w}[t+1]\}$ which is uncorrelated to any linear combination of $\boldsymbol{p}_{0}[t]=\left[\boldsymbol{y}^{T}[t] \boldsymbol{y}^{T}[t-1] \ldots \boldsymbol{y}^{T}[t-s]\right]^{T}$. This implies that there is a zero canonical correlation between $\boldsymbol{f}[t]$ and $\boldsymbol{p}_{0}[t]$.

The procedure may be generalized in order to obtain the true MA order $n c$ by performing sequentially CCA among $\boldsymbol{f}[t]$ and $\boldsymbol{p}_{j}[t]$ by replacing $j$ with $j-1$ at each iteration. The following hypothesis testing problem is considered for the smallest canonical correlation $\rho$ :

$$
\begin{array}{ll}
H_{0}: & \rho=0 \\
H_{1}: & \rho>0 \quad, \quad \text { the model orders are }(n a, n c)=(n, n+j)
\end{array}
$$

Under $H_{0}$ the test statistic below asymptotically follows a $\chi^{2}$ (chi-square) distribution with $(M n y(s+1)-$ $\operatorname{dim}(\boldsymbol{f}[t])+1$ ) degrees of freedom (in analogy to [73] for the conventional VARMA model case ${ }^{9}$ ):

$$
T_{j}=-(N-s-j+1) \ln \left(1-\frac{\widehat{\rho}^{2}}{\widehat{d}_{n, j}}\right) \sim \chi^{2}(M n y(s+1)-\operatorname{dim}(\boldsymbol{f}[t])+1)
$$

with $\widehat{d}_{n, j}=1+2 \sum_{v=1}^{n+j-1} \widehat{\rho}_{x x}(v) \widehat{\rho}_{z z}(v)$.

This leads to the following test at the $\alpha$ risk level:

$$
\begin{array}{cl}
T_{j}<\chi_{(1-\alpha)}^{2}(M n y(s+1)-\operatorname{dim}(\boldsymbol{f}[t])+1) & \Longrightarrow H_{0} \text { is accepted } \\
\text { Else } & \Longrightarrow H_{1} \text { is accepted }
\end{array}
$$

\footnotetext{
${ }^{9}$ Suppose that $\boldsymbol{y}[t]$ is a $(n y \times 1)$ stationary Gaussian conventional VARMA process. Let $\boldsymbol{p}_{j}[t]=\left[\boldsymbol{y}^{T}[t-j] \boldsymbol{y}^{T}[t-j-\right.$ 1] $\left.\ldots \boldsymbol{y}^{T}[t-j-s]\right]^{T}$ be the past vector with pre-specified $s>0$ and $j$, and $\boldsymbol{f}[t]=\left[\ldots y_{j}[t+n]\right]^{T}$ be the sub-vector of future response values, where in application $j \geq 1-n$ and $n=\max \left(h_{1}, \ldots, h_{n y}\right)=\max (n a, n c)$. Then, under the null hypothesis $\left(H_{0}: \rho=0\right)$ that the smallest canonical correlation $\rho$ between $\boldsymbol{f}[t]$ and $\boldsymbol{p}_{j}[t]$ is zero, but all the other canonical correlations are non-zero, the test statistic below asymptotically follows a $\chi^{2}$ (chi-square) distribution with $(n y(s+1)-\operatorname{dim}(\boldsymbol{f}[t])+1)$ degrees of freedom:

$$
T_{j}=-(N-s-j+1) \ln \left(1-\frac{\widehat{\rho}^{2}}{\widehat{d}_{n, j}}\right) \sim \chi^{2}(n y(s+1)-\operatorname{dim}(\boldsymbol{f}[t])+1)
$$
}

where $\widehat{d}_{n, j}=1+2 \sum_{v=1}^{n+j-1} \widehat{\rho}_{x x}(v) \widehat{\rho}_{z z}(v)[73]$. 
If $H_{0}$ is rejected, the model orders corresponding to the $(n y(m-1)+j)$-th row of the echelon FPVARMA model in Eq. (4.34) are $(n, n+j)$ and the procedure is terminated. Otherwise it is repeated if $j>1-n$, whereas the model orders corresponding to the $(n y(m-1)+j)$-th row are $(n, 0)$, if $j=1-n$.

The overall MA order $n c$ of the FP-VARMA model will be the maximum of the identified MA model orders from the rows where zero canonical correlations exist between the vectors $\boldsymbol{p}[t]$ and $\boldsymbol{f}[t]=$ $\left[\ldots, y_{j, k_{m}}\left[t+h_{j, m}\right]\right]^{T}$, for which $h_{j, m}=n$ from the basic scheme.

A simplified example of FP-VARMA model order specification is provided in Appendix 4.D.

\subsubsection{Functional subspace dimensionality specification}

The second step involves the specification of the functional subspace dimensionalities. Once the model orders $(n a, n c)$ are specified and the basis function family is selected, a sensible approach consists of fitting $\operatorname{FP}-\operatorname{VARMA}(n a, n c)_{[p, p]}$ models with increasing basis dimensionality $p$ on the data set $Z^{N M}$, until an adequate model is obtained. To avoid potential over-specification in the AR and MA part functional subspaces, lower dimensionalities are also considered.

Model adequacy may be quantified using proper forms of the Akaike Information Criterion (AIC) or the Bayessian Information Criterion (BIC) (see Chapter 2, Section 2.6). Notice, however, that the number of estimated parameters for a P-VAR model is $d_{\mathcal{M}}=\operatorname{dim}(\boldsymbol{\vartheta})$, whereas for an FP-VARMA model $d_{\mathcal{M}}=$ $\operatorname{dim}(\boldsymbol{\theta})$. Also, in order to determine the most compact FP-VARMA representation, different basis function families should be tested via the AIC and/or BIC criteria.

\subsubsection{Model validation}

The selected FP-VARMA model is accepted as an accurate representation of the system once properly validated. Model validation is generally based upon examining (i) the residual whiteness by means of the sample Cross-Correlation Function (CCF) [44, pp. 160-161] or a "portmanteau" test statistic [44, p. 510], and (ii) the predictive ability of the model within a properly selected validation data set (not previously used in the estimation procedure) [1, p. 510].

\subsection{Monte Carlo study}

A bivariate $\operatorname{FP}-\operatorname{VARMA}(1,1)_{[3,3]}$ model is used to generate data. The functional subspaces consist of the first three shifted Chebyshev polynomials of the second kind [45, p. 782] (see Chapter 2, Appendix 2.D), that is $p=3$. The modeling procedure relies upon $M=11$ operating conditions with $k \in[0,10]$, obtained at unit increments. The innovations for each operating condition $k$ are (i) Gaussian white with zero mean and identity covariance $\boldsymbol{\Sigma}_{k, k}$, and (ii) uncorrelated with those of any other operating condition (that is, $\boldsymbol{\Sigma}_{k, \ell}=$ 0), which implies that no interrelations exist among the obtained data records (groupwise homoscedastic innovations; see Chapter 2, Table 2.1).

The study encompasses 500 Monte Carlo runs, and parameter estimation is based upon $N=2,000$ sample-long data records. An iterated 2SLS estimator, based upon QR implementation [1, pp. 318-320] (see Chapter 2, Appendix 2.E) is utilized. The conditional ML estimation is based upon a quasi-Newton optimization algorithm [46, MATLAB ${ }^{\circledR}$ function “fminunc.m”] and uses the 2SLS parameter vector sample mean estimate from the first iteration as initial conditions (details in Table 4.1).

Model order specification via CCA employs a risk level $\alpha=10^{-2}$ and $s=6$ (since the AIC and BIC were minimized for P-VAR model orders around $4-6$ when fitted to the data in a pilot study). The 
Table 4.1: Details on the Monte Carlo study for FP-VARMA $(1,1)_{[3,3]}$ model identification.

\begin{tabular}{|c|c|}
\hline No. of responses: $n y=2$ & Func. subspaces: first 3 Chebyshev II polynomials \\
\hline No. of Monte Carlo runs: 500 & Innovation dependency: groupwise homoscedastic \\
\hline \multirow{9}{*}{$\begin{array}{c}\text { FP-VARMA } \\
\text { identification details }\end{array}$} & ML: quasi-Newton optimization algorithm \\
\hline & init. values from 2 SLS mean estimate ( $1^{\text {st }}$ iter) \\
\hline & max. number of iterations $=300$ \\
\hline & estimated pars termination tol. $=10^{-6}$ \\
\hline & objective function termination tol. $=10^{-3}$ \\
\hline & convergence criterion: $\left\|\widehat{\boldsymbol{\theta}}_{2 S L S}^{i+1}-\widehat{\boldsymbol{\theta}}_{2 S L S}^{i}\right\|_{2} /\left\|\widehat{\boldsymbol{\theta}}_{2 S L S}^{i}\right\|_{2}<10^{-3}$ \\
\hline & $\|\cdot\|_{2}$ : Euclidean norm of indicated vector; $\widehat{\boldsymbol{\theta}}_{2 S L S}^{i}: 2$ SLS estimate at $i-$ th iteration \\
\hline & $\begin{array}{l}\text { Model structure specification: model orders via CCA scheme }\left(s=6, \alpha=10^{-2}\right) \\
\text { functional subspaces via information criteria (AIC, BIC) }\end{array}$ \\
\hline & Number of estimated parameters: 24 \\
\hline \multirow{6}{*}{$\begin{array}{l}\text { conventional VARMA } \\
\text { identification details }\end{array}$} & ML: quasi-Newton optimization algorithm \\
\hline & init. values from 2 SLS mean estimate \\
\hline & max number of iterations $=300$ \\
\hline & estimated pars termination tol. $=10^{-6}$ \\
\hline & objective function termination tol. \\
\hline & Number of estimated parameters: 88 ( 8 parameters per operating point) \\
\hline
\end{tabular}
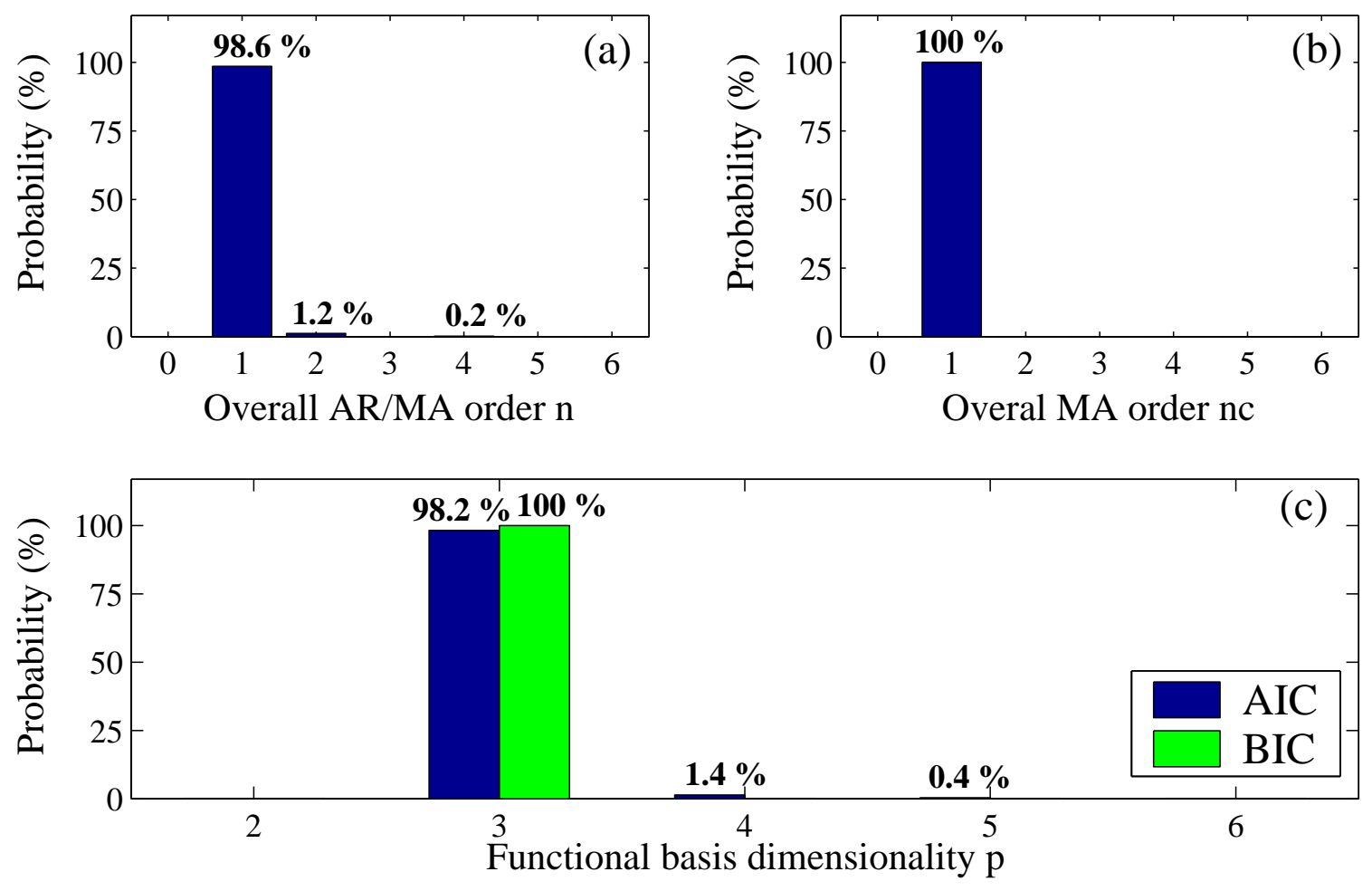

Figure 4.2: FP-VARMA $(1,1)_{[3,3]}$ model structure specification results $\left(s=6, \alpha=10^{-2}, 500\right.$ Monte Carlo runs): (a) basic CCA scheme for FP-VARMA $(n, n)$ model order specification; (b) refined CCA scheme for MA order reduction of FP-VARMA $(1,1)$ model; (c) FP-VARMA $(1,1)_{[p, p]}$ functional subspace dimensionality specification via the AIC/BIC criteria.

obtained results indicate that the CCA scheme specifies an $\operatorname{FP}-\operatorname{VARMA}(1,1)$ in the majority of the cases (see Fig. 4.2(a),(b)). The functional subspace specification problem is treated by fitting successive FP$\operatorname{VARMA}(1,1)_{[p, p]}$ models with increasing basis dimensionality $p=2,3, \ldots, 6$ and computing the corresponding AIC and BIC values. Monte Carlo results depicting the probability of attaining minimum AIC and 

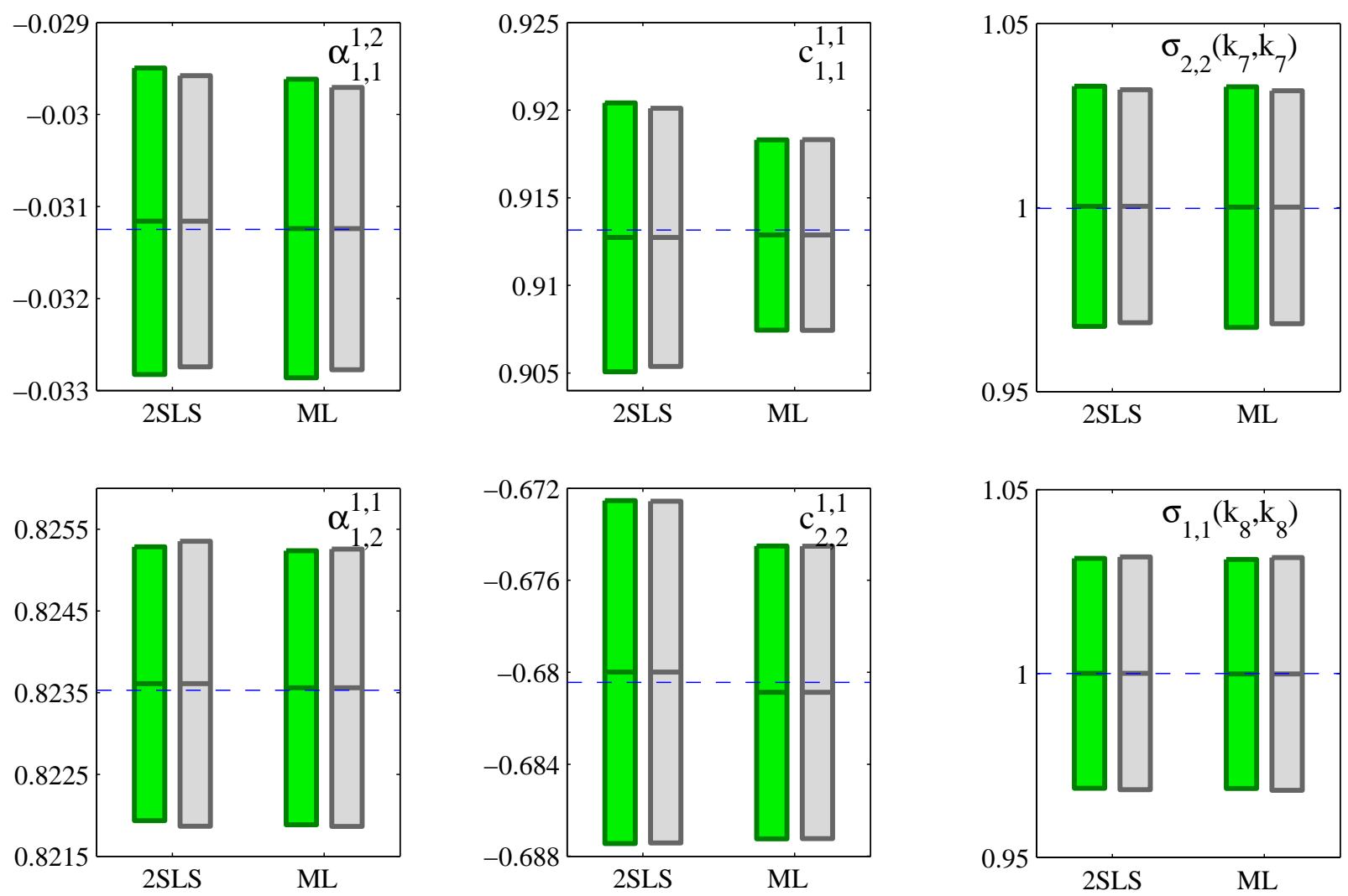

Figure 4.3: FP-VARMA $(1,1)_{[3,3]}$ actual values (--) and Monte Carlo estimates based upon the 2SLS and ML methods for selected projection coefficients and innovation variances (500 Monte Carlo runs per method; $a_{m, n}^{i, j}$ and $c_{m, n}^{i, j}$ designate the $(m, n)$-th elements of $\boldsymbol{A}_{i, j}$ and $\boldsymbol{C}_{i, j}$ respectively; $\sigma_{m, n}(k, \ell)$ designates the $(m, n)$-th element of $\boldsymbol{\Sigma}_{k, \ell}$; the dark green boxes indicate the sample mean \pm sample standard deviation; the light grey boxes indicate the sample mean \pm mean standard deviation computed through the asymptotic distribution).

BIC values versus functional basis dimensionality for the 2SLS estimator are presented in Fig. 4.2(b). The success rates $(\%)$ for both criteria are excellent, as minimal values are attained for $p=3$ in the vast majority of the runs.

The convergence of the iterated 2SLS estimator is rapid, as four iterations are typically required (see Table 4.1). Indicative interval parameter estimates (depicting sample mean \pm sample standard deviation) for the 2SLS and ML estimators along with the actual parameter values are presented in Fig. 4.3. Excellent agreement is observed between the estimates and their actual values. The results indicate that both estimators yield essentially unbiased estimates, with the ML expectedly providing better accuracy. Furthermore, the excellent agreement between the sample standard deviations and the corresponding values from the asymptotic analysis confirms the validity of the derived normal distribution for the projection coefficient and the innovation variance estimators (additional results in Appendix 4.F.1).

Partial results comparing the accuracy of the identified FP-VARMA $(1,1)_{[3,3]}$ model with its multimodel counterpart are shown in Fig. 4.4. For this purpose, conventional VARMA $(1,1)$ models are identified for each operating condition. The parameter estimation of the $\operatorname{FP}-\operatorname{VARMA}(1,1)_{[3,3]}$ and the conventional $\operatorname{VARMA}(1,1)$ models is based upon conditional ML (see Table 4.1 for details). The interval parameter estimates (depicting sample mean $\pm 2 \times$ sample standard deviation) indicate very good agreement among theoretical and estimated values for all operating conditions. However, the FP-VARMA $(1,1)_{[3,3]}$ based estimates, as expected, provide better accuracy than their conventional VARMA $(1,1)$ based counterparts. 

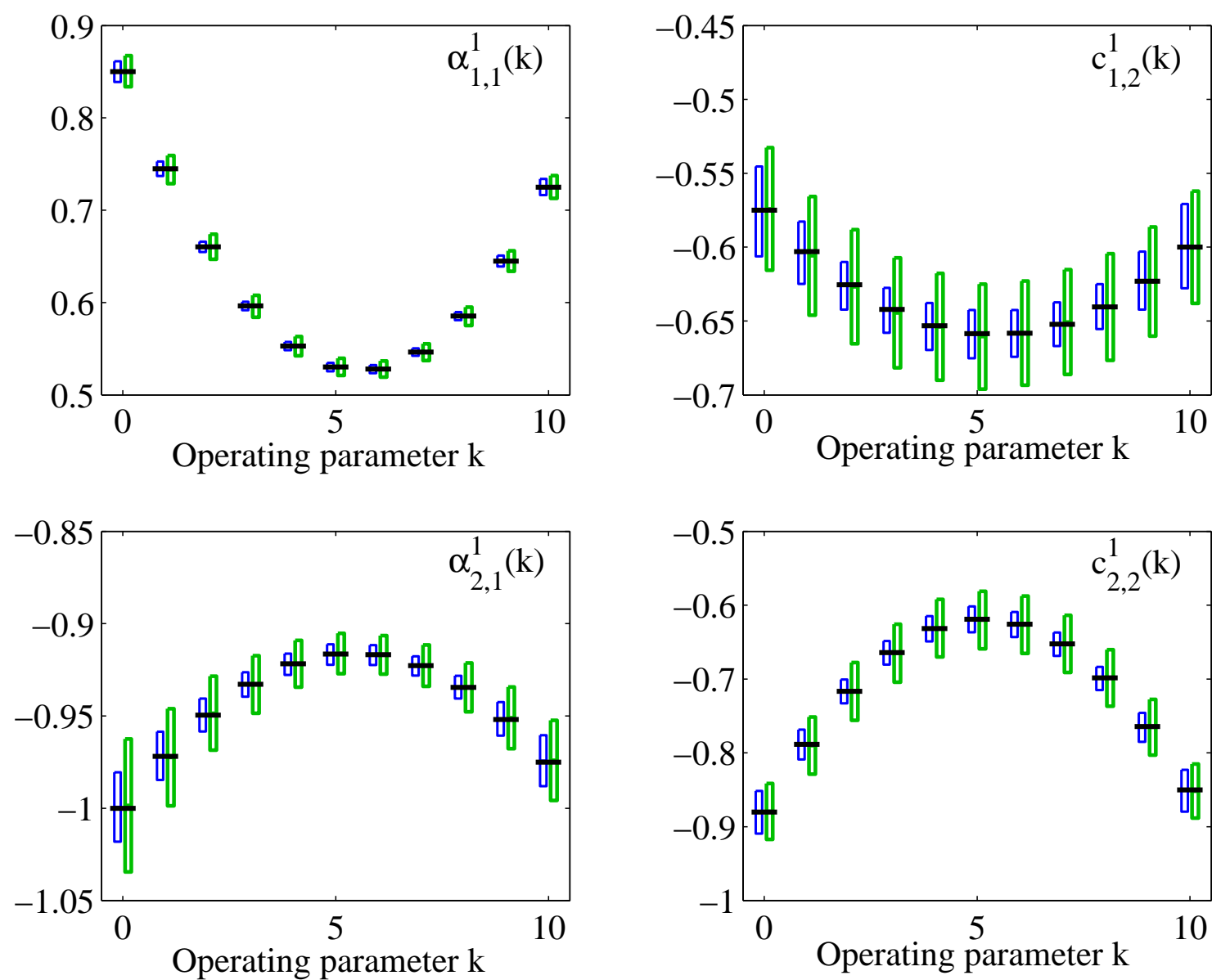

Figure 4.4: Dependence of certain AutoRegressive and Moving Average parameters on the operating parameter $k$ (boxes indicate sample mean estimates $\pm 2 \times$ mean sample standard deviation computed through the asymptotic distribution): (一) actual values; (blue boxes) FP-VARMA $(1,1)_{[3,3]}$ model; (green boxes) conventional VARMA $(1,1)$ models (500 Monte Carlo runs per model).

Since the assumption of groupwise homoscedastic innovations implies that no interrelations exist among the obtained data records, the only source of inaccuracy for the multi-model approach is the large number of estimated parameters for the same amount of data (a total of 88 parameters for the $11 \operatorname{VARMA}(1,1)$ models versus 24 for the $\mathrm{FP}-\operatorname{VARMA}(1,1)_{[3,3]}$ model).

\subsection{Concluding remarks}

The identification of multivariate stochastic systems capable of operating under different conditions was addressed, based on data records corresponding to a sample of such operating conditions. The focus was on the identification of FP-VARMA models within a novel Functional Pooling framework. The 2SLS and ML estimators were formulated, and their consistency and asymptotic normality were established. An effective method for 2SLS model estimation based on Pooled-VAR (P-VAR) modeling was introduced, and conditions ensuring FP-VARMA model identifiability were postulated. Model structure specification was based upon a two-step approach using CCA for model order selection, and proper forms of the AIC and BIC criteria for functional subspace selection. The effectiveness of the identification approach was confirmed via a Monte Carlo study, which also demonstrated the improved accuracy of the ML-based estimates over their 
2SLS counterparts. Finally, the comparison of the FP-VARMA identification approach with its conventional VARMA based multi-model counterpart verified the effectiveness of the novel framework as a useful tool for accurate global system modeling.

\section{Appendix 4.A: Tutorial example of P-VAR parameter estimation}

The identification of a $\mathrm{P}-\operatorname{VAR}(2)$ model with two excitations (that is, $n y=2$ ) is considered. The AR matrices are fully parameterized, and parameter estimation is based upon $M=2$ sets of response data of $N$ samples each (that is, $k=k_{1}, k_{2}$ and $t=1,2, \ldots, N$ ). The model is of the following form:

$$
\underbrace{\left[\begin{array}{l}
y_{1, k}[t] \\
y_{2, k}[t]
\end{array}\right]}_{\boldsymbol{y}_{k}[t]}+\underbrace{\left[\begin{array}{ll}
a_{1,1, k}^{1} & a_{1,2, k}^{1} \\
a_{2,1, k}^{1} & a_{2,2, k}^{1}
\end{array}\right]}_{\widetilde{\boldsymbol{A}}_{1, k}} \underbrace{\left[\begin{array}{c}
y_{1, k}[t-1] \\
y_{2, k}[t-1]
\end{array}\right]}_{\boldsymbol{y}_{k}[t-1]}+\underbrace{\left[\begin{array}{ll}
a_{1,1, k}^{2} & a_{1,2, k}^{2} \\
a_{2,1, k}^{2} & a_{2,2, k}^{2}
\end{array}\right]}_{\widetilde{\boldsymbol{A}}_{2, k}} \underbrace{\left[\begin{array}{c}
y_{1, k}[t-2] \\
y_{2, k}[t-2]
\end{array}\right]}_{\boldsymbol{y}_{k}[t-2]}=\underbrace{\left[\begin{array}{l}
\widetilde{e}_{1, k}[t] \\
\widetilde{e}_{2, k}[t]
\end{array}\right]}_{\widetilde{\boldsymbol{e}}_{k}[t]}
$$

Standard matrix calculus leads to:

$$
\left[\begin{array}{l}
\left.y_{1, k}[t]\right] \\
y_{2, k}[t]
\end{array}\right]=\underbrace{\left(\boldsymbol{I}_{2} \otimes\left[-y_{1, k}[t-1]-y_{2, k}[t-1]-y_{1, k}[t-2]-y_{2, k}[t-2]\right]\right)}_{\widetilde{\boldsymbol{\Phi}}_{k}^{T}[t]} \underbrace{\left[\begin{array}{l}
a_{1,1, k}^{1} \\
a_{1,2, k}^{1} \\
a_{1,1, k}^{2} \\
a_{1,2, k}^{2} \\
a_{2,1, k}^{1} \\
a_{2,2, k}^{1} \\
a_{2,1, k}^{2} \\
a_{2,2, k}^{2}
\end{array}\right]}_{\boldsymbol{\vartheta}_{k}}+\left[\begin{array}{l}
e_{1, k}[t] \\
e_{2, k}[t]
\end{array}\right]
$$

Pooling together the preceding equations which belong to the operating conditions $k=k_{1}, k_{2}$ yields:

$$
\underbrace{\left[\begin{array}{c}
\boldsymbol{y}_{k_{1}}[t] \\
\cdots \\
\boldsymbol{y}_{k_{2}}[t]
\end{array}\right]}_{\boldsymbol{y}[t]}=\underbrace{\left[\begin{array}{cc}
\widetilde{\boldsymbol{\Phi}}_{k_{1}}^{T}[t] & \mathbf{0} \\
\cdots \cdots & \dot{\widetilde{\boldsymbol{\Phi}}}_{k_{2}}^{T}[t]
\end{array}\right]}_{\widetilde{\boldsymbol{\Phi}}^{T}[t]} \underbrace{\left[\begin{array}{c}
\boldsymbol{\vartheta}_{k_{1}} \\
\cdots \\
\boldsymbol{\vartheta}_{k_{2}}
\end{array}\right]}_{\boldsymbol{\vartheta}}+\underbrace{\left[\begin{array}{c}
\boldsymbol{e}_{k_{1}}[t] \\
\cdots \\
\boldsymbol{e}_{k_{2}}[t]
\end{array}\right]}_{\boldsymbol{e}[t]}
$$

Under the assumption of contemporaneously correlated innovations, the corresponding covariance matrix $\boldsymbol{\Sigma}$ is given by:

$$
\boldsymbol{\Sigma}=\left[\begin{array}{c|c}
\boldsymbol{\Sigma}_{k_{1}, k_{1}} & \boldsymbol{\Sigma}_{k_{1}, k_{2}} \\
\hline \boldsymbol{\Sigma}_{k_{2}, k_{1}} & \boldsymbol{\Sigma}_{k_{2}, k_{2}}
\end{array}\right]=\left[\begin{array}{cc|cc}
\sigma_{1,1}\left(k_{1}, k_{1}\right) & \sigma_{1,2}\left(k_{1}, k_{1}\right) & \sigma_{1,1}\left(k_{1}, k_{2}\right) & \sigma_{1,2}\left(k_{1}, k_{2}\right) \\
\sigma_{2,1}\left(k_{1}, k_{1}\right) & \sigma_{2,2}\left(k_{1}, k_{1}\right) & \sigma_{2,1}\left(k_{1}, k_{2}\right) & \sigma_{2,2}\left(k_{1}, k_{2}\right) \\
\hline \sigma_{1,1}\left(k_{2}, k_{1}\right) & \sigma_{1,2}\left(k_{2}, k_{1}\right) & \sigma_{1,1}\left(k_{2}, k_{2}\right) & \sigma_{1,2}\left(k_{2}, k_{2}\right) \\
\sigma_{2,1}\left(k_{2}, k_{1}\right) & \sigma_{2,2}\left(k_{2}, k_{1}\right) & \sigma_{2,1}\left(k_{2}, k_{2}\right) & \sigma_{2,2}\left(k_{2}, k_{2}\right)
\end{array}\right]
$$

The WLS estimators are given by:

$$
\begin{gathered}
\widehat{\boldsymbol{\vartheta}}_{W L S}=\left[\frac{1}{2 N} \sum_{t=1}^{N} \widetilde{\boldsymbol{\Phi}}[t] \boldsymbol{\Sigma}^{-1} \widetilde{\boldsymbol{\Phi}}^{T}[t]\right]^{-1}\left[\frac{1}{2 N} \sum_{t=1}^{N} \widetilde{\boldsymbol{\Phi}}[t] \boldsymbol{\Sigma}^{-1} \boldsymbol{y}[t]\right] \\
\widehat{\boldsymbol{\Sigma}}_{W L S}=\frac{1}{N} \sum_{t=1}^{N} \widetilde{\boldsymbol{e}}\left[t, \widehat{\boldsymbol{\vartheta}}_{W L S}\right] \widetilde{\boldsymbol{e}}^{T}\left[t, \widehat{\boldsymbol{\vartheta}}_{W L S}\right]
\end{gathered}
$$




\section{Appendix 4.B: Additional results on the 2SLS and ML type estimators}

\section{B.1 Proof of Theorem 4.4.1}

\section{B.4.1 Large sample properties of the iterated 2SLS estimator $\widehat{\boldsymbol{\theta}}_{2 S L S}$}

The projection coefficient estimator in Eq. (4.15a) is rewritten in the following recursive form:

$$
\widehat{\boldsymbol{\theta}}_{2 S L S}^{i+1}=\left[\frac{1}{N M} \sum_{t=1}^{N} \boldsymbol{\Phi}\left[t, \widehat{\boldsymbol{\theta}}_{2 S L S}^{i}\right] \boldsymbol{\Sigma}^{-1} \boldsymbol{\Phi}^{T}\left[t, \widehat{\boldsymbol{\theta}}_{2 S L S}^{i}\right]\right]^{-1}\left[\frac{1}{N M} \sum_{t=1}^{N} \boldsymbol{\Phi}\left[t, \widehat{\boldsymbol{\theta}}_{2 S L S}^{i}\right] \boldsymbol{\Sigma}^{-1} \boldsymbol{y}[t]\right]
$$

with $\widehat{\boldsymbol{\theta}}_{2 S L S}^{i}$ designating the $i$-th iteration 2SLS estimate. Note that the estimation of the refined 2SLS estimate $\widehat{\boldsymbol{\theta}}_{2 S L S}^{i+1}$ requires the updating of matrix $\boldsymbol{\Phi}\left[t, \widehat{\boldsymbol{\theta}}_{2 S L S}^{i}\right]$, which is an implicit function of $\widehat{\boldsymbol{\theta}}_{2 S L S}^{i}$.

Convergence analysis of the projection coefficient estimator Consider the recursive form of the 2SLS estimator in Eq. (4.B.1) as $N$ tends to infinity $(N \longrightarrow \infty)$. Under mild conditions (that is, $\boldsymbol{y}_{k}[t]$ and $\boldsymbol{e}_{k}[t, \boldsymbol{\theta}]$ are zero-mean ergodic and stationary responses, and $\boldsymbol{e}_{k}[t, \boldsymbol{\theta}]$ being also an analytic function of $\boldsymbol{\theta}$ ), the sample covariances will tend to their corresponding theoretical expectations ${ }^{10}[2, \mathrm{pp} .547-550]$, thereby ${ }^{11}$ :

$$
\begin{aligned}
\widehat{\boldsymbol{\theta}}_{2 S L S}^{i+1} & =E\left\{\boldsymbol{\Phi}\left[t, \widehat{\boldsymbol{\theta}}_{2 S L S}^{i}\right] \boldsymbol{\Sigma}^{-1} \boldsymbol{\Phi}^{T}\left[t, \widehat{\boldsymbol{\theta}}_{2 S L S}^{i}\right]\right\}^{-1} E\left\{\boldsymbol{\Phi}\left[t, \widehat{\boldsymbol{\theta}}_{2 S L S}^{i}\right] \boldsymbol{\Sigma}^{-1} \boldsymbol{y}[t]\right\} \\
& =\widehat{\boldsymbol{\theta}}_{2 S L S}^{i}+E\left\{\boldsymbol{\Phi}\left[t, \widehat{\boldsymbol{\theta}}_{2 S L S}^{i}\right] \boldsymbol{\Sigma}^{-1} \boldsymbol{\Phi}^{T}\left[t, \widehat{\boldsymbol{\theta}}_{2 S L S}^{i}\right]\right\}^{-1} E\left\{\boldsymbol{\Phi}\left[t, \widehat{\boldsymbol{\theta}}_{2 S L S}^{i}\right] \boldsymbol{\Sigma}^{-1} \boldsymbol{e}\left[t, \widehat{\boldsymbol{\theta}}_{2 S L S}^{i}\right]\right\}
\end{aligned}
$$

Linearizing Eq. (4.B.2) using a first order Taylor series expansion ${ }^{12}$ around the true projection coefficient vector $\boldsymbol{\theta}_{o}$ yields:

$$
\begin{aligned}
& \widehat{\boldsymbol{\theta}}_{2 S L S}^{i+1}- \boldsymbol{\theta}_{o} \approx \widehat{\boldsymbol{\theta}}_{2 S L S}^{i}-\boldsymbol{\theta}_{o}-E\left\{\boldsymbol{\Phi}\left[t, \boldsymbol{\theta}_{o}\right] \boldsymbol{\Sigma}^{-1} \boldsymbol{\Phi}^{T}\left[t, \boldsymbol{\theta}_{o}\right]\right\}^{-1} E\left\{\boldsymbol{\Phi}\left[t, \boldsymbol{\theta}_{o}\right] \boldsymbol{\Sigma}^{-1} \boldsymbol{\Psi}^{T}\left[t, \boldsymbol{\theta}_{o}\right]\right\}\left(\widehat{\boldsymbol{\theta}}_{2 S L S}^{i}-\boldsymbol{\theta}_{o}\right) \\
&=\left(\boldsymbol{I}_{\operatorname{dim}(\boldsymbol{\theta})}-E\left\{\boldsymbol{\Phi}\left[t, \boldsymbol{\theta}_{o}\right] \boldsymbol{\Sigma}^{-1} \boldsymbol{\Phi}^{T}\left[t, \boldsymbol{\theta}_{o}\right]\right\}^{-1} E\left\{\boldsymbol{\Phi}\left[t, \boldsymbol{\theta}_{o}\right] \boldsymbol{\Sigma}^{-1} \boldsymbol{\Psi}^{T}\left[t, \boldsymbol{\theta}_{o}\right]\right\}\right)\left(\widehat{\boldsymbol{\theta}}_{2 S L S}^{i}-\boldsymbol{\theta}_{o}\right)
\end{aligned}
$$

which implies that $\widehat{\boldsymbol{\theta}}^{i}$ is locally linear convergent to $\boldsymbol{\theta}_{o}$ if and only if $E\left\{\boldsymbol{\Phi}\left[t, \boldsymbol{\theta}_{o}\right] \boldsymbol{\Sigma}^{-1} \boldsymbol{\Phi}^{T}\left[t, \boldsymbol{\theta}_{o}\right]\right\}^{-1}$ $E\left\{\boldsymbol{\Phi}\left[t, \boldsymbol{\theta}_{o}\right] \boldsymbol{\Sigma}^{-1} \mathbf{\Psi}^{T}\left[t, \boldsymbol{\theta}_{o}\right]\right\}$, has eigenvalues inside the unit circle centered at $(1,0)$ in the complex plane ${ }^{13}$.

Remark 8. The preceding result is in accordance to the conventional model case [76]. Notice however that in the FP-VARMA case, the covariances in the expectations are weighted by the term $\boldsymbol{\Sigma}^{-1}$.

\footnotetext{
${ }^{10}$ Let the stationary stochastic processes $z_{1}[t]$ and $z_{2}[t]$ be given by $z_{1}[t]=G(B) w_{1}[t]$ and $z_{2}[t]=H(B) w_{2}[t]$, with $G(B)$ and $H(B)$ stable filters, and $\boldsymbol{w}[t]=\left[w_{1}[t] w_{2}[t]\right]^{T}$ a zero-mean white noise with positive-definite covariance matrix and finite fourth order moments. Then, $1 / N \sum_{t=1}^{N} z_{1}[t] z_{2}[t] \stackrel{p}{\longrightarrow} E\left\{z_{1}[t] z_{2}[t]\right\} \quad(N \longrightarrow \infty)$ [2, pp. 547-550].

${ }^{11}$ If $\boldsymbol{x}_{N} \stackrel{p}{\longrightarrow} \boldsymbol{x}$ and $\boldsymbol{A}_{N} \stackrel{p}{\longrightarrow} \boldsymbol{A}$, then $\boldsymbol{A}_{N} \boldsymbol{x}_{N} \stackrel{p}{\longrightarrow} \boldsymbol{A} \boldsymbol{x} \quad(N \longrightarrow \infty)$ [44, p. 683].

${ }^{12}$ Let $\boldsymbol{\theta}_{N}=\boldsymbol{\theta}_{o}+O_{p}\left(r_{N}\right)$ be a $(n \times 1)$ random vector sequence, where $r_{N}=o(1)$, and let $g: \mathbb{R}^{n} \longrightarrow \mathbb{R}$ be a function with continuous partial derivatives of order two at $\boldsymbol{\theta}_{o}$. Then, $g\left(\boldsymbol{\theta}_{N}\right)=g\left(\boldsymbol{\theta}_{o}\right)+\frac{\partial g\left(\boldsymbol{\theta}_{o}\right)}{\partial \boldsymbol{\theta}^{T}}\left(\boldsymbol{\theta}_{N}-\boldsymbol{\theta}_{o}\right)+O_{p}\left(r_{N}^{2}\right)$.

(Let $\left\{a_{N}\right\}$ be a sequence of real numbers and $\left\{b_{N}\right\}$ a sequence of positive real numbers. Then $\left\{a_{N}\right\}$ is said to be of smaller order than $\left\{b_{N}\right\}\left(a_{N}=o\left(b_{N}\right)\right)$ if $a_{N} / b_{N} \longrightarrow 0(N \longrightarrow \infty)$. If $\left\{\boldsymbol{A}_{N}=\left[a_{i j, N}\right]\right\}$ is a sequence of random matrices, then $\boldsymbol{A}_{N}$ is said to be at most of order in probability $\left(\boldsymbol{A}_{N}=O_{p}\left(b_{N}\right)\right)$ if $\forall \epsilon>0, \exists c_{\epsilon}: \forall N \operatorname{Pr}\left(\left|a_{i j, N}\right|>c_{\epsilon} b_{N}\right) \leq \epsilon$ for $i=1,2, \ldots, m$, $j=1,2, \ldots, n)[44$, p. 685].

${ }^{13}$ Consider the $(m \times m)$ matrix $\boldsymbol{A}$ with eigenvalues $\lambda_{1}, \ldots, \lambda_{m}$, then: $\boldsymbol{A}$ is convergent $\Longleftrightarrow \max _{i=1, \ldots, m}\left|\lambda_{i}\right|<1$ [43, p. 119].
} 
Consistency of the projection coefficient estimator If $\widehat{\boldsymbol{\theta}}_{2 S L S}$ denotes the limit point of $\widehat{\boldsymbol{\theta}}_{2 S L S}^{i}$ (that is, $\lim _{i \rightarrow \infty} \widehat{\boldsymbol{\theta}}_{2 S L S}^{i}=\widehat{\boldsymbol{\theta}}_{2 S L S}$ ), then $\widehat{\boldsymbol{\theta}}_{2 S L S}$ is consistent by virtue of Eq. (4.B.3).

Asymptotic distribution of the projection coefficient estimator The iterated 2SLS estimator asymptotic distribution is established by noticing that the limiting points of Eq. (4.B.1) satisfy the following relationship:

$$
\lim _{i \rightarrow \infty} \widehat{\boldsymbol{\theta}}_{2 S L S}^{i}=\widehat{\boldsymbol{\theta}}_{2 S L S}=\operatorname{sol}\left\{\frac{1}{N M} \sum_{t=1}^{N} \boldsymbol{\Phi}\left[t, \widehat{\boldsymbol{\theta}}_{2 S L S}\right] \boldsymbol{\Sigma}^{-1} \boldsymbol{e}\left[t, \widehat{\boldsymbol{\theta}}_{2 S L S}\right]=\mathbf{0}\right\}
$$

with the argument "sol $\{\cdot\}$ " designating the solutions of the indicated equation.

A first order Taylor series expansion ${ }^{12}$ of Eq. (4.B.4) around $\boldsymbol{\theta}_{o}$ yields:

$$
\begin{aligned}
\mathbf{0}=\frac{1}{N M} \sum_{t=1}^{N} \boldsymbol{\Phi}\left[t, \boldsymbol{\theta}_{o}\right] \boldsymbol{\Sigma}^{-1} \boldsymbol{e}\left[t, \boldsymbol{\theta}_{o}\right]+ & {\left[\left.\frac{1}{N M} \sum_{t=1}^{N} \frac{\partial \boldsymbol{\Phi}[t, \boldsymbol{\theta}]}{\partial \boldsymbol{\theta}^{T}}\right|_{\boldsymbol{\theta}=\boldsymbol{\theta}_{o}} \boldsymbol{\Sigma}^{-1} \boldsymbol{e}\left[t, \boldsymbol{\theta}_{o}\right]\right.} \\
& \left.-\frac{1}{N M} \sum_{t=1}^{N} \boldsymbol{\Phi}\left[t, \boldsymbol{\theta}_{o}\right] \boldsymbol{\Sigma}^{-1} \mathbf{\Psi}\left[t, \boldsymbol{\theta}_{o}\right]\right]\left(\widehat{\boldsymbol{\theta}}_{2 S L S}-\boldsymbol{\theta}_{o}\right)
\end{aligned}
$$

To establish the 2SLS estimator asymptotic distribution, Eq. (4.B.5) may be rewritten as follows:

$$
\begin{array}{r}
\sqrt{N M}\left(\widehat{\boldsymbol{\theta}}_{2 S L S}-\boldsymbol{\theta}_{o}\right)=-\left[\left.\frac{1}{N M} \sum_{t=1}^{N} \frac{\partial \boldsymbol{\Phi}[t, \boldsymbol{\theta}]}{\partial \boldsymbol{\theta}^{T}}\right|_{\boldsymbol{\theta}_{=\boldsymbol{\theta}_{o}}} \boldsymbol{\Sigma}^{-1} \boldsymbol{e}\left[t, \boldsymbol{\theta}_{o}\right]-\frac{1}{N M} \sum_{t=1}^{N} \boldsymbol{\Phi}\left[t, \boldsymbol{\theta}_{o}\right] \boldsymbol{\Sigma}^{-1} \mathbf{\Psi}\left[t, \boldsymbol{\theta}_{o}\right]\right]^{-1} \\
\frac{1}{\sqrt{N M}} \sum_{t=1}^{N} \boldsymbol{\Phi}\left[t, \boldsymbol{\theta}_{o}\right] \boldsymbol{\Sigma}^{-1} \boldsymbol{e}\left[t, \boldsymbol{\theta}_{o}\right]
\end{array}
$$

Notice that $\boldsymbol{e}\left[t, \boldsymbol{\theta}_{o}\right] \triangleq \boldsymbol{w}[t]$, and also that the terms $\partial \boldsymbol{\Phi}[t, \boldsymbol{\theta}] /\left.\partial \boldsymbol{\theta}^{T}\right|_{\boldsymbol{\theta}=\boldsymbol{\theta}_{o}}, \boldsymbol{\Phi}\left[t, \boldsymbol{\theta}_{o}\right]$ and $\partial \boldsymbol{e}[t, \boldsymbol{\theta}] /\left.\partial \boldsymbol{\theta}^{T}\right|_{\boldsymbol{\theta}_{=} \boldsymbol{\theta}_{o}}$ depend upon data up to $(t-1)$ (see Eq. (4.12b)-(4.12c) and also Eq. (4.B.12a)-(4.B.12b) in Appendix 4.B.2). Thus, the limit in probability of the bracketed expression in the Eq. (4.B.6) yields ${ }^{10,14}$ :

$$
\begin{array}{r}
-\left[\left.\frac{1}{N M} \sum_{t=1}^{N} \frac{\partial \boldsymbol{\Phi}[t, \boldsymbol{\theta}]}{\partial \boldsymbol{\theta}^{T}}\right|_{\boldsymbol{\theta}=\boldsymbol{\theta}_{o}} \boldsymbol{\Sigma}^{-1} \boldsymbol{w}[t]-\frac{1}{N M} \sum_{t=1}^{N} \boldsymbol{\Phi}\left[t, \boldsymbol{\theta}_{o}\right] \boldsymbol{\Sigma}^{-1} \boldsymbol{\Psi}\left[t, \boldsymbol{\theta}_{o}\right]\right]^{-1} \stackrel{p}{\longrightarrow} \\
M E\left\{\boldsymbol{\Phi}\left[t, \boldsymbol{\theta}_{o}\right] \boldsymbol{\Sigma}^{-1} \boldsymbol{\Psi}^{T}\left[t, \boldsymbol{\theta}_{o}\right]\right\}^{-1}(N \longrightarrow \infty)
\end{array}
$$

The limiting distribution of the rightmost quantity in Eq. (4.B.6) is found by means of a central limit theorem is found by means of a central limit theorem ${ }^{15}$ [2, p. 550], and is given by:

$$
\frac{1}{\sqrt{N M}} \sum_{t=1}^{N} \boldsymbol{\Phi}\left[t, \boldsymbol{\theta}_{o}\right] \boldsymbol{\Sigma}^{-1} \boldsymbol{w}[t] \stackrel{d}{\longrightarrow} \mathcal{N}\left(\mathbf{0}, \frac{1}{M} E\left\{\boldsymbol{\Phi}\left[t, \boldsymbol{\theta}_{o}\right] \boldsymbol{\Sigma}^{-1} \boldsymbol{\Phi}^{T}\left[t, \boldsymbol{\theta}_{o}\right]\right\}\right) \quad(N \longrightarrow \infty)
$$

\footnotetext{
${ }^{14}$ (Slutsky's Theorem) If $\boldsymbol{g}: \mathbb{R}^{k} \longrightarrow \mathbb{R}^{m}$ is a continuous function, then $\boldsymbol{x}_{N} \stackrel{p}{\longrightarrow} \boldsymbol{x} \Longrightarrow \boldsymbol{g}\left(\boldsymbol{x}_{N}\right) \stackrel{p}{\longrightarrow} \boldsymbol{g}(\boldsymbol{x})(N \longrightarrow \infty)[44, \mathrm{p}$. 683].

${ }^{15}$ Consider $\boldsymbol{x}_{N}=(1 / \sqrt{N}) \sum_{t=1}^{N} \boldsymbol{z}[t]$, where $\boldsymbol{z}[t]$ is a zero-mean vector stationary process given by $\boldsymbol{z}[t]=\boldsymbol{\Phi}[t] \boldsymbol{v}[t]$. The entries of $\boldsymbol{\Phi}[t]$ and $\boldsymbol{v}[t]$ are stationary, possibly correlated, ARMA processes with zero means and underlying white noise sequences with finite fourth-order moments. The elements of $\boldsymbol{\Phi}[t]$ may also contain a bounded deterministic term. Assume that the limit $\boldsymbol{P}=\lim _{N \longrightarrow \infty} E\left\{\boldsymbol{x}_{N} \boldsymbol{x}_{N}^{T}\right\}$ exists and is nonsingular. Then $\boldsymbol{x}_{N}$ is asymptotically Gaussian distributed, $\boldsymbol{x}_{N} \stackrel{d}{\longrightarrow} \mathcal{N}(\mathbf{0}, \boldsymbol{P})$ $(N \longrightarrow \infty)[2$, p. 550].
} 
The asymptotic distribution of the 2SLS estimator $\widehat{\boldsymbol{\theta}}_{2 S L S}$ is thus ${ }^{16}$ :

$$
\sqrt{N M}\left(\widehat{\boldsymbol{\theta}}_{2 S L S}-\boldsymbol{\theta}_{o}\right) \stackrel{d}{\longrightarrow} \mathcal{N}\left(\mathbf{0}, \boldsymbol{P}_{2 S L S}\right) \quad(N \longrightarrow \infty)
$$

with $\boldsymbol{P}_{2 S L S}=M E\left\{\boldsymbol{\Phi}\left[t, \boldsymbol{\theta}_{o}\right] \boldsymbol{\Sigma}^{-1} \boldsymbol{\Psi}^{T}\left[t, \boldsymbol{\theta}_{o}\right]\right\}^{-1} E\left\{\boldsymbol{\Phi}\left[t, \boldsymbol{\theta}_{o}\right] \boldsymbol{\Sigma}^{-1} \boldsymbol{\Phi}^{T}\left[t, \boldsymbol{\theta}_{o}\right]\right\} E\left\{\boldsymbol{\Phi}\left[t, \boldsymbol{\theta}_{o}\right] \boldsymbol{\Sigma}^{-1} \mathbf{\Psi}^{T}\left[t, \boldsymbol{\theta}_{o}\right]\right\}^{-T}$.

\section{B.1.2 Large sample properties of the iterated 2SLS estimator $\widehat{\boldsymbol{\Sigma}}_{2 S L S}$}

To establish the large sample properties of $\widehat{\boldsymbol{\Sigma}}_{2 S L S}$, the FP-VARMA $(n a, n c)_{[p a, p c]}$ representations of Eq. (4.3a)-(4.3c), for each distinct (sample) operating condition $\left\{k_{1}, k_{2}, \ldots, k_{M}\right\}$ are stacked one below the other, yielding the following expression:

$$
\boldsymbol{A}(\mathcal{B}) \boldsymbol{y}[t]=\boldsymbol{C}(\mathcal{B}) \boldsymbol{w}[t]
$$

where:

$$
\boldsymbol{A}(\mathcal{B}) \triangleq\left[\begin{array}{cccc}
\boldsymbol{A}\left[\mathcal{B}, k_{1}\right] & \mathbf{0} & \ldots & \mathbf{0} \\
\mathbf{0} & \boldsymbol{A}\left[\mathcal{B}, k_{2}\right] & \ldots & \mathbf{0} \\
\vdots & \vdots & \ddots & \vdots \\
\mathbf{0} & \mathbf{0} & \ldots & \boldsymbol{A}\left[\mathcal{B}, k_{M}\right]
\end{array}\right], \boldsymbol{C}(\mathcal{B}) \triangleq\left[\begin{array}{cccc}
\boldsymbol{C}\left[\mathcal{B}, k_{1}\right] & \mathbf{0} & \ldots & \mathbf{0} \\
\mathbf{0} & \boldsymbol{C}\left[\mathcal{B}, k_{2}\right] \ldots & \mathbf{0} \\
\vdots & \vdots & \ddots & \vdots \\
\mathbf{0} & \mathbf{0} & \ldots & \boldsymbol{C}\left[\mathcal{B}, k_{M}\right]
\end{array}\right]
$$

The preceding matrix polynomials may be written as (see also Eq. (4.3b) and Eq. (4.3c)):

$$
\boldsymbol{A}(\mathcal{B}) \triangleq \boldsymbol{I}_{M n y}+\boldsymbol{A}_{1} \mathcal{B}+\ldots+\boldsymbol{A}_{n a} \mathcal{B}^{n a}, \quad \boldsymbol{C}(\mathcal{B}) \triangleq \boldsymbol{I}_{M n y}+\boldsymbol{C}_{1} \mathcal{B}+\ldots+\boldsymbol{C}_{n c} \mathcal{B}^{n c}
$$

with (see also Eq. (4.2c)):

$$
\boldsymbol{A}_{i} \triangleq\left[\begin{array}{cccc}
\boldsymbol{A}_{i}\left(k_{1}\right) & \mathbf{0} & \ldots & \mathbf{0} \\
\mathbf{0} & \boldsymbol{A}_{i}\left(k_{2}\right) \ldots & \mathbf{0} \\
\vdots & \vdots & \ddots & \vdots \\
\mathbf{0} & \mathbf{0} & \ldots & \boldsymbol{A}_{i}\left(k_{M}\right)
\end{array}\right], \boldsymbol{C}_{i} \triangleq\left[\begin{array}{cccc}
\boldsymbol{C}_{j}\left(k_{1}\right) & \mathbf{0} & \ldots & \mathbf{0} \\
\mathbf{0} & \boldsymbol{C}_{j}\left(k_{2}\right) \ldots & \mathbf{0} \\
\vdots & \vdots & \ddots & \vdots \\
\mathbf{0} & \mathbf{0} & \ldots & \boldsymbol{C}_{j}\left(k_{M}\right)
\end{array}\right], \begin{aligned}
& i=1,2, \ldots, n a \\
& j=1,2, \ldots, n c
\end{aligned}
$$

The following lemma is useful to continue the analysis.

Lemma 4.B.1. Consider the FP-VARMA model of Eq. (4.B.8a)-(4.B.8d), and let $\widehat{\boldsymbol{\theta}}$ be a consistent estimate of the projection coefficient vector with $\sqrt{N M}\left(\widehat{\boldsymbol{\theta}}-\boldsymbol{\theta}_{o}\right)$ converging in distribution. The Taylor series expansions of $\boldsymbol{e}[t, \widehat{\boldsymbol{\theta}}]$ and $\widehat{\boldsymbol{\Sigma}}$ are then given by:

$$
\begin{aligned}
\boldsymbol{e}[t, \widehat{\boldsymbol{\theta}}]=\boldsymbol{w}[t] & +\sum_{i=1}^{n a} \sum_{j=0}^{\infty} \boldsymbol{\Pi}_{j}\left(\widehat{\boldsymbol{A}}_{i}-\boldsymbol{A}_{i}\right) \boldsymbol{y}[t-i-j] \\
& -\sum_{i=1}^{n c} \sum_{j=0}^{\infty} \boldsymbol{\Pi}_{j}\left(\widehat{\boldsymbol{C}}_{i}-\boldsymbol{C}_{i}\right) \boldsymbol{w}[t-i-j]+O_{p}\left(N^{-1}\right)
\end{aligned}
$$

\footnotetext{
${ }^{16}$ Let $\boldsymbol{x}_{N} \stackrel{d}{\longrightarrow} \mathcal{N}(\mathbf{0}, \boldsymbol{P})$ and $\boldsymbol{y}_{N}=\boldsymbol{A}_{N} \boldsymbol{x}_{N}+\boldsymbol{b}_{N}$, with $\boldsymbol{A}_{N} \stackrel{p}{\longrightarrow} \boldsymbol{A}$ and $\boldsymbol{b}_{N} \stackrel{p}{\longrightarrow} \boldsymbol{b}(N \longrightarrow \infty)$. Then, $\boldsymbol{y}_{N} \stackrel{d}{\longrightarrow} \mathcal{N}\left(\boldsymbol{b}, \boldsymbol{A} \boldsymbol{P} \boldsymbol{A}^{T}\right)$ $(N \longrightarrow \infty)[2$, p. 551].
} 


$$
\begin{aligned}
\widehat{\boldsymbol{\Sigma}}=\frac{1}{N} \sum_{t=1}^{N} \boldsymbol{w}[t] \boldsymbol{w}^{T}[t] & +\sum_{i=1}^{n a} \sum_{j=0}^{\infty} \sum_{\ell=1}^{\infty} \boldsymbol{\Pi}_{j}\left(\widehat{\boldsymbol{A}}_{i}-\boldsymbol{A}_{i}\right) \boldsymbol{H}_{\ell}\left(\frac{1}{N} \sum_{t=1}^{N} \boldsymbol{w}[t-i-j-\ell] \boldsymbol{w}^{T}[t]\right) \\
& -\sum_{i=1}^{n c} \sum_{j=0}^{\infty} \boldsymbol{\Pi}_{j}\left(\widehat{\boldsymbol{C}}_{i}-\boldsymbol{C}_{i}\right)\left(\frac{1}{N} \sum_{t=1}^{N} \boldsymbol{w}[t-i-j] \boldsymbol{w}^{T}[t]\right) \\
& +\sum_{i=1}^{n a} \sum_{j=0}^{\infty} \sum_{\ell=1}^{\infty}\left(\frac{1}{N} \sum_{t=1}^{N} \boldsymbol{w}[t] \boldsymbol{w}^{T}[t-i-j-\ell]\right) \boldsymbol{H}_{j}^{T}\left(\widehat{\boldsymbol{A}}_{i}-\boldsymbol{A}_{i}\right)^{T} \boldsymbol{\Pi}_{\ell}^{T} \\
& -\sum_{i=1}^{n c} \sum_{j=0}^{\infty}\left(\frac{1}{N} \sum_{t=1}^{N} \boldsymbol{w}[t-i-j] \boldsymbol{w}^{T}[t]\right)\left(\widehat{\boldsymbol{C}}_{i}-\boldsymbol{C}_{i}\right)^{T} \boldsymbol{\Pi}_{j}^{T}+O_{p}\left(N^{-1}\right)
\end{aligned}
$$

with:

$$
\begin{aligned}
& \boldsymbol{\Pi}[\mathcal{B}, k] \triangleq \boldsymbol{C}^{-1}[\mathcal{B}, k] \boldsymbol{A}[\mathcal{B}, k]=\boldsymbol{I}_{M n y}+\boldsymbol{\Pi}_{1}(k) \mathcal{B}+\boldsymbol{\Pi}_{2}(k) \mathcal{B}^{2}+\ldots \\
& \boldsymbol{H}[\mathcal{B}, k] \triangleq \boldsymbol{A}^{-1}[\mathcal{B}, k] \boldsymbol{C}[\mathcal{B}, k]=\boldsymbol{I}_{M n y}+\boldsymbol{H}_{1}(k) \mathcal{B}+\boldsymbol{H}_{2}(k) \mathcal{B}^{2}+\ldots
\end{aligned}
$$

Proof. The proof is in analogy to the conventional VARMA model case [77].

Consistency of the residual covariance matrix estimator It may be shown that the limit in probability of $\widehat{\Sigma}$ equals the limit in probability of the summed quantities in the right hand side of Eq. (4.B.9b). This follows from the fact that a sequence of random variables $\left\{x_{N}\right\}$ for which $x_{N}=O_{p}\left(N^{q}\right)$ has a limit in probability $N^{-r} x_{N} \stackrel{p}{\longrightarrow} 0$ for $r>q$, as $N \longrightarrow \infty$ (presently $q=-1$, whereas $r=0$; refer to [78, p. 148]).

The consistency of $\widehat{\boldsymbol{\Sigma}}_{2 S L S}$ is established by noticing that $\widehat{\boldsymbol{A}}_{i}$ and $\widehat{\boldsymbol{C}}_{i}$ are continuous functions of $\widehat{\boldsymbol{\theta}}_{2 S L S}$ (refer to Eq. (4.B.8d)), and thus consistent ${ }^{14}$. It then follows directly ${ }^{10}{ }^{11}$ from Eq. (4.B.9b) in Lemma 4.B.1 that $\widehat{\boldsymbol{\Sigma}}_{2 S L S}$ is consistent, since $\boldsymbol{w}[t]$ is white noise (refer to assumption A5 in Section 4.2).

Asymptotic distribution of the residual covariance matrix estimator To establish the asymptotic distribution of $\widehat{\boldsymbol{\Sigma}}_{2 S L S}$, notice that $\widehat{\boldsymbol{A}}_{i}$ and $\widehat{\boldsymbol{C}}_{i}$ in Eq. (4.B.8d) are continuously differentiable functions of $\boldsymbol{\theta}$, hence $\sqrt{N} \operatorname{vec}\left(\widehat{\boldsymbol{A}}_{i}-\boldsymbol{A}_{i}\right)$ and $\sqrt{N} \operatorname{vec}\left(\widehat{\boldsymbol{C}}_{i}-\boldsymbol{C}_{i}\right)$ converge in distribution ${ }^{17}$. totically ${ }^{18}$ :

It then follows from Lemma 4.B.1, by multiplying both sides of Eq. (4.B.9b) with $\sqrt{N}$, that asymp-

$$
\sqrt{N}\left(\widehat{\boldsymbol{\Sigma}}-\frac{1}{N} \sum_{t=1}^{N} \boldsymbol{w}[t] \boldsymbol{w}^{T}[t]\right) \stackrel{p}{\longrightarrow} \mathbf{0} \quad(N \longrightarrow \infty)
$$

The preceding equation implies that $\sqrt{N}\left(\widehat{\boldsymbol{\sigma}}_{2 S L S}-\widehat{\boldsymbol{\sigma}}_{M L}\right) \stackrel{p}{\longrightarrow} \mathbf{0}(N \longrightarrow \infty)$, which means that $\sqrt{N}\left(\widehat{\boldsymbol{\sigma}}_{2 S L S}-\boldsymbol{\sigma}_{o}\right)$ and $\sqrt{N}\left(\widehat{\boldsymbol{\sigma}}_{M L}-\boldsymbol{\sigma}_{o}\right)$ have the same asymptotic distribution ${ }^{19}$, thus (see also Subsection 4.B.3):

$$
\sqrt{N M}\left(\widehat{\boldsymbol{\sigma}}_{2 S L S}-\boldsymbol{\sigma}_{o}\right) \stackrel{d}{\longrightarrow} \mathcal{N}\left(\mathbf{0}, 2 M \boldsymbol{D}_{M n y}^{+}\left(\boldsymbol{\Sigma}_{o} \otimes \boldsymbol{\Sigma}_{o}\right)\left(\boldsymbol{D}_{M n y}^{+}\right)^{T}\right) \quad(N \longrightarrow \infty)
$$

\footnotetext{
${ }^{17}$ Suppose $\widehat{\boldsymbol{\theta}}$ is an estimator of the $(n \times 1)$ vector $\boldsymbol{\theta}$ with $\sqrt{N}(\widehat{\boldsymbol{\theta}}-\boldsymbol{\theta}) \stackrel{d}{\longrightarrow} \mathcal{N}(\mathbf{0}, \boldsymbol{\Sigma})(N \longrightarrow \infty)$. If $\boldsymbol{g}(\boldsymbol{\theta})=$ $\left[g_{1}(\boldsymbol{\theta}) \ldots g_{m}(\boldsymbol{\theta})\right]^{T}$ is a vector-valued continuously differentiable function of $\boldsymbol{\theta}$ with $\partial \boldsymbol{g}(\boldsymbol{\theta}) / \partial \boldsymbol{\theta} \neq \mathbf{0}$ at $\boldsymbol{\theta}$, then $\sqrt{N}(\boldsymbol{g}(\widehat{\boldsymbol{\theta}})-$ $\boldsymbol{g}(\boldsymbol{\theta})) \stackrel{d}{\longrightarrow} \mathcal{N}\left(\mathbf{0}, \frac{\partial \boldsymbol{g}(\boldsymbol{\theta})}{\partial \boldsymbol{\theta}^{T}} \boldsymbol{\Sigma} \frac{\partial \boldsymbol{g}(\boldsymbol{\theta})^{T}}{\partial \boldsymbol{\theta}}\right)(N \longrightarrow \infty)[44$, p. 693].

${ }^{18}$ Let $\boldsymbol{x}_{N} \stackrel{d}{\longrightarrow} \boldsymbol{x}$ and $\boldsymbol{A}_{N} \stackrel{p}{\longrightarrow} \mathbf{0}$, then $\boldsymbol{A}_{N} \boldsymbol{x}_{N} \stackrel{p}{\longrightarrow} \mathbf{0}(N \longrightarrow \infty)$ [44, p. 684].

${ }^{19}$ Let $\boldsymbol{x}_{N} \stackrel{d}{\longrightarrow} \boldsymbol{x}$ and $\left(\boldsymbol{x}_{N}-\boldsymbol{y}_{N}\right) \stackrel{p}{\longrightarrow} \mathbf{0}$, then $\boldsymbol{y}_{N} \stackrel{d}{\longrightarrow} \boldsymbol{x}(N \longrightarrow \infty)$ [44, p. 683].
} 
which completes the proof of Theorem 4.4.1.

\section{B.2 Computation of FP-VARMA derivatives}

Consider the FP-VARMA $(n a, n c)_{[p a, p c]}$ model of Eq. (6.4), differentiating with respect to its AR and MA parameters gives:

$$
\begin{gathered}
\frac{\partial \boldsymbol{e}_{k}[t, \boldsymbol{\theta}]}{\partial a_{m, n}^{i}(k)}=\boldsymbol{C}^{-1}[\mathcal{B}, k, \boldsymbol{\theta}] \boldsymbol{Q}_{m, n} \boldsymbol{y}_{k}[t-i] \\
\frac{\partial \boldsymbol{e}_{k}[t, \boldsymbol{\theta}]}{\partial c_{m, n}^{i}(k)}=-\boldsymbol{C}^{-1}[\mathcal{B}, k, \boldsymbol{\theta}] \boldsymbol{Q}_{m, n} \boldsymbol{e}_{k}[t-i, \boldsymbol{\theta}]
\end{gathered}
$$

with $a_{m, n}^{i}(k)$ and $c_{m, n}^{i}(k)$ designating the $(m, n)$-th elements of $\boldsymbol{A}_{i}(k)$ and $\boldsymbol{C}_{i}(k)$, respectively, and $\boldsymbol{Q}_{m, n}$ is a $(n y \times n y)$ matrix with " 1 " in position $(m, n)$ and " 0 " elsewhere (refer to [79] for the corresponding case of conventional $\operatorname{VARMA}(n a, n c)$ models).

The derivatives with respect to the projection coefficients are found using the chain rule of differentiation $^{20}$ as follows:

$$
\begin{gathered}
\frac{\partial \boldsymbol{e}_{k}[t, \boldsymbol{\theta}]}{\partial a_{m, n}^{i, j}}=\frac{\partial \boldsymbol{e}_{k}[t, \boldsymbol{\theta}]}{\partial a_{m, n}^{i}(k)} \frac{\partial a_{m, n}^{i}(k)}{\partial a_{m, n}^{i, j}}=G_{j}(k) \boldsymbol{C}^{-1}[\mathcal{B}, k, \boldsymbol{\theta}] \boldsymbol{Q}_{m, n} \boldsymbol{y}_{k}[t-i] \\
\frac{\partial \boldsymbol{e}_{k}[t, \boldsymbol{\theta}]}{\partial c_{m, n}^{i, j}}=\frac{\partial \boldsymbol{e}_{k}[t, \boldsymbol{\theta}]}{\partial c_{m, n}^{i}(k)} \frac{\partial c_{m, n}^{i}(k)}{\partial c_{m, n}^{i, j}}=-G_{j}(k) \boldsymbol{C}^{-1}[\mathcal{B}, k, \boldsymbol{\theta}] \boldsymbol{Q}_{m, n} \boldsymbol{e}_{k}[t-i, \boldsymbol{\theta}]
\end{gathered}
$$

with $a_{m, n}^{i, j}$ and $c_{m, n}^{i, j}$ designating the $(m, n)$-th elements of the projection coefficients $\boldsymbol{A}_{i, j}$ and $\boldsymbol{C}_{i, j}$, respectively.

\section{B.3 Proof of Theorem 4.4.2}

The joint log-likelihood function for an FP-VARMA process $\boldsymbol{y}[t]$ with zero mean, independent and normally (Gaussian) distributed, innovations having a covariance matrix $\boldsymbol{\Sigma}$ is given by:

$$
L(\boldsymbol{\theta}, \boldsymbol{\Sigma})=-\frac{N M n y}{2} \ln (2 \pi)-\frac{N}{2} \ln (\operatorname{det}(\boldsymbol{\Sigma}))-\frac{1}{2} \sum_{t=1}^{N} \boldsymbol{e}^{T}[t, \boldsymbol{\theta}] \boldsymbol{\Sigma}^{-1} \boldsymbol{e}[t, \boldsymbol{\theta}]
$$

which is in analogy to the conventional VARMA case [2, p. 210], and the following assumptions hold:

(i) The projection coefficient vector $\boldsymbol{\theta}$ and the covariance matrix $\boldsymbol{\Sigma}$ are independently parametrized.

(ii) All elements of $\boldsymbol{\Sigma}$ are unknown.

The following composite parameter vector $\overline{\boldsymbol{\theta}}=\left[\boldsymbol{\theta}^{T}: \boldsymbol{\sigma}^{T}\right]^{T}$ is considered, with $\boldsymbol{\sigma}$ being a vector comprising of the non-identical elements on and below the main diagonal of $\boldsymbol{\Sigma}$. Care has to be taken not to select all elements on and below the main diagonal of $\boldsymbol{\Sigma}$ as in the conventional VARMA model case, because the covariance $\boldsymbol{\Sigma}$ has a richer structure with identical elements at certain locations below the main diagonal (refer to Eq. (4.A.4) in Appendix 4.A).

\footnotetext{
${ }^{20}$ Let $\boldsymbol{a}$ and $\boldsymbol{\theta}$ be $(m \times 1)$ and $(n \times 1)$ vectors, respectively, and suppose $\boldsymbol{h}(\boldsymbol{a})$ is $(p \times 1)$ and $\boldsymbol{g}(\boldsymbol{\theta})$ is $(m \times 1)$. Then, with $\boldsymbol{a}=\boldsymbol{g}(\boldsymbol{\theta}), \frac{\partial \boldsymbol{h}(\boldsymbol{g}(\boldsymbol{\theta}))}{\partial \boldsymbol{\theta}^{T}}=\frac{\partial \boldsymbol{h}(\boldsymbol{a})}{\partial \boldsymbol{a}^{T}} \frac{\partial \boldsymbol{g}(\boldsymbol{\theta})}{\partial \boldsymbol{\theta}^{T}}(p \times n)$ [43, p. 174].
} 
Consistency of parameter estimator The first order Taylor series expansion ${ }^{12}$ of $\partial L(\overline{\boldsymbol{\theta}}) / \partial \overline{\boldsymbol{\theta}}$ around the system's true parameter vector $\overline{\boldsymbol{\theta}}_{o}$ is given by:

$$
\widehat{\overline{\boldsymbol{\theta}}}_{M L} \approx \overline{\boldsymbol{\theta}}_{o}-\left.\left[\left.\frac{1}{N} \frac{\partial^{2} L(\overline{\boldsymbol{\theta}})}{\partial \overline{\boldsymbol{\theta}} \partial \overline{\boldsymbol{\theta}}^{T}}\right|_{\overline{\boldsymbol{\theta}}=\overline{\boldsymbol{\theta}}_{o}}\right]^{-1} \frac{1}{N} \frac{\partial L(\overline{\boldsymbol{\theta}})}{\partial \overline{\boldsymbol{\theta}}}\right|_{\overline{\boldsymbol{\theta}}_{=} \overline{\boldsymbol{\theta}}_{o}}
$$

Obviously, due to the partitioning of $\overline{\boldsymbol{\theta}}$ the first and second order partial derivatives ${ }^{21}$ are rewritten as:

$$
\frac{\partial L(\overline{\boldsymbol{\theta}})}{\partial \overline{\boldsymbol{\theta}}}=\left[\begin{array}{c}
\frac{\partial L(\overline{\boldsymbol{\theta}})}{\partial \boldsymbol{\theta}} \\
\cdots \cdots \cdots \\
\frac{\partial L(\overline{\boldsymbol{\theta}})}{\partial \boldsymbol{\sigma}}
\end{array}\right], \quad \frac{\partial^{2} L(\overline{\boldsymbol{\theta}})}{\partial \overline{\boldsymbol{\theta}} \partial \overline{\boldsymbol{\theta}}^{T}}=\left[\begin{array}{cc}
\frac{\partial^{2} L(\overline{\boldsymbol{\theta}})}{\partial \boldsymbol{\theta} \partial \boldsymbol{\theta}^{T}} & \frac{\partial^{2} L(\overline{\boldsymbol{\theta}})}{\partial \boldsymbol{\theta} \partial \boldsymbol{\sigma}^{T}} \\
\frac{\partial^{2} L(\overline{\boldsymbol{\theta}})}{\partial \boldsymbol{\sigma} \partial \boldsymbol{\theta}^{T}} & \frac{\partial^{2} L(\overline{\boldsymbol{\theta}})}{\partial \boldsymbol{\sigma} \partial \boldsymbol{\sigma}^{T}}
\end{array}\right]
$$

and their block elements are computed by the following relationships $22,23,24,25,26,27,28,29,30$ :

$$
\begin{gathered}
\frac{\partial L(\overline{\boldsymbol{\theta}})}{\partial \boldsymbol{\theta}}=\sum_{t=1}^{N} \boldsymbol{\Psi}[t, \boldsymbol{\theta}] \boldsymbol{\Sigma}^{-1} \boldsymbol{e}^{T}[t, \boldsymbol{\theta}] \\
\frac{\partial L(\overline{\boldsymbol{\theta}})}{\partial \boldsymbol{\theta} \partial \boldsymbol{\theta}^{T}}=-\sum_{t=1}^{N}\left(\boldsymbol{\Psi}[t, \boldsymbol{\theta}] \boldsymbol{\Sigma}^{-1} \boldsymbol{\Psi}^{T}[t, \boldsymbol{\theta}]-\left(\boldsymbol{e}^{T}[t, \boldsymbol{\theta}] \boldsymbol{\Sigma}^{-1} \otimes \boldsymbol{I}_{\operatorname{dim}(\boldsymbol{\theta})}\right) \frac{\partial \operatorname{vec}(\boldsymbol{\Psi}[t, \boldsymbol{\theta}])}{\partial \boldsymbol{\theta}^{T}}\right) \\
\frac{\partial L(\overline{\boldsymbol{\theta}})}{\partial \operatorname{vec}(\boldsymbol{\Sigma})}=-\frac{N}{2} \operatorname{vec}\left(\boldsymbol{\Sigma}^{-1}\right)+\frac{N}{2} \operatorname{vec}\left[\boldsymbol{\Sigma}^{-1}\left(\frac{1}{N} \sum_{t=1}^{N} \boldsymbol{e}[t, \boldsymbol{\theta}] \boldsymbol{e}^{T}[t, \boldsymbol{\theta}]\right) \boldsymbol{\Sigma}^{-1}\right] \\
\frac{\partial^{2} L(\overline{\boldsymbol{\theta}})}{\partial \operatorname{vec}(\boldsymbol{\Sigma}) \partial \operatorname{vec}(\boldsymbol{\Sigma})^{T}}=\frac{N}{2}\left[\left(\boldsymbol{\Sigma}^{-1} \otimes \boldsymbol{\Sigma}^{-1}\right)-\left(\boldsymbol{\Sigma}^{-1} \otimes \boldsymbol{\Sigma}^{-1}\left(\frac{1}{N} \sum_{t=1}^{N} \boldsymbol{e}[t, \boldsymbol{\theta}] \boldsymbol{e}^{T}[t, \boldsymbol{\theta}]\right) \boldsymbol{\Sigma}^{-1}\right)\right. \\
\left.-\left(\boldsymbol{\Sigma}^{-1}\left(\frac{1}{N} \sum_{t=1}^{N} \boldsymbol{e}[t, \boldsymbol{\theta}] \boldsymbol{e}^{T}[t, \boldsymbol{\theta}]\right) \boldsymbol{\Sigma} \otimes \boldsymbol{\Sigma}^{-1}\right)\right]
\end{gathered}
$$

${ }^{21}$ Let $f(\boldsymbol{\theta})$ be a scalar function that depends on the $(n \times 1)$ vector $\boldsymbol{\theta}$. Then, $\frac{\partial f}{\partial \boldsymbol{\theta}^{T}}=\left[\frac{\partial f}{\partial \boldsymbol{\theta}_{1}} \ldots \frac{\partial f}{\partial \boldsymbol{\theta}_{n}}\right]$ and $\frac{\partial^{2} f}{\partial \boldsymbol{\theta} \partial \boldsymbol{\theta}^{T}}=$ $\left[\begin{array}{ccc}\frac{\partial^{2} f}{\partial \theta_{1} \partial \theta_{1}} & \cdots & \frac{\partial^{2} f}{\theta_{1} \partial \theta_{n}} \\ \vdots & \ddots & \vdots \\ \frac{\partial^{2} f}{\theta_{n} \partial \theta_{1}} & \cdots & \frac{\partial^{2} f}{\theta_{n} \partial \theta_{n}}\end{array}\right][44$, pp. 171-172].

${ }^{22}$ Let $\boldsymbol{\Omega}$ be a symmetric $(n \times n)$ matrix and $\boldsymbol{c}(\boldsymbol{\theta})$ an $(n \times 1)$ vector that depends on the $(m \times 1)$ vector $\boldsymbol{\theta}$. Then, $\frac{\partial \boldsymbol{c}^{T}(\boldsymbol{\theta}) \boldsymbol{\Omega} \boldsymbol{c}(\boldsymbol{\theta})}{\partial \boldsymbol{\theta}^{T}}=$ $2 \boldsymbol{c}^{T}(\boldsymbol{\theta}) \boldsymbol{\Omega} \frac{\partial \boldsymbol{c}(\boldsymbol{\theta})}{\partial \boldsymbol{\theta}^{T}}$, and $\frac{\partial^{2} \boldsymbol{c}^{T}(\boldsymbol{\theta}) \boldsymbol{\Omega} \boldsymbol{c}(\boldsymbol{\theta})}{\partial \boldsymbol{\theta} \partial \boldsymbol{\theta}^{T}}=2\left[\frac{\partial \boldsymbol{c}^{T}(\boldsymbol{\theta})}{\partial \boldsymbol{\theta}} \boldsymbol{\Omega} \frac{\partial \boldsymbol{c}(\boldsymbol{\theta})}{\partial \boldsymbol{\theta}^{T}}+\left[\boldsymbol{c}^{T}(\boldsymbol{\theta}) \boldsymbol{\Omega} \otimes \boldsymbol{I}_{m}\right] \frac{\partial \mathrm{vec}\left(\partial \boldsymbol{c}^{T}(\boldsymbol{\theta}) / \partial \boldsymbol{\theta}\right)}{\partial \boldsymbol{\theta}^{T}}\right]$ [43, p. 175].

${ }^{23}$ Let $\boldsymbol{A}$ be an $(m \times n)$ matrix and $\boldsymbol{\theta}$ be an $(n \times 1)$ vector, then $\frac{\partial \boldsymbol{A} \boldsymbol{\theta}}{\partial \boldsymbol{\theta}^{T}}=\boldsymbol{A}$ and $\frac{\partial \boldsymbol{\theta}^{T} \boldsymbol{A}^{T}}{\partial \boldsymbol{\theta}}=\boldsymbol{A}^{T}$ [43, p. 174].

${ }^{24}$ If $\boldsymbol{A}$ is a non-singular $(m \times m)$ matrix with $\operatorname{det}(\boldsymbol{A})>0$, then $\frac{\partial \ln \operatorname{det}(\boldsymbol{A})}{\partial \boldsymbol{A}}=\left(\boldsymbol{A}^{T}\right)^{-1}$ [43, p. 182].

${ }^{25}$ Let $\boldsymbol{A}, \boldsymbol{B}$ and $\boldsymbol{C}$ be $(m \times m)$ matrices and suppose $\boldsymbol{A}$ is non-singular, then $\frac{\partial \operatorname{tr}\left(\boldsymbol{B} \boldsymbol{A}^{-1} \boldsymbol{C}\right)}{\partial \boldsymbol{A}}=-\left(\boldsymbol{A}^{-1} \boldsymbol{C} \boldsymbol{B} \boldsymbol{A}^{-1}\right)^{T}$ [43, p. 179].

${ }^{26}$ Suppose $\boldsymbol{\theta}$ is $(m \times 1), \boldsymbol{B}(\boldsymbol{\theta})$ is $(n \times p), \boldsymbol{A}$ is $(k \times n)$, and $\boldsymbol{C}$ is $(p \times q)$ and the latter two matrices do not depend on $\boldsymbol{\theta}$. Then, $\frac{\partial \operatorname{vec}(\boldsymbol{A B C})}{\partial \boldsymbol{\theta}^{T}}=\left(\boldsymbol{C}^{T} \otimes \boldsymbol{A}\right) \frac{\partial \operatorname{vec}(\boldsymbol{B})}{\partial \boldsymbol{\theta}^{T}}[43$, p. 188].

${ }^{27}$ If $\boldsymbol{\theta}$ is $(m \times 1), \boldsymbol{A}(\boldsymbol{\theta})$ is $(n \times p), \boldsymbol{D}(\boldsymbol{\theta})$ is $(q \times r)$, and $\boldsymbol{C}$ is $(p \times q)$ and does not depend on $\boldsymbol{\theta}$. Then, $\frac{\partial \operatorname{vec}(\boldsymbol{A C} \boldsymbol{D})}{\partial \boldsymbol{\theta}^{T}}=$ $\left(\boldsymbol{I}_{r} \otimes \boldsymbol{A C}\right) \frac{\partial \mathrm{vec}(\boldsymbol{D})}{\partial \boldsymbol{\theta}^{T}}+\left(\boldsymbol{D}^{T} \boldsymbol{C}^{T} \otimes \boldsymbol{I}_{n}\right) \frac{\partial \mathrm{vec}(\boldsymbol{A})}{\partial \boldsymbol{\theta}^{T}}[43$, p. 196].

${ }^{28}$ If $\boldsymbol{A}$ is a non-singular $(m \times m)$ matrix, then $\frac{\partial \operatorname{vec}(\boldsymbol{A})^{-1}}{\partial \operatorname{vec}(\boldsymbol{A})^{T}}=-\left(\boldsymbol{A}^{-1}\right)^{T} \otimes \boldsymbol{A}^{-1}$ [43, p. 198].

${ }^{29}$ If $\boldsymbol{\theta}$ is $(m \times 1), \boldsymbol{A}(\boldsymbol{\theta})$ is $(n \times p)$ and $\boldsymbol{B}(\boldsymbol{\theta})$ is $(p \times q)$, then, $\frac{\partial \mathrm{vec}(\boldsymbol{A} \boldsymbol{B})}{\partial \boldsymbol{\theta}^{T}}=\left(\boldsymbol{I}_{q} \otimes \boldsymbol{A}\right) \frac{\partial \mathrm{vec}(\boldsymbol{B})}{\partial \boldsymbol{\theta}^{T}}+\left(\boldsymbol{B}^{T} \otimes \boldsymbol{I}_{n}\right) \frac{\partial \mathrm{vec}(\boldsymbol{A})}{\partial \boldsymbol{\theta}^{T}}$ [43, p. 196].

${ }^{30}$ Let, $\boldsymbol{A}, \boldsymbol{B}$ and $\boldsymbol{C}$ be matrices of appropriate dimensions, then $\operatorname{vec}(\boldsymbol{A} \boldsymbol{B} \boldsymbol{C})=\left(\boldsymbol{C}^{T} \otimes \boldsymbol{A}\right) \operatorname{vec}(\boldsymbol{B})=(\boldsymbol{I} \otimes \boldsymbol{A} \boldsymbol{B}) \operatorname{vec}(\boldsymbol{C})=$ $\left(\boldsymbol{C}^{T} \boldsymbol{B}^{T} \otimes \boldsymbol{I}\right) \operatorname{vec}(\boldsymbol{A})[43$, p. 97]. 


$$
\frac{\partial^{2} L(\overline{\boldsymbol{\theta}})}{\partial \operatorname{vec}(\boldsymbol{\Sigma}) \partial \boldsymbol{\theta}^{T}}=-\frac{1}{2}\left(\boldsymbol{\Sigma}^{-1} \otimes \boldsymbol{\Sigma}^{-1}\right) \sum_{t=1}^{N}\left[\left(\boldsymbol{I}_{M n y} \otimes \boldsymbol{e}[t, \boldsymbol{\theta}]\right)+\left(\boldsymbol{e}[t, \boldsymbol{\theta}] \otimes \boldsymbol{I}_{M n y}\right)\right] \boldsymbol{\Psi}^{T}[t, \boldsymbol{\theta}]
$$

Note that $\boldsymbol{\sigma}$ and $\boldsymbol{\Sigma}$ are related via the expression $\operatorname{vec}(\boldsymbol{\Sigma})=\boldsymbol{D}_{M n y} \boldsymbol{\sigma}$, with $\boldsymbol{D}_{M n y}$ being a duplication matrix of proper dimensions, defined in analogy to the conventional VARX model case [44, p. 480], thereby ${ }^{20}$ :

$$
\begin{gathered}
\frac{\partial L(\overline{\boldsymbol{\theta}})}{\partial \boldsymbol{\sigma}}=\boldsymbol{D}_{M n y}^{T} \frac{\partial L(\overline{\boldsymbol{\theta}})}{\partial \operatorname{vec}(\boldsymbol{\Sigma})} \\
\frac{\partial^{2} L(\overline{\boldsymbol{\theta}})}{\partial \boldsymbol{\sigma} \partial \boldsymbol{\sigma}^{T}}=\boldsymbol{D}_{M n y}^{T} \frac{\partial^{2} L(\overline{\boldsymbol{\theta}})}{\partial \operatorname{vec}(\boldsymbol{\Sigma}) \partial \operatorname{vec}(\boldsymbol{\Sigma})^{T}} \boldsymbol{D}_{M n y} \\
\frac{\partial^{2} L(\overline{\boldsymbol{\theta}})}{\partial \boldsymbol{\sigma} \partial \boldsymbol{\theta}^{T}}=\boldsymbol{D}_{M n y}^{T} \frac{\partial^{2} L(\overline{\boldsymbol{\theta}})}{\partial \operatorname{vec}(\boldsymbol{\Sigma}) \partial \boldsymbol{\theta}^{T}}
\end{gathered}
$$

At $\overline{\boldsymbol{\theta}}=\overline{\boldsymbol{\theta}}_{o}$, the following limits in probability hold ${ }^{10}$ (under the assumption that $\boldsymbol{y}_{k}[t]$ and $\boldsymbol{e}_{k}[t, \boldsymbol{\theta}]$ are zero-mean, ergodic and stationary processes):

$$
\begin{gathered}
\left.\frac{1}{N} \frac{\partial L(\overline{\boldsymbol{\theta}})}{\partial \overline{\boldsymbol{\theta}}}\right|_{\overline{\boldsymbol{\theta}}=\overline{\boldsymbol{\theta}}_{o}} \stackrel{p}{\longrightarrow} \mathbf{0} \quad(N \longrightarrow \infty) \\
\left.\frac{1}{N} \frac{\partial^{2} L(\overline{\boldsymbol{\theta}})}{\partial \overline{\boldsymbol{\theta}} \partial \overline{\boldsymbol{\theta}}^{T}}\right|_{\overline{\boldsymbol{\theta}}_{=} \overline{\boldsymbol{\theta}}_{o}} \stackrel{p}{\longrightarrow}\left[\begin{array}{cc}
E\left\{\boldsymbol{\Psi}\left[t, \boldsymbol{\theta}_{o}\right] \boldsymbol{\Sigma}_{o}^{-1} \boldsymbol{\Psi}^{T}\left[t, \boldsymbol{\theta}_{o}\right]\right\} & \mathbf{0} \\
\mathbf{0} & \frac{1}{2} \boldsymbol{D}_{M n y}^{T}\left(\boldsymbol{\Sigma}_{o}^{-1} \otimes \boldsymbol{\Sigma}_{o}^{-1}\right) \boldsymbol{D}_{M n y}
\end{array}\right] \quad(N \longrightarrow \infty)
\end{gathered}
$$

Thus, the probability limit in Eq. (4.B.14) yields ${ }^{11}$ :

$$
\widehat{\overline{\boldsymbol{\theta}}}_{M L} \stackrel{p}{\longrightarrow} \overline{\boldsymbol{\theta}}_{O} \quad(N \longrightarrow \infty)
$$

which is in analogy to the conventional VARMA model case.

Asymptotic distribution of parameter estimator As in the conventional VARMA model case, to derive the asymptotic distribution of $\widehat{\overline{\boldsymbol{\theta}}}_{M L}$, Eq. (4.B.14) is rewritten as follows:

$$
\sqrt{N M}\left(\widehat{\overline{\boldsymbol{\theta}}}_{M L}-\overline{\boldsymbol{\theta}}_{o}\right) \approx-\left[\left.\frac{1}{N M} \frac{\partial^{2} L(\overline{\boldsymbol{\theta}})}{\partial \overline{\boldsymbol{\theta}} \partial \overline{\boldsymbol{\theta}}^{T}}\right|_{\overline{\boldsymbol{\theta}}=\overline{\boldsymbol{\theta}}_{o}}\right]^{-1}\left[\left.\frac{1}{\sqrt{N M}} \frac{\partial L(\overline{\boldsymbol{\theta}})}{\partial \overline{\boldsymbol{\theta}}}\right|_{\overline{\boldsymbol{\theta}}_{=} \overline{\boldsymbol{\theta}}_{o}}\right]
$$

The limiting distribution of the rightmost bracketed quantity is found by means of a central limit theorem ${ }^{15}$ [2, p. 550] yielding:

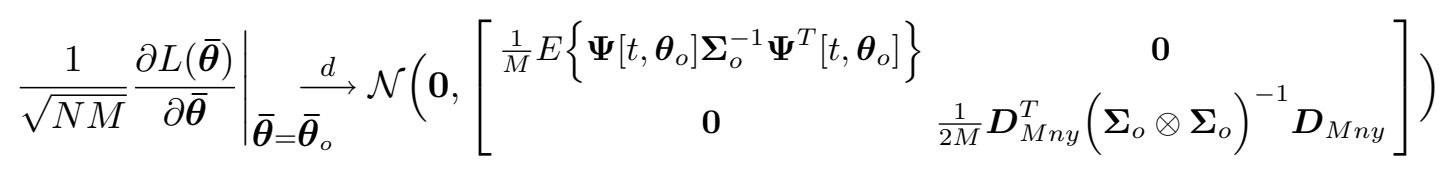

and the asymptotic distribution of the ML estimator ${ }^{16}$ is:

$$
\sqrt{N M}\left(\widehat{\overline{\boldsymbol{\theta}}}_{M L}-\overline{\boldsymbol{\theta}}_{o}\right) \stackrel{d}{\longrightarrow} \mathcal{N}\left(\mathbf{0},\left[\begin{array}{cc}
M E\left\{\boldsymbol{\Psi}\left[t, \boldsymbol{\theta}_{o}\right] \boldsymbol{\Sigma}_{o}^{-1} \boldsymbol{\Psi}^{T}\left[t, \boldsymbol{\theta}_{o}\right]\right\}^{-1} & \mathbf{0} \\
\mathbf{0} & 2 M \boldsymbol{D}_{M n y}^{+}\left(\boldsymbol{\Sigma}_{o} \otimes \boldsymbol{\Sigma}_{o}\right)\left(\boldsymbol{D}_{M n y}^{+}\right)^{T}
\end{array}\right]\right)
$$

which completes the proof of Theorem 4.4.2. 


\section{B.4 Statistical accuracy of 2SLS and ML estimators}

In order to study the statistical accuracy of the 2SLS and ML estimators, the following proposition is useful. Proposition 4.B.1. (Cramér-Rao lower bound for FP-VARMA models): The asymptotic Cramér-Rao lower bound for a consistent and asymptotically normal estimator $\widehat{\overline{\boldsymbol{\theta}}}=\left[\widehat{\boldsymbol{\theta}}^{T}: \widehat{\boldsymbol{\sigma}}^{T}\right]^{T}$ of the FP-VARMA model is:

$$
E\left\{\left(\widehat{\overline{\boldsymbol{\theta}}}-\overline{\boldsymbol{\theta}}_{o}\right)\left(\widehat{\overline{\boldsymbol{\theta}}}-\overline{\boldsymbol{\theta}}_{o}\right)^{T}\right\}=\left[\begin{array}{cc}
\frac{1}{N} E\left\{\boldsymbol{\Psi}\left[t, \boldsymbol{\theta}_{o}\right] \boldsymbol{\Sigma}_{o}^{-1} \boldsymbol{\Psi}^{T}\left[t, \boldsymbol{\theta}_{o}\right]\right\}^{-1} & \mathbf{0} \\
\mathbf{0} & \frac{2}{N} \boldsymbol{D}_{M n y}^{+}\left(\boldsymbol{\Sigma}_{o} \otimes \boldsymbol{\Sigma}_{o}\right)\left(\boldsymbol{D}_{M n y}^{+}\right)^{T}
\end{array}\right]
$$

Proof. By definition, the Cramér-Rao lower bound equals the inverse of the Fisher information matrix, which is given by the following relationship [2, pp. 560-563]:

$$
\mathcal{F}=-\left.E\left\{\frac{\partial^{2} L(\overline{\boldsymbol{\theta}})}{\partial \overline{\boldsymbol{\theta}} \partial \overline{\boldsymbol{\theta}}^{T}}\right\}\right|_{\overline{\boldsymbol{\theta}}=\overline{\boldsymbol{\theta}}_{o}}
$$

By virtue of Eq. (4.B.15a), the Fisher information matrix $\mathcal{F}$ is rewritten as:

$$
\mathcal{F}=-\left.E\left\{\left[\begin{array}{ll}
\frac{\partial^{2} L(\overline{\boldsymbol{\theta}})}{\partial \boldsymbol{\theta} \partial \boldsymbol{\theta}^{T}} & \frac{\partial^{2} L(\overline{\boldsymbol{\theta}})}{\partial \boldsymbol{\theta} \partial \boldsymbol{\sigma}^{T}} \\
\frac{\partial^{2} L(\overline{\boldsymbol{\theta}})}{\partial \boldsymbol{\sigma} \partial \boldsymbol{\theta}^{T}} & \frac{\partial^{2} L(\overline{\boldsymbol{\theta}})}{\partial \boldsymbol{\sigma} \partial \boldsymbol{\sigma}^{T}}
\end{array}\right]\right\}\right|_{\boldsymbol{\theta}=\boldsymbol{\theta}_{o}, \boldsymbol{\sigma}=\boldsymbol{\sigma}_{o}}
$$

It follows from Eq. (4.B.15c), Eq. (4.B.15h) and Eq. (4.B.15i) that $\mathcal{F}$ is block diagonal, since:

$$
\begin{gathered}
-\left.E\left\{\frac{\partial^{2} L(\overline{\boldsymbol{\theta}})}{\partial \boldsymbol{\theta} \partial \boldsymbol{\theta}^{T}}\right\}\right|_{\boldsymbol{\theta}=\boldsymbol{\theta}_{o}}=N E\left\{\boldsymbol{\Psi}\left[t, \boldsymbol{\theta}_{o}\right] \boldsymbol{\Sigma}_{o}^{-1} \mathbf{\Psi}^{T}\left[t, \boldsymbol{\theta}_{o}\right]\right\} \\
-\left.E\left\{\frac{\partial^{2} L(\overline{\boldsymbol{\theta}})}{\partial \boldsymbol{\sigma} \partial \boldsymbol{\sigma}^{T}}\right\}\right|_{\boldsymbol{\sigma}=\boldsymbol{\sigma}_{o}}=\frac{N}{2} \boldsymbol{D}_{M n y}^{T}\left(\boldsymbol{\Sigma}_{o} \otimes \boldsymbol{\Sigma}_{o}\right)^{-1} \boldsymbol{D}_{M n y} \\
-\left.E\left\{\frac{\partial^{2} L(\overline{\boldsymbol{\theta}})}{\partial \boldsymbol{\theta} \partial \boldsymbol{\sigma}^{T}}\right\}\right|_{\boldsymbol{\theta}=\boldsymbol{\theta}_{o},} \boldsymbol{\sigma}_{=} \boldsymbol{\sigma}_{o}=\mathbf{0}
\end{gathered}
$$

whereas subsequent inversion ${ }^{31}$ of $\mathcal{F}$ leads to Eq. (4.B.19), which completes the proof.

It follows from Proposition 4.B.1 that the Cramér-Rao lower bounds of the optimal FP-VARMA estimators for $\boldsymbol{\theta}$ and $\boldsymbol{\sigma}$ are given by:

$$
\begin{aligned}
& E\left\{\sqrt{N M}\left(\widehat{\boldsymbol{\theta}}-\boldsymbol{\theta}_{o}\right)\left(\widehat{\boldsymbol{\theta}}-\boldsymbol{\theta}_{o}\right)^{T} \sqrt{N M}\right\}=M E\left\{\boldsymbol{\Psi}\left[t, \boldsymbol{\theta}_{o}\right] \boldsymbol{\Sigma}_{o}^{-1} \mathbf{\Psi}^{T}\left[t, \boldsymbol{\theta}_{o}\right]\right\}^{-1} \\
& E\left\{\sqrt{N M}\left(\widehat{\boldsymbol{\sigma}}-\boldsymbol{\sigma}_{o}\right)\left(\widehat{\boldsymbol{\sigma}}-\boldsymbol{\sigma}_{o}\right)^{T} \sqrt{N M}\right\}=2 M \boldsymbol{D}_{M n y}^{+}\left(\boldsymbol{\Sigma}_{o} \otimes \boldsymbol{\Sigma}_{o}\right)\left(\boldsymbol{D}_{M n y}^{+}\right)^{T}
\end{aligned}
$$

${ }^{31}$ Let $\boldsymbol{A}_{i}$ be $\left(m_{i} \times m_{i}\right)$ non-singular matrices $(i=1, \ldots, r)$, then $\left[\begin{array}{lll}\boldsymbol{A}_{1} & & \mathbf{0} \\ & \ddots & \\ & & \boldsymbol{A}_{r}\end{array}\right]^{-1}=\left[\begin{array}{ccc}\boldsymbol{A}_{1}^{-1} & & \mathbf{0} \\ & \ddots & \\ & & \boldsymbol{A}_{r}^{-1}\end{array}\right]$ [43, p. 147]. 


\section{B.4.1 Proof of Corollary 4.4.1}

The following matrix is nonnegative-definite by construction:

$$
\frac{1}{M} E\left\{\left[\begin{array}{cc}
\mathbf{\Psi}\left[t, \boldsymbol{\theta}_{o}\right] \boldsymbol{\Sigma}_{o}^{-1} \mathbf{\Psi}^{T}\left[t, \boldsymbol{\theta}_{o}\right] & \left.\left(\boldsymbol{\Phi}\left[t, \boldsymbol{\theta}_{o}\right] \boldsymbol{\Sigma}_{o}^{-1} \mathbf{\Psi}^{T}\left[t, \boldsymbol{\theta}_{o}\right]\right)^{T}\right] \\
\mathbf{\Phi}\left[t, \boldsymbol{\theta}_{o}\right] \boldsymbol{\Sigma}_{o}^{-1} \mathbf{\Psi}^{T}\left[t, \boldsymbol{\theta}_{o}\right] & \mathbf{\Phi}\left[t, \boldsymbol{\theta}_{o}\right] \boldsymbol{\Sigma}_{o}^{-1} \boldsymbol{\Phi}^{T}\left[t, \boldsymbol{\theta}_{o}\right]
\end{array}\right]\right\} \geq \mathbf{0}
$$

Thereby $^{32}$ :

$$
\begin{gathered}
\frac{1}{M} E\left\{\boldsymbol{\Psi}\left[t, \boldsymbol{\theta}_{o}\right] \boldsymbol{\Sigma}_{o}^{-1} \boldsymbol{\Psi}^{T}\left[t, \boldsymbol{\theta}_{o}\right]\right\}-\frac{1}{M} E\left\{\boldsymbol{\Phi}\left[t, \boldsymbol{\theta}_{o}\right] \boldsymbol{\Sigma}_{o}^{-1} \boldsymbol{\Psi}^{T}\left[t, \boldsymbol{\theta}_{o}\right]\right\}^{T} E\left\{\boldsymbol{\Phi}\left[t, \boldsymbol{\theta}_{o}\right] \boldsymbol{\Sigma}_{o}^{-1} \boldsymbol{\Phi}^{T}\left[t, \boldsymbol{\theta}_{o}\right]\right\}^{-1} \\
E\left\{\boldsymbol{\Phi}\left[t, \boldsymbol{\theta}_{o}\right] \boldsymbol{\Sigma}_{o}^{-1} \boldsymbol{\Psi}^{T}\left[t, \boldsymbol{\theta}_{o}\right]\right\} \geq \mathbf{0}
\end{gathered}
$$

The preceding inequality is easily rewritten as ${ }^{33}$ :

$$
\begin{array}{r}
M E\left\{\boldsymbol{\Psi}\left[t, \boldsymbol{\theta}_{o}\right] \boldsymbol{\Sigma}_{o}^{-1} \mathbf{\Psi}^{T}\left[t, \boldsymbol{\theta}_{o}\right]\right\}^{-1} \leq M E\left\{\boldsymbol{\Phi}\left[t, \boldsymbol{\theta}_{o}\right] \boldsymbol{\Sigma}_{o}^{-1} \boldsymbol{\Psi}^{T}\left[t, \boldsymbol{\theta}_{o}\right]\right\}^{-1} E\left\{\boldsymbol{\Phi}\left[t, \boldsymbol{\theta}_{o}\right] \boldsymbol{\Sigma}_{o}^{-1} \boldsymbol{\Phi}^{T}\left[t, \boldsymbol{\theta}_{o}\right]\right\} \\
E\left\{\boldsymbol{\Phi}\left[t, \boldsymbol{\theta}_{o}\right] \boldsymbol{\Sigma}_{o}^{-1} \boldsymbol{\Psi}^{T}\left[t, \boldsymbol{\theta}_{o}\right]\right\}^{-T}=\boldsymbol{P}_{2 S L S}
\end{array}
$$

Eq. (4.B.24c) confirms that $\boldsymbol{P}_{2 S L S}$ is in general asymptotically inefficient. The preceding inequality will in general be strict, meaning that the equality will hold for some special cases. One such important case is when $\boldsymbol{\Phi}[t, \boldsymbol{\theta}]$ is independent of $\boldsymbol{\theta}$, where it easy to verify that $\mathbf{\Phi}[t, \boldsymbol{\theta}]=\boldsymbol{\Psi}[t, \boldsymbol{\theta}] \triangleq \boldsymbol{\Phi}[t]$. Then Eq. (4.13a) corresponds to a pure linear regression (for instance, an FP-VAR(X) model).

Furthermore, the asymptotic efficiency of $\widehat{\boldsymbol{\sigma}}_{2 S L S}$ follows directly from Eq. (4.B.23b).

\section{B.4.3 Proof of Corollary 4.4.2}

The asymptotic efficiency of $\widehat{\boldsymbol{\theta}}_{M L}$, and $\widehat{\boldsymbol{\sigma}}_{M L}$ follows directly from Eq. (4.B.23a)-(4.B.23b), respectively.

\section{Appendix 4.C: Additional results on FP-VARMA model identifiability}

\section{C.1 Proof of Theorem 4.5.1}

The obvious similarity of the FP-VARMA representation in Eq. (4.B.8a)-(4.B.8d) with the conventional VARMA models, suggests that the same reasoning for conventional VARMA model uniqueness of representation may be used. Thus the above FP-VARMA parametrization is unique in the class of matrix fraction descriptions (MFD) of degrees $(n a, n c)$ if and only if [2, pp. 182-183]:

$$
\begin{aligned}
\boldsymbol{A}(\mathcal{B}), \boldsymbol{C}(\mathcal{B}) & : \text { left coprime } \\
\operatorname{rank}\left[\boldsymbol{A}_{n a} \vdots \boldsymbol{C}_{n c}\right] & =\text { Mny }
\end{aligned}
$$

\footnotetext{
${ }^{32}$ Consider the symmetric matrix $\boldsymbol{S}=\left[\begin{array}{cc}\boldsymbol{A} & \boldsymbol{B} \\ \boldsymbol{B}^{T} & \boldsymbol{C}\end{array}\right]$, then $\boldsymbol{S} \geq \mathbf{0} \Longleftrightarrow \boldsymbol{A}-\boldsymbol{B} \boldsymbol{C}^{-1} \boldsymbol{B}^{T} \geq \mathbf{0}$ [48, p. 462].

${ }^{33}$ Let $\boldsymbol{A}$ and $\boldsymbol{B}$ be $(m \times m)$ symmetric matrices, and assume that either $\boldsymbol{A}$ and $\boldsymbol{B}$ are positive-definite or $\boldsymbol{A}$ and $\boldsymbol{B}$ are negativedefinite. If $\boldsymbol{A} \leq \boldsymbol{B}$, then $\boldsymbol{B}^{-1} \leq \boldsymbol{A}^{-1}$. If, in addition $\boldsymbol{A}<\boldsymbol{B}$, then $\boldsymbol{B}^{-1}<\boldsymbol{A}^{-1}$ [48, p. 476].
} 


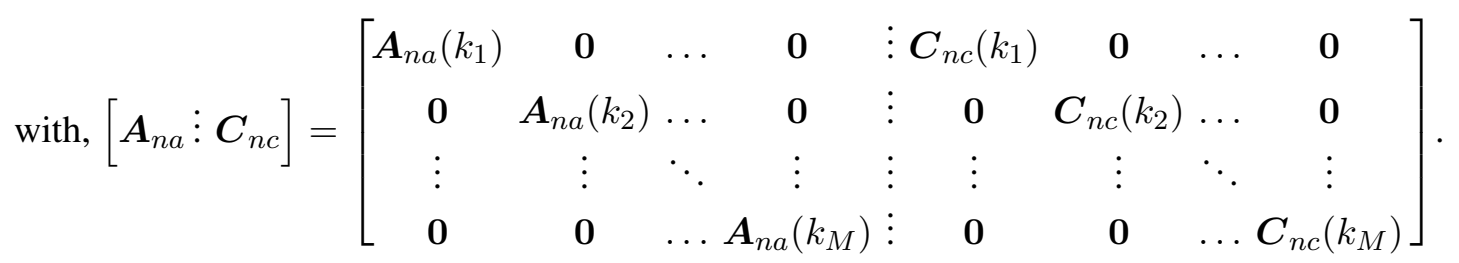

Notice that unlike the conventional VARMA case, the matrices $\boldsymbol{A}(\mathcal{B}), \boldsymbol{C}(\mathcal{B})$ are block diagonal. Hence, there exists a block diagonal unimodular matrix implying that their corresponding block elements $\boldsymbol{A}[\mathcal{B}, k], \boldsymbol{C}[\mathcal{B}, k]\left(k=k_{1}, k_{2}, \ldots, k_{M}\right)$ are left coprime.

Rearranging the columns of the composite matrix $\left[\boldsymbol{A}_{n a} \vdots \boldsymbol{C}_{n c}\right]$ yields:

$$
\operatorname{rank}\left[\boldsymbol{A}_{n a} \vdots \boldsymbol{C}_{n c}\right]=\operatorname{rank}\left[\begin{array}{cccc}
{\left[\boldsymbol{A}_{n a}\left(k_{1}\right) \vdots \boldsymbol{C}_{n c}\left(k_{1}\right)\right]} & \mathbf{0} & \ldots & \mathbf{0} \\
\mathbf{0} & {\left[\boldsymbol{A}_{n a}\left(k_{2}\right) \vdots \boldsymbol{C}_{n c}\left(k_{2}\right)\right]} & \ldots & \mathbf{0} \\
\vdots & \vdots & \ddots & \vdots \\
\mathbf{0} & \mathbf{0} & \ldots\left[\boldsymbol{A}_{n a}\left(k_{M}\right) \vdots \boldsymbol{C}_{n c}\left(k_{M}\right)\right]
\end{array}\right]
$$

implying that ${ }^{34}$ :

$$
\sum_{k=k_{1}}^{k_{M}} \operatorname{rank}\left[\boldsymbol{A}_{n a}(k) \vdots \boldsymbol{C}_{n c}(k)\right]=M n y
$$

which indicates that, $\operatorname{rank}\left[\boldsymbol{A}_{n a}(k) \vdots \boldsymbol{C}_{n c}(k)\right]=n y$ for $k=k_{1}, k_{2}, \ldots, k_{M}{ }^{35}$.

The uniqueness of the AR and MA matrices, $\boldsymbol{A}_{i}(k)$ and $\boldsymbol{C}_{i}(k)\left(k=k_{1}, k_{2}, \ldots, k_{M}\right)$, implies that a unique projection coefficient vector will be obtained from their projection on the functional basis $G_{j}(k)$ $(j=1,2, \ldots, p)$.

\section{Appendix 4.D: Tutorial example of FP-VARMA structure specification}

Assume that an FP-VARMA $(2,1)_{[2,2]}$ model with two excitations (that is, $n y=2$ ) is used to generate data. The AR and MA matrices belong to functional subspaces of dimensionality $p a=p c=2$, spanned by the mutually independent functions $G_{1}(k)$ and $G_{2}(k)$. The FP-VARMA model order specification is based upon $M=2$ sets of response data having $N$ samples each (that is, $k=k_{1}, k_{2}$ and $t=1,2, \ldots, N$ ). The model is of the following form:

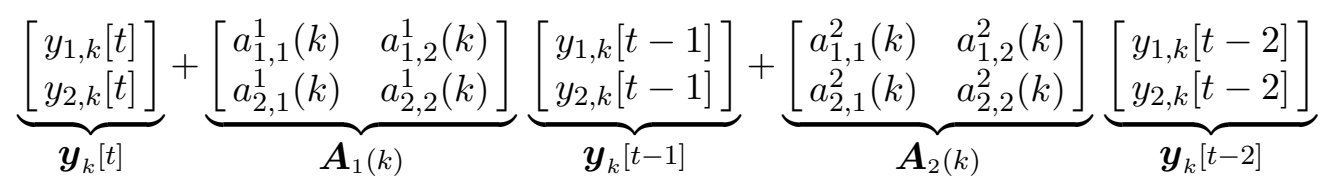

$$
\begin{aligned}
& =\underbrace{\left[\begin{array}{l}
w_{1, k}[t] \\
w_{2, k}[t]
\end{array}\right]}_{\boldsymbol{w}_{k}[t]}+\underbrace{\left[\begin{array}{ll}
c_{1,1}^{1}(k) & c_{1,2}^{1}(k) \\
c_{2,1}^{1}(k) & c_{2,2}^{1}(k)
\end{array}\right]}_{\boldsymbol{C}_{1}(k)} \underbrace{\left[\begin{array}{l}
w_{1, k}[t-1] \\
w_{2, k}[t-1]
\end{array}\right]}_{\boldsymbol{w}_{k}[t-1]}
\end{aligned}
$$

\footnotetext{
${ }^{34}$ Consider the $(m \times n)$ matrix $\boldsymbol{A}$ and the $(\ell \times q)$ matrix $\boldsymbol{B}$, then $\operatorname{rank} \boldsymbol{A}+\operatorname{rank} \boldsymbol{B}=\operatorname{rank}\left[\begin{array}{cc}\boldsymbol{A} & \mathbf{0} \\ \mathbf{0} & \boldsymbol{B}\end{array}\right][43, \mathrm{p} .145]$.

${ }^{35}$ Consider the $(m \times n)$ matrix $\boldsymbol{A}$, then $\operatorname{rank} \boldsymbol{A} \leq \min (m, n)$ [43, p. 61].
} 
with:

$$
\begin{aligned}
& \boldsymbol{A}_{1}(k)=\underbrace{\left[\begin{array}{ll}
a_{1,1}^{1,1} & a_{1,2}^{1,1} \\
a_{2,1}^{1,1} & a_{2,2}^{1,1}
\end{array}\right]}_{\boldsymbol{A}_{1,1}} G_{1}(k)+\underbrace{\left[\begin{array}{ll}
a_{1,1}^{1,2} & a_{1,2}^{1,2} \\
a_{2,1}^{1,2} & a_{2,2}^{1,2}
\end{array}\right]}_{\boldsymbol{A}_{1,2}} G_{2}(k) \\
& \boldsymbol{A}_{2}(k)=\underbrace{\left[\begin{array}{ll}
a_{1,1}^{2,1} & a_{1,2}^{2,1} \\
a_{2,1}^{2,1} & a_{2,1}^{2,1}
\end{array}\right]}_{\boldsymbol{A}_{2,1}} G_{1}(k)+\underbrace{\left[\begin{array}{rr}
a_{1,1}^{2,2} & a_{1,2}^{2,2} \\
a_{2,1}^{2,2} & a_{2,2}^{2,2}
\end{array}\right]}_{\boldsymbol{A}_{2,2}} G_{2}(k) \\
& \boldsymbol{C}_{1}(k)=\underbrace{\left[\begin{array}{ll}
c_{1,1}^{1,1} & c_{1,2}^{1,1} \\
c_{2,1}^{1,1} & c_{2,2}^{1,1}
\end{array}\right]}_{\boldsymbol{C}_{1,1}} G_{1}(k)+\underbrace{\left[\begin{array}{rr}
c_{1,1}^{1,2} & c_{1,2}^{1,2} \\
c_{2,1}^{1} & c_{2,2}^{1,2}
\end{array}\right]}_{\boldsymbol{C}_{1,2}} G_{2}(k)
\end{aligned}
$$

Stacking the FP-VARMA expressions of the form Eq. (4.D.1a) for the operating conditions $k=k_{1}, k_{2}$ yields:

$$
\begin{aligned}
& \underbrace{\left[\begin{array}{c}
\boldsymbol{y}_{k_{1}}[t] \\
\ldots \ldots \\
\boldsymbol{y}_{k_{2}}[t]
\end{array}\right]}_{\boldsymbol{y}[t]}+\underbrace{\left[\begin{array}{cc}
\boldsymbol{A}_{1}\left(k_{1}\right) & \mathbf{0} \\
\ldots \ldots & \ldots \ldots \\
\mathbf{0} & \boldsymbol{A}_{1}\left(k_{2}\right)
\end{array}\right]}_{\boldsymbol{A}_{1}} \underbrace{\left[\begin{array}{c}
\boldsymbol{y}_{k_{1}}[t-1] \\
\ldots \ldots \ldots \\
\boldsymbol{y}_{k_{2}}[t-1]
\end{array}\right]}_{\boldsymbol{y}[t-1]}+\underbrace{\left[\begin{array}{cc}
\boldsymbol{A}_{2}\left(k_{1}\right) & \mathbf{0} \\
\ldots \ldots & \ldots \ldots \\
\mathbf{0} & \boldsymbol{A}_{2}\left(k_{2}\right)
\end{array}\right]}_{\boldsymbol{A}_{2}} \underbrace{\left[\begin{array}{c}
\boldsymbol{y}_{k_{1}}[t-2] \\
\ldots \ldots \ldots \\
\boldsymbol{y}_{k_{2}}[t-2]
\end{array}\right]}_{\boldsymbol{y}[t-2]} \\
& =\underbrace{\left[\begin{array}{c}
\boldsymbol{w}_{k_{1}}[t] \\
\cdots \\
\boldsymbol{w}_{k_{2}}[t]
\end{array}\right]}_{\boldsymbol{w}[t]}+\underbrace{\left[\begin{array}{cc}
\boldsymbol{C}_{1}\left(k_{1}\right) & \mathbf{0} \\
\ldots \ldots & \ldots \ldots \\
\mathbf{0} & \boldsymbol{C}_{1}\left(k_{2}\right)
\end{array}\right]}_{\boldsymbol{C}_{1}} \underbrace{\left[\begin{array}{c}
\boldsymbol{w}_{k_{1}}[t-1] \\
\ldots \ldots \ldots \\
\boldsymbol{w}_{k_{2}}[t-1]
\end{array}\right]}_{\boldsymbol{w}[t-1]}
\end{aligned}
$$

In order to specify the FP-VARMA model orders $(n a, n c)$ from the complete data set $Z^{N M}$, the dimension of the "past" vector in Eq. (4.26a) is determined first by means of the AIC or BIC. For this purpose P-VAR $(n)$ models of increasing orders are fitted on $Z^{N M}$ and the order minimizing the AIC or BIC (refer to Eq. (2.23a)-(2.23b) in Chapter 2) determines the dimension of $\boldsymbol{p}[t]$. For instance, if the P-VAR(6) yields minimum AIC or BIC values, then in analogy to the conventional model case [73], the past vector is $\boldsymbol{p}[t]=\left[\boldsymbol{y}^{T}[t-1] \boldsymbol{y}^{T}[t-2] \boldsymbol{y}^{T}[t-3] \boldsymbol{y}^{T}[t-4] \boldsymbol{y}^{T}[t-5] \boldsymbol{y}^{T}[t-6]\right]^{T}$.

The data tabulated in Table 4.2, present an indicative application of the basic CCA scheme for overall AR/MA order determination of the FP-VARMA model in Eq. (4.D.2). Note that zero canonical correlations are observed for iterations 9-12 and the corresponding rows of the FP-VARMA model in Eq. (4.D.2) have overall AR/MA orders $(2,2)$. Once the overall orders in each row of the FP-VARMA model are determined, possible simplifications in the MA part for each row may be specified by means of the refined scheme.

The data tabulated in Table 4.3, present an indicative application of the refined CCA scheme for MA order specification of the FP-VARMA $(2,2)$ model determined in Table 4.2. The canonical correlations for each "future" vector $\boldsymbol{f}[t]$ yielding AR/MA orders $(2,2)$ with the "past" vector $\boldsymbol{p}_{j}[t](j=0,1, \ldots)$ introduced in Eq. (4.33) suggest that the MA order may be reduced in all four rows of the model in Eq. (4.D.2), thus indicating that an $\operatorname{FP}-\operatorname{VARMA}(2,1)_{[p a, p c]}$ is finally specified.

Once the FP-VARMA model orders are specified, and the basis function family is selected, the corresponding functional subspace dimensionality is determined by means of the AIC or BIC (see Section 4.6.2). 
Table 4.2: Indicative basic CCA scheme for overall AR/MA order determination of an FP-VARMA $(2,1)$ model.

\begin{tabular}{|c|c|c|c|}
\hline Iter. & Entries in "future" vector $\boldsymbol{f}[t]$ & $\begin{array}{l}\text { Smallest canonical } \\
\text { correlation } \rho^{\dagger}\end{array}$ & $\begin{array}{c}\text { Overall } \\
\text { AR/MA orders }\end{array}$ \\
\hline 1 & $y_{1, k_{1}}[t]$ & + & \\
\hline 2 & $y_{1, k_{1}}[t], y_{2, k_{1}}[t]$ & + & \\
\hline 3 & $y_{1, k_{1}}[t], y_{2, k_{1}}[t], y_{1, k_{2}}[t]$ & + & \\
\hline 4 & $y_{1, k_{1}}[t], y_{2, k_{1}}[t], y_{1, k_{2}}[t], y_{2, k_{2}}[t]$ & + & \\
\hline 5 & $y_{1, k_{1}}[t], y_{2, k_{1}}[t], y_{1, k_{2}}[t], y_{2, k_{2}}[t], y_{1, k_{1}}[t+1]$ & + & \\
\hline 6 & $y_{1, k_{1}}[t], y_{2, k_{1}}[t], y_{1, k_{2}}[t], y_{2, k_{2}}[t], y_{1, k_{1}}[t+1], y_{2, k_{1}}[t+1]$ & + & \\
\hline 7 & $y_{1, k_{1}}[t], y_{2, k_{1}}[t], y_{1, k_{2}}[t], y_{2, k_{2}}[t], y_{1, k_{1}}[t+1], y_{2, k_{1}}[t+1], y_{1, k_{2}}[t+1]$ & + & \\
\hline 8 & $y_{1, k_{1}}[t], y_{2, k_{1}}[t], y_{1, k_{2}}[t], y_{2, k_{2}}[t], y_{1, k_{1}}[t+1], y_{2, k_{1}}[t+1], y_{1, k_{2}}[t+1], y_{2, k_{2}}[t+1]$ & + & \\
\hline 9 & $y_{1, k_{1}}[t], y_{2, k_{1}}[t], y_{1, k_{2}}[t], y_{2, k_{2}}[t], y_{1, k_{1}}[t+1], y_{2, k_{1}}[t+1], y_{1, k_{2}}[t+1], y_{2, k_{2}}[t+1], y_{1, k_{1}}[t+2]$ & - & $1^{\text {st }}$ row $(2,2)$ \\
\hline 10 & $y_{1, k_{1}}[t], y_{2, k_{1}}[t], y_{1, k_{2}}[t], y_{2, k_{2}}[t], y_{1, k_{1}}[t+1], y_{2, k_{1}}[t+1], y_{1, k_{2}}[t+1], y_{2, k_{2}}[t+1], y_{2, k_{1}}[t+2]$ & • & $2^{\text {nd }}$ row $(2,2)$ \\
\hline 11 & $y_{1, k_{1}}[t], y_{2, k_{1}}[t], y_{1, k_{2}}[t], y_{2, k_{2}}[t], y_{1, k_{1}}[t+1], y_{2, k_{1}}[t+1], y_{1, k_{2}}[t+1], y_{2, k_{2}}[t+1], y_{1, k_{2}}[t+2]$ & 2] & $3^{\text {rd }}$ row $(2,2)$ \\
\hline 12 & $y_{1, k_{1}}[t], y_{2, k_{1}}[t], y_{1, k_{2}}[t], y_{2, k_{2}}[t], y_{1, k_{1}}[t+1], y_{2, k_{1}}[t+1], y_{1, k_{2}}[t+1], y_{2, k_{2}}[t+1], y_{2, k_{2}}[t+2]$ & - & $4^{\text {th }}$ row $(2,2)$ \\
\hline
\end{tabular}

† Plus signs "+" denote positive values, whereas "•" denote zero values determined by the test statistic $T$.

Table 4.3: Indicative refined CCA scheme for possible MA order reduction of an FP-VARMA $(2,1)$ model.

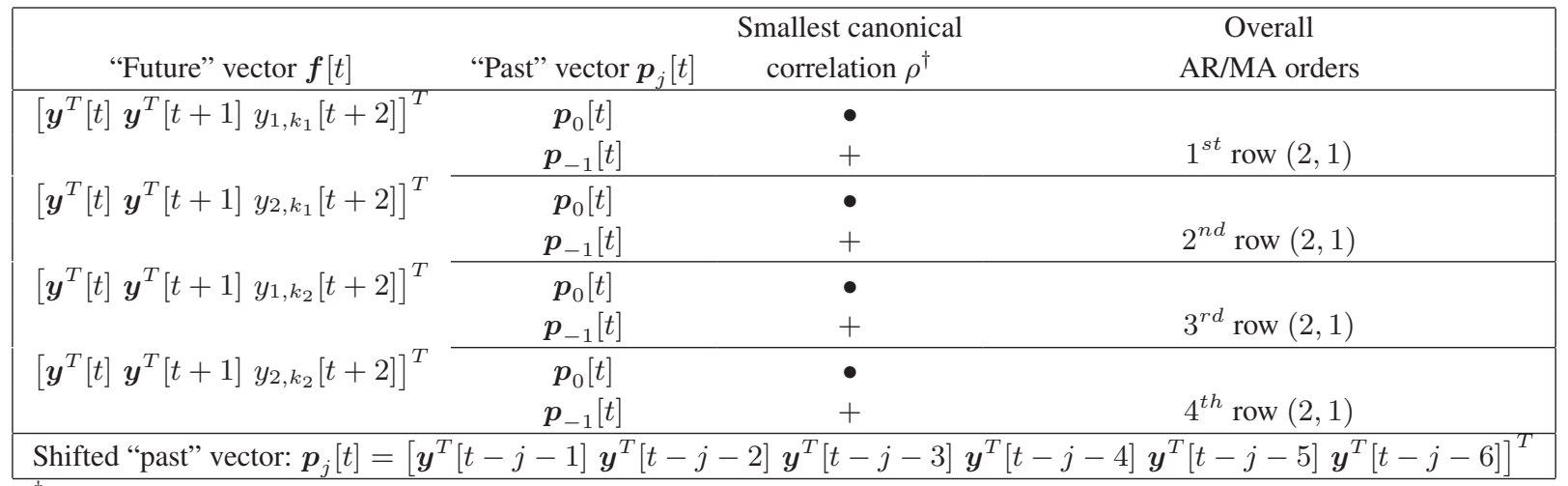

${ }^{\dagger}$ Plus signs "+" denote positive values, whereas "•" denote zero values determined by the test statistic $T_{j}$.

\section{Appendix 4.E: Additional Monte Carlo study results}

\section{E.1 FP-VARMA $(1,1)_{[3,3]}$ model}

Additional FP-VARMA $(1,1)_{[3,3]}$ model identification results from the Monte Carlo study are presented in this section. The data tabulated in Tables 4.4-4.6 correspond to the interval parameter estimates of the AR, MA projection coefficients and cross-covariance matrices as estimated by the 2SLS and ML methods along with their actual values. The results confirm the excellent agreement observed between the actual values and their estimated counterparts. Furthermore, it is verified that both estimators yield essentially unbiased estimates with the ML, expectedly, providing better accuracy for the coefficients of projection.

In Fig. 4.5-4.6, the spectral densities versus frequency and the operating parameter $k$ for the true system are compared to their mean estimated counterparts via the 2SLS and ML estimators. The agreement between each estimate and its true spectral density counterpart is excellent, and confirms the effectiveness of the formulated estimators.

The convergence of the iterated 2SLS estimator is presented in Fig. 4.7. The convergence is rapid as the convergence metric becomes lower than the threshold defined in Table 4.1 within four iterations. 
Table 4.4: Monte Carlo estimation results for the AutoRegressive projection coefficients of the FP$\operatorname{VARMA}(1,1)_{[3,3]}$ model (500 runs per method; sample mean estimate \pm sample standard deviation).

\begin{tabular}{|c|r|r|r|}
\hline Coefficient & Actual Value & \multicolumn{1}{c|}{ 2SLS } & \multicolumn{1}{c|}{ ML } \\
\hline$\alpha_{1,1}^{1,1}$ & 0.59449 & $0.59422 \pm 0.00183$ & $0.59445 \pm 0.00185$ \\
\hline$\alpha_{1,1}^{1,2}$ & -0.03125 & $-0.03116 \pm 0.00167$ & $-0.03124 \pm 0.00162$ \\
\hline$\alpha_{1,1}^{1,3}$ & 0.06434 & $0.06425 \pm 0.00131$ & $0.06440 \pm 0.00126$ \\
\hline$\alpha_{1,2}^{1,1}$ & 0.82353 & $0.82361 \pm 0.00168$ & $0.82356 \pm 0.00168$ \\
\hline$\alpha_{1,2}^{1,2}$ & 0.05000 & $0.05002 \pm 0.00140$ & $0.04997 \pm 0.00133$ \\
\hline$\alpha_{1,2}^{1,3}$ & -0.10784 & $-0.10796 \pm 0.00126$ & $-0.10796 \pm 0.00117$ \\
\hline$\alpha_{2,1}^{1,1}$ & -0.93419 & $-0.93435 \pm 0.00228$ & $-0.93424 \pm 0.00232$ \\
\hline$\alpha_{2,1}^{1,2}$ & 0.00625 & $0.00628 \pm 0.00254$ & $0.00626 \pm 0.00244$ \\
\hline$\alpha_{2,1}^{1,3}$ & -0.01777 & $-0.01757 \pm 0.00222$ & $-0.01750 \pm 0.00208$ \\
\hline$\alpha_{2,2}^{1,1}$ & 0.15993 & $0.15997 \pm 0.00214$ & $0.15986 \pm 0.00219$ \\
\hline$\alpha_{2,2}^{1,2}$ & 0.00625 & $0.00619 \pm 0.00209$ & $0.00623 \pm 0.00214$ \\
\hline$\alpha_{2,2}^{1,3}$ & 0.05086 & $0.05086 \pm 0.00171$ & $0.05086 \pm 0.00172$ \\
\hline
\end{tabular}

Table 4.5: Monte Carlo estimation results for the Moving Average projection coefficients of the FP$\operatorname{VARMA}(1,1)_{[3,3]}$ model (500 runs per method; sample mean estimate \pm sample standard deviation).

\begin{tabular}{|c|r|r|r|}
\hline Coefficient & Actual Value & \multicolumn{1}{c|}{ 2SLS } & \multicolumn{1}{c|}{ ML } \\
\hline$c_{1,1}^{1,1}$ & 0.91316 & $0.91274 \pm 0.00767$ & $0.91287 \pm 0.00543$ \\
\hline$c_{1,1}^{1,2}$ & 0.00125 & $0.00104 \pm 0.00560$ & $0.00109 \pm 0.00433$ \\
\hline$c_{1,1}^{1,3}$ & -0.01189 & $-0.01189 \pm 0.00479$ & $-0.01165 \pm 0.00379$ \\
\hline$c_{1,2}^{1,1}$ & -0.64081 & $-0.64117 \pm 0.00740$ & $-0.64109 \pm 0.00594$ \\
\hline$c_{1,2}^{1,2}$ & -0.00625 & $-0.00567 \pm 0.00579$ & $-0.00588 \pm 0.00473$ \\
\hline$c_{1,2}^{1,3}$ & 0.01777 & $0.01796 \pm 0.00519$ & $0.01784 \pm 0.00405$ \\
\hline$c_{2,1}^{1,1}$ & 0.72500 & $0.72460 \pm 0.00796$ & $0.72459 \pm 0.00678$ \\
\hline$c_{2,1}^{1,2}$ & -0.02500 & $-0.02505 \pm 0.00594$ & $-0.02523 \pm 0.00533$ \\
\hline$c_{2,1}^{1,3}$ & 0.04167 & $0.04201 \pm 0.00513$ & $0.04200 \pm 0.00457$ \\
\hline$c_{2,2}^{1,1}$ & -0.68044 & $-0.67999 \pm 0.00746$ & $-0.68087 \pm 0.00636$ \\
\hline$c_{2,2}^{1,2}$ & 0.00750 & $0.00729 \pm 0.00515$ & $0.00733 \pm 0.00442$ \\
\hline$c_{2,2}^{1,3}$ & -0.06152 & $-0.06145 \pm 0.00511$ & $-0.06165 \pm 0.00422$ \\
\hline
\end{tabular}

The projection of the function minimized in the ML method (equal to the negative value of the concentrated log-likelihood; see Eq. (4.16a)) on selected FP-VARMA $(1,1)_{[3,3]}$ model parameters is shown in Fig. 4.8. The obtained surfaces in Fig. 4.8(a),(b) indicate that the minimization criterion is a smooth function of the model parameters with a single global minimum value. The minimum corresponds to the actual FP-VARMA $(1,1)_{[3,3]}$ projection coefficient values (see contour plots in Fig. 4.8(c),(d)), thus ensuring the convergence of the iterative optimization algorithm used to obtain the ML estimates. 
Table 4.6: Indicative FP-VARMA $(1,1)_{[3,3]}$ noise variance estimation results from Monte Carlo simulations (500 runs per method; sample mean estimate \pm sample standard deviation).

\begin{tabular}{|c|r|c|c|}
\hline Coefficient & Actual Value & 2SLS & ML \\
\hline$\sigma_{1,1}\left(k_{1}, k_{1}\right)$ & 1.00013 & $1.00006 \pm 0.03215$ & $0.99926 \pm 0.03216$ \\
\hline$\sigma_{2,2}\left(k_{1}, k_{1}\right)$ & 1.00037 & $1.00042 \pm 0.03162$ & $0.99952 \pm 0.03164$ \\
\hline$\sigma_{1,1}\left(k_{2}, k_{2}\right)$ & 0.99874 & $0.99877 \pm 0.03077$ & $0.99851 \pm 0.03065$ \\
\hline$\sigma_{2,2}\left(k_{2}, k_{2}\right)$ & 0.99926 & $0.99965 \pm 0.03386$ & $0.99916 \pm 0.03375$ \\
\hline$\sigma_{1,1}\left(k_{3}, k_{3}\right)$ & 1.00084 & $1.00130 \pm 0.03396$ & $1.00116 \pm 0.03397$ \\
\hline$\sigma_{2,2}\left(k_{3}, k_{3}\right)$ & 1.00056 & $1.00098 \pm 0.03394$ & $1.00055 \pm 0.03397$ \\
\hline$\sigma_{1,1}\left(k_{4}, k_{4}\right)$ & 1.00051 & $1.00075 \pm 0.03302$ & $1.00063 \pm 0.03281$ \\
\hline$\sigma_{2,2}\left(k_{4}, k_{4}\right)$ & 1.00204 & $1.00210 \pm 0.03250$ & $1.00203 \pm 0.03242$ \\
\hline$\sigma_{1,1}\left(k_{5}, k_{5}\right)$ & 1.00059 & $1.00089 \pm 0.03004$ & $1.00073 \pm 0.03010$ \\
\hline$\sigma_{2,2}\left(k_{5}, k_{5}\right)$ & 1.00004 & $1.00023 \pm 0.03376$ & $1.00008 \pm 0.03378$ \\
\hline$\sigma_{1,1}\left(k_{6}, k_{6}\right)$ & 1.00077 & $1.00151 \pm 0.02985$ & $1.00110 \pm 0.02964$ \\
\hline$\sigma_{2,2}\left(k_{6}, k_{6}\right)$ & 0.99922 & $0.99899 \pm 0.03284$ & $0.99868 \pm 0.03277$ \\
\hline$\sigma_{1,1}\left(k_{7}, k_{7}\right)$ & 1.00155 & $1.00169 \pm 0.03243$ & $1.00149 \pm 0.03239$ \\
\hline$\sigma_{2,2}\left(k_{7}, k_{7}\right)$ & 0.99982 & $1.00041 \pm 0.03264$ & $1.00015 \pm 0.03270$ \\
\hline$\sigma_{1,1}\left(k_{8}, k_{8}\right)$ & 1.00001 & $1.00008 \pm 0.03127$ & $0.99992 \pm 0.03115$ \\
\hline$\sigma_{2,2}\left(k_{8}, k_{8}\right)$ & 0.99914 & $0.99931 \pm 0.02964$ & $0.99912 \pm 0.02961$ \\
\hline$\sigma_{1,1}\left(k_{9}, k_{9}\right)$ & 1.00232 & $1.00272 \pm 0.03326$ & $1.00246 \pm 0.03316$ \\
\hline$\sigma_{2,2}\left(k_{9}, k_{9}\right)$ & 0.99919 & $0.99944 \pm 0.03270$ & $0.99916 \pm 0.03257$ \\
\hline$\sigma_{1,1}\left(k_{10}, k_{10}\right)$ & 0.99852 & $0.99849 \pm 0.03354$ & $0.99820 \pm 0.03352$ \\
\hline$\sigma_{2,2}\left(k_{10}, k_{10}\right)$ & 1.00034 & $1.00027 \pm 0.03155$ & $0.99984 \pm 0.03132$ \\
\hline$\sigma_{1,1}\left(k_{11}, k_{11}\right)$ & 0.99857 & $0.99882 \pm 0.03253$ & $0.99802 \pm 0.03257$ \\
\hline$\sigma_{2,2}\left(k_{11}, k_{11}\right)$ & 0.99820 & $0.99866 \pm 0.03295$ & $0.99778 \pm 0.03305$ \\
\hline
\end{tabular}
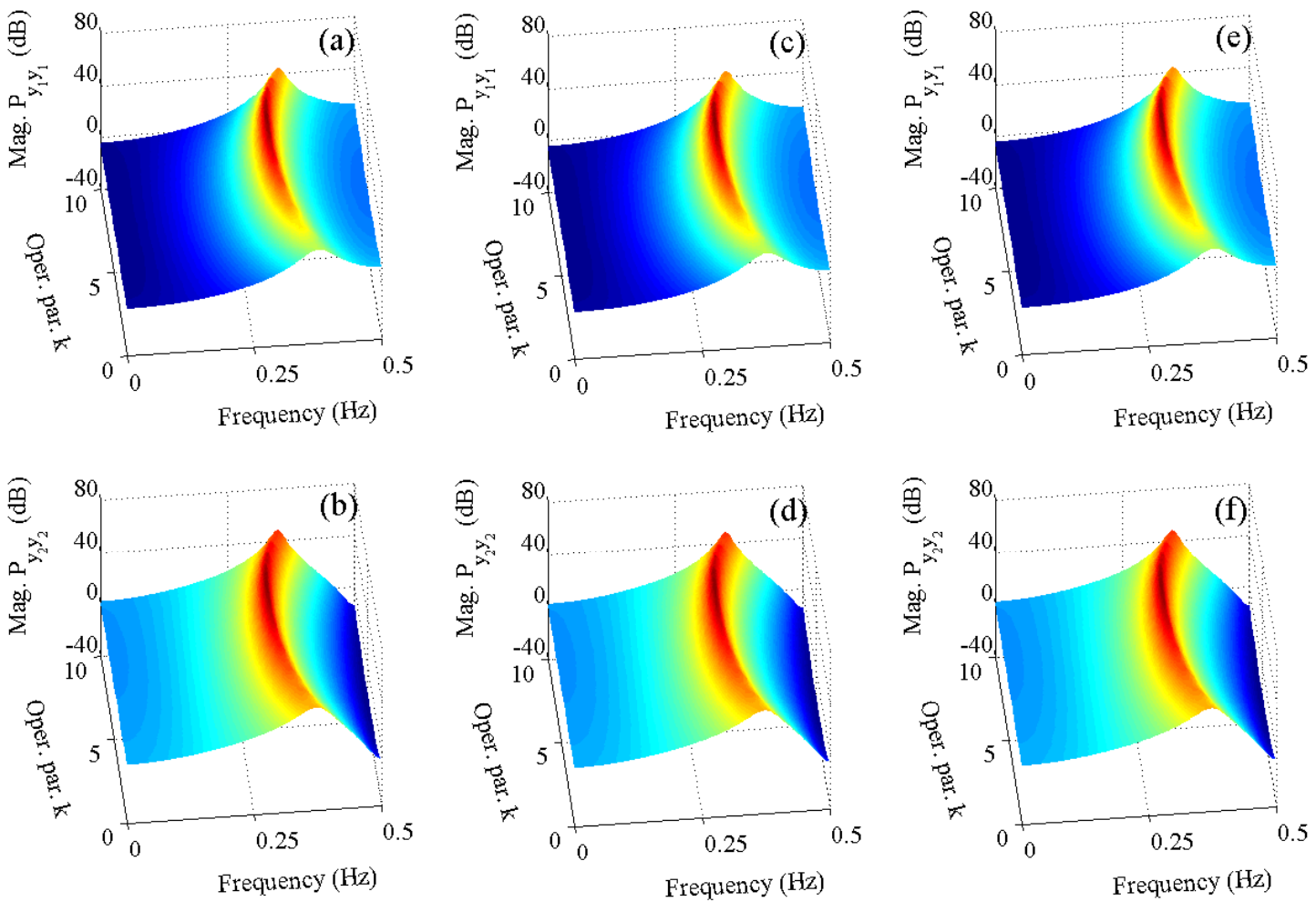

Figure 4.5: FP-VARMA $(1,1)_{[3,3]}$ model based power spectral densities with respect to frequency and operating parameter $k$ : (a),(b) actual model, (c),(d) 2SLS mean estimate and, (e),(f) ML mean estimate. 

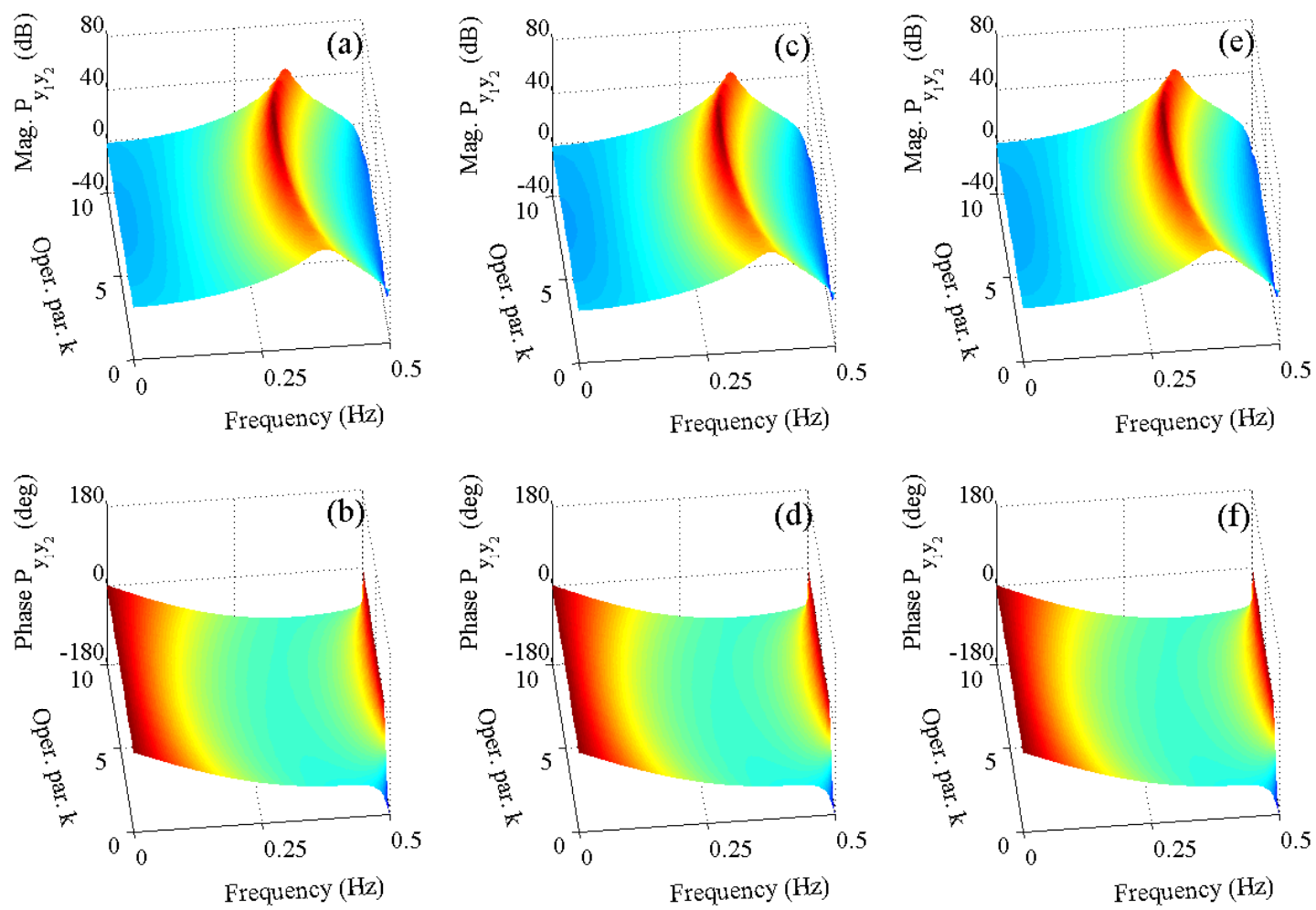

Figure 4.6: FP-VARMA $(1,1)_{[3,3]}$ model based cross-spectral densities with respect to frequency and operating parameter $k$ : (a),(b) actual model, (c),(d) 2SLS mean estimate and, (e),(f) ML mean estimate.

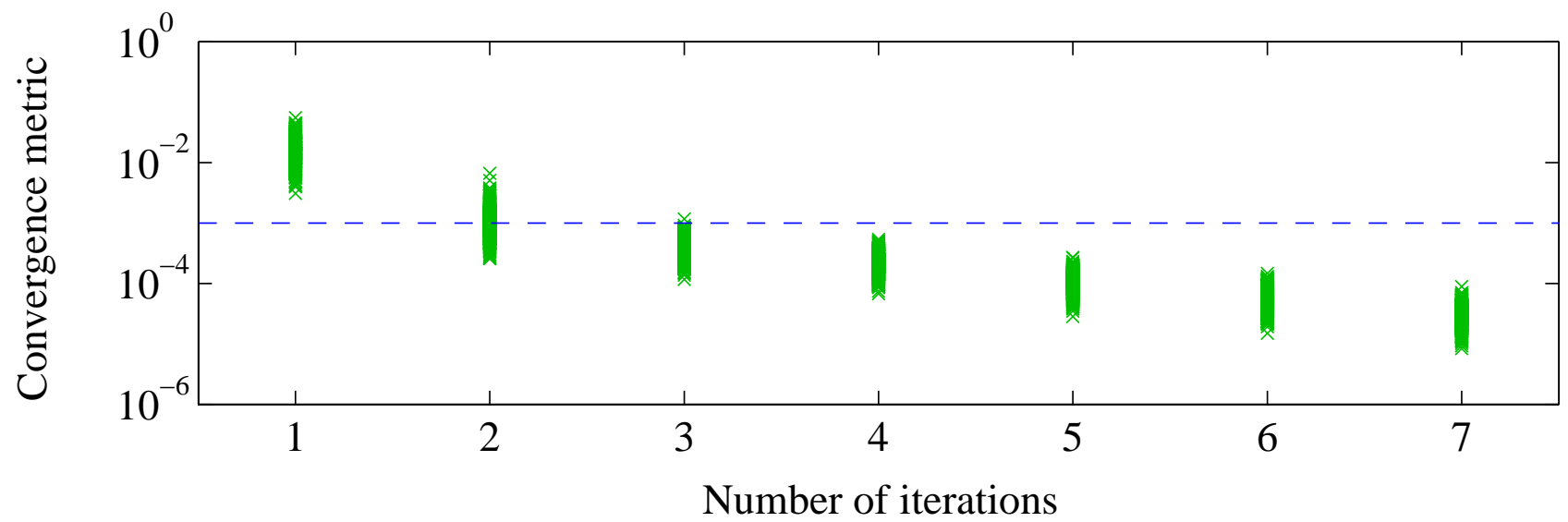

Figure 4.7: Convergence metric versus number of iterations for the 2SLS estimator of the FP$\operatorname{VARMA}(1,1)_{[3,3]}$ model (the horizontal dashed line indicates the convergence criterion limit; the " $\times$ " mark designates the value of a single experiment; 500 Monte Carlo runs). 

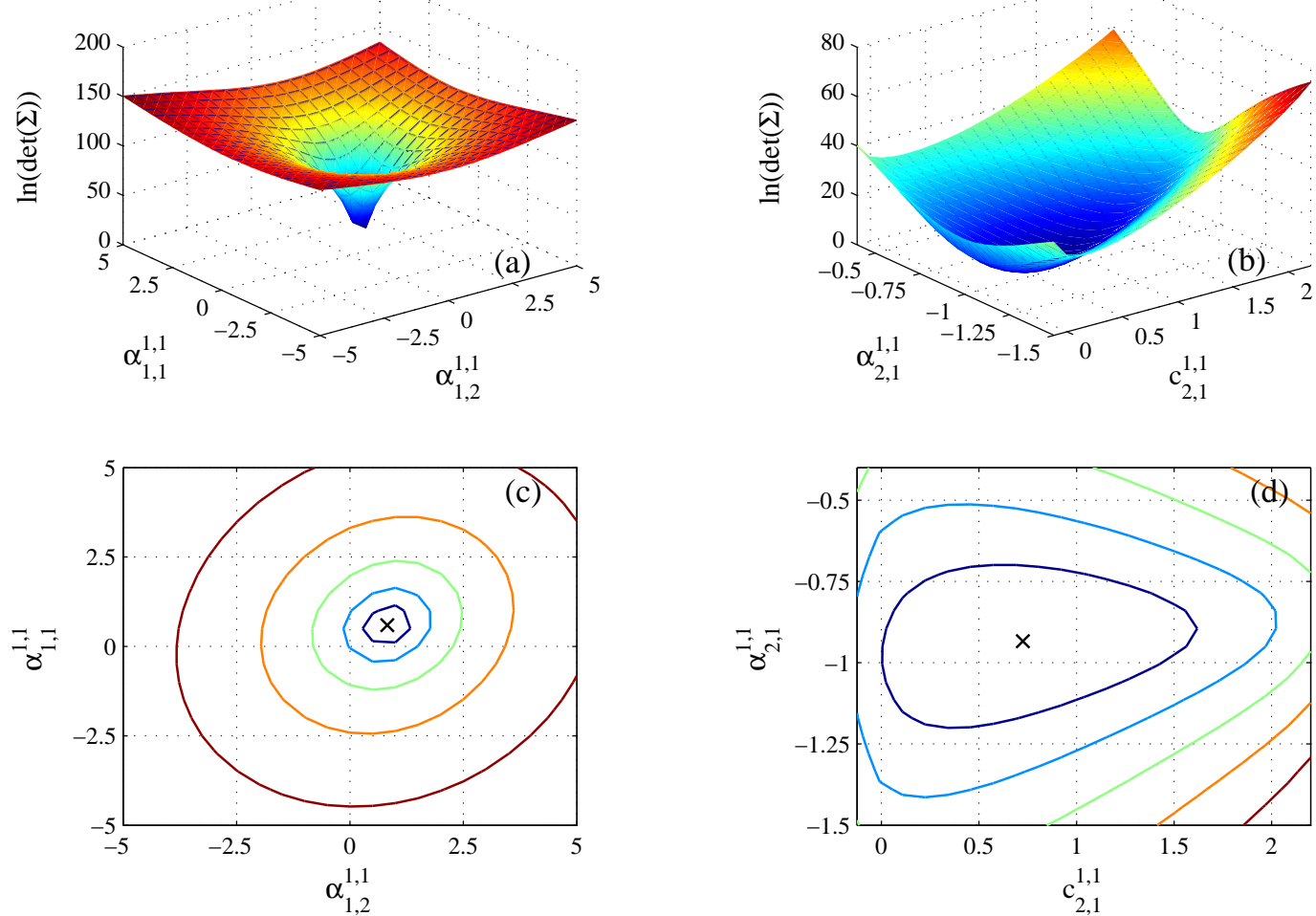

Figure 4.8: Projection of the function minimized in the ML method on selected FP-VARMA $(1,1)_{[3,3]}$ model parameters: (a),(c) surface and contour plots by projecting on $\alpha_{1,1}^{1,1}$ and $\alpha_{1,2}^{1,1}$; (b),(d) surface and contour plots by projecting on $a_{2,1}^{1,1}$ and $c_{2,1}^{1,1}$ (the " $\times$ " marks designate the actual $\operatorname{FP}-\operatorname{VARMA}(1,1)_{[3,3]}$ model parameters).

\section{E.2 FP-VAR $(2)_{[4]}$ model}

The performance characteristics of the two-step approach for the special case of a pure AR process is considered. For this purpose, a bivariate FP-VAR $(2)_{[4]}$ model is used to generate data. The functional subspaces consist of the first four shifted Chebyshev polynomials of the second kind, that is $p=4$. The modeling procedure relies upon $M=11$ operating conditions with $k \in[0,10]$, obtained at unit increments. The innovations for each operating condition $k$ are (i) Gaussian white with zero mean and identity covariance $\boldsymbol{\Sigma}_{k, k}$, and (ii) uncorrelated with those of any other operating condition (that is, $\boldsymbol{\Sigma}_{k, \ell}=\mathbf{0}$ ), which implies that no interrelations exist among the obtained data records (groupwise homoscedastic innovations; see Chapter 2, Table 2.1).

In Fig. 4.9, the spectral densities versus frequency and the operating parameter $k$ is shown for the actual model. The study encompasses 500 Monte Carlo runs, and parameter estimation is based upon $N=$ 3, 500 sample-long data records. A non-iterative WLS estimator, based upon QR implementation [1, pp. 318-320] (see Chapter 2, Appendix 2.E), is utilized for this purpose (details in Table 4.7).

Model order specification via CCA employs a risk level equal to $\alpha=10^{-3}$ and $s=2$ (since the AIC and BIC were minimized for P-VAR model orders around 2 when fitted to the data in a pilot study). The obtained results indicate that the CCA scheme specifies an $\operatorname{FP}-\operatorname{VAR}(2)$ in the vast majority of the cases (see Fig. 4.10(a),(b)). The functional subspace specification problem is treated by fitting successive FP$\operatorname{VAR}(2)_{[p]}$ models with increasing basis dimensionality $p=2,3, \ldots, 8$ and computing the corresponding AIC and BIC values. Monte Carlo results depicting the probability of attaining minimum AIC and BIC values versus functional basis dimensionality are presented in Fig. 4.10(c). The success rates (\%) for both criteria are excellent, as minimal values are attained for $p=4$ in the vast majority of the runs. 

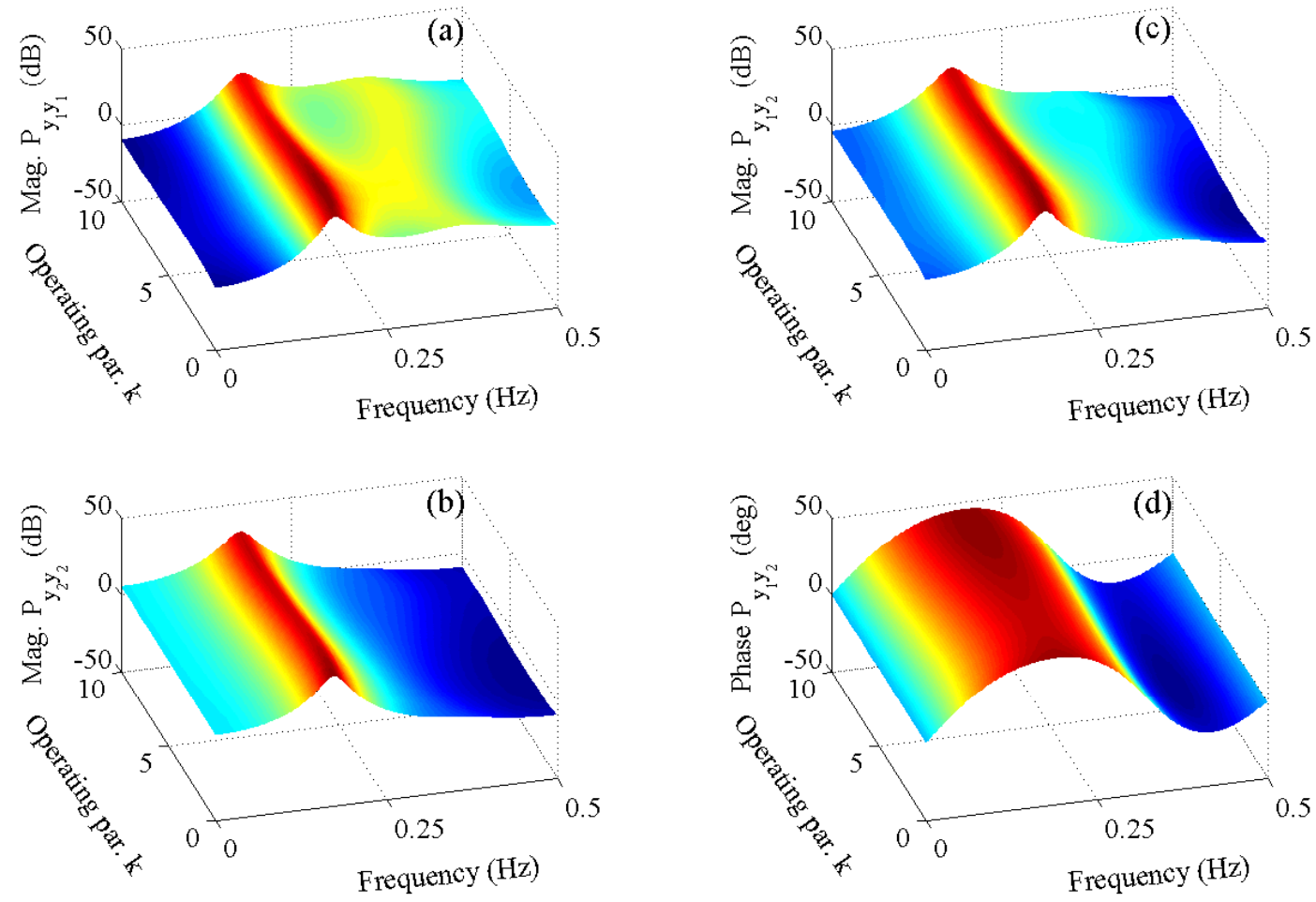

Figure 4.9: FP-VAR $(2)_{[4]}$ model based spectral densities with respect to frequency and operating parameter $k$.

Table 4.7: Details on the Monte Carlo study for FP-VAR $(2)_{[4]}$ model identification.

\begin{tabular}{lll}
\hline No. of responses: $n y=2$ & Operating points: $M=11(k \in[0,10], \Delta k=1)$ & $\begin{array}{l}\text { Functional subspaces: first 4 Chebyshev II polynomials } \\
\text { Innovations dependency: groupwise homoscedastic }\end{array}$ \\
No. of Monte Carlo runs: 500 & Data length: $N=3,500$ samples & \\
\hline Parameter estimation method & non-iterative WLS algorithm $(\mathrm{QR} \mathrm{implementation)}$ & \\
\hline Model structure specification & Model orders: CCA scheme $\left(s=2, \alpha=10^{-3}\right)$ & Functional subspaces: information criteria (AIC, BIC) \\
\hline
\end{tabular}

\section{E.3 Mass-spring-damper system}

A lumped mass-spring-damper system with four degrees of freedom (d.o.f.) is utilized as seen in Fig. 4.11. Each mass is driven by a Gaussian white noise force having zero mean and unit variance, whereas the corresponding displacements are obtained by numerical integration using the Runge-Kutta $(4,5)$ formula. The signals are discretized by means of a zero-order-hold (ZOH) at a sampling frequency of $f_{\mathrm{s}}=30 \mathrm{~Hz}$. The stiffness and damping elements depend linearly on the operating parameter $k$. A total of $M=11$ operating points are considered, with the operating parameter values $k$ lying within the $[0,10]$ range at unit increments (details in Table 4.8). Indicative FRF magnitudes with respect to frequency and operating parameter $k$ are shown in Fig. 4.12.

The study encompasses 500 Monte Carlo runs, and model order specification is based upon $N=$ 5, 000 samples. CCA is used to specify the appropriate FP-VARMA model orders corresponding to the following pairs of responses, $\left\{y_{1}, y_{3}\right\},\left\{y_{2}, y_{3}\right\}$ and $\left\{y_{2}, y_{4}\right\}$ (see also Fig. 4.11). The model order specification procedure via CCA employs a risk level $\alpha=10^{-3}$ and $s=4$ (since the AIC and BIC were minimized for P-VAR model orders around $4-6$ when fitted to the data of each response pair considered in a pilot study). The obtained results indicate that for all three response pairs, the CCA approach specifies an FP$\operatorname{VARMA}(4,3)$ in the majority of cases (see Fig. 4.13), which is also in agreement with theory [80]. The CCA-based results, indicate that the proposed two-step-approach facilitates the decoupling of the model 

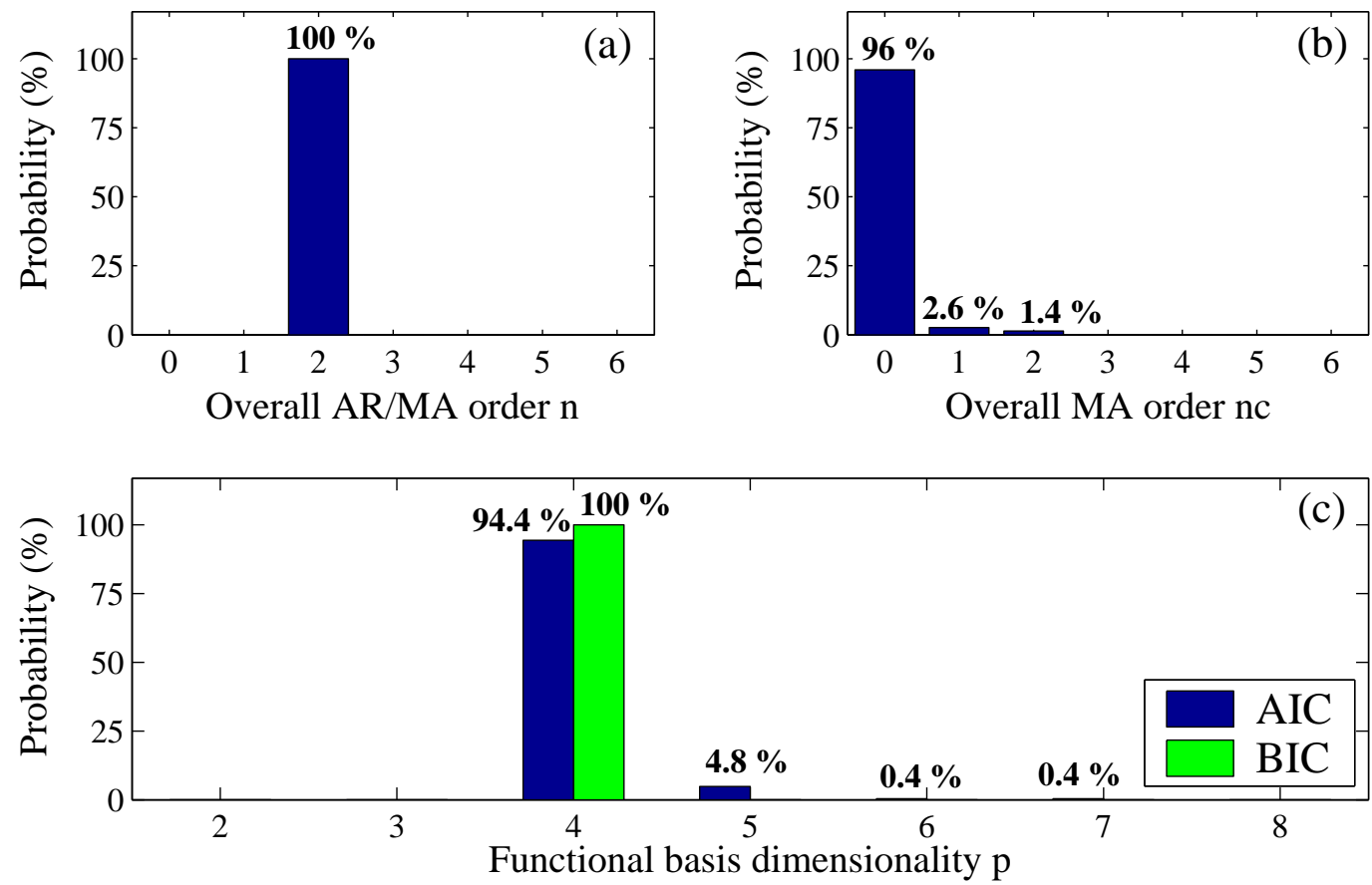

Figure 4.10: $\operatorname{FP}-\operatorname{VAR}(2)_{[4]}$ model structure specification results $\left(s=2, \alpha=10^{-3}, 500\right.$ Monte Carlo runs): (a) basic CCA scheme for $\operatorname{FP}-\operatorname{VARMA}(n, n)$ model order specification; (b) refined CCA scheme for MA order reduction of FP-VARMA $(2,2)$ model; (c) FP-VAR $(2)_{[p]}$ functional subspace dimensionality specification via the AIC/BIC criteria.

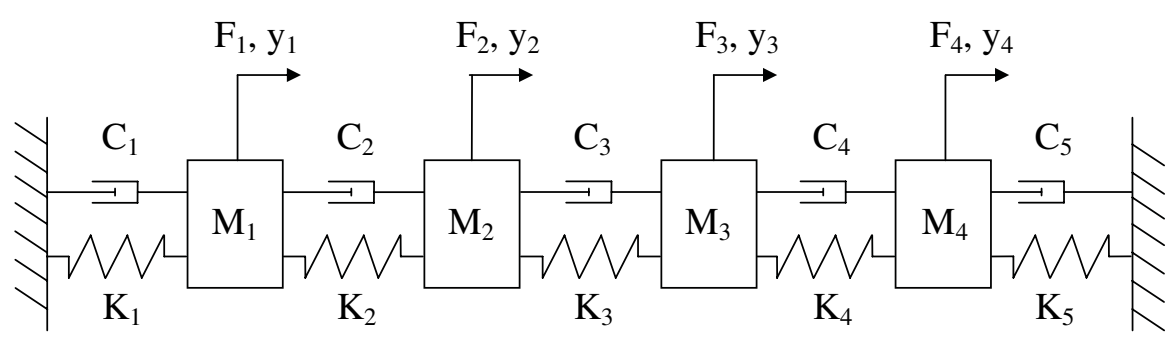

Figure 4.11: The 4 d.o.f. lumped system with linear dependent stiffness and damping on the operating parameter $k$.

Table 4.8: Details on the Monte Carlo study for the 4 d.o.f. mass-spring-damper system.

\begin{tabular}{cll}
\hline No. of responses (displacements): $n y=4$ & No. of excitations (forces): $n x=4$ & Excitation type: Gaussian white noise \\
Operating points: $M=11(k \in[0,10], \Delta k=1)$ & Data length: $N=5,000$ samples & No. of Monte Carlo runs: 500 \\
Responses obtained via Runge-Kutta $(4,5)$ formula & Signals discretized via ZOH & Sampling frequency: $f_{\mathrm{s}}=30 \mathrm{~Hz}$ \\
\hline Masses $(\mathrm{kg})$ & Stiffness $\left(\mathrm{N} \mathrm{m}^{-1}\right)$ dependence on $k$ & Damping $\left(\mathrm{N} \mathrm{s} \mathrm{m}^{-1}\right)$ dependence on $k$ \\
$M_{1}=0.010$ & $K_{1}=0.1 k+15$ & $C_{1}=0.0002 k+0.005$ \\
$M_{2}=0.025$ & $K_{2}=0.3 k+10$ & $C_{2}=0.0005 k+0.010$ \\
$M_{3}=0.075$ & $K_{3}=0.2 k+20$ & $C_{3}=0.0010 k+0.050$ \\
$M_{4}=0.060$ & $K_{4}=0.2 k+25$ & $C_{4}=0.0005 k+0.010$ \\
& $K_{5}=0.2 k+10$ & $C_{5}=0.0005 k+0.020$ \\
\hline CCA performed on response pairs: $\left\{y_{1}, y_{3}\right\},\left\{y_{2}, y_{3}\right\},\left\{y_{2}, y_{4}\right\}$ & model order specification: CCA scheme $\left(s=4, \alpha=10^{-3}\right)$ \\
Selected FP-VARMA model orders: $n a=4, n c=3$ & &
\end{tabular}

order $(n a, n c)$ specification problem from the functional subspace dimensionality ( $p a, p c)$ problem. 

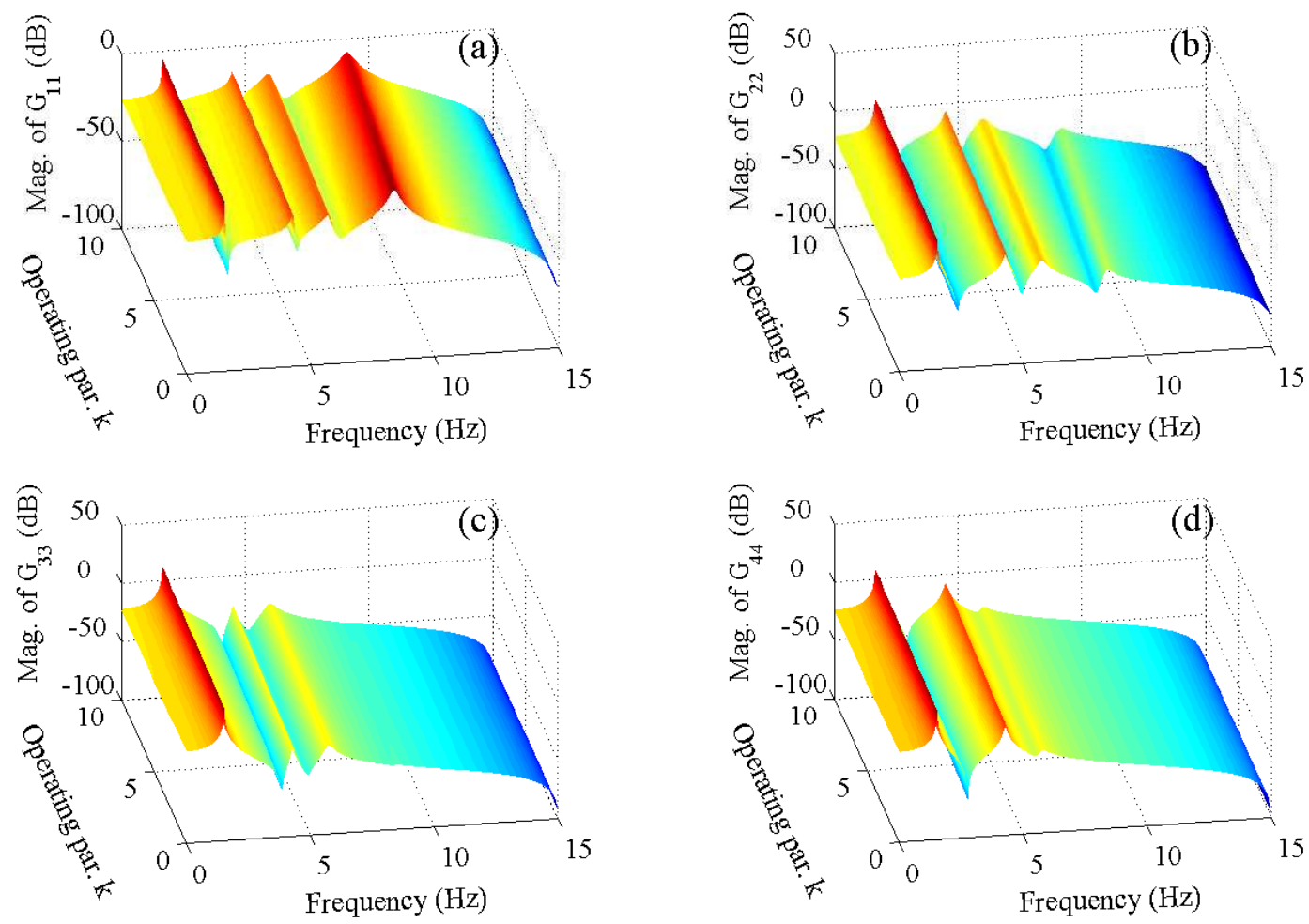

Figure 4.12: Indicative four d.o.f. system frequency response function magnitudes $G_{i j}$ with respect to frequency and operating parameter $k\left(G_{i j}\right.$ is the transfer function between the $i$-th response and the $j$-th excitation).
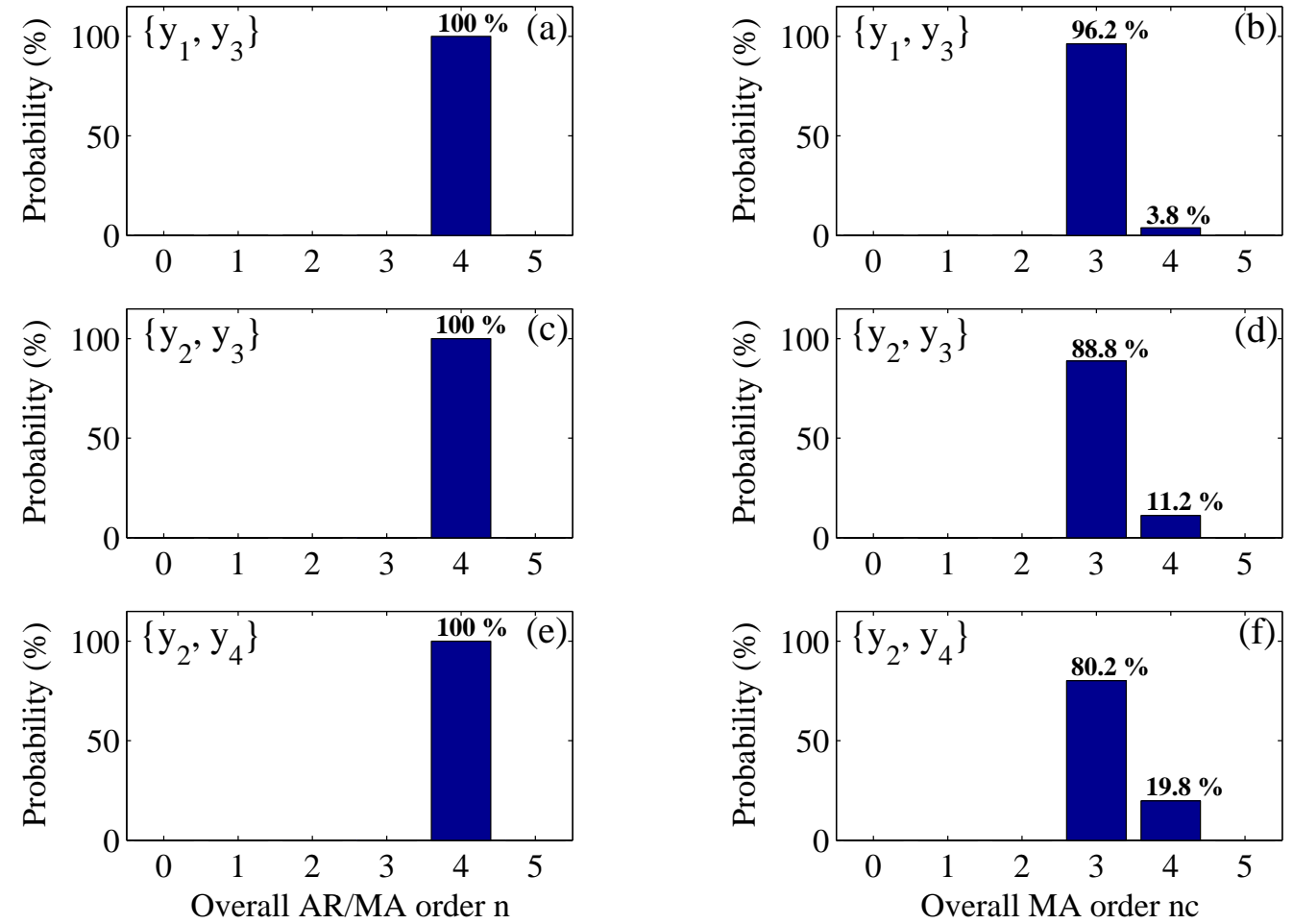

Figure 4.13: Model order specification results on the 4 d.o.f. system $\left(s=4, \alpha=10^{-3}, 500\right.$ Monte Carlo runs): (a),(c),(e) basic CCA scheme for FP-VARMA $(n, n)$ model order specification; (b),(d),(f) refined CCA scheme for MA order reduction of the FP-VARMA $(4,4)$ model. 


\section{Chapter 5}

\section{Output-only vibration-based statistical damage detection in a composite structure under different temperatures: a global model based approach}

\subsection{Introduction}

The influence of environmental factors on the structural dynamics has attracted significant attention by the engineering community in recent years. This issue is critical for vibration-based structural health monitoring applications, as it has been widely acknowledged that these factors - especially temperature - can often mask subtle structural changes caused by damage [3]. Indeed, several studies have focused on developing methods to "separate" the effects of temperature on the structural dynamics from those of damage, and may be classified into two main classes.

The first class relies upon extracting, from the measured data, characteristic quantities (also referred to as features) that are sensitive to damage but insensitive to temperature changes. Statistical pattern recognition techniques are often used to project the selected features (usually being model or modal parameters, although other quantities may be used also) on lower dimensions using variations of Principal Component Analysis [24-29], and Factor Analysis [30] procedures in order to attenuate the temperature effects. The aforementioned procedures treat temperature as an embedded variable and thus temperature measurements are not required, whereas damage is detected when the corresponding changes in the features are somehow "orthogonal" to the changes caused by temperature. Alternatively, subspace identification techniques may be used to model response vibration data and generate residuals for damage detection [31-33]. The temperature effects in [31] are "averaged out" by merging data from the healthy structure at different (though not necessarily measured) temperatures. A conceptually different approach is followed in [32,33], as a physics-based model of the temperature effects on the structural dynamics is also required. Two versions of the approach for damage detection are formulated. The first version utilizes the physics-based model to obtain a temperature adjusted residual vector for damage detection. The second version treats temperature as a "nuisance" parameter and its effects on the residual vector are eliminated with the aid of the physics-based model. Note that these residual-based approaches detect damages producing changes "orthogonal" to the response spaces spanned by the baseline (healthy) data.

The second class is based upon the direct modeling of temperature effects on the features of interest, and may be used when temperature measurements are available. Typically, a conventional structural 
dynamics model is obtained for each temperature condition using customary identification techniques (the "multi-model" approach [40-42]). The models are then linked via polynomial regression or interpolation procedures which approximate the dependence of model parameters on temperature [34]. Alternatively, the modal parameters are extracted and are subsequently related to temperature via additional expressions. Static linear regression models associating modal frequency with temperature are employed in [35]. In order to account for the structure's thermal capacity, linear dynamic models describing the modal frequency dynamic dependence on temperature have also been employed [23,36,37].

The present study aims at introducing an approach for effective vibration-based damage detection under different temperatures by means of novel global models and statistical decision making. The approach belongs to the second class of methods that directly model the temperature effects on the dynamics, while also being a member of the broader family of statistical time series methods (see the overviews [18,21]). The statistical time series methods offer a number of important advantages such as (i) no requirement for physics-based or finite element models, (ii) use of a partial structural model based on a limited number of measurable signals, (iii) inherent accounting for uncertainty, (iv) statistical decision making with specified performance characteristics, and (v) the effective use of natural random vibration data records (no need to interrupt normal operation).

The strengths and advantages of the approach stem from the statistically efficient stochastic global modeling of the dynamics under any temperature, the effective statistical treatment of uncertainties, and statistical decision making to infer damage detection. The global models are of the Functionally Pooled (FP) form [14-17,72] which are presently characterized by model parameters having an explicit functional dependence on temperature. FP modeling is advantageous over the multi-model approach as it (i) treats data records corresponding to various temperatures simultaneously and fully takes cross-dependencies into account, (ii) uses a highly parsimonious representation, and (iii) provides precise information about the system dynamics at any intermediate ("unmodeled") temperature without resorting to customary interpolation schemes. These characteristics lead to highly accurate and efficient stochastic modeling of the temperaturedependent dynamics.

In the present study output-only vibration data are used, as in many practical applications the excitation is difficult or impossible to measure rendering the problem significantly more challenging. FP-VAR (Vector AutoRegressive) models are used for this purpose, and also offer simplicity in estimation. In addition, a novel scheme for global FP model structure selection that manages to simplify the problem and reduce the computational complexity of the FP model structure selection (previously based on exhaustive search procedures) is presently introduced. Also, uncertainties are modeled via proper interval estimates, while damage detection is based on detecting changes in the dynamics via statistical decision making using either the estimated natural frequencies, or the discrete-time model parameters. Information compression techniques, based on Principal Component Analysis (PCA), are also investigated in an effort to streamline damage detection. It is important to stress that the extension of the proposed approach for damage detection to handle input-output vibration data is trivial.

This work builds upon previous studies by Hios and Fassois [40-42] (see also Chapter 3), where FP models have been successfully used for identifying the temperature effects on the dynamics of a composite beam. As this is a proof-of-concept study, a similar beam and test rig are presently used allowing for the incorporation of previous experience and also for a direct comparison with the previous results using fresh experiments and output-only data. Although the proposed approach shares certain aspects with the Functional Model based damage detection and identification approach of Fassois and co-workers [18-21], it is distinct in its structure and unique in its capability of handling temperature effects. Early results on this topic are presented by Hios and Fassois in [38], while an additional type of damage (progressive saw cut) is investigated in [39].

The primary challenges addressed in this study are: 
(a) The use of output-only data for identifying the temperature dependent dynamics. In contrast to the previous studies by Hios and Fassois [40-42], this is a more challenging problem and it is of particular interest to investigate the ability of the output-only approach to potentially match the earlier results.

(b) The use of a subtle damage scenario, which involves the attachment of a small mass (corresponding to an increase of about $4 \%$ ) to the beam. This is a challenging problem, and it is interesting to examine whether the change is detectable under different temperatures.

(c) The use of a limited number of sensors to infer damage detection. The present use of only two signals implies that no mode shape information is employed, thus the challenge is to investigate whether effective damage detection may be achieved with a limited number of sensors.

(d) A final issue relates to the frequency range employed, which is purposely selected at the convenient lower frequency end $(0-1000 \mathrm{~Hz})$. The challenge here is to examine whether damage can be effectively detected in this frequency range.

The novel contributions of the present study may be summarized as follows:

(i) The postulation of an effective statistical approach for structural damage detection under different temperatures.

(ii) The introduction of a new scheme for global (FP) model structure selection which avoids the use of exhaustive search procedures.

(iii) The postulation and testing of two versions of the approach - the first relying on the estimated natural frequencies and the second on the discrete-time model parameters. Techniques for information compression (dimensionality reduction) are also investigated.

(iv) The experimental identification of the temperature-dependent dynamics from output-only data and comparison with those previously identified from input-output data.

(v) The laboratory assessment of the approach with many hundreds of experiments corresponding to different temperatures (proof-of-concept study).

(vi) Extensive comparisons with alternative damage detection methods that attempt potential removal of the temperature effects from the damage-sensitive features.

The rest of this chapter is organized as follows: The experimental set-up is presented in Section 5.2, and the stochastic global model based approach to damage detection in Section 5.3. Experimental damage detection results and assessment are presented in Section 5.4, along with comparisons alternative methods attempting potential removal of the temperature effects from the damage-sensitive features. Concluding remarks are finally summarized in Section 5.5.

\subsection{The experimental set-up}

\subsubsection{The structure}

A composite cantilever beam with integrated piezoceramic transducers (Fig. 5.1(a)) is used in this study. Such structures are referred to as "smart" due to their potential for performing advanced functions, such as vibration control and health monitoring. The beam's effective dimensions are $600 \times 52.5 \times 3 \mathrm{~mm}$, and it is made of a 3-ply glass-polyester composite with fibers oriented along the longitudinal direction, while four $50 \times 25 \times 1 \mathrm{~mm}$ Ferroperm type PZT-5A piezoceramic patches are symmetrically attached to its sides (see Table 5.1 for details). A patch near the clamped end excites the beam and the response is measured by both patches at the free end (Fig. 5.1(b)). The total mass of the structure is $196.3 \mathrm{~g}$, whereas the properties of the composite material and the piezoceramic patches are provided in Chapter 3, Appendix 3.A. Notice that the beam and test rig are similar to those used in [38]. This selection is motivated by the proof-of-concept 


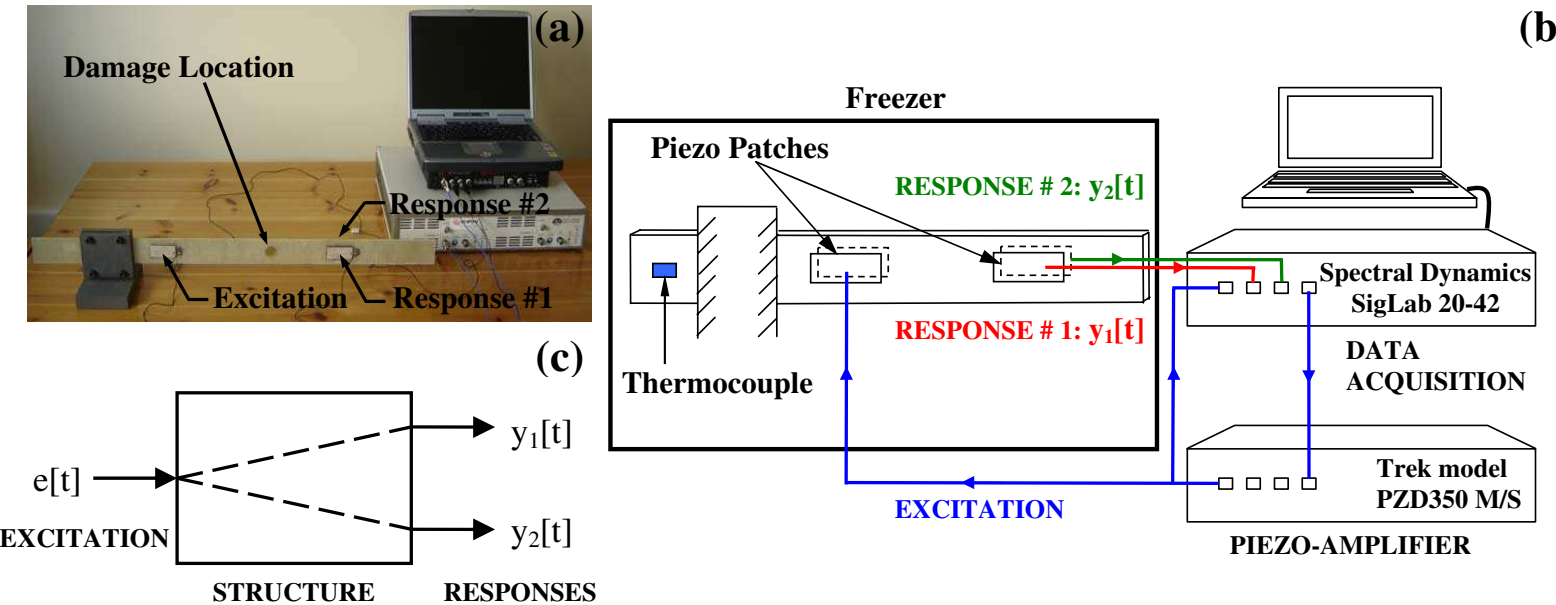

(b)

Figure 5.1: (a) Photo of the experimental set-up; (b) schematic diagram of the experimental set-up; (c) schematic representation of the considered system.

nature of the study, and the benefits of using the previous experience while also allowing for an interesting direct comparison with the previous results using fresh experiments and output-only vibration data.

\subsubsection{The damage}

A non-destructive damage is considered which consists of the attachment of a small mass on the beam as a means of simulating a local elasticity reduction. The added mass is $7.9 \mathrm{~g}$ and is located $270 \mathrm{~mm}$ from the beam's clamped end (Fig. 5.1(a)). As the healthy structure's mass is $196.3 \mathrm{~g}$, the additional mass corresponds to an increase of only $4.0 \%$, and amounts to a minor decrease in the natural frequencies (see Subsection 5.4.1.2 for details). An additional type of damage (progressive saw cut) is reported in [39].

\subsubsection{The experiments}

The smart beam is placed in a freezer, and the experiments are carried out under quasi-static thermal conditions. The smart beam temperature is obtained from a digital thermometer with a K-type bead thermocouple attached behind the clamp to avoid interference with its vibratory motion (Fig. 5.1(b)). The study focuses on the $[-20,+20]^{\circ} \mathrm{C}$ temperature range. The actuator is driven by a properly amplified stochastic, zero-mean, Gaussian and white voltage. The study focuses on the $[0,1,000] \mathrm{Hz}$ bandwidth. All signals are collected by the data acquisition device at a sampling frequency of $f_{\mathrm{s}}=2,560 \mathrm{~Hz}$, and the response signals are designated as $y_{1}[t]$ and $y_{2}[t]$ (Fig. 5.1(c)). Following sample mean subtraction, each signal is scaled to unit variance in order to enhance numerical accuracy. The experimental and data acquisition details are summarized in Table 5.1.

\subsection{The stochastic global model based approach to damage detection}

Like other vibration-based methods (see the surveys $[18,21]$ ), the proposed approach consists of two phases: (a) The baseline phase, which involves the identification of a global model for the healthy structure, and (b) the inspection phase, which includes conventional identification of the current structural state and statistical decision making to test for significant discrepancies between the baseline (healthy) and current modal/model parameters. A schematic representation of the approach is shown in Fig. 5.2. 
Table 5.1: Experimental and data acquisition details.

\begin{tabular}{ll}
\hline $\begin{array}{c}\text { Beam: } 600 \times 52.5 \times 3 \mathrm{~mm} 3-\text { ply glass-polyester composite } \\
\text { with isopthalic polyester resin matrix and fibers } \\
\left.\text { oriented in the longitudinal direction (that is, }[0]_{3}\right)\end{array}$ & \multicolumn{1}{c}{ Actuators/sensors: $50 \times 25 \times 1 \mathrm{~mm}$} \\
perroperm type PZT-5A \\
$\begin{array}{l}\text { Data acquisition device: Spectral Dynamics SigLab } 20-42 \\
\text { Temperature measurements: K-type bead thermocouple }\end{array}$ & Actuator amplifier: Trek PZD350 M/S \\
Signal bandwidth: $[0,1,000] \mathrm{Hz}$ & Temperature range: $[-20,+20]^{\circ} \mathrm{C}$ \\
Measured signal length: $N=76,800$ samples $(\approx 30 \mathrm{~s})$ & Sampling frequency: $f_{\mathrm{s}}=2,560 \mathrm{~Hz}$ \\
& Excitation: Gaussian white noise
\end{tabular}

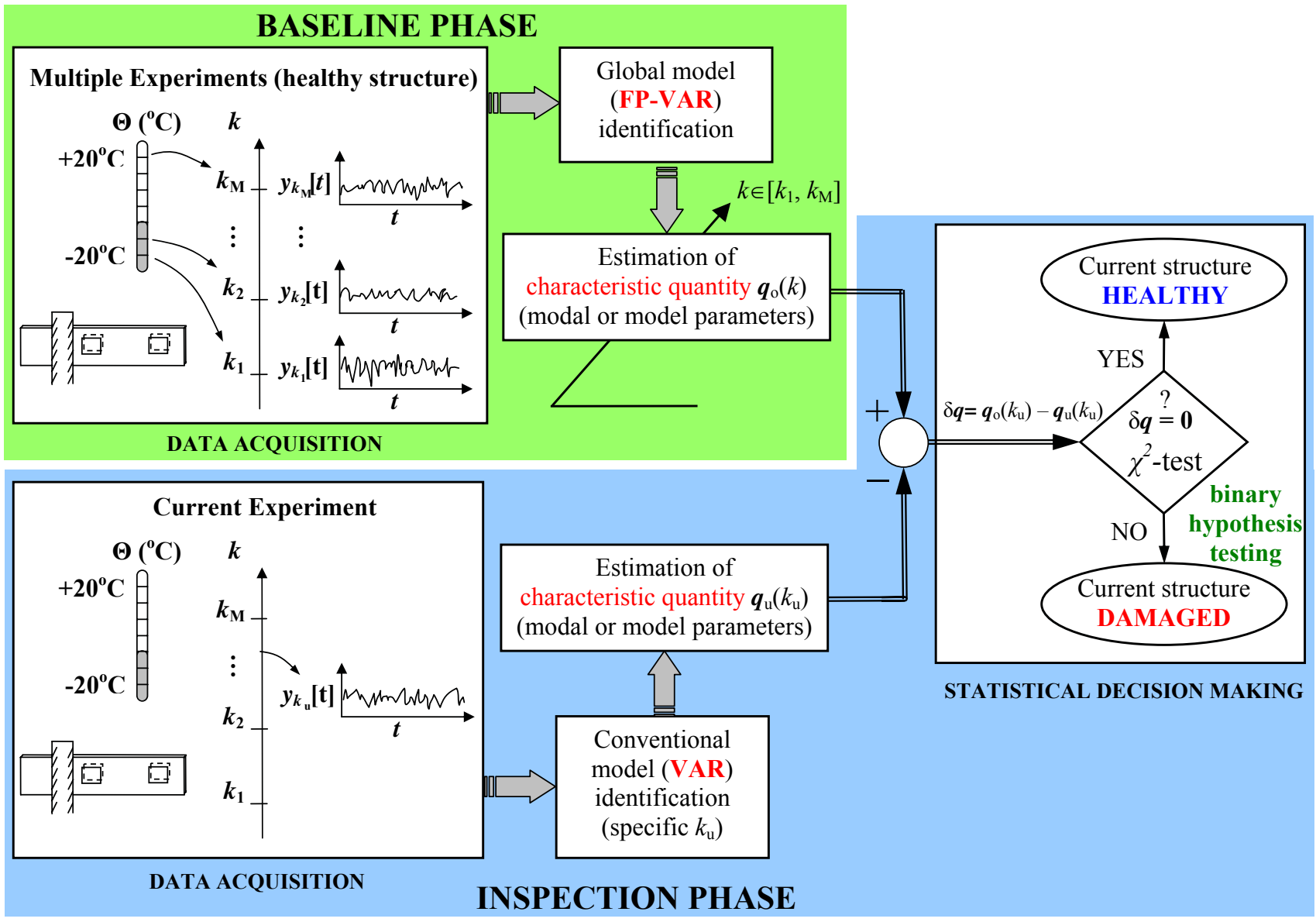

Figure 5.2: Schematic of the stochastic global model based approach to damage detection.

\subsubsection{Baseline phase}

The data set Global model identification is based upon $M$ sets of $N$ sample-long vector signals, each one obtained under a distinct temperature $k$. The set of all possible temperatures belongs to the range $\mathcal{R}=$ $\left[k_{\min }, k_{\max }\right] \subset \mathbb{R}$, while a discretized version $\left\{k_{1}, k_{2}, \ldots, k_{M}\right\}$ of it (referred to as nominal temperatures) is used for data acquisition.

Global model specification The approach is based on Functionally Pooled Vector AutoRegressive (FPVAR) models, which can be thought of as generalizations of their conventional VAR counterparts, with the important distinction being that the AR parameter matrices and innovations covariance depend on the operating condition (presently temperature) - the former being explicitly expressed as a function of it. This 
distinct feature allows for the analytical modeling of temperature dependence on the structural dynamics (also see [38]). An FP-VAR model is of the form ${ }^{1}$ :

$$
\begin{gathered}
\boldsymbol{y}_{k}[t]+\sum_{i=1}^{n} \boldsymbol{A}_{i}(k) \boldsymbol{y}_{k}[t-i]=\boldsymbol{e}_{k}[t, \boldsymbol{\theta}] \\
E\left\{\boldsymbol{e}_{k}[t, \boldsymbol{\theta}] \boldsymbol{e}_{\ell}^{T}[t-\tau, \boldsymbol{\theta}]\right\}=\boldsymbol{\Sigma}_{k, \ell}(\boldsymbol{\theta}) \delta[\tau] \quad k, \ell \in \mathcal{R} \\
\boldsymbol{A}_{i}(k)=\sum_{j=1}^{p} \boldsymbol{A}_{i, j} G_{j}(k), \quad \boldsymbol{\theta}=\operatorname{vec}\left(\left[\boldsymbol{A}_{1,1} \boldsymbol{A}_{1,2} \ldots \boldsymbol{A}_{1, p} \vdots \ldots \vdots \boldsymbol{A}_{n, 1} \boldsymbol{A}_{n, 2} \ldots \boldsymbol{A}_{n, p}\right]^{T}\right)
\end{gathered}
$$

where $t$ is the normalized discrete time, and $n$ designates model order. $\boldsymbol{y}_{k}[t]$ is the $(n y \times 1)$ response vector corresponding to the $k$-th temperature condition (presently $n y=2$ ), whereas $\boldsymbol{e}_{k}[t, \boldsymbol{\theta}]$ is the corresponding $(n y \times 1)$ residual (one-step-ahead prediction error) vector. The residuals should be zero mean and uncorrelated with fully parametrized cross-covariance matrices $\boldsymbol{\Sigma}_{k, \ell}(\boldsymbol{\theta}) . E\{\cdot\}$ designates statistical expectation, and $\delta[\tau]$ the Kronecker delta.

The fully parametrized $(n y \times n y)$ AutoRegressive (AR) parameter matrices, $\boldsymbol{A}_{i}(k)$, are explicit functions of the temperature $k$, with their elements belonging to a functional subspace of dimensionality $p$ and spanned by the mutually independent functions $G_{j}(k)(j=1,2, \ldots, p)$. These functions form a functional basis, with $\boldsymbol{A}_{i, j}$ designating the corresponding, fully parametrized, projection matrices. The model is parametrized in terms of the $\left(n n y^{2} p \times 1\right)$ projection coefficient vector $\boldsymbol{\theta}$, comprising all elements of the projection matrices, whereas the $\operatorname{vec}(\cdot)$ operator transforms the indicated matrix into a vector by stacking its columns. The representation of Eq. (5.1a)-(5.1c) is referred to as an FP-VAR model of order $n$ and functional subspace dimensionality $p$, or in short as an $\operatorname{FP}-\operatorname{VAR}(n)_{[p]}$ model.

Pure FP-VAR models are used presently due to their convenient model structure and estimation simplicity, as linear least squares procedures may be employed effectively to obtain model parameters with optimal statistical accuracy (Chapter 2, Section 2.3). Nonetheless, more complicated FP models of the VARMA (AutoRegressive Moving Average) form may be used if necessary, at the cost of increased model complexity and computational burden (Chapter 4, Subsection 4.3).

Global model identification The FP-VAR model parameter estimation is based on the minimization of the following Weighted Least Squares (WLS) criterion (refer to Chapter 2, Section 2.3):

$$
J(\boldsymbol{\theta})=\frac{1}{N M} \sum_{t=1}^{N} \boldsymbol{e}^{T}[t, \boldsymbol{\theta}] \boldsymbol{\Sigma}^{-1} \boldsymbol{e}[t, \boldsymbol{\theta}]
$$

with $\boldsymbol{\Sigma}$ designating the $(M n y \times M n y)$ innovations covariance matrix, and $\boldsymbol{e}[t, \boldsymbol{\theta}]$ the $(M n y \times 1)$ residual vector which are defined as follows:

$$
\boldsymbol{\Sigma}=\left[\begin{array}{cccc}
\boldsymbol{\Sigma}_{k_{1}, k_{1}} & \boldsymbol{\Sigma}_{k_{1}, k_{2}} & \ldots & \boldsymbol{\Sigma}_{k_{1}, k_{M}} \\
\boldsymbol{\Sigma}_{k_{2}, k_{1}} & \boldsymbol{\Sigma}_{k_{2}, k_{2}} & \ldots & \boldsymbol{\Sigma}_{k_{2}, k_{M}} \\
\vdots & \vdots & \ddots & \vdots \\
\boldsymbol{\Sigma}_{k_{M}, k_{1}} & \boldsymbol{\Sigma}_{k_{M}, k_{2}} & \ldots & \boldsymbol{\Sigma}_{k_{M}, k_{M}}
\end{array}\right], \quad \boldsymbol{e}[t, \boldsymbol{\theta}]=\left[\begin{array}{c}
\boldsymbol{e}_{k_{1}}[t, \boldsymbol{\theta}] \\
\boldsymbol{e}_{k_{2}}[t, \boldsymbol{\theta}] \\
\vdots \\
\boldsymbol{e}_{k_{M}}[t, \boldsymbol{\theta}]
\end{array}\right]
$$

The WLS estimator of the FP-VAR projection coefficient vector, along with its corresponding covariance and the residual covariance are given $b y^{2}$ :

$$
\widehat{\boldsymbol{\theta}}_{W L S}=\left[\sum_{t=1}^{N} \boldsymbol{\Phi}[t] \widehat{\boldsymbol{\Sigma}}^{-1} \boldsymbol{\Phi}^{T}[t]\right]^{-1}\left[\sum_{t=1}^{N} \boldsymbol{\Phi}[t] \widehat{\boldsymbol{\Sigma}}^{-1} \boldsymbol{y}[t]\right]
$$

\footnotetext{
${ }^{1}$ Boldface upper/ lower case symbols designate matrix/ column-vector quantities, respectively.
}

${ }^{2}$ Symbols in hat designate estimators/estimates. 


$$
\begin{gathered}
\widehat{\boldsymbol{P}}\left(\widehat{\boldsymbol{\theta}}_{W L S}\right)=\left[\sum_{t=1}^{N} \boldsymbol{\Phi}[t] \widehat{\boldsymbol{\Sigma}}^{-1} \boldsymbol{\Phi}^{T}[t]\right]^{-1} \\
\widehat{\boldsymbol{\Sigma}}=\frac{1}{N} \sum_{t=1}^{N} \boldsymbol{e}\left[t, \widehat{\boldsymbol{\theta}}_{W L S}\right] \boldsymbol{e}^{T}\left[t, \widehat{\boldsymbol{\theta}}_{W L S}\right]
\end{gathered}
$$

where $\boldsymbol{P}(\cdot)$ designates the covariance matrix of the indicated vector and:

$$
\boldsymbol{y}[t]=\left[\begin{array}{c}
\boldsymbol{y}_{k_{1}}[t] \\
\boldsymbol{y}_{k_{2}}[t] \\
\vdots \\
\boldsymbol{y}_{k_{M}}[t]
\end{array}\right], \boldsymbol{\Phi}^{T}[t]=\left[\begin{array}{c}
\boldsymbol{\Phi}_{k_{1}}^{T}[t] \\
\boldsymbol{\Phi}_{k_{2}}^{T}[t] \\
\vdots \\
\boldsymbol{\Phi}_{k_{M}}^{T}[t]
\end{array}\right], \boldsymbol{\Phi}_{k}[t]=\boldsymbol{I}_{n y} \otimes\left(\left[\begin{array}{c}
-\boldsymbol{y}_{k}[t-1] \\
-\boldsymbol{y}_{k}[t-2] \\
\vdots \\
-\boldsymbol{y}_{k}[t-n]
\end{array}\right] \otimes\left[\begin{array}{c}
G_{1}(k) \\
G_{2}(k) \\
\vdots \\
G_{p}(k)
\end{array}\right]\right)
$$

with $\otimes$ designating the Kronecker product ${ }^{3}$.

Estimation of model parameters Once an FP-VAR model has been obtained, information on the AR parameter matrices for any temperature $k \in \mathcal{R}$ (nominal or non-nominal) is available through the following relationships, that associate the projection coefficient vector estimate $\widehat{\boldsymbol{\theta}}$ to the $\left(n n y^{2} \times 1\right)$ parameter vector $\widehat{\vartheta}(k)$ comprising all elements of the AR matrices corresponding to the $k$-th temperature condition (see also Eq. (5.1c)):

$$
\begin{gathered}
\widehat{\boldsymbol{\vartheta}}(k)=\boldsymbol{T}(k) \widehat{\boldsymbol{\theta}} \quad k \in \mathcal{R} \\
\boldsymbol{P}(\widehat{\boldsymbol{\vartheta}}(k))=\boldsymbol{T}(k) \boldsymbol{P}(\widehat{\boldsymbol{\theta}}) \boldsymbol{T}^{T}(k) \\
\boldsymbol{\vartheta}(k)=\operatorname{vec}\left(\left[\boldsymbol{A}_{1}(k) \boldsymbol{A}_{2}(k) \ldots \boldsymbol{A}_{n}(k)\right]^{T}\right)
\end{gathered}
$$

with $\boldsymbol{T}(k)$ designating a transformation matrix whose entries are zeros and basis functions $G_{j}(k)(j=$ $1, \ldots, p)$ at proper locations (see also Chapter 2, Appendix 2.A).

Estimation of modal parameters Once the information on the AR parameters has been obtained, modal information in the form of natural frequencies, damping factors and mode shapes is available [68]. A measure of modal parameter accuracy is provided by estimating their covariance matrix based on the corresponding discrete-time model parameter covariance and using the Gauss approximation formula (see for instance [1, p. 292] and [70]).

Let $\boldsymbol{\xi}(k)=\boldsymbol{f}(\boldsymbol{\vartheta}(k))$ represent the nonlinear functional relationship between the $(r \times 1)$ modal parameter vector $\boldsymbol{\xi}(k)$ and the $\left(n n y^{2} \times 1\right)$ dicrete-time model parameter vector $\boldsymbol{\vartheta}(k)$ at the $k$-th temperature condition. Linearizing this relationship using a first order Taylor series expansion yields for the corresponding covariance matrices of the estimators $\widehat{\boldsymbol{\xi}}(k)$ and $\widehat{\boldsymbol{\vartheta}}(k)$, respectively:

$$
\boldsymbol{P}(\widehat{\boldsymbol{\xi}}(k)) \approx \boldsymbol{J}(\widehat{\boldsymbol{\vartheta}}(k)) \boldsymbol{P}(\widehat{\boldsymbol{\vartheta}}(k)) \boldsymbol{J}^{T}(\widehat{\boldsymbol{\vartheta}}(k))
$$

where $\boldsymbol{J}(\widehat{\boldsymbol{\vartheta}}(k))=\frac{\partial \boldsymbol{f}\left(\hat{\boldsymbol{\vartheta}}_{(k))}\right.}{\partial \boldsymbol{\vartheta}^{T}(k)}$ is the $\left(r \times n n y^{2}\right)$ Jacobian matrix. Since the covariance matrix $\boldsymbol{P}(\widehat{\boldsymbol{\vartheta}}(k))$ in Eq. (5.5) is usually unavailable, its estimate $\widehat{\boldsymbol{P}}(\widehat{\boldsymbol{\vartheta}}(k))$ is used in practice. A simple way to obtain the Jacobian matrix is via numerical differentiation using the central difference approximation [70]. In this case, the $i$-th column of $\boldsymbol{J}(\widehat{\boldsymbol{\vartheta}}(k))$, designated as $\boldsymbol{j}_{i}(\widehat{\boldsymbol{\vartheta}}(k))$, is approximated as follows:

$$
\boldsymbol{j}_{i}(\widehat{\boldsymbol{\vartheta}}(k)) \approx \frac{\boldsymbol{f}(\widehat{\boldsymbol{\vartheta}}(k)+\boldsymbol{c})-\boldsymbol{f}(\widehat{\boldsymbol{\vartheta}}(k)-\boldsymbol{c})}{2 c_{i}}
$$

\footnotetext{
${ }^{3}$ The Kronecker product of an $(m \times n)$ matrix $\boldsymbol{A}=\left[a_{i, j}\right]$ and a $(p \times q)$ matrix $\boldsymbol{B}=\left[b_{i, j}\right]$ is $\boldsymbol{A} \otimes \boldsymbol{B}=\left[a_{i, j} \boldsymbol{B}\right]$ [43, p. 3].
} 
where $\boldsymbol{c}$ is an $\left(n n y^{2} \times 1\right)$ null vector save for the $i$-th element, designated as $c_{i}$, which holds a properly chosen value causing a small perturbation in the $i$-th element of $\widehat{\vartheta}(k)$.

Global model structure specification The estimation of the model order and the functional subspace dimensionality is achieved via a two-step scheme which aims at splitting the combined problem into two separate, easier to handle, subproblems. In the first step, the FP-VAR model order is specified by fitting on the complete data set auxiliary Pooled VAR (P-VAR) models of the form:

$$
\begin{gathered}
\boldsymbol{y}_{k}[t]+\sum_{i=1}^{n} \tilde{\boldsymbol{A}}_{i, k} \boldsymbol{y}_{k}[t-i]=\boldsymbol{e}_{k}[t, \tilde{\boldsymbol{\vartheta}}] \\
E\left\{\boldsymbol{e}_{k}[t, \tilde{\boldsymbol{\vartheta}}] \boldsymbol{e}_{\ell}^{T}[t-\tau, \tilde{\boldsymbol{\vartheta}}]\right\}=\boldsymbol{\Sigma}_{k, \ell}(\tilde{\boldsymbol{\vartheta}}) \delta[\tau] \quad k, \ell \in\left\{k_{1}, k_{2}, \ldots, k_{M}\right\} \\
\tilde{\boldsymbol{\vartheta}}=\operatorname{vec}\left(\left[\begin{array}{cccc}
\tilde{\boldsymbol{A}}_{1, k_{1}} & \tilde{\boldsymbol{A}}_{2, k_{1}} & \ldots & \tilde{\boldsymbol{A}}_{n, k_{1}} \\
\tilde{\boldsymbol{A}}_{1, k_{2}} & \tilde{\boldsymbol{A}}_{2, k_{2}} & \ldots & \tilde{\boldsymbol{A}}_{n, k_{2}} \\
\vdots & \vdots & \ddots & \vdots \\
\tilde{\boldsymbol{A}}_{1, k_{M}} & \tilde{\boldsymbol{A}}_{2, k_{M}} & \ldots & \tilde{\boldsymbol{A}}_{n, k_{M}}
\end{array}\right]^{T}\right)
\end{gathered}
$$

Notice that these models are not functionally pooled. Although the fully parametrized $(n y \times n y)$ AR parameter matrices $\tilde{\boldsymbol{A}}_{i, k}$ depend on the temperature $k$, they are not expressed as explicit functions of it, thus being of simpler structure and easier to identify. The auxiliary P-VAR models do not explicitly account for each temperature condition belonging in the range $\mathcal{R}$, but instead provide global model representations that are valid only for the nominal temperatures $k=k_{1}, k_{2}, \ldots, k_{M}$. The estimation of these models (that is, the estimation of the $\left(M n n y^{2} \times 1\right)$ parameter vector $\tilde{\vartheta}$ comprising all elements of the AR matrices) follows the same procedure as in the FP-VAR case (see also Chapter 4, Subsection 4.3.1 and Appendix 4.A).

Model adequacy is judged mainly using a properly adapted form of the Bayessian Information Criterion (BIC) (see Chapter 2, Section 2.6). Notice, however, that the number of estimated parameters for a $\mathrm{P}-\operatorname{VAR}(n)$ model is $d_{\mathcal{M}}=\operatorname{dim}(\tilde{\boldsymbol{\vartheta}})$, with $\operatorname{dim}(\cdot)$ designating the indicated vector dimensionality. Frequency stabilization diagrams $[68,81]$ depicting the estimated frequencies for increasing model order, for each nominal temperature $\left(k=k_{1}, k_{2}, \ldots, k_{M}\right)$ are also used.

The second step involves the specification of the functional subspace dimensionality $p$. Once the model order $n$ is specified and the basis function family is selected, a sensible approach consists of fitting $\operatorname{FP}-\operatorname{VAR}(n)_{[p]}$ models with increasing basis dimensionality $p$ on the data set. Typically, the subspace considered is spanned by consecutive basis functions $G_{j}(k)(j=1,2, \ldots, p$; complete functional subspace), nevertheless specific functions may be selected instead (incomplete functional subspace) using a search algorithm.

Model adequacy is judged as in the auxiliary P-VAR case via the BIC criterion (refer to Chapter 4, Subsection 4.3.1). Alternatively, frequency stabilization diagrams depicting the estimated frequencies as functions of the nominal temperatures $\left(k=k_{1}, k_{2}, \ldots, k_{M}\right)$ for increasing basis dimensionality $p$ are also used [38]. Adequacy is in this case is considered to be attained as soon as the estimated frequencies of each temperature condition converge.

The described two-step scheme is a useful alternative over a typical exhaustive search scheme, where for each specific $n(n=1,2, \ldots)$ FP-VAR models are fitted to the data for $p=1,2, \ldots(p<M)$. The use of the auxiliary P-VAR model is justified from a conceptual viewpoint, due to the fact that it is the same system that operates under different temperature conditions each time. The strength of the two-step scheme lies in the fact that the computational burden is significantly reduced even for small-size search spaces (refer to Appendix 5.A for details). 
Table 5.2: Summary of the two versions of the damage detection approach.

\begin{tabular}{lcc}
\hline \multicolumn{1}{c}{ Version } & Principle & Test statistic \\
\hline Modal parameter & $\widehat{\delta \boldsymbol{\xi}}\left(k_{\mathrm{u}}\right)=\widehat{\boldsymbol{\xi}}_{\mathrm{o}}\left(k_{\mathrm{u}}\right)-\widehat{\boldsymbol{\xi}}_{\mathrm{u}}\left(k_{\mathrm{u}}\right) \stackrel{?}{=} \mathbf{0}$ & $\chi_{\boldsymbol{\xi}}^{2}=\widehat{\delta \boldsymbol{\xi}}\left(k_{\mathrm{u}}\right)^{T}\left(\widehat{\boldsymbol{P}}\left(\widehat{\delta \boldsymbol{\xi}}\left(k_{\mathrm{u}}\right)\right)\right)^{-1} \widehat{\delta \boldsymbol{\xi}}\left(k_{\mathrm{u}}\right)$ \\
Model parameter & $\widehat{\delta \boldsymbol{\vartheta}}\left(k_{\mathrm{u}}\right)=\widehat{\boldsymbol{\vartheta}}_{\mathrm{o}}\left(k_{\mathrm{u}}\right)-\widehat{\boldsymbol{\vartheta}}_{\mathrm{u}}\left(k_{\mathrm{u}}\right) \stackrel{?}{=} \mathbf{0}$ & $\chi_{\boldsymbol{\vartheta}}^{2}=\widehat{\delta \boldsymbol{\vartheta}}\left(k_{\mathrm{u}}\right)^{T}\left(\widehat{\boldsymbol{P}}\left(\widehat{\delta \boldsymbol{\vartheta}}\left(k_{\mathrm{u}}\right)\right)\right)^{-1} \widehat{\delta \boldsymbol{\vartheta}}\left(k_{\mathrm{u}}\right)$ \\
\hline $\boldsymbol{\xi}_{\mathrm{o}}\left(k_{\mathrm{u}}\right):$ baseline (healthy) modal parameter vector at temp $k_{\mathrm{u}}$ & (FP-VAR model) \\
$\boldsymbol{\xi}_{\mathrm{u}}\left(k_{\mathrm{u}}\right):$ current (healthy or damaged) modal parameter vector at temp $k_{\mathrm{u}}$ & (VAR model) \\
$\boldsymbol{\vartheta}_{\mathrm{o}}\left(k_{\mathrm{u}}\right):$ baseline (healthy) model parameter vector at temp $k_{\mathrm{u}}$ & (FP-VAR model) \\
$\boldsymbol{\vartheta}_{\mathrm{u}}\left(k_{\mathrm{u}}\right):$ current (healthy or damaged) model parameter vector at temp $k_{\mathrm{u}}$ & (VAR model) \\
\hline
\end{tabular}

\subsubsection{Inspection phase}

The inspection phase is based on identifying conventional VAR models of the form [81]:

$$
\begin{gathered}
\boldsymbol{y}[t]+\sum_{i=1}^{n} \boldsymbol{A}_{i} \boldsymbol{y}[t-i]=\boldsymbol{e}[t, \boldsymbol{\vartheta}] \\
E\left\{\boldsymbol{e}[t, \boldsymbol{\vartheta}] \boldsymbol{e}^{T}[t-\tau, \boldsymbol{\vartheta}]\right\}=\boldsymbol{\Sigma}(\boldsymbol{\vartheta}) \delta[\tau], \quad \boldsymbol{\vartheta}=\operatorname{vec}\left(\left[\boldsymbol{A}_{1} \boldsymbol{A}_{2} \ldots \boldsymbol{A}_{n}\right]^{T}\right)
\end{gathered}
$$

with $\boldsymbol{y}[t]$ designating the $(n y \times 1)$ response vector, and $\boldsymbol{e}[t, \boldsymbol{\vartheta}]$ the $(n y \times 1)$ residual (one-step-ahead prediction error) vector that should be a zero mean uncorrelated sequence with a fully parametrized, positivedefinite, covariance matrix $\boldsymbol{\Sigma}(\boldsymbol{\vartheta})$. The $(n y \times n y)$ AR matrices are fully parametrized and designated as $\boldsymbol{A}_{i}$. The model is parametrized in terms of the parameter vector $\vartheta$, comprising all elements of the AR matrices. The representation of Eq. (5.8a)-(5.8b) is referred to as a VAR model of order $n$, or in short as a $\operatorname{VAR}(n)$ model.

Model estimation is based on the minimization of an Ordinary Least Squares (OLS) criterion [1, p. 206]. Once a model has been estimated, modal information in the form of natural frequencies, damping factors and mode shapes is readily obtained [68]. A measure of modal parameter accuracy is obtained via the Gauss approximation formula, as in the FP-VAR model case (see Subsection 5.3.1).

\subsubsection{The modal parameter-based version}

In this version the characteristic quantity $\boldsymbol{q}(k)$ postulated for damage detection is the VAR modal parameter vector, that is $\boldsymbol{q}(k)=\boldsymbol{\xi}(k)$ (see Table 5.2). Damage detection is accomplished by testing for statistically significant discrepancies between the vectors of the baseline (healthy) and current (healthy or damaged) state of the structure under the current temperature $k_{\mathrm{u}}, \boldsymbol{\xi}_{\mathrm{o}}\left(k_{\mathrm{u}}\right)$ and $\boldsymbol{\xi}_{\mathrm{u}}\left(k_{\mathrm{u}}\right)$, respectively. Notice that $\boldsymbol{\xi}_{\mathrm{o}}\left(k_{\mathrm{u}}\right)$ corresponds to the global FP-VAR model, whereas $\boldsymbol{\xi}_{\mathrm{u}}\left(k_{\mathrm{u}}\right)$ corresponds to the conventional VAR model obtained at temperature $k_{\mathrm{u}}$. The hypothesis testing problem considered is:

$$
\begin{array}{lll}
H_{0}: & \delta \boldsymbol{\xi}\left(k_{\mathrm{u}}\right)=\boldsymbol{\xi}_{\mathrm{o}}\left(k_{\mathrm{u}}\right)-\boldsymbol{\xi}_{\mathrm{u}}\left(k_{\mathrm{u}}\right)=\mathbf{0} & \text { (null hypothesis - healthy structure) } \\
H_{1}: & \delta \boldsymbol{\xi}\left(k_{\mathrm{u}}\right)=\boldsymbol{\xi}_{\mathrm{o}}\left(k_{\mathrm{u}}\right)-\boldsymbol{\xi}_{\mathrm{u}}\left(k_{\mathrm{u}}\right) \neq \mathbf{0} & \text { (alternative hypothesis - damaged structure) }
\end{array}
$$

with $H_{0}$ and $H_{1}$ designating the null and alternative hypothesis, respectively.

The modal parameter vector estimates are mutually independent random vectors following normal (Gaussian) distribution, that is $\widehat{\boldsymbol{\xi}}_{\mathrm{o}}\left(k_{\mathrm{u}}\right) \sim \mathcal{N}\left(\boldsymbol{\xi}_{\mathrm{o}}\left(k_{\mathrm{u}}\right), \boldsymbol{P}\left(\widehat{\boldsymbol{\xi}}_{\mathrm{o}}\left(k_{\mathrm{u}}\right)\right)\right)$ and $\widehat{\boldsymbol{\xi}}_{\mathrm{u}}\left(k_{\mathrm{u}}\right) \sim \mathcal{N}\left(\boldsymbol{\xi}_{\mathrm{u}}\left(k_{\mathrm{u}}\right), \boldsymbol{P}\left(\widehat{\boldsymbol{\xi}_{\mathrm{u}}}\left(k_{\mathrm{u}}\right)\right)\right)$, with $\mathcal{N}(\cdot, \cdot)$ designating the normal distribution with indicated mean and covariance. Owing to vector independence, the difference of the modal parameter vector estimates also follows normal distribution [82]:

$$
\widehat{\delta \boldsymbol{\xi}}\left(k_{\mathrm{u}}\right)=\widehat{\boldsymbol{\xi}}_{\mathrm{o}}\left(k_{\mathrm{u}}\right)-\widehat{\boldsymbol{\xi}}_{\mathrm{u}}\left(k_{\mathrm{u}}\right) \sim \mathcal{N}\left(\delta \boldsymbol{\xi}\left(k_{\mathrm{u}}\right), \boldsymbol{P}\left(\widehat{\delta \boldsymbol{\xi}}\left(k_{\mathrm{u}}\right)\right)\right)
$$


with $\delta \boldsymbol{\xi}\left(k_{\mathrm{u}}\right)=\boldsymbol{\xi}_{\mathrm{o}}\left(k_{\mathrm{u}}\right)-\boldsymbol{\xi}_{\mathrm{u}}\left(k_{\mathrm{u}}\right)$, and $\boldsymbol{P}\left(\widehat{\delta \boldsymbol{\xi}}\left(k_{\mathrm{u}}\right)\right)=\boldsymbol{P}\left(\widehat{\boldsymbol{\xi}_{\mathrm{o}}}\left(k_{\mathrm{u}}\right)\right)+\boldsymbol{P}\left(\widehat{\boldsymbol{\xi}_{\mathrm{u}}}\left(k_{\mathrm{u}}\right)\right)$. Notice that the global FP-VAR modal parameter covariance at any temperature is obtained from Eq. (5.4b) and Eq. (5.5).

Under $H_{0}, \widehat{\delta \boldsymbol{\xi}}\left(k_{\mathrm{u}}\right) \sim \mathcal{N}\left(\mathbf{0}, \boldsymbol{P}\left(\widehat{\delta \boldsymbol{\xi}}\left(k_{\mathrm{u}}\right)\right)\right)$, and the test statistic $\chi_{\boldsymbol{\xi}}^{2}=\widehat{\delta \boldsymbol{\xi}}\left(k_{\mathrm{u}}\right)^{T}\left(\boldsymbol{P}\left(\widehat{\delta \boldsymbol{\xi}}\left(k_{\mathrm{u}}\right)\right)\right)^{-1} \widehat{\delta \boldsymbol{\xi}}\left(k_{\mathrm{u}}\right)$ follows a $\chi^{2}$ (chi-square) distribution having $\operatorname{dim}\left(\delta \boldsymbol{\xi}\left(k_{\mathrm{u}}\right)\right)$ degrees of freedom [82]. Since the covariance $\boldsymbol{P}\left(\widehat{\delta \boldsymbol{\xi}}\left(k_{\mathrm{u}}\right)\right)$ is usually unavailable, its estimate $\widehat{\boldsymbol{P}}\left(\widehat{\delta \boldsymbol{\xi}}\left(k_{\mathrm{u}}\right)\right)$ is used in practice. Treating this sample covariance as a deterministic quantity, that is a quantity characterized by negligible variability (which is reasonable for large $N$ ), leads to the following test at the $\alpha$ risk level (that is, probability of accepting $H_{1}$ while $H_{0}$ is true equals $\alpha$ ) [82]:

$$
\begin{array}{ccc}
\chi_{\boldsymbol{\xi}}^{2}<\chi_{(1-\alpha)}^{2}\left(\operatorname{dim}\left(\delta \boldsymbol{\xi}\left(k_{\mathrm{u}}\right)\right)\right) & \Longrightarrow H_{0} \text { is accepted } \\
\text { Else } & \Longrightarrow H_{1} \text { is accepted }
\end{array}
$$

with $\chi_{(1-\alpha)}^{2}\left(\operatorname{dim}\left(\delta \boldsymbol{\xi}\left(k_{\mathrm{u}}\right)\right)\right)$ designating the distribution's $(1-\alpha)$ critical point (see Fig. 4.1).

Notice that in practical damage detection problems, involving structures with "high" modal density, the large number of modal parameters may pose a difficulty. In such cases, truncated versions of the modal parameter vector may prove beneficial (see Subsection 5.3.2.3).

\subsubsection{The model parameter-based version}

In this version, the characteristic quantity $\boldsymbol{q}(k)$ postulated for damage detection is the discrete-time VAR model parameter vector, that is $\boldsymbol{q}(k)=\boldsymbol{\vartheta}(k)$ (see Table 5.2). The choice is motivated from the fact that changes in physical parameters (for instance, stiffness or damping) due to damage affect the poles and mode shapes of the structural system, which in turn affect the discrete-time VAR model parameters. Damage detection is accomplished by testing for statistically significant discrepancies between the vectors of the baseline and current state of the structure under the current temperature $k_{\mathrm{u}}, \boldsymbol{\vartheta}_{\mathrm{o}}\left(k_{\mathrm{u}}\right)$ and $\boldsymbol{\vartheta}_{\mathrm{u}}\left(k_{\mathrm{u}}\right)$, respectively. Notice that $\boldsymbol{\vartheta}_{\mathrm{o}}\left(k_{\mathrm{u}}\right)$ corresponds to the global FP-VAR model, whereas $\boldsymbol{\vartheta}_{\mathrm{u}}\left(k_{\mathrm{u}}\right)$ corresponds to the conventional VAR model at temperature $k_{\mathrm{u}}$. The hypothesis testing problem considered is:

$$
\begin{array}{lll}
H_{0}: & \delta \boldsymbol{\vartheta}\left(k_{\mathrm{u}}\right)=\boldsymbol{\vartheta}_{\mathrm{o}}\left(k_{\mathrm{u}}\right)-\boldsymbol{\vartheta}_{\mathrm{u}}\left(k_{\mathrm{u}}\right)=\mathbf{0} & \text { (null hypothesis }- \text { healthy structure) } \\
H_{1}: & \delta \boldsymbol{\vartheta}\left(k_{\mathrm{u}}\right)=\boldsymbol{\vartheta}_{\mathrm{o}}\left(k_{\mathrm{u}}\right)-\boldsymbol{\vartheta}_{\mathrm{u}}\left(k_{\mathrm{u}}\right) \neq \mathbf{0} & \text { (alternative hypothesis - damaged structure) }
\end{array}
$$

It may be shown (under mild assumptions) that for sufficiently long signals ( $N$ large) the model parameter vector estimates follow normal distribution [1, p. 282], that is $\widehat{\boldsymbol{\vartheta}}_{\mathrm{o}}\left(k_{\mathrm{u}}\right) \sim \mathcal{N}\left(\boldsymbol{\vartheta}_{\mathrm{o}}\left(k_{\mathrm{u}}\right), \boldsymbol{P}\left(\widehat{\boldsymbol{\vartheta}}_{\mathrm{o}}\left(k_{\mathrm{u}}\right)\right)\right)$ and $\widehat{\boldsymbol{\vartheta}}_{\mathrm{u}}\left(k_{\mathrm{u}}\right) \sim \mathcal{N}\left(\boldsymbol{\vartheta}_{\mathrm{u}}\left(k_{\mathrm{u}}\right), \boldsymbol{P}\left(\widehat{\boldsymbol{\vartheta}_{\mathrm{u}}}\left(k_{\mathrm{u}}\right)\right)\right)$, thereby [18]:

$$
\widehat{\delta \boldsymbol{\vartheta}}\left(k_{\mathrm{u}}\right)=\widehat{\boldsymbol{\vartheta}}_{\mathrm{o}}\left(k_{\mathrm{u}}\right)-\widehat{\boldsymbol{\vartheta}}_{\mathrm{u}}\left(k_{\mathrm{u}}\right) \sim \mathcal{N}\left(\delta \boldsymbol{\vartheta}\left(k_{\mathrm{u}}\right), \boldsymbol{P}\left(\widehat{\delta \boldsymbol{\vartheta}}\left(k_{\mathrm{u}}\right)\right)\right)
$$

with $\delta \boldsymbol{\vartheta}\left(k_{\mathrm{u}}\right)=\boldsymbol{\vartheta}_{\mathrm{o}}\left(k_{\mathrm{u}}\right)-\boldsymbol{\vartheta}_{\mathrm{u}}\left(k_{\mathrm{u}}\right)$, and $\boldsymbol{P}\left(\widehat{\delta \boldsymbol{\vartheta}}\left(k_{\mathrm{u}}\right)\right)=\boldsymbol{P}\left(\widehat{\boldsymbol{\vartheta}_{\mathrm{o}}}\left(k_{\mathrm{u}}\right)\right)+\boldsymbol{P}\left(\widehat{\boldsymbol{\vartheta}_{\mathrm{u}}}\left(k_{\mathrm{u}}\right)\right)$. Note that the global FP-VAR model parameter covariance at any temperature is obtained from Eq. (5.4b).

Under $H_{0}, \widehat{\delta \boldsymbol{\vartheta}}\left(k_{\mathrm{u}}\right) \sim \mathcal{N}\left(\mathbf{0}, \boldsymbol{P}\left(\widehat{\delta \boldsymbol{\vartheta}}\left(k_{\mathrm{u}}\right)\right)\right)$, and the test statistic $\chi_{\boldsymbol{\vartheta}}^{2}=\widehat{\delta \boldsymbol{\vartheta}}\left(k_{\mathrm{u}}\right)^{T}\left(\boldsymbol{P}\left(\widehat{\delta \boldsymbol{\vartheta}}\left(k_{\mathrm{u}}\right)\right)\right)^{-1} \widehat{\delta \boldsymbol{\vartheta}}\left(k_{\mathrm{u}}\right)$ follows a $\chi^{2}$ (chi-square) distribution having $\operatorname{dim}\left(\delta \boldsymbol{\vartheta}\left(k_{\mathrm{u}}\right)\right)$ degrees of freedom [18]. Since the covariance $\boldsymbol{P}\left(\widehat{\delta \boldsymbol{\vartheta}}\left(k_{\mathrm{u}}\right)\right)$ is usually unavailable, its estimate $\widehat{\boldsymbol{P}}\left(\widehat{\delta \boldsymbol{\vartheta}}\left(k_{\mathrm{u}}\right)\right)$ is used in practice, thus leading to the following test at the $\alpha$ risk level [16]:

$$
\begin{array}{ccc}
\chi_{\boldsymbol{\vartheta}}^{2}<\chi_{(1-\alpha)}^{2}\left(\operatorname{dim}\left(\delta \boldsymbol{\vartheta}\left(k_{\mathrm{u}}\right)\right)\right) & \Longrightarrow & H_{0} \text { is accepted } \\
\text { Else } & \Longrightarrow & H_{1} \text { is accepted }
\end{array}
$$


which is in analogy to the modal parameter-based version.

Overall, the model parameter-based version requires very little in terms of model processing and physical insight, as opposed to its modal parameter-based counterpart. Notice however, that the (often) large number of parameters required to identify a proper model of the structural dynamics poses a difficulty. For this reason truncated versions of the discrete-time model parameter vector may prove beneficial (see Subsection 5.3.2.3).

\subsubsection{Truncation of the modal/model parameter vectors}

Truncated versions of the characteristic quantity $\boldsymbol{q}(k)$ (modal or model parameters) may be also used in an effort to simplify and improve the performance of the damage detection procedure. In such cases the truncated characteristic quantity vector may be arbitrarily selected or based upon a systematic procedure, depending on the specific problem addressed.

An arbitrary selection may contain (i) modal parameters from specific damage-sensitive modes, (ii) the structure's natural frequencies (or damping factors, mode shapes) only, or (iii) damage-sensitive model parameters. Damage detection then follows the same procedure as in the modal or model parameter versions described previously. Such an approach proves beneficial when the dimensionality of the characteristic quantity vector $\boldsymbol{q}(k)$ is relatively small, or knowledge on the damage-sensitive features is available.

In order to address the shortcomings associated with the arbitrary truncation of the characteristic quantity vector $\boldsymbol{q}(k)$, a systematic procedure, based on Principal Component Analysis (PCA) [83, pp. 215-249] may be preferred. The idea is to project the baseline (healthy) characteristic quantity vector $\boldsymbol{q}_{\mathrm{o}}(k)$ onto a coordinate system where information compression is possible. A subspace of lower dimensionality, without sacrificing significant information, is subsequently selected, and the discrepancy between the baseline (healthy) and the current (healthy or damaged) vectors $\delta \boldsymbol{q}(k)$ is projected on the same subspace.

Obviously, a different subspace corresponds to each temperature, and in order to circumvent this problem the following procedure is adopted: PCA is employed on the FP-VAR based characteristic quantity estimates $\widehat{\boldsymbol{q}_{\mathrm{o}}}(k)$ belonging to the nominal temperatures $\left(k=k_{1}, k_{2}, \ldots, k_{M}\right)$ of the baseline phase (healthy structure). The objective is to diagonalize the corresponding covariance matrices via Singular Value Decomposition (SVD) as follows:

$$
\boldsymbol{P}\left(\widehat{\boldsymbol{q}_{\mathrm{o}}}(k)\right)=\boldsymbol{U}(k) \boldsymbol{S}(k) \boldsymbol{U}^{T}(k), \quad k=k_{1}, k_{2}, \ldots, k_{M}
$$

with:

$$
\begin{gathered}
\boldsymbol{S}(k)=\left[\begin{array}{cccc}
s_{1}(k) & 0 & \ldots & 0 \\
0 & s_{2}(k) & \ldots & 0 \\
\vdots & \vdots & \ddots & \vdots \\
0 & 0 & \ldots & s_{n n y^{2}}(k)
\end{array}\right] \\
\boldsymbol{U}(k)=\left[\begin{array}{llll}
\boldsymbol{u}_{1}(k) & \boldsymbol{u}_{2}(k) & \ldots & \boldsymbol{u}_{n n y^{2}}(k)
\end{array}\right]
\end{gathered}
$$

where $\boldsymbol{U}(k)$ is an orthonormal matrix (that is, $\boldsymbol{U}(k) \boldsymbol{U}^{T}(k)=\boldsymbol{I}_{n n y^{2}}$ ) the columns of which define the principal components, and form a subspace spanning the vector $\boldsymbol{q}_{\mathrm{o}}(k)$. The singular values $s_{j}(k)(j=$ $\left.1,2, \ldots, n n y^{2}\right)$ are ranked in decreasing order, and represent the active energy of the associated principal components.

The subspace dimensionality selection is then based on bounding the information loss, expressed in terms of active energy contribution, below a certain threshold. A measure of how well the first $m$ principal 
components explain the variance of $\widehat{\boldsymbol{q}_{\mathrm{o}}}(k)$ is given by the relative proportion:

$$
\psi_{m}=\frac{\sum_{j=1}^{m} s_{j}(k)}{\sum_{j=1}^{n n y^{2}} s_{j}(k)} 100(\%)
$$

Selecting only the first $m$ coordinates $\left\{\boldsymbol{u}_{1}(k), \boldsymbol{u}_{2}(k), \ldots, \boldsymbol{u}_{m}(k)\right\}$ in Eq. (5.15c) determines an $m-$ dimensional subspace, and the projection of $\widehat{\boldsymbol{q}}_{\mathrm{o}}(k)$ on this subspace (principal component transformation), is given by:

$$
\widehat{\boldsymbol{q}_{\mathrm{o}}^{P C A}}(k)=\boldsymbol{U}_{m}^{T}(k) \widehat{\boldsymbol{q}_{\mathrm{o}}}(k), \quad k=k_{1}, k_{2}, \ldots, k_{M}
$$

with:

$$
\begin{gathered}
\boldsymbol{U}_{m}(k)=\left[\begin{array}{llll}
\boldsymbol{u}_{1}(k) & \boldsymbol{u}_{2}(k) & \ldots & \boldsymbol{u}_{m}(k)
\end{array}\right] \\
\boldsymbol{P}\left(\widehat{\boldsymbol{q}_{\mathrm{o}}^{P C A}}(k)\right)=\boldsymbol{U}_{m}^{T}(k) \boldsymbol{P}\left(\widehat{\boldsymbol{q}_{\mathrm{o}}}(k)\right) \boldsymbol{U}_{m}(k)=\left[\begin{array}{cccc}
s_{1}(k) & 0 & \ldots & 0 \\
0 & s_{2}(k) & \ldots & 0 \\
\vdots & \vdots & \ddots & \vdots \\
0 & 0 & \ldots & s_{m}(k)
\end{array}\right]
\end{gathered}
$$

Notice that the above procedure yields a set of $M$ principal component transformation matrices $\boldsymbol{U}_{m}(k)$ - one for each nominal temperature $k=k_{1}, k_{2}, \ldots, k_{M}$. This implies that the truncation of the characteristic quantity vector $\boldsymbol{q}(k)$ at any temperature (nominal or non-nominal) needs to be based upon a proper transformation matrix. The following choices may be made:

(i) A "mean" transformation matrix, valid for any arbitrary temperature $k_{\mathrm{u}}$, which is determined by averaging the matrices $\boldsymbol{U}_{m}(k)$ corresponding to the nominal temperatures $\left(k=k_{1}, k_{2}, \ldots, k_{M}\right)$, that is:

$$
\boldsymbol{U}_{\text {mean }}=\frac{1}{M} \sum_{k=k_{1}}^{k_{M}} \boldsymbol{U}_{m}(k)
$$

(ii) An "interpolated" transformation matrix, capable of accounting for the mismatch between an arbitrary temperature $k_{\mathrm{u}}$ and the nominal temperatures $\left\{k_{1}, k_{2}, \ldots, k_{M}\right\}$. The matrix corresponding to the arbitrary temperature $k_{\mathrm{u}} \in\left[k_{i}, k_{i+1}\right]$, with $i=1,2, \ldots,(M-1)$, is determined via linear interpolation of $\boldsymbol{U}_{m}\left(k_{i}\right)$ and $\boldsymbol{U}_{m}\left(k_{i+1}\right)$, as follows [34]:

$$
\boldsymbol{U}_{m}\left(k_{\mathrm{u}}\right)=\lambda \boldsymbol{U}_{m}\left(k_{i+1}\right)+(1-\lambda) \boldsymbol{U}_{m}\left(k_{i}\right) \text { and } \lambda=\frac{k_{\mathrm{u}}-k_{i}}{k_{i+1}-k_{i}}
$$

Once the transformation matrix (either $\boldsymbol{U}_{\text {mean }}$ or $\boldsymbol{U}_{m}\left(k_{\mathrm{u}}\right)$ ) has been computed, the discrepancy between the baseline (healthy) and the current (healthy or damaged) vector estimates for any arbitrary temperature $\widehat{\delta \boldsymbol{q}}\left(k_{\mathrm{u}}\right)$ is transformed accordingly. Damage detection then follows the same procedure as in the modal or model parameter versions described previously.

Notice that the covariance of the characteristic quantity $\boldsymbol{P}\left(\widehat{\boldsymbol{q}_{\mathrm{o}}}(k)\right)$ is usually unavailable, therefore its estimate $\widehat{\boldsymbol{P}}\left(\widehat{\boldsymbol{q}_{\mathrm{o}}}(k)\right)$ is used in practice to obtain $\boldsymbol{U}(k)$ (see Eq. $(5.15 \mathrm{a})-(5.15 \mathrm{c})$ ).

Remark: A critical requirement for the approach to work properly is - quite naturally - that the global and conventional models should be in overall agreement. Significant discrepancies between the characteristic quantities estimated by the FP-VAR and the conventional VAR models for the healthy structure may compromise the performance of the statistical tests in Eq. (5.9) and Eq. (5.12) leading to false alarms. To remedy this situation, the use of excessively low risk level $\alpha$ values would be required in order to set proper statistical limits, at the cost of potential missed detections. 


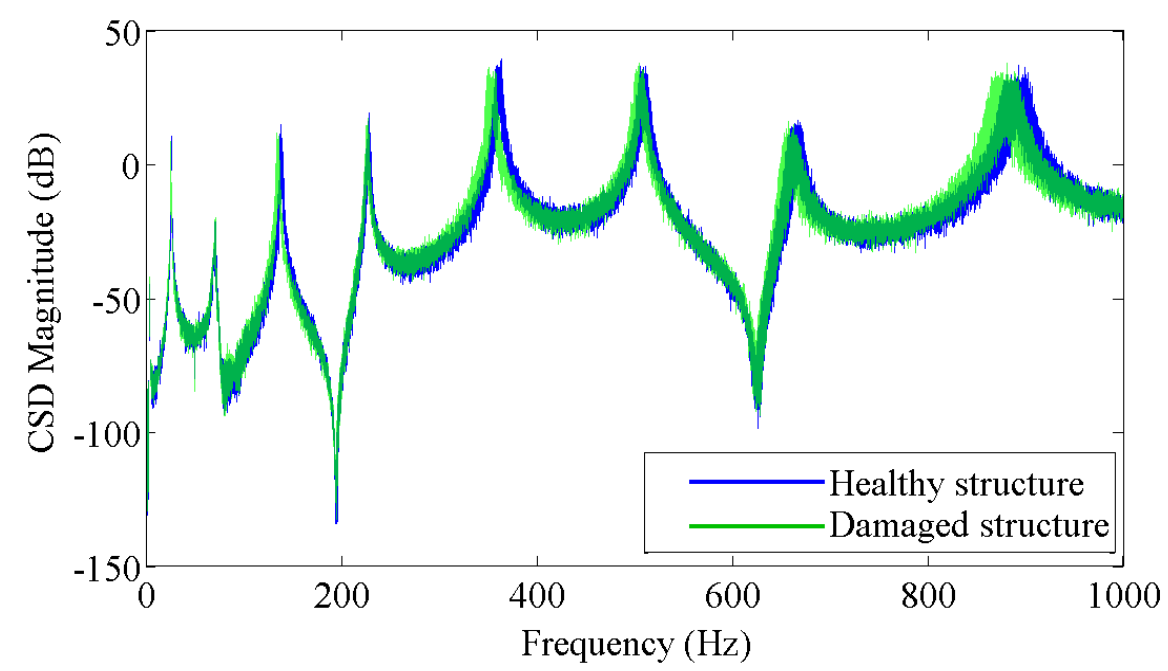

Figure 5.3: Welch-based cross-spectral density (CSD) magnitude estimates at the $M=21$ nominal temperatures belonging to the $[-20,+20]^{\circ} \mathrm{C}$ range: ( - dark blue zone) healthy structure, and ( - transparent light green zone) damaged structure (each zone comprises $M=21$ estimates, that is one CSD curve per each nominal temperature).

\subsection{Experimental damage detection results and assessment}

The effect of temperature on the healthy and the damaged structural dynamics is depicted in Fig. 5.3. Estimates of the Welch-based Cross-Spectral Density (CSD) magnitudes (see [63, pp. 76-77], and [67, MATLAB ${ }^{\circledR}$ function “csd.m"]) are computed for the healthy and the damaged structure at the $M=21$ nominal temperatures (belonging to the $[-20,+20]^{\circ} \mathrm{C}$ range and obtained at $2^{\circ} \mathrm{C}$ increments; see also Table 5.3) based on a single data record at each temperature (data length $N=70,000$ samples, Hanning data segment window length $=10,240$ samples, data segment overlap $=80 \%$, sampling frequency $f_{\mathrm{s}}=2,560 \mathrm{~Hz}$, frequency resolution $\Delta f=0.25 \mathrm{~Hz}$ ). The obtained CSD curves indicate the presence of eight modes within the $[0,1,000] \mathrm{Hz}$ frequency range. The temperature effects are more evident at higher frequencies, with the corresponding modes exhibiting higher temperature dependence. The significant amount of overlapping observed in the healthy and damaged structure CSDs suggests that the effects of damage can be masked by structural changes due to temperature. Similar comments hold for the corresponding Similar comments hold for the corresponding Welch-based Power Spectral Densities (PSDs) shown in Appendix 5.B.

\subsubsection{The stochastic global model based approach}

\subsubsection{Baseline phase}

The FP-VAR modeling procedure uses $N=5,000$ sample-long $(\approx 1.95 \mathrm{~s})$ data records from $M=21$ nominal temperatures (see Table 5.3) to identify a model valid within the $[-20,+20]^{\circ} \mathrm{C}$ range. The AR parameters are projected into a functional subspace spanned by Chebyshev polynomials of the second kind [45, p. 782]. These polynomials are orthogonal over the interval $x \in[-1,+1] \subset \mathbb{R}$ and obey the following recurrence relation:

$$
G_{j+1}(x)=2 x G_{j}(x)-G_{j-1}(x), \text { with } G_{1}(x)=1, G_{2}(x)=2 x .
$$

In the present application $k \in[-20,+20]^{\circ} \mathrm{C}$, therefore the following substitution is necessary, $x=k /(M-$ 1 ), since $k$ is discretized at increments $\Delta k=2^{\circ} C$ ( $M=21$ experiments), thus yielding $k_{1}=-20^{\circ} C$, 

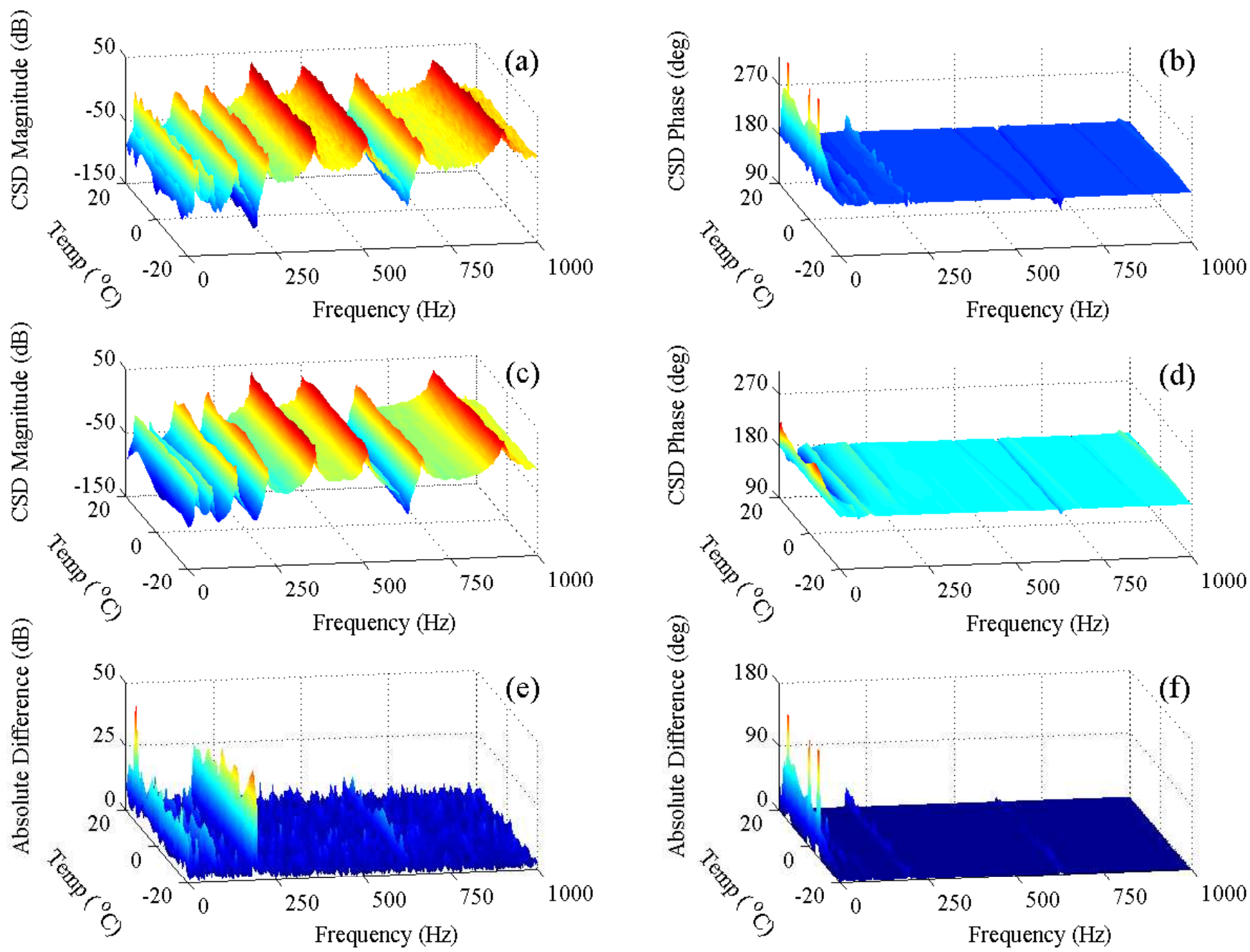

Figure 5.5: Cross-spectral density (CSD) for the healthy beam versus frequency and temperature: (a),(b) Welch-based estimate; (c),(d) FP-VAR(88) ${ }_{[7]}$-based estimate; (e),(f) absolute differences.

der equal to $n=88$ is adequate. A succession of $\operatorname{FP}-\operatorname{VAR}(88)_{[p]}$ models with increasing dimensionality $p=2,3, \ldots, 10$ are subsequently fitted to the data based upon non-iterative WLS estimation (QR implementation; Chapter 2, Appendix 2.E). The corresponding BIC plot shown in Fig. 5.4(b), suggests that an FP-VAR $(88)_{[7]}$ model with a functional subspace spanned by the first seven Chebyshev polynomials is appropriate. The identified FP-VAR(88) ${ }_{[7]}$ model has 2, 464 parameters, yielding an SPP (Samples Per Parameter, that is the ratio of all response signal samples over the number of estimated parameters) value of 85.2 , and condition number $\sim 10^{5}$ for the WLS estimator inverted matrix (see Table 5.3 for details). Notice that the use of the proposed two-step scheme is advantageous in terms of computational complexity as the total number of estimated parameters in the present study is 439,428 , whereas for an exhaustive search the corresponding number would be $1,081,080$. The reduction of estimated parameters is substantial, reaching $59.4 \%$ (see also Appendix 5.A).

The close resemblance of the Welch-based and the FP-VAR $(88)_{[7]}$ model based CSD estimates in Fig. 5.5 builds further confidence on the results, and indicates that the FP-VAR modeling procedure provides an accurate and valid, under all considered temperatures, representation of the dynamics.

Notice that the CSD phase in Fig. 5.5(b),(d) is around $180^{\circ}$, due to the fact that the response signals $y_{1}[t]$ and $y_{2}[t]$ are obtained from collocated sensors (see Fig. 5.1(b)). A potential problem associated with this situation is multicollinearity, which occurs when the obtained signals are linearly dependent, and introduces 

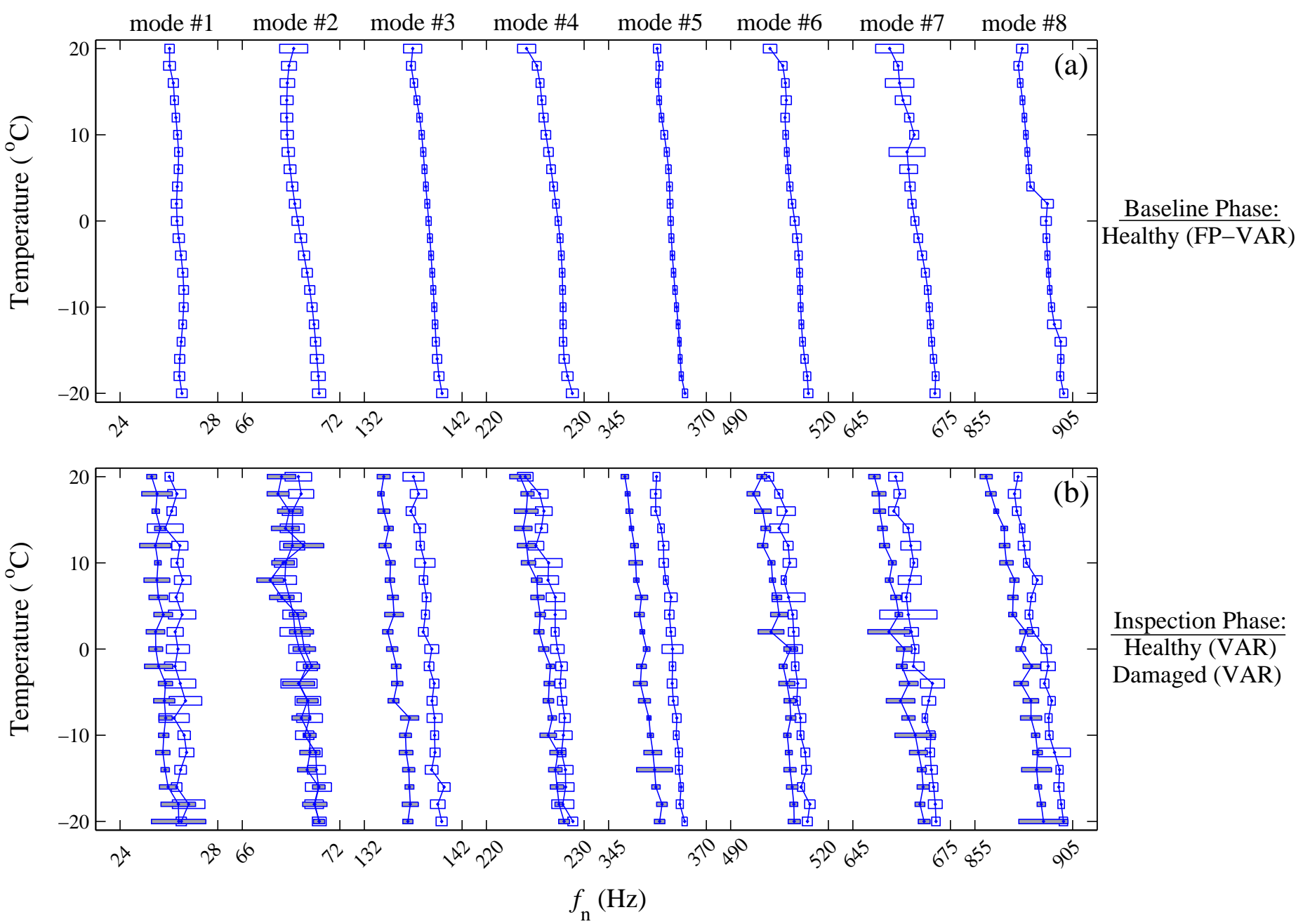

Figure 5.6: Natural frequency $\left(f_{\mathrm{n}}\right)$ point estimates and their estimated uncertainties ( \pm two standard deviations) at the $M=21$ nominal temperatures: (a) baseline phase (healthy structure) FP-VAR $(88)_{[7]}$ estimates, (b) inspection phase (healthy and damaged structure) VAR(88) estimates (the uncertainties are designated by white boxes for the healthy structure and gray boxes for the damaged).

singularities into the model estimation procedure. In the present study this is not an issue, as the FP-VAR and conventional VAR model based LS inverted matrices are well-conditioned (see Table 5.3). Nevertheless, for the sake of completeness, the use of PCA-based diagnostic procedures to detect linear dependencies in the data $[84,85]$ are also employed, and the obtained results confirm that there are no redundant component signals in $\boldsymbol{y}_{k}[t]$ (refer to Appendix 5.B.3 for details).

Figure 5.6(a) presents the natural frequency point estimates and their estimated uncertainties $( \pm$ two standard deviation zones) obtained via the Gauss approximation formula (see subsection 5.3.1 and Table 5.3) for the healthy structure. Only results from the $M=21$ nominal temperatures are displayed, allowing for a temperature resolution of $\Delta k=2^{\circ} C$. The natural frequencies exhibit a decreasing trend with increasing temperature, whereas the dependence on temperature is more pronounced for increasing mode number (slopes ranging from $0.01 \mathrm{~Hz} /{ }^{\circ} \mathrm{C}$ for mode 1 to $0.53 \mathrm{~Hz} /{ }^{\circ} \mathrm{C}$ for mode 8). On the contrary, damping factors (see Appendix 5.B.1) exhibit a weak dependence on temperature. The results are in qualitative agreement with those in Chapter 3, where the same structure with a different sensor configuration is considered within an alternative frequency range. Additional modeling results are presented in Appendix 5.B.1.

Note that the numerical differentiation procedure used to estimate modal parameter uncertainty is 
a possible source of errors. Nevertheless, the alternative use of analytical differentiation [70] is not recommended as it would require the inversion of the parameter vector estimator covariance matrix which is presently characterized by large condition numbers (orders of magnitude between $10^{10}$ and $10^{11}$ - see Table 5.3). Also, the selection of proper perturbation terms for the AR parameters requires a trial-and-error procedure. Presently, the perturbation term $c_{i}$ in Eq. (5.6) is a fraction of the corresponding AR parameter's standard deviation (see Table 5.3).

\subsubsection{Inspection phase (damage detection)}

The testing procedure involves $M_{1}=180$ experiments for the healthy structure and another $M_{2}=188$ experiments for the damaged structure, covering the entire $[-20,+20]^{\circ} \mathrm{C}$ temperature range (see Table 5.3). Notice that in each structural state (healthy/damaged), only 21 of the experiments correspond to the nominal temperatures. For reasons of direct comparison with the FP-VAR $(88)_{[7]}$ model, same order conventional VAR models are identified for each test case using $N=5,000$ sample-long data records. Parameter estimation is based upon Ordinary Least Squares (OLS) with QR implementation (see [1, pp. 318-320] and [47, MATLAB ${ }^{\circledR}$ function "arx.m"]). Each of the identified VAR( 88$)$ models has 352 parameters, yielding an SPP equal to 28.4, and condition number $\sim 10^{5}$ for the OLS estimator inverted matrix (see Table 5.3 for details).

Fig. 5.6(b) presents the healthy and damaged structure natural frequency point estimates and their estimated uncertainties at the $M=21$ nominal temperatures (obtained via conventional VAR modeling). Very good agreement is observed for the FP-VAR and conventional VAR modeling approaches (compare Fig. 5.6(a),(b)).

The modal parameter-based version The comparison of the healthy and damaged structure natural frequencies of Fig. 5.6(b) indicates that damage shifts frequencies downwards (changes $<4 \%$ ). The damage effects are more evident at higher modes, as changes between the healthy and damaged structure become more significant. On the contrary, the influence of damage on the damping factors is very weak, as both structural states exhibit overlapping confidence intervals at all temperatures (refer to Appendix 5.B.2). The preceding observations suggest that natural frequencies alone could be employed in the statistical test of Eq. (5.11) - in principle mode shape information may be of course also used, but is not presently considered due to the limited number of response sensors $(n y=2)$.

Damage detection results for all $M_{1}=180$ and $M_{2}=188$ inspection experiments are presented in Fig. 5.7. The performance is excellent at all temperatures, nominal and non-nominal, since all healthy and damaged test cases are clearly separated. Indeed, the $\chi_{\boldsymbol{\xi}}^{2}$ statistic values (see Eq. (5.11)) corresponding to damage are well above the threshold defined by the $\alpha=10^{-3}$ risk level (note the logarithmic scale on the vertical axis). The results confirm that the natural frequencies are good damage indicators combining effectiveness and robustness, as no missed damage or false alarms are observed in the considered temperature range.

The model parameter-based version Due to the large number of AR parameters (see Table 5.3), truncated versions of the model parameter vector are used for damage detection, along with the complete model parameter vector. In order to obtain the truncated versions, PCA is performed on each of the FP-VAR $(88)_{[7]}$ model parameter vector estimates corresponding to the $M=21$ nominal temperatures. A common subspace dimensionality $m$ for all model parameter vectors is determined by setting a threshold value equal to $99.5 \%$ to the criterion $\psi_{m}$ in Eq. (5.16). This leads to a substantial dimensionality reduction of the transformed discrete-time parameter vectors from 352 to 97 , as shown in Fig. 5.8.

The damage detection results for all $M_{1}=180$ and $M_{2}=188$ inspection experiments are presented 


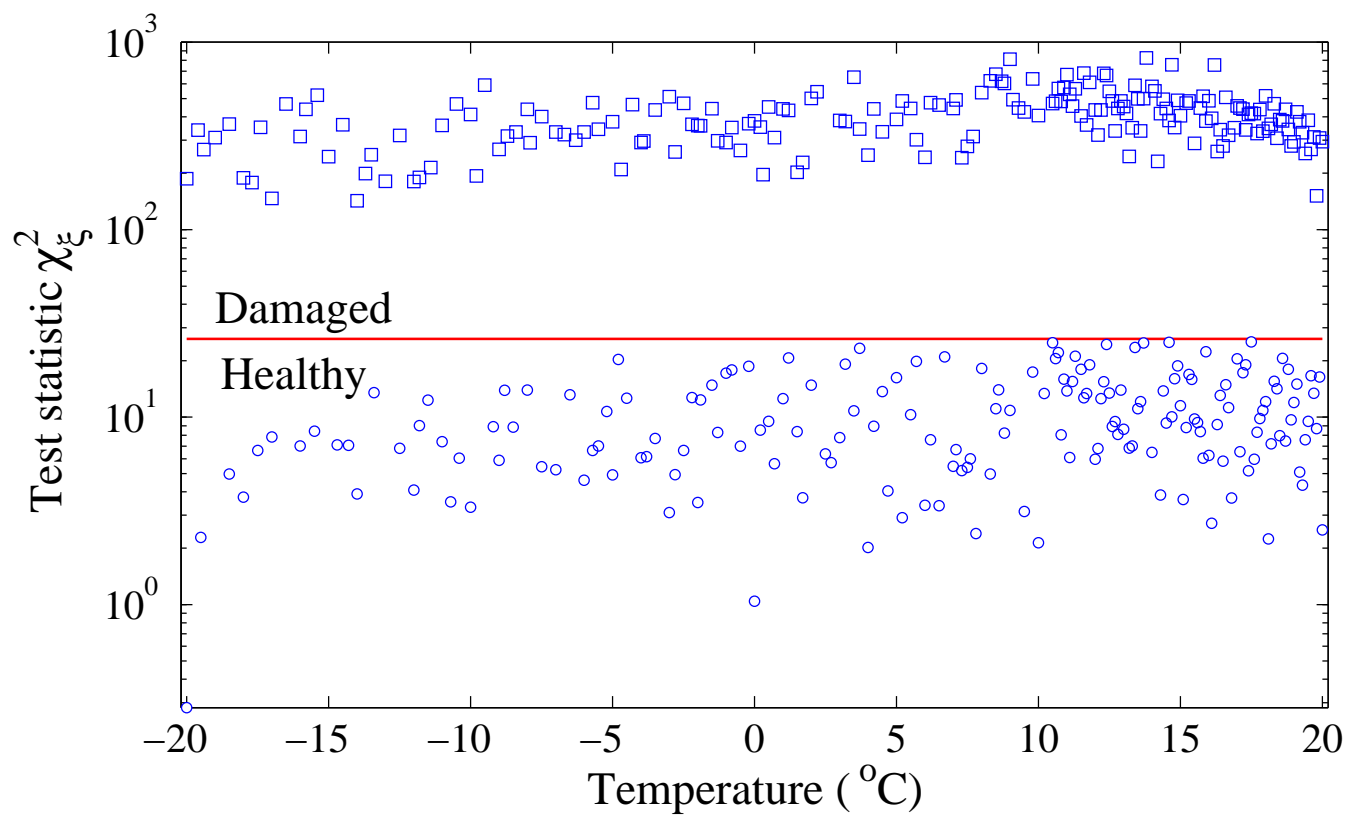

Figure 5.7: Modal parameter-based damage detection results: $\chi_{\boldsymbol{\xi}}^{2}$ statistic for healthy ( $\left.\circ\right)$ and damaged ( $\square$ ) test cases at different temperatures (the horizontal line designates the statistical limit at the $\alpha=10^{-3}$ risk level; damage is detected when $\chi_{\boldsymbol{\xi}}^{2}$ lies above the limit; $M_{1}=180$ healthy and $M_{2}=188$ damaged cases).

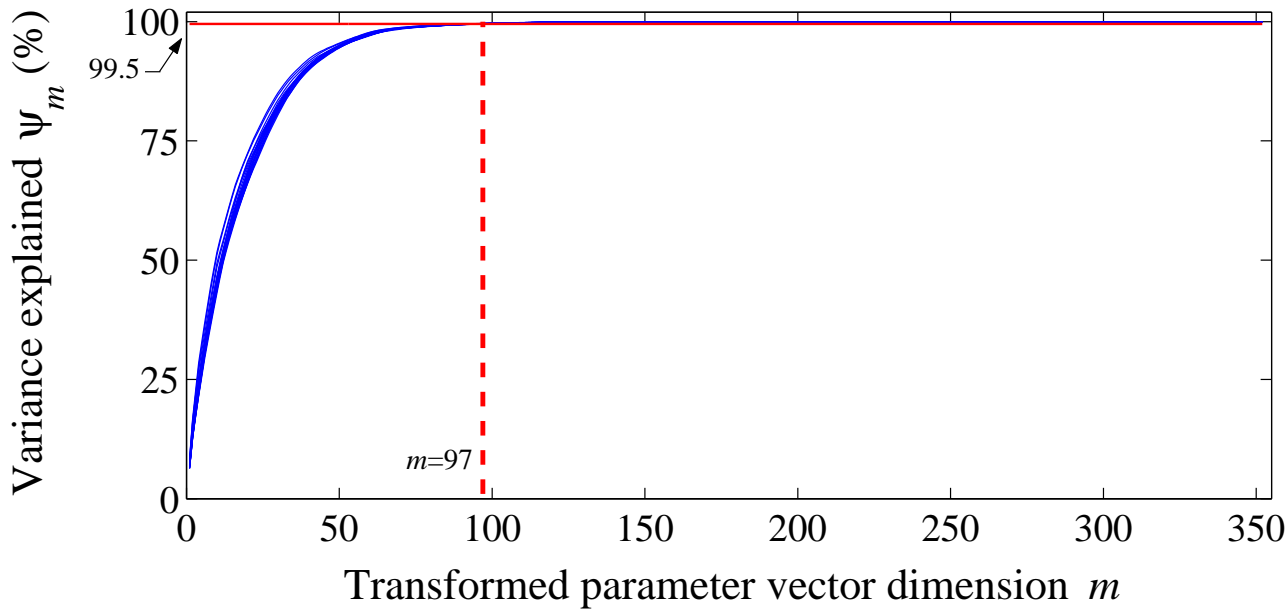

Figure 5.8: Relative proportion of variance explained $\left(\psi_{m}\right)$ for the transformed FP-VAR $(88)_{[7]}$ model parameter vector estimates versus vector dimension $(m)$ at the nominal temperatures (baseline phase, $M=21$ curves; the vertical dashed line indicates the selected dimensionality; the horizontal line indicates the threshold value of variance explained).

in Fig. 5.9. The $\chi_{\vartheta}^{2}$ statistic values (see Eq. (5.14)) based on the complete parameter vector are depicted in Fig. 5.9(a), whereas the PCA-based truncated versions are depicted in Fig. 5.9(b),(c). All healthy and damaged test cases are well separated in the temperature range considered, with the corresponding $\chi_{\vartheta}^{2}$ values lying in separate zones (note the logarithmic scale on the vertical axis). It appears, however, from Fig. 5.9 that setting an analytical damage detection threshold would require extremely low values of the risk level $\alpha$. A possible reason for this is the insufficient modeling of uncertainties. In such cases it is customary to use an empirical threshold (for instance see $[86,87]$ ).

The test statistic based on truncating the parameter vector via the "mean" transformation matrix seems 

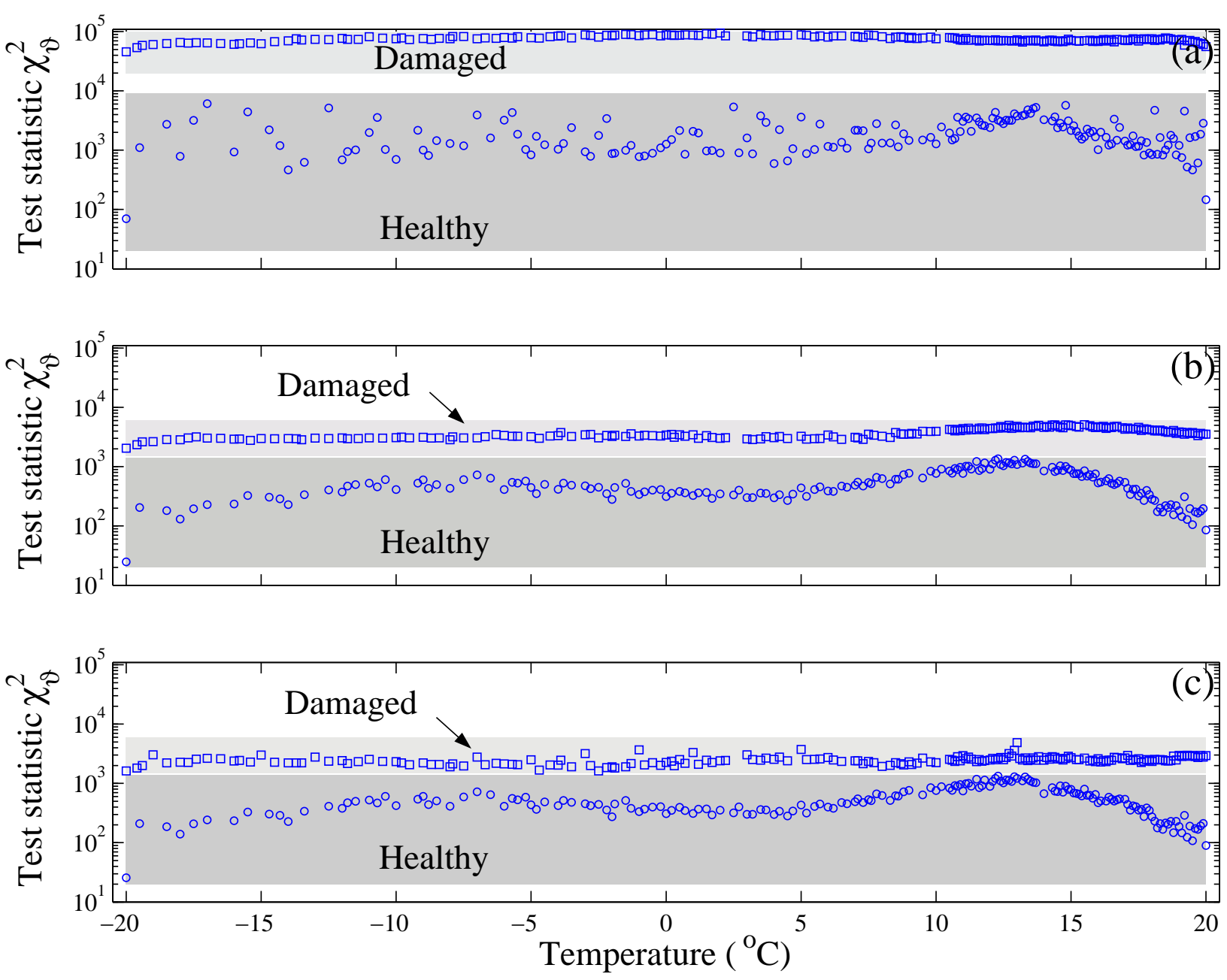

Figure 5.9: Model parameter-based damage detection results: $\chi_{\vartheta}^{2}$ statistic for healthy (o) and damaged ( $\square$ ) test cases at different temperatures; (a) without truncation, (b) truncation via "mean" transformation matrix, (c) truncation via "interpolated" transformation matrix (the shaded areas indicate where $\chi_{\vartheta}^{2}$ lies for the healthy and damaged test cases; $M_{1}=180$ healthy and $M_{2}=188$ damaged cases).

to perform slightly better, as the "distance" between the $\chi_{\vartheta}^{2}$ values for the healthy and damaged test cases is somewhat larger as opposed to the case where the "interpolated" transformation matrix is used (compare Fig. 5.9(b),(c)). Overall the obtained results verify that the discrete-time model parameters provide reliable damage indicators, as neither missed damage nor false alarms are observed.

\subsubsection{Comparisons with alternative methods}

\subsubsection{Comparison with the Principal Component Analysis (PCA) based method [25]}

The characteristic quantity used to infer damage detection in this method is the structure's natural frequencies [25]. Estimates of the frequencies are readily obtained by the conventional $\operatorname{VAR}(88)$ models used in the global model based approach.

In order to facilitate comparisons with the global model based approach, the covariance matrix of the natural frequencies is based upon frequency point estimates corresponding to the $M=21$ nominal temperatures used in the baseline phase (see Table 5.3). Since temperature is the main environmental factor in this study, a single principal component is retained by the PCA-based method [25]. Once the principal compo- 


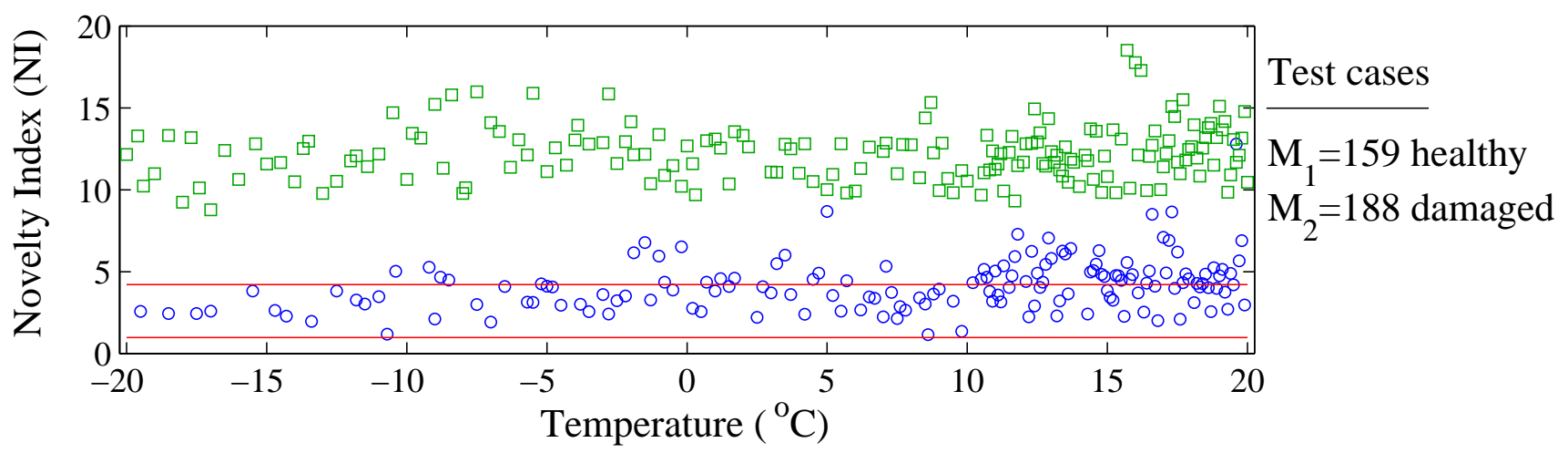

Figure 5.10: PCA-based method damage detection results: $X$-bar control chart for healthy (o) and damaged $(\square)$ test cases at different temperatures (the natural frequency covariance matrix is estimated from $M=21$ nominal temperature experiments; the horizontal lines indicate the $\alpha=10^{-3}$ risk level limits; damage is detected when $N I$ lies above the upper limit).

nent transformation matrix is computed, the residual vectors corresponding to the nominal temperatures are obtained. Estimates of the mean and variance of the associated novelty index are subsequently derived, and statistical limits for the $X$-bar control chart are postulated (see also Appendix 5.C.1).

The damage detection results for $M_{1}=159$ experiments with the healthy structure and another $M_{2}=$ 188 experiments with the damaged structure (using a $99.9 \%$ confidence interval, that is risk level equal to $\alpha=10^{-3}$ ) are presented in Fig. 5.10. The PCA-based method exhibits a large number of false alarms, especially for temperatures in the $[0,+20]^{\circ} \mathrm{C}$ range. This problem may be alleviated by increasing the confidence interval of the $X$-bar control chart, at the cost of having missed detections. Notice however that the damage detection results may be potentially improved once a more accurate estimate of the natural frequency covariance matrix is obtained via data from additional temperature conditions.

\subsubsection{Comparison with the Factor Analysis (FA) based method [30]}

The FA-based method presently utilizes the structure's natural frequencies as the characteristic quantity for damage detection - in this fashion comparisons with its PCA-based counterpart are facilitated. As in the PCA-based method, estimates of the frequencies are obtained by the conventional VAR(88) models used in the global model based approach.

A factor model is identified from the frequency point estimates corresponding to the $M=21$ nominal temperatures used in the baseline phase (see Table 5.3). A single factor is used in the modeling procedure, since temperature is the main environmental factor in this study [30]. The model parameters are presently estimated via the principal component method [83, p. 261], whereas the vector of common factors is estimated via the regression method [83, p. 264]. Once the factor model is identified, residual vectors corresponding to the healthy and damaged structures are obtained. In accordance to [30], PCA is utilized to compress the residual-based information. The residual covariance matrix corresponding to the $M=21$ baseline phase nominal temperatures is diagonalized via SVD, whereas a threshold value equal to $99 \%$ is set to the criterion $\psi_{m}$ in Eq. (5.16), in order to determine the residual vector dimensionality. This procedure leads to a small dimensionality reduction of the transformed residual vectors from 8 to 7 . Once the transformed residuals are obtained, statistical limits for the $T^{2}$ control chart are subsequently postulated (see also Appendix 5.C.2).

The $T^{2}$ control chart damage detection results for $M_{1}=159$ experiments on the healthy and another $M_{2}=188$ experiments on the damaged structure at the $\alpha=10^{-3}$ risk level are presented in Fig. 5.11. The FA-based method in this case performs considerably better than its PCA-based counterpart (Fig. 5.10), 


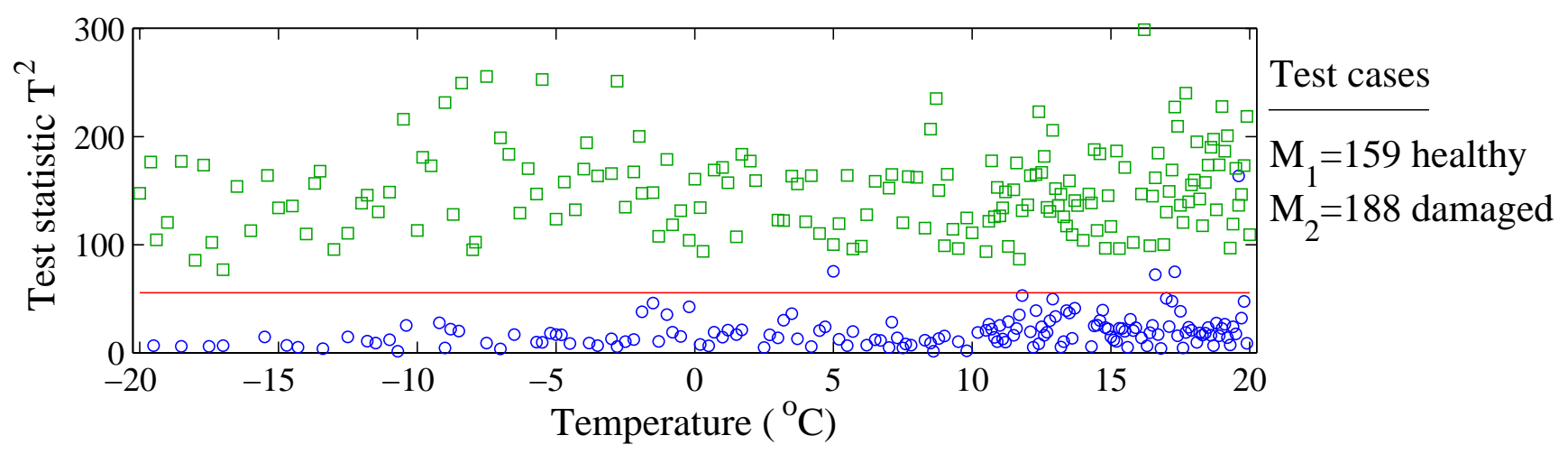

Figure 5.11: FA-based method damage detection results: $T^{2}$ control chart for healthy $(\circ)$ and damaged $(\square)$ test cases at different temperatures (the factor model is identified from $M=21$ nominal temperature experiments; the horizontal line designates the statistical limit at the $\alpha=10^{-3}$ risk level; damage is detected when $T^{2}$ lies above the limit).

exhibiting only a few false alarms. Yet, it is less robust compared to the modal parameter-based version of the global model approach (Fig. 5.7). Indeed, the FA-based method healthy and damaged test cases are not as clearly separated as in the modal parameter-based version (note the logarithmic scale on the vertical axis in Fig. 5.7). This may be attributed to the implicit modeling of the temperature effects on the natural frequencies via the factor model, as opposed to the explicit modeling of the temperature dependent structural dynamics and the effective treatment of uncertainties via the FP-VAR model. Notice however that, in analogy to the PCA-based method, improved damage detection results may be obtained by identifying a more accurate factor model from data corresponding to additional temperature conditions.

\subsection{Concluding remarks}

In this study a stochastic global model based approach for statistical damage detection under different temperatures using output-only vibration signals was introduced. The approach is based on the novel class of global (FP-VAR) models for describing the structural dynamics under any temperature, interval parameter estimation, as well as statistical decision making for damage detection. Two distinct versions were postulated: one based on modal parameter estimates and the other based on discrete-time model parameter estimates. In order to streamline damage detection, PCA-based information compression (characteristic quantity dimensionality reduction) techniques were also explored. As the study primarily focused on proofof-concept, the approach was experimentally validated and its performance assessed with hundreds of experiments using a composite beam set-up in the $[-20,+20]^{\circ} \mathrm{C}$ temperature range. The main conclusions drawn may be summarized as follows:

(i) The global FP-VAR based identification provides a convenient means for the output-only identification of the temperature-dependent structural dynamics. Its achievable accuracy and effectiveness are comparable to those obtained using input-output data in [42].

(ii) Despite the subtle damage scenario considered (local mass increase amounting to $4 \%$ ), the proposed global model approach proved very effective in detecting damage under different temperatures. It is worthwhile noting that this effectiveness was not dependent upon whether or not the temperature of each test was used in the baseline (training) phase. In addition, successful results have been also obtained with a different damage scenario involving progressive saw cuts and are reported in a recent study by the authors [39].

(iii) The effectiveness of the approach was demonstrated under only two vibration response sensors, and 
hence with no mode shape information, combined with a "low" $(0-1000 \mathrm{~Hz})$ frequency range.

(iv) Two versions of the approach, the first based on natural frequencies and the second on discrete-time model parameters, were postulated. The first version exhibited a somewhat better "discriminatory ability" (sensitivity to damage) between the healthy and damaged states. The use of a PCA-based dimensionality reduction in the latter case was effective, although its "discriminatory ability" (sensitivity to damage) was consequently reduced.

(v) The comparison with alternative (a PCA and an FA based) methods that attempt potential removal of the temperature effects from the damage-sensitive features revealed improved performance and improved "discriminatory ability" (sensitivity to damage) for the proposed approach. The FA-based method outperformed its PCA-based counterpart.

Overall, the obtained results confirm the capability of the stochastic global model based approach for damage detection under different temperatures and underscore its usefulness as a tool for structural health monitoring. Issues for further investigation include the assessment of the stochastic global model approach with additional damage scenarios as well as its potential application to practical problems.

\section{Appendix 5.A: FP-VAR model specification: two-step scheme versus exhaus- tive search}

An example comparing the two-step scheme and a typical exhaustive search, in terms of computational complexity expressed as the total number of estimated parameters, is given below.

Two-step scheme Suppose that a succession of $\operatorname{P}-\operatorname{VAR}(n)$ models with $n=1,2, \ldots, n^{*}$ are fitted to the data in the first step, and the BIC indicates that a model order equal to $n=\widehat{n}$ is adequate. The second step consists of fitting to the data a succession of $\operatorname{FP}-\operatorname{VAR}(\widehat{n})_{[p]}$ models with increasing dimensionality, say $p=1,2, \ldots,(M-1)$. The total number of estimated parameters for each step is:

- First step : $\sum_{n=1}^{n^{*}} M n y^{2} n=M n y^{2} \frac{n^{*}\left(n^{*}+1\right)}{2}$

- Second step : $\sum_{p=1}^{M-1} \widehat{n} n y^{2} p=\widehat{n} n y^{2} \frac{(M-1) M}{2}$

Exhaustive search The total number of parameters is: $\sum_{n=1}^{n^{*}} \sum_{p=1}^{M-1} n y^{2} n p=n y^{2} \frac{n^{*}\left(n^{*}+1\right)}{2} \frac{(M-1) M}{2}$

The ratio of estimated parameters is:

$$
\begin{aligned}
\frac{\text { No. of pars 2-step scheme }}{\text { No. of pars exhaustive search }} & =\frac{M n y^{2} \frac{n^{*}\left(n^{*}+1\right)}{2}+\widehat{n} n y^{2} \frac{(M-1) M}{2}}{n y^{2} \frac{n^{*}\left(n^{*}+1\right)}{2} \frac{(M-1) M}{2}}=\frac{2}{M-1}+\frac{2 \widehat{n}}{n^{*}\left(n^{*}+1\right)} \\
& \leq \frac{2}{M-1}+\frac{2}{\left(n^{*}+1\right)}
\end{aligned}
$$

which is generally less than unity even for small-size search spaces (for instance, $M \geq 5$ and $n^{*} \geq 4$ ).

\section{Appendix 5.B: Additional modeling results}

Fig. 5.12 presents the Welch-based power spectral densities (PSDs) of the response signals $y_{1}[t]$ and $y_{2}[t]$, designated as $P_{y_{1} y_{1}}$ and $P_{y_{2} y_{2}}$ respectively, for the healthy and damaged structure at $M=21$ nominal 

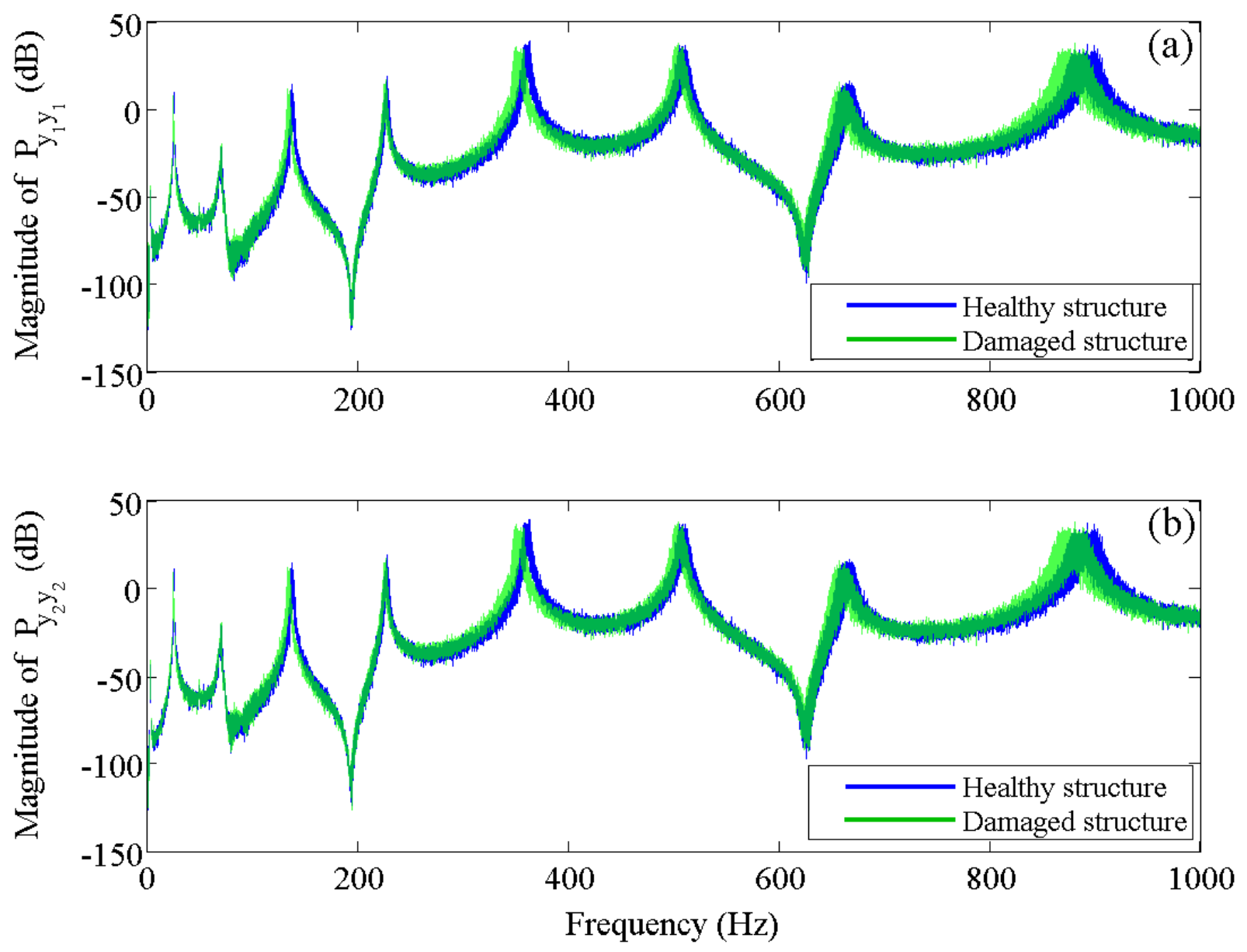

Figure 5.12: Welch-based power spectral density (PSD) estimates at the $M=21$ nominal temperatures belonging to the $[-20,+20]^{\circ} \mathrm{C}$ range: ( - dark blue zone) healthy structure, and ( - transparent light green zone) damaged structure (each zone comprises $M=21$ estimates, that is one CSD curve per each nominal temperature).

temperatures [46, MATLAB ${ }^{\circledR}$ function "psd.m"]. Notice that the similarity of the PSD curves is due to the fact that the response signals are obtained from collocated sensors. As in the CSD case, the obtained curves indicate the presence of eight modes within the $[0,1,000] \mathrm{Hz}$ frequency range (data length $N=70,000$ samples, Hanning data segment window length $=10,240$ samples, data segment overlap $=80 \%$, sampling frequency $f_{\mathrm{s}}=2,560 \mathrm{~Hz}$, frequency resolution $\Delta f=0.25 \mathrm{~Hz}$ ). The temperature exerts its effects at higher frequencies, as the corresponding modes exhibit larger variability. The significant amount of overlapping observed in the healthy and damaged structure PSDs suggests that the effects of damage can be masked by structural changes due to temperature.

\section{B.1 Baseline phase}

The frequency stabilization diagrams of the auxiliary $\operatorname{P}-\operatorname{VAR}(n)$ modeling procedure at certain nominal temperatures are presented in Fig. 5.13. The diagrams suggest that the higher mode frequencies "stabilize" for model orders $n \geq 60$. Fig. 5.14 depicts the convergence of the estimated natural frequency versus temperature curves for the $\operatorname{FP}-\operatorname{VAR}(88)_{[p]}$ models with increasing subspace dimensionality $(p=2,3 \ldots, 10)$. Only the natural frequencies belonging to the $M=21$ nominal temperatures are displayed, allowing for a temperature resolution of $\Delta k=2^{\circ} C$. The convergence is very good and rapid ( $p \geq 4$ ). The preceding 

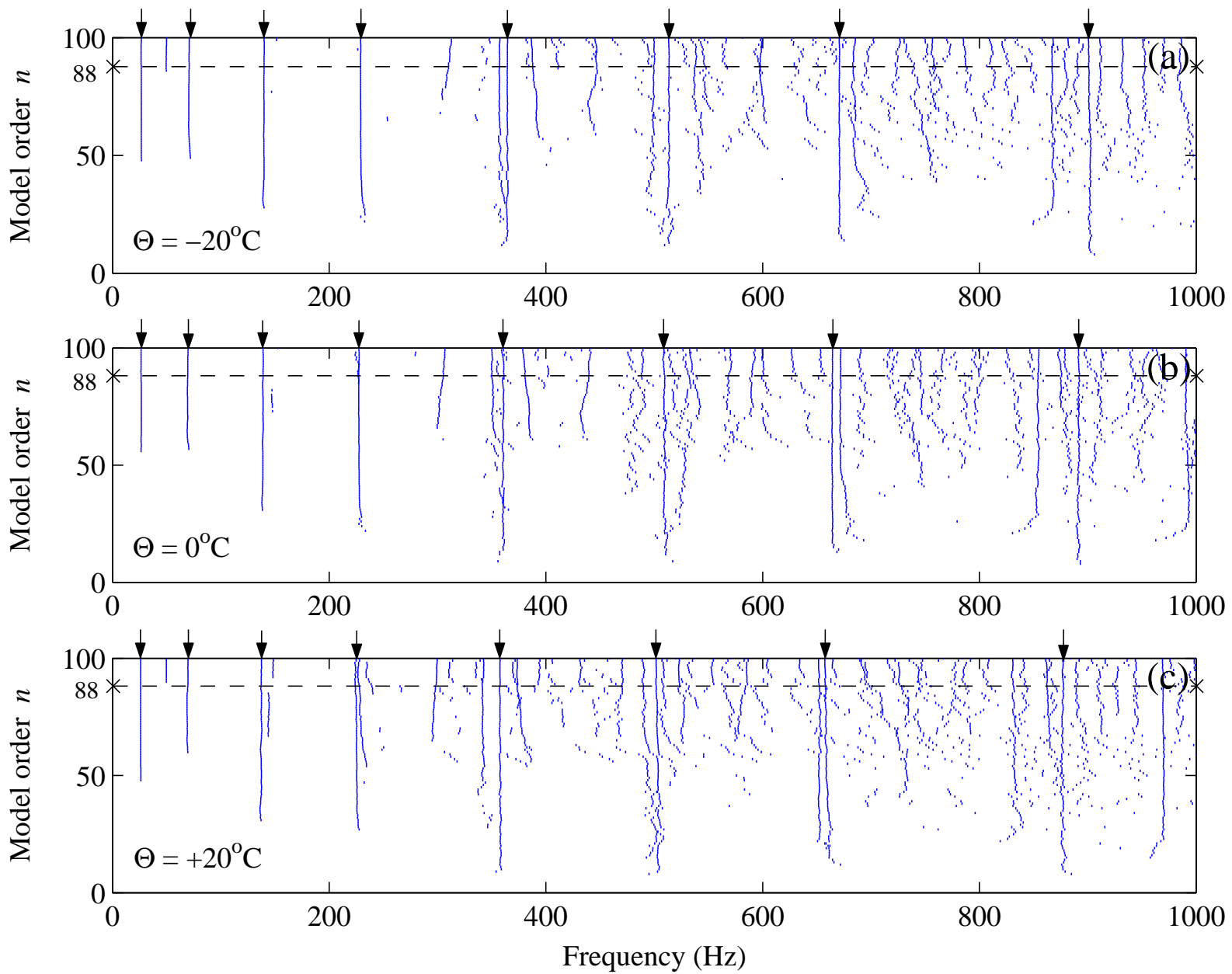

Figure 5.13: $\mathrm{P}-\operatorname{VAR}(n)$ frequency stabilization diagrams for three distinct nominal temperatures (only modes with damping factors $\zeta<3 \%$ are depicted; the arrows at the top indicate the identified modes; frequencies stabilize for $n \geq 60$; the horizontal dashed lines indicate the selected model order via the BIC criterion).

observations suggest that a possibly more compact FP-VAR model representation might have been selected by means of frequency stabilization diagrams.

Fig. 5.15 presents the PSD estimates of the Welch-based method and FP-VAR $(88)_{[7]}$ model. The agreement is very good and confirms the accuracy of the FP-VAR modeling in representing the temperature effects on the structural dynamics. Recall that the similarity of the PSDs is due to the fact that the response signals are obtained from collocated sensors.

Fig. 5.16(a) presents the damping factor point estimates and their estimated uncertainties $( \pm$ two standard deviation zones) via the Gauss approximation formula (see Subsection 5.3.1 and Table 5.3) for the healthy structure at the $M=21$ nominal temperatures. The damping factors exhibit a weak dependence on temperature as they appear sensibly constant. The results are in qualitative agreement with those in Chapter 3 , where the same structure with a different sensor configuration is considered within an alternative frequency range.

The temperature dependence of certain AR parameters from the identified FP-VAR $(88)_{[7]}$ model at the $M=21$ nominal temperatures is presented in Fig. 5.17. The uncertainty associated with each parameter is given by its point estimate \pm two standard deviations. The "smooth" parameter dependence on temperature is compatible to the physics of the problem. The obtained accuracy of the FP-VAR $(88)_{[7]}$ model is also 

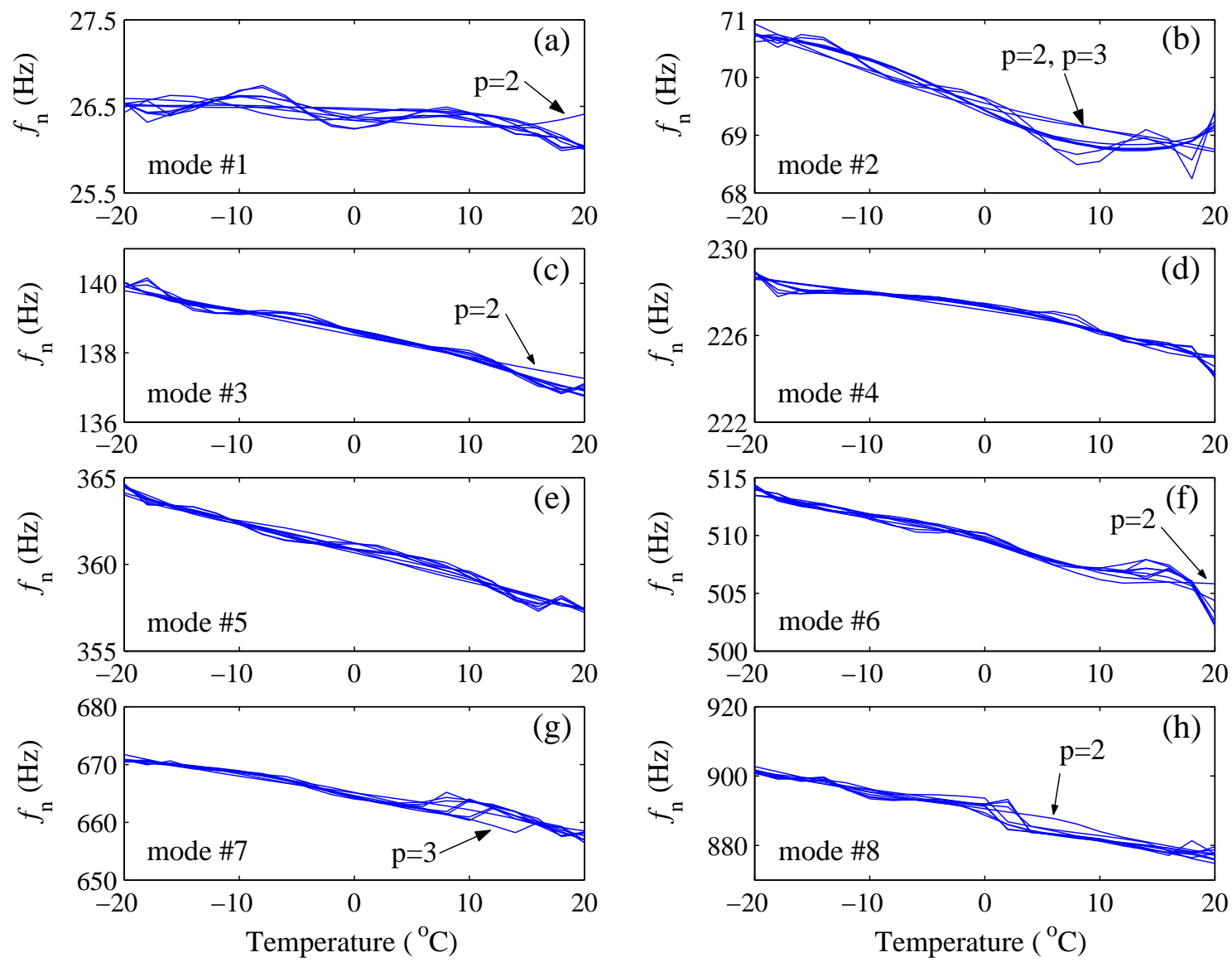

Figure 5.14: Convergence of the $\operatorname{FP}-\operatorname{VAR}(88)_{[p]}$ natural frequency $\left(f_{\mathrm{n}}\right)$ versus temperature curves for increasing functional subspace dimensionality $p=2,3, \ldots, 10$ (frequencies belonging to the $M=21$ nominal temperatures are displayed; the arrows indicate curves for $p=2,3$; convergence occurs for $p \geq 4$ ).

better than its conventional $\operatorname{VAR}(88)$ based counterparts, and may be attributed to the increased statistical accuracy attained via data pooling and the limited parametrization.

\section{B.2 Inspection phase}

Fig. 5.16(b) presents the healthy and damaged structure damping factors point estimates along with their uncertainties ( \pm two standard deviation zones) at the $M=21$ nominal temperatures. The influence of damage is very weak, as both structural states exhibit overlapping confidence intervals at all temperatures.

The temperature dependence of certain AR parameters from the identified $\operatorname{VAR}(88)$ models corresponding to the healthy and damaged structure at the $M=21$ nominal temperatures is presented in Fig. 5.17. The uncertainty associated with each parameter is given by its point estimate \pm two standard deviations. The parameter dependence on temperature is not as smooth as in the FP-VAR case, whereas the corresponding uncertainties are generally larger. Notice that the AR parameters of the FP-VAR $(88)_{[7]}$ and its healthy structure $\operatorname{VAR}(88)$ counterparts are expectedly in a rough overall agreement, whereas the corresponding damaged structure AR parameters exhibit large discrepancies.

\section{B.3 Diagnostic checking for multicollinearity}

The PCA-based diagnostic procedures used to detect linear dependencies in the data search for (i) exact contemporaneous linear relationships, and (ii) exact lagged linear relationships between the observed response 

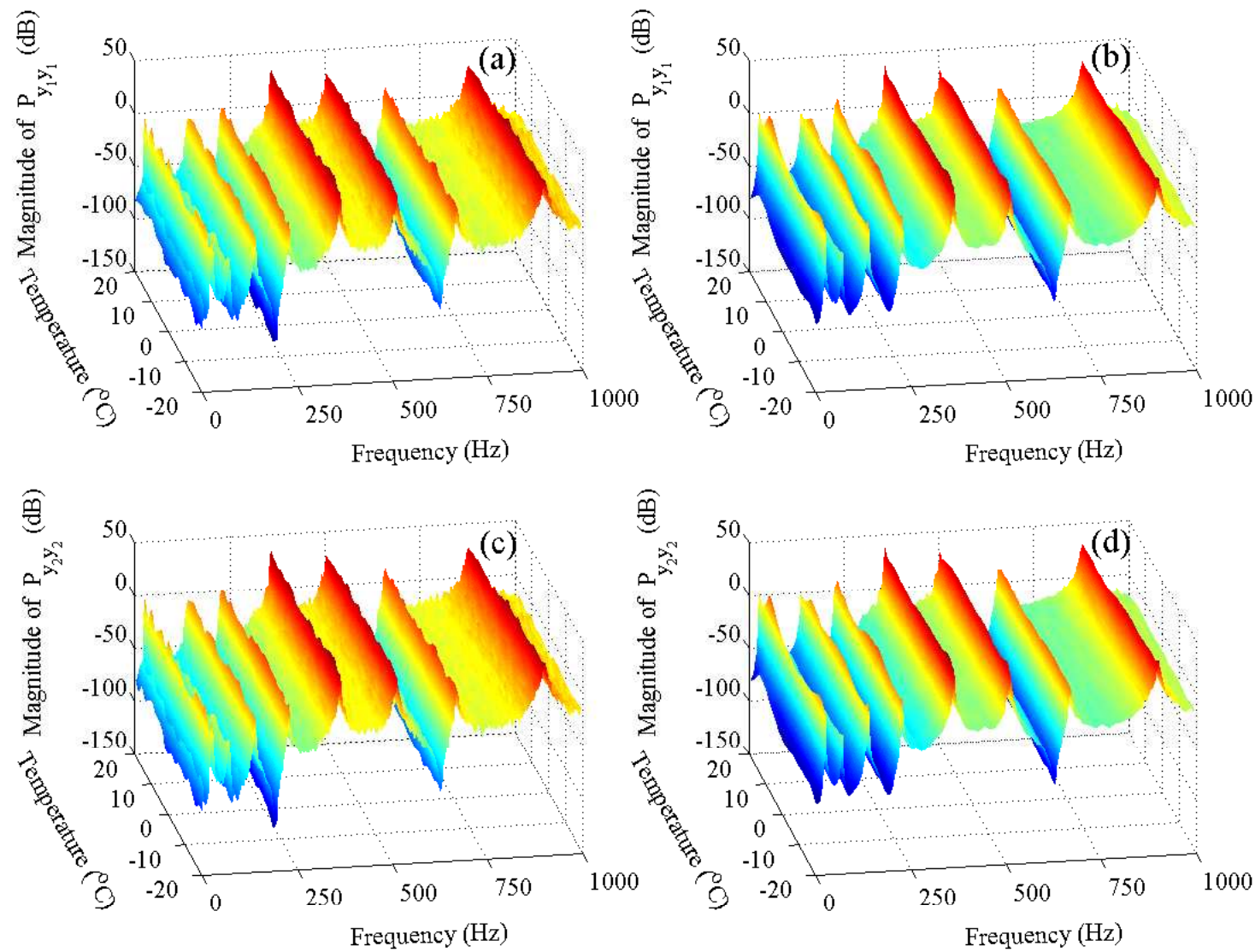

Figure 5.15: Power spectral densities (PSDs) for the healthy beam versus frequency and temperature: (a),(c) Welch-based estimates; (b),(d) FP-VAR(88) ${ }_{[7]}$-based estimates.

signals $[84,85]$.

Exact contemporaneous linear relationships between the component signals of $\boldsymbol{y}_{k}[t]\left(k=k_{1}, k_{2}, \ldots\right.$, $\left.k_{M}\right)$ are detected by searching for singularities in the zero-lag cross-covariance matrix of the response vector, denoted by $\boldsymbol{\Gamma}_{k, k}(0)=E\left\{\boldsymbol{y}_{k}[t] \boldsymbol{y}_{k}^{T}[t]\right\}$. The condition numbers of the matrices $\boldsymbol{\Gamma}_{k, k}(0)$ at the $M=21$ nominal temperatures are presented in Fig. 5.18(a). The matrices are well-conditioned (condition numbers $\sim 10^{3}$ ), suggesting that there are no exact contemporaneous relationships between the component signals of the response vector.

Exact lagged linear relationships are detected by searching for singularities in the model-based residual covariance matrices $\boldsymbol{\Sigma}_{k, k}\left(k=k_{1}, k_{2}, \ldots, k_{M}\right)$. The corresponding condition numbers of the $\operatorname{VAR}(88)$ and FP-VAR $(88)_{[7]}$ model based residual covariance matrices $\boldsymbol{\Sigma}_{k, k}$ at the $M=21$ nominal temperatures are presented in Fig. 5.18(b). The matrices are well-conditioned (condition numbers $\sim 10^{2}$ ), confirming that there are no exact lagged linear relationships between the component signals of the response vector.

\section{Appendix 5.C: Damage detection based on methods attempting removal of temperature effects}

\section{C.1 The Principal Component Analysis (PCA) based method [25]}

The PCA-based method aims at removing the environmental effects by decomposing the covariance matrix of the characteristic quantity $\boldsymbol{q}(k)$, observed under different (but not necessarily measured) environmental 

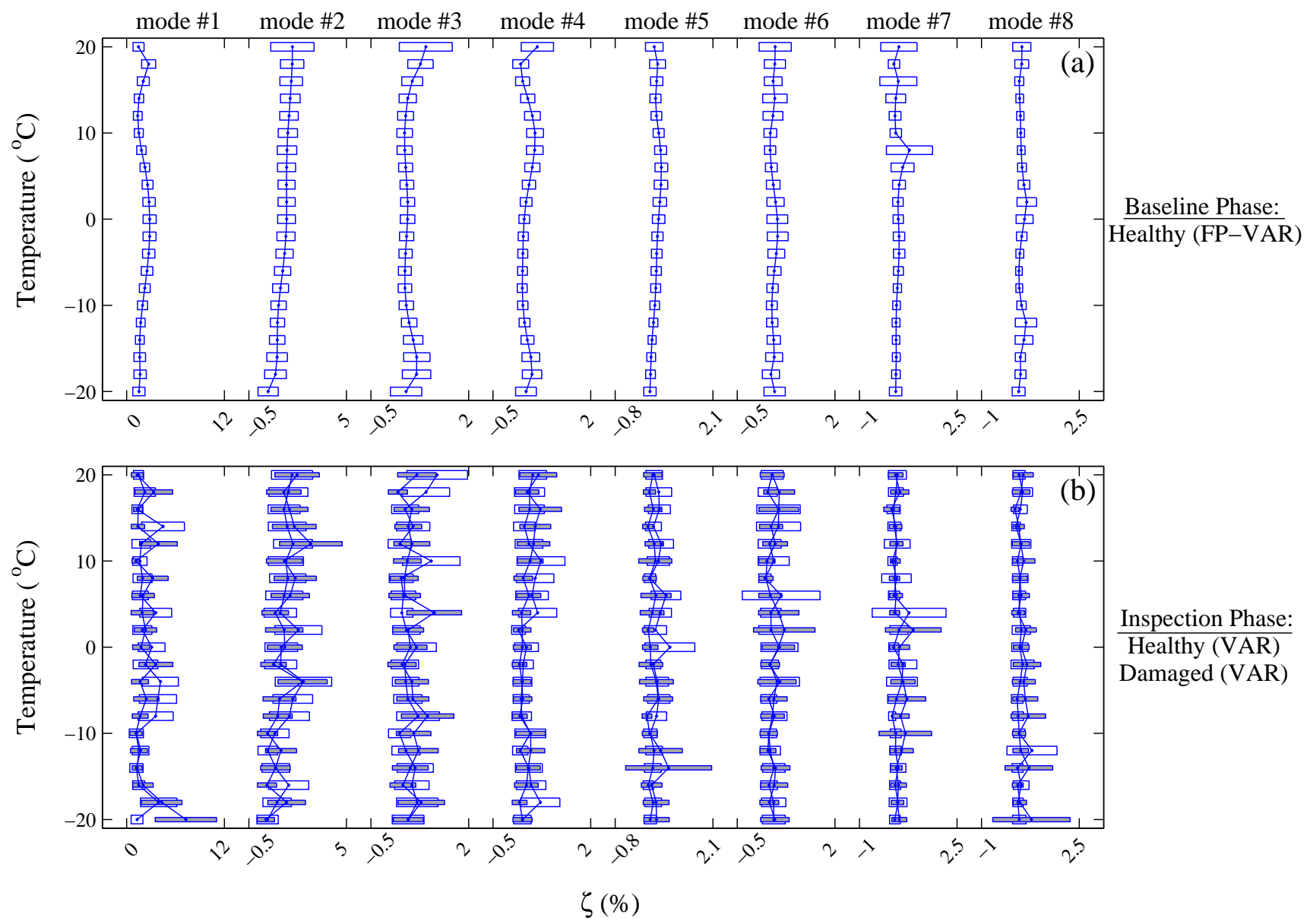

Figure 5.16: Damping factor $(\zeta)$ point estimates and their estimated uncertainties ( \pm two standard deviations) at the $M=21$ nominal temperatures: (a) baseline phase (healthy structure) FP-VAR $(88)_{[7]}$ estimates, (b) inspection phase (healthy and damaged structure) VAR(88) estimates (the uncertainties are designated by white boxes for the healthy structure and gray boxes for the damaged.

conditions. The objective is to identify a linear subspace in which the environmental effects lie, and remove them by projecting $\boldsymbol{q}(k)$ on its orthogonal complement.

The characteristic quantity associated to the baseline (healthy) structure, $\boldsymbol{q}_{\mathrm{o}}(k)$, is assumed to be a random variable with mean $\overline{\boldsymbol{q}}_{\mathrm{o}}$ and covariance $\boldsymbol{\Sigma}_{\boldsymbol{q}_{\mathrm{o}}}$. According to the PCA-based approach, the most important principal components of $\boldsymbol{q}_{\mathrm{o}}(k)$ correspond to the combined environmental factors that affect it. Assuming that the first $m$ principal components are considered important, then an $m$-dimensional subspace is identified by the columns of the corresponding principal component transformation matrix $\boldsymbol{U}_{m}$ (refer to Eq. (5.17a)-(5.17c)).

Once the characteristic quantity corresponding to the current (healthy or damaged) state of the structure at temperature $k_{\mathrm{u}}$ (designated as $\boldsymbol{q}_{\mathrm{u}}\left(k_{\mathrm{u}}\right)$ ) is available, damage detection is based upon the following residual:

$$
\boldsymbol{\epsilon}\left(k_{\mathrm{u}}\right)=\left(\boldsymbol{I}-\boldsymbol{U}_{m} \boldsymbol{U}_{m}^{T}\right)\left(\boldsymbol{q}_{\mathrm{u}}\left(k_{\mathrm{u}}\right)-\overline{\boldsymbol{q}}_{\mathrm{o}}\right)
$$

which describes the part of the characteristic quantity $\boldsymbol{q}_{\mathrm{u}}\left(k_{\mathrm{u}}\right)$ that remains after the removal of the temperature effects (by projecting $\boldsymbol{q}_{\mathrm{u}}\left(k_{\mathrm{u}}\right)$ on the $m$-dimensional subspace).

In order to assess the state (healthy/damaged) of the structure, the following distance metric (also referred to as the novelty index) is commonly used: 

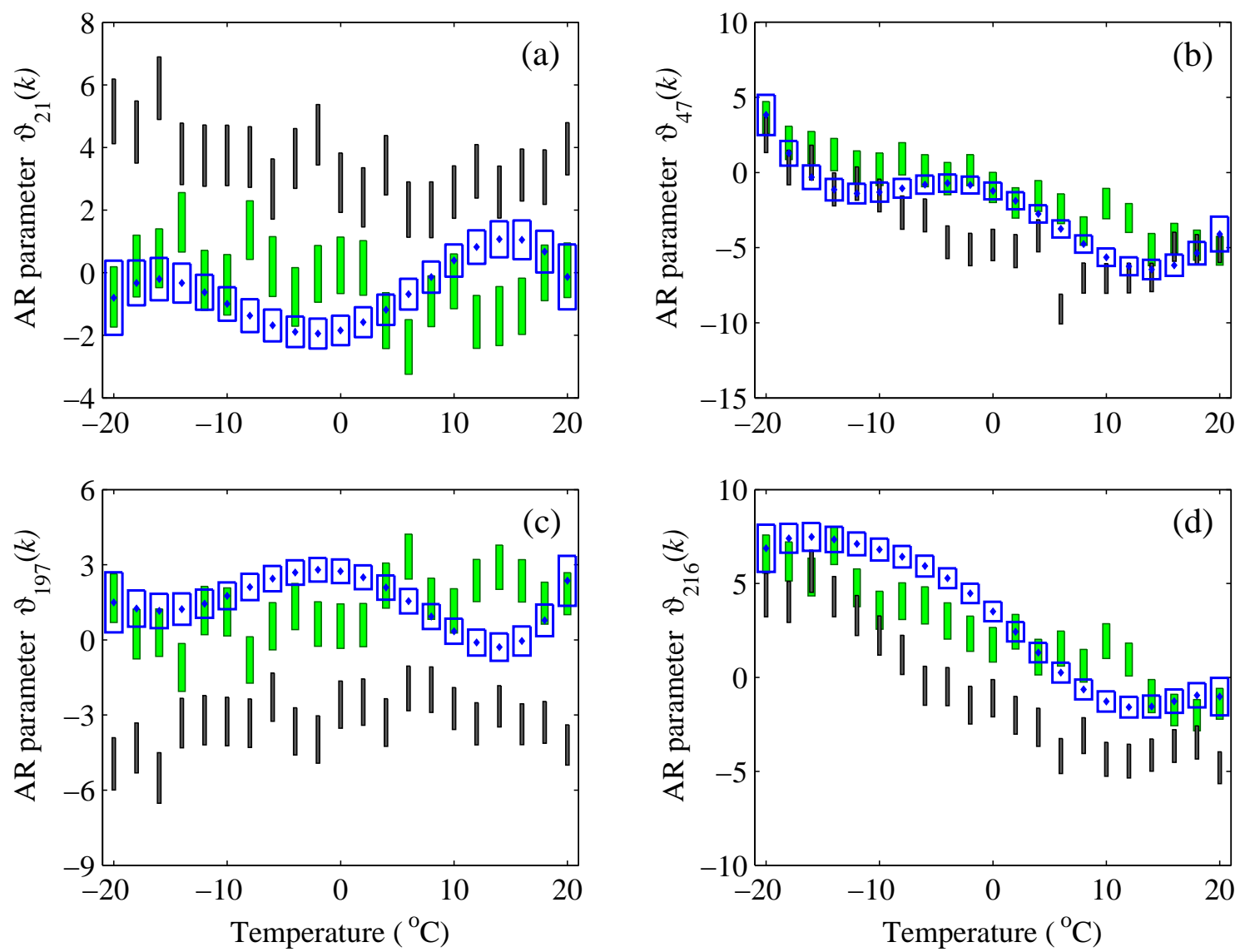

Figure 5.17: Dependence of certain AutoRegressive parameters at the $M=21$ nominal temperatures (the boxes indicate point estimates \pm two standard deviations): (white) baseline phase FP-VAR $(88)_{[7]}$ estimates; (light green) inspection phase healthy $\operatorname{VAR}(88)$ estimates; (dark gray) inspection phase damaged $\operatorname{VAR}(88)$ estimates $\left(\vartheta_{i}(k)\right.$ designates the $i$-th element of the AR parameter vector $\left.\vartheta(k)\right)$.

$$
N I\left(k_{\mathrm{u}}\right)=\sqrt{\boldsymbol{\epsilon}^{T}\left(k_{\mathrm{u}}\right)\left(\boldsymbol{\Sigma}_{\boldsymbol{q}_{\mathrm{o}}}\right)^{-1} \boldsymbol{\epsilon}\left(k_{\mathrm{u}}\right)}
$$

which is assumed to follow a normal (Gaussian) distribution with mean $\overline{N I}$ and variance $\sigma$ for a healthy structure. Damage detection is accomplished by testing for statistically significant discrepancies of the novelty index corresponding to the current (healthy or damaged) state of the structure $N I\left(k_{\mathrm{u}}\right)$ from the mean value $\overline{N I}$. An $X$-bar control chart [88, p. 328] is used for this purpose to test the following:

$$
\begin{array}{cl}
\overline{N I}-\beta \sigma<N I\left(k_{\mathrm{u}}\right)<\overline{N I}+\beta \sigma & \Longrightarrow \text { healthy structure } \\
\text { Else } & \Longrightarrow \text { damaged structure }
\end{array}
$$

with $\beta$ usually set equal to 3 , which corresponds to a $99.9 \%$ confidence interval under normal distribution.

\section{C.2 The Factor Analysis (FA) based method [30]}

The FA-based method is closely related to its PCA-based counterpart, as both methods identify a linear subspace in which the environmental effects lie, and remove them by projecting $\boldsymbol{q}(k)$ on its orthogonal complement.

Similar to the PCA-based method, the analysis assumes that, for the baseline (healthy) structure, $\boldsymbol{q}_{\mathrm{o}}(k)$ is a random variable with mean $\overline{\boldsymbol{q}}_{\mathrm{o}}$ and covariance $\boldsymbol{\Sigma}_{\boldsymbol{q}_{\mathrm{o}}}$. The characteristic quantity $\boldsymbol{q}_{\mathrm{o}}(k)$, however, 

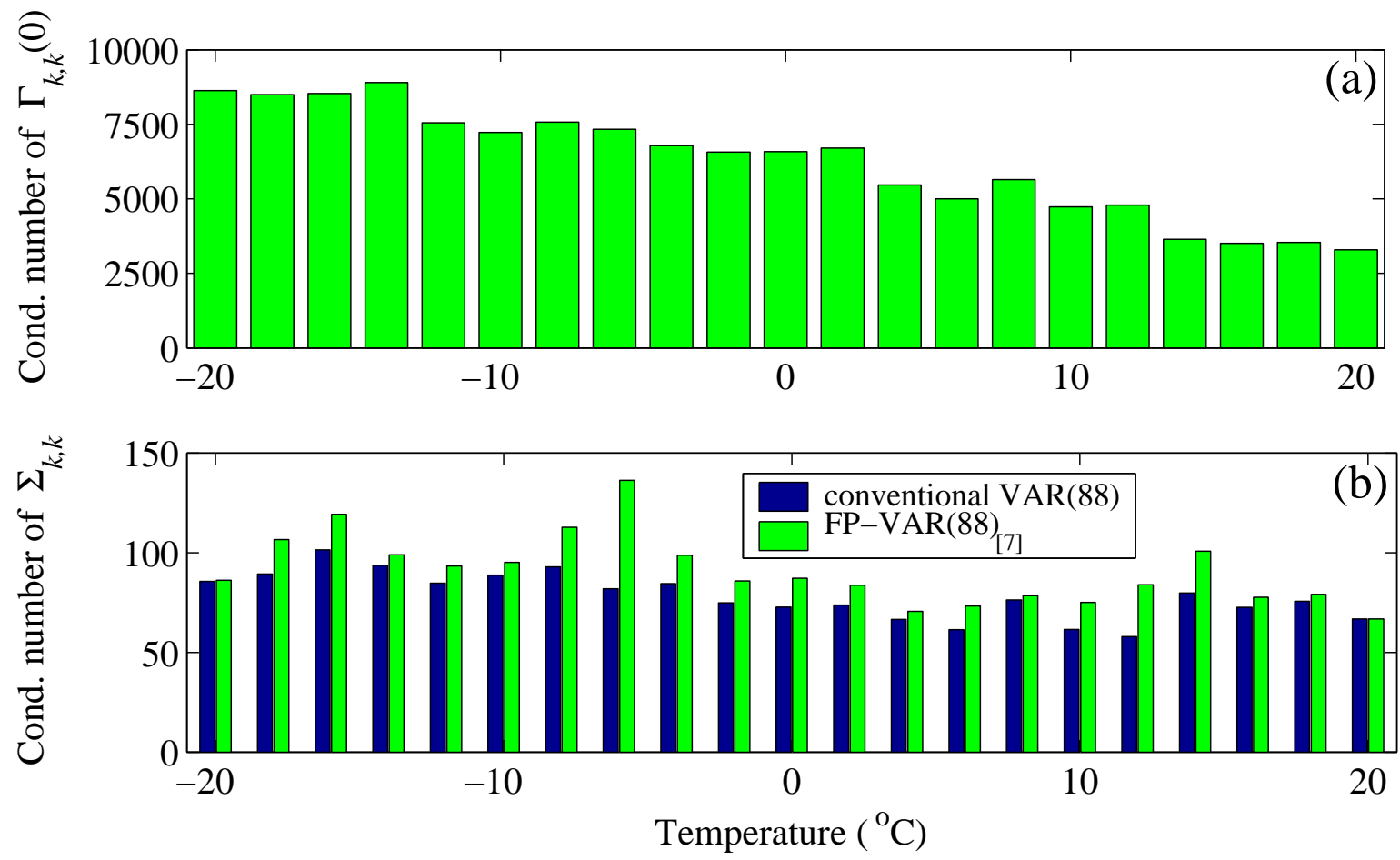

Figure 5.18: Diagnostic checking for multicollinearity in the response data at the $M=21$ nominal temperatures: (a) condition numbers of response data zero-lag cross-covariance matrices, (b) condition numbers of residual covariance matrices; (blue) $\operatorname{VAR}(88)$ estimates; (green) FP-VAR $(88)_{[7]}$ estimates.

is modeled as a linear transformation of a small number of common, yet unobservable, factors (for instance, environmental effects) as follows:

$$
\boldsymbol{q}_{\mathrm{o}}(k)=\boldsymbol{\Lambda} \boldsymbol{f}(k)+\boldsymbol{\varepsilon}(k)+\overline{\boldsymbol{q}}_{\mathrm{o}}
$$

where $\boldsymbol{f}(k)$ designates the $(m \times 1)$ random vector of common factors, which is zero-mean with an identity covariance matrix, and it holds that $m<\operatorname{dim}\left(\overline{\boldsymbol{q}}_{\mathrm{o}}\right)$. $\boldsymbol{\Lambda}$ designates the $\left(\operatorname{dim}\left(\overline{\boldsymbol{q}}_{\mathrm{o}}\right) \times m\right)$ matrix of the nonrandom factor loadings. The $(m \times 1)$ random vector $\varepsilon(k)$ contains the specific factors (residuals), and is zero-mean with a diagonal covariance matrix $\Psi$. The common and the specific factors are mutually uncorrelated, decomposing the covariance of $\boldsymbol{q}_{\mathrm{o}}(k)$ as follows:

$$
\boldsymbol{\Sigma}_{\boldsymbol{q}_{\mathrm{o}}}=\boldsymbol{\Lambda} \boldsymbol{\Lambda}^{T}+\boldsymbol{\Psi}
$$

Damage detection is accomplished by testing for statistically significant discrepancies of the residual $\varepsilon\left(k_{\mathrm{u}}\right)$, corresponding to the current (healthy or damaged) state of the structure, from zero. Under the assumption of residual normality, a multivariate $T^{2}$ control chart [88, p. 331] is used to test the following:

$$
\begin{aligned}
T^{2}\left(k_{\mathrm{u}}\right)=\varepsilon^{T}\left(k_{\mathrm{u}}\right)\left(\widehat{\boldsymbol{\Sigma}}_{\boldsymbol{\varepsilon}_{\mathrm{o}}}\right)^{-1} \varepsilon\left(k_{\mathrm{u}}\right)<\frac{\operatorname{dim}(\boldsymbol{\varepsilon})(M-1)}{(M-\operatorname{dim}(\varepsilon))} \mathcal{F}_{(1-\alpha)}(\operatorname{dim}(\varepsilon),(M-\operatorname{dim}(\varepsilon))) & \Longrightarrow \text { healthy structure } \\
\text { Else } & \Longrightarrow \text { damaged structure }
\end{aligned}
$$

where $\widehat{\Sigma}_{\varepsilon_{\mathrm{o}}}$ is an estimate of the healthy structure residual covariance, obtained from a sample of $M$ measurements. Furthermore, $\mathcal{F}_{(1-\alpha)}(\operatorname{dim}(\varepsilon),(M-\operatorname{dim}(\varepsilon)))$ designates the $\mathcal{F}$-distribution's $(1-\alpha)$ critical point with $(\operatorname{dim}(\varepsilon),(M-\operatorname{dim}(\varepsilon)))$ degrees of freedom. Notice that, rather than using a single residual observation, the $T^{2}$ control chart may be based on observing the mean of a small sample of residuals, as in [30]. 


\section{Chapter 6}

\section{Stochastic identification under multiple operating conditions: assessment of global and conventional VARX modeling approaches}

\subsection{Introduction}

System identification is a well-studied interdisciplinary topic which traditionally addresses the problem of finding a model capable of representing a dynamic system at a specific operating condition, based on measured data. Yet, it is often the case that a system operates under different, though constant, conditions at different occasions (time intervals). Typical examples include mechanical, aerospace or civil structures that operate under different environmental conditions (such as temperature or humidity) on different occasions (period of the day and so on). Such different operating conditions may affect the system characteristics, and therefore its dynamics. Oftentimes, data records, each one corresponding to a distinct operating condition, are obtained. In such situations, it is desirable to use all the available data records in order to identify a single, "global" model that is capable of accurately describing the system dynamics within a specified operating range.

A "feasible" solution to this problem would consist of splitting it into several disjoint problems (one per each operating condition) and identify a model based on a single data record at a time. This procedure is known as the "multi-model" approach. The identified models may be subsequently "interconnected" into a single global model via proper interpolation of their parameters. Nevertheless, such an approach is both inefficient and statistically suboptimal. Inefficiency has to do with the treatment of multiple identification problems instead of a single one. Statistical suboptimality has to do with (i) the ineffective use of the available information as possible interrelations among different data records are ignored, (ii) the increased estimation error resulting from the potentially large number of estimated parameters (several identified models), and (iii) the separate treatment of the parameter interpolation stage which constitutes an additional source of modeling error.

In order to effectively overcome the preceding shortcomings, novel global modeling approaches have been recently introduced by Fassois and co-workers (univariate models are treated in [14-17], whereas multivariate models are treated in [38-42,72]). The global approaches are based on a novel statistical pooling framework, which allows for (i) the postulation of a single, stochastic, global model, and (ii) the simultaneous and statistically optimal treatment of all available data records. 
A case of particular interest concerns the global models of the Vector AutoRegressive with eXogenous excitation (VARX) form, as they have been employed in several practical applications [38-42]. These models may be thought as generalizations of the conventional VARX model class. Their motivation lies on the fact that oftentimes the joint study of several excitation-response signals is required in order to obtain a better understanding of the system dynamics. The study of global VARX models poses certain technical challenges not encountered in the univariate case, as the couplings between the excitation and response signals lead to representations of increased complexity. The model structures involved are richer (many parameters to estimate), whereas their nontrivial parametrization raises issues on model identifiability.

Depending upon the particular set of assumptions with respect to the model parameters, the following classes of global VARX models have been developed:

1. The Pooled VARX (P-VARX) model class $[39,72]$. This class is limited in scope, as the obtained models are valid only for the operating conditions used in the identification procedure - they do not account for the dynamics of any other operating condition. Nonetheless, these models prove valuable in FP-VARX/VARMA model structure specification (refer to Chapter 4, Section 4.6, and Chapter 5 Subsection 5.3.1), as well as in FP-VARMA Least Squares based estimation (refer to Chapter 4, Section 4.3.1).

2. The Constant Coefficient Pooled VARX (CCP-VARX) model class $[41,42,65]$. This class is also limited in scope, since the obtained models are characterized by parameters that do not depend on the operating condition (constant coefficients). As a consequence, they may only be employed for providing a simple, "averaged", description of the dynamics over the entire range of operating conditions.

3. The Functionally Pooled VARX (FP-VARX) model class [38-42]. This class is more elaborate, allowing for the explicit (functional) dependence of the model parameters on the operating condition. The FP-VARX models constitute the essential representation of choice for providing a single, compact (parsimonious), and accurate description of the dynamics under any operating condition within a specified operating range.

Although the P-VARX and CCP-VARX model classes have proven a useful tool for global model identification, their properties remain to a large extent unexplored and deserve further research. In addition, the question of how the preceding model classes compare to the FP-VARX model class remains unanswered, whereas the relation of the global VARX models to their conventional VARX model counterpart is an open problem. These issues are very important, and motivate the present study to provide useful insight on the postulated global modeling approaches.

In view of the above, the aim of the present study is twofold, since: (i) the identification of the $\mathrm{P}-$ VARX and CCP-VARX model classes is treated exhaustively for the first time, whereas (ii) the relationships interconnecting the global VARX model structures, and their association to the conventional VARX model structure are studied in detail.

The novel contributions of the present study may be summarized as follows:

(i) The specification of the novel P-VARX and CCP-VARX model classes.

(ii) The formulation of Least Squares (LS) and conditional Maximum Likelihood (ML) type estimators for the P-VARX and CCP-VARX models, and confirmation of their large sample properties (consistency, asymptotic normality).

(iii) The postulation of conditions ensuring P-VARX and CCP-VARX model identifiability (uniqueness of representation and informative experiments).

(iv) The assessment of the global VARX and conventional VARX model structures in terms of model parsimony and achievable accuracy. The effectiveness and performance characteristics of the novel global modeling approaches are further examined via two Monte Carlo studies. 
The rest of this chapter is organized as follows: The P-VARX model identification is presented in Section 6.2. The CCP-VARX model identification is presented in Section 6.3. In Section 6.4, the global VARX modeling approaches are assessed by means of Monte Carlo studies, whereas the concluding remarks are summarized in Section 6.5.

\subsection{The P-VARX model class}

\subsubsection{The data set and the P-VARX model specification}

Global model identification is based upon $M$ sets of $N$ sample-long data records, each one obtained under a different, constant, operating condition. That is, each data record corresponds to a specific, constant, value of a measurable operating parameter $k$. The set of all possible operating conditions belongs to the range $\mathcal{R}=\left[k_{\min }, k_{\max }\right] \subset \mathbb{R}$ while a discretized version $\left\{k_{1}, k_{2}, \ldots, k_{M}\right\}$ of it is used for data acquisition. The complete data set is designated as ${ }^{1}$ :

$$
Z^{N M} \triangleq\left\{\boldsymbol{x}_{k}[t], \boldsymbol{y}_{k}[t] \mid k=k_{1}, \ldots, k_{M} ; t=1, \ldots, N\right\}
$$

with $t$ designating the normalized discrete time, $\boldsymbol{x}_{k}[t]$ being the $(n x \times 1)$ excitation vector, whereas $\boldsymbol{y}_{k}[t]$ is the $(n y \times 1)$ response vector corresponding to the $k$-th operating condition.

The P-VARX representation postulated is of the form:

$$
\begin{gathered}
\boldsymbol{y}_{k}[t]+\sum_{i=1}^{n a} \boldsymbol{A}_{i, k} \boldsymbol{y}_{k}[t-i]=\sum_{i=0}^{n b} \boldsymbol{B}_{i, k} \boldsymbol{x}_{k}[t-i]+\boldsymbol{w}_{k}[t] \\
E\left\{\boldsymbol{w}_{k}[t] \boldsymbol{w}_{\ell}^{T}[t-\tau]\right\}=\boldsymbol{\Sigma}_{k, \ell} \delta[\tau] \quad k, \ell \in\left\{k_{1}, k_{2}, \ldots, k_{M}\right\}
\end{gathered}
$$

with $n a$ and $n b$ indicating the corresponding AutoRegressive (AR) and eXogenous (X) model orders. $\boldsymbol{w}_{k}[t]$ is a $(n y \times 1)$ innovations vector which is zero mean, serially uncorrelated, with fully parametrized crosscovariance matrices $\boldsymbol{\Sigma}_{k, \ell} . E\{\cdot\}$ designates statistical expectation and $\delta[\tau]$ the Kronecker delta. The AR and $\mathrm{X}$ matrices $\boldsymbol{A}_{i, k}$ and $\boldsymbol{B}_{i, k}$ are fully parametrized and have dimensions $(n y \times n y)$ and $(n y \times n x)$, respectively. The representation of Eq. (6.2a)-(6.2b) is referred to as a P-VARX model of orders $(n a, n b)$, or in short as a P-VARX $(n a, n b)$ model.

Notice that $\boldsymbol{A}_{i, k}$ and $\boldsymbol{B}_{i, k}$ are not explicit functions of the operating parameter $k$. The P-VARX models are valid for the operating conditions $k \in\left\{k_{1}, k_{2}, \ldots, k_{M}\right\}$ - they do not account for any other operating condition belonging to the range $\mathcal{R}$. Their main advantage is the simple and easy to identify structure, at the cost of reduced accuracy due to the increased number of estimated model parameters (see Subsection 6.2.5.2).

The P-VARX $(n a, n b)$ model in Eq. (6.2a)-(6.2b) may be rewritten in the following polynomial form:

$$
\begin{gathered}
\boldsymbol{A}_{k}(\mathcal{B}) \boldsymbol{y}_{k}[t]=\boldsymbol{B}_{k}(\mathcal{B}) \boldsymbol{x}_{k}[t]+\boldsymbol{w}_{k}[t], \quad k \in\left\{k_{1}, k_{2}, \ldots, k_{M}\right\} \\
\boldsymbol{A}_{k}(\mathcal{B}) \triangleq \boldsymbol{I}_{n y}+\boldsymbol{A}_{1, k} \mathcal{B}+\ldots+\boldsymbol{A}_{n a, k} \mathcal{B}^{n a} \\
\boldsymbol{B}_{k}(\mathcal{B}) \triangleq \boldsymbol{B}_{0, k}+\boldsymbol{B}_{1, k} \mathcal{B}+\ldots+\boldsymbol{B}_{n b, k} \mathcal{B}^{n b}
\end{gathered}
$$

with the matrix polynomials $\boldsymbol{A}_{k}(\mathcal{B}), \boldsymbol{B}_{k}(\mathcal{B})$ having degrees $n a, n b$ respectively, whereas $\mathcal{B}$ designates the backward shift operator. The preceding model satisfies the following assumptions.

\footnotetext{
${ }^{1}$ Boldface upper/ lower case symbols designate matrix/ column-vector quantities, respectively.
} 


\section{Assumptions on the model structure}

A1.1. All zeros of $\operatorname{det}\left(\boldsymbol{A}_{k}(\mathcal{B})\right)$ lie outside the unit circle for the distinct (sample) values $\left\{k_{1}, k_{2}, \ldots, k_{M}\right\}$ ("stability condition").

A1.2. The polynomial matrices $\boldsymbol{A}_{k}(\mathcal{B}), \boldsymbol{B}_{k}(\mathcal{B})$ are left coprime for the distinct (sample) values $\left\{k_{1}, k_{2}\right.$, $\left.\ldots, k_{M}\right\}$.

A1.3. $\operatorname{rank}\left[\boldsymbol{A}_{n a, k} \vdots \boldsymbol{B}_{n b, k}\right]=n y$ for the distinct (sample) values $\left\{k_{1}, k_{2}, \ldots, k_{M}\right\}$ ("identifiability condition”).

\section{Assumptions on $\boldsymbol{x}_{k}[t], \boldsymbol{w}_{k}[t]$ and $k$}

A1.4. The sequence $\boldsymbol{x}_{k}[t]$ is ergodic covariance stationary and is persistently exciting of order $(n b+1)$ for the distinct (sample) values $\left\{k_{1}, k_{2}, \ldots, k_{M}\right\}$.

A1.5. $\boldsymbol{w}_{k}[t]$ is a sequence of independent and identically distributed (i.i.d.) random vectors with zero mean and positive-definite covariance $\boldsymbol{\Sigma}_{k, k}$ (white noise). Furthermore, $\boldsymbol{w}_{k}[t]$ is uncorrelated with $\boldsymbol{x}_{\ell}[t-$ $j], \forall j \geq 0$ and $k, \ell \in\left\{k_{1}, k_{2}, \ldots, k_{M}\right\}$.

A1.6. Each value of the operating parameter $k$ corresponds to a specific operating condition. The operating parameter $k$ is measurable upon operation of the system, bounded (that is, $k \in \mathcal{R}=\left[k_{\min }, k_{\text {max }}\right] \subset$ $\mathbb{R})$, and remains constant during data acquisition.

Remark 1. The assumptions A1.1 and A1.4 ensure the covariance stationarity of the response vectors $\boldsymbol{y}_{k}[t]$ for the distinct (sample) values $\left\{k_{1}, k_{2}, \ldots, k_{M}\right\}$. This means that the responses are completely described by their mean and covariance, whereas to derive the large sample properties of the LS and ML estimators (see Subsection 6.2.3) the sums of $\boldsymbol{y}_{k}[t]$ over time may be replaced with their corresponding expectations.

Remark 2. The assumption A1.2 ensures that there are no common left factors in the AR and X polynomials of the model except for unimodular matrices (that is, matrices with constant determinant), whereas assumption A1.3 ensures that the common left factors are constant matrices. When assumptions A1.2 and A1.3 hold, uniqueness of representation for the P-VARX $(n a, n b)$ model in Eq. (6.2a)-(6.2b) is warranted (see Subsection 6.2.4).

Remark 3. The assumption A1.6 poses some practical conditions in order to obtain a global model. It ensures that (i) the system dynamics at two different operating conditions are not described by the same VARX representation, and (ii) data records suitable for P-VARX identification are obtained (see Subsection 6.2.2).

\subsubsection{P-VARX model parameter estimation}

The P-VARX model estimation problem may be stated as: "Given the data record $Z^{N M}$, identify the model $\mathcal{M}_{P}(\boldsymbol{\vartheta})$ from the following set $\mathcal{M}_{P}$ of all candidate P-VARX models that "best" fits the observed responses":

$$
\begin{aligned}
& \mathcal{M}_{P} \triangleq\left\{\mathcal{M}_{P}(\boldsymbol{\vartheta}): \boldsymbol{A}_{k}(\mathcal{B}, \boldsymbol{\vartheta}) \boldsymbol{y}_{k}[t]=\boldsymbol{B}_{k}(\mathcal{B}, \boldsymbol{\vartheta}) \boldsymbol{x}_{k}[t]+\boldsymbol{e}_{k}[t, \boldsymbol{\vartheta}] \mid \boldsymbol{A}_{k}(\mathcal{B}, \boldsymbol{\vartheta}), \boldsymbol{B}_{k}(\mathcal{B}, \boldsymbol{\vartheta})\right. \\
& \text { obey A1.1 - A1.3, } \left.E\left\{\boldsymbol{e}_{k}[t, \boldsymbol{\vartheta}] \boldsymbol{e}_{\ell}^{T}[t-\tau, \boldsymbol{\vartheta}]\right\}=\boldsymbol{\Sigma}_{k, \ell}(\boldsymbol{\vartheta}) \delta[\tau] \quad k, \ell \in\left\{k_{1}, k_{2}, \ldots, k_{M}\right\}\right\}
\end{aligned}
$$

with $\boldsymbol{e}_{k}[t, \boldsymbol{\vartheta}]$ being the $(n y \times 1)$ residual (one-step-ahead prediction error) vector corresponding to the $k$-th operating condition. The residuals, for an accurate model, should be zero mean, serially uncorrelated, with 
positive-definite covariance matrices $\boldsymbol{\Sigma}_{k, k}(\boldsymbol{\vartheta})$ (white noise; see assumption A1.5 in Section 6.2.1). The P-VARX models are parametrized in terms of the parameter vector $\vartheta$ defined as:

$$
\boldsymbol{\vartheta}=\operatorname{vec}\left(\left[\begin{array}{cccccccc}
\boldsymbol{A}_{1, k_{1}} & \boldsymbol{A}_{2, k_{1}} & \ldots & \boldsymbol{A}_{n a, k_{1}} & \boldsymbol{B}_{0, k_{1}} & \boldsymbol{B}_{1, k_{1}} & \ldots & \boldsymbol{B}_{n b, k_{1}} \\
\boldsymbol{A}_{1, k_{2}} & \boldsymbol{A}_{2, k_{2}} & \ldots & \boldsymbol{A}_{n a, k_{2}} & \boldsymbol{B}_{0, k_{2}} & \boldsymbol{B}_{1, k_{2}} & \ldots & \boldsymbol{B}_{n b, k_{2}} \\
\vdots & \vdots & \ddots & \vdots & \vdots & \vdots & \ddots & \vdots \\
\boldsymbol{A}_{1, k_{M}} & \boldsymbol{A}_{2, k_{M}} & \ldots & \boldsymbol{A}_{n a, k_{M}} & \boldsymbol{B}_{0, k_{M}} & \boldsymbol{B}_{1, k_{M}} & \ldots & \boldsymbol{B}_{n b, k_{M}}
\end{array}\right]^{T}\right)
$$

with $\operatorname{vec}(\cdot)$ designating the operator that transforms the indicated matrix into a vector by stacking its columns.

Parameter estimation is presently based on properly formulated Least Squares (LS) and Maximum Likelihood (ML) estimators.

\subsubsection{LS-based estimators}

The P-VARX $(n a, n b)$ model of Eq. (6.4) may be equivalently expressed in a linear regression form as:

$$
\boldsymbol{y}_{k}[t]=\widetilde{\boldsymbol{\Phi}}_{k}^{T}[t] \boldsymbol{\vartheta}_{k}+\boldsymbol{e}_{k}[t, \boldsymbol{\vartheta}]
$$

where:

$$
\begin{gathered}
\widetilde{\boldsymbol{\Phi}}_{k}^{T}[t]=\boldsymbol{I}_{n y} \otimes \widetilde{\boldsymbol{\phi}}_{k}^{T}[t] \\
\widetilde{\boldsymbol{\phi}}_{k}[t]=\left[-\boldsymbol{y}_{k}^{T}[t-1]-\boldsymbol{y}_{k}^{T}[t-2] \ldots-\boldsymbol{y}_{k}^{T}[t-n a] \vdots \boldsymbol{x}_{k}^{T}[t] \boldsymbol{x}_{k}^{T}[t-1] \ldots \boldsymbol{x}_{k}^{T}[t-n b]\right]^{T} \\
\boldsymbol{\vartheta}_{k}=\operatorname{vec}\left(\left[\boldsymbol{A}_{1, k} \boldsymbol{A}_{2, k} \ldots \boldsymbol{A}_{n a, k} \vdots \boldsymbol{B}_{0, k} \boldsymbol{B}_{1, k} \ldots \boldsymbol{B}_{n b, k}\right]^{T}\right)
\end{gathered}
$$

with $\otimes$ designating Kronecker product ${ }^{2}$.

Model parameter estimation requires stacking the equations of the form (6.6a) for each of the distinct operating conditions $\left\{k_{1}, k_{2}, \ldots, k_{M}\right\}$ one below the other. This procedure is referred to as pooling:

$$
\begin{gathered}
\boldsymbol{y}[t]=\widetilde{\boldsymbol{\Phi}}^{T}[t] \boldsymbol{\vartheta}+\boldsymbol{e}[t, \boldsymbol{\vartheta}] \\
\boldsymbol{y}[t]=\left[\begin{array}{c}
\boldsymbol{y}_{k_{1}}[t] \\
\boldsymbol{y}_{k_{2}}[t] \\
\vdots \\
\boldsymbol{y}_{k_{M}}[t]
\end{array}\right], \widetilde{\boldsymbol{\Phi}}^{T}[t]=\left[\begin{array}{cccc}
\widetilde{\boldsymbol{\Phi}}_{k_{1}}^{T}[t] & \mathbf{0} & \ldots & \mathbf{0} \\
\mathbf{0} & \widetilde{\boldsymbol{\Phi}}_{k_{2}}^{T}[t] & \ldots & \mathbf{0} \\
\vdots & \vdots & \ddots & \vdots \\
\mathbf{0} & \mathbf{0} & \ldots & \widetilde{\boldsymbol{\Phi}}_{k_{M}}^{T}[t]
\end{array}\right], \boldsymbol{\vartheta}=\left[\begin{array}{c}
\boldsymbol{\vartheta}_{k_{1}} \\
\boldsymbol{\vartheta}_{k_{2}} \\
\vdots \\
\boldsymbol{\vartheta}_{k_{M}}
\end{array}\right], \boldsymbol{e}[t, \boldsymbol{\vartheta}]=\left[\begin{array}{c}
\boldsymbol{e}_{k_{1}}[t, \boldsymbol{\vartheta}] \\
\boldsymbol{e}_{k_{2}}[t, \boldsymbol{\vartheta}] \\
\vdots \\
\boldsymbol{e}_{k_{M}}[t, \boldsymbol{\vartheta}]
\end{array}\right]
\end{gathered}
$$

Based upon the above linear regression framework, the parameter vector $\vartheta$ may be estimated using a criterion that minimizes a scalar positive function of the residual sequence $\boldsymbol{e}[t]$ with respect to $\boldsymbol{\vartheta}$, that is ${ }^{3}$ :

$$
\widehat{\boldsymbol{\vartheta}}_{L S}=\arg \min _{\boldsymbol{\vartheta}}\left(\frac{1}{N M} \sum_{t=1}^{N} \boldsymbol{e}^{T}[t, \boldsymbol{\vartheta}] \boldsymbol{H} \boldsymbol{e}[t, \boldsymbol{\vartheta}]\right)
$$

where "arg min" designates minimizing argument, and $\boldsymbol{H}$ is a $(M n y \times M n y)$ symmetric, positive-definite weighting matrix.

\footnotetext{
${ }^{2}$ The Kronecker product of an $(m \times n)$ matrix $\boldsymbol{A}=\left[a_{i, j}\right]$ and a $(p \times q)$ matrix $\boldsymbol{B}=\left[b_{i, j}\right]$ is $\boldsymbol{A} \otimes \boldsymbol{B}=\left[a_{i, j} \boldsymbol{B}\right][43$, p. 3].

${ }^{3}$ Symbols in hat designate estimators/estimates.
} 
The Ordinary Least Squares (OLS) estimator The OLS estimator is based upon minimizing the criterion in Eq. (6.8) for $\boldsymbol{H}=\boldsymbol{I}_{M n y}$, which leads to the following estimators for the parameter vector $\boldsymbol{\vartheta}$ and the covariance matrix $\boldsymbol{\Sigma}$ of the innovations $\boldsymbol{w}[t]$ (defined similarly to $\boldsymbol{e}[t, \boldsymbol{\vartheta}]$ in Eq. (6.7b); see also assumption A1.5 in Section 6.2.1, and Table 2.1 in Chapter 2):

$$
\begin{gathered}
\widehat{\boldsymbol{\vartheta}}_{O L S}=\left[\frac{1}{N M} \sum_{t=1}^{N} \widetilde{\boldsymbol{\Phi}}[t] \widetilde{\boldsymbol{\Phi}}^{T}[t]\right]^{-1}\left[\frac{1}{N M} \sum_{t=1}^{N} \widetilde{\boldsymbol{\Phi}}[t] \boldsymbol{y}[t]\right] \\
\widehat{\boldsymbol{\Sigma}}_{O L S}=\frac{1}{N} \sum_{t=1}^{N} \boldsymbol{e}\left[t, \widehat{\boldsymbol{\vartheta}}_{O L S}\right] \boldsymbol{e}^{T}\left[t, \widehat{\boldsymbol{\vartheta}}_{O L S}\right]
\end{gathered}
$$

The Weighted Least Squares (WLS) estimator The WLS estimator is based upon minimizing the criterion in Eq. (6.8) for $\boldsymbol{H}=\boldsymbol{\Sigma}^{-1}$, which leads to the following estimators for $\boldsymbol{\vartheta}$ and $\boldsymbol{\Sigma}$ :

$$
\begin{gathered}
\widehat{\boldsymbol{\vartheta}}_{W L S}=\left[\frac{1}{N M} \sum_{t=1}^{N} \widetilde{\boldsymbol{\Phi}}[t] \boldsymbol{\Sigma}^{-1} \widetilde{\boldsymbol{\Phi}}^{T}[t]\right]^{-1}\left[\frac{1}{N M} \sum_{t=1}^{N} \widetilde{\boldsymbol{\Phi}}[t] \boldsymbol{\Sigma}^{-1} \boldsymbol{y}[t]\right] \\
\widehat{\boldsymbol{\Sigma}}_{W L S}=\frac{1}{N} \sum_{t=1}^{N} \boldsymbol{e}\left[t, \widehat{\boldsymbol{\vartheta}}_{W L S}\right] \boldsymbol{e}^{T}\left[t, \widehat{\boldsymbol{\vartheta}}_{W L S}\right]
\end{gathered}
$$

Remark 4. Note that $\boldsymbol{\Sigma}$ in Eq. (6.10a) is usually unavailable. Thus, to obtain a practically applicable estimator, an OLS-based estimate of the covariance matrix is inserted into Eq. (6.10a) in order to compute $\widehat{\vartheta}_{W L S}$, while the final residual covariance is obtained as in Eq. (6.10b). The procedure may be continued by inserting the resulting $\widehat{\boldsymbol{\Sigma}}_{W L S}$ into Eq. (6.10a) in order to obtain a new WLS estimate, and repeated until $\widehat{\vartheta}_{W L S}$ converges (iterated WLS estimation).

Remark 5. Note that the LS-based parameter estimation of conventional VARX models, with fully parametrized AR and X polynomial matrices, yields identical OLS and WLS estimators [2, pp. 233-235]. When P-VARX models are considered, these estimators are different (compare Eq. (6.9a) and Eq. (6.10a)) except for special cases, which is in analogy to the FP-VARX model case.

In view of the preceding remark, the following proposition holds for the WLS and OLS estimators.

Proposition 6.2.1. Under the assumption of groupwise heteroscedastic and homoscedastic innovations, the $P-V A R X(n a, n b) W L S$ parameter vector estimator is identical to its OLS counterpart, and equivalent to estimating the parameter vectors of disjoint $\operatorname{VARX}(n a, n b)$ models for each distinct operating condition and stacking them one below the other.

Proof. See Appendix 6.A.1.

\subsubsection{The conditional ML estimator}

Let $\boldsymbol{w}[t]$ be a Gaussian white noise process, that is $\boldsymbol{w}[t] \sim \mathcal{N}(\mathbf{0}, \boldsymbol{\Sigma})$, with $\mathcal{N}(\cdot, \cdot)$ designating the normal distribution with indicated mean and covariance. The ML estimators of $\vartheta$ and $\boldsymbol{\Sigma}$ are:

$$
\begin{gathered}
\widehat{\boldsymbol{\vartheta}}_{M L}=\underset{\boldsymbol{\vartheta}}{\arg \min } \ln \left(\operatorname{det}\left(\frac{1}{N} \sum_{t=1}^{N} \boldsymbol{e}[t, \boldsymbol{\vartheta}] \boldsymbol{e}^{T}[t, \boldsymbol{\vartheta}]\right)\right) \\
\widehat{\boldsymbol{\Sigma}}_{M L}=\frac{1}{N} \sum_{t=1}^{N} \boldsymbol{e}\left[t, \widehat{\boldsymbol{\vartheta}}_{M L}\right] \boldsymbol{e}^{T}\left[t, \widehat{\boldsymbol{\vartheta}}_{M L}\right]
\end{gathered}
$$

with $\ln (\cdot)$ designating the natural logarithm and $\operatorname{det}(\cdot)$ the determinant. Notice that obtaining $\widehat{\vartheta}_{M L}$ requires the use of iterative optimization techniques [2, pp. 212-214]. 


\subsubsection{Large sample properties of the LS and ML type estimators}

The consistency and the asymptotic distribution of the LS and conditional ML type estimators are studied under the assumption of exactly known model structure.

\subsubsection{LS-based estimators}

Theorem 6.2.1. (The LS-based estimator consistency and asymptotic distribution): Let $\boldsymbol{\vartheta}_{o}$ be the system's true parameter vector and $\boldsymbol{\Sigma}_{o}$ the true (positive-definite) covariance matrix of $\boldsymbol{w}[t]$, which is additionally assumed to have bounded fourth-order moments. Consider the vector $\sigma_{o}$, comprising of the non-identical elements on and below the main diagonal of $\boldsymbol{\Sigma}_{o}$, that is $\operatorname{vec}\left(\boldsymbol{\Sigma}_{o}\right)=\boldsymbol{D}_{\text {Mny }} \boldsymbol{\sigma}_{o}$, with $\boldsymbol{D}_{\text {Mny }}$ designating a duplication matrix of proper dimensions. Assuming that $E\left\{\widetilde{\boldsymbol{\Phi}}[t] \widetilde{\boldsymbol{\Phi}}^{T}[t]\right\}$ is a finite positive definite matrix, then the estimators $\widehat{\vartheta}_{L S}$ and $\widehat{\boldsymbol{\Sigma}}_{L S}$ are consistent, that is:

$$
\begin{aligned}
& \widehat{\boldsymbol{\vartheta}}_{L S} \stackrel{p}{\longrightarrow} \boldsymbol{\vartheta}_{O} \quad(N \longrightarrow \infty) \\
& \widehat{\boldsymbol{\Sigma}}_{L S} \stackrel{p}{\longrightarrow} \boldsymbol{\Sigma}_{O} \quad(N \longrightarrow \infty)
\end{aligned}
$$

with “ $\stackrel{p}{\longrightarrow}$ ” designating the limit in probability 4 .

The limiting distribution of $\widehat{\vartheta}_{L S}$ is:

$$
\sqrt{N M}\left(\widehat{\boldsymbol{\vartheta}}_{L S}-\boldsymbol{\vartheta}_{o}\right) \stackrel{d}{\longrightarrow} \mathcal{N}\left(\mathbf{0}, \boldsymbol{P}_{L S}\right) \quad(N \longrightarrow \infty)
$$

with $\boldsymbol{P}_{L S}=M E\left\{\widetilde{\boldsymbol{\Phi}}[t] \boldsymbol{H} \widetilde{\boldsymbol{\Phi}}^{T}[t]\right\}^{-1} E\left\{\widetilde{\boldsymbol{\Phi}}[t] \boldsymbol{H} \boldsymbol{\Sigma}_{o} \boldsymbol{H} \widetilde{\boldsymbol{\Phi}}^{T}[t]\right\} E\left\{\widetilde{\boldsymbol{\Phi}}[t] \boldsymbol{H} \widetilde{\boldsymbol{\Phi}}^{T}[t]\right\}^{-1}$ designating the asymptotic normalized covariance matrix and “ $\stackrel{d}{\longrightarrow}$ " the limit in distribution ${ }^{5}$.

The limiting distribution of $\widehat{\boldsymbol{\sigma}}_{L S}$ is:

$$
\sqrt{N M}\left(\widehat{\boldsymbol{\sigma}}_{L S}-\boldsymbol{\sigma}_{o}\right) \stackrel{d}{\longrightarrow} \mathcal{N}\left(\mathbf{0}, 2 M \boldsymbol{D}_{M n y}^{+}\left(\boldsymbol{\Sigma}_{o} \otimes \boldsymbol{\Sigma}_{o}\right)\left(\boldsymbol{D}_{M n y}^{+}\right)^{T}\right) \quad(N \longrightarrow \infty)
$$

with the superscript “+” designating the Moore-Penrose generalized inverse matrix 6 .

Proof. See Appendix 6.A.2.

Remark 6. The definition of $\boldsymbol{\sigma}$ and $\boldsymbol{D}_{M n y}$ for the P-VARX models is the same as in the FP-VARX model case, since the covariance $\Sigma$ has the same structure for both model classes (see Chapter 2, Table 2.1).

The following propositions and corollaries hold in view of Theorem 6.2.1.

Proposition 6.2.2. (OLS estimator large sample properties): Under the assumptions of Theorem 6.2.1, the estimators $\widehat{\boldsymbol{\vartheta}}_{O L S}$ and $\widehat{\boldsymbol{\Sigma}}_{O L S}$ are consistent, and furthermore $\widehat{\boldsymbol{\vartheta}}_{O L S}$ and $\widehat{\boldsymbol{\sigma}}_{O L S}$ are asymptotically normally distributed, that is:

$$
\sqrt{N M}\left(\widehat{\boldsymbol{\vartheta}}_{O L S}-\boldsymbol{\vartheta}_{o}\right) \stackrel{d}{\longrightarrow} \mathcal{N}\left(\mathbf{0}, \boldsymbol{P}_{O L S}\right) \quad(N \longrightarrow \infty)
$$

with $\boldsymbol{P}_{O L S}=M E\left\{\widetilde{\boldsymbol{\Phi}}[t] \widetilde{\boldsymbol{\Phi}}^{T}[t]\right\}^{-1} E\left\{\widetilde{\boldsymbol{\Phi}}[t] \boldsymbol{\Sigma}_{o} \widetilde{\boldsymbol{\Phi}}^{T}[t]\right\} E\left\{\widetilde{\boldsymbol{\Phi}}[t] \widetilde{\boldsymbol{\Phi}}^{T}[t]\right\}^{-1}$, and:

\footnotetext{
${ }^{4}$ Let $\left\{\boldsymbol{x}_{N}\right\} N=1,2, \ldots$ be an indexed sequence of $(n \times 1)$ stochastic vector variables, and $\boldsymbol{x}$ a $(n \times 1)$ stochastic vector variable. Then, $\boldsymbol{x}_{N} \stackrel{p}{\longrightarrow} \boldsymbol{x}$ if $\forall \epsilon>0, \operatorname{Pr}\left(\left|x_{i N}-x_{i}\right|>\epsilon\right) \longrightarrow \mathbf{0}$ as $N \longrightarrow \infty$ for $i=1, \ldots, n$ [44, pp. 681-682]

${ }^{5}$ Let the joint distribution functions of $\boldsymbol{x}_{N}$ and $\boldsymbol{x}$ be $F_{N}$ and $F$, respectively. Then, $\boldsymbol{x}_{N} \stackrel{d}{\longrightarrow} \boldsymbol{x}$ if for all real vectors $\boldsymbol{c}$ for which $F$ is continuous, $F_{N}(\boldsymbol{c}) \longrightarrow F(c)$ as $N \longrightarrow \infty$ [44, p. 682].

${ }^{6}$ The Moore-Penrose generalized inverse of an $(m \times n)$ matrix $\boldsymbol{A}$ is, $\boldsymbol{A}^{+}=\left(\boldsymbol{A}^{T} \boldsymbol{A}\right)^{-1} \boldsymbol{A}^{T}$ [43, p. 35].
} 


$$
\sqrt{N M}\left(\widehat{\boldsymbol{\sigma}}_{O L S}-\boldsymbol{\sigma}_{o}\right) \stackrel{d}{\longrightarrow} \mathcal{N}\left(\mathbf{0}, 2 M \boldsymbol{D}_{M n y}^{+}\left(\boldsymbol{\Sigma}_{o} \otimes \boldsymbol{\Sigma}_{o}\right)\left(\boldsymbol{D}_{M n y}^{+}\right)^{T}\right) \quad(N \longrightarrow \infty)
$$

Proof. The proof follows directly from Theorem 6.2.1 by setting $\boldsymbol{H}=\boldsymbol{I}_{M n y}$.

Estimates of the covariances for $\widehat{\vartheta}_{O L S}$ and $\widehat{\boldsymbol{\sigma}}_{O L S}$ are thus given by:

$$
\begin{aligned}
\widehat{\boldsymbol{P}}\left(\widehat{\boldsymbol{\vartheta}}_{O L S}\right)= & {\left[\sum_{t=1}^{N} \widetilde{\boldsymbol{\Phi}}[t] \widetilde{\boldsymbol{\Phi}}^{T}[t]\right]^{-1}\left[\sum_{t=1}^{N} \widetilde{\boldsymbol{\Phi}}[t] \widehat{\boldsymbol{\Sigma}}_{O L S} \widetilde{\boldsymbol{\Phi}}^{T}[t]\right]\left[\sum_{t=1}^{N} \widetilde{\boldsymbol{\Phi}}[t] \widetilde{\boldsymbol{\Phi}}^{T}[t]\right]^{-1} } \\
& \widehat{\boldsymbol{P}}\left(\widehat{\boldsymbol{\sigma}}_{O L S}\right)=\frac{2}{N} \boldsymbol{D}_{M n y}^{+}\left(\widehat{\boldsymbol{\Sigma}}_{O L S} \otimes \widehat{\boldsymbol{\Sigma}}_{O L S}\right)\left(\boldsymbol{D}_{M n y}^{+}\right)^{T}
\end{aligned}
$$

with $\boldsymbol{P}(\cdot)$ designating the covariance matrix of the indicated vector.

Corollary 6.2.1. (OLS estimator statistical accuracy): Under the assumptions of Theorem 6.2.1, $\widehat{\vartheta}_{\text {OLS }}$ is asymptotically inefficient, as $\boldsymbol{P}_{O L S}$ does not reach the Cramér-Rao lower bound, except for the cases of groupwise hetroscedastic and homoscedastic innovations, whereas $\widehat{\sigma}_{O L S}$ is always asymptotically efficient.

Proof. See Appendix 6.A.4.1.

Proposition 6.2.3. (WLS estimator large sample properties): Under the assumptions of Theorem 6.2.1, the estimators $\widehat{\vartheta}_{W L S}$ and $\widehat{\boldsymbol{\Sigma}}_{W L S}$ are consistent, and furthermore $\widehat{\vartheta}_{W L S}$ and $\widehat{\boldsymbol{\sigma}}_{W L S}$ are asymptotically normally distributed, that is:

$$
\sqrt{N M}\left(\widehat{\boldsymbol{\vartheta}}_{W L S}-\boldsymbol{\vartheta}_{o}\right) \stackrel{d}{\longrightarrow} \mathcal{N}\left(\mathbf{0}, \boldsymbol{P}_{W L S}\right) \quad(N \longrightarrow \infty)
$$

with $\boldsymbol{P}_{W L S}=M E\left\{\widetilde{\boldsymbol{\Phi}}[t] \boldsymbol{\Sigma}_{o}^{-1} \widetilde{\boldsymbol{\Phi}}^{T}[t]\right\}^{-1}$, and:

$$
\sqrt{N M}\left(\widehat{\boldsymbol{\sigma}}_{W L S}-\boldsymbol{\sigma}_{o}\right) \stackrel{d}{\longrightarrow} \mathcal{N}\left(\mathbf{0}, 2 M \boldsymbol{D}_{M n y}^{+}\left(\boldsymbol{\Sigma}_{o} \otimes \boldsymbol{\Sigma}_{o}\right)\left(\boldsymbol{D}_{M n y}^{+}\right)^{T}\right) \quad(N \longrightarrow \infty)
$$

Proof. The proof follows directly from Theorem 6.2.1 by setting $\boldsymbol{H}=\boldsymbol{\Sigma}_{o}^{-1}$.

Estimates of the covariances for $\widehat{\vartheta}_{W L S}$ and $\widehat{\boldsymbol{\sigma}}_{W L S}$ are thus given by:

$$
\begin{gathered}
\widehat{\boldsymbol{P}}\left(\widehat{\boldsymbol{\vartheta}}_{W L S}\right)=\left[\sum_{t=1}^{N} \widetilde{\boldsymbol{\Phi}}[t] \widehat{\boldsymbol{\Sigma}}_{W L S}^{-1} \widetilde{\boldsymbol{\Phi}}^{T}[t]\right]^{-1} \\
\widehat{\boldsymbol{P}}\left(\widehat{\boldsymbol{\sigma}}_{W L S}\right)=\frac{2}{N} \boldsymbol{D}_{M n y}^{+}\left(\widehat{\boldsymbol{\Sigma}}_{W L S} \otimes \widehat{\boldsymbol{\Sigma}}_{W L S}\right)\left(\boldsymbol{D}_{M n y}^{+}\right)^{T}
\end{gathered}
$$

Corollary 6.2.2. (WLS estimator statistical accuracy): Under the assumptions of Theorem 6.2.1, $\widehat{\boldsymbol{\theta}}_{W L S}$ and $\widehat{\boldsymbol{\sigma}}_{W L S}$ are asymptotically statistically efficient, as their corresponding covariance matrices reach the Cramér-Rao lower bound, thereby providing optimal accuracy.

Proof. See Appendix 6.A.4.2. 


\subsubsection{The conditional ML estimator}

Theorem 6.2.2. (The ML-based estimator consistency and asymptotic distribution): Let $\overline{\boldsymbol{\vartheta}}_{o}=\left[\boldsymbol{\vartheta}_{o}^{T} \vdots \boldsymbol{\sigma}_{o}^{T}\right]^{T}$ be the system's true parameter vector. Assuming that $\boldsymbol{\Sigma}_{o}$ and $E\left\{\widetilde{\boldsymbol{\Phi}}[t] \widetilde{\boldsymbol{\Phi}}^{T}[t]\right\}$ are positive-definite matrices, then the estimator $\widehat{\bar{\vartheta}}_{M L}$ is consistent, that is:

$$
\widehat{\overline{\boldsymbol{\vartheta}}}_{M L} \stackrel{p}{\longrightarrow} \overline{\boldsymbol{\vartheta}}_{o}(N \longrightarrow \infty)
$$

The limiting distribution of $\widehat{\overline{\boldsymbol{\theta}}}_{M L}$ is given by:

$$
\begin{gathered}
\sqrt{N M}\left(\widehat{\overline{\boldsymbol{\vartheta}}}_{M L}-\overline{\boldsymbol{\vartheta}}_{o}\right) \stackrel{d}{\longrightarrow} \mathcal{N}\left(\mathbf{0}, \boldsymbol{P}_{M L}\right) \quad(N \longrightarrow \infty) \\
\text { with, } \boldsymbol{P}_{M L}=\left[\begin{array}{cc}
M E\left\{\widetilde{\boldsymbol{\Phi}}[t] \boldsymbol{\Sigma}_{o}^{-1} \widetilde{\boldsymbol{\Phi}}^{T}[t]\right\}^{-1} & \mathbf{0} \\
\mathbf{0} & 2 M \boldsymbol{D}_{M n y}^{+}\left(\boldsymbol{\Sigma}_{o} \otimes \boldsymbol{\Sigma}_{o}\right)\left(\boldsymbol{D}_{M n y}^{+}\right)^{T}
\end{array}\right] .
\end{gathered}
$$

Proof. See Appendix 6.A.3.

Remark 7. Note that the ML estimator properties are in analogy to those of the FP-VARX and the conventional VAR(X) models [44, p. 93]. Also, the upper left block matrix of $\boldsymbol{P}_{M L}$ corresponds to the asymptotic covariance of $\widehat{\vartheta}_{M L}$, and is identical to its WLS counterpart (see Proposition 6.2.3) suggesting that $\widehat{\vartheta}_{M L}$ and $\widehat{\vartheta}_{W L S}$ are asymptotically equivalent.

By virtue of Proposition 6.2.1, a direct consequence of this equivalence is that for groupwise heteroscedastic and homoscedastic innovations (see Chapter 2, Table 2.1) the P-VARX $(n a, n b)$ ML parameter vector estimator is equivalent to estimating the ML-based parameter vectors of disjoint conventional $\operatorname{VARX}(n a, n b)$ models for each distinct operating condition, and stacking them one below the other.

As in the LS-based case, estimates of the covariances for $\widehat{\vartheta}_{M L}$ and $\widehat{\boldsymbol{\sigma}}_{M L}$ are given by:

$$
\begin{gathered}
\widehat{\boldsymbol{P}}\left(\widehat{\boldsymbol{\vartheta}}_{M L}\right)=\left[\sum_{t=1}^{N} \widetilde{\boldsymbol{\Phi}}[t] \widehat{\boldsymbol{\Sigma}}_{M L}^{-1} \widetilde{\boldsymbol{\Phi}}^{T}[t]\right]^{-1} \\
\widehat{\boldsymbol{P}}\left(\widehat{\boldsymbol{\sigma}}_{M L}\right)=\frac{2}{N} \boldsymbol{D}_{M n y}^{+}\left(\widehat{\boldsymbol{\Sigma}}_{M L} \otimes \widehat{\boldsymbol{\Sigma}}_{M L}\right)\left(\boldsymbol{D}_{M n y}^{+}\right)^{T}
\end{gathered}
$$

An immediate result of Theorem 6.2.2 is the following corollary.

Corollary 6.2.3. (ML estimator statistical accuracy): Under the assumptions of Theorem 6.2.2, $\widehat{\overline{\boldsymbol{\theta}}}_{M L}$ is asymptotically statistically efficient, as $\boldsymbol{P}_{M L}$ reaches the Cramér-Rao lower bound, thereby providing optimal accuracy.

Proof. See Appendix 6.A.4.2.

An overview of the asymptotic properties of the LS and ML type parameter estimators for the different types of innovation dependency is provided in Table 6.1. 
Table 6.1: Overview of asymptotic properties for the LS and ML type P-VARX parameter estimators.

\begin{tabular}{|c|c|c|c|}
\hline Innovation dependency type & $\begin{array}{l}\text { Ordinary Least Squares } \\
\text { (OLS) }\end{array}$ & $\begin{array}{l}\text { Weighted Least Squares } \\
\text { (WLS) }\end{array}$ & $\begin{array}{c}\text { Maximum Likelihood } \\
\text { (ML) }\end{array}$ \\
\hline Contemporaneously correlated & $\begin{array}{c}\text { consistent } \\
\text { normally distributed } \\
\text { statistically inefficient }\end{array}$ & $\begin{array}{c}\text { consistent } \\
\text { normally distributed } \\
\text { statistically efficient }\end{array}$ & $\begin{array}{c}\text { consistent } \\
\text { normally distributed } \\
\text { statistically efficient }\end{array}$ \\
\hline Groupwise heteroscedastic $\dagger$ & $\begin{array}{l}\text { consistent } \\
\text { normally distributed } \\
\text { statistically efficient }\end{array}$ & $\begin{array}{l}\text { consistent } \\
\text { normally distributed } \\
\text { statistically efficient }\end{array}$ & $\begin{array}{l}\text { consistent } \\
\text { normally distributed } \\
\text { statistically efficient }\end{array}$ \\
\hline Groupwise homoscedastic $\dagger$ & $\begin{array}{l}\text { consistent } \\
\text { normally distributed } \\
\text { statistically efficient }\end{array}$ & $\begin{array}{l}\text { consistent } \\
\text { normally distributed } \\
\text { statistically efficient }\end{array}$ & $\begin{array}{c}\text { consistent } \\
\text { normally distributed } \\
\text { statistically efficient }\end{array}$ \\
\hline
\end{tabular}

$\dagger$ Equivalent to estimating disjoint conventional VARX models

\subsubsection{P-VARX model identifiability}

An important issue in P-VARX modeling is to ensure that the identification procedure (i) yields a unique parameter vector $\vartheta$ and (ii) the identified model converges to the exact model structure (when this belongs to $\left.\mathcal{M}_{P}(\boldsymbol{\vartheta})\right)$. In this section the conditions under which the parameters of a P-VARX $(n a, n b)$ model may be recovered by means of the complete data set $Z^{N M}$ are studied.

The conditions for uniqueness of the $\mathrm{P}-\operatorname{VARX}(n a, n b)$ parameter vector are provided by the following theorem (compare to [2, pp.182-183] for the conventional VARX model case).

Theorem 6.2.3. (P-VARX model uniqueness of representation): Consider P-VARX (na,nb) model of Eq. (6.3a)-(6.3c). The model's transfer function matrices:

$$
\boldsymbol{G}_{k}(\mathcal{B}) \triangleq \boldsymbol{A}_{k}^{-1}(\mathcal{B}) \boldsymbol{B}_{k}(\mathcal{B}), \quad k=k_{1}, k_{2}, \ldots, k_{M}
$$

are unique in the class of matrix fraction descriptions of degrees $(n a, n b)$ if and only if:

$$
\begin{aligned}
\boldsymbol{A}_{k}(\mathcal{B}), \boldsymbol{B}_{k}(\mathcal{B}) & : \text { left coprime for } k=k_{1}, k_{2}, \ldots, k_{M} \\
\operatorname{rank}\left[\boldsymbol{A}_{n a, k} \vdots \boldsymbol{B}_{n b, k}\right] & =n y \text { for } k=k_{1}, k_{2}, \ldots, k_{M}
\end{aligned}
$$

Proof. The proof is the same as in the FP-VARX model case.

The following lemma delineates the types of excitation that allow for distinguishing between different P-VARX $(n a, n b)$ models belonging to $\mathcal{M}_{P}$ (compare to [1, p. 456] for the conventional VARX model case).

Lemma 6.2.1. (Informative experiments): Consider the P-VARX (na,nb) models belonging to the set $\mathcal{M}_{P}$ in Eq. (6.4). Then the experiments performed at the distinct (sample) operating conditions $\left\{k_{1}, k_{2}, \ldots, k_{M}\right\}$ using persistently exciting inputs $\boldsymbol{x}_{k}[t]$ of order $(n b+1)$ are sufficiently informative with respect to $\mathcal{M}_{P}$, regardless of the AR polynomial degree, provided that the corresponding residuals $\boldsymbol{e}_{k}[t, \vartheta]$ are persistently exciting.

Proof. The proof is in analogy to the FP-VARX model case. 
Remark 8. Notice that the same requirements for distinguishing between different FP-VARX models are necessary for the P-VARX model case.

The following theorem relates the persistent excitation property with the LS and ML estimator consistency.

Theorem 6.2.4. The consistency of the P-VARX $(n a, n b) L S$ and ML based estimators, provided that the residuals $\boldsymbol{e}_{k}[t, \boldsymbol{\vartheta}]$ are persistently exciting, is satisfied if and only if $\boldsymbol{x}_{k}[t]\left(k=k_{1}, k_{2}, \ldots, k_{M}\right)$ is persistently exciting of order $(n b+1)$.

Proof. See Appendix 6.A.5.

\subsubsection{Comparison of P-VARX and FP-VARX model classes.}

A question of interest is how a P-VARX model compares to its FP-VARX counterpart at the distinct operating conditions $\left\{k_{1}, k_{2}, \ldots, k_{M}\right\}$ used for identification. In this section issues regarding their structure and achievable accuracy are addressed.

\subsubsection{The FP-VARX model specification}

The FP-VARX model class has the following form:

$$
\begin{gathered}
\boldsymbol{y}_{k}[t]+\sum_{i=1}^{n a} \boldsymbol{A}_{i}(k) \boldsymbol{y}_{k}[t-i]=\sum_{i=0}^{n b} \boldsymbol{B}_{i}(k) \boldsymbol{x}_{k}[t-i]+\boldsymbol{w}_{k}[t] \\
E\left\{\boldsymbol{w}_{k}[t] \boldsymbol{w}_{\ell}^{T}[t-\tau]\right\}=\boldsymbol{\Sigma}_{k, \ell} \delta[\tau] \quad k, \ell \in \mathcal{R} \\
\boldsymbol{A}_{i}(k)=\sum_{j=1}^{p a} \boldsymbol{A}_{i, j} G_{j}(k), \quad \boldsymbol{B}_{i}(k)=\sum_{j=1}^{p b} \boldsymbol{B}_{i, j} G_{j}(k)
\end{gathered}
$$

where the AR and X matrices, designated as $\boldsymbol{A}_{i}(k)$ and $\boldsymbol{B}_{i}(k)$, are fully parameterized and have dimensions $(n y \times n y)$ and $(n y \times n x)$, respectively. These matrices are expressed as explicit functions of the operating parameter $k$ by belonging to a functional subspace of dimensionality $p=\max (p a, p b)$ spanned by the mutually independent functions $G_{j}(k)(j=1, \ldots, p)$. The preceding functions form a functional basis, with $\boldsymbol{A}_{i, j}$, and $\boldsymbol{B}_{i, j}$ designtating the corresponding, fully parametrized, projection matrices. The representation of Eq. (6.21a)-(6.21c) is referred to as an FP-VARX model of orders $(n a, n b)$ and functional subspace dimensionalities $(p a, p b)$, or in short as an $\operatorname{FP}-\operatorname{VARX}(n a, n b)_{[p a, p b]}$ model. mial form:

The FP-VARX $(n a, n b)_{[p a, p b]}$ model in Eq. (6.21a)-(6.21c) may be rewritten in the following polyno-

$$
\begin{gathered}
\boldsymbol{A}[\mathcal{B}, k] \boldsymbol{y}_{k}[t]=\boldsymbol{B}[\mathcal{B}, k] \boldsymbol{x}_{k}[t]+\boldsymbol{w}_{k}[t], \quad k \in \mathcal{R} \\
\boldsymbol{A}[\mathcal{B}, k] \triangleq \boldsymbol{I}_{n y}+\boldsymbol{A}_{1}(k) \mathcal{B}+\ldots+\boldsymbol{A}_{n a}(k) \mathcal{B}^{n a} \\
\boldsymbol{B}[\mathcal{B}, k] \triangleq \boldsymbol{B}_{0}(k)+\boldsymbol{B}_{1}(k) \mathcal{B}+\ldots+\boldsymbol{B}_{n b}(k) \mathcal{B}^{n b}
\end{gathered}
$$

The preceding model satisfies the following assumptions. 


\section{Assumptions on the model structure}

A2.1. All zeros of $\operatorname{det}(\boldsymbol{A}[\mathcal{B}, k])$ lie outside the unit circle for the distinct (sample) values $\left\{k_{1}, k_{2}, \ldots, k_{M}\right\}$ ("stability condition").

A2.2. The polynomial matrices $\boldsymbol{A}[\mathcal{B}, k], \boldsymbol{B}[\mathcal{B}, k]$ are left coprime for the distinct (sample) values $\left\{k_{1}, k_{2}\right.$, $\left.\ldots, k_{M}\right\}$ ("irreducibility condition").

A2.3. $\operatorname{rank}\left[\boldsymbol{A}_{n a}(k) \vdots \boldsymbol{B}_{n b}(k)\right]=n y$ for the distinct (sample) values $\left\{k_{1}, k_{2}, \ldots, k_{M}\right\}$ ("identifiability condition”).

A2.4. The basis functions $G_{j}(k)$ and subspace dimensionalities $(p a, p b)$ are known.

\section{Assumptions on $\boldsymbol{x}_{k}[t], \boldsymbol{w}_{k}[t]$ and $k$}

A2.5. The sequence $\boldsymbol{x}_{k}[t]$ is ergodic covariance stationary and is persistently exciting of order $(n b+1)$ for the distinct (sample) values $\left\{k_{1}, k_{2}, \ldots, k_{M}\right\}$.

A2.6. $\boldsymbol{w}_{k}[t]$ is a sequence of independent and identically distributed (i.i.d.) random vectors with zero mean and positive-definite covariance $\boldsymbol{\Sigma}_{k, k}$ (white noise). Furthermore, $\boldsymbol{w}_{k}[t]$ is uncorrelated with $\boldsymbol{x}_{\ell}[t-$ $j], \forall j \geq 0$ and $k, \ell \in \mathcal{R}$.

A2.7. Each value of the operating parameter $k$ corresponds to a specific operating condition. The operating parameter $k$ is measurable upon operation of the system, bounded (that is, $k \in \mathcal{R}=\left[k_{\min }, k_{\max }\right] \subset$ $\mathbb{R})$, and remains constant during data acquisition.

The FP-VARX model estimation problem may be stated as: "Given the data record $Z^{N M}$, identify the model $\mathcal{M}_{F P}(\boldsymbol{\theta})$ from the following set $\mathcal{M}_{F P}$ of all candidate FP-VARX models that "best" fits the observed responses":

$$
\begin{array}{r}
\mathcal{M}_{F P} \triangleq\left\{\mathcal{M}_{F P}(\boldsymbol{\theta}): \boldsymbol{A}[\mathcal{B}, k, \boldsymbol{\theta}] \boldsymbol{y}_{k}[t]=\boldsymbol{B}[\mathcal{B}, k, \boldsymbol{\theta}] \boldsymbol{x}_{k}[t]+\boldsymbol{e}_{k}[t, \boldsymbol{\theta}] \mid \boldsymbol{A}[\mathcal{B}, k, \boldsymbol{\theta}], \boldsymbol{B}[\mathcal{B}, k, \boldsymbol{\theta}]\right. \\
\text { obey A2.1-A } \left.2.4, E\left\{\boldsymbol{e}_{k}[t, \boldsymbol{\theta}] \boldsymbol{e}_{\ell}^{T}[t-\tau, \boldsymbol{\theta}]\right\}=\boldsymbol{\Sigma}_{k, \ell}(\boldsymbol{\theta}) \delta[\tau] \quad k, \ell \in \mathcal{R}\right\}
\end{array}
$$

The FP-VARX models are parametrized in terms of the projection coefficient vector $\boldsymbol{\theta}$ defined as:

$$
\boldsymbol{\theta} \triangleq \operatorname{vec}\left(\left[\boldsymbol{A}_{1,1} \boldsymbol{A}_{1,2} \ldots \boldsymbol{A}_{n a, p a} \vdots \boldsymbol{B}_{0,1} \boldsymbol{B}_{0,2} \ldots \boldsymbol{B}_{n b, p b}\right]^{T}\right)
$$

It is easy to verify that $\mathcal{M}_{F P}$ is a subset of $\mathcal{M}_{P}$ (that is, $\mathcal{M}_{F P} \subset \mathcal{M}_{P}$ ), as any model in the set $\mathcal{M}_{F P}$ belongs also to the set $\mathcal{M}_{P}$. For this purpose, the model of equation Eq. (6.21a) may be expressed in the following linear regression form (compare to Eq. (6.6a)-(6.6d)):

$$
\begin{gathered}
\boldsymbol{y}_{k}[t]=\widetilde{\boldsymbol{\Phi}}_{k}^{T}[t] \boldsymbol{\vartheta}(k)+\boldsymbol{w}_{k}[t] \\
\boldsymbol{\vartheta}(k)=\operatorname{vec}\left(\left[\boldsymbol{A}_{1}(k) \boldsymbol{A}_{2}(k) \ldots \boldsymbol{A}_{n a}(k) \vdots \boldsymbol{B}_{0}(k) \boldsymbol{B}_{1}(k) \ldots \boldsymbol{B}_{n b}(k)\right]^{T}\right)
\end{gathered}
$$

Notice that the parameter vector $\boldsymbol{\vartheta}(k)$ is linearly constraint by the following relationships:

$$
\begin{gathered}
\boldsymbol{\vartheta}(k)=\boldsymbol{T}(k) \boldsymbol{\theta} \quad k \in \mathcal{R} \\
\boldsymbol{\theta} \triangleq \operatorname{vec}\left(\left[\boldsymbol{A}_{1,1} \boldsymbol{A}_{1,2} \ldots \boldsymbol{A}_{n a, p a} \vdots \boldsymbol{B}_{0,1} \boldsymbol{B}_{0,2} \ldots \boldsymbol{B}_{n b, p b}\right]^{T}\right)
\end{gathered}
$$

with $\boldsymbol{T}(k)$ designating a transformation matrix whose entries are zeros and basis functions $G_{j}(k)$ at proper locations (see also Eq. (6.21c), and Chapter 2, Appendix 2.A). 


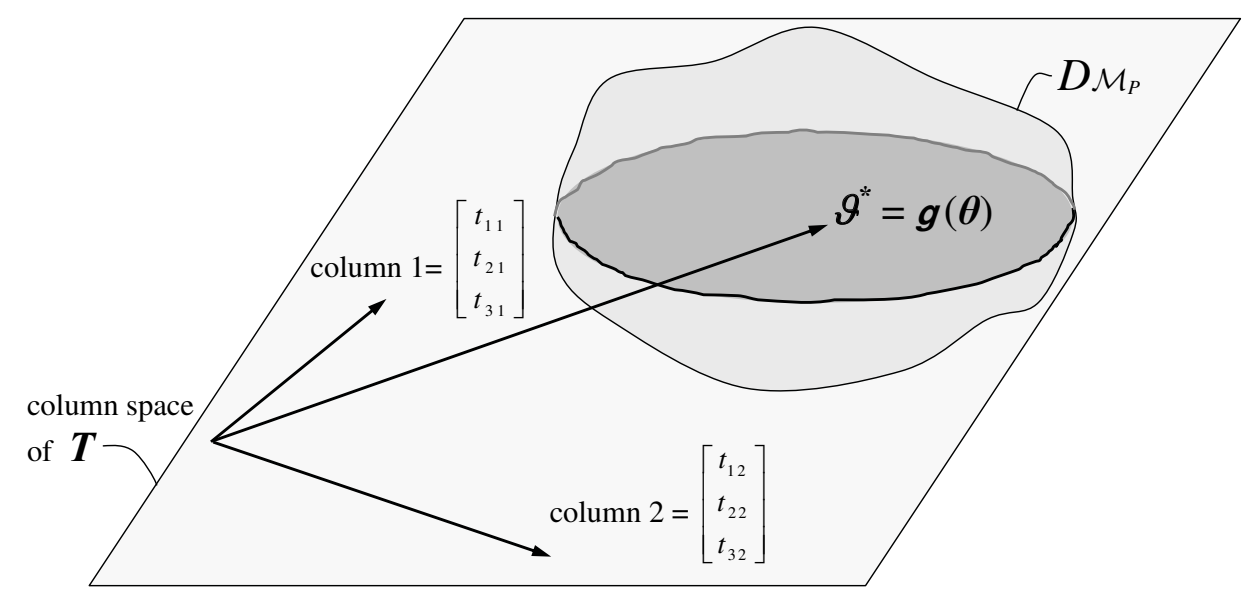

Figure 6.1: Geometric interpretation of relationship between the parameter vectors obtained by the P-VARX (unrestricted) and FP-VARX (restricted) models in a three-dimensional space. The unrestricted $(3 \times 1)$ parameter vector $\boldsymbol{\vartheta}$ belongs to the set $D_{\mathcal{M}_{P}}$, whereas the $(2 \times 1)$ projection coefficient vector $\boldsymbol{\theta}$ belongs to

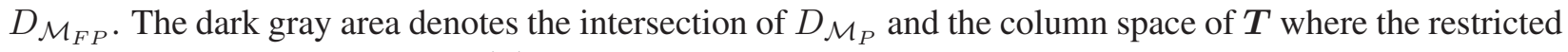
parameter vectors $\boldsymbol{\vartheta}^{*}=\boldsymbol{T} \boldsymbol{\theta}=\boldsymbol{g}(\boldsymbol{\theta})$ lie.

Pooling the expressions of Eq. (6.25a)-(6.25c) for each distinct operating condition $\left\{k_{1}, k_{2}, \ldots, k_{M}\right\}$ yields (compare to (Eq. 6.7a)-(6.7b)):

$$
\boldsymbol{y}[t]=\widetilde{\boldsymbol{\Phi}}^{T}[t] \boldsymbol{\vartheta}^{*}+\boldsymbol{w}[t]
$$

with the linear constraints for the parameter vector $\vartheta^{*}$ given by:

$$
\begin{gathered}
\boldsymbol{\vartheta}^{*}=\boldsymbol{T} \boldsymbol{\theta} \\
\boldsymbol{\vartheta}^{*}=\left[\begin{array}{c}
\boldsymbol{\vartheta}\left(k_{1}\right) \\
\boldsymbol{\vartheta}\left(k_{2}\right) \\
\vdots \\
\boldsymbol{\vartheta}\left(k_{M}\right)
\end{array}\right], \quad \boldsymbol{T}=\left[\begin{array}{c}
\boldsymbol{T}\left(k_{1}\right) \\
\boldsymbol{T}\left(k_{2}\right) \\
\vdots \\
\boldsymbol{T}\left(k_{M}\right)
\end{array}\right]
\end{gathered}
$$

Eq. (6.27b)-(6.27c) imply that for an $\operatorname{FP}-\operatorname{VARX}(n a, n b)_{[p a, p b]}$ model the parameter vector $\boldsymbol{\vartheta}^{*}$ is restricted to lie in the column space of $\boldsymbol{T}$. Hence, the estimation of the FP-VARX $(n a, n b)_{[p a, p b]}$ parameter vector $\vartheta^{*}$ is equivalent to estimating a P-VARX $(n a, n b)$ model with the parameter constraints given in Eq. (6.27b)-(6.27c).

The model classes considered are hierarchical (or nested, see [2, pp. 464-465] and [89] for instance), since the preceding equations imply that $\mathcal{M}_{F P} \subset \mathcal{M}_{P}$. Furthermore, assuming that the FP-VARX projection coefficient vectors $\boldsymbol{\theta}$ satisfying assumptions A2.1-A2.4 belong to the set $D_{\mathcal{M}_{F P}}$, and that the P-VARX parameter vectors satisfying assumptions A1.1-A1.3 belong to the set $D_{\mathcal{M}_{P}}$, then the parameter constraints of Eq. (6.27b)-(6.27c) suggest that the (differentiable) function $\boldsymbol{g}(\boldsymbol{\theta})=\boldsymbol{T} \boldsymbol{\theta}: D_{\mathcal{M}_{F P}} \longrightarrow D_{\mathcal{M}_{P}}$ maps every model in $\mathcal{M}_{F P}$ with projection coefficients $\boldsymbol{\theta}$ to a model in $\mathcal{M}_{P}$ with parameters $\boldsymbol{\vartheta}^{*}$. This result implies that:

$$
\boldsymbol{e}_{k}^{\mathcal{M}_{F P}}[t, \boldsymbol{\theta}]=\boldsymbol{e}_{k}^{\mathcal{M}_{P}}[t, \boldsymbol{g}(\boldsymbol{\theta})] \quad \forall \boldsymbol{\theta} \in D_{\mathcal{M}_{F P}} \text { and } k=k_{1}, k_{2}, \ldots, k_{M}
$$

with $\boldsymbol{e}_{k}^{\mathcal{M}}[t, \cdot]$ designating the residuals corresponding to a model belonging in $\mathcal{M}$. A geometric interpretation of the relationship between the parameter vectors obtained by the FP-VARX and P-VARX models is shown in Fig. 6.1. 


\subsubsection{The parsimony principle for the P-VARX and FP-VARX model classes}

The parsimony principle states that between two identifiable model structures that fit certain data, the one containing the smallest number of parameters will on average give better accuracy [2, p. 464].

In order to study the accuracy of the P-VARX and FP-VARX modeling procedures at the distinct operating conditions $\left\{k_{1}, k_{2}, \ldots, k_{M}\right\}$ used for identification, it is assumed that the true system generating the data set $Z^{N M}$ belongs to $\mathcal{M}_{F P}$. This assumption implies that there exists a unique (true) parameter vector $\boldsymbol{\vartheta}_{o}^{*}=\boldsymbol{g}\left(\boldsymbol{\theta}_{o}\right)$ such that the residuals satisfy: $\boldsymbol{e}_{k}^{\mathcal{M}_{F P}}\left[t, \boldsymbol{\theta}_{o}\right]=\boldsymbol{e}_{k}^{\mathcal{M}_{P}}\left[t, \boldsymbol{g}\left(\boldsymbol{\theta}_{o}\right)\right]=\boldsymbol{w}_{k}[t]$ (white noise) for $k=k_{1}, k_{2}, \ldots, k_{M}$.

In analogy to the conventional model case [2, p. 465], [89] an assessment criterion $W_{\mathcal{M}}(\boldsymbol{\theta})$ is postulated, which is a scalar and differentiable function such that:

$$
\min _{\boldsymbol{\theta}} W_{\mathcal{M}}(\boldsymbol{\theta})=W_{\mathcal{M}}\left(\boldsymbol{\theta}_{o}\right)
$$

Possible choices for $W_{\mathcal{M}}(\boldsymbol{\theta})$ include the determinant of the residual covariance matrix, a weighted norm of the residual vector, and so on.

The accuracy of the model set $\mathcal{M}$ is obtained by means of the accuracy criterion $E\left\{W_{\mathcal{M}}(\widehat{\boldsymbol{\theta}})\right\}$. Notice that the expectation is with respect to $\boldsymbol{\theta}$, so that the criterion is independent of the realization. An asymptotically valid approximation of the accuracy criterion (for consistent parameter estimation methods) is obtained via a second order Taylor expansion ${ }^{7}$ around $\boldsymbol{\theta}_{o}$, that is:

$$
W_{\mathcal{M}}(\widehat{\boldsymbol{\theta}}) \approx W_{\mathcal{M}}\left(\boldsymbol{\theta}_{o}\right)+\left.\frac{\partial W_{\mathcal{M}}(\boldsymbol{\theta})}{\partial \boldsymbol{\theta}^{T}}\right|_{\boldsymbol{\theta}=\boldsymbol{\theta}_{o}}\left(\widehat{\boldsymbol{\theta}}-\boldsymbol{\theta}_{o}\right)+\left.\frac{1}{2}\left(\widehat{\boldsymbol{\theta}}-\boldsymbol{\theta}_{o}\right)^{T} \frac{\partial^{2} W_{\mathcal{M}}(\boldsymbol{\theta})}{\partial \boldsymbol{\theta} \partial \boldsymbol{\theta}^{T}}\right|_{\boldsymbol{\theta}=\boldsymbol{\theta}_{o}}\left(\widehat{\boldsymbol{\theta}}-\boldsymbol{\theta}_{o}\right)
$$

The second term on the right hand of Eq. (6.30) is zero due to Eq. (6.29). Let $\boldsymbol{P}$ be the asymptotic covariance matrix of the estimator $\widehat{\boldsymbol{\theta}}$, then the following expression may be considered as an asymptotic measure of accuracy:

$$
F_{\mathcal{M}}=\lim _{N \longrightarrow \infty} M N E\left\{W_{\mathcal{M}}(\widehat{\boldsymbol{\theta}})-W_{\mathcal{M}}\left(\boldsymbol{\theta}_{o}\right)\right\}=\frac{1}{2} \operatorname{tr}\left(\left.\frac{\partial^{2} W_{\mathcal{M}}(\boldsymbol{\theta})}{\partial \boldsymbol{\theta} \partial \boldsymbol{\theta}^{T}}\right|_{\boldsymbol{\theta}=\boldsymbol{\theta}_{o}} \boldsymbol{P}\right)
$$

with $\operatorname{tr}(\cdot)$ designating the trace of the indicated matrix.

Obviously, for $\mathcal{M}_{F P}$ and $\mathcal{M}_{P}$ it holds that:

$$
W_{\mathcal{M}_{F P}}(\boldsymbol{\theta})=W_{\mathcal{M}_{P}}(\boldsymbol{g}(\boldsymbol{\theta})) \forall \boldsymbol{\theta} \in D_{\mathcal{M}_{F P}}
$$

Hence, the criterion $F_{\mathcal{M}}$ may be used for comparing $\mathcal{M}_{F P}$ and $\mathcal{M}_{P}$, and the following proposition holds:

\footnotetext{
${ }^{7}$ Let $\boldsymbol{\theta}_{N}=\boldsymbol{\theta}_{o}+O_{p}\left(r_{N}\right)$ be a $(n \times 1)$ random vector sequence, where $r_{N}=o(1)$, and let $g: \mathbb{R}^{n} \longrightarrow \mathbb{R}$ be a function with continuous partial derivatives of order two at $\boldsymbol{\theta}_{o}$. Then,

$$
g\left(\boldsymbol{\theta}_{N}\right)=g\left(\boldsymbol{\theta}_{o}\right)+\frac{\partial g\left(\boldsymbol{\theta}_{o}\right)}{\partial \boldsymbol{\theta}^{T}}\left(\boldsymbol{\theta}_{N}-\boldsymbol{\theta}_{o}\right)+O_{p}\left(r_{N}^{2}\right)
$$

If $g$ has continuous partial derivatives of order three,$$
g\left(\boldsymbol{\theta}_{N}\right)=g\left(\boldsymbol{\theta}_{o}\right)+\frac{\partial g\left(\boldsymbol{\theta}_{o}\right)}{\partial \boldsymbol{\theta}^{T}}\left(\boldsymbol{\theta}_{N}-\boldsymbol{\theta}_{o}\right)+\frac{1}{2}\left(\boldsymbol{\theta}_{N}-\boldsymbol{\theta}_{o}\right)^{T} \frac{\partial^{2} g\left(\boldsymbol{\theta}_{o}\right)}{\partial \boldsymbol{\theta} \partial \boldsymbol{\theta}^{T}}\left(\boldsymbol{\theta}_{N}-\boldsymbol{\theta}_{o}\right)+O_{p}\left(r_{N}^{3}\right)
$$

(Let $\left\{a_{N}\right\}$ be a sequence of real numbers and $\left\{b_{N}\right\}$ a sequence of positive real numbers. Then $\left\{a_{N}\right\}$ is said to be of smaller order than $\left\{b_{N}\right\}\left(a_{N}=o\left(b_{N}\right)\right)$ if $a_{N} / b_{N} \longrightarrow 0(N \longrightarrow \infty)$. If $\left\{\boldsymbol{A}_{N}=\left[a_{i j, N}\right]\right\}$ is a sequence of random matrices, then $\boldsymbol{A}_{N}$ is said to be at most of order in probability $\left(\boldsymbol{A}_{N}=O_{p}\left(b_{N}\right)\right)$ if $\forall \epsilon>0, \exists c_{\epsilon}: \forall N \operatorname{Pr}\left(\left|a_{i j, N}\right|>c_{\epsilon} b_{N}\right) \leq \epsilon$ for $i=1,2, \ldots, m$, $j=1,2, \ldots, n)[44$, p. 685].
} 
Proposition 6.2.4. Consider the model sets $\mathcal{M}_{F P}$ and $\mathcal{M}_{P}$ (with $\mathcal{M}_{F P} \subset \mathcal{M}_{P}$ ), and assume that the true system $\mathcal{S}$ generating the data set $Z^{N M}$ at the distinct operating conditions $\left\{k_{1}, k_{2}, \ldots, k_{M}\right\}$ belongs to $\mathcal{M}_{F P}$, so that:

$$
\mathcal{S} \in \mathcal{M}_{F P} \subset \mathcal{M}_{P}
$$

Furthermore, assume that parameter estimation is based on a statistically efficient (LS or ML) method. Then, the FP-VARX modeling leads on the average to more accurate results than the P-VARX modeling at the distinct operating conditions $\left\{k_{1}, k_{2}, \ldots, k_{M}\right\}$ in the sense that:

$$
F_{\mathcal{M}_{F P}} \leq F_{\mathcal{M}_{P}}
$$

Proof. See Appendix 6.A.6.

Remark 9. Notice that the parsimony principle is not valid when inefficient parameter estimation methods are employed. Such situations occur when FP-VARX parameter estimation is based on OLS and the innovations are contemporaneously correlated or groupwise heteroscedastic. In such cases additional requirements are imposed to relax the assumptions of Proposition 6.2.4 (refer to Appendix 6.A.6).

The implication of the parsimony principle on the obtained accuracy of the efficient (LS and ML based) P-VARX and FP-VARX estimators may also be addressed within a constraint parameter estimation framework. This is possible since the FP-VARX model estimation at the distinct operating conditions $\left\{k_{1}, k_{2}, \ldots, k_{M}\right\}$ is equivalent to P-VARX model estimation with parameter constraints, and the following proposition is obtained.

Proposition 6.2.5. Consider the model sets $\mathcal{M}_{F P}$ and $\mathcal{M}_{P}$ (with $\mathcal{M}_{F P} \subset \mathcal{M}_{P}$ ), and assume that the true system $\mathcal{S}$ generating the data set $Z^{N M}$ at the distinct operating conditions $\left\{k_{1}, k_{2}, \ldots, k_{M}\right\}$ belongs to $\mathcal{M}_{F P}$, so that $\mathcal{S} \in \mathcal{M}_{F P} \subset \mathcal{M}_{P}$. If parameter estimation is based on a statistically efficient (LS or $M L$ ) method, then the FP-VARX modeling leads on parameter estimation uncertainties smaller or at most equal to those of $P-V A R X$ modeling at the distinct operating conditions $\left\{k_{1}, k_{2}, \ldots, k_{M}\right\}$.

Proof. See Appendix 6.A.7.

The important result of Propositions 6.2.4 and 6.2.5 is that in the cases of groupwise heteroscedastic and homoscedastic innovations, the FP-VARX models will in general be more accurate than their conventional VARX model counterparts at the same operating conditions.

\subsection{The CCP-VARX model class}

\subsubsection{The CCP-VARX model specification}

The Constant Coefficient Pooled VARX (CCP-VARX) models may be thought of as generalizations of their conventional VARX counterparts in that they simultaneously consider the complete data set $Z^{N M}$. Their main characteristic is the use of (fully parametrized) common and constant over the complete operating range $\mathrm{AR}$ and $\mathrm{X}$ parameter matrices. The models are of the form:

$$
\begin{gathered}
\boldsymbol{y}_{k}[t]+\sum_{i=1}^{n a} \boldsymbol{A}_{i} \boldsymbol{y}_{k}[t-i]=\sum_{i=0}^{n b} \boldsymbol{B}_{i} \boldsymbol{x}_{k}[t-i]+\boldsymbol{w}_{k}[t] \\
E\left\{\boldsymbol{w}_{k}[t] \boldsymbol{w}_{\ell}^{T}[t-\tau]\right\}=\boldsymbol{\Sigma}_{k, \ell} \delta[\tau] \quad k, \ell \in \mathcal{R}
\end{gathered}
$$


with $\boldsymbol{A}_{i}$ and $\boldsymbol{B}_{i}$ designating the fully parametrized AR and X matrices of dimensions $(n y \times n y)$ and $(n y \times$ $n x)$, respectively. The representation of Eq. (6.35a)-(6.35b) is referred to as a CCP-VARX model of orders $(n a, n b)$, or in short as a CCP-VARX $(n a, n b)$ model.

It is worth noting that the assumption of common and constant $\mathrm{AR}$ and $\mathrm{X}$ parameter matrices over the complete operating range $\mathcal{R}$, implies that the CCP-VARX models do not explicitly account for the dynamics of each operating condition but rather "average out" their effects (see also Subsection 6.3.5).

The CCP-VARX $(n a, n b)$ model in Eq. (6.35a)-(6.35b) may be rewritten in the following polynomial form:

$$
\begin{gathered}
\boldsymbol{A}(\mathcal{B}) \boldsymbol{y}_{k}[t]=\boldsymbol{B}(\mathcal{B}) \boldsymbol{x}_{k}[t]+\boldsymbol{w}_{k}[t], \quad k \in \mathcal{R} \\
\boldsymbol{A}(\mathcal{B}) \triangleq \boldsymbol{I}_{n y}+\boldsymbol{A}_{1} \mathcal{B}+\ldots+\boldsymbol{A}_{n a} \mathcal{B}^{n a} \\
\boldsymbol{B}(\mathcal{B}) \triangleq \boldsymbol{B}_{0}+\boldsymbol{B}_{1} \mathcal{B}+\ldots+\boldsymbol{B}_{n b} \mathcal{B}^{n b}
\end{gathered}
$$

and the following assumptions are satisfied.

\section{Assumptions on the model structure}

A3.1. All zeros of $\operatorname{det}(\boldsymbol{A}(\mathcal{B}))$ lie outside the unit circle ("stability condition").

A3.2. The polynomial matrices $\boldsymbol{A}(\mathcal{B}), \boldsymbol{B}(\mathcal{B})$ are left coprime ("irreducibility condition").

A3.3. $\operatorname{rank}\left[\boldsymbol{A}_{n a} \vdots \boldsymbol{B}_{n b}\right]=n y$ (“identifiability condition”).

Assumptions on $\boldsymbol{x}_{k}[t], \boldsymbol{w}_{k}[t]$ and $k$

A3.4. The sequence $\boldsymbol{x}_{k}[t]$ is ergodic covariance stationary and possibly persistently exciting of order $(n b+1)$ at the distinct (sample) values $\left\{k_{1}, k_{2}, \ldots, k_{M}\right\}$.

A3.5. $\boldsymbol{w}_{k}[t]$ is a sequence of independent and identically distributed (i.i.d.) random vectors with zero mean and positive-definite covariance $\boldsymbol{\Sigma}_{k, k}$ (white noise). Furthermore, $\boldsymbol{w}_{k}[t]$ is uncorrelated with $\boldsymbol{x}_{\ell}[t-$ $j], \forall j \geq 0$ and $k, \ell \in \mathcal{R}$.

A3.6. Each value of the operating parameter $k$ corresponds to a specific operating condition. The operating parameter $k$ is measurable upon operation of the system, bounded (that is, $k \in \mathcal{R}=\left[k_{\text {min }}, k_{\text {max }}\right] \subset$ $\mathbb{R})$, and remains constant during data acquisition.

\subsubsection{CCP-VARX model parameter estimation}

The CCP-VARX model estimation problem may be stated as: "Given the data record $Z^{N M}$, identify the model $\mathcal{M}_{C C P}\left(\vartheta^{\#}\right)$ from the following set $\mathcal{M}_{C C P}$ of all candidate P-VARX models that "best" fits the observed responses":

$$
\begin{aligned}
& \mathcal{M}_{C C P} \triangleq\left\{\mathcal{M}_{C C P}\left(\boldsymbol{\vartheta}^{\#}\right): \boldsymbol{A}\left(\mathcal{B}, \boldsymbol{\vartheta}^{\#}\right) \boldsymbol{y}_{k}[t]=\boldsymbol{B}\left(\mathcal{B}, \boldsymbol{\vartheta}^{\#}\right) \boldsymbol{x}_{k}[t]+\boldsymbol{e}_{k}\left[t, \boldsymbol{\vartheta}^{\#}\right] \mid \boldsymbol{A}\left(\mathcal{B}, \boldsymbol{\vartheta}^{\#}\right), \boldsymbol{B}\left(\mathcal{B}, \boldsymbol{\vartheta}^{\#}\right)\right. \\
& \text { obey A3.1 - A3.3, } \left.E\left\{\boldsymbol{e}_{k}\left[t, \boldsymbol{\vartheta}^{\#}\right] \boldsymbol{e}_{\ell}^{T}\left[t-\tau, \boldsymbol{\vartheta}^{\#}\right]\right\}=\boldsymbol{\Sigma}_{k, \ell}\left(\boldsymbol{\vartheta}^{\#}\right) \delta[\tau] \quad k, \ell \in \mathcal{R}\right\}
\end{aligned}
$$

The CCP-VARX models are parametrized in terms of the parameter vector $\vartheta^{\#}$ defined as:

$$
\boldsymbol{\vartheta}^{\#}=\operatorname{vec}\left(\left[\boldsymbol{A}_{1} \boldsymbol{A}_{2} \ldots \boldsymbol{A}_{n a} \vdots \boldsymbol{B}_{0} \boldsymbol{B}_{1} \ldots \boldsymbol{B}_{n b}\right]^{T}\right)
$$

and parameter estimation is presently based on properly formulated Least Squares (LS) and Maximum Likelihood (ML) estimators. 


\subsubsection{LS-based estimators}

The CCP-VARX $(n a, n b)$ model of (6.37) may be equivalently expressed in a linear regression form (compare to Eq. (6.6a)-(6.6d)) as:

$$
\boldsymbol{y}_{k}[t]=\widetilde{\boldsymbol{\Phi}}_{k}^{T}[t] \boldsymbol{\vartheta}^{\#}+\boldsymbol{e}_{k}\left[t, \boldsymbol{\vartheta}^{\#}\right]
$$

Pooling together the equations of the form Eq. (6.39) for each distinct operating condition $\left\{k_{1}, k_{2}\right.$, $\left.\ldots, k_{M}\right\}$, yields (compare to Eq. (6.7a)-(6.7b)):

$$
\begin{gathered}
\boldsymbol{y}[t]=\overline{\boldsymbol{\Phi}}^{T}[t] \boldsymbol{\vartheta}^{\#}+\boldsymbol{e}\left[t, \boldsymbol{\vartheta}^{\#}\right] \\
\boldsymbol{y}[t]=\left[\begin{array}{c}
\boldsymbol{y}_{k_{1}}[t] \\
\boldsymbol{y}_{k_{2}}[t] \\
\vdots \\
\boldsymbol{y}_{k_{M}}[t]
\end{array}\right], \quad \overline{\boldsymbol{\Phi}}^{T}[t]=\left[\begin{array}{c}
\widetilde{\boldsymbol{\Phi}}_{k_{1}}^{T}[t] \\
\widetilde{\boldsymbol{\Phi}}_{k_{2}}^{T}[t] \\
\vdots \\
\widetilde{\boldsymbol{\Phi}}_{k_{M}}^{T}[t]
\end{array}\right], \quad \boldsymbol{e}\left[t, \boldsymbol{\vartheta}^{\#}\right]=\left[\begin{array}{c}
\boldsymbol{e}_{k_{1}}\left[t, \boldsymbol{\vartheta}^{\#}\right] \\
\boldsymbol{e}_{k_{2}}\left[t, \boldsymbol{\vartheta}^{\#}\right] \\
\vdots \\
\boldsymbol{e}_{k_{M}}\left[t, \boldsymbol{\vartheta}^{\#}\right]
\end{array}\right]
\end{gathered}
$$

Based upon the above linear regression framework, the parameter vector $\vartheta^{\#}$ may be estimated using a LS criterion as in Eq. (6.8).

The Ordinary Least Squares (OLS) estimator The OLS estimators for the parameter vector $\vartheta^{\#}$ and the covariance matrix $\boldsymbol{\Sigma}$ of the innovations $\boldsymbol{w}[t]$ are given by:

$$
\begin{gathered}
\widehat{\boldsymbol{\vartheta}}_{O L S}^{\#}=\left[\frac{1}{N M} \sum_{t=1}^{N} \overline{\boldsymbol{\Phi}}[t] \overline{\boldsymbol{\Phi}}^{T}[t]\right]^{-1}\left[\frac{1}{N M} \sum_{t=1}^{N} \overline{\boldsymbol{\Phi}}[t] \boldsymbol{y}[t]\right] \\
\widehat{\boldsymbol{\Sigma}}_{O L S}=\frac{1}{N} \sum_{t=1}^{N} \boldsymbol{e}\left[t, \widehat{\boldsymbol{\vartheta}}_{O L S}^{\#}\right] \boldsymbol{e}^{T}\left[t, \widehat{\boldsymbol{\vartheta}}_{O L S}^{\#}\right]
\end{gathered}
$$

The Weighted Least Squares (WLS) estimator The WLS estimators for $\vartheta^{\#}$ and $\boldsymbol{\Sigma}$ are given by:

$$
\begin{gathered}
\widehat{\boldsymbol{\vartheta}}_{W L S}^{\#}=\left[\frac{1}{N M} \sum_{t=1}^{N} \overline{\mathbf{\Phi}}[t] \boldsymbol{\Sigma}^{-1} \overline{\boldsymbol{\Phi}}^{T}[t]\right]^{-1}\left[\frac{1}{N M} \sum_{t=1}^{N} \overline{\mathbf{\Phi}}[t] \boldsymbol{\Sigma}^{-1} \boldsymbol{y}[t]\right] \\
\widehat{\boldsymbol{\Sigma}}_{W L S}=\frac{1}{N} \sum_{t=1}^{N} \boldsymbol{e}\left[t, \widehat{\boldsymbol{\vartheta}}_{W L S}^{\#}\right] \boldsymbol{e}^{T}\left[t, \widehat{\boldsymbol{\vartheta}}_{W L S}^{\#}\right]
\end{gathered}
$$

Remark 10. As in the P-VARX model case (see Section 6.2.2.1) $\boldsymbol{\Sigma}$ in Eq. (6.42a) is usually unavailable. Thus, to obtain a practically applicable estimator, an OLS-based estimate of the covariance matrix is inserted into Eq. (6.42a) in order to compute $\widehat{\vartheta}_{W L S}^{\#}$, while the final residual covariance is obtained as in Eq. (6.42b). The procedure may be continued by inserting the resulting $\widehat{\Sigma}_{W L S}$ into Eq. (6.42a) in order to obtain a new WLS estimate, and repeated until $\widehat{\vartheta}_{W L S}^{\#}$ converges (iterated WLS estimation).

Remark 11. In analogy to the P-VARX and FP-VARX model case, the CCP-VARX OLS and WLS estimators are not equivalent except for special cases.

In view of the preceding remark, the following proposition holds for the WLS and OLS estimators.

Proposition 6.3.1. Under the assumption of groupwise heteroscedastic innovations, the WLS parameter vector estimator is identical to its OLS counterpart.

Proof. The proof is in analogy to the corresponding FP-VARX model case. 


\subsubsection{The conditional ML estimator}

Let $\boldsymbol{w}[t]$ be a Gaussian white noise process, that is $\boldsymbol{w}[t] \sim \mathcal{N}(\mathbf{0}, \boldsymbol{\Sigma})$, with $\mathcal{N}(\cdot, \cdot)$ designating the normal distribution with indicated mean and covariance. The ML estimators of $\vartheta^{\#}$ and $\boldsymbol{\Sigma}$ are:

$$
\begin{gathered}
\widehat{\boldsymbol{\vartheta}}_{M L}^{\#}=\underset{\boldsymbol{\vartheta}^{\#}}{\arg \min } \ln \left(\operatorname{det}\left(\frac{1}{N} \sum_{t=1}^{N} \boldsymbol{e}\left[t, \boldsymbol{\vartheta}^{\#}\right] \boldsymbol{e}^{T}\left[t, \boldsymbol{\vartheta}^{\#}\right]\right)\right) \\
\widehat{\boldsymbol{\Sigma}}_{M L}=\frac{1}{N} \sum_{t=1}^{N} \boldsymbol{e}\left[t, \widehat{\boldsymbol{\vartheta}}_{M L}^{\#}\right] \boldsymbol{e}^{T}\left[t, \widehat{\boldsymbol{\vartheta}}_{M L}^{\#}\right]
\end{gathered}
$$

Notice that in analogy to the P-VARX and FP-VARX model case, the computation of $\widehat{\boldsymbol{\vartheta}}_{M L}^{\#}$ requires the use of iterative optimization techniques [2, pp. 212-214].

\subsubsection{Large sample properties of the LS and ML type estimators}

The consistency and the asymptotic distribution of the LS and conditional ML type estimators are studied under the assumption of exactly known structure.

\subsubsection{LS-based estimators}

Theorem 6.3.1. (The LS-based estimator consistency and asymptotic distribution): Let $\boldsymbol{\vartheta}_{O}^{\#}$ be the system's true parameter vector and $\boldsymbol{\Sigma}_{o}$ the true (positive-definite) covariance matrix of $\boldsymbol{w}[t]$, which is additionally assumed to have bounded fourth-order moments. Consider the vector $\sigma_{o}$, comprising of the non-identical elements on and below the main diagonal of $\boldsymbol{\Sigma}_{o}$. Assuming that $E\left\{\overline{\mathbf{\Phi}}[t] \overline{\boldsymbol{\Phi}}^{T}[t]\right\}$ is a finite positive definite matrix, then the estimators $\widehat{\vartheta}_{L S}^{\#}$ and $\widehat{\boldsymbol{\Sigma}}_{L S}$ are consistent, that is:

$$
\begin{aligned}
& \widehat{\boldsymbol{\vartheta}}_{L S}^{\#} \stackrel{p}{\longrightarrow} \boldsymbol{\vartheta}_{o}^{\#} \quad(N \longrightarrow \infty) \\
& \widehat{\boldsymbol{\Sigma}}_{L S} \stackrel{p}{\longrightarrow} \boldsymbol{\Sigma}_{o} \quad(N \longrightarrow \infty)
\end{aligned}
$$

The limiting distribution of $\widehat{\vartheta}_{L S}^{\#}$ is:

$$
\sqrt{N M}\left(\widehat{\boldsymbol{\vartheta}}_{L S}^{\#}-\boldsymbol{\vartheta}_{o}^{\#}\right) \stackrel{d}{\longrightarrow} \mathcal{N}\left(\mathbf{0}, \boldsymbol{P}_{L S}\right) \quad(N \longrightarrow \infty)
$$

with $\boldsymbol{P}_{L S}=M E\left\{\overline{\boldsymbol{\Phi}}[t] \boldsymbol{H} \overline{\boldsymbol{\Phi}}^{T}[t]\right\}^{-1} E\left\{\overline{\boldsymbol{\Phi}}[t] \boldsymbol{H} \boldsymbol{\Sigma}_{o} \boldsymbol{H} \overline{\boldsymbol{\Phi}}^{T}[t]\right\} E\left\{\overline{\mathbf{\Phi}}[t] \boldsymbol{H} \overline{\boldsymbol{\Phi}}^{T}[t]\right\}^{-1}$ designating the asymptotic normalized covariance matrix.

The limiting distribution of $\widehat{\sigma}_{L S}$ is:

$$
\sqrt{N M}\left(\widehat{\boldsymbol{\sigma}}_{L S}-\boldsymbol{\sigma}_{o}\right) \stackrel{d}{\longrightarrow} \mathcal{N}\left(\mathbf{0}, 2 M \boldsymbol{D}_{M n y}^{+}\left(\boldsymbol{\Sigma}_{o} \otimes \boldsymbol{\Sigma}_{o}\right)\left(\boldsymbol{D}_{M n y}^{+}\right)^{T}\right) \quad(N \longrightarrow \infty)
$$

Proof. The proof is in analogy to the corresponding FP-VARX model case.

Remark 12. The definition of $\boldsymbol{\sigma}$ and $\boldsymbol{D}_{M n y}$ for the CCP-VARX models is the same as in the P-VARX and FP-VARX model case, since all global model classes share the same structure for the covariance matrix $\boldsymbol{\Sigma}$ (see Chapter 2, Table 2.1).

The following propositions and corollaries hold in view of Theorem 6.3.1. 
Proposition 6.3.2. (OLS estimator large sample properties): Under the assumptions of Theorem 6.3.1, the estimators $\widehat{\boldsymbol{\vartheta}}_{O L S}^{\#}$ and $\widehat{\boldsymbol{\Sigma}}_{O L S}$ are consistent, and furthermore $\widehat{\boldsymbol{\vartheta}}_{O L S}^{\#}$ and $\widehat{\boldsymbol{\sigma}}_{O L S}$ are asymptotically normally distributed, that is:

$$
\sqrt{N M}\left(\widehat{\boldsymbol{\vartheta}}_{O L S}^{\#}-\boldsymbol{\vartheta}_{O}^{\#}\right) \stackrel{d}{\longrightarrow} \mathcal{N}\left(\mathbf{0}, \boldsymbol{P}_{O L S}\right) \quad(N \longrightarrow \infty)
$$

with $\boldsymbol{P}_{O L S}=M E\left\{\overline{\boldsymbol{\Phi}}[t] \overline{\mathbf{\Phi}}^{T}[t]\right\}^{-1} E\left\{\overline{\mathbf{\Phi}}[t] \boldsymbol{\Sigma}_{o} \overline{\boldsymbol{\Phi}}^{T}[t]\right\} E\left\{\overline{\boldsymbol{\Phi}}[t] \overline{\boldsymbol{\Phi}}^{T}[t]\right\}^{-1}$, and:

$$
\sqrt{N M}\left(\widehat{\boldsymbol{\sigma}}_{O L S}-\boldsymbol{\sigma}_{o}\right) \stackrel{d}{\longrightarrow} \mathcal{N}\left(\mathbf{0}, 2 M \boldsymbol{D}_{M n y}^{+}\left(\boldsymbol{\Sigma}_{o} \otimes \boldsymbol{\Sigma}_{o}\right)\left(\boldsymbol{D}_{M n y}^{+}\right)^{T}\right) \quad(N \longrightarrow \infty)
$$

Proof. The proof follows directly from Theorem 6.3 .1 by setting $\boldsymbol{H}=\boldsymbol{I}_{M n y}$.

Estimates of the covariances for $\widehat{\boldsymbol{\vartheta}}_{O L S}^{\#}$ and $\widehat{\boldsymbol{\sigma}}_{O L S}$ are thus given by:

$$
\begin{aligned}
& \widehat{\boldsymbol{P}}\left(\widehat{\boldsymbol{\vartheta}}_{O L S}^{\#}\right)=\left[\sum_{t=1}^{N} \overline{\mathbf{\Phi}}[t] \overline{\mathbf{\Phi}}^{T}[t]\right]^{-1}\left[\sum_{t=1}^{N} \overline{\mathbf{\Phi}}[t] \widehat{\boldsymbol{\Sigma}}_{O L S} \overline{\mathbf{\Phi}}^{T}[t]\right]\left[\sum_{t=1}^{N} \overline{\mathbf{\Phi}}[t] \overline{\mathbf{\Phi}}^{T}[t]\right]^{-1} \\
& \widehat{\boldsymbol{P}}\left(\widehat{\boldsymbol{\sigma}}_{O L S}\right)=\frac{2}{N} \boldsymbol{D}_{M n y}^{+}\left(\widehat{\boldsymbol{\Sigma}}_{O L S} \otimes \widehat{\boldsymbol{\Sigma}}_{O L S}\right)\left(\boldsymbol{D}_{M n y}^{+}\right)^{T}
\end{aligned}
$$

Corollary 6.3.1. (OLS estimator statistical accuracy): Under the assumptions of Theorem 6.3.1, $\widehat{\vartheta}_{O L S}^{\#}$ is asymptotically inefficient, as $\boldsymbol{P}_{O L S}$ does not reach the Cramér-Rao lower bound, except for the cases of groupwise hetroscedastic and homoscedastic innovations, whereas $\widehat{\sigma}_{O L S}$ is always asymptotically efficient.

Proof. The proof is in analogy to the corresponding FP-VARX model case.

Proposition 6.3.3. (WLS estimator large sample properties): Under the assumptions of Theorem 6.3.1, the estimators $\widehat{\boldsymbol{\vartheta}}_{W L S}^{\#}$ and $\widehat{\boldsymbol{\Sigma}}_{W L S}$ are consistent, and furthermore $\widehat{\boldsymbol{\vartheta}}_{W L S}^{\#}$ and $\widehat{\boldsymbol{\sigma}}_{W L S}$ are asymptotically normally distributed, that is:

$$
\sqrt{N M}\left(\widehat{\boldsymbol{\vartheta}}_{W L S}^{\#}-\boldsymbol{\vartheta}_{o}^{\#}\right) \stackrel{d}{\longrightarrow} \mathcal{N}\left(\mathbf{0}, \boldsymbol{P}_{W L S}\right) \quad(N \longrightarrow \infty)
$$

with $\boldsymbol{P}_{W L S}=M E\left\{\overline{\boldsymbol{\Phi}}[t] \boldsymbol{\Sigma}_{o}^{-1} \overline{\boldsymbol{\Phi}}^{T}[t]\right\}^{-1}$, and:

$$
\sqrt{N M}\left(\widehat{\boldsymbol{\sigma}}_{W L S}-\boldsymbol{\sigma}_{o}\right) \stackrel{d}{\longrightarrow} \mathcal{N}\left(\mathbf{0}, 2 M \boldsymbol{D}_{M n y}^{+}\left(\boldsymbol{\Sigma}_{o} \otimes \boldsymbol{\Sigma}_{o}\right)\left(\boldsymbol{D}_{M n y}^{+}\right)^{T}\right) \quad(N \longrightarrow \infty)
$$

Proof. The proof follows directly from Theorem 6.3 .1 by setting $\boldsymbol{H}=\boldsymbol{\Sigma}_{o}^{-1}$.

Estimates of the covariances for $\widehat{\boldsymbol{\vartheta}}_{W L S}^{\#}$ and $\widehat{\boldsymbol{\sigma}}_{W L S}$ are thus given by:

$$
\begin{gathered}
\widehat{\boldsymbol{P}}\left(\widehat{\boldsymbol{\vartheta}}_{W L S}^{\#}\right)=\left[\sum_{t=1}^{N} \overline{\boldsymbol{\Phi}}[t] \widehat{\boldsymbol{\Sigma}}_{W L S}^{-1} \overline{\boldsymbol{\Phi}}^{T}[t]\right]^{-1} \\
\widehat{\boldsymbol{P}}\left(\widehat{\boldsymbol{\sigma}}_{W L S}\right)=\frac{2}{N} \boldsymbol{D}_{M n y}^{+}\left(\widehat{\boldsymbol{\Sigma}}_{W L S} \otimes \widehat{\boldsymbol{\Sigma}}_{W L S}\right)\left(\boldsymbol{D}_{M n y}^{+}\right)^{T}
\end{gathered}
$$

Corollary 6.3.2. (WLS estimator statistical accuracy): Under the assumptions of Theorem 6.3.1, $\widehat{\boldsymbol{\vartheta}}_{W L S}^{\#}$ and $\widehat{\boldsymbol{\sigma}}_{W L S}$ are asymptotically statistically efficient, as their corresponding covariance matrices reach the Cramér-Rao lower bound, thereby providing optimal accuracy.

Proof. The proof is in analogy to the corresponding FP-VARX model case. 


\subsubsection{The conditional ML estimator}

Theorem 6.3.2. (The ML-based estimator consistency and asymptotic distribution): Let $\overline{\boldsymbol{\vartheta}}_{o}^{\#}=\left[\boldsymbol{\vartheta}_{o}^{\# T}: \boldsymbol{\sigma}_{o}^{T}\right]^{T}$ be the system's true parameter vector. Assuming that $\boldsymbol{\Sigma}_{o}$ and $E\left\{\overline{\boldsymbol{\Phi}}[t] \overline{\boldsymbol{\Phi}}^{T}[t]\right\}$ are positive-definite matrices, then the estimator $\widehat{\bar{\vartheta}}_{M L}^{\#}$ is consistent, that is:

$$
\widehat{\overline{\boldsymbol{\vartheta}}}_{M L}^{\#} \stackrel{p}{\longrightarrow} \overline{\boldsymbol{\vartheta}}_{o}^{\#} \quad(N \longrightarrow \infty)
$$

The limiting distribution of the ML estimator $\widehat{\overline{\boldsymbol{\theta}}}_{M L}$ is given by:

$$
\sqrt{N M}\left(\widehat{\overline{\boldsymbol{\vartheta}}}_{M L}^{\#}-\overline{\boldsymbol{\vartheta}}_{o}^{\#}\right) \stackrel{d}{\longrightarrow} \mathcal{N}\left(\mathbf{0}, \boldsymbol{P}_{M L}\right) \quad(N \longrightarrow \infty)
$$

with, $\boldsymbol{P}_{M L}=\left[\begin{array}{cc}M E\left\{\overline{\boldsymbol{\Phi}}[t] \boldsymbol{\Sigma}_{o}^{-1} \overline{\boldsymbol{\Phi}}^{T}[t]\right\}^{-1} & \mathbf{0} \\ \mathbf{0} & 2 M \boldsymbol{D}_{M n y}^{+}\left(\boldsymbol{\Sigma}_{o} \otimes \boldsymbol{\Sigma}_{o}\right)\left(\boldsymbol{D}_{M n y}^{+}\right)^{T}\end{array}\right]$.

Proof. The proof is in analogy to the corresponding FP-VARX model case.

Remark 13. Note that the ML estimator properties are in analogy to those of the other global (P-VARX and FP-VARX) and conventional VAR(X) models [44, p. 93]. Also, the upper left block matrix of $\boldsymbol{P}_{M L}$ corresponds to the asymptotic covariance of $\widehat{\vartheta}_{M L}^{\#}$, and is identical to its WLS counterpart (see Proposition 6.3.3) suggesting that $\widehat{\boldsymbol{\vartheta}}_{M L}^{\#}$ and $\widehat{\boldsymbol{\vartheta}}_{W L S}^{\#}$ are asymptotically equivalent.

As in the LS-based case, estimates of the covariances for $\widehat{\overline{\boldsymbol{\vartheta}}}_{M L}^{\#}$ and $\widehat{\boldsymbol{\sigma}}_{M L}$ are given by:

$$
\begin{gathered}
\widehat{\boldsymbol{P}}\left(\widehat{\overline{\boldsymbol{\vartheta}}}_{M L}^{\#}\right)=\left[\sum_{t=1}^{N} \overline{\boldsymbol{\Phi}}[t] \widehat{\boldsymbol{\Sigma}}_{M L}^{-1} \overline{\boldsymbol{\Phi}}^{T}[t]\right]^{-1} \\
\widehat{\boldsymbol{P}}\left(\widehat{\boldsymbol{\sigma}}_{M L}\right)=\frac{2}{N} \boldsymbol{D}_{M n y}^{+}\left(\widehat{\boldsymbol{\Sigma}}_{M L} \otimes \widehat{\boldsymbol{\Sigma}}_{M L}\right)\left(\boldsymbol{D}_{M n y}^{+}\right)^{T}
\end{gathered}
$$

An immediate result of Theorem 6.3.2 is the following corollary.

Corollary 6.3.3. (ML estimator statistical accuracy): Under the assumptions of Theorem 6.3.2, $\widehat{\overline{\boldsymbol{\vartheta}}}_{M L}^{\#}$ is asymptotically statistically efficient, as $\boldsymbol{P}_{M L}$ reaches the Cramér-Rao lower bound, thereby providing optimal accuracy.

Proof. The proof is in analogy to the corresponding FP-VARX model case.

An overview of the asymptotic properties of the LS and ML type parameter estimators for the different types of innovation dependency is provided in Table 6.2.

\subsubsection{CCP-VARX model identifiability}

An important issue in CCP-VARX modeling is to ensure that the identification procedure (i) yields a unique parameter vector $\vartheta^{\#}$ and (ii) the identified model converges to the exact model structure (when this belongs to $\mathcal{M}\left(\vartheta^{\#}\right)$ ). In this section the conditions under which the parameters of a CCP-VARX $(n a, n b)$ model may be recovered by means of the complete data set $Z^{N M}$ are studied.

The conditions for uniqueness of the CCP-VARX $(n a, n b)$ parameter vector are provided by the following corollary. 
Table 6.2: Overview of asymptotic properties for the LS and ML type CCP-VARX parameter estimators.

\begin{tabular}{|c|c|c|c|}
\hline Innovation dependency type & $\begin{array}{l}\text { Ordinary Least Squares } \\
\text { (OLS) }\end{array}$ & $\begin{array}{l}\text { Weighted Least Squares } \\
\text { (WLS) }\end{array}$ & $\begin{array}{l}\text { Maximum Likelihood } \\
\text { (ML) }\end{array}$ \\
\hline Contemporaneously correlated & $\begin{array}{c}\text { consistent } \\
\text { normally distributed } \\
\text { statistically inefficient }\end{array}$ & $\begin{array}{l}\text { consistent } \\
\text { normally distributed } \\
\text { statistically efficient }\end{array}$ & $\begin{array}{l}\text { consistent } \\
\text { normally distributed } \\
\text { statistically efficient }\end{array}$ \\
\hline Groupwise heteroscedastic $\dagger$ & $\begin{array}{c}\text { consistent } \\
\text { normally distributed } \\
\text { statistically inefficient }\end{array}$ & $\begin{array}{l}\text { consistent } \\
\text { normally distributed } \\
\text { statistically efficient }\end{array}$ & $\begin{array}{l}\text { consistent } \\
\text { normally distributed } \\
\text { statistically efficient }\end{array}$ \\
\hline Groupwise homoscedastic $\ddagger$ & $\begin{array}{l}\text { consistent } \\
\text { normally distributed } \\
\text { statistically efficient }\end{array}$ & $\begin{array}{l}\text { consistent } \\
\text { normally distributed } \\
\text { statistically efficient }\end{array}$ & $\begin{array}{l}\text { consistent } \\
\text { normally distributed } \\
\text { statistically efficient }\end{array}$ \\
\hline
\end{tabular}

$\dagger$ WLS/ML estimate equivalent to a weighted average of disjoint conventional VARX models

$\ddagger$ OLS/WLS/ML estimates equivalent to a weighted average of disjoint conventional VARX models

Corollary 6.3.4. (CCP-VARX model uniqueness of representation): Consider the CCP-VARX(na,nb) model of Eq. (6.36a)-(6.36c). The model's transfer function matrix:

$$
\boldsymbol{G}(\mathcal{B}) \triangleq \boldsymbol{A}^{-1}(\mathcal{B}) \boldsymbol{B}(\mathcal{B})
$$

is unique in the class of matrix fraction descriptions of degrees $(n a, n b)$ if and only if:

$$
\begin{aligned}
\boldsymbol{A}(\mathcal{B}), \boldsymbol{B}(\mathcal{B}) & : \text { left coprime } \\
\operatorname{rank}\left[\boldsymbol{A}_{n a} \vdots \boldsymbol{B}_{n b}\right] & =n y
\end{aligned}
$$

Proof. The proof is the same as in the case of conventional VARX models [2, pp. 182-183].

The following theorem relates the "persistence of excitation" concept of the conventional VARX model case [1, pp. 411-415 and p. 456] to the consistency of CCP-VARX model LS and ML based estimators.

Theorem 6.3.3. The consistency of the CCP-VARX (na,nb) LS and ML based estimators, provided that the residuals $\boldsymbol{e}_{k}[t, \vartheta]$ are persistently exciting, is satisfied if and only if:

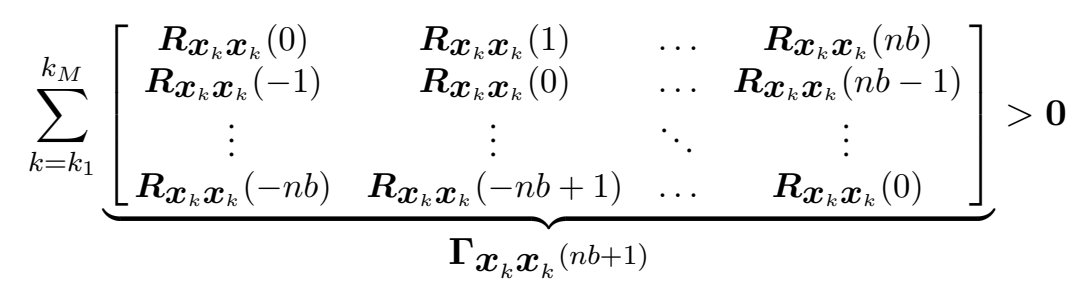

with $\boldsymbol{R}_{\boldsymbol{x}_{k} \boldsymbol{x}_{k}}[\tau]=E\left\{\boldsymbol{x}_{k}[t] \boldsymbol{x}_{k}[t-\tau]^{T}\right\}$.

Proof. See Appendix 6.B.1.

\subsubsection{Comparison of CCP-VARX and conventional VARX models}

Consider the complete data set $Z^{N M}$ and assume that a conventional $\operatorname{VARX}(n a, n b)$ model with fully parametrized AR and $\mathrm{X}$ matrices is estimated for each distinct (sample) operating condition $\left\{k_{1}, k_{2}, \ldots, k_{M}\right\}$. 
Let $\widehat{\boldsymbol{\vartheta}}_{k}$ designate the $\operatorname{VARX}(n a, n b)$ parameter vector estimate of the $k$-th operating condition, and $\widehat{\boldsymbol{P}}\left(\widehat{\boldsymbol{\vartheta}}_{k}\right)$ the corresponding covariance matrix estimate. The data records are assumed to be "well-separated", so that the different estimates may be regarded as independent. Then, the optimal combination of these conventional $\operatorname{VARX}(n a, n b)$ based estimates, yielding an estimate of smallest variance, is given by the following relationships [1, p. 465]:

$$
\begin{aligned}
& \widehat{\boldsymbol{\vartheta}}^{\#}=\widehat{\boldsymbol{P}}\left(\widehat{\boldsymbol{\vartheta}}^{\#}\right) \sum_{k=k_{1}}^{k_{M}} \widehat{\boldsymbol{P}}\left(\widehat{\boldsymbol{\vartheta}}_{k}\right)^{-1} \widehat{\boldsymbol{\vartheta}}_{k} \\
& \widehat{\boldsymbol{P}}\left(\widehat{\boldsymbol{\vartheta}}^{\#}\right)=\left[\sum_{k=k_{1}}^{k_{M}} \widehat{\boldsymbol{P}}\left(\widehat{\boldsymbol{\vartheta}}_{k}\right)^{-1}\right]^{-1}
\end{aligned}
$$

The LS estimates for each distinct operating condition $k\left(k=k_{1}, k_{2}, \ldots, k_{M}\right)$, are given by:

$$
\begin{gathered}
\widehat{\boldsymbol{\vartheta}}_{k}=\left[\sum_{t=1}^{N} \widetilde{\boldsymbol{\Phi}}_{k}[t] \widetilde{\boldsymbol{\Phi}}_{k}^{T}[t]\right]^{-1}\left[\sum_{t=1}^{N} \widetilde{\boldsymbol{\Phi}}_{k}[t] \boldsymbol{y}_{k}[t]\right] \\
\widehat{\boldsymbol{P}}\left(\widehat{\boldsymbol{\vartheta}}_{k}\right)=\left[\sum_{t=1}^{N} \widetilde{\boldsymbol{\Phi}}_{k}[t] \widehat{\boldsymbol{\Sigma}}_{k, k}^{-1} \widetilde{\boldsymbol{\Phi}}_{k}^{T}[t]\right]^{-1} \\
\widehat{\boldsymbol{\Sigma}}_{k, k}=\frac{1}{N} \sum_{t=1}^{N} \boldsymbol{e}_{k}\left[t, \widehat{\boldsymbol{\vartheta}}_{k}\right] \boldsymbol{e}_{k}^{T}\left[t, \widehat{\boldsymbol{\vartheta}}_{k}\right]
\end{gathered}
$$

Notice that the OLS and WLS estimators are identical in the conventional VARX model case [2, pp. 233-235].

Inserting the $\operatorname{VARX}(n a, n b)$ LS-based estimates into Eq. (6.53a)-(6.53b), yields for the case of groupwise heteroscedastic innovations (that is, $\boldsymbol{\Sigma}_{k_{1}, k_{1}} \neq \boldsymbol{\Sigma}_{k_{2}, k_{2}} \neq \ldots \neq \boldsymbol{\Sigma}_{k_{M}, k_{M}}$; see also Chapter 2, Table 2.1) the following estimators:

$$
\begin{gathered}
\widehat{\boldsymbol{\vartheta}}^{\#}=\left[\sum_{k=k_{1}}^{k_{M}} \sum_{t=1}^{N} \widetilde{\boldsymbol{\Phi}}_{k}[t] \widehat{\boldsymbol{\Sigma}}_{k, k}^{-1} \widetilde{\boldsymbol{\Phi}}_{k}^{T}[t]\right]^{-1}\left[\sum_{k=k_{1}}^{k_{M}} \sum_{t=1}^{N} \widetilde{\boldsymbol{\Phi}}_{k}[t] \widehat{\boldsymbol{\Sigma}}_{k, k}^{-1} \boldsymbol{y}_{k}[t]\right] \\
\widehat{\boldsymbol{P}}\left(\widehat{\boldsymbol{\vartheta}}^{\#}\right)=\left[\sum_{k=k_{1}}^{k_{M}} \sum_{t=1}^{N} \widetilde{\boldsymbol{\Phi}}_{k}[t] \widehat{\boldsymbol{\Sigma}}_{k, k}^{-1} \widetilde{\boldsymbol{\Phi}}_{k}^{T}[t]\right]^{-1}
\end{gathered}
$$

which are identical to the corresponding WLS-based CCP-VARX $(n a, n b)$ estimators.

The case of groupwise homoscedastic innovations (that is, $\boldsymbol{\Sigma}_{k, k}=\overline{\mathbf{\Sigma}}$ for $k=k_{1}, k_{2}, \ldots, k_{M}$; see also Chapter 2, Table 2.1) yields the following estimators:

$$
\begin{gathered}
\widehat{\boldsymbol{\vartheta}}^{\#}=\left[\sum_{k=k_{1}}^{k_{M}} \sum_{t=1}^{N} \widetilde{\boldsymbol{\Phi}}_{k}[t] \widetilde{\boldsymbol{\Phi}}_{k}^{T}[t]\right]^{-1}\left[\sum_{k=k_{1}}^{k_{M}} \sum_{t=1}^{N} \widetilde{\boldsymbol{\Phi}}_{k}[t] \boldsymbol{y}_{k}[t]\right] \\
\widehat{\boldsymbol{P}}\left(\widehat{\boldsymbol{\vartheta}}^{\#}\right)=\left[\sum_{k=k_{1}}^{k_{M}} \sum_{t=1}^{N} \widetilde{\boldsymbol{\Phi}}_{k}[t] \widehat{\overline{\boldsymbol{\Sigma}}}_{k, k}^{-1} \widetilde{\boldsymbol{\Phi}}_{k}^{T}[t]\right]^{-1}
\end{gathered}
$$

which are identical to the corresponding OLS and WLS-based CCP-VARX $(n a, n b)$ estimators.

Remark 14. In view of the preceding results it follows that when groupwise heteroscedastic or homoscedastic innovations are considered and statistically efficient (LS or ML) parameter estimation methods are employed, then CCP-VARX $(n a, n b)$ model estimated at the distinct operating conditions $\left\{k_{1}, k_{2}, \ldots, k_{M}\right\}$ is 
equivalent to a weighted average of disjoint conventional $\operatorname{VARX}(n a, n b)$ models yielding the smallest estimator variance.

Under the assumption that the conventional LS based VARX model parameter estimates converge in probability to the same parameter vector (that is, $\widehat{\vartheta}_{k} \stackrel{p}{\longrightarrow} \boldsymbol{\vartheta}_{o}$, as $N \longrightarrow \infty$ for $k=k_{1}, k_{2}, \ldots, k_{M}$ ), their optimal combination yields consistent, asymptotically normally distributed and efficient estimators identical to those obtained from the CCP-VARX modeling procedure.

In practice however, such an assumption is often unrealistic as the complete data set $Z^{N M}$ comprises of data records belonging to distinct operating conditions. Hence, the resulting CCP-VARX model will provide an "averaged" representation of the system dynamics. An exception to this case occurs when, for safety or production reasons, data records are collected from the system by conducting separate experiments where one input at a time is manipulated.

The superiority of the CCP-VARX model class over the optimal combination of disjoint conventional VARX models is threefold, since:

(i) CCP-VARX modeling is computationally more efficient, as it minimizes only one criterion rather than $M$ separate ones.

(ii) Increased numerical accuracy and robustness is obtained, as all data are used simultaneously.

(iii) The CCP-VARX models are more general, as they account for potential interrelations between the data obtained from different operating conditions (contemporaneously correlated innovations; see Chapter 2, Table 2.1).

\subsubsection{Comparison of CCP-VARX and FP-VARX model classes.}

A question of interest is how an identified CCP-VARX model compares to its FP-VARX counterpart for any operating condition $k \in \mathcal{R}$. In this section issues regarding their structure and achievable accuracy are addressed.

\subsubsection{The FP-VARX model specification}

An alternative way to think of the CCP-VARX model class is as a special case of the FP-VARX model class with parameter matrices independent of the operating condition (compare to Subsection 6.3.1). Indeed, a $\mathrm{CCP}-\operatorname{VARX}(n a, n b)$ model is derived by re-writing the FP-VARX $(n a, n b)_{[p a, p b]}$ model of Eq. (6.21a) in the following linear regression form:

$$
\boldsymbol{y}_{k}[t]=\boldsymbol{\Phi}_{k}^{T}[t] \boldsymbol{\theta}^{\#}+\boldsymbol{w}_{k}[t]
$$

where:

$$
\left.\begin{array}{c}
\boldsymbol{\Phi}_{k}^{T}[t]=\boldsymbol{I} \otimes \boldsymbol{\phi}_{k}^{T}[t] \\
\boldsymbol{\phi}_{k}[t]=\left[\begin{array}{c}
-\boldsymbol{y}_{k}[t-1] \\
\vdots \\
-\boldsymbol{y}_{k}[t-n a]
\end{array}\right] \otimes\left[\begin{array}{c}
G_{1}(k) \\
\vdots \\
G_{p a}(k)
\end{array}\right] \\
{\left[\begin{array}{c}
\boldsymbol{x}_{k}[t] \\
\vdots \\
\boldsymbol{x}_{k}[t-n b]
\end{array}\right] \otimes\left[\begin{array}{c}
G_{1}(k) \\
\vdots \\
G_{p b}(k)
\end{array}\right]}
\end{array}\right]
$$


under the following restrictions: $\boldsymbol{A}_{i, j}=\mathbf{0}(i=1,2, \ldots, n a$, and $j=2, \ldots, p a)$, and $\boldsymbol{B}_{i, j}=\mathbf{0}(i=0,1, \ldots, n b$, and $j=2, \ldots, p b)$. Note that the restrictions on the projection coefficients hold for $j \geq 2$, since $G_{1}(k)=1$ for trigonometric functions and most families of orthogonal polynomials [45, p. 777]. yields:

Pooling the expressions of Eq. (6.57a)-(6.57d) for each distinct operating condition $\left\{k_{1}, k_{2}, \ldots, k_{M}\right\}$

$$
\boldsymbol{y}[t]=\boldsymbol{\Phi}^{T}[t] \boldsymbol{\theta}^{\#}+\boldsymbol{w}[t]
$$

with $\boldsymbol{\Phi}^{T}[t]$ defined in analogy to $\boldsymbol{y}[t]$ and $\boldsymbol{w}[t]$, whereas the linear constraints for the projection coefficient vector $\boldsymbol{\theta}^{\#}$ are given by:

$$
\boldsymbol{\theta}^{\#}=\boldsymbol{R} \vartheta^{\#}
$$

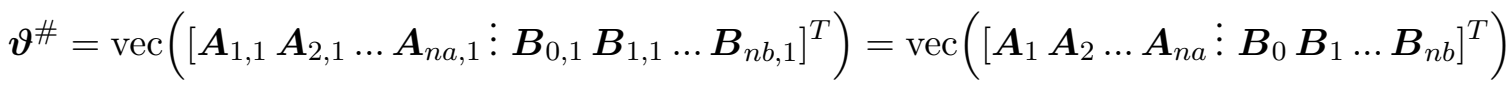

where $\boldsymbol{R}$ designates a matrix whose entries are zeros and ones at proper locations, since the CCP-VARX projection coefficient vector is equivalent to its corresponding parameter vector.

The preceding equations imply that the estimation of the CCP-VARX $(n a, n b)$ parameter vector $\boldsymbol{\vartheta}^{\#}$ is equivalent to estimating an $\operatorname{FP}-\operatorname{VARX}(n a, n b)_{[p a, p b]}$ model with the parameter constraints given in Eq. (6.58b)-(6.58c). Furthermore, the model classes considered are hierarchical, since the preceding equations imply that $\mathcal{M}_{C C P} \subset \mathcal{M}_{F P}$. Assuming that the CCP-VARX parameter vectors $\vartheta^{\#}$ satisfying assumptions A3.1-A3.3 belong to the set $D_{\mathcal{M}_{C C P}}$, and that the FP-VARX projection coefficients satisfying assumptions A2.1-A2.3 belong to the set $D_{\mathcal{M}_{F P}}$, then the parameter constraints of Eq. (6.58b)-(6.58c) suggest that the (differentiable) function $\boldsymbol{g}\left(\boldsymbol{\vartheta}^{\#}\right)=\boldsymbol{R} \boldsymbol{\vartheta}^{\#}: D_{\mathcal{M}_{C C P}} \longrightarrow D_{\mathcal{M}_{F P}}$ maps every model in $\mathcal{M}_{C C P}$ with parameters $\boldsymbol{\vartheta}^{\#}$ to a model in $\mathcal{M}_{F P}$ with projection coefficients $\boldsymbol{\theta}^{\#}$. This result implies that:

$$
\boldsymbol{e}_{k}^{\mathcal{M}_{C C P}}\left[t, \boldsymbol{\vartheta}^{\#}\right]=\boldsymbol{e}_{k}^{\mathcal{M}_{F P}}\left[t, \boldsymbol{g}\left(\boldsymbol{\vartheta}^{\#}\right)\right] \forall \boldsymbol{\vartheta}^{\#} \in D_{\mathcal{M}_{C C P}} \text { and } k \in \mathcal{R}
$$

\subsubsection{The parsimony principle for the CCP-VARX and FP-VARX model classes}

In order to study the accuracy of the CCP-VARX and FP-VARX modeling procedures, it is assumed that the true system generating the data set $Z^{N M}$ belongs to $\mathcal{M}_{C C P}$. This assumption implies that there exists a unique (true) projection coefficient vector $\boldsymbol{\theta}_{o}^{\#}=\boldsymbol{g}\left(\boldsymbol{\vartheta}_{o}^{\#}\right)$ such that the residuals satisfy: $\boldsymbol{e}_{k}^{\mathcal{M}_{F P}}\left[t, \boldsymbol{\vartheta}_{o}^{\#}\right]=$ $\boldsymbol{e}_{k}^{\mathcal{M}_{P}}\left[t, \boldsymbol{g}\left(\boldsymbol{\vartheta}_{o}^{\#}\right)\right]=\boldsymbol{w}_{k}[t]$ (white noise) for $k \in \mathcal{R}$.

In analogy to the case of P-VARX and FP-VARX models (refer to Subsection 6.2.5.2), an assessment criterion $W_{\mathcal{M}}\left(\boldsymbol{\vartheta}^{\#}\right)$ is postulated, which is a scalar and differentiable function of the residuals such that:

$$
\min _{\boldsymbol{\vartheta}^{\#}} W_{\mathcal{M}}\left(\boldsymbol{\vartheta}^{\#}\right)=W_{\mathcal{M}}\left(\boldsymbol{\vartheta}_{o}^{\#}\right)
$$

and the following asymptotic measure of accuracy is derived:

$$
F_{\mathcal{M}}=\lim _{N \longrightarrow \infty} M N E\left\{W_{\mathcal{M}}\left(\widehat{\boldsymbol{\vartheta}}^{\#}\right)-W_{\mathcal{M}}\left(\widehat{\boldsymbol{\vartheta}}_{o}^{\#}\right)\right\}=\frac{1}{2} \operatorname{tr}\left(\left.\frac{\partial^{2} W_{\mathcal{M}}\left(\boldsymbol{\vartheta}^{\#}\right)}{\partial \boldsymbol{\vartheta}^{\#} \partial \boldsymbol{\vartheta}^{\# T}}\right|_{\boldsymbol{\vartheta}^{\#}=\boldsymbol{\vartheta}_{o}^{\#}} \boldsymbol{P}\right)
$$

Obviously, for the model sets $\mathcal{M}_{C C P}$ and $\mathcal{M}_{F P}$ it holds that:

$$
W_{\mathcal{M}_{C C P}}\left(\boldsymbol{\vartheta}^{\#}\right)=W_{\mathcal{M}_{F P}}\left(\boldsymbol{g}\left(\boldsymbol{\vartheta}^{\#}\right)\right) \forall \boldsymbol{\vartheta}^{\#} \in D_{\mathcal{M}_{C C P}}
$$

and the following proposition is obtained: 
Proposition 6.3.4. Consider the model sets $\mathcal{M}_{C C P}$ and $\mathcal{M}_{F P}$ (with $\mathcal{M}_{C C P} \subset \mathcal{M}_{F P}$ ), and assume that the true system $\mathcal{S}$ generating the data set $Z^{N M}$ is a CCP-VARX model belonging to $\mathcal{M}_{C C P}$, so that:

$$
\mathcal{S} \in \mathcal{M}_{C C P} \subset \mathcal{M}_{F P}
$$

Furthermore, assume that parameter estimation is based on a statistically efficient ( $L S$ or ML) method. Then, the $C C P-V A R X$ modeling leads on the average to more accurate results than FP-VARX modeling for $k \in \mathcal{R}$, in the sense that:

$$
F_{\mathcal{M}_{C C P}} \leq F_{\mathcal{M}_{F P}}
$$

Proof. See Appendix 6.B.2.

Remark 15. In analogy to the case where the P-VARX and FP-VARX model classes were treated, the parsimony principle is not valid when inefficient parameter estimation methods are employed. Such situations occur when CCP-VARX parameter estimation is based on OLS and the innovations are contemporaneously correlated or groupwise heteroscedastic. In such cases additional requirements are imposed to relax the assumptions of Proposition 6.3.4 (refer to Appendix 6.B.2).

The implication of the parsimony principle on the obtained accuracy of the efficient (LS and ML based) FP-VARX and CCP-VARX estimators may also be addressed within a constraint parameter estimation framework. This is possible since the CCP-VARX model estimation at the distinct operating conditions $\left\{k_{1}, k_{2}, \ldots, k_{M}\right\}$ is equivalent to FP-VARX model estimation with parameter constraints, and the following proposition is obtained.

Proposition 6.3.5. Consider the model sets $\mathcal{M}_{C C P}$ and $\mathcal{M}_{F P}$ (with $\mathcal{M}_{C C P} \subset \mathcal{M}_{F P}$ ), and assume that the true system $\mathcal{S}$ generating the data set $Z^{N M}$ at the distinct operating conditions $\left\{k_{1}, k_{2}, \ldots, k_{M}\right\}$ belongs to $\mathcal{M}_{C C P}$, so that $\mathcal{S} \in \mathcal{M}_{C C P} \subset \mathcal{M}_{F P}$. If parameter estimation is based on a statistically efficient ( $L S$ or $M L)$ method, then the CCP-VARX modeling leads on parameter estimation uncertainties smaller or at most equal to those of FP-VARX modeling for any operating condition $k \in \mathcal{R}$.

Proof. See Appendix 6.B.3.

The important result of Propositions 6.3.4 and 6.3.5 is that the CCP-VARX models will in general be more accurate than their FP-VARX model counterparts at any operating condition $k \in \mathcal{R}$.

Furthermore, from Propositions 6.2.5 and 6.3.5 it follows that in the cases of groupwise heteroscedastic and homoscedastic innovations the CCP-VARX modeling leads on parameter estimation uncertainties smaller or at most equal to those of conventional VARX models at any operating condition $k \in \mathcal{R}$, which is also confirmed from Eq. (6.53b) in Subsection 6.3.5.

\subsection{Monte Carlo studies}

The performance characteristics of the novel global (P, CCP, FP) modeling approaches are assessed, by means of two Monte Carlo studies. The focus is on the estimation uncertainties obtained by the P-VARX and CCP-VARX models when the true system generating the data belongs to the FP-VARX model class, since the latter class constitutes the choice of preference for describing systems operating under different, though constant, conditions at different occasions. 
Table 6.3: Details on the Monte Carlo study for FP-VARX $(3,1)_{[4,4]}$ model identification.

\begin{tabular}{|c|c|c|}
\hline $\begin{array}{l}\text { No. of excitations: } n x=2 \\
\text { No. of responses: } n y=2 \\
\text { Innovations dependency type: }\end{array}$ & $\begin{array}{l}\text { Operating points: } M=11(k \in[0,10], \Delta k=1) \\
\text { No. of Monte Carlo runs: } 500 \\
\text { groupwise heteroscedastic }\end{array}$ & $\begin{array}{l}\text { Func. subspace: spanned by Chebyshev II polynomials } \\
\text { Data length: } N=2,000 \text { samples } \\
\text { Innovation sequence type: Gaussian white noise } \\
\text { Excitation sequence type: Gaussian white noise }\end{array}$ \\
\hline Global model & Identification details & \\
\hline (i) CCP-VARX $(3,1)^{\dagger}$ & $\begin{array}{l}\text { Par. estimation: non-iterative WLS via QR implen } \\
\text { Number of estimated parameters: } 20\end{array}$ & entation \\
\hline (ii) $\mathrm{FP}-\operatorname{VARX}(3,1)_{[4,4]}$ & $\begin{array}{l}\text { Par. estimation: non-iterative WLS via QR implen } \\
\text { Number of estimated parameters: } 80\end{array}$ & entation \\
\hline (iii) $\mathrm{P}-\operatorname{VARX}(3,1)^{\ddagger}$ & $\begin{array}{l}\text { Par. estimation: OLS via QR implementation } \\
\text { Number of estimated parameters: } 220 \text { ( } 20 \text { paramet }\end{array}$ & rs per operating point) \\
\hline
\end{tabular}

\subsubsection{Case study I: An FP-VARX $(3,1)_{[4,4]}$ model}

An FP-VARX $(3,1)_{[4,4]}$ model with two excitations $(n x=2)$, two responses $(n y=2)$, and zero excitation delay (that is, $\boldsymbol{B}_{0}(k) \neq \mathbf{0}$ ) is considered. The functional subspaces consist of the first four shifted Chebyshev polynomials of the second kind [45, p. 782] (see Chapter 2, Appendix 2.D), that is $p=4$. The modeling procedure relies upon $M=11$ operating conditions with $k \in[0,10]$, obtained at unit increments. All excitation sequences are Gaussian white, mutually independent, with zero mean and unit variance. The innovations for each operating condition $k$ are (i) zero mean Gaussian white, and (ii) uncorrelated with those of any other operating condition (that is, $\boldsymbol{\Sigma}_{k, \ell}=\mathbf{0}$ ), which implies that no interrelations exist among the obtained data records (groupwise heteroscedastic innovations; see Chapter 2, Table 2.1). Moreover, for each response, the innovations' standard deviation over the model's noise-free response standard deviation is set to $10 \%$ (that is, $\sigma_{w_{i k}} / \sigma_{y_{i k}}=10 \%, i=1,2$; see Chapter 2, Fig. 2.1).

The study encompasses 500 Monte Carlo runs, and parameter estimation is based upon $N=2,000$ sample-long data records. The CCP-VARX $(3,1)$ and $\operatorname{FP}-\operatorname{VARX}(3,1)_{[4,4]}$ modeling is based upon noniterative WLS via QR implementation [1, pp. 318-320] (see Chapter 2, Appendix 2.E), which results in statistically efficient parameter estimates. Also note that due to the assumption of groupwise heteroscedastic innovations, $\mathrm{P}-\operatorname{VARX}(3,1)$ modeling is equivalent to estimating conventional $\operatorname{VARX}(3,1)$ models for each operating condition $k$ (refer to Table 6.1). As a result, P-VARX $(3,1)$ model parameter estimation relies upon MATLAB ${ }^{\circledR}$ function “arx.m” [47], which also yields statistically efficient parameter estimates. Details on the Monte Carlo study are shown in Table 6.3.

Partial results depicting the interval parameter estimates (depicting sample mean \pm two sample standard deviations) for the identified models along with the actual parameter values are presented in Fig. 6.2. Very good agreement is observed between the P-VARX $(3,1)$ and $\operatorname{FP}-\operatorname{VARX}(3,1)_{[4,4]}$ estimates and the actual values for all operating conditions. Nevertheless, the $\operatorname{FP}-\operatorname{VARX}(3,1)_{[4,4]}$ based estimates, as expected, provide better accuracy than their P-VARX $(3,1)$ based counterparts. Since the assumption of groupwise heteroscedastic innovations implies that no interrelations exist among the obtained data records, the only source of inaccuracy for the P-VARX modeling approach is the large number of estimated parameters for the same amount of data (a total of 220 parameters for the 11 conventional $\operatorname{VARX}(3,1)$ models versus 80 parameters of the FP-VARX $(3,1)_{[4,4]}$ model). On the other hand, the CCP-VARX $(3,1)$ estimates are, as expected, statistically "biased" (see also Section 6.3.5). This is a direct consequence of the constant AR and X parameter matrices, which results in "averaging out" the operating parameter dependent dynamics. Although the dependence of the system dynamics on the operating parameter is transformed by the CCP$\operatorname{VARX}(3,1)$ model into uncertainty of the estimated parameters, Fig. 6.2 shows that the CCP model fails to 

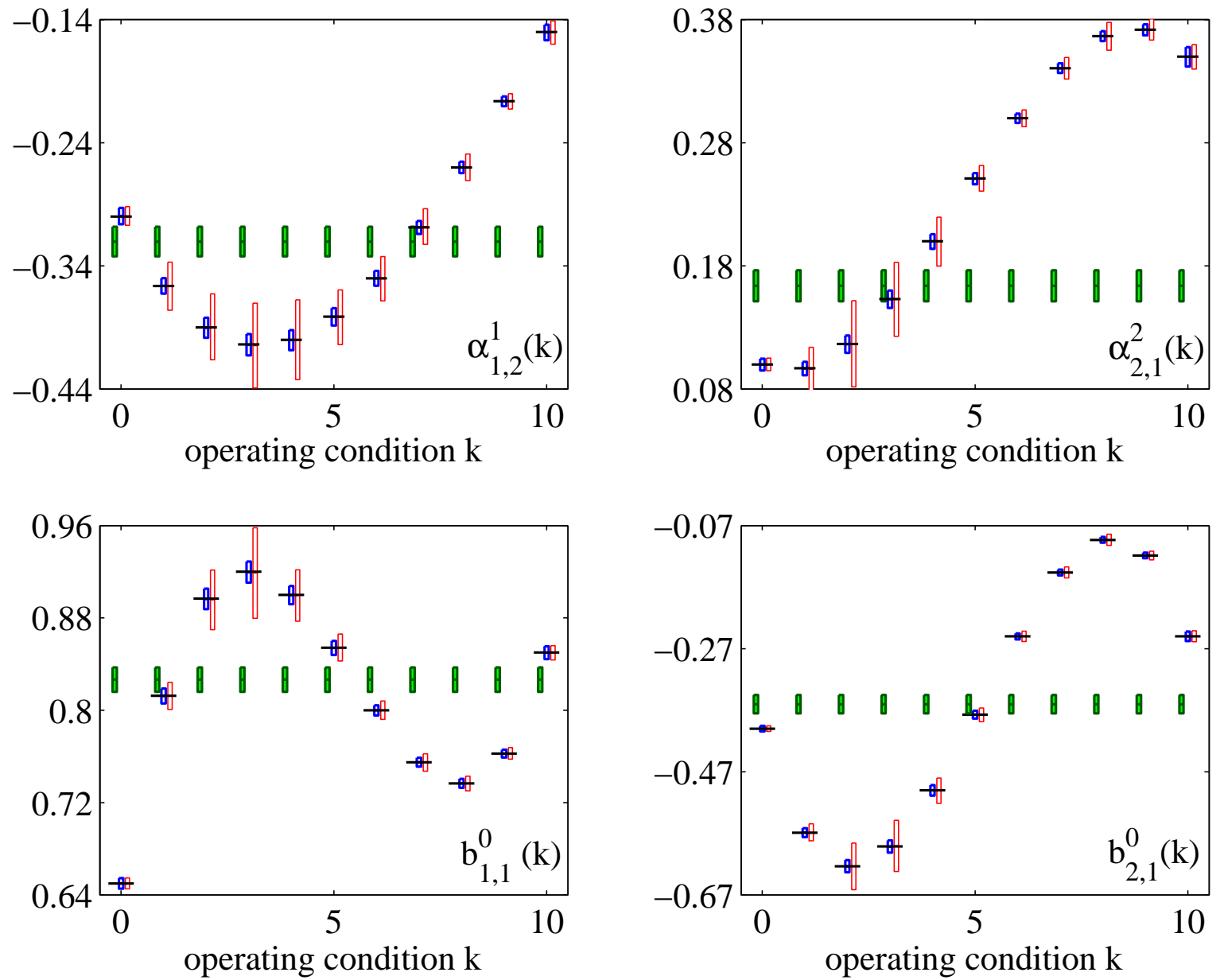

Figure 6.2: Dependence of certain AutoRegressive and eXogenous parameters on the operating condition $k$ (boxes indicate sample mean $\pm 2 \times$ sample standard deviation): $(-)$ theoretical values; (filled green boxes) CCP-VARX $(3,1)$ model; (thick lined blue boxes) FP-VARX $(3,1)_{[4,4]}$ model; (thin lined red boxes) P$\operatorname{VARX}(3,1)$ model $\left(500\right.$ Monte Carlo runs per model; $a_{m, n}^{i}(k)$ and $b_{m, n}^{i}(k)$ designate the $(m, n)$-th elements of $\boldsymbol{A}_{i}(k)$ and $\boldsymbol{B}_{i}(k)$, respectively).

explain the observed variability of the true system. The CCP-VARX $(3,1)$ interval parameter estimates are confined in narrow zones, as opposed to the theoretical values of the true system - a fact attributed to the CCP model compactness (20 estimated parameters). Similar results have also been obtained in a recent experimental study [41,42], where the problem of identifying the dynamics of a composite beam under various temperatures via global (FP and CCP) and conventional VARX modeling approaches is treated.

\subsubsection{Case study II: An FP-VAR $(2)_{[4]}$ model}

A bivariate FP-VAR $(2)_{[4]}$ model is used to generate data in the second study - no exogenous excitation is present. The functional subspace consists of the first four shifted Chebyshev polynomials of the second kind [45, p. 782] (see Chapter 2, Appendix 2.D), that is $p=4$. The modeling procedure relies upon $M=11$ operating conditions with $k \in[0,10]$, obtained at unit increments. The innovations for each operating condition $k$ are (i) Gaussian white with zero mean and identity covariance $\boldsymbol{\Sigma}_{k, k}$ and (ii) uncorrelated with those of any other operating condition (that is, $\boldsymbol{\Sigma}_{k, \ell}=\mathbf{0}$ ), which implies that no interrelations exist among the obtained data records (groupwise homoscedastic innovations; see Chapter 2, Table 2.1). 
Table 6.4: Details on the Monte Carlo study for FP-VAR $(2)_{[4]}$ model identification.

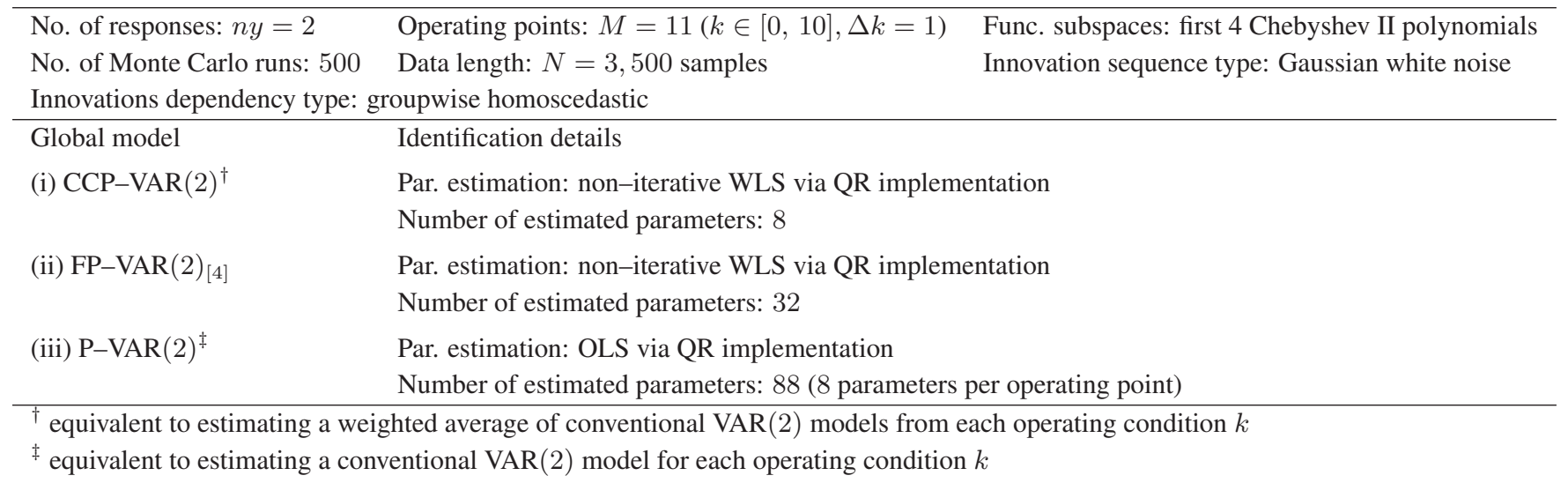

The study encompasses 500 Monte Carlo runs, and global model parameter estimation is based upon $N=3,500$ sample-long data records. The CCP-VAR $(2)$ and FP-VAR $(2)_{[4]}$ modeling is based upon noniterative WLS via QR implementation [1, pp. 318-320] (see Chapter 2, Appendix 2.E), which results in statistically efficient parameter estimates. Also note that due to the assumption of groupwise homoscedastic innovations, $\mathrm{P}-\operatorname{VAR}(2)$ modeling is equivalent to estimating conventional $\operatorname{VAR}(2)$ models for each operating condition $k$ (refer to Table 6.1). As a result, $\mathrm{P}-\operatorname{VAR}(2)$ model parameter estimation relies upon MATLAB ${ }^{\circledR}$ function "arx.m" [47], which also yields statistically efficient parameter estimates. Details on the Monte Carlo study are shown in Table 6.4.

Partial results depicting the interval parameter estimates (depicting sample mean \pm two sample standard deviations) for the identified models along with the actual parameter values are presented in Fig. 6.3. Very good agreement is observed between the P-VAR $(2)$ and $\operatorname{FP}-\operatorname{VAR}(2)_{[4]}$ estimates and the actual values for all operating conditions. Nevertheless, the FP-VAR $(2)_{[4]}$ based estimates, as expected, provide better accuracy than their P-VAR (2) based counterparts. Since the assumption of groupwise homoscedastic innovations implies that no interrelations exist among the obtained data records, the only source of inaccuracy for the P-VAR modeling approach is the large number of estimated parameters for the same amount of data (a total of 88 parameters for the 11 conventional $\operatorname{VAR}(2)$ models versus 32 parameters of the FP-VAR $(2)_{\text {[4] }}$ model). On the other hand, the CCP-VAR(2) estimates are, as expected, statistically "biased" (see also Section 6.3.5), due to the common and constant AR parameter matrices for all operating conditions. The $\mathrm{CCP}-\operatorname{VAR}(2)$ interval parameter estimates are confined in narrow zones, as observed in Fig. 6.3, which may be attributed to the CCP model compactness (8 estimated parameters).

\subsection{Concluding remarks}

The identification of multivariate stochastic systems capable of operating under different conditions, was addressed via novel global modeling approaches. The global model classes postulated in this study were of the P-VARX, CCP-VARX and FP-VARX form. The primary focus was on the identification of the P-VARX and CCP-VARX model classes. LS and conditional ML type estimators were formulated, and their consistency along with their asymptotic normality was established. Furthermore, conditions ensuring P-VARX and CCP-VARX identifiability were postulated. A comparison of the global VARX and the conventional VARX model structures was also performed to assess the preceding structures in terms of model parsimony and achievable accuracy. The effectiveness and performance characteristics of the novel global modeling approaches were further examined via two Monte Carlo studies.

The main conclusion drawn were: 

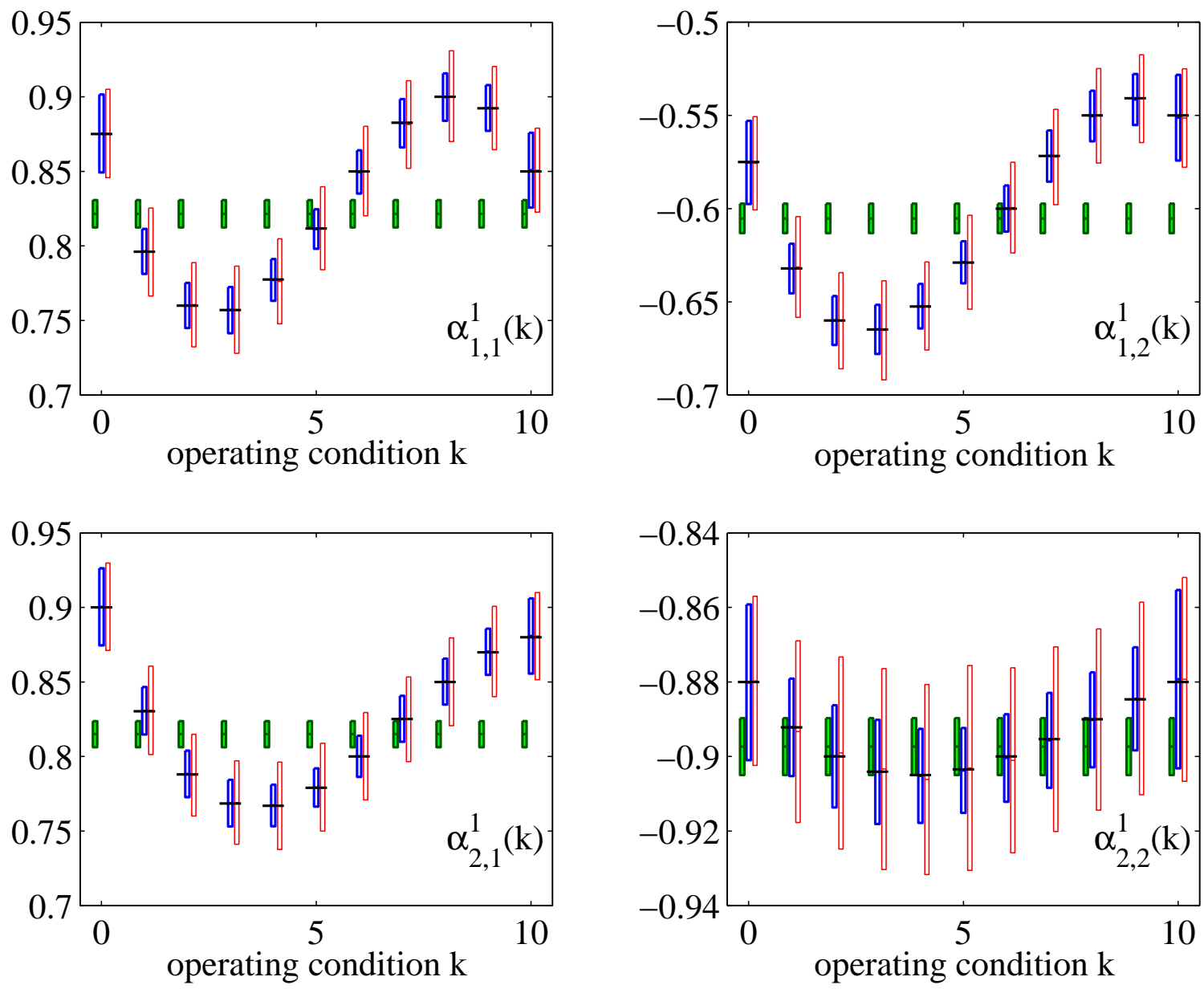

Figure 6.3: Dependence of certain AutoRegressive parameters on the operating condition $k$ (boxes indicate sample mean $\pm 2 \times$ sample standard deviation): (-) theoretical values; (filled green boxes) CCP-VAR $(2)$ model; (thick lined blue boxes) FP-VAR $(2)_{[4]}$ model; (thin lined red boxes) P-VAR(2) model (500 Monte Carlo runs per model; $a_{m, n}^{i}(k)$ designates the $(m, n)$-th element of $\left.\boldsymbol{A}_{i}(k)\right)$.

(i) The P-VARX and CCP-VARX model classes provide interesting global descriptions of the system, although limited in scope. The P-VARX model class provides valid models for the operating conditions used in the identification procedure only, whereas the CCP-VARX model class provides simple, "averaged" descriptions of the system dynamics over the entire range of operating conditions.

(ii) The WLS and ML estimators of the global VARX models are statistically efficient for all types of innovations dependency. The OLS estimates are in general inefficient except for special cases, depending on the specified model class.

(iii) The P-VARX modeling procedure is equivalent to estimating disjoint conventional VARX models when groupwise heteroscedastic or homoscedastic innovations are considered.

(iv) The CCP-VARX modeling procedure is equivalent to estimating a weighted average of disjoint conventional VARX models yielding the smallest estimator variance when groupwise heteroscedastic or homoscedastic innovations are considered and efficient (LS or ML) parameter estimation methods are employed.

(v) The estimation of FP-VARX models at the distinct operating conditions used for identification is equivalent to estimating a P-VARX model with parameter constraints. On the other hand, the estimation of CCP-VARX models from the distinct operating conditions used for identification is equivalent 
to estimating an FP-VARX model with parameter constraints.

(vi) The parsimony principle when comparing (a) P-VARX and FP-VARX models or (b) FP-VARX and CCP-VARX models, holds for efficient (LS and ML based) parameter estimation methods. When parameter estimation is based on inefficient methods, additional requirements are necessary.

\section{Appendix 6.A: Additional results on P-VARX modeling}

\section{A.1 Proof of Proposition 6.2.1}

Let the LS criterion in Eq. (6.8) be minimized using a block diagonal weighting matrix $\boldsymbol{H}$ that does not take into account the interrelations between the obtained data records. Then, the obtained LS estimator may be rewritten as follows:

$$
\begin{aligned}
& \widehat{\boldsymbol{\vartheta}}_{L S}=\left[\sum_{t=1}^{N} \widetilde{\boldsymbol{\Phi}}[t] \boldsymbol{H} \widetilde{\boldsymbol{\Phi}}^{T}[t]\right]^{-1}\left[\sum_{t=1}^{N} \widetilde{\boldsymbol{\Phi}}[t] \boldsymbol{H} \boldsymbol{y}[t]\right] \\
& =\left[\sum_{t=1}^{N}\left[\begin{array}{ccc}
\widetilde{\boldsymbol{\Phi}}_{k_{1}}[t] & & \mathbf{0} \\
& \ddots & \\
\mathbf{0} & & \widetilde{\boldsymbol{\Phi}}_{k_{M}}[t]
\end{array}\right]\left[\begin{array}{ccc}
\boldsymbol{H}_{k_{1}, k_{1}} & & \mathbf{0} \\
& \ddots & \\
\mathbf{0} & & \boldsymbol{H}_{k_{M}, k_{M}}
\end{array}\right]\left[\begin{array}{ccc}
\widetilde{\boldsymbol{\Phi}}_{k_{1}}^{T}[t] & & \mathbf{0} \\
& \ddots & \\
\mathbf{0} & & \widetilde{\boldsymbol{\Phi}}_{k_{M}}^{T}[t]
\end{array}\right]\right]^{-1} \\
& \times\left[\sum_{t=1}^{N}\left[\begin{array}{ccc}
\widetilde{\boldsymbol{\Phi}}_{k_{1}}[t] & & \mathbf{0} \\
& \ddots & \\
\mathbf{0} & & \widetilde{\boldsymbol{\Phi}}_{k_{M}}[t]
\end{array}\right]\left[\begin{array}{ccc}
\boldsymbol{H}_{k_{1}, k_{1}} & & \mathbf{0} \\
& \ddots & \\
\mathbf{0} & & \boldsymbol{H}_{k_{M}, k_{M}}
\end{array}\right]\left[\begin{array}{c}
\boldsymbol{y}_{k_{1}}[t] \\
\vdots \\
\boldsymbol{y}_{k_{M}}[t]
\end{array}\right]\right]
\end{aligned}
$$

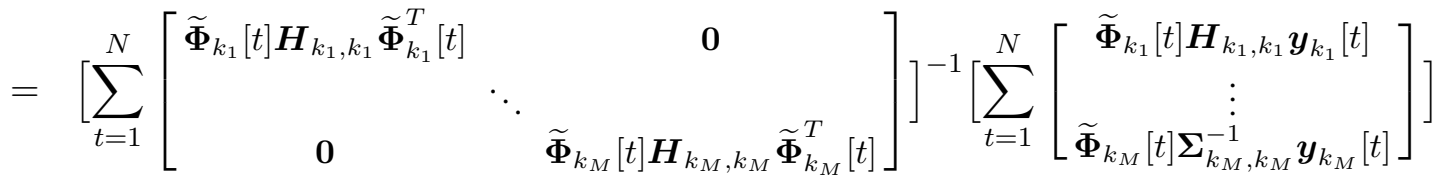

$$
\begin{aligned}
& =\left[\begin{array}{c}
{\left[\sum_{t=1}^{N} \widetilde{\boldsymbol{\Phi}}_{k_{1}}[t] \boldsymbol{H}_{k_{1}, k_{1}} \widetilde{\boldsymbol{\Phi}}_{k_{1}}^{T}[t]\right]^{-1}\left[\sum_{t=1}^{N} \widetilde{\boldsymbol{\Phi}}_{k_{1}}[t] \boldsymbol{H}_{k_{1}, k_{1}} \boldsymbol{y}_{k_{1}}[t]\right]} \\
\vdots \\
{\left[\sum_{t=1}^{N} \widetilde{\boldsymbol{\Phi}}_{k_{M}}[t] \boldsymbol{H}_{k_{M}, k_{M}} \widetilde{\boldsymbol{\Phi}}_{k_{M}}^{T}[t]\right]^{-1}\left[\sum_{t=1}^{N} \widetilde{\boldsymbol{\Phi}}_{k_{M}}[t] \boldsymbol{H}_{k_{M}, k_{M}} \boldsymbol{y}_{k_{M}}[t]\right]}
\end{array}\right]
\end{aligned}
$$

Using standard calculus, the following quantity may be rewritten as ${ }^{8,9,10}$ :

$$
\begin{aligned}
{\left[\sum_{t=1}^{N} \widetilde{\boldsymbol{\Phi}}_{k}[t] \boldsymbol{H}_{k, k} \widetilde{\boldsymbol{\Phi}}_{k}^{T}[t]\right]^{-1} } & =\left[\sum_{t=1}^{N}\left(\boldsymbol{I}_{n y} \otimes \widetilde{\boldsymbol{\phi}}_{k}[t]\right)\left(\boldsymbol{H}_{k, k} \otimes 1\right)\left(\boldsymbol{I}_{n y} \otimes \widetilde{\boldsymbol{\phi}}_{k}^{T}[t]\right)\right]^{-1} \\
& =\left[\sum_{t=1}^{N}\left(\boldsymbol{H}_{k, k} \otimes \widetilde{\boldsymbol{\phi}}_{k}[t] \widetilde{\boldsymbol{\phi}}_{k}^{T}[t]\right)\right]^{-1} \\
& =[\boldsymbol{H}_{k, k} \otimes \underbrace{\left(\sum_{t=1}^{N} \widetilde{\boldsymbol{\phi}}_{k}[t] \boldsymbol{\phi}_{k}^{T}[t]\right)}_{\mathbf{\Phi}_{k}}]^{-1}
\end{aligned}
$$

\footnotetext{
${ }^{8}$ Let $\boldsymbol{A}, \boldsymbol{B}, \boldsymbol{C}$ and $\boldsymbol{D}$ be matrices of compatible dimensions, then $(\boldsymbol{A} \otimes \boldsymbol{B})(\boldsymbol{C} \otimes \boldsymbol{D})=\boldsymbol{A} \boldsymbol{C} \otimes \boldsymbol{B} \boldsymbol{D}$ [43, p. 19].

${ }^{9}$ Let $\boldsymbol{A}, \boldsymbol{B}, \boldsymbol{C}$ and $\boldsymbol{D}$ be matrices of compatible dimensions, $\boldsymbol{A} \otimes(\boldsymbol{B} \pm \boldsymbol{C})=\boldsymbol{A} \otimes \boldsymbol{B} \pm \boldsymbol{A} \otimes \boldsymbol{C}$ [43, p. 19].

${ }^{10}$ Let the $(m \times m)$ matrix $\boldsymbol{A}$ and the $(n \times n)$ matrix $\boldsymbol{B}$ be non-singular, then $(\boldsymbol{A} \otimes \boldsymbol{B})^{-1}=\boldsymbol{A}^{-1} \otimes \boldsymbol{B}^{-1}$ [43, p. 19].
} 


$$
=\boldsymbol{H}_{k, k}^{-1} \otimes \boldsymbol{\Phi}_{k}^{-1}
$$

and also $^{8-10}$ :

$$
\begin{aligned}
\sum_{t=1}^{N} \widetilde{\boldsymbol{\Phi}}_{k}[t] \boldsymbol{H}_{k, k} \boldsymbol{y}_{k}[t] & =\sum_{t=1}^{N}\left(\boldsymbol{I}_{n y} \otimes \widetilde{\boldsymbol{\phi}}_{k}[t]\right)\left(\boldsymbol{H}_{k, k} \otimes 1\right) \boldsymbol{y}_{k}[t] \\
& =\sum_{t=1}^{N}\left(\boldsymbol{H}_{k, k} \otimes \widetilde{\boldsymbol{\phi}}_{k}[t]\right) \boldsymbol{y}_{k}[t]
\end{aligned}
$$

Multiplying Eq. (6.A.2) and Eq. (6.A.3) yields:

$$
\begin{aligned}
{\left[\sum_{t=1}^{N} \widetilde{\boldsymbol{\Phi}}_{k}[t] \boldsymbol{H}_{k, k} \widetilde{\boldsymbol{\Phi}}_{k}^{T}[t]\right]^{-1} \sum_{t=1}^{N} \widetilde{\boldsymbol{\Phi}}_{k}[t] \boldsymbol{H}_{k, k} \boldsymbol{y}_{k}[t] } & =\sum_{t=1}^{N}\left(\boldsymbol{I}_{n y} \otimes \boldsymbol{\Phi}_{k}^{-1} \widetilde{\boldsymbol{\phi}}_{k}[t]\right) \boldsymbol{y}_{k}[t] \\
& =\left[\sum_{t=1}^{N} \widetilde{\boldsymbol{\Phi}}_{k}[t] \widetilde{\boldsymbol{\Phi}}_{k}^{T}[t]\right]^{-1} \sum_{t=1}^{N} \widetilde{\boldsymbol{\Phi}}_{k}[t] \boldsymbol{y}_{k}[t]=\widehat{\boldsymbol{\vartheta}}_{k}
\end{aligned}
$$

which is equal to the LS estimator of a conventional $\operatorname{VARX}(n a, n b)$ model at the $k$-th operating condition.

By virtue of Eq. (6.A.4), the LS estimator in Eq. (6.A.1) may be rewritten as follows:

$$
\widehat{\boldsymbol{\vartheta}}_{L S}=\left[\begin{array}{c}
{\left[\sum_{t=1}^{N} \widetilde{\boldsymbol{\Phi}}_{k_{1}}[t] \widetilde{\boldsymbol{\Phi}}_{k_{1}}^{T}[t]\right]^{-1}\left[\sum_{t=1}^{N} \widetilde{\boldsymbol{\Phi}}_{k_{1}}[t] \boldsymbol{y}_{k_{1}}[t]\right]} \\
{\left[\sum_{t=1}^{N} \widetilde{\boldsymbol{\Phi}}_{k_{2}}[t] \widetilde{\boldsymbol{\Phi}}_{k_{2}}^{T}[t]\right]^{-1}\left[\sum_{t=1}^{N} \widetilde{\boldsymbol{\Phi}}_{k_{2}}[t] \boldsymbol{y}_{k_{2}}[t]\right]} \\
\vdots \\
{\left[\sum_{t=1}^{N} \widetilde{\boldsymbol{\Phi}}_{k_{M}}[t] \widetilde{\boldsymbol{\Phi}}_{k_{M}}^{T}[t]\right]^{-1}\left[\sum_{t=1}^{N} \widetilde{\boldsymbol{\Phi}}_{k_{M}}[t] \boldsymbol{y}_{k_{M}}[t]\right]}
\end{array}\right]=\left[\begin{array}{c}
\widehat{\boldsymbol{\vartheta}}_{k_{1}} \\
\widehat{\boldsymbol{\vartheta}}_{k_{2}} \\
\vdots \\
\widehat{\boldsymbol{\vartheta}}_{k_{M}}
\end{array}\right]
$$

thus suggesting that when groupwise heteroscedastic or homoscedastic innovations are considered

(i) the LS estimator $\widehat{\vartheta}_{L S}$ is equivalent to estimating the LS based parameter vectors of disjoint conventional VARX models for each distinct operating condition $\left\{k_{1}, k_{2}, \ldots, k_{M}\right\}$ and stacking them one below the other, and

(ii) the LS estimator is independent of the covariance matrix $\boldsymbol{\Sigma}$, hence $\widehat{\boldsymbol{\vartheta}}_{O L S}=\widehat{\boldsymbol{\vartheta}}_{W L S}$.

\section{A.2 Proof of Theorem 6.2.1}

The consistency and asymptotic distribution of the LS-based estimators is derived in the following paragraphs.

Consistency of parameter vector estimator To establish consistency, the LS-based estimator is written as:

$$
\widehat{\boldsymbol{\vartheta}}_{L S}=\boldsymbol{\vartheta}_{o}+\left[\frac{1}{N M} \sum_{t=1}^{N} \widetilde{\boldsymbol{\Phi}}[t] \boldsymbol{H} \widetilde{\boldsymbol{\Phi}}^{T}[t]\right]^{-1}\left[\frac{1}{N M} \sum_{t=1}^{N} \widetilde{\boldsymbol{\Phi}}[t] \boldsymbol{H} \boldsymbol{w}[t]\right]
$$

The inverted matrix in the expression above is assumed to be a finite positive definite matrix (refer to Appendix 6.A.5 for details). Furthermore, under fairly general conditions on $\boldsymbol{y}_{k}[t]$ and $\boldsymbol{x}_{k}[t]$ (that is 
zero-mean, ergodic and stationary processes [2, pp. 547-550]), it holds that ${ }^{11}$ :

$$
\begin{gathered}
\frac{1}{N M} \sum_{t=1}^{N} \widetilde{\boldsymbol{\Phi}}[t] \boldsymbol{H} \widetilde{\boldsymbol{\Phi}}^{T}[t] \stackrel{p}{\longrightarrow} \frac{1}{M} E\left\{\widetilde{\boldsymbol{\Phi}}[t] \boldsymbol{H} \widetilde{\boldsymbol{\Phi}}^{T}[t]\right\} \quad(N \longrightarrow \infty) \\
\frac{1}{N M} \sum_{t=1}^{N} \widetilde{\boldsymbol{\Phi}}[t] \boldsymbol{H} \boldsymbol{w}[t] \stackrel{p}{\longrightarrow} \mathbf{0} \quad(N \longrightarrow \infty)
\end{gathered}
$$

which implies that ${ }^{12}$ :

$$
\widehat{\boldsymbol{\theta}}_{L S} \stackrel{p}{\longrightarrow} \boldsymbol{\theta}_{O} \quad(N \longrightarrow \infty)
$$

Remark 16. Note that in contrast to the conventional VARX model case, the proof of consistency involves the weighting matrix $\boldsymbol{H}$. Yet, the consistency result is not affected, which is in analogy to the FP-VARX model case.

Asymptotic distribution of parameter vector estimator To establish the LS-based estimator asymptotic distribution, Eq. (6.A.6) may be rewritten as follows:

$$
\sqrt{N M}\left(\widehat{\boldsymbol{\vartheta}}_{L S}-\boldsymbol{\vartheta}_{o}\right)=\left[\frac{1}{N M} \sum_{t=1}^{N} \widetilde{\boldsymbol{\Phi}}[t] \boldsymbol{H} \widetilde{\boldsymbol{\Phi}}^{T}[t]\right]^{-1}\left[\frac{1}{\sqrt{N M}} \sum_{t=1}^{N} \widetilde{\boldsymbol{\Phi}}[t] \boldsymbol{H} \boldsymbol{w}[t]\right]
$$

Under the assumption that $\boldsymbol{y}_{k}[t]$ and $\boldsymbol{x}_{k}[t]$ are zero-mean, ergodic and stationary processes, the limiting distribution of the rightmost quantity in Eq. (6.A.8) is found by means of a central limit theorem ${ }^{13}[2$, p. 550], and is given by:

$$
\frac{1}{\sqrt{N M}} \sum_{t=1}^{N} \widetilde{\boldsymbol{\Phi}}[t] \boldsymbol{H} \boldsymbol{w}[t] \stackrel{d}{\longrightarrow} \mathcal{N}\left(\mathbf{0}, \frac{1}{M} E\left\{\widetilde{\boldsymbol{\Phi}}[t] \boldsymbol{H} \boldsymbol{\Sigma}_{o} \boldsymbol{H} \widetilde{\boldsymbol{\Phi}}^{T}[t]\right\}\right) \quad(N \longrightarrow \infty)
$$

By virtue of Eq. (6.A.7a) and Eq. (6.A.9), the asymptotic distribution of the LS estimator $\widehat{\boldsymbol{\theta}}_{L S}$ is ${ }^{14}$ :

$$
\sqrt{N M}\left(\widehat{\boldsymbol{\vartheta}}_{L S}-\boldsymbol{\vartheta}_{o}\right) \stackrel{d}{\longrightarrow} \mathcal{N}\left(\mathbf{0}, \boldsymbol{P}_{L S}\right) \quad(N \longrightarrow \infty)
$$

with $\boldsymbol{P}_{L S}=M E\left\{\widetilde{\boldsymbol{\Phi}}[t] \boldsymbol{H} \widetilde{\boldsymbol{\Phi}}^{T}[t]\right\}^{-1} E\left\{\widetilde{\boldsymbol{\Phi}}[t] \boldsymbol{H} \boldsymbol{\Sigma}_{o} \boldsymbol{H} \widetilde{\boldsymbol{\Phi}}^{T}[t]\right\} E\left\{\widetilde{\boldsymbol{\Phi}}[t] \boldsymbol{H} \widetilde{\boldsymbol{\Phi}}^{T}[t]\right\}^{-1}$.

Remark 17. Note, that unlike the case of conventional VARX models [44, p. 196 and p. 397], the asymptotic covariance matrix $\boldsymbol{P}_{L S}$ is presently affected from the choice of $\boldsymbol{H}$, which is in analogy to the FP-VARX model case. The cases where optimal statistical accuracy is obtained by the LS estimator is discussed in Appendix 6.A.4.

\footnotetext{
${ }^{11}$ Let the stationary stochastic processes $z_{1}[t]$ and $z_{2}[t]$ be given by $z_{1}[t]=G(B) w_{1}[t]$ and $z_{2}[t]=H(B) w_{2}[t]$, with $G(B)$ and $H(B)$ stable filters, and $\boldsymbol{w}[t]=\left[w_{1}[t] w_{2}[t]\right]^{T}$ a zero-mean white noise with positive-definite covariance matrix and finite fourth order moments. Then, $1 / N \sum_{t=1}^{N} z_{1}[t] z_{2}[t] \stackrel{p}{\longrightarrow} E\left\{z_{1}[t] z_{2}[t]\right\} \quad(N \longrightarrow \infty)$ [2, pp. 547-550].

${ }^{12}$ If $\boldsymbol{x}_{N} \stackrel{p}{\longrightarrow} \boldsymbol{x}$ and $\boldsymbol{A}_{N} \stackrel{p}{\longrightarrow} \boldsymbol{A}$, then $\boldsymbol{A}_{N} \boldsymbol{x}_{N} \stackrel{p}{\longrightarrow} \boldsymbol{A} \boldsymbol{x} \quad(N \longrightarrow \infty)$ [44, p. 683].

${ }^{13}$ Consider $\boldsymbol{x}_{N}=(1 / \sqrt{N}) \sum_{t=1}^{N} \boldsymbol{z}[t]$, where $\boldsymbol{z}[t]$ is a zero-mean vector stationary process given by $\boldsymbol{z}[t]=\boldsymbol{\Phi}[t] \boldsymbol{v}[t]$. The entries of $\boldsymbol{\Phi}[t]$ and $\boldsymbol{v}[t]$ are stationary, possibly correlated, ARMA processes with zero means and underlying white noise sequences with finite fourth-order moments. The elements of $\boldsymbol{\Phi}[t]$ may also contain a bounded deterministic term. Assume that the limit $\boldsymbol{P}=\lim _{N \longrightarrow \infty} E\left\{\boldsymbol{x}_{N} \boldsymbol{x}_{N}^{T}\right\}$ exists and is nonsingular. Then $\boldsymbol{x}_{N}$ is asymptotically Gaussian distributed, $\boldsymbol{x}_{N} \stackrel{d}{\longrightarrow} \mathcal{N}(\mathbf{0}, \boldsymbol{P})$ $(N \longrightarrow \infty)[2$, p. 550].

${ }^{14}$ Let $\boldsymbol{x}_{N} \stackrel{d}{\longrightarrow} \mathcal{N}(\mathbf{0}, \boldsymbol{P})$ and $\boldsymbol{y}_{N}=\boldsymbol{A}_{N} \boldsymbol{x}_{N}+\boldsymbol{b}_{N}$, with $\boldsymbol{A}_{N} \stackrel{p}{\longrightarrow} \boldsymbol{A}$ and $\boldsymbol{b}_{N} \stackrel{p}{\longrightarrow} \boldsymbol{b}(N \longrightarrow \infty)$. Then, $\boldsymbol{y}_{N} \stackrel{d}{\longrightarrow} \mathcal{N}\left(\boldsymbol{b}, \boldsymbol{A} \boldsymbol{P} \boldsymbol{A}^{T}\right)$ $(N \longrightarrow \infty)$ [2, p. 551].
} 
Consistency of the residual covariance matrix estimator The consistency of $\widehat{\boldsymbol{\Sigma}}_{L S}$ follows directly from the following lemma (compare to the corresponding case for conventional $\operatorname{VAR}(X)$ models; [44, pp. 75-76]).

Lemma 6.A.1. Let $\boldsymbol{\vartheta}_{o}$ be the P-VARX model true parameter vector and $\boldsymbol{\Sigma}_{o}$ the true covariance matrix of $\boldsymbol{w}[t]$, which is additionally assumed to have bounded fourth-order moments. Then, for any consistent parameter vector estimator $\widehat{\vartheta}$, the following covariance matrix estimator is consistent:

$$
\widehat{\boldsymbol{\Sigma}}=\frac{1}{N} \sum_{t=1}^{N} \boldsymbol{e}[t, \widehat{\boldsymbol{\vartheta}}] \boldsymbol{e}^{T}[t, \widehat{\boldsymbol{\vartheta}}]=\frac{1}{N} \sum_{t=1}^{N}\left(\boldsymbol{y}[t]-\widetilde{\boldsymbol{\Phi}}^{T}[t] \widehat{\boldsymbol{\vartheta}}\right)\left(\boldsymbol{y}[t]-\widetilde{\boldsymbol{\Phi}}^{T}[t] \widehat{\boldsymbol{\vartheta}}\right)^{T} \stackrel{p}{\longrightarrow} \boldsymbol{\Sigma}_{o}(N \longrightarrow \infty)
$$

Proof. The proof is in analogy to the conventional VAR(X) model case [44, pp. 75-76].

Asymptotic distribution of the residual covariance matrix estimator To establish the asymptotic distribution of $\widehat{\boldsymbol{\Sigma}}_{L S}$ the following lemma is useful (compare to the corresponding case for conventional VAR(X) models; [44, pp. 75-76]).

Lemma 6.A.2. Let $\widehat{\boldsymbol{\vartheta}}$ be a consistent estimator of the P-VARX parameter vector $\boldsymbol{\vartheta}_{o}$ so that $\sqrt{N}\left(\widehat{\boldsymbol{\vartheta}}-\boldsymbol{\vartheta}_{o}\right)$ converges in distribution. Consider the covariance matrix estimator:

$$
\widehat{\boldsymbol{\Sigma}}=\frac{1}{N} \sum_{t=1}^{N} \boldsymbol{e}[t, \widehat{\boldsymbol{\vartheta}}] \boldsymbol{e}^{T}[t, \widehat{\boldsymbol{\vartheta}}]
$$

then, the following limit in probability holds:

$$
\sqrt{N}\left(\widehat{\boldsymbol{\Sigma}}-\frac{1}{N} \sum_{t=1}^{N} \boldsymbol{w}[t] \boldsymbol{w}^{T}[t]\right) \stackrel{p}{\longrightarrow} \mathbf{0} \quad(N \longrightarrow \infty)
$$

Proof. The P-VARX linear regression equation of Eq. (6.6a) is rewritten as:

$$
\boldsymbol{y}_{k}[t]=\boldsymbol{\Theta}_{k}^{T} \widetilde{\boldsymbol{\phi}}_{k}[t]+\boldsymbol{e}_{k}[t]
$$

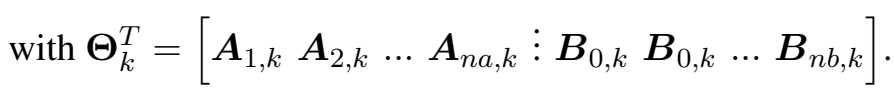

Stacking the available observations for time samples $t=1,2, \ldots N$ one besides the other yields:

$$
\boldsymbol{Y}_{k}=\boldsymbol{\Theta}_{k}^{T} \widetilde{\boldsymbol{\Phi}}_{k}+\boldsymbol{E}_{k}
$$

where:

$$
\begin{aligned}
\boldsymbol{Y}_{k} & =\left[\begin{array}{llll}
\boldsymbol{y}_{k}[1] & \boldsymbol{y}_{k}[2] & \ldots & \boldsymbol{y}_{k}[N]
\end{array}\right] \\
\widetilde{\boldsymbol{\Phi}}_{k} & =\left[\begin{array}{llll}
\widetilde{\boldsymbol{\phi}}_{k}[1] & \widetilde{\boldsymbol{\phi}}_{k}[2] & \ldots & \widetilde{\boldsymbol{\phi}}_{k}[N]
\end{array}\right] \\
\boldsymbol{E}_{k} & =\left[\begin{array}{llll}
\boldsymbol{e}_{k}[1] & \boldsymbol{e}_{k}[2] & \ldots & \boldsymbol{e}_{k}[N]
\end{array}\right]
\end{aligned}
$$

The following cross-covariance estimator:

$$
\widehat{\boldsymbol{\Sigma}}_{k, \ell}=\frac{1}{N} \sum_{t=1}^{N} \boldsymbol{e}_{k}[t, \widehat{\boldsymbol{\theta}}] \boldsymbol{e}_{\ell}^{T}[t, \widehat{\boldsymbol{\theta}}]=\frac{1}{N}\left(\boldsymbol{Y}_{k}-\widehat{\boldsymbol{\Theta}}_{k}^{T} \widetilde{\boldsymbol{\Phi}}_{k}\right)\left(\boldsymbol{Y}_{\ell}-\widehat{\boldsymbol{\Theta}}_{\ell}^{T} \widetilde{\boldsymbol{\Phi}}_{\ell}\right)^{T} \quad k, \ell \in\left\{k_{1}, k_{2}, \ldots, k_{M}\right\}
$$

may be rewritten as:

$$
\begin{aligned}
\frac{1}{N}\left(\boldsymbol{Y}_{k}-\widehat{\boldsymbol{\Theta}}_{k}^{T} \widetilde{\boldsymbol{\Phi}}_{k}\right)\left(\boldsymbol{Y}_{\ell}-\widehat{\boldsymbol{\Theta}}_{\ell}^{T} \widetilde{\boldsymbol{\Phi}}_{\ell}\right)^{T}= & \left(\widehat{\boldsymbol{\Theta}}_{k}-\boldsymbol{\Theta}_{k, o}\right)^{T}\left[\frac{\widetilde{\boldsymbol{\Phi}}_{k} \widetilde{\boldsymbol{\Phi}}_{\ell}^{T}}{N}\right]\left(\widehat{\boldsymbol{\Theta}}_{\ell}-\boldsymbol{\Theta}_{\ell, o}\right)+\frac{\boldsymbol{W}_{k} \boldsymbol{W}_{\ell}^{T}}{N} \\
& +\left(\widehat{\boldsymbol{\Theta}}_{k}-\boldsymbol{\Theta}_{k, o}\right)^{T} \frac{\widetilde{\boldsymbol{\Phi}}_{k} \boldsymbol{W}_{\ell}^{T}}{N}+\frac{\boldsymbol{W}_{k} \widetilde{\boldsymbol{\Phi}}_{\ell}^{T}}{N}\left(\widehat{\boldsymbol{\Theta}}_{\ell}-\boldsymbol{\Theta}_{\ell, o}\right)
\end{aligned}
$$


with $\boldsymbol{W}_{k}$ designating the innovations matrix, defined similar to $\boldsymbol{E}_{k}$ in Eq. (6.A.14d), whereas $\boldsymbol{\Theta}_{k, o}^{T}$ and $\boldsymbol{\Theta}_{\ell, o}^{T}$ designate the true parameter matrices corresponding to the operating conditions $k$ and $\ell$ respectively.

Under the assumption that $\boldsymbol{y}_{k}[t]$ and $\boldsymbol{x}_{k}[t]$ are zero-mean, ergodic and stationary processes, the following limits in probability are zero ${ }^{13}, 15$ :

$$
\begin{gathered}
\left(\widehat{\boldsymbol{\Theta}}_{k}-\boldsymbol{\Theta}_{k, o}\right)^{T}\left[\frac{\widetilde{\boldsymbol{\Phi}}_{k} \widetilde{\boldsymbol{\Phi}}_{\ell}^{T}}{N}\right] \sqrt{N}\left(\widehat{\boldsymbol{\Theta}}_{\ell}-\boldsymbol{\Theta}_{\ell, o}\right) \stackrel{p}{\longrightarrow} \mathbf{0} \quad(N \longrightarrow \infty) \\
\left(\widehat{\boldsymbol{\Theta}}_{k}-\boldsymbol{\Theta}_{k, o}\right)^{T} \frac{\widetilde{\boldsymbol{\Phi}}_{k} \boldsymbol{W}_{\ell}^{T}}{\sqrt{N}} \stackrel{p}{\longrightarrow} \mathbf{0} \quad(N \longrightarrow \infty) \\
\frac{\boldsymbol{W}_{k} \widetilde{\boldsymbol{\Phi}}_{\ell}^{T}}{\sqrt{N}}\left(\widehat{\boldsymbol{\Theta}}_{\ell}-\boldsymbol{\Theta}_{\ell, o}\right) \stackrel{p}{\longrightarrow} \mathbf{0} \quad(N \longrightarrow \infty)
\end{gathered}
$$

Thereby, Eq. (6.A.16) yields ${ }^{16}$ :

$$
\sqrt{N}\left(\widehat{\boldsymbol{\Sigma}}_{k, \ell}-\frac{\boldsymbol{W}_{k} \boldsymbol{W}_{\ell}^{T}}{N}\right)=\sqrt{N}\left(\widehat{\boldsymbol{\Sigma}}_{k, \ell}-\frac{1}{N} \sum_{t=1}^{N} \boldsymbol{w}_{k}[t] \boldsymbol{w}_{\ell}^{T}[t]\right) \stackrel{p}{\longrightarrow} \mathbf{0} \quad(N \longrightarrow \infty)
$$

The preceding result implies that, $\sqrt{N}\left(\widehat{\boldsymbol{\Sigma}}-\frac{1}{N} \sum_{t=1}^{N} \boldsymbol{w}[t] \boldsymbol{w}^{T}[t]\right) \stackrel{p}{\longrightarrow} \mathbf{0}(N \longrightarrow \infty)$, which completes the proof of Lemma 6.A.2.

By virtue of Lemma 6.A.2, $\sqrt{N}\left(\widehat{\boldsymbol{\sigma}}_{L S}-\widehat{\boldsymbol{\sigma}}_{M L}\right) \stackrel{p}{\longrightarrow} \mathbf{0}(N \longrightarrow \infty)$, implying that $\sqrt{N}\left(\widehat{\boldsymbol{\sigma}}_{L S}-\boldsymbol{\sigma}_{o}\right)$ and $\sqrt{N}\left(\widehat{\boldsymbol{\sigma}}_{M L}-\boldsymbol{\sigma}_{o}\right)$ have the same asymptotic distribution ${ }^{17}$, thus (see also Appendix 6.A.3):

$$
\sqrt{N M}\left(\widehat{\boldsymbol{\sigma}}_{L S}-\boldsymbol{\sigma}_{o}\right) \stackrel{d}{\longrightarrow} \mathcal{N}\left(\mathbf{0}, 2 M \boldsymbol{D}_{M n y}^{+}\left(\boldsymbol{\Sigma}_{o} \otimes \boldsymbol{\Sigma}_{o}\right)\left(\boldsymbol{D}_{M n y}^{+}\right)^{T}\right) \quad(N \longrightarrow \infty)
$$

hence, the proof of Theorem 6.2.1 is completed.

\section{A.3 Proof of Theorem 6.2.2}

The joint log-likelihood function for a P-VARX process $\boldsymbol{y}[t]$ with zero mean, independent and normally (Gaussian) distributed, innovations having a covariance matrix $\boldsymbol{\Sigma}$ is given by:

$$
L(\boldsymbol{\vartheta}, \boldsymbol{\Sigma})=-\frac{N M n y}{2} \ln (2 \pi)-\frac{N}{2} \ln (\operatorname{det}(\boldsymbol{\Sigma}))-\frac{1}{2} \sum_{t=1}^{N} \boldsymbol{e}^{T}[t, \boldsymbol{\vartheta}] \boldsymbol{\Sigma}^{-1} \boldsymbol{e}[t, \boldsymbol{\vartheta}]
$$

which is in analogy to the conventional VARX case [2, p. 210], and the following assumptions hold:

(i) The parameter vector $\vartheta$ and the covariance matrix $\boldsymbol{\Sigma}$ are independently parametrized.

(ii) All elements of $\boldsymbol{\Sigma}$ are unknown.

The following composite parameter vector $\overline{\boldsymbol{\vartheta}}=\left[\boldsymbol{\vartheta}^{T} \vdots \boldsymbol{\sigma}^{T}\right]^{T}$ is considered, with $\boldsymbol{\sigma}$ being a vector comprising of the non-identical elements on and below the main diagonal of $\boldsymbol{\Sigma}$ as in the FP-VARX case.

\footnotetext{
${ }^{15}$ Let $\boldsymbol{x}_{N} \stackrel{d}{\longrightarrow} \boldsymbol{x}$ and $\boldsymbol{A}_{N} \stackrel{p}{\longrightarrow} \mathbf{0}$, then $\boldsymbol{A}_{N} \boldsymbol{x}_{N} \stackrel{p}{\longrightarrow} \mathbf{0}(N \longrightarrow \infty)$ [44, p. 684].

${ }^{16}$ Let $\boldsymbol{x}_{N} \stackrel{p}{\longrightarrow} \boldsymbol{x}$ and $\boldsymbol{y}_{N} \stackrel{p}{\longrightarrow} \boldsymbol{y}$, then $\left(\boldsymbol{x}_{N} \pm \boldsymbol{y}_{N}\right) \stackrel{p}{\longrightarrow}(\boldsymbol{x} \pm \boldsymbol{y})(N \longrightarrow \infty)$ [44, p. 683].

${ }^{17}$ Let $\boldsymbol{x}_{N} \stackrel{d}{\longrightarrow} \boldsymbol{x}$ and $\left(\boldsymbol{x}_{N}-\boldsymbol{y}_{N}\right) \stackrel{p}{\longrightarrow} \mathbf{0}$, then $\boldsymbol{y}_{N} \stackrel{d}{\longrightarrow} \boldsymbol{x}(N \longrightarrow \infty)$ [44, p. 683].
} 
Consistency of parameter estimator The first order Taylor series expansion ${ }^{7}$ of $\partial L(\overline{\boldsymbol{\vartheta}}) / \partial \overline{\boldsymbol{\vartheta}}$ around the system's true parameter vector $\overline{\boldsymbol{\vartheta}}_{o}$ is given by:

$$
\widehat{\overline{\boldsymbol{\vartheta}}}_{M L} \approx \overline{\boldsymbol{\vartheta}}_{o}-\left.\left[\left.\frac{1}{N} \frac{\partial^{2} L(\overline{\boldsymbol{\vartheta}})}{\partial \overline{\boldsymbol{\vartheta}} \partial \overline{\boldsymbol{\vartheta}}^{T}}\right|_{\overline{\boldsymbol{\vartheta}}_{=} \overline{\boldsymbol{\vartheta}}_{o}}\right]^{-1} \frac{1}{N} \frac{\partial L(\overline{\boldsymbol{\vartheta}})}{\partial \overline{\boldsymbol{\vartheta}}}\right|_{\overline{\boldsymbol{\vartheta}}_{=}=\overline{\boldsymbol{\vartheta}}_{o}}
$$

Following straightforward calculations, similar to the FP-VARX model case, at $\bar{\vartheta}=\bar{\vartheta}_{o}$, the following limits in probability hold ${ }^{11}$ (under the assumption that $\boldsymbol{y}_{k}[t]$ and $\boldsymbol{x}_{k}[t]$ are zero-mean, ergodic and stationary processes):

$$
\begin{gathered}
\left.\frac{1}{N} \frac{\partial L(\overline{\boldsymbol{\vartheta}})}{\partial \overline{\boldsymbol{\vartheta}}}\right|_{\overline{\boldsymbol{\vartheta}}=\overline{\boldsymbol{\vartheta}}_{o}} \stackrel{p}{\longrightarrow} \mathbf{0} \quad(N \longrightarrow \infty) \\
\left.\frac{1}{N} \frac{\partial^{2} L(\overline{\boldsymbol{\vartheta}})}{\partial \overline{\boldsymbol{\vartheta}} \partial \overline{\boldsymbol{\vartheta}}^{T}}\right|_{\overline{\boldsymbol{\vartheta}}_{=} \overline{\boldsymbol{\vartheta}}_{o}} \stackrel{p}{\longrightarrow}\left[\begin{array}{cc}
E\left\{\widetilde{\boldsymbol{\Phi}}[t] \boldsymbol{\Sigma}_{o}^{-1} \widetilde{\boldsymbol{\Phi}}^{T}[t]\right\} & \mathbf{0} \\
\mathbf{0} & \frac{1}{2} \boldsymbol{D}_{M n y}^{T}\left(\boldsymbol{\Sigma}_{o}^{-1} \otimes \boldsymbol{\Sigma}_{o}^{-1}\right) \boldsymbol{D}_{M n y}
\end{array}\right](N \longrightarrow \infty)
\end{gathered}
$$

Thus, the probability limit in Eq. (6.A.20) yields ${ }^{12}$ :

$$
\widehat{\overline{\boldsymbol{\vartheta}}}_{M L} \stackrel{p}{\longrightarrow} \overline{\boldsymbol{\vartheta}}_{o}(N \longrightarrow \infty)
$$

which is in analogy to the FP-VARX and the conventional VARX model cases.

Asymptotic distribution of parameter estimator Following the FP-VARX and the conventional VARX model cases, in order to derive the asymptotic distribution of $\widehat{\bar{\vartheta}}_{M L}$, Eq. (6.A.20) is rewritten as follows:

$$
\sqrt{N M}\left(\widehat{\overline{\boldsymbol{\vartheta}}}_{M L}-\overline{\boldsymbol{\vartheta}}_{o}\right) \approx-\left[\left.\frac{1}{N M} \frac{\partial^{2} L(\overline{\boldsymbol{\vartheta}})}{\partial \overline{\boldsymbol{\vartheta}} \partial \overline{\boldsymbol{\vartheta}}^{T}}\right|_{\overline{\boldsymbol{\vartheta}}_{=} \overline{\boldsymbol{\vartheta}}_{o}}\right]^{-1}\left[\left.\frac{1}{\sqrt{N M}} \frac{\partial L(\overline{\boldsymbol{\vartheta}})}{\partial \overline{\boldsymbol{\vartheta}}}\right|_{\overline{\boldsymbol{\vartheta}}_{=} \overline{\boldsymbol{\vartheta}}_{o}}\right]
$$

The limiting distribution of the rightmost bracketed quantity is found by means of a central limit theorem ${ }^{13}[2$, p. 550] yielding:

$$
\left.\frac{1}{\sqrt{N M}} \frac{\partial L(\overline{\boldsymbol{\vartheta}})}{\partial \overline{\boldsymbol{\vartheta}}}\right|_{\overline{\boldsymbol{\vartheta}}_{=} \overline{\boldsymbol{\vartheta}}_{o}} \stackrel{d}{\longrightarrow} \mathcal{N}\left(\mathbf{0},\left[\begin{array}{cc}
\frac{1}{M} E\left\{\widetilde{\boldsymbol{\Phi}}[t] \boldsymbol{\Sigma}_{o}^{-1} \widetilde{\boldsymbol{\Phi}}^{T}[t]\right\} & \mathbf{0} \\
\mathbf{0} & \frac{1}{2 M} \boldsymbol{D}_{M n y}^{T}\left(\boldsymbol{\Sigma}_{o} \otimes \boldsymbol{\Sigma}_{o}\right)^{-1} \boldsymbol{D}_{M n y}
\end{array}\right]\right)
$$

and the asymptotic distribution of the ML estimator ${ }^{14}$ is:

$$
\sqrt{N M}\left(\widehat{\overline{\boldsymbol{\vartheta}}}_{M L}-\overline{\boldsymbol{\vartheta}}_{o}\right) \stackrel{d}{\longrightarrow} \mathcal{N}\left(\mathbf{0},\left[\begin{array}{cc}
M E\left\{\widetilde{\boldsymbol{\Phi}}[t] \boldsymbol{\Sigma}_{o}^{-1} \widetilde{\boldsymbol{\Phi}}^{T}[t]\right\}^{-1} & \mathbf{0} \\
\mathbf{0} & 2 M \boldsymbol{D}_{M n y}^{+}\left(\boldsymbol{\Sigma}_{o} \otimes \boldsymbol{\Sigma}_{o}\right)\left(\boldsymbol{D}_{M n y}^{+}\right)^{T}
\end{array}\right]\right) \quad(N \longrightarrow \infty)
$$

which completes the proof of Theorem 6.2.2.

\section{A.4 Statistical accuracy of LS and ML estimators}

In order to study the statistical accuracy of the LS and ML estimators, the following proposition is useful.

Proposition 6.A.1. (Cramér-Rao lower bound for P-VARX models): The asymptotic Cramér-Rao lower bound for a consistent and asymptotically normal estimator $\widehat{\overline{\boldsymbol{\vartheta}}}=\left[\widehat{\boldsymbol{\vartheta}}^{T}: \widehat{\boldsymbol{\sigma}}^{T}\right]^{T}$ of the P-VARX model is:

$$
E\left\{\left(\widehat{\overline{\boldsymbol{\vartheta}}}-\overline{\boldsymbol{\vartheta}}_{o}\right)\left(\widehat{\overline{\boldsymbol{\vartheta}}}-\overline{\boldsymbol{\vartheta}}_{o}\right)^{T}\right\}=\left[\begin{array}{cc}
\frac{1}{N} E\left\{\widetilde{\boldsymbol{\Phi}}[t] \boldsymbol{\Sigma}_{o}^{-1} \widetilde{\boldsymbol{\Phi}}^{T}[t]\right\}^{-1} & \mathbf{0} \\
\mathbf{0} & \frac{2}{N} \boldsymbol{D}_{M n y}^{+}\left(\boldsymbol{\Sigma}_{o} \otimes \boldsymbol{\Sigma}_{o}\right)\left(\boldsymbol{D}_{M n y}^{+}\right)^{T}
\end{array}\right]
$$


Proof. The proof is in analogy to the FP-VARX model case.

It follows from Proposition 6.A.1 that the Cramér-Rao lower bounds of the optimal P-VARX estimators for $\vartheta$ and $\boldsymbol{\sigma}$ are given by:

$$
\begin{array}{r}
E\left\{\sqrt{N M}\left(\widehat{\boldsymbol{\vartheta}}-\boldsymbol{\vartheta}_{o}\right)\left(\widehat{\boldsymbol{\vartheta}}-\boldsymbol{\vartheta}_{o}\right)^{T} \sqrt{N M}\right\}=M E\left\{\widetilde{\boldsymbol{\Phi}}[t] \boldsymbol{\Sigma}_{o}^{-1} \widetilde{\boldsymbol{\Phi}}^{T}[t]\right\}^{-1} \\
E\left\{\sqrt{N M}\left(\widehat{\boldsymbol{\sigma}}-\boldsymbol{\sigma}_{o}\right)\left(\widehat{\boldsymbol{\sigma}}-\boldsymbol{\sigma}_{o}\right)^{T} \sqrt{N M}\right\}=2 M \boldsymbol{D}_{M n y}^{+}\left(\boldsymbol{\Sigma}_{o} \otimes \boldsymbol{\Sigma}_{o}\right)\left(\boldsymbol{D}_{M n y}^{+}\right)^{T}
\end{array}
$$

\section{A.4.1 Proof of Corollary 6.2.1}

The following matrix is nonnegative-definite by construction:

$$
\frac{1}{M} E\left\{\left[\begin{array}{cc}
\widetilde{\boldsymbol{\Phi}}[t] \boldsymbol{\Sigma}_{o}^{-1} \widetilde{\boldsymbol{\Phi}}^{T}[t] & \widetilde{\boldsymbol{\Phi}}[t] \widetilde{\boldsymbol{\Phi}}^{T}[t] \\
\widetilde{\boldsymbol{\Phi}}[t] \widetilde{\boldsymbol{\Phi}}^{T}[t] & \widetilde{\boldsymbol{\Phi}}[t] \boldsymbol{\Sigma}_{o} \widetilde{\boldsymbol{\Phi}}^{T}[t]
\end{array}\right]\right\} \geq \mathbf{0}
$$

Thereby ${ }^{18}$ :

$$
\frac{1}{M} E\left\{\widetilde{\boldsymbol{\Phi}}[t] \boldsymbol{\Sigma}_{o}^{-1} \widetilde{\boldsymbol{\Phi}}^{T}[t]\right\}-\frac{1}{M} E\left\{\widetilde{\boldsymbol{\Phi}}[t] \widetilde{\boldsymbol{\Phi}}^{T}[t]\right\} E\left\{\widetilde{\boldsymbol{\Phi}}[t] \boldsymbol{\Sigma}_{o} \widetilde{\boldsymbol{\Phi}}[t]\right\}^{-1} E\left\{\widetilde{\boldsymbol{\Phi}}[t] \widetilde{\boldsymbol{\Phi}}^{T}[t]\right\} \geq \mathbf{0}
$$

The above inequality is easily rewritten as ${ }^{19}$ :

$$
M E\left\{\widetilde{\boldsymbol{\Phi}}[t] \boldsymbol{\Sigma}_{o}^{-1} \widetilde{\boldsymbol{\Phi}}^{T}[t]\right\}^{-1} \leq M E\left\{\widetilde{\boldsymbol{\Phi}}[t] \widetilde{\boldsymbol{\Phi}}^{T}[t]\right\}^{-1} E\left\{\widetilde{\boldsymbol{\Phi}}[t] \boldsymbol{\Sigma}_{o} \widetilde{\boldsymbol{\Phi}}[t]\right\} E\left\{\widetilde{\boldsymbol{\Phi}}[t] \widetilde{\boldsymbol{\Phi}}^{T}[t]\right\}^{-1}=\boldsymbol{P}_{O L S}
$$

which implies that $\widehat{\vartheta}_{O L S}$ is in general statistically inefficient (compare Eq. (6.A.25a) and Eq. (6.A.28)).

The equality in Eq. (6.A.28) holds under the assumption of groupwise heteroscedastic innovations (see also Chapter 2, Table 2.1), as straightforward calculations on $\boldsymbol{P}_{O L S}$, similar to the proof of Proposition 6.2.1 (see Appendix 6.A.1), yield:

$$
\begin{aligned}
& \boldsymbol{P}_{O L S}=M E\left\{\widetilde{\boldsymbol{\Phi}}[t] \widetilde{\boldsymbol{\Phi}}^{T}[t]\right\}^{-1} E\left\{\widetilde{\boldsymbol{\Phi}}[t] \boldsymbol{\Sigma}_{o} \widetilde{\boldsymbol{\Phi}}^{T}[t]\right\} E\left\{\widetilde{\boldsymbol{\Phi}}[t] \widetilde{\boldsymbol{\Phi}}^{T}[t]\right\}^{-1} \\
& =M\left[\begin{array}{ccc}
E\left\{\widetilde{\boldsymbol{\Phi}}_{k_{1}}[t] \boldsymbol{\Sigma}_{k_{1}, k_{1}}^{-1} \widetilde{\boldsymbol{\Phi}}_{k_{1}}^{T}[t]\right\}^{-1} & \mathbf{0} \\
\mathbf{0} & \ddots & E\left\{\widetilde{\boldsymbol{\Phi}}_{k_{M}}[t] \boldsymbol{\Sigma}_{k_{M}, k_{M}}^{-1} \widetilde{\boldsymbol{\Phi}}_{k_{M}}^{T}[t]\right\}^{-1}
\end{array}\right]
\end{aligned}
$$

The Cramér-Rao lower bound in Eq. (6.A.25a) becomes:

$$
\begin{aligned}
E\left\{\sqrt{N M}\left(\widehat{\boldsymbol{\vartheta}}-\boldsymbol{\vartheta}_{o}\right)\left(\widehat{\boldsymbol{\vartheta}}-\boldsymbol{\vartheta}_{o}\right)^{T} \sqrt{N M}\right\}=M E\left\{\widetilde{\boldsymbol{\Phi}}[t] \boldsymbol{\Sigma}_{o}^{-1} \widetilde{\boldsymbol{\Phi}}^{T}[t]\right\}^{-1} \\
=M\left[\begin{array}{ccc}
E\left\{\widetilde{\boldsymbol{\Phi}}_{k_{1}}[t] \boldsymbol{\Sigma}_{k_{1}, k_{1}}^{-1} \widetilde{\boldsymbol{\Phi}}_{k_{1}}^{T}[t]\right\}^{-1} & \mathbf{0} \\
\mathbf{0} & \ddots & E\left\{\widetilde{\boldsymbol{\Phi}}_{k_{M}}[t] \boldsymbol{\Sigma}_{k_{M}, k_{M}}^{-1} \widetilde{\boldsymbol{\Phi}}_{k_{M}}^{T}[t]\right\}^{-1}
\end{array}\right]
\end{aligned}
$$

\footnotetext{
${ }^{18}$ Consider the symmetric matrix $\boldsymbol{S}=\left[\begin{array}{cc}\boldsymbol{A} & \boldsymbol{B} \\ \boldsymbol{B}^{T} & \boldsymbol{C}\end{array}\right]$, then $\boldsymbol{S} \geq \mathbf{0} \Longleftrightarrow \boldsymbol{A}-\boldsymbol{B C}^{-1} \boldsymbol{B}^{T} \geq \mathbf{0}$ [48, p. 462].

${ }^{19}$ Let $\boldsymbol{A}$ and $\boldsymbol{B}$ be $(m \times m)$ symmetric matrices, and assume that either $\boldsymbol{A}$ and $\boldsymbol{B}$ are positive-definite or $\boldsymbol{A}$ and $\boldsymbol{B}$ are negativedefinite. If $\boldsymbol{A} \leq \boldsymbol{B}$, then $\boldsymbol{B}^{-1} \leq \boldsymbol{A}^{-1}$. If, in addition $\boldsymbol{A}<\boldsymbol{B}$, then $\boldsymbol{B}^{-1}<\boldsymbol{A}^{-1}$ [48, p. 476].
} 
In view of Eq. (6.A.29)-(6.A.30) the statistical optimality of $\widehat{\vartheta}_{O L S}$ for the case of groupwise heteroscedastic innovations is confirmed.

The statistical optimality of $\widehat{\vartheta}_{O L S}$ for the case of groupwise homoscedastic innovations is obtained similarly by replacing $\boldsymbol{\Sigma}_{k_{M}, k_{M}}$ in Eq. (6.A.29)-(6.A.30) with $\overline{\boldsymbol{\Sigma}}$.

The asymptotic efficiency of $\widehat{\boldsymbol{\sigma}}_{O L S}$ follows directly from Eq. (6.A.25b).

\section{A.4.2 Proof of Corollary 6.2.2}

The asymptotic efficiency of $\widehat{\vartheta}_{W L S}$, and $\widehat{\boldsymbol{\sigma}}_{W L S}$ follows directly from Eq. (6.A.25a)-(6.A.25b).

\section{A.4.3 Proof of Corollary 6.2.3}

The asymptotic efficiency of $\widehat{\vartheta}_{M L}$, and $\widehat{\boldsymbol{\sigma}}_{M L}$ follows directly from Eq. (6.A.25a)-(6.A.25b).

\section{A.5 Proof of Theorem 6.2.4}

To establish the consistency of the WLS (the results apply directly on the OLS case) or the ML estimator, the second order derivatives of the corresponding minimization criteria have to be asymptotically positivedefinite. It is easy to observe that the following inequality holds ${ }^{20}$ :

$$
E\left\{\widetilde{\boldsymbol{\Phi}}[t] \boldsymbol{\Sigma}^{-1} \widetilde{\boldsymbol{\Phi}}^{T}[t]\right\} \geq \lambda_{\min }\left(\boldsymbol{\Sigma}^{-1}\right) E\left\{\widetilde{\boldsymbol{\Phi}}[t] \widetilde{\boldsymbol{\Phi}}^{T}[t]\right\} \geq 0
$$

with $\lambda_{\min }(\cdot)$ denoting the minimum eigenvalue of the indicated matrix. The last inequality implies that the second order derivative is positive-definite when the right hand expectation is also positive-definite (note that $\lambda_{\min }\left(\boldsymbol{\Sigma}^{-1}\right)>0$, by virtue of assumption A1.5 in Subsection 6.2.1). Therefore, the problem is simplified into finding the conditions for which the right hand expectation in Eq. (6.A.31) is positive-definite.

Using Eq. (6.6b)-(6.6c), simple algebraic calculations lead to ${ }^{8,9}$ :

$$
E\left\{\widetilde{\boldsymbol{\Phi}}[t] \widetilde{\boldsymbol{\Phi}}^{T}[t]\right\}=\left[\begin{array}{ccc}
\boldsymbol{I}_{n y} \otimes E\left\{\widetilde{\boldsymbol{\phi}}_{k_{1}}[t] \widetilde{\boldsymbol{\phi}}_{k_{1}}^{T}[t]\right\} & \mathbf{0} \\
\mathbf{0} & & \boldsymbol{I}_{n y} \otimes E\left\{\widetilde{\boldsymbol{\phi}}_{k_{M}}[t] \widetilde{\boldsymbol{\phi}}_{k_{M}}^{T}[t]\right\}
\end{array}\right] \geq \mathbf{0}
$$

which imply that ${ }^{21}$ :

$$
\boldsymbol{\Gamma}_{k}=E\left\{\widetilde{\boldsymbol{\phi}}_{k}[t] \widetilde{\boldsymbol{\phi}}_{k}^{T}[t]\right\} \geq \mathbf{0} \quad k=k_{1}, k_{2}, \ldots, k_{M}
$$

Thus, what remains is to find the conditions under which the matrices in Eq. (6.A.33) are positive-definite.

Designating as $\boldsymbol{R}_{\boldsymbol{u}_{k}} \boldsymbol{v}_{k}(\tau)=E\left\{\boldsymbol{u}_{k}[t] \boldsymbol{v}_{k}^{T}[t-\tau]\right\}$ the cross-correlation between $\boldsymbol{u}_{k}[t]$ and $\boldsymbol{v}_{k}[t]$ at lag $\tau$, the matrix $\boldsymbol{\Gamma}_{k}$ may be alternatively written as:

\footnotetext{
${ }^{20}$ Consider the $(m \times m)$ symmetric matrix $\boldsymbol{A}$, then $\lambda_{\min }(\boldsymbol{A}) \boldsymbol{I}_{m} \leq \boldsymbol{A} \leq \lambda_{\max }(\boldsymbol{A}) \boldsymbol{I}_{m}$ [48, p. 467].

${ }^{21}$ Consider the $(m \times m)$ matrices $\boldsymbol{A}_{1}, \boldsymbol{A}_{2}, \boldsymbol{B}_{1}, \boldsymbol{B}_{2} \geq \mathbf{0}$, and assume that $\mathbf{0} \leq \boldsymbol{A}_{1} \leq \boldsymbol{B}_{1}$ and $\mathbf{0} \leq \boldsymbol{A}_{2} \leq \boldsymbol{B}_{2}$, then $\mathbf{0} \leq \boldsymbol{A}_{1} \otimes \boldsymbol{A}_{2} \leq \boldsymbol{B}_{1} \otimes \boldsymbol{B}_{2}[48$, p. 589].
} 


$$
\begin{aligned}
& \boldsymbol{\Gamma}_{k}=\left[\begin{array}{ccc|ccc}
\boldsymbol{R}_{\boldsymbol{y}_{k}} \boldsymbol{y}_{k}(0) & \ldots & \boldsymbol{R}_{\boldsymbol{y}_{k} \boldsymbol{y}_{k}(n a-1)} & -\boldsymbol{R}_{\boldsymbol{y}_{k} \boldsymbol{x}_{k}(-1)} & \ldots & -\boldsymbol{R}_{\boldsymbol{y}_{k} \boldsymbol{x}_{k}}(n b-1) \\
\vdots & \ddots & \vdots & \vdots & \ddots & \vdots \\
\boldsymbol{R}_{\boldsymbol{y}_{k} \boldsymbol{y}_{k}(-n a+1)} \ldots & \boldsymbol{R}_{\boldsymbol{y}_{k} \boldsymbol{y}_{k}(0)} & -\boldsymbol{R}_{\boldsymbol{y}_{k}} \boldsymbol{x}_{k}(-n a) & \ldots & -\boldsymbol{R}_{\boldsymbol{y}_{k} \boldsymbol{x}_{k}(-n a+n b)} \\
\hline-\boldsymbol{R}_{\boldsymbol{x}_{k} \boldsymbol{y}_{k}(1)} & \ldots & -\boldsymbol{R}_{\boldsymbol{x}_{k} \boldsymbol{y}_{k}(n a)} & \boldsymbol{R}_{\boldsymbol{x}_{k} \boldsymbol{x}_{k}(0)} & \ldots & \boldsymbol{R}_{\boldsymbol{x}_{k} \boldsymbol{x}_{k}(n b)}(n b \\
\vdots & \ddots & \vdots & \vdots & \ddots & \vdots \\
-\boldsymbol{R}_{\boldsymbol{x}_{k} \boldsymbol{y}_{k}(-n b+1)} \ldots & -\boldsymbol{R}_{\boldsymbol{x}_{k} \boldsymbol{y}_{k}(-n b+n a)} & \boldsymbol{R}_{\boldsymbol{x}_{k} \boldsymbol{x}_{k}(-n b)} \ldots & \boldsymbol{R}_{\boldsymbol{x}_{k} \boldsymbol{x}_{k}(0)}
\end{array}\right] \\
& =\left[\begin{array}{c|c}
\boldsymbol{\Gamma}_{\boldsymbol{y}_{k}} \boldsymbol{y}_{k}(n a) & -\boldsymbol{\Gamma} \boldsymbol{y}_{k} \boldsymbol{x}_{k}(n a, n b+1) \\
\hline-\left(\boldsymbol{\Gamma} \boldsymbol{y}_{k} \boldsymbol{x}_{k}(n a, n b+1)\right)^{T} & \boldsymbol{\Gamma}_{\boldsymbol{x}_{k}} \boldsymbol{x}_{k}(n b+1)
\end{array}\right]
\end{aligned}
$$

Therefore, the asymptotic positive-definiteness of the second derivative implies the following equivalence:

$$
\begin{aligned}
& E\left\{\widetilde{\boldsymbol{\Phi}}[t] \boldsymbol{\Sigma}^{-1} \widetilde{\boldsymbol{\Phi}}^{T}[t]\right\}>\mathbf{0} \Longleftrightarrow \\
& {\left[\begin{array}{cc}
\boldsymbol{\Gamma} \boldsymbol{y}_{k} \boldsymbol{y}_{k}(n a) & -\boldsymbol{\Gamma} \boldsymbol{y}_{k} \boldsymbol{x}_{k}(n a, n b+1) \\
-\left(\boldsymbol{\Gamma} \boldsymbol{y}_{k} \boldsymbol{x}_{k}(n a, n b+1)\right)^{T} & \boldsymbol{\Gamma} \boldsymbol{x}_{k} \boldsymbol{x}_{k}(n b+1)
\end{array}\right]>\mathbf{0} \quad k=k_{1}, k_{2}, \ldots, k_{M} }
\end{aligned}
$$

In order to continue the analysis, the P-VARX $(n a, n b)$ model in Eq. (6.3a) may be rewritten as follows:

$$
\boldsymbol{y}_{k}[t]=\underbrace{\boldsymbol{A}_{k}^{-1}(\mathcal{B}) \boldsymbol{B}_{k}(\mathcal{B}) \boldsymbol{x}_{k}[t]}_{\boldsymbol{y}_{k}^{*}[t]}+\underbrace{\boldsymbol{A}_{k}^{-1}(\mathcal{B}) \boldsymbol{e}_{k}[t]}_{\boldsymbol{n}_{k}[t]}
$$

with $\boldsymbol{y}_{k}^{*}[t]$ and $\boldsymbol{n}_{k}[t]$ designating the part of the response due to the excitation and the additional noise respectively.

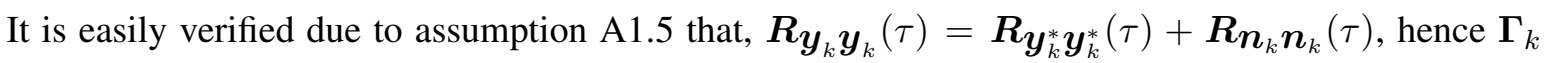
may be alternatively written as:

$\boldsymbol{\Gamma}_{k}=\left[\begin{array}{ccc|ccc}\boldsymbol{R}_{\boldsymbol{y}_{k}^{*} \boldsymbol{y}_{k}^{*}(0)} & \ldots & \boldsymbol{R}_{\boldsymbol{y}_{k}^{*} \boldsymbol{y}_{k}^{*}(n a-1)} & -\boldsymbol{R}_{\boldsymbol{n}_{k} \boldsymbol{x}_{k}(-1)} & \ldots & -\boldsymbol{R}_{\boldsymbol{n}_{k}} \boldsymbol{x}_{k}(n b-1) \\ \vdots & \ddots & \vdots & \vdots & \ddots & \vdots \\ \boldsymbol{R}_{\boldsymbol{y}_{k}^{*} \boldsymbol{y}_{k}^{*}(-n a+1)} \ldots & \boldsymbol{R}_{\boldsymbol{y}_{k}^{*} \boldsymbol{y}_{k}^{*}(0)} & -\boldsymbol{R}_{\boldsymbol{n}_{k} \boldsymbol{x}_{k}(-n a)} \ldots & -\boldsymbol{R}_{\boldsymbol{n}_{k}} \boldsymbol{x}_{k}(-n a+n b) \\ \hline-\boldsymbol{R}_{\boldsymbol{x}_{k}} \boldsymbol{n}_{k}(1) & \ldots & -\boldsymbol{R}_{\boldsymbol{x}_{k} \boldsymbol{n}_{k}(n a)} & \boldsymbol{R}_{\boldsymbol{x}_{k} \boldsymbol{x}_{k}(0)}(0) & \ldots & \boldsymbol{R}_{\boldsymbol{x}_{k} \boldsymbol{x}_{k}(n b)} \\ \vdots & \ddots & \vdots & \vdots & \ddots & \vdots \\ -\boldsymbol{R}_{\boldsymbol{x}_{k} \boldsymbol{n}_{k}(-n b+1)}(-n & -\boldsymbol{R}_{\boldsymbol{x}_{k} \boldsymbol{n}_{k}(-n b+n a)} & \boldsymbol{R}_{\boldsymbol{x}_{k} \boldsymbol{x}_{k}(-n b)} \ldots & \boldsymbol{R}_{\boldsymbol{x}_{k} \boldsymbol{x}_{k}(0)}\end{array}\right]$

$$
\begin{aligned}
& +\left[\begin{array}{ccc|ccc}
\boldsymbol{R}_{\boldsymbol{n}_{k} \boldsymbol{n}_{k}(0)} & \ldots & \boldsymbol{R} \boldsymbol{n}_{k} \boldsymbol{n}_{k}(n a-1) & \mathbf{0} & \ldots & \mathbf{0} \\
\vdots & \ddots & \vdots & \vdots & \ddots & \vdots \\
\boldsymbol{R}_{\boldsymbol{n}_{k} \boldsymbol{n}_{k}(-n a+1)} \ldots & \boldsymbol{R}_{\boldsymbol{n}_{k}} \boldsymbol{n}_{k}(0) & \mathbf{0} & \ldots & \mathbf{0} \\
\hline \mathbf{0} & \ldots & \mathbf{0} & \mathbf{0} & \ldots & \mathbf{0} \\
\vdots & \ddots & \vdots & \vdots & \ddots & \vdots \\
\mathbf{0} & \ldots & \mathbf{0} & \mathbf{0} & \ldots & \mathbf{0}
\end{array}\right] \\
& =\left[\begin{array}{c|c|c}
\boldsymbol{\Gamma}_{\boldsymbol{y}_{k}^{*} \boldsymbol{y}_{k}^{*}}(n a) & -\boldsymbol{\Gamma}_{\boldsymbol{n}_{k} \boldsymbol{x}_{k}(n a, n b+1)} \\
\hline-\left(\boldsymbol{\Gamma} \boldsymbol{n}_{k} \boldsymbol{x}_{k}(n a, n b+1)\right)^{T} & \boldsymbol{\Gamma} \boldsymbol{x}_{k} \boldsymbol{x}_{k}(n b+1)
\end{array}\right]+\left[\begin{array}{c|c}
\boldsymbol{\Gamma}_{\boldsymbol{n}_{k}} \boldsymbol{n}_{k}(n a) & \mathbf{0} \\
\hline \mathbf{0} & \mathbf{0}
\end{array}\right]
\end{aligned}
$$


It then follows that the asymptotic positive-definiteness of the second order derivative implies that ${ }^{22}$ :

$$
E\left\{\widetilde{\boldsymbol{\Phi}}[t] \boldsymbol{\Sigma}^{-1} \widetilde{\boldsymbol{\Phi}}^{T}[t]\right\}>\mathbf{0} \Longleftrightarrow \mid \begin{array}{ll}
\boldsymbol{\Gamma} \boldsymbol{x}_{k} \boldsymbol{x}_{k}(n b+1)>\mathbf{0} & k=k_{1}, k_{2}, \ldots, k_{M} \\
\left(\boldsymbol{\Gamma}_{\boldsymbol{y}_{k}^{*}} \boldsymbol{y}_{k}^{*}(n a)+\boldsymbol{\Gamma}_{\boldsymbol{n}_{k}} \boldsymbol{n}_{k}(n a)\right)>\mathbf{0} & k=k_{1}, k_{2}, \ldots, k_{M}
\end{array}
$$

In view of Lemma 6.2.1, condition (I) is satisfied when $\boldsymbol{x}_{k}[t]\left(k=k_{1}, k_{2}, \ldots, k_{M}\right)$ is persistently exciting of order $(n b+1)$. Condition (II) requires that $\boldsymbol{\Gamma}_{\boldsymbol{n}_{k}} \boldsymbol{n}_{k}(n a)$ is positive definite ${ }^{23}$, which is satisfied when $\boldsymbol{e}_{k}[t]\left(k=k_{1}, k_{2}, \ldots, k_{M}\right)$ is persistently exciting.

Thereby, under weak conditions on the residuals (that is, $\boldsymbol{e}_{k}[t]$ being persistently exciting), the consistency of the LS and ML based estimators implies the following equivalence:

$$
E\left\{\widetilde{\boldsymbol{\Phi}}[t] \boldsymbol{\Sigma}^{-1} \widetilde{\boldsymbol{\Phi}}^{T}[t]\right\}>\mathbf{0} \Longleftrightarrow \boldsymbol{x}_{k}[t]\left(k=k_{1}, k_{2}, \ldots, k_{M}\right) \text { is persistently exciting of order }(n b+1) . \square
$$

\section{A.6 Proof of Proposition 6.2.4}

A straightforward differentiation of Eq. (6.32) yields ${ }^{24}$ :

$$
\frac{\partial W_{\mathcal{M}_{F P}}(\boldsymbol{\theta})}{\partial \boldsymbol{\theta}^{T}}=\frac{\partial W_{\mathcal{M}_{P}}(\boldsymbol{g}(\boldsymbol{\theta}))}{\partial \boldsymbol{\theta}^{T}}=\frac{\partial W_{\mathcal{M}_{P}}(\boldsymbol{g}(\boldsymbol{\theta}))}{\partial \boldsymbol{g}(\boldsymbol{\theta})^{T}} \frac{\partial \boldsymbol{g}(\boldsymbol{\theta})}{\partial \boldsymbol{\theta}^{T}}
$$

and the second derivative is ${ }^{25}$ :

$$
\frac{\partial^{2} W_{\mathcal{M}_{F P}}(\boldsymbol{\theta})}{\partial \boldsymbol{\theta} \partial \boldsymbol{\theta}^{T}}=\left(\frac{\partial \boldsymbol{g}(\boldsymbol{\theta})}{\partial \boldsymbol{\theta}^{T}}\right)^{T} \frac{\partial^{2} W_{\mathcal{M}_{P}}(\boldsymbol{g}(\boldsymbol{\theta}))}{\partial g(\boldsymbol{\theta}) \partial \boldsymbol{g}(\boldsymbol{\theta})^{T}} \frac{\partial \boldsymbol{g}(\boldsymbol{\theta})}{\partial \boldsymbol{\theta}^{T}}+\left(\frac{\partial W_{\mathcal{M}_{P}}(g(\boldsymbol{\theta}))}{\partial \boldsymbol{g}(\boldsymbol{\theta})^{T}} \otimes \boldsymbol{I}\right) \frac{\partial \operatorname{vec}\left(\frac{\partial \boldsymbol{g}(\boldsymbol{\theta})}{\partial \boldsymbol{\theta}}\right)}{\partial \boldsymbol{\theta}^{T}}
$$

At $\boldsymbol{\vartheta}_{o}^{*}=\boldsymbol{g}\left(\boldsymbol{\theta}_{o}\right)$, the rightmost term in Eq. (6.A.39) is zero by virtue of Eq. (6.29), hence:

$$
\left.\frac{\partial^{2} W_{\mathcal{M}_{F P}}(\boldsymbol{\theta})}{\partial \boldsymbol{\theta} \partial \boldsymbol{\theta}^{T}}\right|_{\boldsymbol{\theta}=\boldsymbol{\theta}_{o}}=\left.\left.\left.\left(\frac{\partial \boldsymbol{g}(\boldsymbol{\theta})}{\partial \boldsymbol{\theta}^{T}}\right)^{T}\right|_{\boldsymbol{\theta}=\boldsymbol{\theta}_{o}} \frac{\partial^{2} W_{\mathcal{M}_{P}}(\boldsymbol{g}(\boldsymbol{\theta}))}{\partial \boldsymbol{g}(\boldsymbol{\theta}) \partial \boldsymbol{g}(\boldsymbol{\theta})^{T}}\right|_{\boldsymbol{\theta}=\boldsymbol{\theta}_{o}} \frac{\partial \boldsymbol{g}(\boldsymbol{\theta})}{\partial \boldsymbol{\theta}^{T}}\right|_{\boldsymbol{\theta}=\boldsymbol{\theta}_{o}}=\left.\boldsymbol{T}^{T} \frac{\partial^{2} W_{\mathcal{M}_{P}\left(\boldsymbol{\vartheta}^{*}\right)}}{\partial \boldsymbol{\vartheta}^{*} \partial \boldsymbol{\vartheta}^{* T}}\right|_{\boldsymbol{\vartheta}^{*} \boldsymbol{\vartheta}_{o}^{*}} \boldsymbol{T}
$$

The Cramér-Rao lower bound for the optimal FP-VARX estimators of $\boldsymbol{\theta}$ equals the inverse of the Fisher information matrix, which is given by the following relationship [2, pp. 560-563]:

$$
\mathcal{F}=-\left.E\left\{\frac{\partial^{2} L(\boldsymbol{\theta})}{\partial \boldsymbol{\theta} \partial \boldsymbol{\theta}^{T}}\right\}\right|_{\boldsymbol{\theta}=\boldsymbol{\theta}_{o}}=-\left.\boldsymbol{T}^{T} E\left\{\frac{\partial^{2} L\left(\boldsymbol{\vartheta}^{*}\right)}{\partial \boldsymbol{\vartheta}^{*} \partial \boldsymbol{\vartheta}^{* T}}\right\}\right|_{\boldsymbol{\vartheta}^{*}=\boldsymbol{\vartheta}_{o}^{*}} \boldsymbol{T}=N \boldsymbol{T}^{T} E\left\{\widetilde{\boldsymbol{\Phi}}[t] \boldsymbol{\Sigma}_{o}^{-1} \widetilde{\boldsymbol{\Phi}}^{T}[t]\right\} \boldsymbol{T}
$$

\footnotetext{
${ }^{22}$ Consider the symmetric matrix $\boldsymbol{S}=\left[\begin{array}{cc}\boldsymbol{A} & \boldsymbol{B} \\ \boldsymbol{B}^{T} & \boldsymbol{C}\end{array}\right]$, then $\boldsymbol{S}>\mathbf{0} \Longleftrightarrow \boldsymbol{A}>\mathbf{0}, \boldsymbol{C}>\mathbf{0}$ [48, p. 462].

${ }^{23}$ Consider the $(m \times m)$ symmetric matrices $\boldsymbol{A}$ and $\boldsymbol{B}$, if $\boldsymbol{A} \geq \mathbf{0}$ and $\boldsymbol{B}>\mathbf{0}$, then $\boldsymbol{A}+\boldsymbol{B}>\mathbf{0}$ [48, p. 460].

${ }^{24}$ Let $\boldsymbol{a}$ and $\boldsymbol{\theta}$ be $(m \times 1)$ and $(n \times 1)$ vectors, respectively, and suppose $\boldsymbol{h}(\boldsymbol{a})$ is $(p \times 1)$ and $\boldsymbol{g}(\boldsymbol{\theta})$ is $(m \times 1)$. Then, with $\boldsymbol{a}=\boldsymbol{g}(\boldsymbol{\theta}), \frac{\partial \boldsymbol{h}(\boldsymbol{g}(\boldsymbol{\theta}))}{\partial \boldsymbol{\theta}^{T}}=\frac{\partial \boldsymbol{h}(\boldsymbol{a})}{\partial \boldsymbol{a}^{T}} \frac{\partial \boldsymbol{g}(\boldsymbol{\theta})}{\partial \boldsymbol{\theta}^{T}}(p \times n)[43, \mathrm{p} .174]$.

${ }^{25}$ If $\boldsymbol{\theta}$ is $(m \times 1), \boldsymbol{A}(\boldsymbol{\theta})$ is $(n \times p)$ and $\boldsymbol{B}(\boldsymbol{\theta})$ is $(p \times q)$, then, $\frac{\partial \mathrm{vec}(\boldsymbol{A} \boldsymbol{B})}{\partial \boldsymbol{\theta}^{T}}=\left(\boldsymbol{I}_{q} \otimes \boldsymbol{A}\right) \frac{\partial \mathrm{vec}(\boldsymbol{B})}{\partial \boldsymbol{\theta}^{T}}+\left(\boldsymbol{B}^{T} \otimes \boldsymbol{I}_{n}\right) \frac{\partial \operatorname{vec}(\boldsymbol{A})}{\partial \boldsymbol{\theta}^{T}}[43, \mathrm{p}$. 196].
} 
thus, the corresponding asymptotic covariance matrix is given by:

$$
\boldsymbol{P}=M\left(\boldsymbol{T}^{T} E\left\{\widetilde{\boldsymbol{\Phi}}[t] \boldsymbol{\Sigma}_{o}^{-1} \widetilde{\boldsymbol{\Phi}}^{T}[t]\right\} \boldsymbol{T}\right)^{-1}
$$

Inserting Eq. (6.A.40) and Eq. (6.A.42) into Eq. (6.31) yields ${ }^{26}$ :

$$
\begin{aligned}
F_{\mathcal{M}_{F P}} & =\frac{M}{2} \operatorname{tr}\left(\left(\left.\boldsymbol{T}^{T} \frac{\partial^{2} W_{\mathcal{M}_{P}}\left(\boldsymbol{\vartheta}^{*}\right)}{\partial \boldsymbol{\vartheta}^{*} \partial \boldsymbol{\vartheta}^{* T}}\right|_{\boldsymbol{\vartheta}^{*}=\boldsymbol{\vartheta}_{o}^{*}} \boldsymbol{T}\right)\left(\boldsymbol{T}^{T} E\left\{\widetilde{\boldsymbol{\Phi}}[t] \boldsymbol{\Sigma}_{o}^{-1} \widetilde{\boldsymbol{\Phi}}^{T}[t]\right\} \boldsymbol{T}\right)^{-1}\right) \\
& \leq \frac{M}{2} \operatorname{tr}\left(\left.\frac{\partial^{2} W_{\mathcal{M}_{P}}\left(\boldsymbol{\vartheta}^{*}\right)}{\partial \boldsymbol{\vartheta}^{*} \partial \boldsymbol{\vartheta}^{* T}}\right|_{\boldsymbol{\vartheta}^{*}=\boldsymbol{\vartheta}_{o}^{*}} E\left\{\widetilde{\boldsymbol{\Phi}}[t] \boldsymbol{\Sigma}_{o}^{-1} \widetilde{\boldsymbol{\Phi}}^{T}[t]\right\}^{-1}\right)=F_{\mathcal{M}_{P}}
\end{aligned}
$$

Notice that the parsimony principle is not valid when inefficient parameter estimation methods are employed. Such situations occur when FP-VARX parameter estimation is based on OLS and the innovations are contemporaneously correlated or groupwise heteroscedastic. It is easy to verify that the asymptotic covariance matrix is then given by:

$$
\boldsymbol{P}=M\left(\boldsymbol{T}^{T} E\left\{\widetilde{\boldsymbol{\Phi}}[t] \widetilde{\boldsymbol{\Phi}}^{T}[t]\right\} \boldsymbol{T}\right)^{-1}\left(\boldsymbol{T}^{T} E\left\{\widetilde{\boldsymbol{\Phi}}[t] \boldsymbol{\Sigma}_{o} \widetilde{\boldsymbol{\Phi}}^{T}[t]\right\} \boldsymbol{T}\right)\left(\boldsymbol{T}^{T} E\left\{\widetilde{\boldsymbol{\Phi}}[t] \widetilde{\boldsymbol{\Phi}}^{T}[t]\right\} \boldsymbol{T}\right)^{-1}
$$

In that case additional requirements are necessary so that Proposition 6.2.4 holds true, as seen below.

(i) The accuracy criterion is equal to the trace of the residual covariance matrix, that is:

$$
W_{\mathcal{M}_{F P}}=\operatorname{tr}\left(E\left\{\boldsymbol{e}^{\mathcal{M}_{F P}}[t]\left(\boldsymbol{e}^{\mathcal{M}_{F P}}[t]\right)^{T}\right\}\right)=E\left\{\left(\boldsymbol{e}^{\mathcal{M}_{F P}}[t]\right)^{T} \boldsymbol{e}^{\mathcal{M}_{F P}}[t]\right\}
$$

(ii) The same set of experimental conditions underlies Eq. (6.A.43)-(6.A.44). Then, by virtue of Eq. (6.A.40) the second derivative of $W_{\mathcal{M}_{F P}}$ at $\boldsymbol{\vartheta}_{o}^{*}=g\left(\boldsymbol{\theta}_{o}\right)$ is:

$$
\left.\frac{\partial^{2} W_{\mathcal{M}_{F P}}(\boldsymbol{\theta})}{\partial \boldsymbol{\theta} \partial \boldsymbol{\theta}^{T}}\right|_{\boldsymbol{\theta}=\boldsymbol{\theta}_{o}}=2 \boldsymbol{T}^{T} E\left\{\widetilde{\boldsymbol{\Phi}}[t] \widetilde{\boldsymbol{\Phi}}^{T}[t]\right\} \boldsymbol{T}
$$

Thereby, inserting Eq. (6.A.43) and Eq. (6.A.45) into Eq. (6.31) yields ${ }^{26}$ :

$$
\begin{aligned}
F_{\mathcal{M}_{F P}} & =M \operatorname{tr}\left(\left(\boldsymbol{T}^{T} E\left\{\widetilde{\boldsymbol{\Phi}}[t] \boldsymbol{\Sigma}_{o} \widetilde{\boldsymbol{\Phi}}^{T}[t]\right\} \boldsymbol{T}\right)\left(\boldsymbol{T}^{T} E\left\{\widetilde{\boldsymbol{\Phi}}[t] \widetilde{\boldsymbol{\Phi}}^{T}[t]\right\} \boldsymbol{T}\right)^{-1}\right) \\
& \leq M \operatorname{tr}\left(E\left\{\widetilde{\boldsymbol{\Phi}}[t] \boldsymbol{\Sigma}_{o} \widetilde{\boldsymbol{\Phi}}^{T}[t]\right\} E\left\{\widetilde{\boldsymbol{\Phi}}[t] \widetilde{\boldsymbol{\Phi}}^{T}[t]\right\}^{-1}\right)=F_{\mathcal{M}_{P}}
\end{aligned}
$$

Remark 18. Note that requirement (ii) ensures that the probability characteristics of the data used to estimate the models and the data used to assess the models are the same, so that the second order properties of the regressor matrix $\widetilde{\mathbf{\Phi}}[t]$ are identical in Eq. (6.A.43) and Eq. (6.A.45).

\footnotetext{
${ }^{26}$ Let $\boldsymbol{A}$ and $\boldsymbol{B}$ be $(m \times m)$ positive definite matrices, and $\boldsymbol{R}$ a $(m \times n)$ matrix, then $\operatorname{tr}\left(\boldsymbol{A} \boldsymbol{B}^{-1}\right) \geq \operatorname{tr}\left(\left(\boldsymbol{R}^{T} \boldsymbol{A} \boldsymbol{R}\right)\left(\boldsymbol{R}^{T} \boldsymbol{B} \boldsymbol{R}\right)^{-1}\right)$ [2, pp. 466-467].
} 


\section{A.7 Proof of Proposition 6.2.5}

The asymptotic distribution of the FP-VARX model parameter vector estimator $\widehat{\vartheta}^{*}$ for an efficient (LS or ML based) estimation method is obtained by means of Eq. (6.27b) and Eq. (6.A.42), and is ${ }^{27}$ :

$$
\sqrt{N M}\left(\widehat{\boldsymbol{\vartheta}}^{*}-\boldsymbol{\vartheta}_{o}^{*}\right) \stackrel{d}{\longrightarrow} \mathcal{N}\left(\mathbf{0}, \boldsymbol{P}^{*}\right)
$$

with $\boldsymbol{P}^{*}=M \boldsymbol{T}\left(\boldsymbol{T}^{T} E\left\{\widetilde{\boldsymbol{\Phi}}[t] \boldsymbol{\Sigma}_{o}^{-1} \widetilde{\boldsymbol{\Phi}}^{T}[t]\right\} \boldsymbol{T}\right)^{-1} \boldsymbol{T}^{T}$.

In order to study the efficient estimators of the restricted P-VARX model in Eq. (6.27a)-(6.27c), a Lagrangian procedure is employed (refer to [44, pp. 199-200], for the corresponding case of conventional $\operatorname{VAR}(\mathrm{X})$ models). Assuming that the true system $\mathcal{S}$ generating the data set $Z^{N M}$ belongs to $\mathcal{M}_{F P}$, then there exists a unique projection coefficient $\boldsymbol{\theta}_{o}$, so that $\boldsymbol{\vartheta}_{o}^{*}=\boldsymbol{T} \boldsymbol{\theta}_{o}$ and the parameter constraints in Eq. (6.27b)-(6.27c) may be rewritten in the following form:

$$
\boldsymbol{C \vartheta ^ { * }}=\boldsymbol{\theta}_{o}
$$

with $\boldsymbol{C}=\left(\boldsymbol{T}^{T} \boldsymbol{T}\right)^{-1} \boldsymbol{T}^{T}$.

For the sake of convenience the WLS estimator of the restricted P-VARX model is examined first, and the results are subsequently extended to the other efficient estimators. The Lagrange function associated to this problem [44, p. 671] is:

$$
\mathcal{L}(\boldsymbol{\vartheta}, \boldsymbol{\gamma})=\frac{1}{N M} \sum_{t=1}^{N} \boldsymbol{e}^{T}[t] \boldsymbol{\Sigma}_{o}^{-1} \boldsymbol{e}[t]-\boldsymbol{\gamma}^{T}\left(\boldsymbol{C \vartheta}-\boldsymbol{\theta}_{o}\right)
$$

where $\gamma$ is a vector of Lagrange multipliers. The restricted minimum of the WLS criterion with respect to $\vartheta$ is known to be attained at a point where the first order partial derivatives of $\mathcal{L}(\vartheta, \gamma)$ are zero, and equals:

$$
\widehat{\boldsymbol{\vartheta}}_{W L S}^{*}=\widehat{\boldsymbol{\vartheta}}_{W L S}+\left(\frac{1}{N} \sum_{t=1}^{N} \widetilde{\boldsymbol{\Phi}}[t] \boldsymbol{\Sigma}_{o}^{-1} \widetilde{\boldsymbol{\Phi}}^{T}[t]\right)^{-1} \boldsymbol{C}^{T}\left[\boldsymbol{C}\left(\frac{1}{N} \sum_{t=1}^{N} \widetilde{\boldsymbol{\Phi}}[t] \boldsymbol{\Sigma}_{o}^{-1} \widetilde{\boldsymbol{\Phi}}^{T}[t]\right)^{-1} \boldsymbol{C}^{T}\right]^{-1}
$$

$$
\left(\boldsymbol{\theta}_{o}-\boldsymbol{C} \widehat{\boldsymbol{\vartheta}}_{W L S}\right)
$$

with $\boldsymbol{\vartheta}_{W L S}$ designating the WLS estimator of the unrestricted P-VARX model. Noting that $\boldsymbol{\theta}_{o}-\boldsymbol{C} \boldsymbol{\vartheta}_{o}^{*}=\mathbf{0}$, subtracting $\boldsymbol{\vartheta}_{o}^{*}$ from both sides of Eq. (6.A.50) and multiplying by $\sqrt{N M}$ yields:

$$
\begin{array}{r}
\sqrt{N M}\left(\widehat{\boldsymbol{\vartheta}}_{W L S}^{*}-\boldsymbol{\vartheta}_{o}^{*}\right)=\left(\boldsymbol{I}-\left(\frac{1}{N} \sum_{t=1}^{N} \widetilde{\boldsymbol{\Phi}}[t] \boldsymbol{\Sigma}_{o}^{-1} \widetilde{\boldsymbol{\Phi}}^{T}[t]\right)^{-1} \boldsymbol{C}^{T}\left[\boldsymbol{C}\left(\frac{1}{N} \sum_{t=1}^{N} \widetilde{\boldsymbol{\Phi}}[t] \boldsymbol{\Sigma}_{o}^{-1} \widetilde{\boldsymbol{\Phi}}^{T}[t]\right)^{-1} \boldsymbol{C}^{T}\right]^{-1} \boldsymbol{C}\right) \\
\sqrt{N M}\left(\widehat{\boldsymbol{\vartheta}}_{W L S}^{*}-\boldsymbol{\vartheta}_{o}^{*}\right)
\end{array}
$$

Under the assumption that $\boldsymbol{y}_{k}[t]$ and $\boldsymbol{x}_{k}[t]$ are zero-mean, ergodic and stationary processes ${ }^{11}$, the limiting distribution of Eq. (6.A.51) is ${ }^{28}$ :

$$
\sqrt{N M}\left(\widehat{\boldsymbol{\vartheta}}_{W L S}^{*}-\boldsymbol{\vartheta}_{o}^{*}\right) \stackrel{d}{\longrightarrow} \mathcal{N}\left(\mathbf{0}, \boldsymbol{P}_{W L S}^{*}\right)
$$

where $\boldsymbol{P}_{W L S}^{*}=(\boldsymbol{I}-\boldsymbol{F}) \boldsymbol{P}_{W L S}(\boldsymbol{I}-\boldsymbol{F})^{T}$, with $\boldsymbol{P}_{W L S}=M E\left\{\widetilde{\boldsymbol{\Phi}}[t] \boldsymbol{\Sigma}_{o}^{-1} \widetilde{\boldsymbol{\Phi}}^{T}[t]\right\}^{-1}$ the covariance matrix of the unrestricted case, and $\boldsymbol{F}=E\left\{\widetilde{\boldsymbol{\Phi}}[t] \boldsymbol{\Sigma}_{o}^{-1} \widetilde{\boldsymbol{\Phi}}^{T}[t]\right\}^{-1} \boldsymbol{C}^{T}\left[\boldsymbol{C E}\left[\widetilde{\boldsymbol{\Phi}}[t] \boldsymbol{\Sigma}_{o}^{-1} \widetilde{\boldsymbol{\Phi}}^{T}[t]\right\}^{-1} \boldsymbol{C}^{T}\right]^{-1} \boldsymbol{C}$.

\footnotetext{
${ }^{27}$ Suppose $\widehat{\boldsymbol{\theta}}$ is an estimator of the $(n \times 1)$ vector $\boldsymbol{\theta}$ with $\sqrt{N}(\widehat{\boldsymbol{\theta}}-\boldsymbol{\theta}) \stackrel{d}{\longrightarrow} \mathcal{N}(\mathbf{0}, \boldsymbol{\Sigma}) \quad(N \longrightarrow \infty)$. If $\boldsymbol{R} \neq \mathbf{0}$ is an $(m \times n)$ matrix, then $\sqrt{N}(\boldsymbol{R} \widehat{\boldsymbol{\theta}}-\boldsymbol{R} \boldsymbol{\theta}) \stackrel{d}{\longrightarrow} \mathcal{N}\left(\mathbf{0}, \boldsymbol{R} \boldsymbol{\Sigma} \boldsymbol{R}^{T}\right) \quad(N \longrightarrow \infty)[44$, p. 693].

${ }^{28}$ Suppose $\widehat{\boldsymbol{\theta}}$ is an estimator of the $(n \times 1)$ vector $\boldsymbol{\theta}$ with $\sqrt{N}(\widehat{\boldsymbol{\theta}}-\boldsymbol{\theta}) \stackrel{d}{\longrightarrow} \mathcal{N}(\mathbf{0}, \boldsymbol{\Sigma}) \quad(N \longrightarrow \infty)$. If $\widehat{\boldsymbol{A}} \stackrel{p}{\longrightarrow} \boldsymbol{A} \quad(N \longrightarrow \infty)$, then $\sqrt{N} \widehat{\boldsymbol{A}}(\widehat{\boldsymbol{\theta}}-\boldsymbol{\theta}) \stackrel{d}{\longrightarrow} \mathcal{N}\left(\mathbf{0}, \boldsymbol{A} \boldsymbol{\Sigma} \boldsymbol{A}^{T}\right) \quad(N \longrightarrow \infty)[44$, p. 693].
} 
Straightforward calculations on the restricted WLS asymptotic covariance matrix $\boldsymbol{P}_{W L S}^{*}$ leads to the following inequality:

$$
M E\left\{\widetilde{\boldsymbol{\Phi}}[t] \boldsymbol{\Sigma}_{o}^{-1} \widetilde{\boldsymbol{\Phi}}^{T}[t]\right\}^{-1} \geq(\boldsymbol{I}-\boldsymbol{F}) M E\left\{\widetilde{\boldsymbol{\Phi}}[t] \boldsymbol{\Sigma}_{o}^{-1} \widetilde{\boldsymbol{\Phi}}^{T}[t]\right\}^{-1}(\boldsymbol{I}-\boldsymbol{F})^{T}
$$

which implies that the asymptotic variances of the restricted WLS estimator are smaller than or at most equal to those of any other unrestricted and efficient estimator. Also, due to the equivalence of the restrictions in Eq. (6.A.48) and Eq. (6.27b)-(6.27c), the following inequality holds:

$$
M E\left\{\widetilde{\boldsymbol{\Phi}}[t] \boldsymbol{\Sigma}_{o}^{-1} \widetilde{\boldsymbol{\Phi}}^{T}[t]\right\}^{-1} \geq M \boldsymbol{T}\left(\boldsymbol{T}^{T} E\left\{\widetilde{\boldsymbol{\Phi}}[t] \boldsymbol{\Sigma}_{o}^{-1} \widetilde{\boldsymbol{\Phi}}^{T}[t]\right\} \boldsymbol{T}\right)^{-1} \boldsymbol{T}^{T}
$$

suggesting that the efficient (LS or ML) parameter estimators of the FP-VARX model are superior to their P-VARX counterparts, provided that the restrictions imposed are valid.

\section{Appendix 6.B: Additional results on CCP-VARX modeling}

\section{B.1 Proof of Theorem 6.3.3}

In analogy to the P-VARX and FP-VARX model cases, to establish the consistency of the LS and ML based estimators, the second order derivatives of the corresponding minimization criteria have to be asymptotically positive-definite. It is easy to observe that the following inequality holds ${ }^{20}$ :

$$
E\left\{\overline{\mathbf{\Phi}}[t] \boldsymbol{\Sigma}^{-1} \overline{\mathbf{\Phi}}^{T}[t]\right\} \geq \lambda_{\min }\left(\boldsymbol{\Sigma}^{-1}\right) E\left\{\overline{\mathbf{\Phi}}[t] \overline{\mathbf{\Phi}}^{T}[t]\right\} \geq 0
$$

which implies that the second order derivative is positive-definite when the right hand expectation is also positive-definite (note that $\lambda_{\min }\left(\boldsymbol{\Sigma}^{-1}\right)>0$, by virtue of assumption A3.5 in Subsection 6.3.1). Therefore, the problem is simplified into finding the conditions for which the right hand expectation in Eq. (6.B.1) is positive-definite.

Using Eq. (6.57b)-(6.57c), simple algebraic calculations lead to ${ }^{8,9}$ :

$$
E\left\{\overline{\boldsymbol{\Phi}}[t] \overline{\boldsymbol{\Phi}}^{T}[t]\right\}=\boldsymbol{I}_{n y} \otimes \sum_{k=k_{1}}^{k_{M}} E\left\{\widetilde{\boldsymbol{\phi}}_{k}[t] \widetilde{\boldsymbol{\phi}}_{k}^{T}[t]\right\} \geq \mathbf{0}
$$

Thus, what remains is to find the conditions under which the sum of expectations in Eq. (6.B.2) is positivedefinite. For this purpose, the following matrices are introduced:

$$
\boldsymbol{\Gamma}_{k}=E\left\{\widetilde{\boldsymbol{\phi}}_{k}[t] \widetilde{\boldsymbol{\phi}}_{k}^{T}[t]\right\} \geq 0 \quad k=k_{1}, k_{2}, \ldots, k_{M}
$$

In analogy to the P-VARX model case (see Eq. (6.A.34)), $\boldsymbol{\Gamma}_{k}$ may be alternatively written as:

$$
\boldsymbol{\Gamma}_{k}=\left[\begin{array}{cc}
\boldsymbol{\Gamma}_{\boldsymbol{y}_{k}} \boldsymbol{y}_{k}(n a) & -\boldsymbol{\Gamma} \boldsymbol{y}_{k} \boldsymbol{x}_{k}(n a, n b+1) \\
-\left(\boldsymbol{\Gamma} \boldsymbol{y}_{k} \boldsymbol{x}_{k}(n a, n b+1)\right)^{T} & \boldsymbol{\Gamma}_{\boldsymbol{x}_{k}} \boldsymbol{x}_{k}(n b+1)
\end{array}\right]
$$

Therefore, the asymptotic positive-definiteness of the second derivative implies the following equivalence $^{21}$ :

$$
E\left\{\overline{\boldsymbol{\Phi}}[t] \boldsymbol{\Sigma}^{-1} \overline{\boldsymbol{\Phi}}^{T}[t]\right\}>\mathbf{0} \Longleftrightarrow \sum_{k=k_{1}}^{k_{M}}\left[\begin{array}{cc}
\boldsymbol{\Gamma} \boldsymbol{y}_{k} \boldsymbol{y}_{k}(n a) & -\boldsymbol{\Gamma} \boldsymbol{y}_{k} \boldsymbol{x}_{k}(n a, n b+1) \\
-\left(\boldsymbol{\Gamma} \boldsymbol{y}_{k} \boldsymbol{x}_{k}(n a, n b+1)\right)^{T} & \boldsymbol{\Gamma} \boldsymbol{x}_{k} \boldsymbol{x}_{k}(n b+1)
\end{array}\right]>\mathbf{0}
$$


In order to continue the analysis, the CCP-VARX model in Eq. (6.36a) may be rewritten in the following form:

$$
\boldsymbol{y}_{k}[t]=\underbrace{\boldsymbol{A}^{-1}(\mathcal{B}) \boldsymbol{B}(\mathcal{B}) \boldsymbol{x}_{k}[t]}_{\boldsymbol{y}_{k}^{*}[t]}+\underbrace{\boldsymbol{A}^{-1}(\mathcal{B}) \boldsymbol{e}_{k}[t]}_{\boldsymbol{n}_{k}[t]}
$$

with $\boldsymbol{y}_{k}^{*}[t]$ and $\boldsymbol{n}_{k}[t]$ designating the part of the response due to the excitation and the additional noise respectively.

Following the P-VARX model case (see Eq. (6.A.37)), $\boldsymbol{\Gamma}_{k}$ may be alternatively written as:

$$
\boldsymbol{\Gamma}_{k}=\left[\begin{array}{cc}
\boldsymbol{\Gamma} \boldsymbol{y}_{k}^{*} \boldsymbol{y}_{k}^{*}(n a) & -\boldsymbol{\Gamma} \boldsymbol{n}_{k} \boldsymbol{x}_{k}(n a, n b+1) \\
-\left(\boldsymbol{\Gamma} \boldsymbol{n}_{k} \boldsymbol{x}_{k}(n a, n b+1)\right)^{T} & \boldsymbol{\Gamma} \boldsymbol{x}_{k} \boldsymbol{x}_{k}(n b+1)
\end{array}\right]+\left[\begin{array}{cc}
\boldsymbol{\Gamma}_{\boldsymbol{n}_{k}} \boldsymbol{n}_{k}(n a) & \mathbf{0} \\
\mathbf{0} & \mathbf{0}
\end{array}\right]
$$

Thereby, the asymptotic positive-definiteness of the second derivative implies that ${ }^{22}$ :

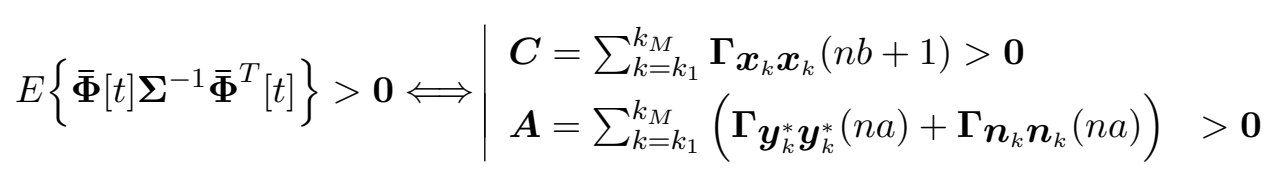

Note that condition (I) is satisfied in two cases:

(a) when there is poor persistence of excitation at all operating conditions $k$, that is $\boldsymbol{\Gamma} \boldsymbol{x}_{k} \boldsymbol{x}_{k}(n b+1) \geq \mathbf{0}$ for $k=k_{1}, k_{2}, \ldots, k_{M}$, yet all excitations considered together yield $\boldsymbol{C}>\mathbf{0}$, or

(b) when the inputs of at least one operating condition are persistently exciting, so that $\boldsymbol{C}>\mathbf{0}$ holds $^{23}$.

Condition (II), on the other hand, is satisfied under weak conditions on the noise process, that is, $e_{k}[t]$ being persistently exciting for $k=k_{1}, k_{2}, \ldots, k_{M}$.

Thereby, under weak conditions on the residuals (that is, $\boldsymbol{e}_{k}[t]$ being persistently exciting), the consistency of the LS and ML based estimators implies the following equivalence:

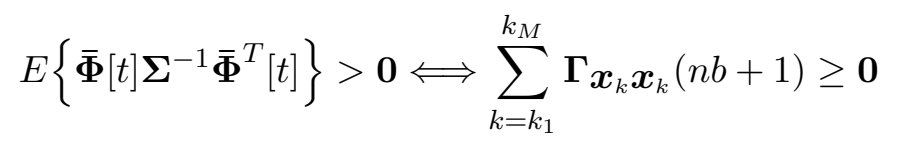

\section{B.2 Proof of Proposition 6.3.4}

In analogy to the proof of Proposition 6.2.4, at $\boldsymbol{\theta}_{o}^{\#}=\boldsymbol{g}\left(\boldsymbol{\vartheta}_{O}^{\#}\right)$ the second order derivative of $W_{\mathcal{M}_{C C P}}\left(\boldsymbol{\theta}^{\#}\right)$ is (compare to Eq. (6.A.40)):

$$
\left.\frac{\partial^{2} W_{\mathcal{M}_{C C P}}\left(\boldsymbol{\vartheta}^{\#}\right)}{\partial \boldsymbol{\vartheta}^{\#} \partial \boldsymbol{\vartheta}^{\# T}}\right|_{\boldsymbol{\vartheta}^{\#}=\boldsymbol{\vartheta}_{o}^{\#}}=\left.\boldsymbol{R}^{T} \frac{\partial^{2} W_{\mathcal{M}_{F P}}\left(\boldsymbol{\theta}^{\#}\right)}{\partial \boldsymbol{\theta}^{\#} \partial \boldsymbol{\theta}^{\# T}}\right|_{\boldsymbol{\theta}^{\#}=\boldsymbol{\theta}_{o}^{\#}} \boldsymbol{R}
$$

Similarly, the asymptotic covariance matrix of the optimal CCP-VARX parameter estimators is given by (compare to Eq. (6.A.42)):

$$
\boldsymbol{P}=M\left(\boldsymbol{R}^{T} E\left\{\boldsymbol{\Phi}[t] \boldsymbol{\Sigma}_{o}^{-1} \boldsymbol{\Phi}^{T}[t]\right\} \boldsymbol{R}\right)^{-1}
$$


Inserting Eq. (6.B.8) and Eq. (6.B.9) into Eq. (6.61) yields ${ }^{26}$ :

$$
\begin{aligned}
& F_{\mathcal{M}_{C C P}}=\frac{M}{2} \operatorname{tr}\left(\left(\left.\boldsymbol{R}^{T} \frac{\partial^{2} W_{\mathcal{M}_{F P}}\left(\boldsymbol{\theta}^{\#}\right)}{\partial \boldsymbol{\theta}^{\#} \partial \boldsymbol{\theta}^{\# T}}\right|_{\boldsymbol{\theta}^{\#}=\boldsymbol{\theta}_{o}^{\#}} \boldsymbol{R}\right)\left(\boldsymbol{R}^{T} E\left\{\boldsymbol{\Phi}[t] \boldsymbol{\Sigma}_{o}^{-1} \boldsymbol{\Phi}^{T}[t]\right\} \boldsymbol{R}\right)^{-1}\right) \\
& \leq \frac{M}{2} \operatorname{tr}\left(\left.\frac{\partial^{2} W_{\mathcal{M}_{F P}}\left(\boldsymbol{\theta}^{\#}\right)}{\partial \boldsymbol{\theta}^{\#} \partial \boldsymbol{\theta}^{\# T}}\right|_{\boldsymbol{\theta}^{\#}=\boldsymbol{\theta}_{o}^{\#}} E\left\{\boldsymbol{\Phi}[t] \boldsymbol{\Sigma}_{o}^{-1} \boldsymbol{\Phi}^{T}[t]\right\}^{-1}\right)=F_{\mathcal{M}_{F P}}
\end{aligned}
$$

When statistically inefficient parameter estimates are obtained via OLS (contemporaneously correlated or groupwise heteroscedastic innovations; refer to CCP-VARX asymptotic properties in Table 6.2), the asymptotic covariance matrix is given by:

$$
\boldsymbol{P}=M\left(\boldsymbol{R}^{T} E\left\{\boldsymbol{\Phi}[t] \boldsymbol{\Phi}^{T}[t]\right\} \boldsymbol{R}\right)^{-1}\left(\boldsymbol{R}^{T} E\left\{\boldsymbol{\Phi}[t] \boldsymbol{\Sigma}_{o} \boldsymbol{\Phi}^{T}[t]\right\} \boldsymbol{R}\right)\left(\boldsymbol{R}^{T} E\left\{\boldsymbol{\Phi}[t] \boldsymbol{\Phi}^{T}[t]\right\} \boldsymbol{R}\right)^{-1}
$$

In analogy to the case of the P-VARX and FP-VARX model classes, the following requirements are imposed so that Proposition 6.3.4 holds true.

(i) The accuracy criterion is equal to the trace of the residual covariance matrix, that is:

$$
W_{\mathcal{M}_{C C P}}=\operatorname{tr}\left(E\left\{\boldsymbol{e}^{\mathcal{M}_{C C P}}[t]\left(\boldsymbol{e}^{\left.\mathcal{M}_{C C P}[t]\right)^{T}}\right\}\right)=E\left\{\left(\boldsymbol{e}^{\mathcal{M}_{C C P}}[t]\right)^{T} \boldsymbol{e}^{\mathcal{M}_{C C P}}[t]\right\}\right.
$$

(ii) The same set of experimental conditions underlies Eq. (6.B.10)-(6.B.11). Then, by virtue of Eq. (6.B.8) the second derivative of $W_{\mathcal{M}_{C C P}}$ at $\boldsymbol{\theta}_{O}^{\#}=\boldsymbol{g}\left(\boldsymbol{\vartheta}_{O}^{\#}\right)$ is:

$$
\left.\frac{\partial^{2} W_{\mathcal{M}_{C C P}}\left(\boldsymbol{\vartheta}^{\#}\right)}{\partial \boldsymbol{\vartheta}^{\#} \partial \boldsymbol{\vartheta}^{\# T}}\right|_{\boldsymbol{\vartheta}^{\#}=\boldsymbol{\vartheta}_{o}^{\#}}=2 \boldsymbol{R}^{T} E\left\{\boldsymbol{\Phi}[t] \boldsymbol{\Phi}^{T}[t]\right\} \boldsymbol{R}
$$

Thereby, inserting Eq. (6.B.10) and Eq. (6.B.12) into Eq. (6.61) yields ${ }^{26}$ :

$$
\begin{aligned}
F_{\mathcal{M}_{C C P}} & =M \operatorname{tr}\left(\left(\boldsymbol{R}^{T} E\left\{\boldsymbol{\Phi}[t] \boldsymbol{\Sigma}_{o} \boldsymbol{\Phi}^{T}[t]\right\} \boldsymbol{R}\right)\left(\boldsymbol{R}^{T} E\left\{\boldsymbol{\Phi}[t] \boldsymbol{\Phi}^{T}[t]\right\} \boldsymbol{R}\right)^{-1}\right) \\
& \leq M \operatorname{tr}\left(E\left\{\boldsymbol{\Phi}[t] \boldsymbol{\Sigma}_{o} \boldsymbol{\Phi}^{T}[t]\right\} E\left\{\boldsymbol{\Phi}[t] \boldsymbol{\Phi}^{T}[t]\right\}^{-1}\right)=F_{\mathcal{M}_{F P}}
\end{aligned}
$$

\section{B.3 Proof of Proposition 6.3.5}

The asymptotic distribution of the CCP-VARX model projection coefficient vector estimator $\widehat{\boldsymbol{\theta}}^{\#}$ for an efficient estimation method (LS or ML based; see Table 6.2) is obtained by means of Eq. (6.58b) and Eq. (6.B.9), and is ${ }^{27}$ :

$$
\sqrt{N M}\left(\widehat{\boldsymbol{\theta}}^{\#}-\boldsymbol{\theta}_{o}^{\#}\right) \stackrel{d}{\longrightarrow} \mathcal{N}\left(\mathbf{0}, \boldsymbol{P}^{\#}\right)
$$

with $\boldsymbol{P}^{\#}=M \boldsymbol{R}\left(\boldsymbol{R}^{T} E\left\{\boldsymbol{\Phi}[t] \boldsymbol{\Sigma}_{o}^{-1} \boldsymbol{\Phi}^{T}[t]\right\} \boldsymbol{R}\right)^{-1} \boldsymbol{R}^{T}$.

Similar to the proof of Proposition 6.2.5, a Lagrangian procedure is employed in order to obtain the efficient estimators of restricted FP-VARX model in Eq. (6.58a)-(6.58c). Assuming that the true system $\mathcal{S}$ generating the data set $Z^{N M}$ belongs to $\mathcal{M}_{C C P}$, then the parameter constraints in Eq. (6.58b)-(6.58c) may be rewritten in the following equivalent form [44, pp. 194-195]:

$$
C \boldsymbol{\theta}^{\#}=\boldsymbol{c}
$$


with $\boldsymbol{C}$ a known $\left(m \times \operatorname{dim}\left(\boldsymbol{\theta}^{\#}\right)\right)$ matrix of rank $m$ and $\boldsymbol{c}$ a known $(m \times 1)$ vector.

For the sake of convenience the WLS estimator of the restricted FP-VARX model is examined first, and the results are subsequently extended to the other efficient estimators. The Lagrange function associated to this problem $[44$, p. 671$]$ is:

$$
\mathcal{L}\left(\boldsymbol{\theta}^{\#}, \boldsymbol{\gamma}\right)=\frac{1}{N M} \sum_{t=1}^{N} \boldsymbol{e}^{T}[t] \boldsymbol{\Sigma}_{o}^{-1} \boldsymbol{e}[t]-\boldsymbol{\gamma}^{T}\left(\boldsymbol{C} \boldsymbol{\theta}^{\#}-\boldsymbol{c}\right)
$$

and in analogy to the proof of Proposition 6.2.5, the following limiting distribution is obtained:

$$
\sqrt{N M}\left(\widehat{\boldsymbol{\theta}}_{W L S}^{\#}-\boldsymbol{\theta}_{o}^{\#}\right) \stackrel{d}{\longrightarrow} \mathcal{N}\left(\mathbf{0}, \boldsymbol{P}_{W L S}^{\#}\right)
$$

where $\boldsymbol{P}_{W L S}^{\#}=(\boldsymbol{I}-\boldsymbol{F}) \boldsymbol{P}_{W L S}(\boldsymbol{I}-\boldsymbol{F})^{T}$, with $\boldsymbol{P}_{W L S}=M E\left\{\boldsymbol{\Phi}[t] \boldsymbol{\Sigma}_{o}^{-1} \boldsymbol{\Phi}^{T}[t]\right\}^{-1}$ the covariance matrix of the unrestricted case, and $\boldsymbol{F}=E\left\{\boldsymbol{\Phi}[t] \boldsymbol{\Sigma}_{o}^{-1} \boldsymbol{\Phi}^{T}[t]\right\}^{-1} \boldsymbol{C}^{T}\left[\boldsymbol{C E}\left\{\boldsymbol{\Phi}[t] \boldsymbol{\Sigma}_{o}^{-1} \boldsymbol{\Phi}^{T}[t]\right\}^{-1} \boldsymbol{C}^{T}\right]^{-1} \boldsymbol{C}$.

Straightforward calculations on the restricted WLS asymptotic covariance matrix $\boldsymbol{P}_{W L S}^{\#}$ leads to the following inequality:

$$
M E\left\{\boldsymbol{\Phi}[t] \boldsymbol{\Sigma}_{o}^{-1} \boldsymbol{\Phi}^{T}[t]\right\}^{-1} \geq(\boldsymbol{I}-\boldsymbol{F}) M E\left\{\boldsymbol{\Phi}[t] \boldsymbol{\Sigma}_{o}^{-1} \boldsymbol{\Phi}^{T}[t]\right\}^{-1}(\boldsymbol{I}-\boldsymbol{F})^{T}
$$

which implies that the asymptotic variances of the restricted WLS estimator are smaller that or at most equal to those of any other unrestricted and efficient estimator. Also, due to the equivalence of the restrictions in Eq. (6.B.14) and Eq. (6.58b)-(6.58c), the following inequality holds:

$$
M E\left\{\boldsymbol{\Phi}[t] \boldsymbol{\Sigma}_{o}^{-1} \boldsymbol{\Phi}^{T}[t]\right\}^{-1} \geq M \boldsymbol{R}\left(\boldsymbol{R}^{T} E\left\{\boldsymbol{\Phi}[t] \boldsymbol{\Sigma}_{o}^{-1} \boldsymbol{\Phi}^{T}[t]\right\} \boldsymbol{R}\right)^{-1} \boldsymbol{R}^{T}
$$

suggesting that the efficient (LS or ML) estimators of the CCP-VARX model are superior to their FP-VARX counterparts, provided that the restrictions imposed are valid. 


\section{Chapter 7}

\section{Conclusions and future research}

The identification of multivariate stochastic systems capable of operating under different conditions was addressed, based on data records corresponding to a sample of such operating conditions. For this purpose, novel global modeling approaches were developed, and the main focus was on the identification of global models of the FP-VARX/VARMA form via the novel Functional Pooling framework. These models may be thought as generalizations of their conventional VARX/VARMA counterparts, with the important distinction being that the model parameters are explicit functions of the operating parameter.

An effective framework for identifying FP-VARX/VARMA models was developed. The estimators of the FP-VARX/VARMA models were formulated by means of the Least Squares (LS) and conditional Maximum Likelihood (ML) principles, and their large sample properties were studied. Conditions ensuring the FP-VARX/VARMA model identifiability were also postulated. The critical problem of FPVARX/VARMA model structure specification was tackled via effective strategies based on information criteria, and statistical decision making tools. The performance characteristics of the FP-VARX/VARMA identification approach were assessed via Monte Carlo studies, which also demonstrated the effectiveness of the proposed framework over conventional VARX/VARMA identification.

An experimental study, aiming at identifying the temperature effects on the dynamics of a smart composite beam, was also treated. Global modeling approaches (CCP-VARX and FP-VARX), and multi-model approaches (non-parametric Welch based and parametric VARX representations) were used to identify the system dynamics under various temperatures. The obtained models were subsequently assessed in terms of compactness and achievable accuracy, which demonstrated the advantages of the Functional Pooling framework over conventional multi-model approaches.

An approach based on the novel FP models and statistical hypothesis testing for damage detection under different operating conditions was also introduced. Two versions of the approach were formulated: the first was based upon the obtained modal parameters, whereas the second was based upon the discrete-time model parameters. The approach presently used response-only vibration data, and the FP-VAR model form for describing the structural dynamics under any operating condition. Its effectiveness was verified via a large number of experiments performed on a smart composite beam under different temperatures, whereas comparisons with alternative methods attempting removal of the temperature effects from the damage-sensitive features were also made.

Finally, the special global models of the P-VARX and the CCP-VARX form were addressed. In analogy to the FP-VARX/VARMA case, the LS and conditional ML type estimators were studied for both model classes, whereas conditions ensuring model identifiability were also postulated. The relationships interconnecting the P-VARX and CCP-VARX models to the FP-VARX models in terms of compactness and achievable accuracy were studied, whereas their association to the conventional VARX models was also 
investigated. The effectiveness and performance characteristics of the novel global modeling approaches were finally assessed via Monte Carlo studies.

The conclusions of this work address both theoretical and practical aspects. The theoretical aspects concern the analysis of the global modeling approaches, whereas the practical aspects refer to the identification of temperature effects on the dynamics of the smart composite beam, as well as the approach for statistical damage detection under different temperatures.

\subsection{Conclusions on theoretical aspects}

(a) The main conclusions drawn from the identification of the FP-VARX and FP-VARMA model classes are:

(i) The asymptotic analysis of the LS and conditional ML type estimators confirmed their consistency and asymptotic normality. The results obtained from the FP-VARX identification suggest that the ML and WLS type estimators attain better accuracy over their OLS counterpart, whereas for the FP-VARMA case the ML estimator generally attains better accuracy over its 2SLS counterpart.

(ii) The model structure specification strategy based on properly forms of the AIC and BIC criteria led to the correct FP-VARX model structure in the majority of cases. The same conclusions are drawn from the application of the two-step approach relying on CCA and proper forms of the preceding information criteria for the FP-VARMA models.

(iii) The comparison of the FP-VARX and FP-VARMA modeling approach with their conventional VARX and VARMA based multi-model counterparts demonstrated the effectiveness of the novel Functional Pooling framework in terms of compactness and achievable accuracy.

(b) The main conclusions drawn from the comparison of the global and conventional VARX model classes are:

(i) The P-VARX and CCP-VARX model classes provide interesting global descriptions of the system, although limited in scope. The P-VARX model class provides valid models for the operating conditions used in the identification procedure only, whereas the CCP-VARX model class provides simple, "averaged" descriptions of the system dynamics over the entire range of operating conditions.

(ii) The estimation of FP-VARX models at the distinct operating conditions used for identification is equivalent to estimating a P-VARX model with parameter constraints. On the other hand, the estimation of CCP-VARX models from the distinct operating conditions used for identification is equivalent to estimating an FP-VARX model with parameter constraints.

(iii) The P-VARX modeling procedure is equivalent to estimating disjoint conventional VARX models when groupwise heteroscedastic or homoscedastic innovations are considered. As for the CCPVARX modeling procedure, it is equivalent to estimating a weighted average of disjoint conventional VARX models yielding the smallest estimator variance when groupwise heteroscedastic or homoscedastic innovations are considered, and efficient LS or ML type parameter estimation methods are employed.

\subsection{Conclusions on practical aspects}

(a) The main conclusions drawn from the identification of temperature effects on the dynamics of a smart composite beam are: 
(i) The multi-model and global representations identified are in rough overall agreement. Yet, the global models are advantageous in that they simultaneously treat all available data records, thus offering improved numerical robustness and compact representations of the structural dynamics.

(ii) The FP models constitute the essential representations of choice as they allow for the explicit, analytical, modeling of temperature dependence. Along with their compactness and improved numerical robustness, they achieve improved estimation accuracy over their multi-model counterparts, which is reflected in significantly smaller modal parameter estimate uncertainties.

(iii) In accordance with previous studies, the natural frequency estimates decrease with temperature in a weakly nonlinear or approximately linear fashion. This dependence becomes more evident for higher frequency modes. Nevertheless, the damping factor estimates remain sensibly constant, indicating a dependence on temperature that is weaker, but of potentially more complex nature.

(b) The main conclusions drawn from the application of the global model based approach for statistical damage detection on a smart composite beam are:

(i) Despite the subtle damage scenario considered (local mass increase amounting to 4\%), the proposed global model approach proved very effective in detecting damage under different temperatures. This effectiveness was not dependent upon whether or not the temperature of each test was used in the baseline (training) phase.

(ii) The natural frequencies provide for an effective and robust characteristic quantity for damage detection, whereas the discrete-time model parameters also provide for a reliable characteristic quantity. The damage detection procedure may be simplified by reduction of the characteristic quantity dimensionality via PCA-based information techniques without compromising severely its performance.

(iii) The comparison with alternative (a PCA and an FA based) methods attempting removal of the temperature effects from the damage-sensitive features revealed improved performance and improved sensitivity to damage for the proposed approach. The FA-based method outperformed its PCA-based counterpart.

Overall, the global modeling approach - and specifically the one based on FP-VARX/VARMA representations - provides a convenient means of identifying systems operating under different conditions and performing uncertainty analysis. The FP models are recommended for achieving the highest accuracy and maximum information extraction from experimental data. Indeed, their advantages over the conventional multi-model approach in terms of achievable accuracy, numerical robustness, physical insight, interpolation capability, and, last but not least, compactness of representation are very important. These features underscore the usefulness of FP models as a tool for precise mathematical modeling, improved physical understanding, structural health monitoring, and automatic control.

\subsection{Future research recommendations}

The novel Functional Pooling identification framework and its applications in practical problems have drawn the attention of the engineering community in recent years. The present thesis addressed some interesting topics on this subject, yet many issues of theoretical and practical nature still need further study. Obviously, the results of the present thesis provide motivation for future research, and some useful ideas toward this direction are given below:

- The extension of the Functional Pooling framework to the case of multivariate models (for instance, 
FP-VARX/ VARMA) characterized by multiple (vector) operating parameters, so that stochastic systems depending on more than one operating parameter are accounted for.

- The extension of the Functional Pooling framework to other interesting model forms, such as the state-space models via subspace identification algorithms.

- The identification of FP models with parameters whose functional dependency on the operating parameter is described by generalized polynomials (such as B-splines), or piece-wise continuous basis functions, in order to account for potential discontinuities.

- The design of optimal experiments for FP model identification, that is to define the number of experiments and the "location" of the corresponding operating conditions (values of operating parameters) so that the identified FP model achieves maximum accuracy.

- The formulation of a functionally model based damage detection, identification and localization method for multivariate FP models.

- The assessment of the stochastic global model approach for damage detection on more realistic damage scenarios of different severity, and its application on "real world" structures with realistic operating conditions. 


\section{Bibliography}

[1] L. Ljung, System Identification: Theory for the User, Second ed., Prentice-Hall PTR, Englewood Cliffs, NJ; 1999.

[2] T. Söderström and P. Stoica, System Identification, Prentice-Hall International, UK; 1989.

[3] H. Sohn, "Effects of Environmental and Operational Variability on Structural Health Monitoring", Philosophical Transactions of the Royal Society - Series A, vol. 365, no. 1851, 2007, pp. 539-560.

[4] R. Tóth, Modeling and Identification of Linear Parameter-Varying Systems, Lecture Notes in Control and Information Sciences, Vol. 403, Springer-Verlag, Berlin, Heidelberg; 2010.

[5] G. Belforte, F. Dabbene and P. Gay, "LPV Approximation of Distributed Parameter Systems in Environmental Modelling", Environmental Modelling \& Software, vol. 20, no. 8, 2005, pp. 1063-1070.

[6] F. Casella and M. Lovera, "LPV/LFT Modelling and Identification: Overview, Synergies and a Case Study", in International Symposium on Computer-Aided Control System Design, San Antonio, Texas, USA, September 3-5, 2008, pp. 852-857.

[7] B. Paijmans, W. Symens, H.V. Brussel and J. Swevers, "Experimental Identification of Affine LPV Models for Mechatronic Systems with One Varying Parameter", European Journal of Control, vol. 14, no. 1,2008 , pp. 16-29.

[8] J. De Caigny, J.F. Camino and J. Swevers, "Interpolating Model Identification for SISO Linear Parameter-Varying Systems", Mechanical Systems and Signal Processing, vol. 23, no. 8, 2009, pp. 2395-2417.

[9] J. De Caigny, J.F. Camino and J. Swevers, "Interpolation-Based Modeling of MIMO LPV Systems", IEEE Transactions on Control Systems Technology, vol. 19, no. 1, 2011, pp. 46-63.

[10] S.A. Billings and S. Chen, "Extended Model Set, Global Data and Threshold Model Identification of Severely Non-Linear Systems", International Journal of Control, vol. 50, no. 5, 1989, pp. 1897-1923.

[11] T.E. Dielman, Pooled Cross-Sectional and Time Series Data Analysis, Marcel Dekker, Inc.; 1989.

[12] N. Beck, "Time-Series-Cross-Section Data", Statistica Neerlandica, vol. 55, no. 2, 2001, pp. 111-133.

[13] C. Horváth and J.E. Wieringa, Combining Time Series and Cross Sectional Data for the Analysis of Dynamic Marketing Systems, SOM Research Report No. 03F13, University of Groningen, 2003.

[14] F.P. Kopsaftopoulos and S.D. Fassois, "Identification of Stochastic Systems Under Multiple Operating Conditions: The Vector Dependent FP-ARX Parametrization", in Proceedings of the $14^{\text {th }}$ Mediterranean Conference on Control and Automation (MED'06), Ancona, Italy, June 28-30, 2006, Paper WM4-4. 
[15] J.S. Sakellariou and S.D. Fassois, "A Functional Pooling Framework for the Identification of Systems Under Multiple Operating Conditions", in Proceedings of the $15^{\text {th }}$ Mediterranean Conference on Control and Automation (MED'07), Athens, Greece, June 27-29, 2007, Paper T21-020-040.

[16] J.S. Sakellariou and S.D. Fassois, "Identification of Dynamical Systems Under Multiple Operating Conditions via Functionally Pooled ARMAX Models", in Proceedings of the $15^{\text {th }}$ Mediterranean Conference on Control and Automation (MED'07), Athens, Greece, June 27-29, 2007, Paper T21-026-021.

[17] F.P. Kopsaftopoulos and S.D. Fassois, "Vector-dependent Functionally Pooled ARX Models for the Identification of Systems Under Multiple Operating Conditions", in Proceedings of the $16^{\text {th }}$ IFAC Symposium on System Identification (SYSID 2012), Brussels, Belgium, July 11-13, 2012.

[18] S.D. Fassois and J.S. Sakellariou, "Time Series Methods for Fault Detection and Identification in Vibrating Structures", Philosophical Transactions of the Royal Society - Series A, vol. 365, no. 1851, 2007, pp. 411-448.

[19] F.P. Kopsaftopoulos and S.D. Fassois, "Vibration-Based Structural Damage Detection and Precise Assessment via Stochastic Functionally Pooled Models", Key Engineering Materials, vol. 347, 2007, pp. $127-132$.

[20] J.S. Sakellariou and S.D. Fassois, "Vibration Based Fault Detection and Identification in an Aircraft Skeleton Structure via a Stochastic Functional Model Based Method", Mechanical Systems and Signal Processing, vol. 22, no. 3, 2008, pp. 557-573.

[21] S.D. Fassois, J.S. Sakellariou, Statistical time series methods for structural health monitoring, in: C. Boller, F. Chang, Y. Fujino (Eds.), Encyclopedia of Structural Health Monitoring, John Wiley \& Sons Ltd., Chichester, U.K., 2009, pp. 443-472.

[22] C.S. Sakaris, J.S. Sakellariou and S.D. Fassois, "Damage Detection and Precise Localization via a Vibration Based Functional Model Method - Application to a 3D Truss Structure", in Proceedings of the $6^{\text {th }}$ European Workshop on Structural Health Monitoring (EWSHM 2012), Dresden, Germany, July 3-6, 2012.

[23] B. Peeters, J. Maeck and G. De Roeck, "Vibration-Based Damage Detection in Civil Engineering: Excitation Sources and Temperature Effects", Smart Materials and Structures, vol. 10, no. 3, 2001, pp. 518-527.

[24] H. Sohn, K. Worden and C.R. Farrar, "Statistical Damage Classification Under Changing Environmental and Operational Conditions", Smart Materials and Structures, vol. 10, no. 9, 2002, pp. 561-574.

[25] A.M. Yan, G. Kerschen, P. De Boe and J.C. Golinval, "Structural Damage Diagnosis Under Varying Environmental Conditions - Part I: A Linear Analysis", Mechanical Systems and Signal Processing, vol. 19 , no. 4, 2005, pp. 847-864.

[26] A.M. Yan, G. Kerschen, P. De Boe and J.C. Golinval, "Structural Damage Diagnosis Under Varying Environmental Conditions - Part II: Local PCA for Non-Linear Cases", Mechanical Systems and Signal Processing, vol. 19, no. 4, 2005, pp. 865-880.

[27] C.K. Oh, H. Soon and I.H. Bae, "Statistical Novelty Detection Within the Yeongjong Suspension Bridge Under Environmental and Operational Variations", Smart Materials and Structures, vol. 18, no. 12, 2009, 125022 doi:10.1088/0964-1726/18/12/125022.

[28] T.Y. Hsu and C.H. Loh, "Damage Detection Accommodating Nonlinear Environmental Effects by Nonlinear Principal Component Analysis", Structural Control and Health Monitoring, vol. 17, no. 3, 2010, pp. 338-354. 
[29] H.F. Zhu, Y.Q Ni and J.M. Ko, "Structural Damage Alarming Using Auto-Associative Neural Network Technique: Exploration of Environment-Tolerant Capacity and Setup of Alarming Threshold", Mechanical Systems and Signal Processing, vol. 25, no. 5, 2011, pp. 1508-1526.

[30] A. Deraemaeker, E. Reynders, G. De Roeck and J. Kullaa, "Vibration-Based Structural Health Monitoring Using Output-Only Measurements Under Changing Environment", Mechanical Systems and Signal Processing, vol. 22, no. 1, 2008, pp. 34-56.

[31] É. Balmès, M. Basseville, F. Bourquin, L. Mevel, H. Nasser and F. Treyssède, "Merging Sensor Data from Multiple Temperature Scenarios for Vibration Monitoring of Civil Structures", Structural Health Monitoring, vol. 7, no. 2, 2008, pp. 129-142.

[32] É. Balmès, M. Basseville, L. Mevel and H. Nasser, "Handling the Temperature Effect in Vibration Monitoring of Civil Structures: A Combined Subspace-Based and Nuisance Rejection Approach", Control Engineering Practice, vol. 17, no. 1, 2009, pp. 80-87.

[33] M. Basseville, F. Bourquin, L. Mevel, H. Nasser and F. Treyssède, "Handling the Temperature Effect in Vibration Monitoring: Two Subspace-Based Analytical Approaches", Journal of Engineering Mechanics, vol. 136, no. 3, 2010, pp. 367-378.

[34] K. Worden, H. Sohn and C.R. Farrar, "Novelty Detection in a Changing Environment: Regression and Interpolation Approaches", Journal of Sound and Vibration, vol. 258, no. 4, 2002, pp. 741-761.

[35] J.T. Kim, J.H. Park and B.J. Lee, "Vibration-Based Damage Monitoring in Model Plate-Girder Bridges Under Uncertain Temperature Conditions”, Engineering Structures, vol. 29, no. 7, 2007, pp. 13541365.

[36] H. Sohn, M. Dzwonczyk, E.G. Straser, A.S. Kiremidjian, K.H. Law and T. Meng, "An Experimental Study of Temperature Effect on Modal Parameters of the Alamosa Canyon Bridge", Earthquake Engineering and Structural Dynamics, vol. 28, no. 8, 1999, pp. 879-897.

[37] F. Magalhães, A. Cunha and E. Caetano, "Vibration Based Structural Health Monitoring of an Arch Bridge: From Automated OMA to Damage Detection", Mechanical Systems and Signal Processing, vol. 28, 2012, pp. 212-228.

[38] J.D. Hios and S.D. Fassois, "Statistical Damage Detection in a Smart Structure Under Different Temperatures via Vibration Testing: A Global Model Based Approach", in Proceedings of the $8^{\text {th }}$ International Conference on Damage Assesment of Structures (DAMAS 2009), Beijing, China, August 3-5, 2009, Paper 042. Also in Key Engineering Materials, vol. 413-414, 2009, pp. 261-268.

[39] A.M. Lekkas, J.D. Hios and S.D. Fassois, "Output-only damage detection in a composite beam under varying temperatures via vector stochastic models", in Proceedings of the $5^{\text {th }}$ European Workshop on Structural Health Monitoring (EWSHM 2010), Sorrento, Italy, June 28 - July 4, 2010, pp. 815-822.

[40] J.D. Hios and S.D. Fassois, "Identification of a Global Model Describing the Temperature Effects on the Dynamics of a Smart Composite Beam", in Proceedings of the International Conference on Noise and Vibration Engineering (ISMA 2006), Leuven, Belgium, September 18-20 2006, CD-ROM; Paper ID 230 .

[41] J.D. Hios and S.D. Fassois, "Stochastic Identification of Temperature Effects on the Dynamics of a Smart Composite Beam: Assessment of Multi-Model and Global Approaches", in Proceedings of the International Conference on Noise and Vibration Engineering (ISMA 2008), Leuven, Belgium, September 15-17, 2008, CD-ROM; Paper ID 131. 
[42] J.D. Hios and S.D. Fassois, "Stochastic Identification of Temperature Effects on the Dynamics of a Smart Composite Beam: Assessment of Multi-Model and Global Model Approaches", Smart Materials and Structures, vol. 18035011 (15pp), 2009. doi:10.1088/0964-1726/18/3/035011.

[43] H. Lütkepohl, Handbook of Matrices, John Willey \& Sons, Chichester; 1996.

[44] H. Lütkepohl, New Introduction to Multiple Time Series Analysis, Springer-Verlag, Berlin, Heidelberg; 2005.

[45] M. Abramowitz and I.A. Stegun, Handbook of Mathematical Functions, New York: Dover; 1970.

[46] The MathWorks ${ }^{\mathrm{TM}}$, Optimization Toolbox, ver. 2.1.1, in http://www . mathworks.com.

[47] The MathWorks ${ }^{\mathrm{TM}}$, System Identification Toolbox, ver. 5, in http://www. mathworks.com.

[48] D.S. Bernstein, Matrix Mathematics: Theory, Facts, and Formulas with Application to Linear Systems Theory, Second ed., Princeton University Press; 2009.

[49] C. L. Lawson and R. J. Hanson, Solving Least Squares Problems, Prentice-Hall Inc., Englewood Cliffs, NJ; 1974.

[50] M.D. Rao, R. Echempati and S. Nadella, "Dynamic Analysis and Damping of Composite Structures Embedded with Viscoelastic Layers", Composites Part B, vol. 28, no. 5-6, 1997, pp. 547-554.

[51] P. Vangipuram and N. Ganesan, "Buckling and Vibration of Rectangular Composite Viscoelastic Sandwhich Plates Under Thermal Loads”, Composite Structures, vol. 77, no. 4, 2007, pp. 419-429.

[52] J. Avsec and M. Oblak, "Thermal Vibrational Analysis for Simply Supported Beam and Clamped Beam", Journal of Sound and Vibration, vol. 308, no. 3-5, 2007, pp. 514-525.

[53] H. Matsunaga, "Free Vibration and Stability of Angle-Ply Laminated Composite and Sandwich Plates Under Thermal Loading", Composite Structures, vol. 77, no. 2, 2007, pp. 249-262.

[54] B.J. Dobson and R. Drew, "Experimental Investigation of the Effects of Temperature on the Dynamic Properties of a Carbon Fibre-Reinforced Plate", Composites, vol. 22, no. 3, 1991, pp. 199-203.

[55] S. Galea and R. White, "The Effect of Temperature on the Natural Frequencies and Acoustically Induced Strains in CFRP Plates", Journal of Sound and Vibration, vol. 164, no. 3, 1993, pp. 399-424.

[56] P.Q. Zhang, J.H. Ruan and W.Z. Lee, "Influence of Some Factors on the Damping Property of FiberReinforced Epoxy Composites at Low Temperature", Cryogenics, vol. 41, no. 4, 2001, pp. 245-251.

[57] N.A. Chrysochoidis, S. Tzoutzouli and D.A. Saravanos, "Identifying the Temperature Effect on the Damped Response of Composite Beams Using Piezoceramic Actuators and Sensors", in Proceedings of the $11^{\text {th }}$ European Conference on Composite Materials (ECCM-11), Rhodes, Greece, May 31 June 03, 2004, CD-ROM; B129.

[58] Y. Sefrani and J. Berthelot, "Temperature Effect on the Damping Properties of Unidirectional Glass Fibre Composites", Composites Part B, vol. 37, no. 4-5, 2006, pp. 364-355.

[59] G.M. Loyd, M.L. Wang and V. Sigh, "Observed Variations of Mode Frequencies of a Prestressed Concrete Bridge with Temperature", in Proceedings of the $14^{\text {th }}$ Engineering Mechanics Conference of the American Society of Civil Engineers (EM 2000), Austin, Texas, 2000, pp. 1-11. 
[60] J.M. Ko, K.K. Chak, J.Y. Wang, Y.Q. Ni and T.H.T. Chan, "Formulation of an Uncertainty Model Relating Modal Parameters and Environmental Factors by Using Long-Term Monitoring Data", in Proceedings of SPIE, Smart Systems and Nondestructive Evaluation for Civil Infrastructures, San Diego, CA, USA, 2003, pp. 298-307.

[61] Y.Q. Ni, X.G. Hua, K.Q. Fan and J.M. Ko, "Correlating Modal Properties with Temperature Using Long-Term Monitoring Data and Support Vector Machine Technique”, Engineering Structures, vol. 27, no. 12, 2005, pp. 1762-1773.

[62] Y. Xia, H. Hao, G. Zanardo and A. Deeks, "Long Term Monitoring of an RC Slab: Temperature and Humidity Effect”, Engineering Structures, vol. 28, no. 3, 2006, pp. 441-452.

[63] S.M. Kay, Modern Spectral Estimation, Prentice-Hall Inc., NJ; 1998

[64] K.A. Florakis, S.D. Fassois and F.M. Hemez, "MIMO LMS-ARMAX Identification of Vibrating Stuctures - Part II: A Critical Assessment", Mechanical Systems and Signal Processing, vol. 15, no. 4, 2001, pp. 737-758.

[65] P.G. Michaelides and S.D. Fassois, "Stochastic Identification of Structural Dynamics from Multiple Experiments - Experimental Variability Analysis", in International Conference on Noise and Vibration Engineering (ISMA 2008), Leuven, Belgium, September 15-17, 2008, pp. 3927-3941.

[66] J. He and Z.F. Fu, Modal Analysis, Butterworth-Heinemann, Oxford, UK; 2001.

[67] The MathWorks ${ }^{\mathrm{TM}}$, Signal Processing Toolbox, ver. 5, in http://www . mathworks.com.

[68] S.D. Fassois, "Parametric Identification of Vibrating Structures", Encyclopedia of Vibration, S.G. Braun, D.J. Ewins, S.S. Rao (eds.), Academic Press, 2001, pp. 673-685.

[69] K.A. Petsounis and S.D. Fassois, "Parametric Time-Domain Methods for the Identification of Vibrating Structures - A Critical Comparison and Assessment", Mechanical Systems and Signal Processing, vol. 15, no. 6, 2001, pp. 1031-1060.

[70] P. Andersen and R. Brincker, "Estimation of Modal Parameters and their Uncertainties", in Proceedings of the $17^{\text {th }}$ International Modal Analysis Conference (IMAC 17), Kissimmee, FL, USA, February 8-11, 1999.

[71] T.S. Plagianakos and D.A. Saravanos, "Hybrid Multidamped Composite Plates with Viscoelastic Composite Plies and Shunted Piezoelectric Layers", Journal of Intelligent Material Systems and Structures, vol. 14, no. 1, 2003, pp. 57-66.

[72] J.D. Hios and S.D. Fassois, "Stochastic Identification Under Multiple Operating Conditions: Functionally Pooled VARMA Methods", in Proceedings of the $15^{\text {th }}$ IFAC Symposium on System Identification (SYSID 2009), Saint-Malo, France, July 6-8, 2009, pp. 1626-1631.

[73] R.S. Tsay, "Identifying Multivariate Time-Series Models", Journal of Time Series Analysis, vol. 10, no. 4, 1989, pp. 357-372.

[74] G.C. Reinsel, Elements of Multivariate Time Series Analysis, Springer, New York; 1993.

[75] G.H. Golub and C.F. Van Loan, Matrix Computations, Third ed., The John Hopkins University Press, Baltimore; 1996.

[76] P. Stoica, T. Söderström, A. Ahlén and G. Solbrand, "On the Asymptotic Accuracy of Pseudo-Linear Regression Algorithms", International Journal of Control, vol. 39, no. 1, 1984, pp. 115-126. 
[77] J.R.M. Hosking, "The Multivariate Portmanteau Statistic", Journal of the American Statistical Association, vol. 75, no. 371, 1980, pp. 602-608.

[78] G.C. Judge, W.E. Griffiths, R.C. Hill, H. Lütkepohl and T.-C. Lee, The Theory and Practice of Econometrics, Second ed., John Willey \& Sons Inc., USA; 1985.

[79] H. Spliid, "A Fast Estimation Method for the Vector Autoregressive Moving Average Model with Exogenous Variables", Journal of the American Statistical Association, vol. 78, no. 384, 1983, pp. 843849 .

[80] P. Andersen, R. Brincker and P.H. Kirkegaard, "Theory of Covariance Equivalent ARMAV Models of Civil Engineering Structures", in Proceedings of the $14^{\text {th }}$ International Modal Analysis Conference (IMAC 14), Dearborn, Michigan, USA, February 12-15, 1996, pp. 518-524.

[81] V. Papakos and S.D. Fassois, "Multichannel Identification of Aircraft Skeleton Structures Under Unobservable Excitation: A Vector AR/ARMA Framework", Mechanical Systems and Signal Processing, vol. 17 , no. 6, 2003, pp. 1271-1290.

[82] D.D. Rizos, S.D. Fassois, Z.P. Marioli-Riga and A.N. Karanika, "Vibration-Based Skin Damage Statistical Detection and Restoration Assessment in a Stiffened Aircraft Panel", Mechanical Systems and Signal Processing, vol. 22, no. 2, 2008, pp. 315-337.

[83] W. Härdle and L. Simar, Applied Multivariate Statistical Analysis, Second ed., Springer-Verlag, Berlin Heidelberg; 2007.

[84] G.E.P. Box and G.C. Tiao, "A Canonical Analysis of Multiple Time Series”, Biometrika, vol. 64, no. 2, 1977, pp. 355-365.

[85] G.C. Tiao and G.E.P. Box, "Modeling Multiple Time Series With Applications", Journal of the American Statistical Association, vol. 76, no. 376, 1981, pp. 802-816.

[86] L. Mevel, L. Hermans and H. van der Auweraer, "Application of a Subspace-Based Fault Detection Method to Industrial Structures", Mechanical Systems and Signal Processing, vol. 13, no. 6, 1999, pp. 823-838.

[87] A.M Yan and J.C. Golinval, "Null Subspace-Based Damage Detection of Structures Using Vibration Measurements", Mechanical Systems and Signal Processing, vol. 20, no. 3, 2006, pp. 611-626.

[88] H. Pham (Ed.), Handbook of Engineering Statitstics, Springer-Verlag, London Ltd.; 2006.

[89] P. Stoica and T. Söderström, "On the Parsimony Principle", International Journal of Control, vol. 36, no. 3, 1982, pp. 409-418. 


\section{List of Figures}

1.1 Schematic of the global model identification problem. Each operating condition $\boldsymbol{k}$ of the system (not varying with time) is depicted as a point in a generic operating space. The set of all possible operating conditions forms the operating range, whereas for each value of $k$ a data record $Z_{\boldsymbol{k}}$ is obtained which is depicted as a point in a generic data space. . . . . . . 2

1.2 Schematic of global model identification based on the "multi-model" approach and interpolation procedures. For every data record $Z_{\boldsymbol{k}}$ belonging to the generic data space an individual model $\mathcal{M}_{\boldsymbol{k}}$ is identified, which corresponds to a point in a generic model space. The set of individual models is subsequently interpolated in order to obtain a "global" model $\mathcal{M}(\boldsymbol{k})$. . .

1.3 Schematic of the Functional Pooling framework for global model identification. The available data records $Z_{\boldsymbol{k}}$ belonging to the generic data space are treated simultaneously using data pooling techniques in order to obtain a "global", functionally pooled, model $\mathcal{M}(\boldsymbol{k})$. . . 4

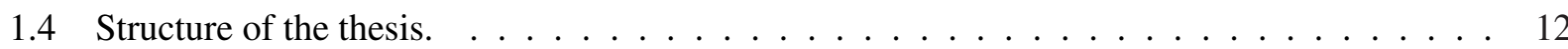

2.1 Schematic of the FP-VARX model structure. . . . . . . . . . . . . . . . . . . 26

2.2 Presence of noise on the data generated by the $\operatorname{FP}-\operatorname{VARX}(2,0)_{[3,3]}$ model for each operating condition $k$; (a),(b) ratio of colored noise standard deviation over the model's response standard deviation; (c),(d) ratio of noise-free response standard deviation over the model's response standard deviation (" $\times$ " marks designate the value of a single experiment; 500 Monte Carlo runs). . . . . . . . . . . . . . . . . . . . .

2.3 FP-VARX $(2,0)_{[p, p]}$ model structure specification results for the WLS-based estimator: (a) residual covariance matrix trace (" $\times$ " marks designate the value of a single experiment and "o" their corresponding sample mean), (b) functional subspace dimensionality specification via the AIC and BIC (500 Monte Carlo runs per model). . . . . . . . . . . . . . . . .

2.4 $\operatorname{FP}-\operatorname{VARX}(2,0)_{[3,3]}$ actual values (--) and Monte Carlo estimates based upon the OLS, WLS, and ML methods for selected projection coefficients and innovation variances (500 Monte Carlo runs per method; $a_{m, n}^{i, j}$ and $b_{m, n}^{i, j}$ designate the $(m, n)$-th elements of $\boldsymbol{A}_{i, j}$ and $\boldsymbol{B}_{i, j}$ respectively; $\sigma_{m, n}(k, \ell)$ designates the $(m, n)$-th element of $\boldsymbol{\Sigma}_{k, \ell}$; the dark green boxes indicate the sample mean \pm sample standard deviation; the light grey boxes indicate the sample mean \pm mean standard deviation computed through the asymptotic distribution).

2.5 Dependence of certain AutoRegressive and eXogenous parameters on the operating parameter $k$ (boxes indicate sample mean estimates $\pm 2 \times$ mean sample standard deviation computed through the asymptotic distribution): (-) theoretical values; (blue boxes) FP$\operatorname{VARX}(2,0)_{[p, p]}$ model; (green boxes) conventional $\operatorname{VARX}(2,0)$ models (500 Monte Carlo runs per model; $a_{m, n}^{i}(k)$ and $b_{m, n}^{i}(k)$ designate the $(m, n)$-th elements of $\boldsymbol{A}_{i}(k)$ and $\boldsymbol{B}_{i}(k)$,

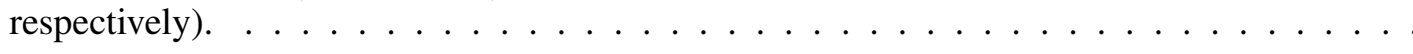


2.6 Presence of noise on the data generated by the FP-VARX $(3,1)_{[4,4]}$ model for each operating condition $k$; (a),(b) ratio of colored noise standard deviation over the model's response standard deviation; (c),(d) ratio of noise-free response standard deviation over the model's response standard deviation (" $x$ " marks designate the value of a single experiment; 500 Monte Carlo runs). . . . . . . . . . . . . . . . . . . . . . . .

2.7 $\operatorname{FP}-\operatorname{VARX}(3,1)_{[p, p]}$ model structure specification results for the WLS-based estimator: (a) residual covariance matrix trace (" $\times$ " marks designate the value of a single experiment and "o" their corresponding sample mean), (b) functional subspace dimensionality specification via the AIC and BIC (500 Monte Carlo runs per model).

2.8 FP-VARX $(3,1)_{[4,4]}$ actual values (--) and Monte Carlo estimates based upon the OLS, WLS, and ML methods for selected projection coefficients and innovation variances (500 Monte Carlo runs per method; $a_{m, n}^{i, j}$ and $b_{m, n}^{i, j}$ designate the $(m, n)$-th elements of $\boldsymbol{A}_{i, j}$ and $\boldsymbol{B}_{i, j}$ respectively; $\sigma_{m, n}(k, \ell)$ designates the $(m, n)$-th element of $\boldsymbol{\Sigma}_{k, \ell}$; the dark green boxes indicate the sample mean \pm sample standard deviation; the light grey boxes indicate the sample mean \pm mean standard deviation computed through the asymptotic distribution).

2.9 Dependence of certain AutoRegressive and eXogenous parameters on the operating parameter $k$ (boxes indicate sample mean estimates $\pm 2 \times$ mean sample standard deviation computed through the asymptotic distribution): (一) actual values; (blue boxes) FP-VARX $(3,1)_{[p, p]}$ model; (green boxes) conventional VARX $(3,1)$ models (500 Monte Carlo runs per model; $a_{m, n}^{i}(k)$ and $b_{m, n}^{i}(k)$ designate the $(m, n)$-th elements of $\boldsymbol{A}_{i}(k)$ and $\boldsymbol{B}_{i}(k)$, respectively). .

2.10 Indicative shifted Chebyshev polynomials of the second kind. . . . . . . . . . . .

$2.11 \mathrm{FP}-\operatorname{VARX}(2,0)_{[3,3]}$ model based frequency response magnitude for $G_{11}$ with respect to frequency and operating parameter $k$ : (a) actual system, (b) OLS mean estimate, (c) WLS mean estimate and, (d) ML mean estimate. . . . . . . . . . . . . . . . . . . . . . . .

2.12 FP-VARX $(2,0)_{[3,3]}$ model based frequency response phase for $G_{11}$ with respect to frequency and operating parameter $k$ : (a) actual system, (b) OLS mean estimate, (c) WLS mean estimate and, (d) ML mean estimate. . . . . . . . . . . . . . . . . . . . . . .

2.13 Projection of the function minimized in the ML method on selected FP-VARX $(2,0)_{[3,3]}$ model parameters: (a),(c) surface and contour plots by projecting on $\alpha_{1,1}^{1,1}$ and $\alpha_{1,1}^{1,2}$; (b),(d) surface and contour plots by projecting on $\alpha_{1,1}^{1,3}$ and $\alpha_{1,2}^{1,3}$ (the " $\times$ " marks designate the actual $\operatorname{FP}-\operatorname{VARX}(2,0)_{[3,3]}$ model parameters $) . \ldots \ldots \ldots \ldots$

$2.14 \mathrm{FP}-\operatorname{VARX}(3,1)_{[4,4]}$ model based frequency response magnitude for $G_{22}$ with respect to frequency and operating parameter $k$ : (a) actual system, (b) OLS mean estimate, (c) WLS mean estimate and, (d) ML mean estimate. . . . . . . . . . . . . . . . . . . . . . . .

2.15 FP-VARX $(3,1)_{[4,4]}$ model based frequency response phase for $G_{22}$ with respect to frequency and operating parameter $k$ : (a) actual system, (b) OLS mean estimate, (c) WLS mean estimate and, (d) ML mean estimate. . . . . . . . . . . . . . . . . . . .

2.16 Projection of the function minimized in the ML method on selected FP-VARX $(3,1)_{[4,4]}$ model parameters: (a),(c) surface and contour plots by projecting on $\alpha_{1,1}^{2,1}$ and $\alpha_{1,1}^{2,2}$; (b),(d) surface and contour plots by projecting on $b_{2,2}^{1,3}$ and $b_{2,2}^{1,4}$ (the " $\times$ " marks designate the actual

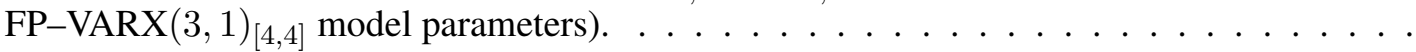

3.1 (a) Photo of the smart beam; (b) schematic representation of the considered transfer functions $G_{1}, G_{2} ;$ (c) schematic diagram of the laboratory setup. . . . . . . . . . . . 
3.2 Parametric VARX-based multi-model approach: $\operatorname{VARX}(n, n)$ model order specification for three distinct temperatures; (a),(c),(e) frequency stabilization diagrams (the arrows at the top indicate identified modes); (b),(d),(f) condition numbers for the OLS estimator inverted matrix versus model order. (In all cases the vertical/horizontal dashed lines indicate the selected model order) . . . . . . . . . . . . . . . . . . . . . . . . . .

3.3 Schematic representation of the global model identification problem, showing the operating parameter $k$ (temperature), the excitation/response data records corresponding to different temperatures, and the FP-VARX model class. . . . . . . . . . . . . . . . . . .

3.4 FP-VARX $(23,23)_{[p, p]}$ structure specification: (a) condition number of the WLS estimator inverted matrix versus functional basis dimensionality $p$; (b)- $(\mathrm{g})$ convergence of the natural frequency $\left(f_{\mathrm{n}}\right)$ versus temperature curves for increasing functional subspace dimensionality $p=2,3, \ldots, 8$ (the arrows indicate curves for $p=2$; convergence occurs for $p \geq 3$ ). . . .

3.5 FRF magnitude and coherence versus frequency and temperature $\left(G_{2}\right.$ transfer function): (a),(b) non-parametric Welch-based multi-model estimates; (c),(d) parametric $\operatorname{VARX}(23,23)$ based multi-model estimates; (e),(f) CCP-VARX $(23,23)$ estimates; (g),(h) FP-VARX $(23,23)_{[4,3]}$

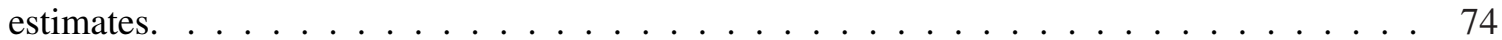

3.6 Dependence of indicative AutoRegressive and eXogenous parameters on temperature (point estimate $\pm 1.96 \times$ standard deviation; $a_{m, n}^{i}(k)$ and $b_{m, n}^{i}(k)$ designate the $(m, n)$-th elements of $\boldsymbol{A}_{i}(k)$ and $\boldsymbol{B}_{i}(k)$, respectively): parametric VARX $(23,23)$-based multi-model representation $(\nabla), \mathrm{CCP}-\operatorname{VARX}(23,23)$ representation $(-)$, and FP-VARX $(23,23)_{[4,3]}$ rep-

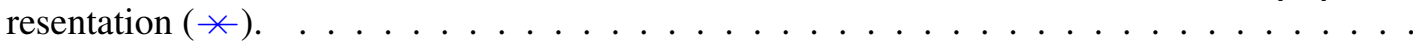

3.7 Dependence of the estimated natural frequencies $\left(f_{\mathrm{n}}\right)$ on temperature: non-parametric Welchbased multi-model representation $\left(-\circ-G_{1} ; \multimap-G_{2}\right)$, VARX $(23,23)$ multi-model representation $(-\nabla-), \mathrm{CCP}-\operatorname{VARX}(23,23)$ representation $(--)$, and FP-VARX $(23,23)_{[4,3]}$ rep-

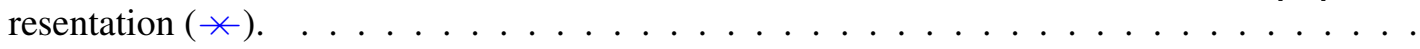

3.8 Dependence of the estimated damping factors $(\zeta)$ on temperature: non-parametric Welchbased multi-model representation $\left(-\circ-G_{1} ; \multimap-G_{2}\right)$, VARX $(23,23)$ multi-model representation $(-\nabla-)$, CCP-VARX $(23,23)$ representation $(--)$, and FP-VARX $(23,23)_{[4,3]}$ rep-

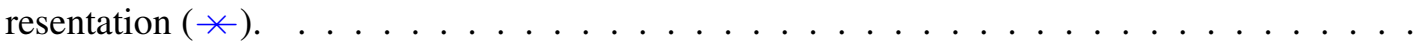

3.9 Natural frequency $\left(f_{\mathrm{n}}\right)$ estimates and their uncertainties ( \pm one standard deviation): (a) $\operatorname{VARX}(23,23)$ multi-model representation, (b) $\operatorname{FP}-\operatorname{VARX}(23,23)_{[4,3]}$ representation, and (c) CCP-VARX $(23,23)$ representation (the horizontal arrows indicate large uncertainty es-

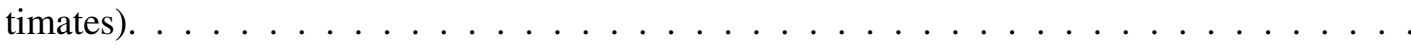

3.10 Damping factor $(\zeta)$ estimates and their uncertainties ( \pm one standard deviation): (a) VARX $(23,23)$ multi-model representation, (b) FP-VARX $(23,23)_{[4,3]}$ representation, and (c) CCP-VARX $(23,23)$ representation (the horizontal arrows indicate large uncertainty estimates) . . . . . . . . 79

4.1 Statistical hypothesis testing based upon a $\chi^{2}$-distributed statistic (one-tailed test; $\alpha=0.05$,

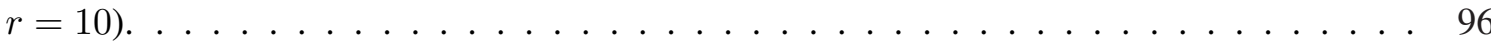

4.2 FP-VARMA $(1,1)_{[3,3]}$ model structure specification results $\left(s=6, \alpha=10^{-2}, 500\right.$ Monte Carlo runs): (a) basic CCA scheme for $\operatorname{FP}-\operatorname{VARMA}(n, n)$ model order specification; (b) refined CCA scheme for MA order reduction of FP-VARMA $(1,1)$ model; (c) FP-VARMA $(1,1)_{[p, p]}$ functional subspace dimensionality specification via the AIC/BIC criteria. . . . . . . . . . 99 
4.3 FP-VARMA $(1,1)_{[3,3]}$ actual values (--) and Monte Carlo estimates based upon the 2SLS and ML methods for selected projection coefficients and innovation variances (500 Monte Carlo runs per method; $a_{m, n}^{i, j}$ and $c_{m, n}^{i, j}$ designate the $(m, n)$-th elements of $\boldsymbol{A}_{i, j}$ and $\boldsymbol{C}_{i, j}$ respectively; $\sigma_{m, n}(k, \ell)$ designates the $(m, n)$-th element of $\boldsymbol{\Sigma}_{k, \ell}$; the dark green boxes indicate the sample mean \pm sample standard deviation; the light grey boxes indicate the sample mean \pm mean standard deviation computed through the asymptotic distribution).

4.4 Dependence of certain AutoRegressive and Moving Average parameters on the operating parameter $k$ (boxes indicate sample mean estimates $\pm 2 \times$ mean sample standard deviation computed through the asymptotic distribution): (-) actual values; (blue boxes) FP-VARMA $(1,1)_{[3,3]}$ model; (green boxes) conventional VARMA $(1,1)$ models (500 Monte Carlo runs per model). 101

4.5 FP-VARMA $(1,1)_{[3,3]}$ model based power spectral densities with respect to frequency and operating parameter $k$ : (a),(b) actual model, (c),(d) 2SLS mean estimate and, (e),(f) ML mean estimate. . . . . . . . . . . . . . . . . . . . . 116

4.6 FP-VARMA $(1,1)_{[3,3]}$ model based cross-spectral densities with respect to frequency and operating parameter $k$ : (a),(b) actual model, (c),(d) 2SLS mean estimate and, (e),(f) ML mean estimate. . . . . . . . . . . . . . . . . . . . . . . . . 117

4.7 Convergence metric versus number of iterations for the 2SLS estimator of the FP-VARMA $(1,1)_{[3,3]}$ model (the horizontal dashed line indicates the convergence criterion limit; the " $\times$ " mark designates the value of a single experiment; 500 Monte Carlo runs). . . . . . . . . . . . .

4.8 Projection of the function minimized in the ML method on selected FP-VARMA $(1,1)_{[3,3]}$ model parameters: (a),(c) surface and contour plots by projecting on $\alpha_{1,1}^{1,1}$ and $\alpha_{1,2}^{1,1}$; (b),(d) surface and contour plots by projecting on $a_{2,1}^{1,1}$ and $c_{2,1}^{1,1}$ (the " $\times$ " marks designate the actual

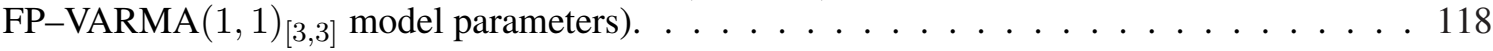

4.9 FP-VAR $(2)_{[4]}$ model based spectral densities with respect to frequency and operating pa-

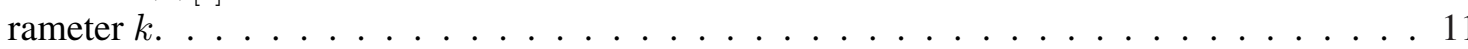

4.10 FP-VAR $(2)_{[4]}$ model structure specification results $\left(s=2, \alpha=10^{-3}, 500\right.$ Monte Carlo runs): (a) basic CCA scheme for $\operatorname{FP}-\operatorname{VARMA}(n, n)$ model order specification; (b) refined CCA scheme for MA order reduction of FP-VARMA $(2,2)$ model; (c) FP-VAR $(2)_{[p]}$ functional subspace dimensionality specification via the AIC/BIC criteria. . . . . . . . . . . . 120

4.11 The 4 d.o.f. lumped system with linear dependent stiffness and damping on the operating

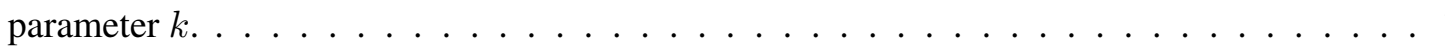

4.12 Indicative four d.o.f. system frequency response function magnitudes $G_{i j}$ with respect to frequency and operating parameter $k\left(G_{i j}\right.$ is the transfer function between the $i$-th response and the $j$-th excitation). . . . . . . . . . . . . . . . .

4.13 Model order specification results on the 4 d.o.f. system $\left(s=4, \alpha=10^{-3}, 500\right.$ Monte Carlo runs): (a),(c),(e) basic CCA scheme for $\operatorname{FP}-\operatorname{VARMA}(n, n)$ model order specification; (b),(d),(f) refined CCA scheme for MA order reduction of the $\operatorname{FP}-\operatorname{VARMA}(4,4)$ model. . . 121

5.1 (a) Photo of the experimental set-up; (b) schematic diagram of the experimental set-up; (c) schematic representation of the considered system. . . . . . . . . . . . 126

5.2 Schematic of the stochastic global model based approach to damage detection. . . . . . . . 127 
5.3 Welch-based cross-spectral density (CSD) magnitude estimates at the $M=21$ nominal temperatures belonging to the $[-20,+20]^{\circ} \mathrm{C}$ range: ( - dark blue zone) healthy structure, and ( - transparent light green zone) damaged structure (each zone comprises $M=21$ estimates, that is one CSD curve per each nominal temperature) . . . . . . . . . . . . . 135

5.4 FP-VAR $(n)_{[p]}$ structure specification (healthy beam): (a) Pooled $\operatorname{VAR}(n)$ model order specification via BIC; (b) FP-VAR $(88)_{[p]}$ subspace dimensionality specification via BIC (the arrows indicate the selected model order and subspace dimensionality) . . . . . . . . . . 136

5.5 Cross-spectral density (CSD) for the healthy beam versus frequency and temperature: (a),(b) Welch-based estimate; (c),(d) FP-VAR $(88)_{[7]}$-based estimate; (e),(f) absolute differences. . 137

5.6 Natural frequency $\left(f_{\mathrm{n}}\right)$ point estimates and their estimated uncertainties ( \pm two standard deviations) at the $M=21$ nominal temperatures: (a) baseline phase (healthy structure) $\operatorname{FP}-\operatorname{VAR}(88)_{[7]}$ estimates, (b) inspection phase (healthy and damaged structure) $\operatorname{VAR}(88)$ estimates (the uncertainties are designated by white boxes for the healthy structure and gray boxes for the damaged) . . . . . . . . . . . . . . . . . . . . . . . . . . . 138

5.7 Modal parameter-based damage detection results: $\chi_{\boldsymbol{\xi}}^{2}$ statistic for healthy (o) and damaged $(\square)$ test cases at different temperatures (the horizontal line designates the statistical limit at the $\alpha=10^{-3}$ risk level; damage is detected when $\chi_{\boldsymbol{\xi}}^{2}$ lies above the limit; $M_{1}=180$ healthy

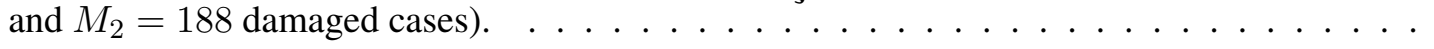

5.8 Relative proportion of variance explained $\left(\psi_{m}\right)$ for the transformed FP-VAR $(88)_{[7]}$ model parameter vector estimates versus vector dimension $(m)$ at the nominal temperatures (baseline phase, $M=21$ curves; the vertical dashed line indicates the selected dimensionality; the horizontal line indicates the threshold value of variance explained) . . . . . . . . . . . . 140

5.9 Model parameter-based damage detection results: $\chi_{\vartheta}^{2}$ statistic for healthy (o) and damaged ( $\square$ ) test cases at different temperatures; (a) without truncation, (b) truncation via "mean" transformation matrix, (c) truncation via "interpolated" transformation matrix (the shaded areas indicate where $\chi_{\vartheta}^{2}$ lies for the healthy and damaged test cases; $M_{1}=180$ healthy and $M_{2}=188$ damaged cases). . . . . . . . . . . . . . . . . . . . . 1

5.10 PCA-based method damage detection results: $X$-bar control chart for healthy (o) and damaged $(\square)$ test cases at different temperatures (the natural frequency covariance matrix is estimated from $M=21$ nominal temperature experiments; the horizontal lines indicate the $\alpha=10^{-3}$ risk level limits; damage is detected when $N I$ lies above the upper limit). . . . . .

5.11 FA-based method damage detection results: $T^{2}$ control chart for healthy $(\circ)$ and damaged ( $\square$ ) test cases at different temperatures (the factor model is identified from $M=21$ nominal temperature experiments; the horizontal line designates the statistical limit at the $\alpha=10^{-3}$ risk level; damage is detected when $T^{2}$ lies above the limit). . . . . . . . . . . . . . . . .

5.12 Welch-based power spectral density (PSD) estimates at the $M=21$ nominal temperatures belonging to the $[-20,+20]^{\circ} \mathrm{C}$ range: ( - dark blue zone) healthy structure, and ( - transparent light green zone) damaged structure (each zone comprises $M=21$ estimates, that is one CSD curve per each nominal temperature) . . . . . . . . . . . . . . . . 145

5.13 $\mathrm{P}-\operatorname{VAR}(n)$ frequency stabilization diagrams for three distinct nominal temperatures (only modes with damping factors $\zeta<3 \%$ are depicted; the arrows at the top indicate the identified modes; frequencies stabilize for $n \geq 60$; the horizontal dashed lines indicate the selected model order via the BIC criterion). . . . . . . . . . . . . . . . . . . . 146 
5.14 Convergence of the $\mathrm{FP}-\operatorname{VAR}(88)_{[p]}$ natural frequency $\left(f_{\mathrm{n}}\right)$ versus temperature curves for increasing functional subspace dimensionality $p=2,3, \ldots, 10$ (frequencies belonging to the $M=21$ nominal temperatures are displayed; the arrows indicate curves for $p=2,3$; convergence occurs for $p \geq 4) \ldots \ldots \ldots$. . . . . . . . . . . . . . . 147

5.15 Power spectral densities (PSDs) for the healthy beam versus frequency and temperature: (a),(c) Welch-based estimates; (b),(d) FP-VAR(88) ${ }_{[7]}$-based estimates. . . . . . . . . . . . . 148

5.16 Damping factor $(\zeta)$ point estimates and their estimated uncertainties ( \pm two standard deviations) at the $M=21$ nominal temperatures: (a) baseline phase (healthy structure) FP$\operatorname{VAR}(88)_{[7]}$ estimates, (b) inspection phase (healthy and damaged structure) $\operatorname{VAR}(88)$ estimates (the uncertainties are designated by white boxes for the healthy structure and gray boxes for the damaged. . . . . . . . . . . . . . . . . . . . . . . . . 149

5.17 Dependence of certain AutoRegressive parameters at the $M=21$ nominal temperatures (the boxes indicate point estimates \pm two standard deviations): (white) baseline phase FP$\operatorname{VAR}(88)_{[7]}$ estimates; (light green) inspection phase healthy $\operatorname{VAR}(88)$ estimates; (dark gray) inspection phase damaged $\operatorname{VAR}(88)$ estimates $\left(\vartheta_{i}(k)\right.$ designates the $i$-th element of the AR parameter vector $\boldsymbol{\vartheta}(k)) \ldots \ldots \ldots \ldots$. . . . . . . . . . . . . . . 150

5.18 Diagnostic checking for multicollinearity in the response data at the $M=21$ nominal temperatures: (a) condition numbers of response data zero-lag cross-covariance matrices, (b) condition numbers of residual covariance matrices; (blue) VAR(88) estimates; (green) FP$\operatorname{VAR}(88)_{[7]}$ estimates. . . . . . . . . . . . . . . . . . . .

6.1 Geometric interpretation of relationship between the parameter vectors obtained by the $\mathrm{P}-$ VARX (unrestricted) and FP-VARX (restricted) models in a three-dimensional space. The unrestricted $(3 \times 1)$ parameter vector $\vartheta$ belongs to the set $D_{\mathcal{M}_{P}}$, whereas the $(2 \times 1)$ projection coefficient vector $\boldsymbol{\theta}$ belongs to $D_{\mathcal{M}_{F P}}$. The dark gray area denotes the intersection of $D_{\mathcal{M}_{P}}$ and the column space of $\boldsymbol{T}$ where the restricted parameter vectors $\boldsymbol{\vartheta}^{*}=\boldsymbol{T} \boldsymbol{\theta}=\boldsymbol{g}(\boldsymbol{\theta})$ lie.165

6.2 Dependence of certain AutoRegressive and eXogenous parameters on the operating condition $k$ (boxes indicate sample mean $\pm 2 \times$ sample standard deviation): (-) theoretical values; (filled green boxes) CCP-VARX $(3,1)$ model; (thick lined blue boxes) FP-VARX $(3,1)_{[4,4]}$ model; (thin lined red boxes) P-VARX $(3,1)$ model (500 Monte Carlo runs per model; $a_{m, n}^{i}(k)$ and $b_{m, n}^{i}(k)$ designate the $(m, n)$-th elements of $\boldsymbol{A}_{i}(k)$ and $\boldsymbol{B}_{i}(k)$, respectively). . 179

6.3 Dependence of certain AutoRegressive parameters on the operating condition $k$ (boxes indicate sample mean $\pm 2 \times$ sample standard deviation): $(-)$ theoretical values; (filled green boxes) CCP-VAR(2) model; (thick lined blue boxes) FP-VAR $(2)_{[4]}$ model; (thin lined red boxes) P-VAR (2) model (500 Monte Carlo runs per model; $a_{m, n}^{i}(k)$ designates the $(m, n)$-th element of $\left.\boldsymbol{A}_{i}(k)\right) \ldots \ldots \ldots \ldots \ldots \ldots \ldots$ 


\section{List of Tables}

2.1 Overview of innovation dependency types. $\ldots \ldots \ldots \ldots \ldots \ldots \ldots$

2.2 Overview of asymptotic properties for the LS and ML type FP-VARX parameter estimators. 23

2.3 Details on the Monte Carlo study for FP-VARX $(2,0)_{[3,3]}$ model identification. . . . . . . 27

2.4 Details on the Monte Carlo study for FP-VARX $(3,1)_{[4,4]}$ model identification. . . . . . . 30

2.5 Monte Carlo estimation results for the AutoRegressive and eXogenous projection coefficients of the $\operatorname{FP}-\operatorname{VARX}(2,0)_{[3,3]}$ model (500 runs per method; sample mean estimate \pm sample standard deviation) . . . . . . . . . . . . . . . . 55

2.6 Monte Carlo estimation results for innovations variance of the $\operatorname{FP}-\operatorname{VARX}(2,0)_{[3,3]}$ model (500 runs per method; sample mean estimate \pm sample standard deviation) . . . . . . . . 56

2.7 Monte Carlo estimation results for the AutoRegressive projection coefficients of the FP$\operatorname{VARX}(3,1)_{[4,4]}$ model (500 runs per method; sample mean estimate \pm sample standard

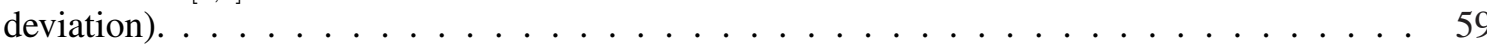

2.8 Monte Carlo estimation results for the eXogenous projection coefficients of the FP-VARX $(3,1)_{[4,4]}$ model (500 runs per method; mean estimate \pm standard deviation) . . . . . . . . . . 60

2.9 Monte Carlo estimation results for innovations variance of the $\operatorname{FP}-\operatorname{VARX}(3,1)_{[4,4]}$ model (500 runs per method; sample mean estimate \pm sample standard deviation). . . . . . . . . 61

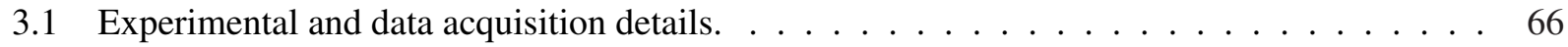

3.2 Conventional multi-model and global model identification approaches and estimation details.

3.3 Natural frequency $\left(f_{\mathrm{n}}\right)$ estimates and their uncertainties ( \pm one standard deviation) for the VARX-based multi-model and FP-VARX approaches. . . . . . . . . . . . . . . 8

4.1 Details on the Monte Carlo study for FP-VARMA $(1,1)_{[3,3]}$ model identification. . . . . . . 99

4.2 Indicative basic CCA scheme for overall AR/MA order determination of an FP-VARMA $(2,1)$

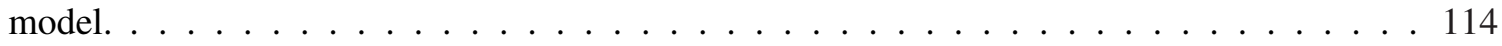

4.3 Indicative refined CCA scheme for possible MA order reduction of an FP-VARMA $(2,1)$ model. . . . . . . . . . . . . . . . . . . . . . 114

4.4 Monte Carlo estimation results for the AutoRegressive projection coefficients of the FP$\operatorname{VARMA}(1,1)_{[3,3]}$ model (500 runs per method; sample mean estimate \pm sample standard deviation). 
4.5 Monte Carlo estimation results for the Moving Average projection coefficients of the FP$\operatorname{VARMA}(1,1)_{[3,3]}$ model (500 runs per method; sample mean estimate \pm sample standard deviation) . . . . . . . . . . . . . . . . . . . . . 115

4.6 Indicative FP-VARMA $(1,1)_{[3,3]}$ noise variance estimation results from Monte Carlo simulations (500 runs per method; sample mean estimate \pm sample standard deviation). . . . . 116

4.7 Details on the Monte Carlo study for FP-VAR $(2)_{[4]}$ model identification. . . . . . . . . . . 119

4.8 Details on the Monte Carlo study for the 4 d.o.f. mass-spring-damper system. . . . . . . 120

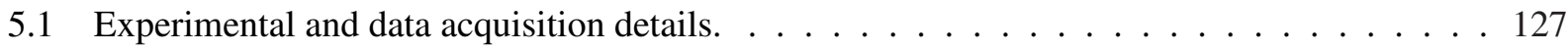

5.2 Summary of the two versions of the damage detection approach. . . . . . . . . . . 131

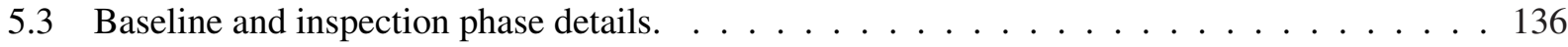

6.1 Overview of asymptotic properties for the LS and ML type P-VARX parameter estimators. 162

6.2 Overview of asymptotic properties for the LS and ML type CCP-VARX parameter estimators. . . . . . . . . . . . . . . . . . . . . . . . . 173

6.3 Details on the Monte Carlo study for $\operatorname{FP}-\operatorname{VARX}(3,1)_{[4,4]}$ model identification. . . . . . . 178

6.4 Details on the Monte Carlo study for FP-VAR $(2)_{[4]}$ model identification. . . . . . . . . . . 180 


\section{Vita}

John D. Hios received his Diploma from the Department of Mechanical Engineering \& Aeronautics at the University of Patras, Greece, in 2002. He is currently a Ph.D student in the same department, at the Stochastic Mechanical Systems and Automation (SMSA) Laboratory.

\section{RESEARCH INTERESTS}

Linear/nonlinear stochastic system identification, stochastic control, fault detection and isolation (FDI) in mechanical \& aerospace systems via statistical methods, and modal analysis.

\section{WORK EXPERIENCE}

Nov. 2010 - present Ilida Consulting Engineers

Stravonos 3 St, GR 14234 Nea Ionia Athens, Greece

External Associate

Conducting energy audits and electromechanical designs for road-lighting, outdoor lighting, and building services for public works

Preparation of proposals for public works and documentation of procurement services

\section{RESEARCH EXPERIENCE}

\section{Oct. 2004 - Jun. 2007 Project: Innovative Future Air Transport System (IFATS)}

Department of Mechanical Engineering \& Aeronautics, University of Patras

Research Associate

Contributed to the development of (i) fault detection and identification (FDI) schemes for actuators, (ii) FDI schemes for autonomous aircraft engines, and (iii) control algorithms with application to aircraft systems

Sep. 2002 - Jan. 2004 Project: Affordable Digital Flight Control System II (ADFCS - II)

Department of Mechanical Engineering \& Aeronautics, University of Patras Research Associate

Contributed to the development of (i) virtual sensors, and (ii) pilot monitoring methods for small commercial aircrafts

\section{TEACHING EXPERIENCE}

Oct. 2002

Jan. 2005 - Dec. 2005
Department of Mechanical Engineering \& Aeronautics, University of Patras Teaching Assistant

Conducted laboratory exercises for the "Systems \& Automation" undergraduate course $\left(7^{\text {th }}\right.$ semester) 


\section{PublicATIONS}

\section{Journal Papers}

1. D.G. Dimogianopoulos, J.D. Hios and S.D. Fassois, "Aircraft Engine Health Management via Stochastic Modelling of Flight Data Interrelations", Aerospace Science and Technology, vol. 16, pp. 70-81, 2012.

2. J.D. Hios and S.D. Fassois, "Stochastic Identification of Temperature Effects on the Dynamics of a Smart Composite Beam: Assessment of Multi-Model and Global Model Approaches", Smart Materials and Structures, vol. 18035011 (15pp), 2009. doi:10.1088/0964-1726/18/3/035011.

3. D.G. Dimogianopoulos, J.D. Hios and S.D. Fassois, "FDI for Aircraft Systems Using Stochastic Pooled NARMAX Representations: Design and Assessment", IEEE Transactions on Control Systems Technology, vol. 17, no. 6, 2009.

4. D.G. Dimogianopoulos, J.D. Hios and S.D. Fassois, "Aircraft Fault Detection and Identification by Stochastic Functionally Pooled Modelling of Relationships Among Attitude Data", Proceedings of the Institution of Mechanical Engineers, Part G, Journal of Aerospace Engineering, vol. 222, no. 6, pp. 801-816, 2008 (invited paper).

5. D.G Dimogianopoulos, J.D. Hios and S. D. Fassois, "Integral Minimum Variance-Like Control for Pooled Nonlinear Representations with Application to an Aircraft System", International Journal of Control, vol. 80, no. 5, pp. 721-730, 2007.

6. D.G Dimogianopoulos, J.D. Hios and S. D. Fassois, "On-board Statistical Detection and Compensation of Anomalous Pilot Aircraft Interactions", IEEE Transactions on Aerospace and Electronic Systems, vol. 43, no. 4, pp. 1385-1396, 2007.

\section{Conference Papers}

7. D.G. Dimogiannopoulos, J.D. Hios and S.D. Fassois, "Onboard Aircraft Engine FDI via Stochastic Nonlinear Modeling of Flight Variable Interrelations", in Proceedings of the International Unmanned Vehicles Workshop (UVW 2010), Istanbul, Turkey, June 10-12, 2010, Paper 64.

8. A.M. Lekkas, J.D. Hios and S.D. Fassois, "Output-Only Damage Detection in a Composite Beam Under Varying Temperatures via Vector Stochastic Models", in Proceedings of the $5^{\text {th }}$ European Workshop on Structural Health Monitoring (EWSHM 2010), Sorrento, Italy, June 28 - July 2, 2010, pp. 815-822.

9. J.D. Hios and S.D. Fassois, "Statistical Damage Detection in a Smart Structure Under Different Temperatures via Vibration Testing: A Global Model Based Approach", in Proceedings of the $8^{\text {th }}$ International Conference on Damage Assessment of Structures (DAMAS 2009), Beijing, China, August 3-5, 2009, Paper 042. Also in Key Engineering Materials, vol. 413-414, 2009, pp. 261-268.

10. J.D. Hios and S.D. Fassois, "Stochastic Identification Under Multiple Operating Conditions: Functionally Pooled VARMA Methods", in Proceedings of the $15^{\text {th }}$ IFAC Symposium on System Identification (SYSID 2009), Saint-Malo, France, July 6-8, 2009, pp. 1626-1631.

11. J.D. Hios and S.D. Fassois, "Stochastic Identification of Temperature Effects on the Dynamics of a Smart Composite Beam: Assessment of Multi-Model and Global Approaches", in Proceedings of the International Conference on Noise and Vibration Engineering (ISMA 2008), Leuven, Belgium, September 15-17, 2008, CD-ROM; Paper ID 131.

12. J.D. Hios and S.D. Fassois, "Stochastic Vector Identification and Uncertain Modal Parameter Estimation for a Smart Composite Beam", in Proceedings of the $3^{\text {rd }}$ International Conference Smart Materials Structures Systems (CIMTEC 2008), Acireale, Italy, June 8-13, 2008. Also in Advances in Science 
and Technology (Emboding Intelligence in Structures and Integrated Systems), vol. 56, pp. 453-458, 2008.

13. D.G. Dimogianopoulos, J.D. Hios and S.D. Fassois, "On-Board Engine FDI in Autonomous Aircrafts Using Compact Stochastic Nonlinear Modelling of Flight Signal Dependencies", in Proceedings of the European Control Conference (ECC'07), Kos, Greece, July 2-5, 2007, pp. 3422-3429.

14. J.D. Hios and S.D. Fassois, "Identification of a Global Model Describing the Temperature Effects on the Dyanmics of a Smart Composite Beam", in Proceedings of the International Conference on Noise and Vibration Engineering (ISMA 2006), Leuven, Belgium, September 18-20, 2006, CD-ROM; Paper ID 230 .

15. D.G Dimogianopoulos, J.D. Hios and S.D. Fassois, "Statistical Fault Detection and Identification in Aircraft Systems via Functionally Pooled Nonlinear Modelling of Flight Data Dependencies", in Proceedings of the $25^{\text {th }}$ International Congress of the Aeronautical Sciences (ICAS 2006), Hamburg, Germany, September 3-8, 2006.

16. D.G Dimogianopoulos, J.D. Hios and S.D. Fassois, "Fault Detection and Isolation in Aircraft Systems Using Stochastic Nonlinear Modelling of Flight Data Dependencies", in Proceedings of the $14^{\text {th }}$ Mediterranean Conference on Control and Automation (MED'06), Ancona, Italy, June 28-30, 2006.

17. D.G Dimogianopoulos, J.D. Hios and S.D. Fassois, "Nonlinear Integral Minimum Variance-Like Control with Application to an Aircraft System", in Proceedings of the $14^{\text {th }}$ Mediterranean Conference on Control and Automation (MED'06), Ancona, Italy, June 28-30, 2006.

18. D.G Dimogianopoulos, J.D. Hios and S.D. Fassois, "On-board Statistical Detection and Control of Anomalous Pilot Aircraft Interactions", in Proceedings of the $16^{\text {th }}$ IFAC World Congress, Prague, Czech Republic, July 4-8, 2005. 